\title{
The Calculation of Sparticle and Higgs Decays in the Minimal and Next-to-Minimal Supersymmetric Standard Models: SOFTSUSY4 0
}

\author{
B.C. Allanach ${ }^{\mathrm{a}}$, T. Cridge
}

${ }^{a}$ DAMTP, CMS, University of Cambridge, Wilberforce road, Cambridge, CB3 OWA, United Kingdom

\begin{abstract}
We describe a major extension of the SOFTSUSY spectrum calculator to include the calculation of the decays, branching ratios and lifetimes of sparticles into lighter sparticles, covering the next-to-minimal supersymmetric standard model (NMSSM) as well as the minimal supersymmetric standard model (MSSM). This document acts as a manual for the new version of SOFTSUSY, which includes the calculation of sparticle decays. We present a comprehensive collection of explicit expressions used by the program for the various partial widths of the different decay modes in the appendix.
\end{abstract}

Keywords: MSSM, NMSSM, branching ratio, lifetime

PACS: $12.60 . \mathrm{Jv}$

PACS: 14.80.Ly

\section{Program Summary}

Program title: SOFTSUSY

Program obtainable from: http://softsusy.hepforge.org/

Distribution format: tar.gz

Programming language: $\mathrm{C}++$, fortran

Computer: Personal computer.

Operating system: Tested on Linux 4.4.0-36-generic, Linux 3.13.0-93-generic

Word size: 64 bits.

External routines: None

Typical running time: $0.1-1$ seconds per parameter point.

Nature of problem: Calculating supersymmetric particle partial decay widths in the MSSM or the NMSSM, given the parameters and spectrum which has already been calculated by SOFTSUSY.

Solution method: Analytic expressions for tree-level 2 body decays and loop-level decays and one-dimensional numerical integration for 3 body decays.

Restrictions: Decays are calculated in the real $R$-parity conserving MSSM or the real $R$-parity conserving NMSSM only. No additional charge-parity violation (CPV) relative to the Standard Model (SM). Sfermion mixing has only been accounted for in the third generation of sfermions in the decay calculation. Decays in the MSSM are 2-body and 3-body, whereas decays in the NMSSM are 2-body only.

CPC Classification: 11.1 and 11.6.

Does the new version supersede the previous version?: Yes.

Reasons for the new version: Significantly extended functionality. The decay rates and branching ratios of sparticles are particularly useful for collider searches. Decays calculated in the NMSSM will be a particularly useful check of

\footnotetext{
${ }^{*}$ Corresponding author

Email address: t.cridge@damtp.cam.ac.uk (T. Cridge)
} 
the other programs in the literature, of which there are few.

Summary of revisions: Addition of the calculation of sparticle and Higgs decays. All 2-body and important 3-body tree-level decays, including phenomenologically important loop-level decays (notably, Higgs decays to $g g, \gamma \gamma$ and $Z \gamma$ ). Next-to-leading order corrections are added to neutral Higgs decays to $q \bar{q}$ for quarks $q$ of any flavour and to the neutral Higgs decays to $g g$.

\section{Introduction}

The phenomenology of simple supersymmetric extensions of the Standard Model has become something of an industry in particle physics of late. The potential of such models to explain the technical hierarchy problem (i.e. the relative smallness of the Higgs mass as compared to the Planck or gauge unification scale) has motivated its study. The models have potential implications for cosmology as well as for collider physics. Several different computational tools are necessary for the study of the models' phenomenology [1]. The initial step is a calculation of the supersymmetric spectrum, as well as particle couplings. This is the step that the computer program SOFTSUSY has previously performed, in the $R$-parity conserving MSSM [2], the $R$-parity conserving NMSSM [3] and the $R$-parity violating MSSM [4]. By adding one-loop corrections to neutrino masses, the program has been extended [5] to calculate neutrino masses and mixings in the presence of lepton number violating $R$-parity violation. Further non-trivial additions of higher order corrections result in increased precision for the gauge and Yukawa couplings [6] and the squark and gluino pole masses [7]. Other publicly available computer programs exist which perform the task of computing the supersymmetric spectrum: for the MSSM, there is FLEXIBLESUSY [8], ISASUSY [9], SUSEFLAV, SUSPECT [10] and sPHENO [11], whilst FEYNHIGGS can calculate the higgs masses [12]. In the NMSSM, there is only one stand-alone dedicated tool for spectrum calculation: NMSSMTools [13, 14], whereas the SARAH [15] framework can be combined with FLEXIBLESUSY or SPHENO in order to calculate the spectrum. Meanwhile, NMSSMCALC [16] can be used for the computation of the Higgs masses and decays in the NMSSM. The plethora of computer programs is useful: some of them use different approximations and extend to different models. Even the programs having the same apparent approximations differ in their numerical output because the higher order corrections not included are implicitly different: both for the sparticle spectrum [17] and the Higgs masses [18, 19, 20]. Thus, the size of differences between the programs for observables calculated at the same order or approximation serve as an rough estimate of the size of higher order corrections. In some cases, different approximations are used and these can help investigate different régimes of parameter space. For example, one can deal with sparticle threshold effects differently: either at fixed order at say $M_{Z}$ using the MSSM or NMSSM as an effective field theory above $M_{Z}$ (this is the approach taken by SOFTSUSY, sPHENO and SUSPECT for instance), or one could integrate the sparticles out in the renormalisation group equations at some higher scale (the effective field theory approach taken by ISAJET and NMSSMTools). The former approach includes finite terms of order $M_{Z}^{2} /\left(16 \pi^{2} M_{p}^{2}\right)$, where $p$ is the mass of some sparticle, but takes the sparticle mass splittings $\Delta M_{p}$ into account at some level approximation, typically $\sim O\left(\log \left[\Delta M_{p}^{2}\right] /\left(16 \pi^{2}\right)\right)$, whereas the latter approach often misses the finite terms but re-sums the mass splitting terms. One generically expects the former approach to be more accurate when sparticles are not too heavy, and the latter when the sparticles are very heavy and when the splittings between them are large. Which one is more accurate given current lower bounds upon sparticle masses is a quantitative question that is observable dependent.

In order to provide predictions for future sparticle and Higgs searches at colliders, or indeed in order to interpret searches at them in terms of the MSSM and NMSSM, cross-section estimates as well as simulations of collisions are required, in order to estimate acceptances and efficiencies. For the collision simulations, estimates of the various decay partial widths for the sparticles and Higgs particles are required. Some Monte-Carlo event generators perform this task in the MSSM, for example PYTHIA [21] or HERWIG7 [22], but there also exist dedicated tools like SUSYHIT [23] (a combination of HDECAY [24] and SDECAY [25]) and FeynHiggs [12] (the latter specialising in the Higgs boson decays). SPHENO also contains a decay calculation for the MSSM. In the NMSSM however, the options for calculating sparticle and Higgs decays are rather slim: NMSSMTools is the only stand-alone option, whereas SARAF 1 can be combined with sPHENO. For calculating Higgs decays in the NMSSM (including CP violation) though, one can use NMSSMCALC [16],

\footnotetext{
${ }^{1}$ After submission of the present paper to the electronic arXiv, a new calculation in the SARAH framework was presented for the generic calculation of two-body partial decay widths at the full one-loop level [26]
} 
which calculates Higgs decay widths and branching ratios including some dominant QCD loop corrections. Previous versions of SOFTSUSY contained an interface to NMSSMTools so that the NMSSM spectrum and couplings could then be fed into the program in order to predict sparticle and Higgs boson partial decay widths.

The present paper describes a significant extension in functionality in SOFTSUSY: to calculate and output the various partial widths for the decays of sparticles and Higgs bosons in the MSSM and in the NMSSM. Emphasis has been placed on speed of execution, preferring to perform as much of the calculation analytically as is practicable. We hope that this addition of functionality to SOFTSUSY will facilitate collider studies of sparticle and supersymmetric Higgs searches: both through the study of differences with the other programs as an estimate of the size of theoretical uncertainty in the prediction, and through a fast and unified computation.

The rest of the paper is organised as follows: in Section 3. we specify the conventions used in this paper and the assumptions made in the decay calculator and we provide some details about the method of computation. Following this, in section 4, we list the decay modes included in the program. Next, in section 5, we provide a few examples of comparison tests with a couple of other publicly available tools for the MSSM and the NMSSM, before summarising in Section 6. We show how to run the program and provide explicit flags for controlling its behaviour in Appendix A. providing some sample output in Appendix B. We provide explicit formulae for the partial widths in Appendix D to Appendix J, these have not been collected together in one reference before.

\section{Conventions, Assumptions and Method}

Throughout $\tilde{Z}_{i}$ and $\tilde{W}_{j}$ are used for neutralinos $(i=1,2,3,4$ in the MSSM or $i=1,2,3,4,5$ in the NMSSM) and charginos $(j=1,2)$, respectively. This is different to the commonly used $\tilde{\chi}_{i}^{0}$ and $\tilde{\chi}_{j}^{+}$notation for ease of reading, particularly when they appear in subscripts. The notation for the mass-ordered CP even and CP odd neutral Higgs bosons is that $h_{i} \in\{h, H, H 3\}$ for $i=1,2,3$ are the CP even neutral Higgs bosons in order of increasing mass, whilst $A_{i} \in\{A, A 2\}$ for $i=1,2$ are the $\mathrm{CP}$ odd neutral Higgs bosons again in order of increasing mass, remembering that $H 3$ and $A 2$ occur only in the NMSSM.

The partial width formulae for all of the decay modes included in the SOFTSUSY decay calculation ${ }^{2}$ are listed in Appendix D onwards, many of these were rederived and have been written in one consistent set of conventions.

\subsection{MSSM}

While the conventions used in the decays code are largely those used in SOFTSUSY [2], there are differences in a few places in order to allow easier comparison with partial width (PW) formulae provided elsewhere. The few differences with respect to Ref. [2] are as listed below ${ }^{3}$

- In our calculations, it is convenient to work in a basis where the third generation sfermions are mass ordered with $m_{\tilde{f}_{1}}<m_{\tilde{f}_{2}}$. In order to ensure this, the mixing angle $\theta_{f}$ is transformed accordingly $\left(\theta_{f} \rightarrow \theta_{f}+\pi / 2\right)$ in the case where the SOFTSUSY spectrum generator has $m_{\tilde{f}_{1}}>m_{\tilde{f}_{2}}$.

- The mixing angles for the charginos are transformed with respect to the SOFTSUSY spectrum generator in order to match conventions used elsewhere (e.g. [10]). Therefore $\theta_{L / R}$ as indicated below is given by $\theta_{L / R}^{\text {decays }}=$ $-\theta_{L / R}^{\text {spectrum }}+\pi / 2$.

\subsection{NMSSM}

The conventions used in the decay code are predominantly those described previously in the SOFTSUSY NMSSM manual [3], but there are differences in a few places. As well as those listed above, there are a few changes specific to the NMSSM, to allow straightforward comparison with NMSSMTools [13, 14, 27, 28]:

\footnotetext{
${ }^{2}$ The source code for the calculations is in the file src/decays.cpp, which is in the $\mathrm{C++}$ programming language.

${ }^{3}$ In the decay code itself, the neutralino mixing matrix used ( $N$ in SOFTSUSY notation) is transposed.
} 
- The Charge Parity (CP) even neutral Higgs mixing matrix is altered relative to the matrix $R$ provided by SOFTSUSY [3]. The matrix $S$ used in the decay formulae is obtained via an orthogonal transformation exchanging eigenstates:

$$
S=R\left(\begin{array}{lll}
0 & 1 & 0 \\
1 & 0 & 0 \\
0 & 0 & 1
\end{array}\right)=\left(\begin{array}{lll}
R(1,2) & R(1,1) & R(1,3) \\
R(2,2) & R(2,1) & R(2,3) \\
R(3,2) & R(3,1) & R(3,3)
\end{array}\right)
$$

i.e. the first two columns are interchanged

- The CP odd neutral Higgs mixing matrix is altered relative to the matrix provided by SOFTSUSY [3], the matrix $P$ detailed in the decay formulae (different to the $P$ in Ref. [3] which we write here as $P^{\text {prov }}$ ) is given below. The differences are that the first row of $P^{\text {prov }}$ is dropped (as this refers to the goldstone boson) and the first and second columns are interchanged. The ratio of the Higgs vacuum expectation values (vevs) $\beta$ and the mixing angle $\theta_{A}$ are as used elsewhere in SOFTSUSY [2, 3]:

$$
P=\left(\begin{array}{ccc}
P^{\text {prov }}(2,2) & P^{\text {prov }}(2,1) & P^{\text {prov }}(2,3) \\
P^{\text {prov }}(3,2) & P^{\text {prov }}(3,1) & P^{\text {prov }}(3,3) \\
0 & 0 & 0
\end{array}\right)=\left(\begin{array}{ccc}
\cos \beta \cos \theta_{A} & \sin \beta \cos \theta_{A} & \sin \theta_{A} \\
\cos \beta \sin \theta_{A} & \sin \beta \sin \theta_{A} & -\cos \theta_{A} \\
0 & 0 & 0
\end{array}\right)
$$

\subsection{Mass Choices and Scales Used}

The input parameters from the SOFTSUSY spectrum generator [2, 3] can be evaluated at a variety of different scales. The choice of the renormalisation scale used is an important consideration and can result in differences between decay calculator predictions. Different scales effectively correspond to including different higher order terms in the calculation. For example, consider the decay of a gluino into a top and a stop. One must choose a renormalisation scale for the coupling. The masses of the particles involved could be running masses evaluated at different scales, or pole masses. Each choice affects the numerical value of the PW, but are all equivalent at tree-level. In SOFTSUSY the following choices are made:

- In general, unless explicitly stated otherwise, the masses of the supersymmetric (SUSY) and Higgs particles and other parameters, such as mixing angles and gauge couplings, are evaluated at the scale $M_{S U S Y}=$ $x \sqrt{m_{\tilde{t}_{1}}\left(M_{S U S Y}\right) m_{\tilde{t}_{2}}\left(M_{S U S Y}\right)}$, where $\mathrm{x}$ can be set by the user but by default is taken to be 1 . Here, $m_{\tilde{t}_{i}}\left(M_{S U S Y}\right)$ is the running $i^{\text {th }}$ stop mass evaluated at a modified dimensional reduction [29] $(\overline{D R})$ renormalisation scale $M_{S U S Y}$.

- For Higgs loop decays the gauge coupling strengths $\alpha_{s}$ and $\alpha$ are evaluated at the mass of the decaying Higgs.

- For Higgs loop decays to $\gamma \gamma$ or $Z \gamma$ the masses of the important quarks (i.e. $m_{t}, m_{b}, m_{c}$ ) are evaluated at the mass of the decaying Higgs. Below $M_{Z}$, these are run in 3 loop QCD and 1 loop in QED. In the calculation of decays of $H, H 3, A$ and $A 2$ quark masses are run to $m_{H}, m_{H 3}, m_{A}, m_{A 2}$ in the (N)MSSM as appropriate, whilst for the lightest CP even Higgs $h$ the $m_{t}, m_{b}$ and $m_{c}$ are run to $m_{h}$ in 1 loop QED and 3 loop QCD.

- Throughout the program, unless otherwise stated here, we generally use two different quark masses; "kinematic masses" for the kinematics (i.e. for masses of particles in the initial or final states) and "running masses" for the evaluation of couplings. This hopefully allows a large part of some higher order corrections to be incorporated into the quark legs via the mass running. The way in which these masses are evaluated is listed in Table 1 below.

- In addition to the above quark masses, there are extra masses mcpole and mspole defined in decays.h which are set to particular values used in the QCD corrections to neutral Higgs boson decays to $q \bar{q}$ or $g g$.

- If the QCD corrections to these decays are turned off in the program then the running masses for the quarks are used in order to attempt to hopefully incorporate some of the Next-to-Leading-Order (NLO) corrections to the quark legs. 


\begin{tabular}{|c|c|c|c|}
\hline \multicolumn{2}{|c|}{ kinematic masses } & \multicolumn{2}{c|}{ running (coupling) masses } \\
\hline mtPole & pole mass from propagator & runmt & $\overline{D R}$ mass at $M_{Z}$ \\
\hline mbPole & pole mass from propagator & runmb & $\overline{D R}$ mass at $M_{Z}$ \\
\hline mtauPole & pole mass from propagator & runmtau & $\overline{D R}$ mass at $M_{Z}$ \\
\hline $\mathrm{mc}$ & $\overline{M S}$ mass at $M_{Z}$ & runmc & Yukawa-extracted mass at $M_{Z}$ \\
\hline $\mathrm{ms}$ & $\overline{M S}$ mass at $M_{Z}$ & runms & Yukawa-extracted mass at $M_{Z}$ \\
\hline $\mathrm{mup}$ & $\overline{M S}$ mass at $M_{Z}$ & runmu & Yukawa-extracted mass at $M_{Z}$ \\
\hline $\mathrm{mdo}$ & $\overline{M S}$ mass at $M_{Z}$ & runmd & Yukawa-extracted mass at $M_{Z}$ \\
\hline $\mathrm{mel}$ & $\overline{M S}$ mass at $M_{Z}$ & runmel & Yukawa-extracted mass at $M_{Z}$ \\
\hline $\mathrm{mmu}$ & $\overline{M S}$ mass at $M_{Z}$ & runmmu & Yukawa-extracted mass at $M_{Z}$ \\
\hline polemw & pole mass from propagator & runmw & running $W$ mass at $M_{S U S Y}$ \\
\hline polemz & pole mass from propagator & runmz & running $Z$ mass at $M_{S U S Y}$ \\
\hline
\end{tabular}

Table 1: The two different types of masses used for the fermions and gauge bosons. The names given are those used in the code (src/decays . cpp). "kinematic" masses are used for the masses of initial and final state particles in the decay formulae whilst "running (coupling)" masses are used where masses appear in expressions involving couplings in the partial width formulae. Note that within SOFTSUSY, the $\overline{M S}$ masses include only SM corrections whilst the Yukawa-extracted masses ( $\overline{D R}$ masses) include SM and SUSY corrections.

As detailed in Table 1, for the third generation sfermions the "kinematic" masses are pole masses obtained from the propagators whilst the "running (coupling)" masses are in the $\overline{D R}$ scheme. For the "kinematic" masses of the first two generation fermions, the $\overline{M S}$ mass at $M_{Z}$ is used, whilst the "running" masses are extracted from the running Yukawa couplings. For the electron and muon, the running is again small (only QED). The "kinematic" masses for the vector bosons are pole masses, whilst for the "running(coupling)" masses they are running $\overline{D R}$ masses evaluated at $M_{S U S Y}$.

- $\overline{M S}$ masses include only SM corrections within SOFTSUSY; 3-loop QCD and 1-loop QED corrections are included.

- Yukawa-extracted masses are in the $\overline{D R}$ scheme and include SM and SUSY corrections.

- Quark input masses can be reset by the user within the SMINPUTS block of the SLHA/SLHA2 input file. The kinematic and running masses used will then change accordingly.

The different choices of scales for the input parameters is one of the key sources of differences between different decay calculator programmes. It is worth noting that an experimental value of Fermi's constant, $G_{F}$, is also used, this is inconsistent with the tree-level expression $\frac{G_{F}}{\sqrt{2}}=\frac{g^{2}}{8 m_{W}^{2}}$ as it is an empirical quantity and so incorporates higher order terms.

\subsection{Assumptions Made}

The following assumptions are made in the decay calculator:

- $R$-parity conservation in the MSSM and in the NMSSM.

- No additional CP violation relative to the SM.

- No additional flavour violation relative to the SM.

- Sfermion mixing has only been accounted for in the third generation of sfermions as it is proportional to the Yukawa couplings, which are negligible for the first two generations. 


\subsection{Method}

In our description in this section and the rest of the paper, we classify decay modes according to both the number of daughter particles (with $N$ body meaning $N$ decay products) and the order of the corrections included; i.e. tree-level, 1-loop or 2-loop.

For 2 body tree-level decay modes, the analytical expressions for the partial widths are explicitly used in order to provide fast evaluation. Similarly, for the 2 body 1-loop decays the loop integrals were performed analytically and the resulting formulae used. For 3 body decay modes (all tree-level), the phase space integral has been analytically reduced to a one-dimensional integral, which is then performed using adaptive Gaussian numerical integration [30]. The exception to this is the neutral CP even lightest Higgs decays to a vector boson and an off-shell vector boson, which then decays into a fermion anti-fermion pair, this calculation was performed explicitly and all integrals were evaluated analytically.

The tree-level 3 body decay modes were therefore where most complications arose. In general for an $N$-body tree-level decay there are $N$ integrals to perform, one over the three-momenta of each of the final state particles. One of these integrals is always trivial to perform using the momentum-conserving delta function. For the 2 body tree-level decay widths this leaves one remaining integral with the energy delta function, this can then be performed using the standard result for the integral of a function of a variable multiplied by a delta function containing another function of the same variable. For tree-level 3 body decay widths however, one has two remaining integrals to perform and in general they are non-trivial to determine analytically. In certain cases the symmetry of the integrands, along with certain assumptions, may allow them to be performed. For example, in the cases of the SM-like neutral CP even higgs decays to a vector boson and fermion anti-fermion pair via an off-shell vector boson, $h \rightarrow W W^{*} \rightarrow W f^{\prime} \bar{f}$ and $h \rightarrow Z Z^{*} \rightarrow Z f \bar{f}$, the mass of the Higgs boson ensures that the outgoing fermions may not be top quarks. Therefore one can neglect the masses of the outgoing fermions and greatly simplify the calculation. Passarino-Veltman reduction [31] can then allow reduction of the integrals to a one-dimensional integral, which in this case may be determined explicitly analytically; the result given in both Appendix F.6 and in the "Higgs Hunter's Guide" [32]. For a general 3 body decay mode the calculations are however considerably more involved. There are two approaches that can be taken once the first trivial integral using the momentum-conserving delta function is performed; at this stage the partial width can be written as a double differential decay rate in two (reduced) Mandelstam variables as is the case in SUSYHIT-1 . 4, following the work performed in reference [33], these two dimensional integrals can then be performed numerically. Alternatively, often one of the two integrals (remaining after the first trivial integral is performed) may be evaluated analytically, leaving a single one-dimensional integral to be performed numerically. This is the approach used in the work in reference [34] and is the method adopted in SPHENO [11, 35], from which the expressions we use for the 3 body decays originate. The Feynman diagrams involved, effects included and any assumptions made for each of the 3 body decays are given in detail in Appendix G with the corresponding partial width formulae.

The situation is of course more complicated for loop decays than at tree-level, each loop provides an additional loop integral to be performed, in the case of the 1-loop decays included in SOFTSUSY, they were performed explicitly with the help of Passarino-Veltman reduction [31].

\section{Decay Modes Included}

The following section provides a list of all the decay modes included in the decay part of the SOFTSUSY package; they are split into MSSM SUSY tree-level 2 body decays, MSSM Next-to-Lightest Supersymmetric Particle (NLSP) decays to the gravitino LSP (Lightest SUSY Particle), MSSM Higgs tree-level 2 body decays, MSSM Higgs 1-loop 2 body decays, MSSM tree-level 3 body decays, NMSSM SUSY and Higgs tree-level 2 body decays, NMSSM 1-loop 2 body decays and decays for which QCD corrections have been included. A comprehensive list of the formulae for all of the decays included is given explicitly in Appendix D to Appendix J for ease of reference. To summarise, we include:

- All MSSM 2 body decays at (at least) tree-level, both sparticle and Higgs boson decays.

- Next-to-Lightest SUSY Particle (NLSP) 2 body decays to gravitinos in the MSSM at tree level.

- The phenomenologically most relevant three-body decays of gluinos, charginos and neutralinos. 
- Higgs decays to $\gamma \gamma$ and $Z \gamma$ at leading order (i.e. one-loop) in the MSSM and NMSSM.

- QCD corrections to neutral Higgs decays to quarks (1-loop) and to gluons (2-loop) in the MSSM and NMSSM.

- All NMSSM 2 body decays at tree-level, including both the extended neutralino and extended Higgs sectors.

Whilst the majority of the decay modes are therefore calculated at tree-level, some effects of higher order corrections are approximated via the use of running masses and couplings, as calculated using the SOFTSUSY spectrum generator [2], the details of the mass choices were given in Section 3.3. In Appendix D there are a series of tables indicating all the modes included, along with appendix references for their partial width formulae as used in SOFTSUSY.

The branching ratios for each mode are grouped into decay tables for each parent SUSY or Higgs particle and are all printed to standard output in the SLHA/SLHA2 convention [36, 37] in order to allow it to be passed straightforwardly to other programs (such as PYTHIA [21], HERWIG7 [22], MadGraph [38], for instance).

\subsection{MSSM SUSY Tree-Level Two Body Decays}

The detailed formulae for these modes are in Appendix F. The gluino $\tilde{g}$ decays included are:

$$
\tilde{g} \rightarrow q \tilde{q}_{L / R}^{*}, \bar{q} \tilde{q}_{L / R}, t \tilde{t}_{1 / 2}^{*}, \bar{t} \tilde{t}_{1 / 2}, b \tilde{b}_{1 / 2}^{*}, \bar{b} \tilde{b}_{1 / 2},
$$

where $1 / 2$ means 1 or 2 , and $L / R$ means $L$ or $R$. The sfermion $\tilde{f}$ decays included are, for the first two generations where there is no sfermion mixing:

( $f^{\prime}$ indicates a fermion in the same generation as the $f$ fermion but with opposite third component of weak isospin, i.e. $f$ and $f^{\prime}$ could be $u$ and $d$ or $v_{e}$ and $e^{-}$):

$$
\begin{aligned}
\tilde{q}_{L / R} & \rightarrow \tilde{g} q, \\
\tilde{f}_{L} & \rightarrow \tilde{W}_{j} f^{\prime}, \\
\tilde{f}_{L / R} & \rightarrow \tilde{Z}_{i} f .
\end{aligned}
$$

For the third generation sfermions:

$$
\begin{aligned}
\tilde{b}_{1 / 2} & \rightarrow \tilde{g} b, \tilde{W}_{j} t, \tilde{Z}_{i} b, \tilde{t}_{1 / 2} W^{-}, \tilde{t}_{1 / 2} H^{-}, \\
\tilde{t}_{1 / 2} & \rightarrow \tilde{g} t, \tilde{W}_{j} b, \tilde{Z}_{i} t, \tilde{b}_{1 / 2} W^{+}, \tilde{b}_{1 / 2} H^{+}, \\
\tilde{b}_{2} & \rightarrow \tilde{b}_{1} Z, \tilde{b}_{1} h / H / A, \\
\tilde{t}_{2} & \rightarrow \tilde{t}_{1} Z, \tilde{t}_{1} h / H / A, \\
\tilde{\tau}_{1 / 2} & \rightarrow \tilde{W}_{j} v_{\tau}, \tilde{Z}_{i} \tau, \tilde{v}_{\tau} W^{-}, \tilde{v}_{\tau} H^{-}, \\
\tilde{v}_{\tau} & \rightarrow \tilde{W}_{j} \tau, \tilde{Z}_{i} v_{\tau}, \tilde{\tau}_{1 / 2} W^{+}, \tilde{\tau}_{1 / 2} H^{+}, \\
\tilde{\tau}_{2} & \rightarrow \tilde{\tau}_{1} Z, \tilde{\tau}_{1} h / H / A .
\end{aligned}
$$

For charginos, the two-body decay modes included are:

$$
\begin{aligned}
& \tilde{W}_{j} \rightarrow \tilde{q}_{L} \bar{q}^{\prime}, \tilde{q}_{1 / 2} \bar{q}^{\prime}, \tilde{l}_{L} \bar{v}_{l}, \tilde{v}_{l} \bar{l}, \tilde{\tau}_{1 / 2} \bar{v}_{\tau}, \tilde{v}_{\tau_{L}} \bar{\tau}, \tilde{Z}_{i} W^{+}, \tilde{Z}_{i} H^{+}, \\
& \tilde{W}_{2} \rightarrow \tilde{W}_{1} Z, \tilde{W}_{1} h / H / A .
\end{aligned}
$$

For neutralinos the two-body decay modes are ( $k>i$ as the neutralinos are mass ordered):

$$
\begin{aligned}
\tilde{Z}_{i} & \rightarrow \tilde{f}_{L / R} \bar{f}, \tilde{f}_{1 / 2} \bar{f}, \tilde{W}_{j} W^{+}, \tilde{W}_{j} H^{+}, \\
\tilde{Z}_{k} & \rightarrow \tilde{Z}_{i} Z, \tilde{Z}_{i} h / H / A .
\end{aligned}
$$

\subsection{MSSM Decays to Gravitinos}

The following NLSP $\rightarrow \tilde{G}+S M$ decays are included, for when the gravitino $\tilde{G}$ is the lightest supersymmetric particle (LSP):

$$
\tilde{g} \rightarrow g \tilde{G}, \quad \tilde{q}_{i} \rightarrow q \tilde{G}, \quad \tilde{l} \rightarrow l \tilde{G}, \quad \tilde{Z}_{i} \rightarrow \gamma \tilde{G}, \quad \tilde{Z}_{i} \rightarrow Z \tilde{G}, \quad \tilde{Z}_{i} \rightarrow \phi \tilde{G},
$$

where $\phi$ denotes one of the neutral Higgs bosons $h, H$ or $A$. The formulae for the partial widths are in Appendix $\mathrm{H}$ 


\subsection{MSSM Higgs Tree-Level Two Body Decays}

The tree-level 2 body decay modes included for the Higgs particles in the MSSM are as follows (see later for the 1-loop Higgs decays included), the formulae for the partial widths are explicitly given in Appendix F.6 Note that for the decays to sfermions any combination of handedness is allowed, $L L, L R, R L, R R$. Similarly, for the third generation where there is squark mixing, and for decays to charginos, all combinations 11, 12, 21, 22 are possible:

$$
\begin{aligned}
h / H / A & \rightarrow \tilde{f}_{L / R} \tilde{f}_{L / R}^{*}, \tilde{f}_{1 / 2} \tilde{f}_{1 / 2}^{*}, \tilde{W}_{1 / 2} \tilde{W}_{1 / 2}, \tilde{Z}_{i} \tilde{Z}_{l}, l^{+} l^{-}, \\
H^{+} & \rightarrow \tilde{Z}_{i} \tilde{W}_{j}, q \bar{q}^{\prime}, v_{l} l, \tilde{f}_{L / R} \tilde{f}_{L / R}^{*}, \tilde{f}_{1 / 2} \tilde{b}_{1 / 2}^{*}, v_{\tau} \tilde{\tau}_{1 / 2}^{*}, h W^{+}, \\
h / H & \rightarrow A A, A Z, \\
H & \rightarrow H^{+} H^{-}, h h, \\
A & \rightarrow h / H Z .
\end{aligned}
$$

The neutral Higgs decays to quarks are not included in this list as QCD corrections have been incorporated for these, see subsection 4.8 For $\mathrm{H}^{+}$, decays to CKM suppressed combinations of $q$ and $q^{\prime}$ are also considered, for example $H^{+} \rightarrow u \bar{s}$. Note also that the decays $H^{+} \rightarrow H / A W^{+}$are not included as they are kinematically forbidden in the MSSM (assuming tree-level mass formulae), these modes are included in the NMSSM.

\subsection{MSSM Higgs 1-loop Two body decays}

The key Higgs 1-loop decays are also included as these are very important channels for LHC Higgs discovery and measurement. The explicit expressions for their partial widths are in Appendix F.6.

$$
h / H / A \rightarrow \gamma \gamma, Z \gamma .
$$

The important loop decay to two gluons $g g$ incorporate QCD corrections and so are listed in subsection 4.8

\subsection{MSSM Tree-Level Three Body Decays}

The phenomenologically most important three-body decays in the MSSM are included, for the neutralino decays to another neutralino and a fermion anti-fermion pair then $i>j$ as the neutralinos are mass-ordered.:

$$
\begin{aligned}
h & \rightarrow V f \bar{f} . \\
\tilde{g} & \rightarrow \tilde{Z}_{i} q \bar{q}, \tilde{W}_{i} q \bar{q}^{\prime} . \\
\tilde{Z}_{i} & \rightarrow \tilde{Z}_{j} f \bar{f}, \tilde{W}_{j} f \bar{f}^{\prime} . \\
\tilde{W}_{j} & \rightarrow \tilde{Z}_{i} f \bar{f}^{\prime} .
\end{aligned}
$$

As of yet, there are no three-body decays of sfermions included; this may be resolved in future versions. The explicit formulae used, for which sPHENO [11] provided a useful reference, are given in Appendix G In our decay calculator the 3 body decay modes are only calculated where no tree level 2 body modes are available for the particular decaying SUSY or Higgs particle to the specific daughter(s) considered. Often 3 body modes will also not be output, even if they have been calculated, as in scenarios where other 2 body decay modes are kinematically available to the decaying parent particle these 2 body modes will dominate the total decay width. In such cases the 3 body modes often have branching ratios smaller than BRTol, which is the minimum BR output in the decay tables and may be set by the user in the input file.

\subsection{NMSSM SUSY and Higgs Tree-Level Two Body decays}

In the NMSSM, decays not involving the extended Higgs or neutralino sectors are the same as in the MSSM. For the extended neutralino and Higgs sectors the allowed decays are largely as before with the exception that now the neutralino index $i$ runs from 1 to 5, whilst there is an additional CP even neutral Higgs and an additional CP odd neutral Higgs. These additional states mix with the MSSM states with the same quantum numbers thereby resulting in 5 neutralinos, $3 \mathrm{CP}$ even neutral Higgs bosons and $2 \mathrm{CP}$ odd neutral Higgs Bosons, all of which we label in order of increasing mass. Therefore there are now additional mass eigenstates $H 3, A 2$ and $\tilde{Z}_{5}$. We use the notation that 
we label the CP even neutral Higgs states as $h_{i} \in\{h, H, H 3\}$ for $i=1,2,3$ and the CP odd neutral Higgs states as $A_{i} \in\{A, A 2\}$ for $i=1,2$. These states are of course mixtures of the original MSSM states and the new NMSSM states, therefore the most "NMSSM-type" state need not necessarily be the heaviest. The $\tilde{Z}_{1,2,3,4}, h$ and $H$ (which we now use to label the lightest two CP even neutral Higgs bosons) and $A$ have the same available modes as listed before; therefore we now list the decay modes of the additional states. As a guide, the same decays which can occur for the heaviest of the two CP even Higgs bosons of the MSSM, the $H$, may now also occur for the $H 3$; similarly we can extend the decays of the $A$ to the $A 2$, and of the $\tilde{Z}_{1,2,3,4}$ of the MSSM to the $\tilde{Z}_{5}$. Additional decay modes in the NMSSM (other than simply extending decays into neutralinos to include $\tilde{Z}_{5}$ or decays into $H 3, A 2$ ) are the decays of the $\tilde{Z}_{5}, H 3$ and $A 2$. Here, $n=1,2,3,4$ since the $\tilde{Z}_{5}$ decays into lighter neutralinos:

$$
\begin{aligned}
\tilde{Z}_{5} & \rightarrow W \tilde{W}_{1 / 2}, Z \tilde{Z}_{n}, H^{ \pm} \tilde{W}_{1 / 2}, \tilde{Z}_{n} h / H / H 3 / A / A 2, \tilde{f}_{L / R} \bar{f}, \tilde{f}_{1 / 2} \bar{f}, \\
H 3 & \rightarrow \tilde{f}_{L / R} \tilde{f}_{L / R}^{*}, \tilde{f}_{1 / 2} \tilde{f}_{1 / 2}^{*}, \tilde{W}_{1 / 2} \tilde{W}_{1 / 2}, \tilde{Z}_{i} \tilde{Z}_{l}, l^{+} l^{-}, A A, A A 2, \\
H 3 & \rightarrow A 2 A 2, Z A / A 2, H^{+} H^{-}, h h, h H, H H, W^{-} H^{+}, V V, \\
A 2 & \rightarrow \tilde{f}_{L} \tilde{f}_{R}^{*}, \tilde{f}_{1} \tilde{f}_{2}^{*}, \tilde{W}_{1 / 2} \tilde{W}_{1 / 2}, \tilde{Z}_{i} \tilde{Z}_{l}, l^{+} l^{-}, Z h / H / H 3, A h / H / H 3, W^{-} H^{+},
\end{aligned}
$$

where $V V \in\left\{W^{+} W^{-}, Z Z\right\}$. As before, for the decays to two sfermions, any combination of handedness is permitted $L L, L R, R L, R R$; similarly for the decays to mixed sfermions or to charginos 11, 12, 21, 22 are all allowed. For the decays to quarks only $c, s, t, b$ are considered. For the $A 2$ there are fewer decays than the $H 3$ as many decays are ruled out by $\mathrm{CP}$ conservation. Decays of the $H 3$ or $A 2$ to $q \bar{q}$ or $g g$ are listed in subsection 4.8 as QCD corrections are included in these channels.

The explicit partial width expressions used within the decay calculator SOFTSUSY are given in Appendix I, the expressions in NMSSMTools [13, 27, 28] were used, with appropriate changes.

\subsection{NMSSM 1-loop Two Body Decays}

Just as in the MSSM, in the NMSSM the phenomenologically important 1-loop decays of Higgs bosons are included:

$$
h / H / H 3 / A / A 2 \rightarrow \gamma \gamma, Z \gamma
$$

Again the decay to two gluons is listed later as it includes QCD corrections, see subsection 4.8. See Appendix Ifor the detailed formulae used within the code for the partial widths of these modes.

\subsection{QCD Corrected Decays}

NLO QCD corrections have been incorporated for the decays in which such effects are most important in both the MSSM and NMSSM, these are the neutral Higgs decays to quarks and decays to gluons:

$$
h / H / H 3 / A / A 2 \rightarrow q \bar{q}, g g
$$

The expressions used are given in Appendix J] and are based on those provided in the calculations in [39, 40]. Note that the quarks which are considered for neutral Higgs decays are only $c, s, b$ for the lightest CP even neutral higgs $h$, whilst $t$ is also included for the heavier CP even neutral Higgs Boson(s) and for the CP odd neutral Higgs Boson(s) of the (N)MSSM. Decays to $u$ and $d$ are negligible.

\section{Validation and Comparison with other programs}

We implemented a number of different approaches to validate the program and the formulae used.

1. Most of the formulae used in the SOFTSUSY program were rederived in order to determine any issues, the exception being the 3 body decays for which there are very complicated expressions for the integrals to be performed, for these the formulae are as in sPHENO-3.3.8 [11]. Meanwhile, NMSSM decay expressions were generalised from MSSM expressions and checked against NMSSMTools. 


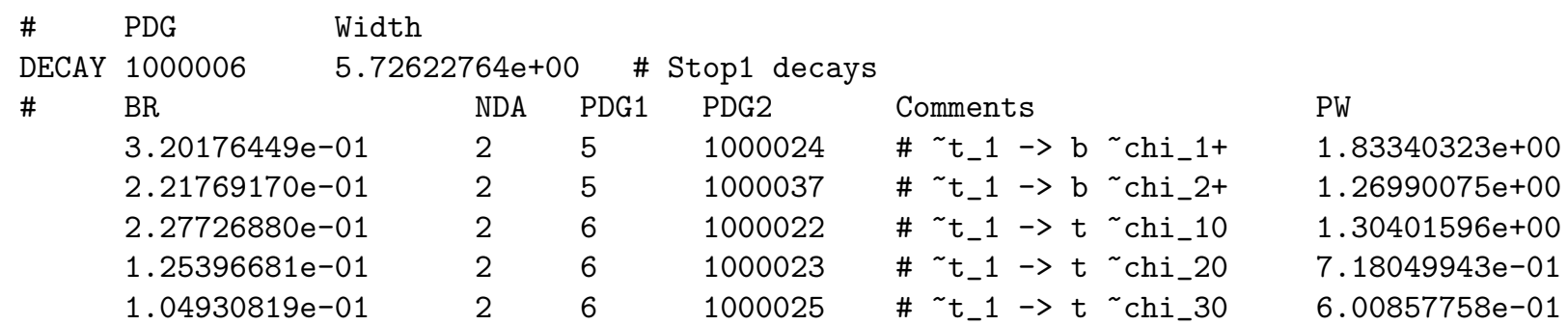

Table 2: The decays of a $\tilde{t}_{1}$ at the parameter point given by the lesHouchesInput file provided with SOFTSUSY. For reference this has $m_{\tilde{t}_{1}}=808.7$ $\mathrm{GeV}, m_{\tilde{W}_{1}}=385.0 \mathrm{GeV}, m_{\tilde{W}_{2}}=637.5 \mathrm{GeV}, m_{\tilde{Z}_{1}}=204.0 \mathrm{GeV}, m_{\tilde{Z}_{2}}=385.0 \mathrm{GeV}, m_{\tilde{Z}_{3}}=-622.7 \mathrm{GeV}, m_{\tilde{Z}_{4}}=637.2 \mathrm{GeV}$. The partial widths are given in $\mathrm{GeV}$ units.

2. MSSM 2 body partial widths were also checked against both Baer and Tata "Weak Scale Supersymmetry" [41] and The Higgs Hunter's Guide [32]. Some small differences were found, in particular relative to Baer and Tata, these differences were largely simple typographical errors although there were several other differences in the 3 body decay formulae, where additional contributions are included. The full formulae for the partial widths used in SOFTSUSY are given in Appendix D to Appendix J

3. The partial width of every channel was compared against corresponding channels in other programs where possible; for example the 2 body MSSM decays were compared against results from SUSYHIT, the 3 body MSSM decays against sPHENO and the 2 body NMSSM decays against NMSSMTools. This ensured that the formulae were correct and any differences were down to different input parameters resulting from different schemes of running, different mass choices and other similar effects. These checks were performed at several parameter space points for each partial width.

4. Once all the decay modes of a given initial state were implemented the branching ratios were compared against those of a relevant program, i.e. SUSYHIT for the MSSM 2 body decays, sPHENO for MSSM 3 body decays, and NMSSMTools (as linked with the SOFTSUSY spectrum generator) for NMSSM decays.

5. Many of the modes have been analysed in detail by scanning over the mass of the decaying particle and comparing the branching ratio structure with known results and with corresponding results in other decay calculator programs.

As described above we therefore performed specific and fairly extensive tests for particular benchmark points with SPHENO and SUSYHIT. Comparisons for some of these benchmark points are provided here for a selection of decaying SUSY and Higgs particles, in addition scans over the mass of the decaying particle are given for the decays of the lightest SM-like Higgs and for the decays of a gluino $\tilde{g}$. This allowed both a qualitative check of the behaviour of the decays in the program and a quantitative comparison of the level of agreement with other programs. In particular the level of agreement with the same input parameters and with our set of input parameters is detailed in some specific cases. This method uses the results the SOFTSUSY program produces to validate it.

\subsection{Supersymmetric Decays - $\tilde{t}_{1}$}

First of all consider the decays of the lightest stop, $\tilde{t}_{1}$, the verbatim output of the SOFTSUSY decay calculator is given in Table 2 and was generated with the lesHouches Input file. The comparison of the results for this benchmark point between the new SOFTSUSY decay calculator and those of SUSYHIT-1. 4 is given in Table 3

In this the input values used for the various masses are: top pole mass mtPole $=174.3 \mathrm{GeV}$, bottom pole mass mbPole $=4.985 \mathrm{GeV}$, running top mass runmt $=145.555 \mathrm{GeV}$ and running bottom mass runmb $=2.576 \mathrm{GeV}$. These differ from the default values used for these quantities in SUSYHIT, Table 3 illustrates the differences observed between SOFTSUSY and SUSYHIT-1.4, as well as the differences when SOFTSUSY has the SUSYHIT mass inputs inserted by hand. This demonstrates that the level of agreement between the programs is around $10 \%$, dropping down to $1 \%$ when the same input masses and coupling constants are used in both programs. These differences result from the different mass and scheme choices, as outlined in Section 3.3 


\begin{tabular}{|c|c|c|c|c|c|c|}
\hline \multicolumn{2}{|c|}{ SOFTSUSY standard inputs } & \multicolumn{2}{c|}{ SOFTSUSY SUSYHIT's inputs } & \multicolumn{2}{c|}{ SUSYHIT } & mode \\
\hline PW/GeV & BR & PW/GeV & BR & PW/GeV & BR & \\
\hline $1.8334 \mathrm{e}+00$ & $3.2018 \mathrm{e}-01$ & $1.7080 \mathrm{e}+00$ & $3.2111 \mathrm{e}-01$ & $1.7080 \mathrm{e}+00$ & $3.2181 \mathrm{e}-01$ & $\tilde{t}_{1} \rightarrow b \tilde{W}_{1}$ \\
\hline $1.2699 \mathrm{e}+00$ & $2.2177 \mathrm{e}-01$ & $1.1027 \mathrm{e}+00$ & $2.0730 \mathrm{e}-01$ & $1.1027 \mathrm{e}+00$ & $2.0775 \mathrm{e}-01$ & $\tilde{t}_{1} \rightarrow b \tilde{W}_{2}$ \\
\hline $1.3040 \mathrm{e}+00$ & $2.2773 \mathrm{e}-01$ & $1.2986 \mathrm{e}+00$ & $2.4414 \mathrm{e}-01$ & $1.2992 \mathrm{e}+00$ & $2.4478 \mathrm{e}-01$ & $\tilde{t}_{1} \rightarrow t \tilde{Z}_{1}$ \\
\hline $7.1805 \mathrm{e}-01$ & $1.2540 \mathrm{e}-01$ & $6.8479 \mathrm{e}-01$ & $1.2874 \mathrm{e}-01$ & $6.7286 \mathrm{e}-01$ & $1.2677 \mathrm{e}-01$ & $\tilde{t}_{1} \rightarrow t \tilde{Z}_{2}$ \\
\hline $6.0086 \mathrm{e}-01$ & $1.0493 \mathrm{e}-01$ & $5.2503 \mathrm{e}-01$ & $9.8706 \mathrm{e}-02$ & $5.2485 \mathrm{e}-01$ & $9.8887 \mathrm{e}-02$ & $\tilde{t}_{1} \rightarrow t \tilde{Z}_{3}$ \\
\hline
\end{tabular}

Table 3: The $\tilde{t}_{1}$ decay partial widths and branching ratios as output by SOFTSUSY with our mass choices (and corresponding Yukawa couplings) and with the masses and Yukawa couplings in SUSYHIT, compared with the results of SUSYHIT-1.4. This illustrates the differences of order $10 \%$ or more that may arise depending upon mass ("kinematic" and "running") choices, the differences here reduce to order $1 \%$ once the same masses are taken. The lesHouches Input file provided with SOFTSUSY gives the parameter point here, it has a common scalar mass $m_{0}=125 \mathrm{GeV}$, a common gaugino mass $m_{1 / 2}=500 \mathrm{GeV}$, ratio of Higgs vacuum expectation values $\tan \beta=10$, sign of the superpotential $\mu$ parameter $\operatorname{sign}(\mu)=+1$ and common soft SUSY breaking trilinear parameter $A_{0}=0$ in the constrained MSSM (CMSSM). Here, as elsewhere in this paper, we present numerical results in mantissa-exponent notation (i.e. e- $0 \mathrm{a}=\ldots \times 10^{-a}$ and $\mathrm{e}+0 \mathrm{~b}=\ldots \times 10^{b}$ for $a, b \in\{0,1,2,3, \ldots\}$ ).

\subsection{Higgs Decays - $h$}

Now, in Table 5, we can perform similar comparisons between SOFTSUSY and HDECAY-3.4 of SUSYHIT-1.4 for Higgs decays. Here we have taken a SM-like Higgs, in the decoupling limit so all the SUSY decays are kinematically forbidden, given by a point in the pMSSM parameter space which has Higgs mass $125 \mathrm{GeV}$, this point we call pmssm1 and the SLHA [36] form of the input file is given verbatim in Table 4 The results of our decay calculator without QCD corrections included, with QCD corrections included, and with the same input quark and gauge boson masses and same input gauge couplings as SUSYHIT, again with QCD corrections, are compared with HDECAY-3.4. Note that the comparisons are done against the non-current version HDECAY-3.4 as this is the version included in the SUSYHIT-1.4 package. This allowed straightforward comparisons to be done between the new decay calculator and SUSYHIT'S version of HDECAY as one can input the spectrum as calculated by SOFTSUSY straight into SUSYHIT. This allowed the effects of the spectrum generator to be isolated as much as possible from the decay calculator which is being tested.

In Table 5 the comparison of the partial widths with QCD corrections switched on and switched off clearly demonstrates the significant difference these corrections make to neutral Higgs decays to quarks and to gluons, as is widely known in the literature [40, 42, 43, 44]. Furthermore, it is clear that the main source of differences in partial widths between the decay calculator of SOFTSUSY-4. 0 and HDECAY is in the choice of masses used. Remaining differences tend to be small and are due largely to differences in other inputs, the exception being the decays to two vector bosons, where order $10 \%$ differences are observed. This is due to HDECAY incorporating additional effects such as the width of the resonance and NLO corrections which are not included in SOFTSUSY. It should also be noted here that HDECAY performs a numerical integration whilst SOFTSUSY has an explicit expression with no integration required so the calculation methods are different. A comparison of the branching ratios output for this SM-like Higgs are given in Figure 1

In order to provide a qualitative demonstration that the decay calculator is functioning correctly one may also scan the mass of the decaying particle and investigate how the partial widths and branching ratios change. Figure 2 shows how the branching ratios of a SM-like Higgs change as its mass is scanned from the $Z^{0}$ boson mass up to $200 \mathrm{GeV}$ as calculated in (a) SOFTSUSY and in (b) a well-known plot produced by the LHC Higgs Cross Section Working Group [45] in 2011. This shows good level of agreement, with small differences due to effects detailed previously in the quantitative comparison at $m_{h}=125 \mathrm{GeV}$.

So far we have demonstrated the validation of SUSY 2 body and Higgs MSSM decays, including the loop-decays, QCD corrections and Higgs 3 body decays. Similar validation and comparison was also performed for the MSSM 3 body decays and the NMSSM decays.

\subsection{Three-Body Decays - $\tilde{g}$}

First, consider the MSSM 3 body decays: an explicit comparison can be performed for the gluino 3 body decays with the spectrum given in Figure 3 , the gluino three-body decays to neutralinos and quark anti-quark pairs are indi- 


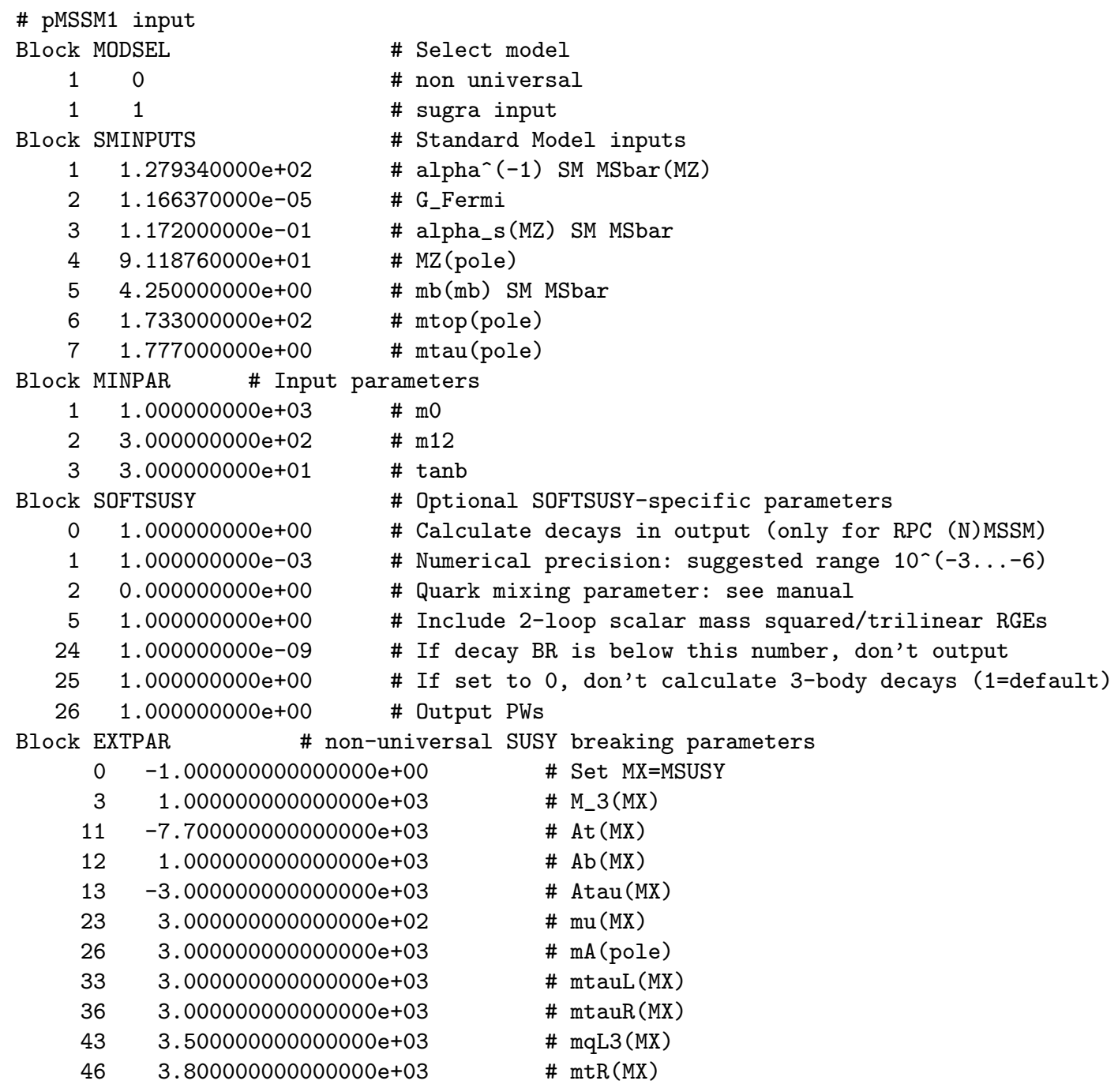

\# Optional SOFTSUSY-specific parameters

\# Calculate decays in output (only for RPC (N)MSSM)

\# Numerical precision: suggested range $10^{\wedge}(-3 \ldots-6)$

\# Quark mixing parameter: see manual

\# Include 2-loop scalar mass squared/trilinear RGEs

\# If decay BR is below this number, don't output

\# If set to 0 , don't calculate 3 -body decays (1=default)

\# Output PWs

Block EXTPAR \# non-universal SUSY breaking parameters
$0-1.000000000000000 e+00$
$1.000000000000000 e+03$
$-7.700000000000000 e+03$
\# M_3(MX)
$1.000000000000000 \mathrm{e}+03$
\# At (MX)
$-3.000000000000000 \mathrm{e}+03$
\# $\mathrm{Ab}(\mathrm{MX})$
\# Atau(MX)
\# mu(MX)
$3.000000000000000 \mathrm{e}+02$
\# mA (pole)
$3.000000000000000 e+03$
\# mtauL(MX)
$3.000000000000000 \mathrm{e}+03$
\# mtauR(MX)
$3.500000000000000 e+03$
\# mqL3(MX)
$3.800000000000000 \mathrm{e}+03$
$\# \operatorname{mtR}(\mathrm{MX})$

\# Set MX=MSUSY

Table 4: The pMSSM parameter space point used for the $h$ decay comparisons in Table 5 and Figure 1 given in SLHA form [36]. 


\begin{tabular}{|c|c|c|c|c|c|c|c|c|}
\hline \multicolumn{2}{|c|}{$\begin{array}{c}\text { SOFTSUSY no QCD } \\
\text { corrections }\end{array}$} & \multicolumn{2}{c|}{$\begin{array}{c}\text { SOFTSUSY with } \\
\text { QCD corrections }\end{array}$} & \multicolumn{2}{c|}{$\begin{array}{c}\text { SOFTSUSY with } \\
\text { SUSYHIT's masses } \\
\text { and QCD corrections }\end{array}$} & \multicolumn{2}{c|}{$\begin{array}{c}\text { HDECAY-3.4 with } \\
\text { same QCD } \\
\text { corrections }\end{array}$} & mode \\
\hline PW/GeV & BR & PW/GeV & BR & PW/GeV & BR & PW/GeV & BR & \\
\hline $1.04 \mathrm{e}-04$ & $3.30 \mathrm{e}-02$ & $2.25 \mathrm{e}-04$ & $4.03 \mathrm{e}-02$ & $2.25 \mathrm{e}-04$ & $4.31 \mathrm{e}-02$ & $2.25 \mathrm{e}-04$ & $4.24 \mathrm{e}-02$ & $h \rightarrow c c$ \\
\hline $8.00 \mathrm{e}-07$ & $2.55 \mathrm{e}-04$ & $1.62 \mathrm{e}-06$ & $2.91 \mathrm{e}-04$ & $1.62 \mathrm{e}-06$ & $3.11 \mathrm{e}-04$ & $1.63 \mathrm{e}-06$ & $3.06 \mathrm{e}-04$ & $h \rightarrow s s$ \\
\hline $1.75 \mathrm{e}-03$ & $5.56 \mathrm{e}-01$ & $3.96 \mathrm{e}-03$ & $7.10 \mathrm{e}-01$ & $3.60 \mathrm{e}-03$ & $6.90 \mathrm{e}-01$ & $3.61 \mathrm{e}-03$ & $6.80 \mathrm{e}-01$ & $h \rightarrow b b$ \\
\hline $8.52 \mathrm{e}-07$ & $2.71 \mathrm{e}-04$ & $8.52 \mathrm{e}-07$ & $1.53 \mathrm{e}-04$ & $9.17 \mathrm{e}-07$ & $1.76 \mathrm{e}-04$ & $9.19 \mathrm{e}-07$ & $1.73 \mathrm{e}-04$ & $h \rightarrow \mu \mu$ \\
\hline $2.61 \mathrm{e}-04$ & $8.30 \mathrm{e}-02$ & $2.61 \mathrm{e}-04$ & $4.67 \mathrm{e}-02$ & $2.59 \mathrm{e}-04$ & $4.97 \mathrm{e}-02$ & $2.60 \mathrm{e}-04$ & $4.90 \mathrm{e}-02$ & $h \rightarrow \tau \tau$ \\
\hline $1.06 \mathrm{e}-05$ & $3.36 \mathrm{e}-03$ & $1.06 \mathrm{e}-05$ & $1.89 \mathrm{e}-03$ & $9.24 \mathrm{e}-06$ & $1.77 \mathrm{e}-03$ & $9.24 \mathrm{e}-06$ & $1.74 \mathrm{e}-03$ & $h \rightarrow \gamma \gamma$ \\
\hline $1.65 \mathrm{e}-04$ & $5.27 \mathrm{e}-02$ & $2.71 \mathrm{e}-04$ & $4.86 \mathrm{e}-02$ & $2.72 \mathrm{e}-04$ & $5.22 \mathrm{e}-02$ & $2.72 \mathrm{e}-04$ & $5.13 \mathrm{e}-02$ & $h \rightarrow g g$ \\
\hline $6.74 \mathrm{e}-06$ & $2.15 \mathrm{e}-03$ & $6.74 \mathrm{e}-06$ & $1.21 \mathrm{e}-03$ & $5.88 \mathrm{e}-06$ & $1.13 \mathrm{e}-03$ & $6.11 \mathrm{e}-06$ & $1.15 \mathrm{e}-03$ & $h \rightarrow Z \gamma$ \\
\hline $7.61 \mathrm{e}-04$ & $2.42 \mathrm{e}-01$ & $7.61 \mathrm{e}-04$ & $1.36 \mathrm{e}-01$ & $7.61 \mathrm{e}-04$ & $1.46 \mathrm{e}-01$ & $8.22 \mathrm{e}-04$ & $1.55 \mathrm{e}-01$ & $h \rightarrow W W^{*}$ \\
\hline $8.44 \mathrm{e}-05$ & $2.69 \mathrm{e}-02$ & $8.44 \mathrm{e}-05$ & $1.51 \mathrm{e}-02$ & $8.44 \mathrm{e}-05$ & $1.62 \mathrm{e}-02$ & $1.02 \mathrm{e}-04$ & $1.92 \mathrm{e}-02$ & $h \rightarrow Z Z^{*}$ \\
\hline
\end{tabular}

Table 5: The $h$ decay partial widths and branching ratios as output by SOFTSUSY without QCD corrections, with QCD corrections, with SUSYHIT's quark and gauge boson masses and gauge couplings and with QCD corrections, and the results of HDECAY-3.4 from SUSYHIT-1.4. This illustrates the necessity of including QCD corrections for decays to quarks and decays to gluons, as well as the fact that differences in mass choices are the primary source of differences between SOFTSUSY and HDECAY-3.4. This is for a pMSSM point given by pmssm1 listed in SLHA [36] format in Table 4 it has $m_{h}=125 \mathrm{GeV}$. Note that the masses and gauge couplings are taken from SUSYHIT and inserted into the SOFTSUSY decay calculator in columns 5 and 6 are $\alpha_{s}=0.11$ and $m_{c}=1.40 \mathrm{GeV}, m_{s}=0.19 \mathrm{GeV}, m_{b}=4.77 \mathrm{GeV}, m_{t}=173.30 \mathrm{GeV}$ for the $h \rightarrow q q$ and $h \rightarrow g g$ decays; $m_{\mu}=0.11 \mathrm{GeV}, m_{\tau}=1.78 \mathrm{GeV}$ for $h \rightarrow l^{+} l^{-}$decays; $\alpha\left(M_{Z}\right)=7.29 \times 10^{-3}$ and $m_{W}=80.35 \mathrm{GeV}, m_{t}=188.72 \mathrm{GeV}, m_{b}=3.47 \mathrm{GeV}, m_{c}=0.74 \mathrm{GeV}$ and $m_{\tau}=1.78 \mathrm{GeV}$ for $h \rightarrow \gamma \gamma ; m_{Z}=91.19 \mathrm{GeV}, m_{t}=173.30 \mathrm{GeV}, m_{b}=4.77 \mathrm{GeV}, \alpha=7.29 \times 10^{-3}$ and $m_{W}=80.36 \mathrm{GeV}$ for $h \rightarrow Z \gamma$ and for $h \rightarrow V V^{*}$.

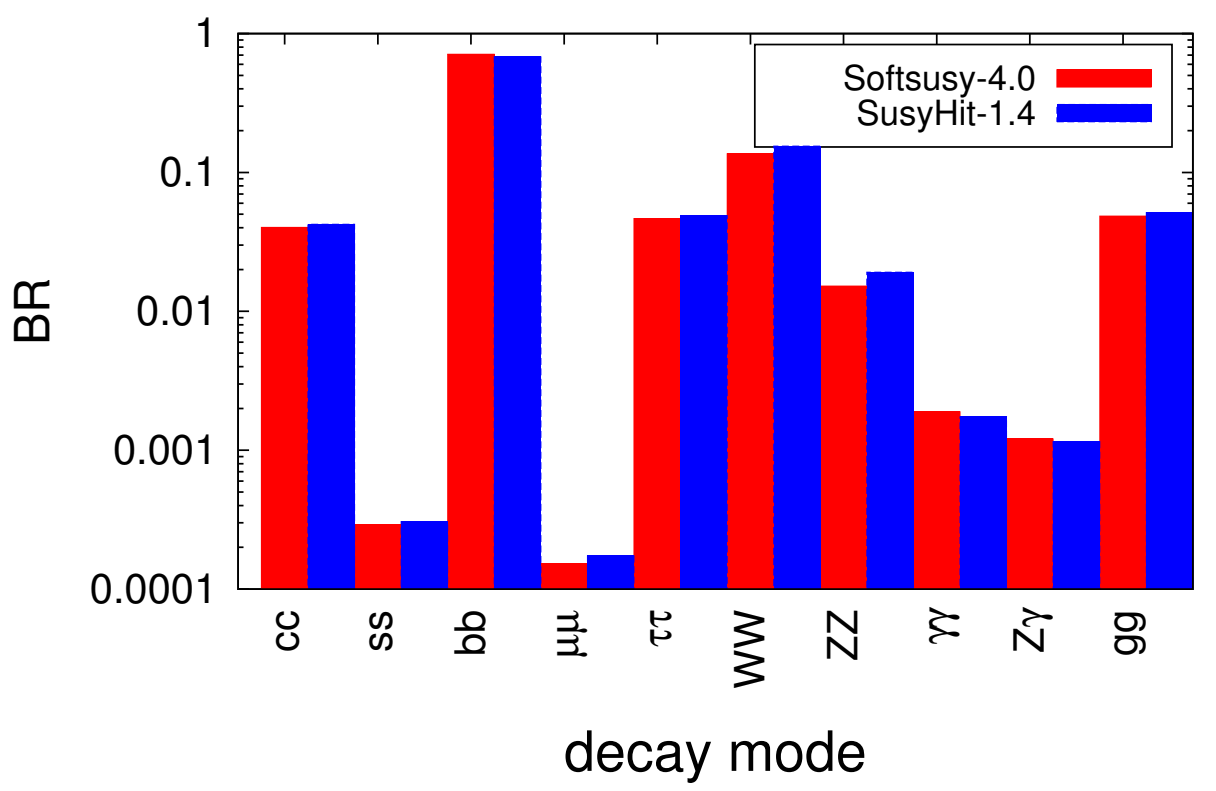

Figure 1: Branching ratios for a SM-like Higgs predicted by SOFTSUSY and by HDECAY-3.4 in SUSYHIT-1.4 for $m_{h}=125$ GeV. This is for a pMSSM point given by pmssm1, see Table 4 


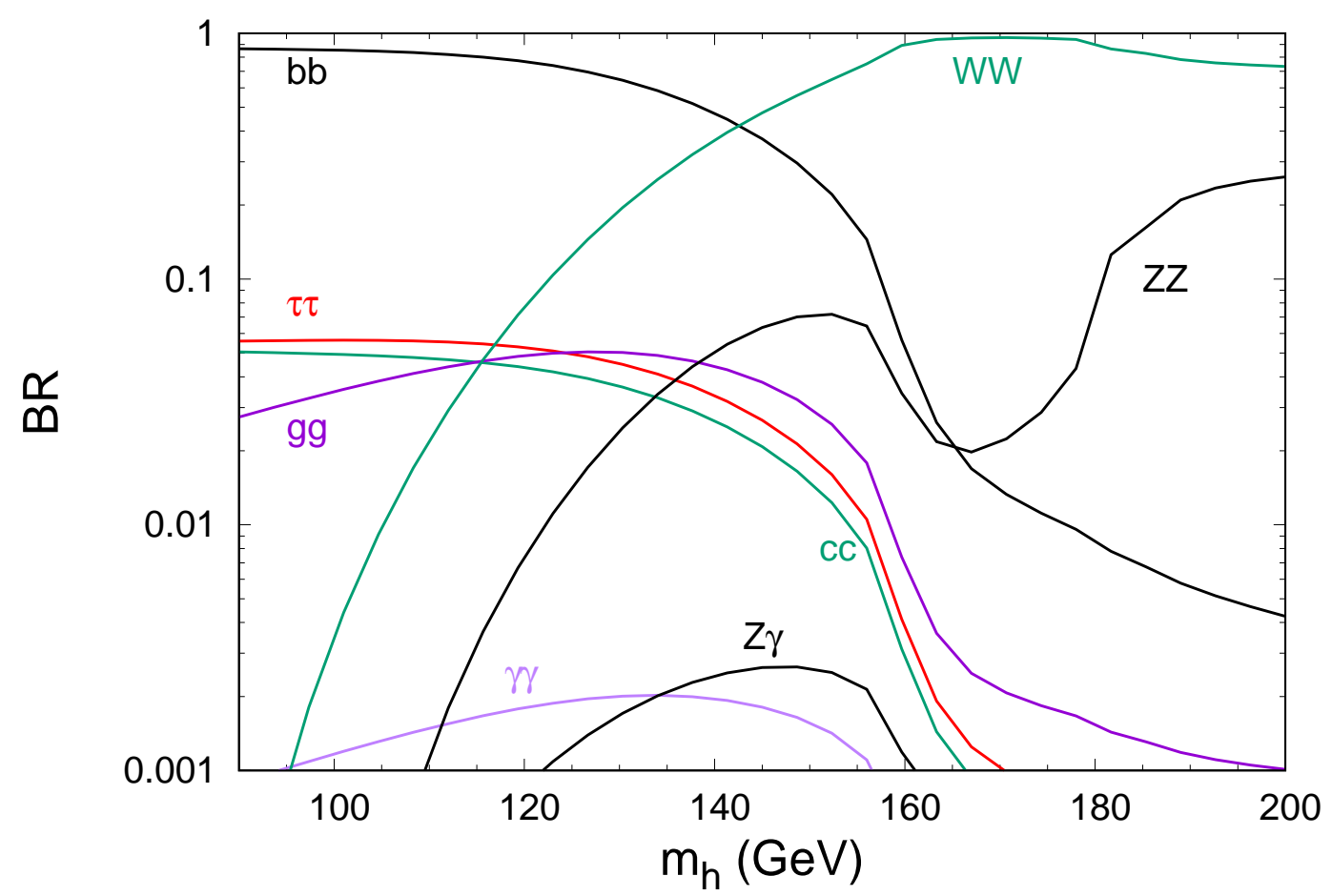

(a) SOFTSUSY

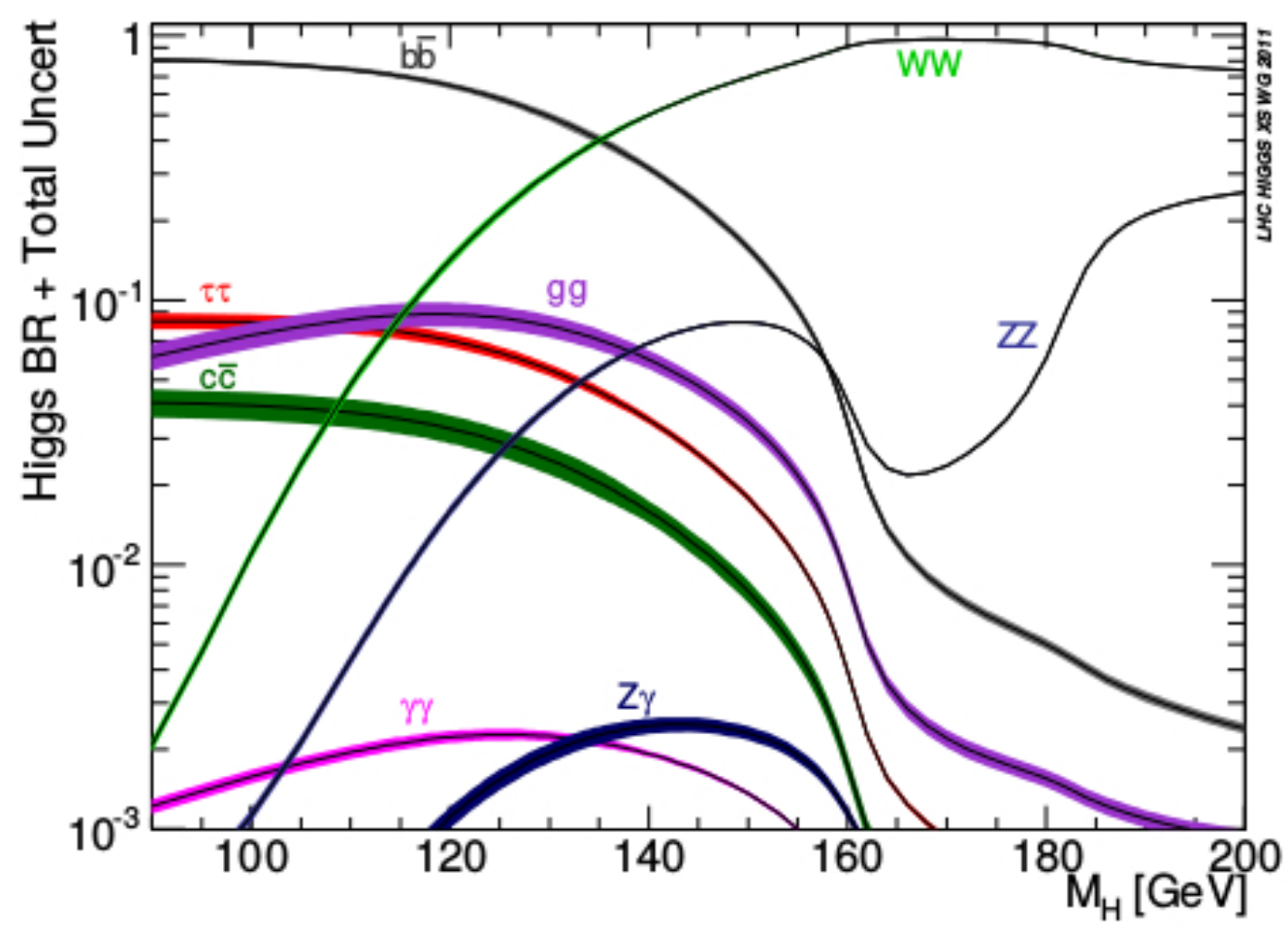

(b) LHC Higgs Cross-Section Working Group

Figure 2: Branching ratios for a SM(-like) Higgs as calculated in (a) by SOFTSUSY and in (b) by the LHC Higgs Cross-Section Working Group in [45]. This demonstrates a verification of the partial widths output by SOFTSUSY. 


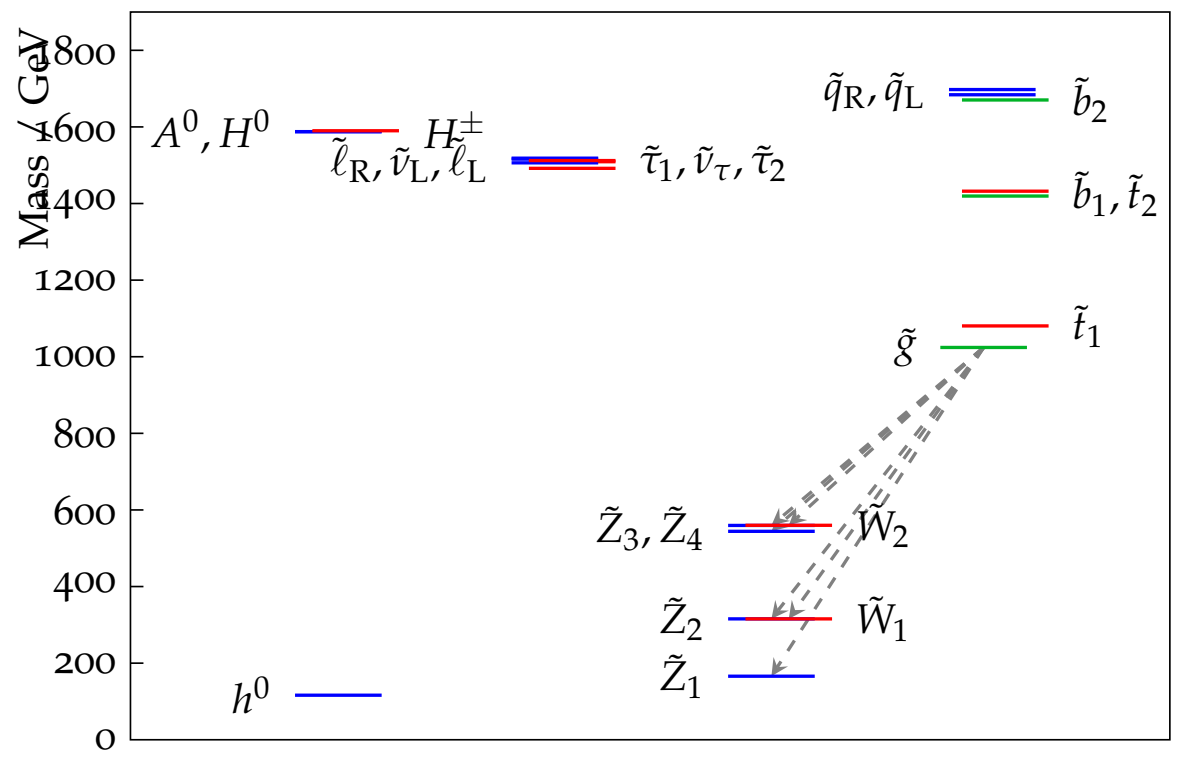

Figure 3: Spectrum used for quantitative comparison of gluino $\tilde{g}$ three body decays. Here the arrows indicate only the 3 body decay modes of the gluino: these are those investigated. This CMSSM spectrum has $m_{0}=1500 \mathrm{GeV}, m_{1 / 2}=400 \mathrm{GeV}, \tan \beta=10.37, \operatorname{sign}(\mu)=+1, A_{0}=-80 \mathrm{GeV}$ and was generated in sPHENO. The figure was produced using a modified version slhaplot-3.0.4 from PySLHA [46].

cated. A comparison of the partial widths and branching ratios given by SOFTSUSY, sPHENO-3.3.8 and SUSYHIT-1.4 for this spectrum is presented in Table 6. This was performed taking the mass, coupling and other input decay parameters from SPHENO and inputting these directly by hand into the SOFTSUSY decay calculator in order to evaluate only differences due to the decay calculation, not any differences which might arise as a result of differing parameters from the spectrum generators. The agreement between the three programs is generally very good, in particular the agreement between SOFTSUSY and SPHENO-3.3.8, upon which the calculations of the 3 body decays is based, is usually between 1 and 5\% with the larger differences often occurring where there are larger differences between SUSYHIT-1.4 and sPHENO-3.3.8. The exceptions to this are the decays to third generation quark-anti-quark pairs and the third and fourth heaviest neutralinos; i.e. $\tilde{g} \rightarrow t \bar{t} \tilde{Z}_{3}, \tilde{g} \rightarrow t \bar{t} \tilde{Z}_{4}, \tilde{g} \rightarrow b \bar{b} \tilde{Z}_{3}$ and $\tilde{g} \rightarrow b \bar{b} \tilde{Z}_{4}$. Here the differences observed are $10-20 \%$ and they arise because of differences in the Yukawa couplings taken, for example for the $b$ quark here the Yukawa coupling used in SOFTSUSY is determined by a running bottom mass of runmb=2.63 GeV, whereas sPHENO has a Yukawa coupling corresponding to a mass of runmb $=2.37 \mathrm{GeV}$. In order to show this results in the differences observed, the running $b$ mass in SOFTSUSY was temporarily set to that of sPHENO and the comparison for $\tilde{g} \rightarrow b \bar{b} \tilde{Z}_{i}$ is provided in Table 7 This demonstrates that the decays to $\tilde{Z}_{1}$ and $\tilde{Z}_{2}$ are not significantly altered by the new Yukawa coupling whereas the decays to $\tilde{Z}_{3}$ and $\tilde{Z}_{4}$ (i.e. those which showed differences with respect to sPHENO) now have significantly altered partial widths which are in much closer agreement with sPHENO, back down to the few percent level agreement seen in the other 3 body decays.

A scan over the mass of the gluino to demonstrate the expected suppression of 3 body decays relative to 2 body decays was also performed, see Figure 4 The result of this is that phenomenologically, 3 body modes are only important when 2 body tree level modes are unavailable. For this reason, SOFTSUSY only calculates 3 body modes when there are no two-body modes kinematically available and does not output the 3 body modes if the total 3 body decay width (sum of all the 3 body decay widths available) is less than the BR tolerance. More details on the other 3 body modes, the contributions included, approximations made and the level of agreement seen between SOFTSUSY and other decay calculators for each mode are given in Appendix $G$. There, the relevant expressions used by our decay calculator to determine their partial widths are also provided. 


\begin{tabular}{|c|c|c|c|c|c|c|}
\hline \multicolumn{2}{|c|}{ SOFTSUSY } & \multicolumn{2}{|c|}{ SPHENO-3.38 } & \multicolumn{2}{|c|}{ SUSYHIT-1.4 } & \multirow[t]{2}{*}{ mode } \\
\hline $\mathrm{PW} / \mathrm{GeV}$ & BR & $\mathrm{PW} / \mathrm{GeV}$ & BR & $\mathrm{PW} / \mathrm{GeV}$ & BR & \\
\hline $2.90 \mathrm{e}-04$ & $2.26 \mathrm{e}-02$ & $2.89 \mathrm{e}-04$ & $2.32 \mathrm{e}-02$ & $2.89 \mathrm{e}-04$ & $2.32 \mathrm{e}-02$ & $\tilde{g} \rightarrow \tilde{Z}_{1} u \bar{u}$ \\
\hline $3.21 \mathrm{e}-04$ & $2.51 \mathrm{e}-02$ & $3.19 \mathrm{e}-04$ & $2.56 \mathrm{e}-02$ & $3.19 \mathrm{e}-04$ & $2.56 \mathrm{e}-02$ & $\tilde{g} \rightarrow \tilde{Z}_{2} u \bar{u}$ \\
\hline $1.35 \mathrm{e}-07$ & $1.06 \mathrm{e}-05$ & $1.35 \mathrm{e}-07$ & $1.08 \mathrm{e}-05$ & $1.35 \mathrm{e}-07$ & $1.08 \mathrm{e}-05$ & $\tilde{g} \rightarrow \tilde{Z}_{3} u \bar{u}$ \\
\hline $5.52 \mathrm{e}-06$ & $4.31 \mathrm{e}-04$ & $5.49 \mathrm{e}-06$ & $4.40 \mathrm{e}-04$ & $5.49 \mathrm{e}-06$ & $4.40 \mathrm{e}-04$ & $\tilde{g} \rightarrow \tilde{Z}_{4} u \bar{u}$ \\
\hline $9.06 \mathrm{e}-05$ & $7.07 e-03$ & $9.02 \mathrm{e}-05$ & $7.22 \mathrm{e}-03$ & $9.02 \mathrm{e}-05$ & $7.24 \mathrm{e}-03$ & $\tilde{g} \rightarrow \tilde{Z}_{1} d \bar{d}$ \\
\hline $3.07 \mathrm{e}-04$ & $2.40 \mathrm{e}-02$ & $3.06 \mathrm{e}-04$ & $2.45 \mathrm{e}-02$ & $3.06 \mathrm{e}-04$ & $2.45 e-02$ & $\tilde{g} \rightarrow \tilde{Z}_{2} d \bar{d}$ \\
\hline $1.75 \mathrm{e}-07$ & $1.36 \mathrm{e}-05$ & $1.74 \mathrm{e}-07$ & $1.39 \mathrm{e}-05$ & $1.74 \mathrm{e}-07$ & $1.40 \mathrm{e}-05$ & $\tilde{g} \rightarrow \tilde{Z}_{3} d \bar{d}$ \\
\hline $6.67 \mathrm{e}-06$ & $5.21 \mathrm{e}-04$ & $6.64 \mathrm{e}-06$ & $5.31 \mathrm{e}-04$ & $6.64 \mathrm{e}-06$ & $5.33 \mathrm{e}-04$ & $\tilde{g} \rightarrow \tilde{Z}_{4} d \bar{d}$ \\
\hline $2.90 \mathrm{e}-04$ & $2.26 \mathrm{e}-02$ & $2.89 \mathrm{e}-04$ & $2.32 \mathrm{e}-02$ & $2.89 \mathrm{e}-04$ & $2.32 \mathrm{e}-02$ & $\tilde{g} \rightarrow \tilde{Z}_{1} c \bar{c}$ \\
\hline $3.21 \mathrm{e}-04$ & $2.51 \mathrm{e}-02$ & $3.19 \mathrm{e}-04$ & $2.56 \mathrm{e}-02$ & $3.19 \mathrm{e}-04$ & $2.56 \mathrm{e}-02$ & $\tilde{g} \rightarrow \tilde{Z}_{2} c \bar{c}$ \\
\hline $1.35 \mathrm{e}-07$ & $1.05 \mathrm{e}-05$ & $1.41 \mathrm{e}-07$ & $1.13 \mathrm{e}-05$ & $1.35 \mathrm{e}-07$ & $1.08 \mathrm{e}-05$ & $\tilde{g} \rightarrow \tilde{Z}_{3} c \bar{c}$ \\
\hline $5.52 \mathrm{e}-06$ & $4.31 \mathrm{e}-04$ & $5.50 \mathrm{e}-06$ & $4.40 \mathrm{e}-04$ & $5.49 \mathrm{e}-06$ & $4.40 \mathrm{e}-04$ & $\tilde{g} \rightarrow \tilde{Z}_{4} c \bar{c}$ \\
\hline $9.06 \mathrm{e}-05$ & $7.07 e-03$ & $9.02 \mathrm{e}-05$ & $7.22 \mathrm{e}-03$ & $9.02 \mathrm{e}-05$ & $7.24 \mathrm{e}-03$ & $\tilde{g} \rightarrow \tilde{Z}_{1} s \bar{s}$ \\
\hline $3.07 \mathrm{e}-04$ & $2.40 \mathrm{e}-02$ & $3.06 \mathrm{e}-04$ & $2.45 \mathrm{e}-02$ & $3.06 \mathrm{e}-04$ & $2.45 \mathrm{e}-02$ & $\tilde{g} \rightarrow \tilde{Z}_{2} s \bar{s}$ \\
\hline $1.75 \mathrm{e}-07$ & $1.36 \mathrm{e}-05$ & $1.77 \mathrm{e}-07$ & $1.42 \mathrm{e}-05$ & $1.74 \mathrm{e}-07$ & $1.40 \mathrm{e}-05$ & $\tilde{g} \rightarrow \tilde{Z}_{3} s \bar{s}$ \\
\hline $6.67 \mathrm{e}-06$ & $5.21 \mathrm{e}-04$ & $6.64 \mathrm{e}-06$ & $5.32 \mathrm{e}-04$ & $6.64 \mathrm{e}-06$ & 5.33e-04 & $\tilde{g} \rightarrow \tilde{Z}_{4} s \bar{s}$ \\
\hline $1.47 \mathrm{e}-03$ & $1.15 \mathrm{e}-01$ & $1.47 \mathrm{e}-03$ & $1.17 \mathrm{e}-01$ & $1.44 \mathrm{e}-03$ & $1.15 \mathrm{e}-01$ & $\tilde{g} \rightarrow \tilde{Z}_{1} t \bar{t}$ \\
\hline $2.56 \mathrm{e}-04$ & $1.99 \mathrm{e}-02$ & $2.46 \mathrm{e}-04$ & $1.97 \mathrm{e}-02$ & $2.67 \mathrm{e}-04$ & $2.15 \mathrm{e}-02$ & $\tilde{g} \rightarrow \tilde{Z}_{2} t \bar{t}$ \\
\hline $3.48 \mathrm{e}-04$ & $2.71 \mathrm{e}-02$ & $3.10 \mathrm{e}-04$ & $2.48 \mathrm{e}-02$ & $3.34 \mathrm{e}-04$ & $2.68 \mathrm{e}-02$ & $\tilde{g} \rightarrow \tilde{Z}_{3} t \bar{t}$ \\
\hline $6.13 \mathrm{e}-04$ & $4.79 \mathrm{e}-02$ & $5.66 \mathrm{e}-04$ & $4.53 \mathrm{e}-02$ & $5.21 \mathrm{e}-04$ & $4.18 \mathrm{e}-02$ & $\tilde{g} \rightarrow \tilde{Z}_{4} t \bar{t}$ \\
\hline $1.27 \mathrm{e}-04$ & $9.93 \mathrm{e}-03$ & $1.25 \mathrm{e}-04$ & $1.00 \mathrm{e}-02$ & $1.25 \mathrm{e}-04$ & $1.00 \mathrm{e}-02$ & $\tilde{g} \rightarrow \tilde{Z}_{1} b \bar{b}$ \\
\hline $7.80 \mathrm{e}-04$ & $6.09 \mathrm{e}-02$ & $7.74 \mathrm{e}-04$ & $6.20 \mathrm{e}-02$ & $7.74 \mathrm{e}-04$ & $6.21 \mathrm{e}-02$ & $\tilde{g} \rightarrow \tilde{Z}_{2} b \bar{b}$ \\
\hline $2.20 \mathrm{e}-05$ & $1.72 \mathrm{e}-03$ & $1.77 \mathrm{e}-05$ & $1.42 \mathrm{e}-03$ & $1.78 \mathrm{e}-05$ & $1.43 \mathrm{e}-03$ & $\tilde{g} \rightarrow \tilde{Z}_{3} b \bar{b}$ \\
\hline $3.48 \mathrm{e}-05$ & $2.72 \mathrm{e}-03$ & $3.24 \mathrm{e}-05$ & $2.60 \mathrm{e}-03$ & $3.23 \mathrm{e}-05$ & $2.60 \mathrm{e}-03$ & $\tilde{g} \rightarrow \tilde{Z}_{4} b \bar{b}$ \\
\hline $6.28 \mathrm{e}-04$ & $4.90 \mathrm{e}-02$ & $6.24 \mathrm{e}-04$ & $5.00 \mathrm{e}-02$ & $6.24 \mathrm{e}-04$ & $5.01 \mathrm{e}-02$ & $\tilde{g} \rightarrow \tilde{W}_{1}^{-} u \bar{d}$ \\
\hline $6.28 \mathrm{e}-04$ & $4.90 \mathrm{e}-02$ & $6.24 \mathrm{e}-04$ & $5.00 \mathrm{e}-02$ & $6.24 \mathrm{e}-04$ & $5.01 \mathrm{e}-02$ & $\tilde{g} \rightarrow \tilde{W}_{1}^{+} d \bar{u}$ \\
\hline $6.28 \mathrm{e}-04$ & $4.90 \mathrm{e}-02$ & $6.24 \mathrm{e}-04$ & $5.00 \mathrm{e}-02$ & $6.24 \mathrm{e}-04$ & $5.01 \mathrm{e}-02$ & $\tilde{g} \rightarrow \tilde{W}_{1}^{-} c \bar{s}$ \\
\hline $6.28 \mathrm{e}-04$ & $4.90 \mathrm{e}-02$ & $6.24 \mathrm{e}-04$ & $5.00 \mathrm{e}-02$ & $6.24 \mathrm{e}-04$ & $5.01 \mathrm{e}-02$ & $\tilde{g} \rightarrow \tilde{W}_{1}^{+} s \bar{c}$ \\
\hline $1.20 \mathrm{e}-05$ & $9.36 \mathrm{e}-04$ & $1.19 \mathrm{e}-05$ & $9.56 \mathrm{e}-04$ & $1.19 \mathrm{e}-05$ & $9.58 \mathrm{e}-04$ & $\tilde{g} \rightarrow \tilde{W}_{2}^{-} u \bar{d}$ \\
\hline $1.20 \mathrm{e}-05$ & $9.36 \mathrm{e}-04$ & $1.19 \mathrm{e}-05$ & $9.56 \mathrm{e}-04$ & $1.19 \mathrm{e}-05$ & $9.58 \mathrm{e}-04$ & $\tilde{g}_{\tilde{g}} \rightarrow \tilde{W}_{2}^{+} d \bar{u}$ \\
\hline $1.20 \mathrm{e}-05$ & $9.36 \mathrm{e}-04$ & $1.19 \mathrm{e}-05$ & $9.56 \mathrm{e}-04$ & $1.19 \mathrm{e}-05$ & $9.58 \mathrm{e}-04$ & $\tilde{g} \rightarrow \tilde{W}_{2}^{-} c \bar{s}$ \\
\hline $1.20 \mathrm{e}-05$ & $9.36 \mathrm{e}-04$ & $1.19 \mathrm{e}-05$ & $9.56 \mathrm{e}-04$ & $1.19 \mathrm{e}-05$ & $9.58 \mathrm{e}-04$ & $\tilde{g} \rightarrow \tilde{W}_{2}^{+} s \bar{c}$ \\
\hline $9.29 \mathrm{e}-04$ & $7.25 \mathrm{e}-02$ & $9.21 \mathrm{e}-04$ & $7.38 \mathrm{e}-02$ & $9.21 \mathrm{e}-04$ & $7.39 \mathrm{e}-02$ & $\tilde{g} \rightarrow \tilde{W}_{1}^{-} t \bar{b}$ \\
\hline $9.29 \mathrm{e}-04$ & $7.25 \mathrm{e}-02$ & $9.21 \mathrm{e}-04$ & $7.38 \mathrm{e}-02$ & $9.21 \mathrm{e}-04$ & $7.39 \mathrm{e}-02$ & $\tilde{g} \rightarrow \tilde{W}_{1}^{+} b \bar{t}$ \\
\hline $1.35 \mathrm{e}-03$ & $1.05 \mathrm{e}-01$ & $1.27 \mathrm{e}-03$ & $1.01 \mathrm{e}-01$ & $1.27 \mathrm{e}-03$ & $1.02 \mathrm{e}-01$ & $\tilde{g} \rightarrow \tilde{W}_{2}^{-} t \bar{b}$ \\
\hline $1.35 \mathrm{e}-03$ & $1.05 \mathrm{e}-01$ & $1.27 \mathrm{e}-03$ & $1.01 \mathrm{e}-01$ & $1.27 \mathrm{e}-03$ & $1.02 \mathrm{e}-01$ & $\tilde{g} \rightarrow \tilde{W}_{2}^{+} b \bar{t}$ \\
\hline
\end{tabular}

Table 6: The $\tilde{g}$ decay partial widths and branching ratios as output by SOFTSUSY, sPHENO-3.3.8 [11] and SUSYHIT-1.4 [23] for the spectrum given in Figure 3 for which the gluino only has 3 body decay modes kinematically available. This CMSSM spectrum has $m_{0}=1500 \mathrm{GeV}$, $m_{1 / 2}=400 \mathrm{GeV}, \tan \beta=10.37, \operatorname{sign}(\mu)=+1, A_{0}=-80 \mathrm{GeV}$ and was generated in sPHENO. 


\begin{tabular}{|c|c|c|c|c|}
\hline \multicolumn{2}{|c|}{$\begin{array}{c}\text { SOFTSUSY with } \\
\text { altered runmb }\end{array}$} & \multicolumn{2}{c|}{ sPHENO-3.38 } & mode \\
\hline PW/GeV & BR & PW/GeV & BR & \\
\hline $1.27 \mathrm{e}-04$ & $9.93 \mathrm{e}-03$ & $1.25 \mathrm{e}-04$ & $1.00 \mathrm{e}-02$ & $\tilde{g} \rightarrow \tilde{Z}_{1} b \bar{b}$ \\
\hline $7.78 \mathrm{e}-04$ & $6.10 \mathrm{e}-02$ & $7.74 \mathrm{e}-04$ & $6.20 \mathrm{e}-02$ & $\tilde{g} \rightarrow \tilde{Z}_{2} b \bar{b}$ \\
\hline $1.81 \mathrm{e}-05$ & $1.42 \mathrm{e}-03$ & $1.77 \mathrm{e}-05$ & $1.42 \mathrm{e}-03$ & $\tilde{g} \rightarrow \tilde{Z}_{3} b \bar{b}$ \\
\hline $3.18 \mathrm{e}-05$ & $2.50 \mathrm{e}-03$ & $3.24 \mathrm{e}-05$ & $2.60 \mathrm{e}-03$ & $\tilde{g} \rightarrow \tilde{Z}_{4} b \bar{b}$ \\
\hline
\end{tabular}

Table 7: The $\tilde{g}$ decay partial widths and branching ratios to $\tilde{Z}_{i} b \bar{b}$ as output by SOFTSUSY with runmb taken so that the $b$ Yukawa coupling in SOFTSUSY matches that in sPHENO. These decays showed significant differences between the two programs, particularly for $\tilde{Z}_{3}$ and $\tilde{Z}_{4}$, see Table 6 The agreement is now much improved, demonstrating that the differences result from a choice of the running $b$ mass runmb and hence in the Yukawa coupling. This again used the parameters from sPHENO's spectrum file directly in the SOFTSUSY decay calculator, with the addition relative to Table 6 of sPHENO's running $b$ mass.
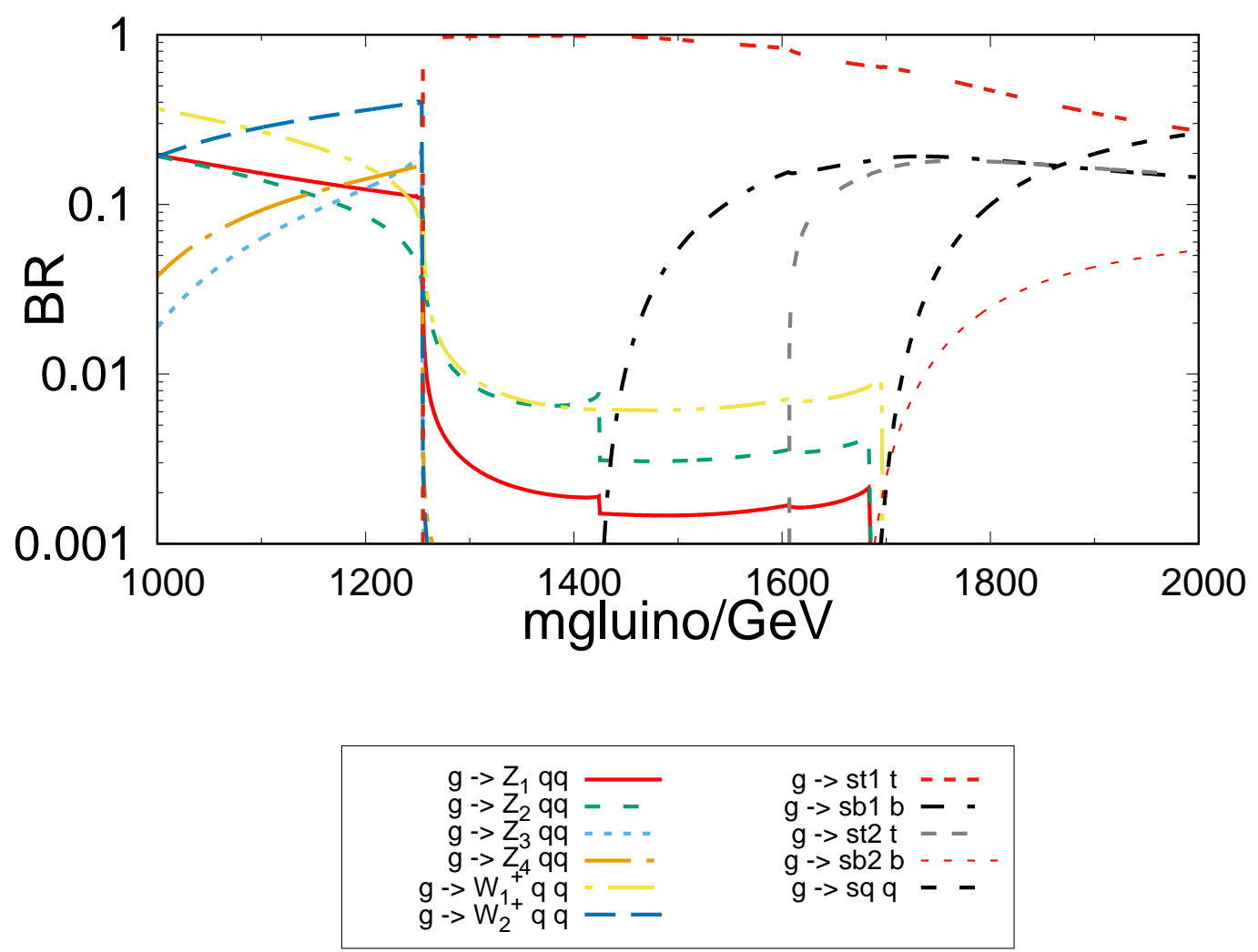

Figure 4: Branching ratios for the gluino $\tilde{g}$ as its mass is increased from 1 to $2 \mathrm{TeV}$. The suppression of 3 body modes relative to 2 body tree level modes is clearly evident in the sudden drop in the 3 body branching ratios once the first 2 body mode $\tilde{g} \rightarrow \tilde{t}_{1} t$ is kinematically available. Note the " $g$ " indicated in the plot are $\tilde{g}$ (i.e. gluinos), whilst " $Z_{i}$ " are $\tilde{Z}_{i}$ (i.e. neutralinos) and " $W_{j}$ " are $\tilde{W}_{j}$ (i.e. charginos). "st" indicates stops $\tilde{t}_{i}$, "sb" indicate sbottoms $\tilde{b}_{i}$, "sq" are $\tilde{q}$ squarks of the first two generations and " $q$ " here are quarks of the first two generations. 


\subsection{NMSSM Decays - H}

Similar detailed checks to those above were performed in the NMSSM fields' sector and we provide some details here. In Table 8 we present a quantitative comparison of the decays of the second heaviest neutral CP even Higgs of the NMSSM, the $H$. The spectrum is given in Figure 5 with decay modes of branching ratios (BRs) greater than 0.1 indicated by arrows, with thicker, bolder arrows representing larger BRs. For this parameter point, $H$ is the $\mathrm{CP}$ even Higgs which has the largest singlet component. Although validation was carried out for all the particle decays of the extended Higgs and neutralinos, for brevity we just provide that of the $H$ here. The comparison in Table 8 demonstrates that the level of agreement is usually better than $10 \%$ with the exception of a few of the decay modes. The decay modes which show larger differences are the decays to "down-type" fermions, i.e. fermions with third component of weak isospin $T_{3}=-\frac{1}{2}$ and the 1-loop decay to two photons $H \rightarrow \gamma \gamma$. Note that the decays to quarks and to two gluons here show good agreement with NMSSMTools: the scale of the decaying Higgs $m_{H}=519.3 \mathrm{GeV}$ is relatively close to $M_{S U S Y}=\sqrt{m_{\tilde{t}_{1}} m_{\tilde{t}_{2}}}=675.5 \mathrm{GeV}$ so any differences in the running between the two programs have little effect. SOFTSUSY and NMSSMTools both run the gauge couplings to $m_{H}$ : however there are potential differences in the running order and scheme. SOFTSUSY matches at $m_{Z}$ and then runs $\alpha_{s}$ in the full NMSSM at 2-loops with 1-loop threshold corrections at $m_{Z}$. For the case of the decays $H \rightarrow s \bar{s}, b \bar{b}, \mu^{+} \mu^{-}$and $\tau^{+} \tau^{-}$, differences are seen between the default SOFTSUSY partial widths and those of NMSSMTools. Some of these differences can be explained by the use of different values for the masses from which Higgs couplings are extracted, particularly in the case of the decays to $b, \mu$ and $\tau$ pairs. SOFTSUSY uses $m_{b}($ pole $)=4.97 \mathrm{GeV}, m_{\mu}\left(M_{S U S Y}\right)=0.103 \mathrm{GeV}$ and $m_{\tau}\left(M_{S U S Y}\right)=1.80 \mathrm{GeV}$; meanwhile NMSSMTools uses $m_{b}=4.54 \mathrm{GeV}, m_{\mu}=0.106 \mathrm{GeV}$ and $m_{\tau}=1.78 \mathrm{GeV}$. However, most of the differences are due to the definition of the $\mathrm{CP}$ even mixing matrix $S$ : the coupling of the singlet-like $H$ to "down-type" fermions is given by $[S(2,2) / \cos (\beta)]^{2}$. SOFTSUSY obtains $S(2,2)=2.71 \times 10^{-2}$, whilst NMSSMTools has $S(2,2)=2.87 \times 10^{-2}$. Given that the partial widths are proportional to the square of the mixing matrix element, this results in an approximate $12 \%$ difference. The SOFTSUSY decay calculation uses the tree-level value $S\left(M_{S U S Y}\right)$, whereas NMSSMTools uses $S$ as extracted from the loop-corrected pole mass matrix. The two choices are equivalent to leading order, and so the numerical difference between the programs is simply a higher order effect. For this reason in columns 5 and 6 of Table 8 , the CP even mixing matrix elements have also been set to those of NMSSMTools to demonstrate that this makes up much of the remaining differences. The other significant difference observed in the partial widths between the default SOFTSUSY results and those of NMSSMTools is in the $\gamma \gamma$ channel. By default SOFTSUSY runs $\alpha$ and quark masses, whereas NMSSMTools runs $\alpha$ but not the quark masses to calculate the Higgs couplings. The quark masses used by SOFTSUSY for this point are $m_{t}\left(m_{H}\right)=144.5 \mathrm{GeV}, m_{b}\left(m_{H}\right)=2.40 \mathrm{GeV}, m_{c}\left(m_{H}\right)=0.57$ $\mathrm{GeV}$ whereas NMSSMTools uses $m_{t}=170.9 \mathrm{GeV}, m_{b}=4.54 \mathrm{GeV}, m_{c}=1.40 \mathrm{GeV}$; meanwhile SOFTSUSY uses $\alpha\left(m_{H}\right)=7.88 \times 10^{-3}$ whereas NMSSMTools obtains $\alpha\left(m_{H}\right)=7.30 \times 10^{-3}$. The difference in the values of $\alpha\left(m_{H}\right)$ is presumably due to a difference in the scheme ${ }^{4}$. With the quark masses and $\alpha$ used by NMSSMTools inserted into the SOFTSUSY decay code the difference between the two programs is dramatically reduced, with them now showing excellent agreement. This clearly demonstrates that the difference observed is due to different quark masses and coupling constants taken, in particular it is the quark masses which have the largest effect here. The reason for such sensitivity to the masses taken is that for this parameter point there is a large cancellation between the $t, W$ and other loop contributions. The degree of the cancellation is consequently heavily dependent upon the top mass used. With SOFTSUSY's choices then the real part of the top loop contribution is $\mathcal{R}\left[I_{t}\right]=8.99 \times 10^{-2}$ and the real part of the $W$ loop contribution $\mathcal{R}\left[I_{W}\right]=-0.114$ whilst the other significant contribution is that of the heaviest chargino $\tilde{W}_{2}$ : $\mathcal{R}\left[I_{\tilde{W}_{2}}\right]=5.53 \times 10^{-2}$, resulting in significant cancellation such that the total of all the particle loop contributions is $(2.65-6.62 i) \times 10^{-2}$. With the quark mass choices of NMSSMTools instead one obtains $\mathcal{R}\left[I_{t}\right]=0.135$ and so the total cancellation is much smaller and the total of all the loop contributions is $(7.16-7.30 i) \times 10^{-2}$, which has a modulus much larger than that obtained using the usual SOFTSUSY choices. Once these are squared this explains the significant discrepancy. Differences seen between the two programs for this channel should be interpreted as an indication of a large theoretical error in the calculation at this order for this parameter point. Note that the comparison is carried out against an old version of NMSSMTools (NMSSMTools-4.2.1) since there only exists an interface between the SOFTSUSY spectrum generator and this old version of NMSSMTools. Therefore comparing with NMSSMTools -4.2 .1$ allowed the effects of the spectrum generator to be isolated as much as possible from other differences in the decay calculations for validation.

${ }^{4}$ SOFTSUSY matches at $m_{Z}$ and then runs $\alpha$ in the full NMSSM at 2-loops. 


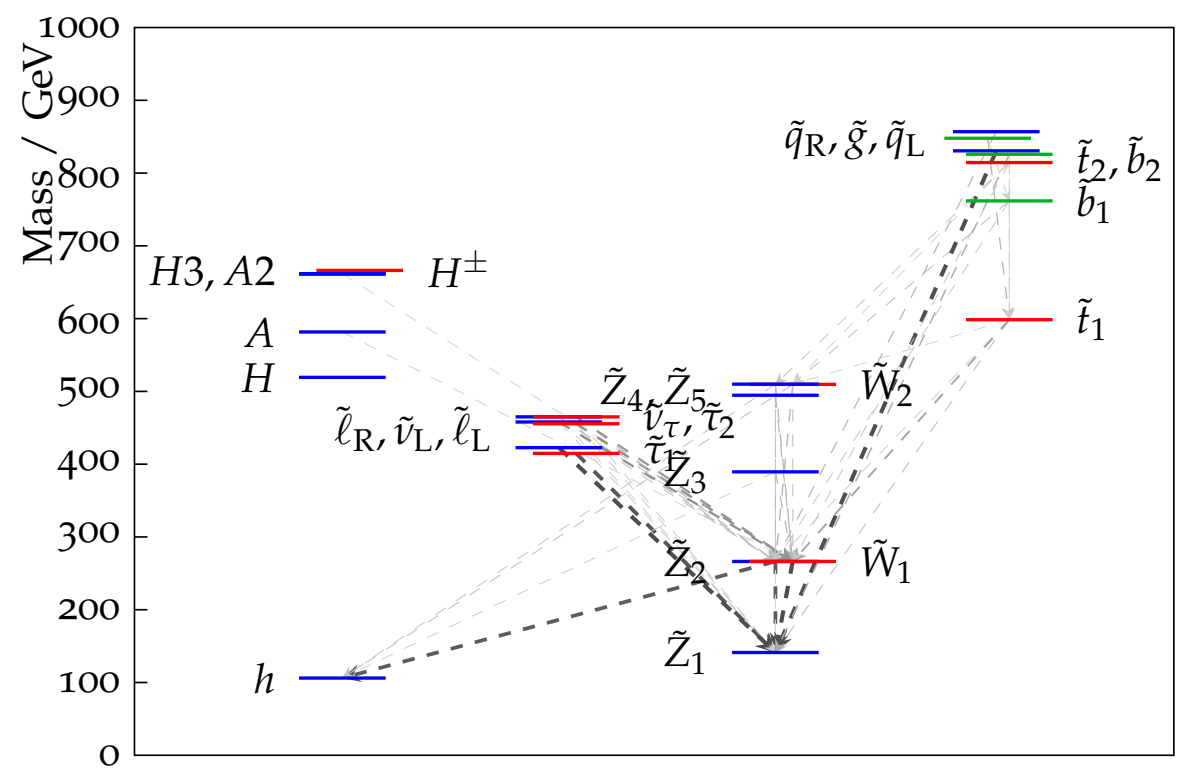

Figure 5: Mass spectrum and branching ratios for the constrained NMSSM parameter point in Table 8 with $m_{0}=400 \mathrm{GeV}, m_{1 / 2}=350 \mathrm{GeV}$, $\tan \beta=10, \operatorname{sign}(\mu)=+1, A_{0}=-300 \mathrm{GeV}, \lambda=0.1, \kappa=0.1, \lambda\langle S\rangle=200 \mathrm{GeV}$ and $\xi_{F}=100 \mathrm{GeV}$ used for the comparison with NMSSMTools. The arrows represent decay modes of branching ratios (BRs) greater than 0.1 , with thicker, bolder arrows representing larger BRs. The figure was produced with the aid of slhaplot-3.0.4 of PySLHA [46].

\section{Summary}

The fast automated computation of the spectrum and decays of particles in the MSSM and the NMSSM is now possible all within SOFTSUSY, and they are necessary steps in the simulation of collider signatures, required for both the prediction and interpretation of the collider signatures. The inclusion of sparticle and Higgs decay partial widths and branching ratios should aid in estimating theoretical uncertainties, particularly in regard to decays in the NMSSM, where there are few other publicly available tools. SOFTSUSY has been routinely used by the ATLAS and CMS experiments to interpret their searches for supersymmetric particles, and so having the decays calculated within the same package as the spectrum should make their calculation easier. The usual SLHA and SLHA2 conventions for input and output have been followed in order to facilitate 'joining up' SOFTSUSY with other observable calculating programs in a bug-free manner, for example with programs that perform Monte-Carlo event simulation.

\section{Acknowledgements}

This work has been partially supported by STFC consolidated grant ST/L000385/1. We thank the Cambridge SUSY working group for helpful discussions and to F Staub for helpful communications regarding SARAH and sPHENO.

\section{Appendix A. Running SOFTSUSY to Calculate Sparticle Decays}

SOFTSUSY produces an executable called softpoint. $x$. It can be run by the command

./softpoint.x leshouches < inOutFiles/lesHouchesInput

where the file inOutFiles/lesHouchesInput contains an ASCII file for input prepared in SUSY Les Houches Accord (SLHA) [36] or SLHA2 [37] format. A SOFTSUSY-specific Block of the SLHA input file is provided in the case that decays are required: 


\begin{tabular}{|c|c|c|c|c|c|c|c|c|}
\hline \multicolumn{2}{|c|}{$\begin{array}{l}\text { SOFTSUSY default } \\
\text { with its quark } \\
\text { masses and coupling } \\
\text { constants run to } m_{H}\end{array}$} & \multicolumn{2}{|c|}{$\begin{array}{c}\text { SOFTSUSY with } \\
\text { NMSSMTools }-4.2 .1 \\
\text { quark masses and } \\
\text { running coupling } \\
\text { constants }\end{array}$} & \multicolumn{2}{|c|}{$\begin{array}{l}\text { SOFTSUSY with } \\
\text { NMSSMTools }-4.2 .1 \\
\text { quark masses, } \\
\text { running coupling } \\
\text { constants and } S\end{array}$} & \multicolumn{2}{|c|}{$\begin{array}{l}\text { NMSSMTools }-4.2 .1 \\
\text { with same QCD } \\
\text { corrections }\end{array}$} & \multirow[t]{2}{*}{ mode } \\
\hline $\mathrm{PW} / \mathrm{GeV}$ & BR & $\mathrm{PW} / \mathrm{GeV}$ & BR & $\mathrm{PW} / \mathrm{GeV}$ & BR & $\mathrm{PW} / \mathrm{GeV}$ & BR & \\
\hline $2.98 \mathrm{e}-06$ & $9.22 \mathrm{e}-06$ & $2.99 \mathrm{e}-06$ & $9.24 \mathrm{e}-06$ & $3.04 \mathrm{e}-06$ & $9.22 \mathrm{e}-06$ & $3.04 \mathrm{e}-06$ & $9.21 \mathrm{e}-06$ & $H \rightarrow c \bar{c}$ \\
\hline $3.65 \mathrm{e}-07$ & $1.13 \mathrm{e}-06$ & $3.67 e-07$ & $1.14 \mathrm{e}-06$ & $4.10 \mathrm{e}-07$ & $1.24 \mathrm{e}-06$ & $4.33 \mathrm{e}-07$ & $1.31 \mathrm{e}-06$ & $H \rightarrow s \bar{s}$ \\
\hline $9.44 \mathrm{e}-04$ & $2.92 \mathrm{e}-03$ & $7.73 \mathrm{e}-04$ & $2.39 \mathrm{e}-03$ & $8.64 \mathrm{e}-04$ & $2.62 \mathrm{e}-03$ & $8.93 \mathrm{e}-04$ & $2.71 \mathrm{e}-03$ & $H \rightarrow b \bar{b}$ \\
\hline $5.58 \mathrm{e}-02$ & $1.73 \mathrm{e}-01$ & $5.58 \mathrm{e}-02$ & $1.73 \mathrm{e}-01$ & $5.68 \mathrm{e}-02$ & $1.72 \mathrm{e}-01$ & $5.68 \mathrm{e}-02$ & $1.72 \mathrm{e}-01$ & $H \rightarrow t \bar{t}$ \\
\hline $2.52 \mathrm{e}-07$ & $7.79 \mathrm{e}-07$ & $2.68 \mathrm{e}-07$ & $8.27 \mathrm{e}-07$ & $2.99 \mathrm{e}-07$ & $9.06 \mathrm{e}-07$ & $3.16 \mathrm{e}-07$ & $9.56 \mathrm{e}-07$ & $H \rightarrow \mu^{-} \mu^{+}$ \\
\hline $7.75 e-05$ & $2.40 \mathrm{e}-04$ & $7.57 \mathrm{e}-05$ & $2.34 \mathrm{e}-04$ & $8.45 e-05$ & $2.56 \mathrm{e}-04$ & $8.92 \mathrm{e}-05$ & $2.70 \mathrm{e}-04$ & $H \rightarrow \tau^{-} \tau^{+}$ \\
\hline $1.21 \mathrm{e}-05$ & $3.73 \mathrm{e}-05$ & $1.21 \mathrm{e}-05$ & $3.74 \mathrm{e}-05$ & $1.33 \mathrm{e}-05$ & $4.04 \mathrm{e}-05$ & $1.22 \mathrm{e}-05$ & $3.70 \mathrm{e}-05$ & $H \rightarrow \tilde{Z}_{1} \tilde{Z}_{1}$ \\
\hline $3.25 \mathrm{e}-05$ & $1.00 \mathrm{e}-04$ & $3.25 \mathrm{e}-05$ & $1.01 \mathrm{e}-04$ & $3.60 \mathrm{e}-05$ & $1.09 \mathrm{e}-04$ & $3.44 \mathrm{e}-05$ & $1.04 \mathrm{e}-04$ & $H \rightarrow \tilde{Z}_{1} \tilde{Z}_{2}$ \\
\hline $2.00 \mathrm{e}-02$ & $6.18 \mathrm{e}-02$ & $2.00 \mathrm{e}-02$ & $6.18 \mathrm{e}-02$ & $2.00 \mathrm{e}-02$ & $6.06 \mathrm{e}-02$ & $2.07 \mathrm{e}-02$ & $6.27 \mathrm{e}-02$ & $H \rightarrow h h$ \\
\hline $9.03 \mathrm{e}-08$ & $2.97 \mathrm{e}-07$ & $1.61 \mathrm{e}-07$ & $4.97 \mathrm{e}-07$ & $1.62 \mathrm{e}-07$ & $4.91 \mathrm{e}-07$ & $1.68 \mathrm{e}-07$ & $5.09 \mathrm{e}-07$ & $H \rightarrow \gamma \gamma$ \\
\hline $1.47 \mathrm{e}-04$ & $4.54 \mathrm{e}-04$ & $1.47 \mathrm{e}-04$ & $4.54 \mathrm{e}-04$ & $1.49 \mathrm{e}-04$ & $4.53 \mathrm{e}-04$ & $1.53 \mathrm{e}-04$ & $4.63 \mathrm{e}-04$ & $H \rightarrow g g$ \\
\hline $2.07 \mathrm{e}-06$ & $6.39 \mathrm{e}-06$ & $1.93 \mathrm{e}-06$ & $5.98 \mathrm{e}-06$ & $2.14 \mathrm{e}-06$ & $6.47 \mathrm{e}-06$ & $2.21 \mathrm{e}-06$ & $6.69 \mathrm{e}-06$ & $H \rightarrow Z \gamma$ \\
\hline $1.67 \mathrm{e}-01$ & $5.15 \mathrm{e}-01$ & $1.67 \mathrm{e}-01$ & $5.15 \mathrm{e}-01$ & $1.70 \mathrm{e}-01$ & $5.16 \mathrm{e}-01$ & $1.70 \mathrm{e}-01$ & $5.15 \mathrm{e}-01$ & $H \rightarrow W^{+} W^{-}$ \\
\hline $8.00 \mathrm{e}-02$ & $2.47 \mathrm{e}-01$ & $8.00 \mathrm{e}-02$ & $2.47 \mathrm{e}-01$ & $8.17 \mathrm{e}-02$ & $2.48 \mathrm{e}-01$ & $8.16 \mathrm{e}-02$ & $2.47 \mathrm{e}-01$ & $H \rightarrow Z Z$ \\
\hline $3.24 \mathrm{e}-01$ & $1.00 \mathrm{e}+00$ & $3.23 \mathrm{e}-01$ & $1.00 \mathrm{e}+00$ & $3.30 \mathrm{e}-01$ & $1.00 \mathrm{e}+00$ & $3.30 \mathrm{e}-01$ & $1.00 \mathrm{e}+00$ & Column totals \\
\hline
\end{tabular}

Table 8: $H$ decay partial widths and branching ratios as output by SOFTSUSY with the quark masses and coupling constants run to $m_{H}$ as is the default (except in cases where QCD corrections are applied - here pole masses must be used), SOFTSUSY with the quark masses and coupling constants set temporarily to those of NMSSMTools for comparison of results, SOFTSUSY with the quark masses and coupling constants and CP even Higgs mixing matrix $(S)$ set temporarily to that of NMSSMTools for comparison of results and NMSSMTools-4.2.1 [13. 27 28]. For columns 3 and 4 this meant setting $\alpha_{s}\left(m_{H}\right)=9.41 \times 10^{-2}, m_{b}=4.54 \mathrm{GeV}$ for $H \rightarrow b b, m_{\mu}=0.106 \mathrm{GeV}$ for $H \rightarrow \mu \mu, m_{\tau}=1.78 \mathrm{GeV}$ for $H \rightarrow \tau \tau$, and the same values were used appropriately for $H \rightarrow g g$. These should be compared with the values SOFTSUSY obtains from running to the $H$ mass of $\alpha_{s}\left(m_{H}\right)=9.42 \times 10^{-2}, m_{\mu}=0.103 \mathrm{GeV}, m_{\tau}=1.80 \mathrm{GeV}$ and its $m_{b}($ pole $)=4.97 \mathrm{GeV}$. Meanwhile for $H \rightarrow \gamma \gamma$, SOFTSUSY uses $m_{t}\left(m_{H}\right)=144.5 \mathrm{GeV}, m_{b}\left(m_{H}\right)=2.40 \mathrm{GeV}, m_{c}\left(m_{H}\right)=0.57 \mathrm{GeV}$ whereas NMSSMTools has $m_{t}=170.9 \mathrm{GeV}, m_{b}=4.54 \mathrm{GeV}, m_{c}=1.40 \mathrm{GeV}$. These were therefore input into SOFTSUSY for columns 3 and 4. In SOFTSUSY $\alpha\left(m_{H}\right)=7.88 \times 10^{-3}$ whereas NMSSMTools obtains $\alpha\left(m_{H}\right)=7.30 \times 10^{-3}$. This is therefore also set in the decay calculator code for columns 3 and 4. Meanwhile it was noticed there were small differences in the smaller elements of the CP even mixing matrix $(S)$ between the two codes, therefore $S$ was additionally set to that of NMSSMTools, on top of the changes described for columns 3 and 4 , in columns 5 and 6 . The parameter point considered is the constrained NMSSM parameter point with $m_{0}=400$ $\mathrm{GeV}, m_{1 / 2}=350 \mathrm{GeV}, \tan \beta=10, \operatorname{sign}(\mu)=+1, A_{0}=-300 \mathrm{GeV}, \lambda=0.1, \kappa=0.1, \lambda\langle S\rangle=200 \mathrm{GeV}$ and $\xi_{F}=100 \mathrm{GeV}$ used for the comparison with NMSSMTools. There is good agreement between the two programs with differences around $10 \%$. Differences can be larger for the decays to quarks and 1-loop decays to $\gamma \gamma$ and $g g$ due to differences in the quark masses and coupling constants taken, or indeed due to small differences in elements of the CP even mixing matrix $S$. Using the same values for these as in NMSSMTools significantly reduces these differences to around 5\% or less. This is illustrated in the improved agreement of the 3rd and 4th columns, and 5th and 6th columns, with the 7th and 8th columns. A more detailed description of the level of agreement and source of differences is given in the text. 
Block SOFTSUSY

$0 \quad 1.000000000 \mathrm{e}+00$
\# Optional SOFTSUSY-specific parameters

\# Calculate decays in output (only for RPC (N)MSSM)

If this block is absent, or if the numerical value is instead 0.000000000 e +00 , then decays will not be calculated. Other input options are available for changing the behaviour of the program as regards sparticle decays:

$\begin{array}{rr}\text { Block } & \text { SOFTSUSY } \\ 24 & 1.000000000 \mathrm{e}-06 \\ 25 & 1.000000000 \mathrm{e}+00 \\ 26 & 0.000000000 \mathrm{e}+00\end{array}$

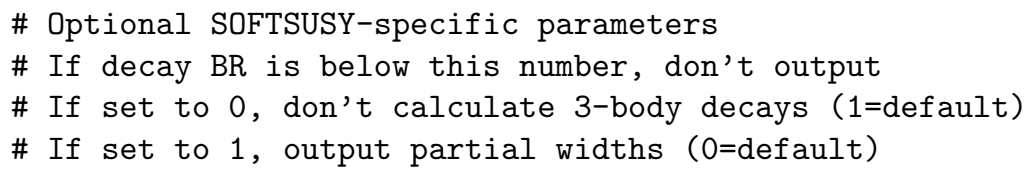

Thus, parameter 24 under this block sets the smallest decay branching ratio that will be output (the default is, as listed, $10^{-6}$ ) whereas parameter 25 can be used to instruct SOFTSUSY not to calculate the 3-body modes, which are more computationally intensive, requiring numerical integration. It should be noted that 3-body modes are not currently included in decays of the NMSSM so for such calculations, regardless of the value of 25, there will be no 3-body modes calculated. Parameter 26 is used to instruct SOFTSUSY to output the partial widths in addition to the branching ratios, this may be useful in making theoretical calculations or performing comparisons with other codes. These partial widths are output as a column beyond the comments \# column so as to not interfere with the SLHA conventions.

If command-line input is required (see Refs. [2, 3] for a full description), the user can use the argument --decays to tell SOFTSUSY to perform the decay calculation. The command line argument -- minBR $=\langle$ value $>$ tells SOFTSUSY which minimum branching ratio to print out in each decay table (where $\langle$ value $\rangle$ is replaced by a numerical value between 0 and 1), whereas the command line argument--dontCalculateThreeBody tells SOFTSUSY not to calculate the 3-body decays of sparticles in order to save time. In addition the command line argument--outputPartialWidths is used to instruct SOFTSUSY to output the partial widths in the final column of the decay tables, beyond the comments so as to allow the output to be read directly into SLHA programs.

In the decay program SOFTSUSY itself there are also additional flags and switches which may be helpful to the user. There are flags at the start of the code named $f l a g<$ name $>$ where one must replace $<$ name $>$ with the particle name, when these flags have value 1 the particle decays are calculated, therefore by default all such flags are set to 1 . These flags allow the user to turn off irrelevant decays for their analyses; for example in producing the scanning plots, such as Figure 4 in Section 5.3, all decays apart from those of the gluino $\tilde{g}$ were turned off by setting all such flags to 0 apart from flaggluino, this ensured all decays output were gluino decays, allowing the plots to be produced more straightforwardly. Similarly there is a Boolean variable QCDcorr, which by default is true, which may be used to turn off QCD corrections. In case the user should want to run the parameters used to different scales, for example in performing comparisons with other decay calculators, it should be noted that running in SOFTSUSY is implemented using MssmSoftsusy and NmssmSoftsusy objects (detailed in references [2] and [3] respectively) and the runto command. If one alters the running scales within SOFTSUSY one must remember to instruct SOFTSUSY to recalculate the $\overline{D R}$ parameters at this scale using calcDRbarPars (). Nonetheless, any changes made to the code are at the user's risk. Finally, given the dependence of many of the partial widths on the input parameters, and in particular on the quark masses used in the cases highlighted previously, users may wish to alter the quark masses $m_{q}$, this may be done in the BLOCK SMINPUTS of the SLHA/SLHA2 input file, the masses used within the decay calculator ("kinematic" and "running") will change accordingly.

\section{Appendix B. Sample Output}

The output comes in the standard SLHA/SLHA2 format [36, 37]. A sample of the part relevant for decays is shown in Table B.9. In concordance with SLHA conventions, the widths/partial widths (PW) are output in units of $\mathrm{GeV}$. The partial widths are output after the comments column so as not to interfere with reading in the decay tables into other programs; this outputting of partial widths can be switched off, as described in Appendix A, so the final column of partial widths in Table B.9 would then not appear. NDA lists the number of daughter particles and PDGi lists the Particle Data Group (PDG) code of daughter i (Section 43 of Ref. [47]). The comment at the end of each line after \# lists the decay mode for easy perusal by the eye. 


\begin{tabular}{|c|c|c|c|c|c|c|}
\hline \# & PDG & & & & & \\
\hline DECAY & 1000021 & $020 \mathrm{e}-$ & 01 & Gluino decays & & \\
\hline$\#$ & $\mathrm{BR}$ & NDA & PDG1 & PDG2 & Comments & PW \\
\hline & $2.13728751 \mathrm{e}-02$ & 2 & 1 & -1000001 & $\# \sim g \rightarrow d \sim d \_L *$ & $3.02052200 \mathrm{e}-01$ \\
\hline & $2.13728751 e-02$ & 2 & -1 & 1000001 & $\# \sim g \rightarrow d b \sim d_{-} L$ & $3.02052200 \mathrm{e}-01$ \\
\hline & $4.53465485 e-02$ & 2 & 1 & -2000001 & $\# \sim g \rightarrow d \sim d_{-} R *$ & $6.40860189 \mathrm{e}-01$ \\
\hline & $4.53465485 e-02$ & 2 & -1 & 2000001 & $\# \sim g \rightarrow d b \sim d_{-} R$ & $6.40860189 \mathrm{e}-01$ \\
\hline & $2.26614800 \mathrm{e}-02$ & 2 & 2 & -1000002 & $\# \sim \mathrm{g} \rightarrow \mathrm{u} \sim \mathrm{u}_{-} \mathrm{L} *$ & $3.20263412 \mathrm{e}-01$ \\
\hline & $2.26614800 \mathrm{e}-02$ & 2 & -2 & 1000002 & $\# \sim$ g $\rightarrow$ ub $u_{-} L$ & $3.20263412 \mathrm{e}-01$ \\
\hline & $4.32957172 e-02$ & 2 & 2 & -2000002 & $\# \sim g \rightarrow u \sim u_{-} R *$ & $6.11876811 \mathrm{e}-01$ \\
\hline & $4.32957172 e-02$ & 2 & -2 & 2000002 & $\# \sim g->$ ub $u_{-} R$ & $6.11876811 \mathrm{e}-01$ \\
\hline & $2.13739410 \mathrm{e}-02$ & 2 & 3 & -1000003 & $\# \sim \tilde{g} \rightarrow \mathrm{s}^{\sim} \mathrm{s}_{-} \mathrm{L} *$ & $3.02067264 \mathrm{e}-01$ \\
\hline & $2.13739410 \mathrm{e}-02$ & 2 & -3 & 1000003 & $\# \sim g->$ sb $\sim s_{-} L$ & $3.02067264 \mathrm{e}-01$ \\
\hline & $4.53479787 e-02$ & 2 & 3 & -2000003 & $\# \sim g->s^{\sim} s_{-} R *$ & $6.40880401 \mathrm{e}-01$ \\
\hline & $4.53479787 e-02$ & 2 & -3 & 2000003 & $\# \sim \tilde{g} \rightarrow s b^{\sim} s_{-} R$ & $6.40880401 \mathrm{e}-01$ \\
\hline & $2.26621068 e-02$ & 2 & 4 & -1000004 & $\# \sim g \rightarrow c \sim c_{-} L *$ & $3.20272270 \mathrm{e}-01$ \\
\hline & $2.26621068 \mathrm{e}-02$ & 2 & -4 & 1000004 & $\# \sim g \rightarrow c b \sim c_{-} L$ & $3.20272270 \mathrm{e}-01$ \\
\hline & $4.32969654 e-02$ & 2 & 4 & -2000004 & $\# \sim g \rightarrow c \sim c_{-} R *$ & $6.11894452 \mathrm{e}-01$ \\
\hline & $4.32969654 e-02$ & 2 & -4 & 2000004 & $\# \sim \mathrm{g} \rightarrow \mathrm{cb} \sim \mathrm{C}_{-} R$ & $6.11894452 \mathrm{e}-01$ \\
\hline & $7.37354606 \mathrm{e}-02$ & 2 & 5 & -1000005 & $\# \sim g->\quad b \quad \sim b_{-} 1 *$ & $1.04206655 \mathrm{e}+00$ \\
\hline & $7.37354606 e-02$ & 2 & -5 & 1000005 & $\# \sim g \rightarrow$ bb $\quad \sim b_{-} 1$ & $1.04206655 \mathrm{e}+00$ \\
\hline & $4.83477101 e-02$ & 2 & 5 & -2000005 & 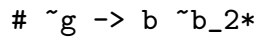 & $6.83274111 \mathrm{e}-01$ \\
\hline & $4.83477101 e-02$ & 2 & -5 & 2000005 & $\# \sim g \rightarrow b b \quad \sim b_{2} 2$ & $6.83274111 \mathrm{e}-01$ \\
\hline & $1.12559217 \mathrm{e}-01$ & 2 & 6 & -1000006 & $\# \sim g \rightarrow t \sim t_{-} 1 *$ & $1.59074336 \mathrm{e}+00$ \\
\hline & $1.12559217 \mathrm{e}-01$ & 2 & -6 & 1000006 & $\# \sim g \rightarrow t b \sim t_{-} 1$ & $1.59074336 \mathrm{e}+00$ \\
\hline
\end{tabular}

$[\ldots]$

Table B.9: Sample Output produced by the SOFTSUSY decay calculator, here only the gluino, $\tilde{g}$, decay table is shown. The input file used was lesHouches Input which is provided with the SOFTSUSY package and has $m_{0}=125 \mathrm{GeV}, m_{1 / 2}=500 \mathrm{GeV}, \tan \beta=10, \operatorname{sign}(\mu)=+1$ and $A_{0}=0$ in the CMSSM. 


\begin{tabular}{|c|c|}
\hline data variable & description \\
\hline string name & particle name \\
\hline double mass & particle mass \\
\hline double PDG & particle PDG code \\
\hline double No_of_Decays & Total Number of possible decays of particle \\
\hline double No_1to2_Decays & Total Number of possible 2 body decays of particle \\
\hline double No_1to3_Decays & Total Number of possible 3 body decays of particle \\
\hline double No_grav_Decays & Total Number of possible decays of particle to LSP gravitinos \\
\hline double No_NMSSM_Decays & Total Number of possible decays of particle in the NMSSM \\
\hline double total_width & Total Decay Width of the particle \\
\hline double two_width & Two-body decay partial width of the particle \\
\hline double three_width & Three-body decay partial width of the particle \\
\hline $\begin{array}{l}\text { vector } \\
<\text { vector }<\text { double }>> \\
\text { Array_Decays }\end{array}$ & $\begin{array}{l}\text { A Nx6 array, where } \mathrm{N}=\text { No_of Decays, containing the PDGs of the daughter } \\
\text { particles in columns } 0 \text { and } 1 \text { (and } 4 \text { for } 3 \text {-body decays), the partial widths of } \\
\text { the decay modes in column } 2 \text {, the number of daughters (NDA) in column } 3 \\
\text { and the branching ratio for each decay mode in column } 5 \text {. }\end{array}$ \\
\hline $\begin{array}{c}\text { vector <string }> \\
\text { Array_Comments }\end{array}$ & $\begin{array}{l}\text { A Nx1 array (vector), where } \mathrm{N}=\text { No_of _Decays, containing a comment for } \\
\text { each decay mode which is output with the decay table, detailing the decay } \\
\text { mode involved, e.g. } \tilde{g} \rightarrow \bar{u} \tilde{u}_{L}\end{array}$ \\
\hline
\end{tabular}

Table C.10: The information contained in the Particle object for each of the decaying particles. PDG codes are given in the reference [37]. Note the numbers of decays contained in double No_..._. Decays are the total number of such decays assuming non are kinematically forbidden. All these decays are checked by the program to see if they are allowed kinematically and calculated if so. All the numbers of decays are in the MSSM unless stated otherwise.

\section{Appendix C. Particle Class}

As part of the extension of SOFTSUSY to include decay calculation a new class has been written, Particle. This is a container for all the relevant decay information of a particle and is used to output the decay tables. We display the class in Table C.10

\section{Appendix D. Glossary: Reference Tables for Decays}

We begin by listing the various decay modes included in SOFTSUSY in some tables, along with equation numbers for quick reference. We group the listings into several tables, each comprising a set of decays: in Table D.11. we list 2 body MSSM tree-level decays, in Table D.12, we list the MSSM Higgs boson decays, in Table D.13, the MSSM 3 body decays included, in Table D.14 we list the decays into gravitinos, in Table D.15 we list the NMSSM decays of neutralinos not already listed above and in Table D.16 we list NMSSM Higgs decay modes. 


\begin{tabular}{|c|c|c|c|}
\hline \multicolumn{2}{|c|}{ Gluino Decays } & \multicolumn{2}{|c|}{ Slepton of first 2 generations decays } \\
\hline$\tilde{g} \rightarrow q \tilde{q}_{L / R}$ & F.1 & $\tilde{l}_{L} \rightarrow l \tilde{Z}_{i}$ & F.27 \\
\hline$\tilde{g} \rightarrow q \tilde{q}_{1 / 2}$ & $\overline{F .2}$ & $\tilde{l}_{R} \rightarrow l \tilde{Z}_{i}$ & F.28 \\
\hline \multicolumn{2}{|c|}{ Squark of first 2 generations decays } & $\tilde{v}_{l} \rightarrow v_{l} \tilde{Z}_{i}$ & $\overline{F .29}$ \\
\hline$\tilde{q}_{L} \rightarrow \tilde{Z}_{i} q$ & F.9 & $\tilde{l}_{L} \rightarrow v_{l} \tilde{W}_{j}^{-}$ & F.30 \\
\hline$\tilde{q}_{L / R} \rightarrow q \tilde{g}$ & F.3 & $\tilde{v}_{l} \rightarrow l \tilde{W}_{j}^{+}$ & F.31 \\
\hline$\tilde{q}_{L} \rightarrow q^{\prime} \tilde{W}_{1 / 2}^{-}$ & F.5 & & \\
\hline$\tilde{q}_{R} \rightarrow \tilde{Z}_{i} q$ & F.10 & & \\
\hline \multicolumn{2}{|c|}{ Squark of 3rd generation decays } & \multicolumn{2}{|c|}{ Slepton of 3rd generation decays } \\
\hline$\tilde{q}_{1 / 2} \rightarrow q \tilde{g}$ & F.4 & $\tilde{\tau}_{1 / 2} \rightarrow \tau \tilde{Z}_{i}$ & F.32 \\
\hline$\tilde{b}_{1 / 2} \rightarrow \tilde{W}_{i}^{-} t$ & F.6 & $\tilde{\tau}_{1 / 2} \rightarrow v_{\tau} \tilde{W}_{i}^{-}$ & F.36 \\
\hline$\tilde{t}_{1 / 2} \rightarrow \tilde{W}_{j}^{+} b$ & F.7 & $\tilde{v}_{\tau} \rightarrow \tau \tilde{W}_{j}^{+}$ & F.37 \\
\hline$\tilde{t}_{1 / 2} \rightarrow \tilde{Z}_{i} t$ & F.11 & $\tilde{\tau}_{1 / 2} \rightarrow \tilde{v}_{\tau} H^{-}$ & F.38 \\
\hline$\tilde{b}_{1 / 2} \rightarrow \tilde{Z}_{i} b$ & F.14 & $\tilde{\tau}_{1 / 2} \rightarrow \tilde{v}_{\tau} W^{-}$ & $\overline{F .39}$ \\
\hline$\tilde{t}_{1 / 2} \rightarrow \tilde{b}_{1 / 2} W^{+}$ & F.17 & $\tilde{v}_{\tau} \rightarrow \tilde{Z}_{i} v_{\tau}$ & F.29 \\
\hline$\tilde{t}_{1 / 2} \rightarrow \tilde{b}_{1 / 2} H^{+}$ & F.18 & $\tilde{v}_{\tau} \rightarrow \tilde{\tau}_{1 / 2} W^{+}$ & $\overline{F .39}$ \\
\hline$\tilde{t}_{2} \rightarrow \phi \tilde{t}_{1}$ & F.20 & $\tilde{v}_{\tau} \rightarrow \tilde{\tau}_{1 / 2} H^{+}$ & F.38 \\
\hline$\tilde{b}_{2} \rightarrow \phi \tilde{b}_{1}$ & F.23 & $\tilde{\tau}_{2} \rightarrow \tilde{\tau}_{1} Z$ & F.40 \\
\hline$\tilde{q}_{2} \rightarrow Z \tilde{q}_{1}$ & F.26 & $\tilde{\tau}_{2} \rightarrow \tilde{\tau}_{1} \phi$ & $\overline{\mathrm{F} .41}$ \\
\hline \multicolumn{2}{|c|}{ Chargino decays } & \multicolumn{2}{|c|}{ Neutralino decays } \\
\hline$\tilde{W}_{i}^{+} \rightarrow \bar{q} \tilde{q}_{L}^{\prime}$ & F.44 & $\tilde{Z}_{i} \rightarrow \bar{q} \tilde{q}_{L} / R$ & F.69 \\
\hline$\tilde{W}_{i}^{+} \rightarrow \bar{b} \tilde{t}_{1 / 2}$ & F.45 & $\tilde{Z}_{i} \rightarrow \tilde{l}_{L} / R$ & F.71 \\
\hline$\tilde{W}_{i}^{+} \rightarrow \tilde{t} \tilde{b}_{1 / 2}$ & F.48 & $\tilde{Z}_{i} \rightarrow \tilde{t}_{1 / 2}$ & $\overline{F .73}$ \\
\hline$\tilde{W}_{i}^{+} \rightarrow \bar{l}_{L}$ & F.52 & $\tilde{Z}_{i} \rightarrow \bar{b} \tilde{b}_{1 / 2}$ & F.77 \\
\hline$\tilde{W}_{i}^{+} \rightarrow \bar{\tau} \tilde{v}_{\tau}$ & F.54 & $\tilde{Z}_{i} \rightarrow \bar{\tau} \tilde{\tau}_{1 / 2}$ & F.80 \\
\hline$\tilde{W}_{i}^{+} \rightarrow \tilde{\tilde{\tau}}_{1 / 2} v_{\tau}$ & F.57 & $\tilde{Z}_{i} \rightarrow W \tilde{W}_{1 / 2}$ & F.85 \\
\hline$\tilde{W}_{1}^{+} \rightarrow W \tilde{Z}_{j}$ & F.59 & $\tilde{Z}_{j} \rightarrow H^{+} \tilde{W}_{1 / 2}$ & F.87 \\
\hline$\tilde{W}_{1}^{+} \rightarrow H^{-} \tilde{Z}_{j}$ & F.61 & $\tilde{Z}_{i} \rightarrow Z \tilde{Z}_{j}$ & F.88 \\
\hline$\tilde{W}_{2} \rightarrow Z \tilde{W}_{1}$ & F.64 & $\tilde{Z}_{i} \rightarrow h \tilde{Z}_{j}$ & F.89 \\
\hline$\tilde{W}_{2} \rightarrow \phi \tilde{W}_{1}$ & F.66 & $\tilde{Z}_{i} \rightarrow A \tilde{Z}_{j}$ & F.91 \\
\hline
\end{tabular}

Table D.11: MSSM 2 body decays included in the SOFTSUSY decay program, the references for the formulae in the appendices are given. $\phi$ here is $h / H / A$ i.e. any of the neutral Higgs bosons. The same references may be given for different decays in cases where the underlying formulae are the same and the necessary replacements for different outgoing particles are given with the formulae. 


\begin{tabular}{|c|c|c|c|}
\hline \multicolumn{2}{|c|}{ CP Even Higgs decays } & \multicolumn{2}{|c|}{ CP Odd Higgs decays } \\
\hline$h / H \rightarrow f \bar{f}$ & \begin{tabular}{|l|l|} 
F.93 & J.1 \\
\end{tabular} & $A \rightarrow f \bar{f}$ & F.95, J.4 \\
\hline$h / H \rightarrow \tilde{Z}_{i} \tilde{Z}_{j}$ & F.97 & $A \rightarrow \tilde{Z}_{i} \tilde{Z}_{j}$ & F.98 \\
\hline$h / H \rightarrow A Z$ & F.107 & $A \rightarrow h Z$ & F.108 \\
\hline$h \rightarrow A A$ & F.103 & $A \rightarrow f_{i} f_{j}^{*}$ & F.124 \\
\hline$H \rightarrow h h$ & F.104 & \multicolumn{2}{|c|}{ CP Even/Odd Higgs decays } \\
\hline$H \rightarrow A A$ & F.105 & $\phi \rightarrow \tilde{W}_{i}^{+} \tilde{W}_{i}^{-}$ & F.99 \\
\hline$H \rightarrow H^{+} H^{-}$ & F.106 & $\phi \rightarrow \tilde{W}_{i}^{+} \tilde{W}_{j}^{-}$ & F.101 \\
\hline$h \rightarrow \tilde{q}_{L / R} \tilde{q}_{L / R}^{*}$ & F.109 & $\phi \rightarrow \gamma \gamma$ & F.145 \\
\hline$H \rightarrow \tilde{q}_{L / R} \tilde{q}_{L / R}^{*}$ & F.111 & $\phi \rightarrow g g$ & \begin{tabular}{|l|l|l|} 
F.190.10 & J.11 \\
\end{tabular} \\
\hline$h \rightarrow \tilde{l}_{L / R} \tilde{l}_{L / R}^{*}$ & F.113 & $\phi \rightarrow Z \gamma$ & F.191 \\
\hline$h \rightarrow \tilde{t}_{i} \tilde{\tau}_{j}^{*}$ & F.115 & \multicolumn{2}{|c|}{ Charged Higgs decays } \\
\hline$h \rightarrow \tilde{b}_{i} \tilde{b}_{j}^{*}$ & F.118 & $H^{+} \rightarrow f \bar{f}^{\prime}$ & F.126 \\
\hline$h \rightarrow \tilde{\tau}_{i} \tilde{\tau}_{j}^{*}$ & F.121 & $H^{+} \rightarrow \tilde{Z}_{i} \tilde{W}_{j}$ & F.127 \\
\hline$h / H \rightarrow Z Z^{*}$ & F.139 & $H^{+} \rightarrow W^{+} h$ & F.134 \\
\hline$h / H \rightarrow W W^{*}$ & F.140 & $H^{+} \rightarrow \tilde{f}_{L / R} \tilde{f}_{L / R}^{\prime}$ & F.135 \\
\hline$h / H \rightarrow W W$ & F.143 & $H^{+} \rightarrow \tilde{f}_{i} \tilde{f}_{j}^{\prime}$ & F.137 \\
\hline$h / H \rightarrow Z Z$ & F.144 & & \\
\hline
\end{tabular}

Table D.12: Higgs decays included in the SOFTSUSY decay program, the references for the formulae in the appendices are given. The same references may be given for different decays in cases where the underlying formulae are the same and the necessary replacements for different outgoing particles are described with the formulae. Multiple references are given for decays where QCD corrections are included, the first reference is the non-QCD corrected decay and the remainder are once QCD corrections are included.

\begin{tabular}{|c|c|c|c|}
\hline \multicolumn{2}{|c|}{ Gluino $1 \rightarrow 3$ decays } & \multicolumn{2}{|c|}{ Neutralino $1 \rightarrow 3$ decays } \\
\hline$\tilde{g} \rightarrow q \bar{q} \tilde{Z}_{i}$ & G.1 & $\tilde{Z}_{i} \rightarrow \tilde{Z}_{j} f \bar{f}$ & G.45 \\
\hline$\tilde{g} \rightarrow t \bar{t} \tilde{Z}_{i}$ & $\overline{\text { G.6 }}$ & $\tilde{Z}_{i} \rightarrow \tilde{W}_{j} f^{\prime} \bar{f}$ & $\overline{\text { G.176 }}$ \\
\hline$\tilde{g} \rightarrow b \bar{b} \tilde{Z}_{i}$ & $\overline{\text { G.6 }}$ & \multicolumn{2}{|c|}{ Chargino $1 \rightarrow 3$ decays } \\
\hline$\tilde{g} \rightarrow q \bar{q}^{\prime} \tilde{W}_{i}^{-}$ & $\overline{\text { G. } 24}$ & $\tilde{W}_{j} \rightarrow \tilde{Z}_{i} \bar{f}^{\prime} f$ & G.176 \\
\hline$\tilde{g} \rightarrow t \bar{b} \tilde{W}_{i}^{-}$ & $\overline{\text { G.24 }}$ & & \\
\hline
\end{tabular}

Table D.13: Three body decays included in the SOFTSUSY decay program, the references for the formulae in the appendices are given. Not all 3 body decays are included as they are naturally suppressed with respect to the 2 body tree level decays. For this reason we have aimed only to incorporate the most phenomenologically relevant 3 body decays, however more may be added in future versions. The same reference is given for neutralino decays to a chargino, fermion and anti-fermion as for the "reverse" decays of a chargino to a neutralino, fermion and anti-fermion as this just results in minus signs in several places in the partial width formulae, which are given in the appendix.

\begin{tabular}{|c|c|c|c|}
\hline$\tilde{g} \rightarrow g \tilde{G}$ & $\mathrm{H} .1$ & $\tilde{q} \rightarrow q \tilde{G}$ & $\mathrm{H} .2$ \\
\hline$\tilde{Z}_{i} \rightarrow \gamma \tilde{G}$ & $\overline{\mathrm{H} .3}$ & $\tilde{l} \rightarrow l \tilde{G}$ & $\mathrm{H} .2$ \\
\hline$\tilde{Z}_{i} \rightarrow \phi \tilde{G}$ & $\overline{\mathrm{H} .5}$ & $\tilde{Z}_{i} \rightarrow Z \tilde{G}$ & $\overline{\mathrm{H} .4}$ \\
\hline
\end{tabular}

Table D.14: The Next-to-Lightest Susy Particle (NLSP) decays to gravitinos included in the program along with the appendix references for their formulae. 


\begin{tabular}{|c|c|c|c|}
\hline \multicolumn{2}{|c|}{ Neutralino Decays } & \multicolumn{2}{|c|}{ Decays into Neutralinos } \\
\hline$\tilde{Z}_{i} \rightarrow \tilde{q}_{L / R} \bar{q}$ & I.151 & $\tilde{q}_{L / R} \rightarrow q \tilde{Z}_{i}$ & I.176 \\
\hline$\tilde{Z}_{i} \rightarrow \tilde{t}_{1 / 2} \bar{t}$ & $\mathrm{I} .155$ & $\tilde{t}_{1 / 2} \rightarrow t \tilde{Z}_{i}$ & I.179 \\
\hline$\tilde{Z}_{i} \rightarrow \tilde{b}_{1 / 2} \bar{b}$ & I.158 & $\tilde{b}_{1 / 2} \rightarrow b \tilde{Z}_{i}$ & I.182 \\
\hline$\tilde{Z}_{i} \rightarrow \tilde{\tau}_{1 / 2} \bar{\tau}$ & I.161 & $\tilde{\tau}_{1 / 2} \rightarrow \tau \tilde{Z}_{i}$ & I.185 \\
\hline$\tilde{Z}_{i} \rightarrow W \tilde{W}_{1 / 2}$ & I.164 & $\tilde{v}_{\tau_{1 / 2}} \rightarrow v_{\tau} \tilde{Z}_{i}$ & I.188 \\
\hline$\tilde{Z}_{i} \rightarrow H^{ \pm} \tilde{W}_{1 / 2}$ & I.167 & $\tilde{W}_{1} \rightarrow H^{ \pm} \tilde{Z}_{j}$ & I.189 \\
\hline$\tilde{Z}_{i} \rightarrow Z \tilde{Z}_{j}$ & I.170 & $\tilde{W}_{1} \rightarrow W \tilde{Z}_{j}$ & $\overline{\mathrm{I} .192}$ \\
\hline$\tilde{Z}_{i} \rightarrow h_{k} \tilde{\bar{Z}}_{j}$ & $\mathrm{I} .172$ & & \\
\hline$\tilde{Z}_{i} \rightarrow A_{k} \tilde{Z}_{j}$ & $\overline{I .174}$ & & \\
\hline
\end{tabular}

Table D.15: The NMSSM decays involving neutralinos that are included in the SOFTSUSY decay program. Note any decays not involving neutralinos or neutral Higgs Bosons are the same as in the MSSM.

\begin{tabular}{|c|c|c|c|}
\hline \multicolumn{2}{|c|}{ CP Even Higgs Decays } & \multicolumn{2}{|c|}{ CP Odd Higgs Decays } \\
\hline$h_{i} \rightarrow f \bar{f}$ & \begin{tabular}{|l|l|} 
I.1 & J.1 \\
\end{tabular} & $A_{i} \rightarrow f \bar{f}$ & \begin{tabular}{|l|l|} 
I.95 & J.4 \\
\end{tabular} \\
\hline$h_{i} \rightarrow \tilde{f}_{L / R} \tilde{f}_{L / R}$ & 1.3 & $A_{i} \rightarrow \tilde{f}_{L} \tilde{f}_{R}$ & I.97 \\
\hline$h_{i} \rightarrow \tilde{f}_{L / R} \tilde{f}_{R / L}$ & I.6 & $A_{i} \rightarrow \tilde{Z}_{j} \tilde{Z}_{k}$ & I.99 \\
\hline$h_{i} \rightarrow \tilde{t}_{j} \tilde{t}_{j}$ & I.8 & $A_{i} \rightarrow \tilde{W}_{j} \tilde{W}_{j}$ & I.101 \\
\hline$h_{i} \rightarrow \tilde{t}_{1} \tilde{t}_{2}$ & 1.10 & $A_{i} \rightarrow \tilde{W}_{1} \tilde{W}_{2}$ & I.103 \\
\hline$h_{i} \rightarrow \tilde{b}_{j} \tilde{b}_{j}$ & II.8 & $A_{i} \rightarrow h_{j} Z$ & I.106 \\
\hline$h_{i} \rightarrow \tilde{b}_{1} \tilde{b}_{2}$ & I.10 & $A_{i} \rightarrow H^{ \pm} W$ & I.108 \\
\hline$h_{i} \rightarrow \tilde{\tau}_{j} \tilde{\tau}_{k}$ & I.14 & $A_{i} \rightarrow \gamma \gamma$ & I.112 \\
\hline$h_{i} \rightarrow \tilde{W}_{j} \tilde{W}_{j}$ & I.18 & $A_{i} \rightarrow Z \gamma$ & I.118 \\
\hline$h_{i} \rightarrow \tilde{W}_{1} \tilde{W}_{2}$ & I. 20 & $A_{i} \rightarrow g g$ & \begin{tabular}{l|l|} 
I. 125.18 \\
\end{tabular} \\
\hline$h_{i} \rightarrow \tilde{Z}_{j} \tilde{Z}_{k}$ & I.23 & \multicolumn{2}{|c|}{ Decays into Higgs Bosons } \\
\hline$h_{i} \rightarrow A_{j} A_{k}$ & I.25 & $\tilde{b}_{2} \rightarrow \tilde{b}_{1} h_{i}$ & I.129 \\
\hline$h_{i} \rightarrow A_{j} Z$ & I.27 & $\tilde{t}_{2} \rightarrow \tilde{t}_{1} h_{i}$ & $\overline{I .133}$ \\
\hline$h_{i} \rightarrow H^{+} H^{-}$ & I.29 & $\tilde{\tau}_{2} \rightarrow \tilde{\tau}_{1} h_{i}$ & I.137 \\
\hline$h_{i} \rightarrow W^{+} H^{-}$ & I.31 & $\tilde{b}_{2} \rightarrow \tilde{b}_{1} A_{i}$ & I.141 \\
\hline$h_{i} \rightarrow Z Z^{*}$ & 1.35 & $\tilde{t}_{2} \rightarrow \tilde{t}_{1} A_{i}$ & I.141 \\
\hline$h_{i} \rightarrow W W^{*}$ & 1.36 & $\tilde{\tau}_{2} \rightarrow \tilde{\tau}_{1} A_{i}$ & $\overline{1.141}$ \\
\hline$h_{i} \rightarrow \mathrm{ZZ}$ & I.35 & $\tilde{W}_{2} \rightarrow \tilde{W}_{1} h_{i}$ & I.145 \\
\hline$h_{i} \rightarrow W W$ & I.36 & $\tilde{W}_{2} \rightarrow \tilde{W}_{1} A_{i}$ & I.148 \\
\hline$h_{i} \rightarrow \gamma \gamma$ & I.44 & $h_{i} \rightarrow h_{j} h_{k}$ & I.32 \\
\hline$h_{i} \rightarrow g g$ & I.83 J.15 & $h_{i} \rightarrow h_{j} h_{k}$ & I.32 \\
\hline$h_{i} \rightarrow Z \gamma$ & I.71 & $A_{2} \rightarrow A h_{i}$ & I.109 \\
\hline
\end{tabular}

Table D.16: The NMSSM decays involving neutral Higgs Bosons that are included in the SOFTSUSY decay program, the references for the formulae in the appendices are given, where two references are given the first is for the leading order case and the second for the QCD-corrected case. Note any decays not involving neutralinos or neutral Higgs Bosons are the same as in the MSSM. 


\section{Appendix E. Kinematic Functions}

Here we begin the list of partial width expressions used in calculating the decay branching ratios in SOFTSUSY, we hope this provides a useful reference. With the exception of the 3 body decays, the majority of these widths were rederived as a form of validation.

The following are a list of commonly occurring functions that arise from the kinematics of the decays: $\tilde{\lambda}^{1 / 2}$ appears as a result of the phase space integration:

$$
\tilde{\lambda}^{1 / 2}\left(m_{1}, m_{2}, m_{3}\right)=\sqrt{\left(1-\left(\frac{m_{2}+m_{3}}{m_{1}}\right)^{2}\right)\left(1-\left(\frac{m_{2}-m_{3}}{m_{1}}\right)^{2}\right)} .
$$

For loop integrals the real and imaginary parts of the loop give the following kinetic factor, where $\tau_{a}=4\left(\frac{m_{a}}{m_{h_{i}}}\right)^{2}$ :

$$
f(\tau)= \begin{cases}{\left[\sin ^{-1}\left(\frac{1}{\sqrt{\tau}}\right)\right]^{2}, \text { for }} & \tau \geq 1 \\ -\frac{1}{4}\left[\ln \left(\frac{1+\sqrt{1-\tau}}{1-\sqrt{1-\tau}}\right)-i \pi\right]^{2}, \text { for } & \tau<1\end{cases}
$$

this is where the real and imaginary parts come from.

For the $Z \gamma$ decay loops, the kinetic factor $g(\tau)$ also occurs:

$$
g(\tau)= \begin{cases}\sqrt{\tau-1} \sin ^{-1}\left(\frac{1}{\sqrt{\tau}}\right), \text { for } & \tau \geq 1, \\ \frac{1}{2} \sqrt{1-\tau}\left[\ln \left(\frac{1+\sqrt{1-\tau}}{1-\sqrt{1-\tau}}\right)-i \pi\right], \text { for } & \tau<1\end{cases}
$$

\section{Appendix F. MSSM Two Body Decay Formulae}

Here we list for ease of reference the formulae for the partial widths of each of the $1 \rightarrow 2$ decay modes incorporated into the decay calculator SOFTSUSY. The $1 \rightarrow 2$ decay widths were all rederived, the book by Baer and Tata [41] was used as a guide, however differences exist relative to their formulae. The formulae provided in SUSYHIT [23, 24, 25] also provided a useful check.

\section{Appendix F.1. Gluinos}

The partial widths for the decays of the gluinos to squarks and quarks are:

$$
\begin{gathered}
\Gamma\left(\tilde{g} \rightarrow q \tilde{q}_{L / R}\right)=\frac{\alpha_{S}}{4} \frac{1}{2 m_{\tilde{g}}}\left(1+\frac{m_{q}^{2}}{m_{\tilde{g}}^{2}}-\frac{m_{\tilde{q}_{L / R}}^{2}}{m_{\tilde{g}}^{2}}\right) \tilde{\lambda}^{1 / 2}\left(m_{\tilde{g}}, m_{q}, m_{\tilde{q}_{L / R}}\right), \\
\Gamma\left(\tilde{g} \rightarrow q \tilde{q}_{1 / 2}\right)=\frac{\alpha_{S}}{4} \frac{1}{2 m_{\tilde{g}}}\left[1+\frac{m_{q}^{2}}{m_{\tilde{g}}^{2}}-\frac{m_{\tilde{q}_{L / R}}^{2}}{m_{\tilde{g}}^{2}} \mp 2 \sin 2 \theta_{q} \frac{m_{q}}{m_{\tilde{g}}}\right] \tilde{\lambda}^{1 / 2}\left(m_{\tilde{g}}, m_{q}, m_{\tilde{q}_{L / R}}\right),
\end{gathered}
$$

where the minus/plus sign applies for $\tilde{q}_{1} / \tilde{q}_{2}$ respectively.

\section{Appendix F.2. Squarks}

The partial widths for the decays of the squarks to quarks are:

$$
\begin{gathered}
\Gamma\left(\tilde{q}_{L / R} \rightarrow q \tilde{g}\right)=\frac{4 \alpha_{S}}{3} \frac{1}{2 m_{\tilde{q}_{L / R}}}\left(1-\frac{m_{q}^{2}}{m_{\tilde{q}_{L / R}^{2}}^{2}}-\frac{m_{\tilde{g}}^{2}}{m_{\tilde{q}_{L / R}^{2}}^{2}}\right) \tilde{\lambda}^{1 / 2}\left(m_{\tilde{q}_{L, R}}, m_{q}, m_{\tilde{g}}\right), \\
\Gamma\left(\tilde{q}_{1 / 2} \rightarrow q \tilde{g}\right)=\frac{4 \alpha_{S}}{3} \frac{1}{2 m_{\tilde{q}_{1 / 2}}}\left(1-\frac{m_{q}^{2}}{m_{\tilde{q}_{1 / 2}^{2}}}-\frac{m_{\tilde{g}}^{2}}{m_{\tilde{q}_{1 / 2}^{2}}^{2}} \pm 2 \sin 2 \theta_{q} \frac{m_{q} m_{\tilde{g}}}{m_{\tilde{q}_{1,2}}^{2}}\right) \tilde{\lambda}^{1 / 2}\left(m_{\tilde{q}_{1,2}}, m_{q}, m_{\tilde{g}}\right),
\end{gathered}
$$


where the minus/plus sign applies for $\tilde{q}_{1} / \tilde{q}_{2}$ respectively.

$$
\Gamma\left(\tilde{q}_{L} \rightarrow q^{\prime} \tilde{W}_{1}^{-}\right)=\frac{g^{2}}{\sin \theta_{L / R}^{2}} \frac{m_{\tilde{q}_{L}}}{16 \pi}\left(1-\frac{m_{\tilde{W}_{1}^{-}}^{2}}{m_{\tilde{q}_{L}}^{2}}-\frac{m_{q}^{2}}{m_{\tilde{q}_{L}}^{2}}\right) \tilde{\lambda}^{1 / 2}\left(m_{\tilde{q}_{L}}, m_{q}, m_{\tilde{W}_{1}^{-}}\right) .
$$

Note here the ' indicates that the quark produced is the opposite type to the squark (so $\tilde{d}_{L}$ produces an up quark for example) and $\theta_{L}$ is for when up-type quarks (i.e. up or charm) are produced and $\theta_{R}$ is for when down-type quarks are produced (i.e. down or strange). The expression (F.5) applies for the first two generations of quarks as no mixing has been accounted for. The formula for decay to $\tilde{W}_{2}^{-}$is $\operatorname{similar}$ but $\left.\sin \theta_{L / R} \rightarrow \cos \theta_{L / R}\right)$. The expressions with sfermion mixing, for the third generation of squarks, are given below.

$$
\begin{aligned}
\Gamma\left(\tilde{b}_{1} \rightarrow \tilde{W}_{1}^{-} t\right)= & \frac{m_{\tilde{b}_{1}}}{16 \pi}\left[\left\{\left(-g \sin \theta_{L} \cos \theta_{b}+f_{b} \cos \theta_{L} \sin \theta_{b}\right)^{2}+\left(-f_{t} \cos \theta_{R} \cos \theta_{b}\right)^{2}\right\}\right. \\
& \times\left(1-\frac{m_{\tilde{W}_{1}}^{2}}{m_{\tilde{b}_{1}}^{2}}-\frac{m_{t}^{2}}{m_{\tilde{b}_{1}}^{2}}\right)+4 \frac{m_{t} m_{\tilde{W}_{1}}}{m_{\tilde{b}_{1}}^{2}}\left(-g \sin \theta_{L} \cos \theta_{b}+f_{b} \cos \theta_{L} \sin \theta_{b}\right) \\
& \left.\times\left(-f_{t} \cos \theta_{R} \cos \theta_{b}\right)\right] \tilde{\lambda}^{1 / 2}\left(m_{\tilde{b}_{1}}, m_{t}, m_{\tilde{W}_{1}}\right), \\
\Gamma\left(\tilde{t}_{1} \rightarrow \tilde{W}_{1}^{+} b\right)= & \frac{m_{\tilde{t}_{1}}}{16 \pi}\left[\left\{\left(-g \sin \theta_{R} \cos \theta_{t}+f_{t} \cos \theta_{R} \sin \theta_{t}\right)^{2}+\left(-f_{b} \cos \theta_{L} \cos \theta_{t}\right)^{2}\right\}\right. \\
& \times\left(1-\frac{m_{\tilde{W}_{1}}^{2}}{m_{\tilde{t}_{1}}^{2}}-\frac{m_{b}^{2}}{m_{\tilde{t}_{1}}^{2}}\right)+4 \frac{m_{b} m_{\tilde{W}_{1}}}{m_{\tilde{t}_{1}}^{2}}\left(-g \sin \theta_{R} \cos \theta_{t}+f_{t} \cos \theta_{R} \sin \theta_{t}\right) \\
& \left.\times\left(-f_{b} \cos \theta_{L} \cos \theta_{t}\right)\right] \tilde{\lambda}^{1 / 2}\left(m_{\tilde{t}_{1}}, b, m_{\tilde{W}_{1}}\right),
\end{aligned}
$$

where

$$
f_{t}=\frac{g m_{t}^{r u n}}{\sqrt{2} m_{W} \sin \beta}, \quad f_{b}=\frac{g m_{b}^{r u n}}{\sqrt{2} m_{W} \cos \beta} .
$$

(For decays of stops and sbottoms to $\tilde{W}_{2}^{+}, \sin \theta_{L / R} \rightarrow \cos \theta_{L / R}$ and $\cos \theta_{L / R} \rightarrow-\sin \theta_{L / R}$, and for decays of $\tilde{b}_{2}$ and $\tilde{t}_{2}$, $\sin \theta_{t / b} \rightarrow \cos \theta_{t / b}$ and $\cos \theta_{t / b} \rightarrow-\sin \theta_{t / b}$.) The squark decays to neutralinos are given by:

$$
\begin{gathered}
\Gamma\left(\tilde{q}_{L} \rightarrow \tilde{Z}_{i} q\right)=\frac{1}{2}\left( \pm g N_{2 i}+\frac{g^{\prime}}{3} N_{1 i}\right) \frac{m_{\tilde{q}_{L}}}{16 \pi}\left(1-\frac{m_{\tilde{Z}_{i}}^{2}}{m_{\tilde{q}_{L}}^{2}}-\frac{m_{q}^{2}}{m_{\tilde{q}_{L}}^{2}}\right) \tilde{\lambda}^{1 / 2}\left(m_{\tilde{q}_{L}}, m_{q}, m_{\tilde{Z}_{i}}\right), \\
\Gamma\left(\tilde{q}_{R} \rightarrow \tilde{Z}_{i} q\right)=\frac{1}{2}\left(\frac{a}{3} g^{\prime} N_{1 i}\right) \frac{m_{\tilde{q}_{R}}}{16 \pi}\left(1-\frac{m_{\tilde{Z}_{i}}^{2}}{m_{\tilde{q}_{R}}^{2}}-\frac{m_{q}^{2}}{m_{\tilde{q}_{R}}^{2}}\right) \tilde{\lambda}^{1 / 2}\left(m_{\tilde{q}_{R}}, m_{q}, m_{\tilde{Z}_{i}}\right),
\end{gathered}
$$

where $a=4$ for up type squarks and $a=-2$ for down type squarks). $N_{j i}$ are neutralino mixing matrix elements. Decays of $\tilde{t}_{1}, \tilde{b}_{1}, \tilde{t}_{2}$ and $\tilde{b}_{2}$ are similar except for the mixing of the $L$ and $R$ parts so we get a linear combination of the two pre-factors involving the neutralino mixing matrix elements $N_{j i}$ with weights which are sines and cosines of the mixing angle $\theta_{t / b}$. In addition the Higgsino components of the neutralinos become important:

$$
\Gamma\left(\tilde{t}_{1} \rightarrow \tilde{Z}_{i} t\right)=\frac{m_{\tilde{t}_{1}}}{8 \pi} \tilde{\lambda}^{1 / 2}\left(m_{\tilde{t}_{1}}, m_{t}, m_{\tilde{Z}_{i}}\right)\left[\frac{1}{2}\left\{a^{2}\left(1-\left(\frac{m_{t}+m_{\tilde{Z}_{i}}}{m_{\tilde{t}_{1}}}\right)^{2}\right)+b^{2}\left(1-\left(\frac{m_{t}-m_{\tilde{Z}_{i}}}{m_{\tilde{t}_{1}}}\right)^{2}\right)\right\}\right],
$$

where

Whilst

$$
\begin{aligned}
& a=\frac{1}{2}\left[\frac{1}{\sqrt{2}} \cos \theta_{t}\left[-g N_{2 i}-\frac{g^{\prime}}{3} N_{1 i}\right]-f_{u} \sin \theta_{t} N_{4 i}+\frac{4}{3 \sqrt{2}} g^{\prime} N_{1 i} \sin \theta_{t}-f_{u} N_{4 i} \cos \theta_{t}\right], \\
& b=\frac{1}{2}\left[\frac{1}{\sqrt{2}} \cos \theta_{t}\left[-g N_{2 i}-\frac{g^{\prime}}{3} N_{1 i}\right]-f_{u} \sin \theta_{t} N_{4 i}-\frac{4}{3 \sqrt{2}} g^{\prime} N_{1 i} \sin \theta_{t}+f_{u} N_{4 i} \cos \theta_{t}\right] .
\end{aligned}
$$

$$
\Gamma\left(\tilde{b}_{1} \rightarrow \tilde{Z}_{i} b\right)=\frac{m_{\tilde{b}_{1}}}{8 \pi} \tilde{\lambda}^{1 / 2}\left(m_{\tilde{b}_{1}}, m_{b}, m_{\tilde{Z}_{i}}\right)\left[a^{2}\left(1-\left(\frac{m_{\tilde{Z}_{i}}+m_{b}}{m_{\tilde{b}_{1}}}\right)^{2}\right)+b^{2}\left(1-\left(\frac{m_{\tilde{Z}_{i}}-m_{b}}{m_{\tilde{b}_{1}}}\right)^{2}\right)\right],
$$


where

$$
\begin{aligned}
& a=\frac{1}{2}\left[\frac{1}{\sqrt{2}}\left(\cos \theta_{b}\left[-N_{1 i} \frac{g^{\prime}}{3}+N_{3 i} g\right)\right]-\sin \theta_{b} N_{3 i} f_{d}-\frac{2}{3 \sqrt{2}} \sin \theta_{b} N_{1 i} g^{\prime}-\cos \theta_{b} f_{d} N_{3 i}\right], \\
& b=\frac{1}{2}\left[\frac{1}{\sqrt{2}}\left(\cos \theta_{b}\left[-N_{1 i} \frac{g^{\prime}}{3}+N_{3 i} g\right)\right]-\sin \theta_{b} N_{3 i} f_{d}+\frac{2}{3 \sqrt{2}} \sin \theta_{b} N_{1 i} g^{\prime}+\cos \theta_{b} f_{d} N_{3 i}\right] .
\end{aligned}
$$

As usual if we instead consider $\tilde{q}_{2}$, make the changes $m_{q_{1}} \rightarrow m_{q_{2}}, \cos \theta_{q} \rightarrow \sin \theta_{q}$ and $\sin \theta_{q} \rightarrow-\cos \theta_{q}$.

$$
\Gamma\left(\tilde{t}_{1} \rightarrow \tilde{b}_{1} W^{+}\right)=\frac{g^{2}}{32 \pi} \frac{m_{\tilde{t}_{1}}^{3}}{m_{W}^{2}} \tilde{\lambda}^{3 / 2}\left(m_{\tilde{t}_{1}}, m_{W}, m_{\tilde{b}_{1}}\right) \cos ^{2} \theta_{t} \cos ^{2} \theta_{b} .
$$

For $\tilde{t}_{2}, \cos \theta_{t} \rightarrow \sin \theta_{t}$, whereas for $\tilde{b}_{2}$ then $\cos \theta_{b} \rightarrow \sin \theta_{b}$. If the sbottoms are the initial states and stops are in the final state then exchange $m_{\tilde{t}_{i}}$ and $m_{\tilde{b}_{i}}$. For the decays to charged Higgs bosons:

$$
\Gamma\left(\tilde{t}_{1} \rightarrow \tilde{b}_{1} H^{+}\right)=\frac{g^{2}}{32 \pi m_{\tilde{t}_{1}} m_{W}^{2}} A^{2} \tilde{\lambda}^{1 / 2}\left(m_{\tilde{t}_{1}}, m_{H^{+}}, m_{\tilde{b}_{1}}\right),
$$

where here

$$
\begin{aligned}
A= & m_{t} m_{b}(\tan \beta+\cot \beta) \sin \theta_{t} \sin \theta_{b}+m_{t}\left(\mu+A_{t} \cot \beta\right) \sin \theta_{t} \cos \theta_{b}+m_{b}\left(\mu+A_{b} \tan \beta\right) \sin \theta_{b} \cos \theta_{t} \\
& +\left(m_{b}^{2} \tan \beta+m_{t}^{2} \cot \beta-m_{W}^{2} \sin 2 \beta\right) \cos \theta_{t} \cos \theta_{b} .
\end{aligned}
$$

If instead we have $\tilde{t}_{2}$ then $\cos \theta_{t} \rightarrow \sin \theta_{t}$ and if we have $\tilde{b}_{2}$ then $\cos \theta_{b} \rightarrow \sin \theta_{b}$ and again if the sbottoms are the initial states and the stops are the final state then exchange $m_{\tilde{t}_{i}}$ and $m_{\tilde{b}_{i}}$.

$$
\Gamma\left(\tilde{t}_{2} \rightarrow \phi \tilde{t}_{1}\right)=\frac{A_{\phi}^{2}}{16 \pi m_{\tilde{t}_{2}}} \tilde{\lambda}^{1 / 2}\left(m_{\tilde{t}_{2}}, m_{\phi}, m_{\tilde{t}_{1}}\right)
$$

where

$$
A_{h}=\frac{g m_{W}}{4} \sin (\beta+\alpha)\left[1-\frac{5}{3} \frac{g^{\prime 2}}{g^{2}}\right] \sin 2 \theta_{t}+\frac{g m_{t}}{2 m_{W} \sin \beta} \cos 2 \theta_{t}\left(A_{t} \cos \alpha+\mu \sin \alpha\right),
$$

$A_{H}$ is similar but we must transform $\cos \alpha \rightarrow-\sin \alpha$ and $\sin \alpha \rightarrow \cos \alpha$, whilst

$$
A_{A}=\frac{g m_{t}}{2 m_{W}}\left(A_{t} \cot \beta+\mu\right) \text {. }
$$

For $\tilde{b}_{2}$ decaying to a Higgs and $\tilde{b}_{1}$ :

$$
\Gamma\left(\tilde{b}_{2} \rightarrow \phi \tilde{b}_{1}\right)=\frac{B_{\phi}^{2}}{16 \pi m_{\tilde{b}_{2}}} \tilde{\lambda}^{1 / 2}\left(m_{\tilde{b}_{2}}, m_{\phi}, m_{\tilde{b}_{1}}\right),
$$

where

$$
B_{h}=g m_{W} \sin (\alpha+\beta) \frac{1}{4}\left[-1+\frac{1}{3} \frac{g^{\prime 2}}{g^{2}} \sin 2 \theta_{b}\right]+g m_{b} \cos 2 \theta_{b} \frac{1}{2 m_{W} \cos \beta}\left[-A_{b} \sin \alpha-\mu \cos \alpha\right],
$$

$B_{H}$ is similar but again we must transform $\cos \alpha \rightarrow-\sin \alpha$ and $\sin \alpha \rightarrow \cos \alpha$, whilst

$$
B_{A}=\frac{g m_{b}}{2 m_{W}}\left(A_{b} \tan \beta+\mu\right) .
$$

For third generation squark decays to $\mathrm{Z}$ bosons we have the following (note that the amplitude is proportional to the sine squared of the mixing so this does not occur for the first two generations):

$$
\Gamma\left(\tilde{q}_{2} \rightarrow Z \tilde{q}_{1}\right)=\frac{g^{2} m_{\tilde{q}_{2}}^{3}}{64 \pi m_{Z}^{2} \cos ^{2} \theta_{W}} \tilde{\lambda}^{3 / 2}\left(m_{\tilde{q}_{2}}, m_{\tilde{q}_{1}}, m_{Z}\right) \cos ^{2} \theta_{q} \sin ^{2} \theta_{q} .
$$


Appendix F.3. Sleptons

$$
\begin{gathered}
\Gamma\left(\tilde{l}_{L} \rightarrow l \tilde{Z}_{i}\right)=\frac{1}{2}\left[g N_{2 i}+g^{\prime} N_{1 i}\right]^{2} \frac{m_{\tilde{l}_{L}}}{16 \pi}\left(1-\frac{m_{\tilde{Z}_{i}}^{2}}{m_{\tilde{l}_{L}}^{2}}-\frac{m_{l}^{2}}{m_{\tilde{l}_{L}}^{2}}\right) \tilde{\lambda}^{1 / 2}\left(m_{\tilde{l}_{L}}, m_{l}, m_{\tilde{Z}_{i}}\right) . \\
\Gamma\left(\tilde{l}_{R} \rightarrow l \tilde{Z}_{i}\right)=\frac{1}{2}\left[g^{\prime} N_{1 i}\right]^{2} \frac{m_{\tilde{l}_{R}}}{16 \pi}\left(1-\frac{m_{\tilde{Z}_{i}}^{2}}{m_{\tilde{l}_{R}}^{2}}-\frac{m_{l}^{2}}{m_{\tilde{l}_{R}}^{2}}\right) \tilde{\lambda}^{1 / 2}\left(m_{\tilde{l}_{R}}, m_{l}, m_{\tilde{Z}_{i}}\right) . \\
\Gamma\left(\tilde{v}_{l} \rightarrow v_{l} \tilde{Z}_{i}\right)=\frac{1}{2}\left[g N_{2 i}-g^{\prime} N_{1 i}\right]^{2} \frac{m_{\tilde{v}_{l}}}{16 \pi}\left(1-\frac{m_{\tilde{Z}_{i}}^{2}}{m_{\tilde{v}_{l}}^{2}}\right)^{2} . \\
\Gamma\left(\tilde{l}_{L} \rightarrow v_{l} \tilde{W}_{1}^{-}\right)=\frac{g^{2} \sin ^{2} \theta_{L}}{16 \pi} m_{\tilde{l}_{L}}\left(1-\frac{m_{\tilde{W}_{1}}^{2}}{m_{\tilde{l}_{L}}^{2}}\right)^{2} .
\end{gathered}
$$

For decays to $\tilde{W}_{2}$ make the replacement $\sin \theta_{L} \rightarrow \cos \theta_{L}$.

$$
\Gamma\left(\tilde{v}_{l} \rightarrow l \tilde{W}_{1}^{+}\right)=\frac{g^{2} \sin ^{2} \theta_{R}}{16 \pi} m_{\tilde{v}_{l}}\left(1-\frac{m_{\tilde{W}_{1}}^{2}}{m_{\tilde{v}_{l}}^{2}}-\frac{m_{l}^{2}}{m_{\tilde{v}_{l}}^{2}}\right)^{2} \tilde{\lambda}^{1 / 2}\left(m_{\tilde{v}_{l}}, m_{l}, m_{\tilde{W}_{1}}\right) .
$$

For decays to $\tilde{W}_{2}$ make the replacement $\sin \theta_{R} \rightarrow \cos \theta_{R}$.

$$
\Gamma\left(\tilde{\tau}_{1} \rightarrow \tau \tilde{Z}_{i}\right)=\frac{m_{\tilde{\tau}_{1}}}{8 \pi}\left[a^{2}\left(1-\left(\frac{m_{\tau}+m_{\tilde{Z}_{i}}}{m_{\tilde{\tau}_{1}}}\right)^{2}\right)+b^{2}\left(1-\left(\frac{m_{\tau}-m_{\tilde{Z}_{i}}}{m_{\tilde{\tau}_{1}}}\right)^{2}\right)\right] \tilde{\lambda}^{1 / 2}\left(m_{\tilde{\tau}_{1}}, m_{\tau}, m_{\tilde{Z}_{i}}\right),
$$

where

$$
\begin{aligned}
& a=\frac{1}{2}\left[\frac{1}{\sqrt{2}} \sin \theta_{\tau}\left(g N_{2 i}+g^{\prime} N_{1 i}\right)+f_{\tau} N_{3 i} \cos \theta \tau-\sqrt{2} g^{\prime} N_{1 i} \cos \theta \tau+f_{\tau} N_{3 i} \sin \theta \tau\right], \\
& b=\frac{1}{2}\left[\frac{1}{\sqrt{2}} \sin \theta_{\tau}\left(g N_{2 i}+g^{\prime} N_{1 i}\right)+f_{\tau} N_{3 i} \cos \theta \tau+\sqrt{2} g^{\prime} N_{1 i} \cos \theta \tau+f_{\tau} N_{3 i} \sin \theta \tau\right],
\end{aligned}
$$

and

$$
f_{\tau}=\frac{g m_{\tau}}{\sqrt{2} m_{W} \cos \beta} .
$$

For $\tilde{\tau}_{2}$ decaying replace $m_{\tilde{\tau}_{1}} \rightarrow m_{\tilde{\tau}_{2}}, \cos \theta_{\tau} \rightarrow \sin \theta_{\tau}$ and $\sin \theta \tau \rightarrow-\cos \theta \tau$.

$$
\Gamma\left(\tilde{\tau}_{1} \rightarrow v_{\tau} \tilde{W}_{1}^{-}\right)=\left[-g \sin \theta_{L} \sin \theta_{\tau}-f_{\tau} \cos \theta_{L} \cos \theta_{\tau}\right]^{2} m_{\tilde{\tau}_{1}}\left(1-\frac{m_{\tilde{W}_{1}}^{2}}{m_{\tilde{\tau}_{1}}^{2}}\right)^{2}
$$

For decays to $\tilde{W}_{2}$ make the replacements $m_{\tilde{W}_{1}} \rightarrow m_{\tilde{W}_{2}}, \sin \theta_{L} \rightarrow \cos \theta_{L}$ and $\cos \theta_{L} \rightarrow-\sin \theta_{L}$, meanwhile for $\tilde{\tau}_{2}$ decays change $m_{\tilde{\tau}_{1}} \rightarrow m_{\tilde{\tau}_{2}}, \cos \theta_{\tau} \rightarrow \sin \theta_{\tau}$ and $\sin \theta_{\tau} \rightarrow-\cos \theta_{\tau}$.

$$
\begin{aligned}
\Gamma\left(\tilde{v}_{\tau} \rightarrow \tau \tilde{W}_{1}^{+}\right)=\frac{m_{\tilde{v}_{\tau}}}{16 \pi}[ & \left(g^{2} \sin ^{2} \theta_{R}+f_{\tau}^{2} \cos ^{2} \theta_{L}\right)\left(1-\frac{m_{\tilde{W}_{1}}}{m_{\tilde{v}_{\tau}}^{2}}-\frac{m_{\tau}^{2}}{m_{\tilde{v}_{\tau}}^{2}}\right) \\
& \left.-4 \frac{m_{\tau} m_{\tilde{W}_{1}}}{m_{\tilde{v}_{\tau}}^{2}} g \sin \theta_{R} f_{\tau} \cos \theta_{L}\right] \tilde{\lambda}^{1 / 2}\left(m_{\tilde{v}_{\tau}}, m_{\tau}, m_{\tilde{W}_{1}}\right) .
\end{aligned}
$$

For decays to $\tilde{W}_{2}$ then make the replacements $m_{\tilde{W}_{1}} \rightarrow m_{\tilde{W}_{2}}, \sin \theta_{R} \rightarrow \cos \theta_{R}$ and $\cos \theta_{L} \rightarrow-\sin \theta_{L}$.

$$
\Gamma\left(\tilde{\tau}_{1} \rightarrow \tilde{v}_{\tau} H^{-}\right)=\frac{g^{2}}{32 \pi m_{W}^{2} m_{\tilde{\tau}_{1}}}\left[m_{\tau}^{2} \tan \beta \sin \theta_{\tau}-m_{\tau}\left(\mu+A_{\tau} \tan \beta\right) \cos \theta_{\tau}\right]^{2} \tilde{\lambda}^{1 / 2}\left(m_{\tilde{\tau}_{1}}, m_{\tilde{\nu}_{\tau}}, m_{H}^{-}\right) .
$$


For $\tilde{\tau}_{2}$ decays then one must make the changes $m_{\tilde{\tau}_{1}} \rightarrow m_{\tilde{\tau}_{2}}, \cos \theta_{\tau} \rightarrow \sin \theta_{\tau}$ and $\sin \theta_{\tau} \rightarrow-\cos \theta_{\tau}$ as usual.

$$
\Gamma\left(\tilde{\tau}_{1} \rightarrow \tilde{v}_{\tau} W^{-}\right)=\frac{g^{2} \sin ^{2} \theta_{\tau} m_{\tilde{\tau}_{1}}^{3}}{32 \pi m_{W}^{2}} \tilde{\lambda}^{3 / 2}\left(m_{\tilde{\tau}_{1}}, m_{\tilde{\nu}_{\tau}}, m_{W}\right)
$$

The equations for $\tilde{\tau}_{1 / 2} \rightarrow \tilde{v}_{\tau} W^{-}$and $\tilde{\tau}_{1 / 2} \rightarrow \tilde{v}_{\tau} H^{-}$can be used for $\tilde{v}_{\tau} \rightarrow \tilde{\tau}_{1 / 2} W^{-}$and $\tilde{v}_{\tau} \rightarrow \tilde{\tau}_{1 / 2} H^{-}$be interchanging the $m_{\tilde{\tau}_{1 / 2}} \leftrightarrow m_{\tilde{v}_{\tau}}$.

$$
\begin{gathered}
\Gamma\left(\tilde{\tau}_{2} \rightarrow \tilde{\tau}_{1} Z\right)=\frac{g^{2} \sin ^{2} \theta_{\tau} \cos ^{2} \theta_{\tau} m_{\tilde{\tau}_{2}}^{3}}{64 \pi m_{Z}^{2} \cos ^{2} \theta_{W}} \tilde{\lambda}^{3 / 2}\left(m_{\tilde{\tau}_{2}} \cdot m_{\tilde{\tau}_{1}}, m_{Z}\right), \\
\Gamma\left(\tilde{\tau}_{2} \rightarrow \tilde{\tau}_{1} \phi\right)=\frac{\tilde{A}_{\phi}^{2}}{16 \pi m_{\tilde{\tau}_{2}}} \tilde{\lambda}^{1 / 2}\left(m_{\tilde{\tau}_{2}}, m_{\tilde{\tau}_{1}}, m_{\phi}\right),
\end{gathered}
$$

where $\tilde{A}_{h}$ is

$$
\tilde{A}_{h}=\frac{-g m_{w}}{4} \sin (\alpha+\beta) \sin 2 \theta_{\tau}\left[-1+3 \frac{g^{\prime 2}}{g^{2}}\right]+\frac{g m_{\tau}}{2 m_{W} \cos \beta} \cos 2 \theta_{\tau}\left(\mu \cos \alpha+A_{\tau} \sin \alpha\right),
$$

$\tilde{A}_{H}$ is the same as $\tilde{A}_{h}$ but with the changes $\cos \alpha \rightarrow-\sin \alpha$ and $\sin \alpha \rightarrow \cos \alpha$, meanwhile $\tilde{A}_{A}$ is:

$$
\tilde{A}_{A}=\frac{g m_{\tau}}{2 m_{W}}\left(\mu+A_{\tau} \tan \beta\right)
$$

Appendix F.4. Charginos

$$
\Gamma\left(\tilde{W}_{1} \rightarrow \bar{q} \tilde{q}_{L}^{\prime}\right)=\frac{3 m_{\tilde{W}_{1}}}{32 \pi}\left(g^{2} \sin ^{2} \theta_{L / R}\right)\left(1-\frac{m_{\tilde{q}_{L}}^{2}}{m_{\tilde{W}_{1}}^{2}}+\frac{m_{q}^{2}}{m_{\tilde{W}_{1}}^{2}}\right) \tilde{\lambda}^{1 / 2}\left(m_{\tilde{W}_{1}}, m_{q}, m_{\tilde{q}_{L}}\right) .
$$

Note here the' on the squark indicates it's of the opposite $S U(2)_{L}$ type to the quark, e.g. if the quark is an up then the squark is a $\tilde{d}_{L}$. Also note that $\theta_{L}$ occurs when up-type quarks (i.e. up or charm) are produced and $\theta_{R}$ is when down-type quarks are produced (i.e. down or strange). (The formula for decay of $\tilde{W}_{2}^{-}$is similar but we must change $\left.\sin \theta_{L / R} \rightarrow \cos \theta_{L / R}\right)$.

$$
\Gamma\left(\tilde{W}_{1}^{+} \rightarrow \bar{b} \tilde{t}_{1}\right)=\frac{3 m_{\tilde{W}_{1}}}{32 \pi}\left[\left(\mathcal{A}^{2}+C^{2} \sin ^{2} \theta_{t}\right)\left(1-\frac{m_{\tilde{t}_{1}}^{2}}{m_{\tilde{W}_{1}}^{2}}+\frac{m_{b}^{2}}{m_{\tilde{W}_{1}}^{2}}\right)+4 \mathcal{A} C \sin \theta_{t} \frac{m_{b}}{m_{\tilde{W}_{1}}}\right] \tilde{\lambda}^{1 / 2}\left(m_{\tilde{W}_{1}}, m_{b}, m_{\tilde{t}_{1}}\right),
$$

where

$$
\begin{gathered}
\mathcal{A}=g \sin \theta_{R} \cos \theta_{t}-f_{u} \cos \theta_{R} \sin \theta_{t}, \\
C=-f_{d} \cos \theta_{L} .
\end{gathered}
$$

For $\tilde{t}_{2}$ take $\cos \theta_{t} \rightarrow \sin \theta_{t}, \sin \theta_{t} \rightarrow-\cos \theta_{t}$ and $m_{\tilde{t}_{1}} \rightarrow m_{\tilde{t}_{2}}$. For $\tilde{W}_{2}$ take $\cos \theta_{R} \rightarrow-\sin \theta_{R}, \cos \theta_{L} \rightarrow-\sin \theta_{L}$ and $\sin \theta_{R} \rightarrow \cos \theta_{R}$, and also $m_{\tilde{W}_{1}} \rightarrow m_{\tilde{W}_{2}}$.

$$
\Gamma\left(\tilde{W}_{1}^{+} \rightarrow \tilde{t} \tilde{b}_{1}\right)=\frac{3 m_{\tilde{W}_{1}}}{32 \pi}\left[\left(\mathcal{A}^{2}+C^{2} \cos ^{2} \theta_{b}\right)\left(1-\frac{m_{\tilde{b}_{1}}^{2}}{m_{\tilde{W}_{1}}^{2}}+\frac{m_{t}^{2}}{m_{\tilde{W}_{1}}^{2}}\right)+4 \mathcal{A C} \cos \theta_{b} \frac{m_{t}}{m_{\tilde{W}_{1}}}\right] \tilde{\lambda}^{1 / 2}\left(m_{\tilde{W}_{1}}, m_{t}, m_{\tilde{b}_{1}}\right),
$$

where now

$$
\begin{gathered}
\mathcal{A}=-g \sin \theta_{L} \cos \theta_{b}+f_{d} \cos \theta_{L} \sin \theta_{b}, \\
C=f_{u} \cos \theta_{R}, \\
f_{t}=\frac{g m_{t}^{r u n}}{\sqrt{2} m_{W} \sin \beta}, \quad f_{b}=\frac{g m_{b}^{r u n}}{\sqrt{2} m_{W} \cos \beta} .
\end{gathered}
$$


For $\tilde{b}_{2}$ take $\cos \theta_{b} \rightarrow \sin \theta_{b}, \sin \theta_{b} \rightarrow-\cos \theta_{b}$ and o $m_{\tilde{b}_{1}} \rightarrow m_{\tilde{b}_{2}}$. For $\tilde{W}_{2}$ take $\cos \theta_{R} \rightarrow-\sin \theta_{R}, \cos \theta_{L} \rightarrow-\sin \theta_{L}$ and $\sin \theta_{L} \rightarrow \cos \theta_{L}$, and also $m_{\tilde{W}_{1}} \rightarrow m_{\tilde{W}_{2}}$.

$$
\Gamma\left(\tilde{W}_{i}^{+} \rightarrow \widetilde{l}_{L}\right)=\frac{m_{\tilde{W}_{i}}}{32 \pi} A^{2}\left(1-\frac{m_{\tilde{L}_{L}}^{2}}{m_{\tilde{W}_{i}}^{2}}+\frac{m_{l}^{2}}{m_{\tilde{W}_{i}}^{2}}\right) \tilde{\lambda}^{1 / 2}\left(m_{\tilde{W}_{i}}, m_{l}, m_{\tilde{L}_{L}}\right),
$$

where

$$
A= \begin{cases}-g \sin \theta_{L / R}, & \text { for } \tilde{W}_{1} \\ -g \cos \theta_{L / R}, & \text { for } \tilde{W}_{2}\end{cases}
$$

$\theta_{L}$ is used for decays to $v_{l}$ and $\theta_{R}$ for decays to $\tilde{v}_{l_{L}}$.

$$
\Gamma\left(\tilde{W}_{i}^{+} \rightarrow \bar{\tau} \tilde{v}_{\tau}\right)=\frac{m_{\tilde{W}_{i}}}{32 \pi}\left[\left(A^{2}+B^{2}\right)\left(1-\frac{m_{\tilde{v}_{\tau}}^{2}}{m_{\tilde{W}_{i}}^{2}}+\frac{m_{\tau}^{2}}{m_{\tilde{W}_{i}}^{2}}\right)+4 A B \frac{m_{\tau}}{m_{\tilde{W}_{i}}}\right] \tilde{\lambda}^{1 / 2}\left(m_{\tilde{W}_{i}}, m_{\tau}, m_{\tilde{V}_{\tau}}\right)
$$

where

$$
\begin{gathered}
A= \begin{cases}g \sin \theta_{R}, & \text { for } \tilde{W}_{1}, \\
g \cos \theta_{R}, & \text { for } \tilde{W}_{2},\end{cases} \\
B= \begin{cases}-f_{\tau} \cos \theta_{L}, & \text { for } \tilde{W}_{1}, \\
f_{\tau} \sin \theta_{L}, & \text { for } \tilde{W}_{2},\end{cases}
\end{gathered}
$$

and $f_{\tau}$ has been given before in $\overline{\text { F.35 }}$.

$$
\Gamma\left(\tilde{W}_{i}^{+} \rightarrow \bar{\tau}_{1} v_{\tau}\right)=\frac{m_{\tilde{W}_{i}}}{32 \pi} \mathcal{A}^{2}\left(1-\frac{m_{\tilde{\tau}_{1}}^{2}}{m_{\tilde{W}_{i}}^{2}}\right)^{2},
$$

where

$$
\mathcal{A}=-g \sin \theta_{L} \sin \theta_{\tau}-f_{\tau} \cos \theta_{L} \cos \theta_{\tau} .
$$

For $\tilde{W}_{2}$ make the replacements $\cos \theta_{L} \rightarrow-\sin \theta_{L}, \sin \theta_{L} \rightarrow \cos \theta_{L}$ and $m_{\tilde{W}_{1}} \rightarrow m_{\tilde{W}_{2}}$. For $\tilde{\tau}_{2}$ make the replacements $\cos \theta_{\tau} \rightarrow \sin \theta_{\tau}, \sin \theta_{\tau} \rightarrow-\cos \theta_{\tau}$ and $m_{\tilde{\tau}_{1}} \rightarrow m_{\tilde{\tau}_{2}}$.

$$
\begin{array}{r}
\Gamma\left(\tilde{W}_{1}^{+} \rightarrow W \tilde{Z}_{j}\right)=\frac{g^{2}}{16 \pi\left|m_{\tilde{W}_{1}}\right|} \tilde{\lambda}^{1 / 2}\left(m_{\tilde{W}_{1}}, m_{W}, m_{\tilde{Z}_{j}}\right)\left[( X ^ { 2 } + Y ^ { 2 } ) \left(m_{\tilde{W}_{1}}^{2}+m_{\tilde{Z}_{j}}^{2}-m_{W}^{2}\right.\right. \\
\left.\left.\left.+\frac{1}{m_{W}^{2}}\left\{\left(m_{\tilde{W}_{1}}^{2}-m_{\tilde{Z}_{j}}^{2}\right)^{2}-m_{W}^{4}\right\}\right)-6\left(X^{2}-Y^{2}\right) m_{\tilde{W}_{1}} m_{\tilde{Z}_{j}}\right)\right],
\end{array}
$$

where

$$
X=\frac{1}{2}\left[\cos \theta_{R} N_{4 j} \frac{1}{\sqrt{2}}-\sin \theta_{R} N_{2 j}-\cos \theta_{L} N_{3 j} \frac{1}{\sqrt{2}}-\sin \theta_{L} N_{2 j}\right] .
$$

$Y$ is the same as $X$ except the first two terms change sign. For $\tilde{W}_{2}$ transform $\cos \theta_{L} \rightarrow-\sin \theta_{L}, \sin \theta_{L} \rightarrow \cos \theta_{L}$, $\cos \theta_{R} \rightarrow-\sin \theta_{R}, \sin \theta_{R} \rightarrow \cos \theta_{R}$ and change $m_{\tilde{W}_{1}} \rightarrow m_{\tilde{W}_{2}}$.

$$
\begin{array}{r}
\Gamma\left(\tilde{W}_{1}^{+} \rightarrow H^{+} \tilde{Z}_{j}\right)=\frac{1}{16 \pi\left|m_{\tilde{W}_{1}}\right|} \tilde{\lambda}^{1 / 2}\left(m_{\tilde{W}_{1}}, m_{H^{-}}, m_{\tilde{Z}_{j}}\right)\left[\left(a^{2}+b^{2}\right)\left(m_{\tilde{W}_{1}}^{2}+m_{\tilde{Z}_{j}}^{2}-m_{H^{-}}^{2}\right)\right. \\
\left.+2\left(a^{2}-b^{2}\right) m_{\tilde{W}_{1}} m_{\tilde{Z}_{j}}\right],
\end{array}
$$

where

$$
\begin{aligned}
& a=\frac{1}{2}\left(-\cos \beta A_{2}+\sin \beta A_{4}\right), \\
& b=\frac{1}{2}\left(-\cos \beta A_{2}-\sin \beta A_{4}\right),
\end{aligned}
$$


and

$$
\begin{aligned}
& A_{2}=-\frac{1}{\sqrt{2}}\left[g N_{2 j}+g^{\prime} N_{1 j}\right] \cos \theta_{R}-g N_{4 j} \sin \theta_{R}, \\
& A_{4}=-\frac{1}{\sqrt{2}}\left[g N_{2 j}+g^{\prime} N_{1 j}\right] \cos \theta_{L}+g N_{3 j} \sin \theta_{L} .
\end{aligned}
$$

For $\tilde{W}_{2}$ change $\cos \theta_{L} \rightarrow-\sin \theta_{L}, \sin \theta_{L} \rightarrow \cos \theta_{L}, \cos \theta_{R} \rightarrow-\sin \theta_{R}, \sin \theta_{R} \rightarrow \cos \theta_{R}$ and $m_{\tilde{W}_{1}} \rightarrow m_{\tilde{W}_{2}}$.

$$
\begin{array}{r}
\Gamma\left(\tilde{W}_{2} \rightarrow Z \tilde{W}_{1}\right)=\frac{1}{64 \pi m_{\tilde{W}_{2}}} \tilde{\lambda}^{1 / 2}\left(m_{\tilde{W}_{2}}, m_{Z}, m_{\tilde{W}_{1}}\right) \frac{\left(g g^{\prime}\right)^{2}}{g^{2}+g^{\prime 2}}\left[( \frac { g } { g ^ { \prime } } + \frac { g ^ { \prime } } { g } ) ^ { 2 } \left[\left(x^{2}+y^{2}\right)\left(m_{\tilde{W}_{2}}^{2}+m_{\tilde{W}_{1}}^{2}-m_{Z}^{2}+\frac{1}{m_{W}^{2}}\left(\left(m_{\tilde{W}_{1}}^{2}-m_{\tilde{W}_{2}}^{2}\right)^{2}-m_{Z}^{4}\right)\right)\right.\right. \\
\left.+6\left(x^{2}-y^{2}\right) m_{\tilde{W}_{1}} m_{\tilde{W}_{2}}\right], \quad \text { (F.64) }
\end{array}
$$

where

$$
x=\frac{1}{2}\left(\sin \theta_{L} \cos \theta_{L}-\sin \theta_{R} \cos \theta_{R}\right),
$$

and $y$ is the same as $x$ except the second term changes sign.

$$
\Gamma\left(\tilde{W}_{2} \rightarrow \phi \tilde{W}_{1}\right)=\frac{g^{2}}{32 \pi m_{\tilde{W}_{2}}} \tilde{\lambda}^{1 / 2}\left(m_{\tilde{W}_{2}}, m_{\phi}, m_{\tilde{W}_{1}}\right)\left[\left(S_{\phi}^{2}+P_{\phi}^{2}\right)\left(m_{\tilde{W}_{2}}^{2}+m_{\tilde{W}_{1}}^{2}-m_{\phi}^{2}\right)+2\left(S_{\phi}^{2}-P_{\phi}^{2}\right) m_{\tilde{W}_{1}} m_{\tilde{W}_{2}}\right],
$$

where

$$
S_{h}=\frac{1}{2}\left(-\sin \theta_{R} \sin \theta_{L} \sin \alpha-\cos \theta_{L} \cos \theta_{R} \cos \alpha+\sin \theta_{L} \sin \theta_{R} \cos \alpha+\cos \theta_{L} \cos \theta_{R} \sin \alpha\right),
$$

$P_{h}$ is the same as $S_{h}$ except the first and second terms gain an extra minus sign (become + ). $S_{H}, P_{H}$ are the same as $S_{h}$ and $P_{h}$ if you take $\sin \alpha \rightarrow-\cos \alpha$ and $\cos \alpha \rightarrow \sin \alpha$.

$$
S_{A}=\frac{1}{2}\left(\sin \theta_{R} \sin \theta_{L} \sin \beta-\cos \theta_{L} \cos \theta_{R} \cos \beta-\sin \theta_{L} \sin \theta_{R} \cos \beta+\cos \theta_{L} \cos \theta_{R} \sin \beta\right) .
$$

Again $P_{A}$ is the same as $S_{A}$ except the first two terms gain an additional minus sign.

\section{Appendix F.5. Neutralinos}

$$
\Gamma\left(\tilde{Z}_{i} \rightarrow \bar{u} \tilde{u}_{L} / R\right)=\frac{3 C^{2}\left|m_{\tilde{Z}_{i}}\right|}{32 \pi} \tilde{\lambda}^{1 / 2}\left(m_{\tilde{Z}_{i}}, m_{u}, m_{\tilde{u}_{L / R}}\right)\left(1+\frac{m_{u}^{2}}{m_{\tilde{Z}_{i}}^{2}}-\frac{m_{\tilde{u}_{L / R}}^{2}}{m_{\tilde{Z}_{i}}^{2}}\right),
$$

where

$$
C= \begin{cases}\frac{1}{\sqrt{2}}\left(-g N_{2 i}-\frac{g^{\prime}}{3} N_{1 i}\right), & \text { for } \tilde{u}_{L}, \\ \frac{-4}{3 \sqrt{2}} g^{\prime} N_{1 i}, & \text { for } \tilde{u}_{R} .\end{cases}
$$

Neutralino decays to charm and $\tilde{c}_{L / R}$ have a similar expression. For decays to down and $\tilde{d}_{L / R}$ or to strange and $\tilde{s}_{L / R}$ then $C$ for the $L$ component is the same as above except $g \rightarrow-g$ and $C$ for the $R$ component has a factor of 2 rather than -4 in the numerator of the pre-factor. The masses must also be changed appropriately. Note the difference between the left-handed ( $\mathrm{LH})$ and right-handed (RH) squark comes from the LH squark coupling to both the zino and wino components of the neutralinos whereas the RH squark couples only to the zino components.

$$
\Gamma\left(\tilde{Z}_{i} \rightarrow \tilde{l}_{L} / R\right)=\frac{C^{2}\left|m_{\tilde{Z}_{i}}\right|}{32 \pi} \tilde{\lambda}^{1 / 2}\left(m_{\tilde{Z}_{i}}, m_{l}, m_{\tilde{L}_{L / R}}\right)\left(1+\frac{m_{l}^{2}}{m_{\tilde{Z}_{i}}^{2}}-\frac{m_{\tilde{l}_{L / R}}^{2}}{m_{\tilde{Z}_{i}}^{2}}\right),
$$

where

$$
C= \begin{cases}\frac{1}{\sqrt{2}}\left(g N_{2 i}+g^{\prime} N_{1 i}\right), & \text { for } \tilde{l}_{L}, \\ \sqrt{2} g^{\prime} N_{1 i}, & \text { for } \tilde{l}_{R} .\end{cases}
$$


Again the difference here between the $L$ and $R$ sleptons is due to the $L$ sleptons coupling to the wino and zino components of the neutralinos whilst the $R$ sleptons couple only to the zino components.

$$
\Gamma\left(\tilde{Z}_{i} \rightarrow \tilde{t}_{1}\right)=\frac{3\left|m_{\tilde{Z}_{i}}\right|}{16 \pi} \tilde{\lambda}^{1 / 2}\left(m_{\tilde{Z}_{i}}, m_{t}, m_{\tilde{t}_{1}}\right)\left[a^{2}\left\{\left(1+\frac{m_{t}}{m_{\tilde{Z}_{i}}}\right)^{2}-\left(\frac{m_{\tilde{t}_{1}}}{m_{\tilde{Z}_{i}}}\right)^{2}\right\}+b^{2}\left\{\left(1-\frac{m_{t}}{m_{\tilde{Z}_{i}}}\right)^{2}-\left(\frac{m_{\tilde{t}_{1}}}{\tilde{Z}_{i}}\right)^{2}\right\}\right],
$$

where

$$
\begin{gathered}
a=\frac{1}{2}(\alpha+\beta), \quad b=\frac{1}{2}(\alpha-\beta), \\
\alpha=\cos \theta_{t} \frac{1}{\sqrt{2}}\left[-g N_{2 i}-\frac{g^{\prime}}{3} N_{1 i}\right]-f_{t} \sin \theta_{t} N_{4 i}, \\
\beta=\frac{4}{3 \sqrt{2}} g^{\prime} N_{1 i} \sin \theta_{t}-f_{t} \cos \theta_{t} N_{4 i} .
\end{gathered}
$$

For $\tilde{t}_{2}$ take $\cos \theta_{t} \rightarrow \sin \theta_{t}, \sin \theta_{t} \rightarrow-\cos \theta_{t}$ and $m_{\tilde{t}_{1}} \rightarrow m_{\tilde{t}_{2}}$.

$$
\Gamma\left(\tilde{Z}_{i} \rightarrow \bar{b} \tilde{b}_{1}\right)=\frac{3\left|m_{\tilde{Z}_{i}}\right|}{16 \pi} \tilde{\lambda}^{1 / 2}\left(m_{\tilde{Z}_{i}}, m_{b}, m_{\tilde{b}_{1}}\right)\left[a^{2}\left\{\left(1+\frac{m_{b}}{m_{\tilde{Z}_{i}}}\right)^{2}-\left(\frac{m_{\tilde{b}_{1}}}{m_{\tilde{Z}_{i}}}\right)^{2}\right\}+b^{2}\left\{\left(1-\frac{m_{b}}{m_{\tilde{Z}_{i}}}\right)^{2}-\left(\frac{m_{\tilde{b}_{1}}}{\tilde{Z}_{i}}\right)^{2}\right\}\right],
$$

where $a$ and $b$ are as before but the $\alpha$ and $\beta$ are different:

$$
\begin{gathered}
\alpha=\cos \theta_{b} \frac{1}{\sqrt{2}}\left[-\frac{g^{\prime}}{3} N_{1 i}+g N_{2 i}\right]-f_{b} \sin \theta_{b} N_{3 i}, \\
\beta=-\sin \theta_{b} \frac{2}{3 \sqrt{2}} g^{\prime} N_{1 i}-\cos \theta_{b} f_{b} N_{3 i} .
\end{gathered}
$$

For $\tilde{b}_{2}$ take $\cos \theta_{b} \rightarrow \sin \theta_{b}, \sin \theta_{b} \rightarrow-\cos \theta_{b}$ and $m_{\tilde{b}_{1}} \rightarrow m_{\tilde{b}_{2}}$.

$$
\Gamma\left(\tilde{Z}_{i} \rightarrow \bar{\tau} \tilde{\tau}_{1}\right)=\frac{\left|m_{\tilde{Z}_{i}}\right|}{16 \pi} \tilde{\lambda}^{1 / 2}\left(m_{\tilde{Z}_{i}}, m_{\tau}, m_{\tilde{\tau}_{1}}\right)\left[a ^ { 2 } \left\{\left(1-\left(\frac{m_{\tau}+m_{\tilde{\tau}_{1}}}{m_{\tilde{Z}_{i}}}\right)^{2}\right\}+b^{2}\left\{\left(1-\left(\frac{m_{\tau}-m_{\tilde{\tau}_{1}}}{m_{\tilde{Z}_{i}}}\right)^{2}\right\}\right],\right.\right.
$$

where

$$
\begin{gathered}
a=\frac{1}{2}(\alpha+\beta), \\
b=\frac{1}{2}(\beta-\alpha), \\
\alpha=\frac{1}{\sqrt{2}} \sin \theta_{\tau}\left[g N_{2 i}+g^{\prime} N_{1 i}\right]+f_{\tau} N_{3 i} \cos \theta_{\tau}, \\
\beta=-\sqrt{2} g^{\prime} N_{1 i} \cos \theta_{\tau}+f_{\tau} N_{3 i} \sin \theta_{\tau} .
\end{gathered}
$$

For $\tilde{\tau}_{2}$ make the replacements $\cos \theta_{\tau} \rightarrow \sin \theta_{\tau}, \sin \theta_{\tau} \rightarrow-\cos \theta_{\tau}$ and $m_{\tilde{\tau}_{1}} \rightarrow m_{\tilde{\tau}_{2}}$.

$$
\begin{array}{r}
\Gamma\left(\tilde{Z}_{i} \rightarrow W \tilde{W}_{1}\right)=\frac{g^{2}}{16 \pi\left|m_{\tilde{Z}_{i}}\right|} \tilde{\lambda}^{1 / 2}\left(m_{\tilde{Z}_{i}}, m_{W}, m_{\tilde{W}_{1}}\right)\left[\left(X^{2}+Y^{2}\right)\left(m_{\tilde{Z}_{i}}^{2}+m_{\tilde{W}_{1}}^{2}-m_{W}^{2}+\frac{1}{m_{W}^{2}}\left\{\left(m_{\tilde{Z}_{i}}^{2}-m_{\tilde{W}_{1}}^{2}\right)^{2}-m_{W}^{4}\right\}\right)\right. \\
\left.-6\left(X^{2}-Y^{2}\right) m_{\tilde{Z}_{i}} m_{\tilde{W}_{1}}\right]
\end{array}
$$

where

$$
X=\frac{1}{2}\left[\cos \theta_{R} N_{4 i} \frac{1}{\sqrt{2}}-\sin \theta_{R} N_{2 i}-\cos \theta_{L} N_{3 i} \frac{1}{\sqrt{2}}-\sin \theta_{L} N_{2 i}\right]
$$

and $Y$ is the same as $X$ except the first two terms get an extra minus sign. For $\tilde{W}_{2}$ change $\cos \theta_{L} \rightarrow-\sin \theta_{L}, \sin \theta_{L} \rightarrow$ $\cos \theta_{L}, \cos \theta_{R} \rightarrow-\sin \theta_{R}, \sin \theta_{R} \rightarrow \cos \theta_{R}$ and $m_{\tilde{W}_{1}} \rightarrow m_{\tilde{W}_{2}}$.

$$
\Gamma\left(\tilde{Z}_{j} \rightarrow H^{+} \tilde{W}_{1}\right)=\frac{1}{16 \pi\left|m_{\tilde{Z}_{i}}\right|} \tilde{\lambda}^{1 / 2}\left(m_{\tilde{Z}_{j}}, m_{H^{+}}, m_{\tilde{W}_{1}}\right)\left[\left(a^{2}+b^{2}\right)\left(m_{\tilde{Z}_{j}}^{2}+m_{\tilde{W}_{1}}^{2}-m_{H^{+}}^{2}\right)+2\left(a^{2}-b^{2}\right) m_{\tilde{Z}_{j}} m_{\tilde{W}_{1}}\right],
$$


where $a$ and $b$ and then $A_{2}$ and $A_{4}$ are exactly as given for the decay $\tilde{W}_{1} \rightarrow H^{+} Z_{j}$ in (F.61].

$$
\begin{gathered}
\Gamma\left(\tilde{Z}_{i} \rightarrow Z \tilde{Z}_{j}\right)=\frac{g^{2}+g^{\prime 2}}{64 \pi\left|m_{\tilde{Z}_{i}}\right|} \tilde{\lambda}^{1 / 2}\left(m_{\tilde{Z}_{i} j}, m_{Z}, m_{\tilde{Z}_{j}}\right)\left\{N_{4 i} N_{4 j}-N_{3 i} N_{3 j}\right\}^{2} \\
\times\left[m_{\tilde{Z}_{i}}^{2}+m_{\tilde{Z}_{j}}^{2}-m_{Z}^{2}+\frac{1}{m_{Z}^{2}}\left[\left(m_{\tilde{Z}_{i}}^{2}-m_{\tilde{Z}_{j}}^{2}\right)^{2}-m_{Z}^{4}\right]+6 m_{\tilde{z}_{i}} m_{\tilde{Z}_{j}}\right] . \\
\Gamma\left(\tilde{Z}_{i} \rightarrow h \tilde{Z}_{j}\right)=\frac{\left(X_{i j}^{h}+X_{j i}^{h}\right)^{2}}{16 \pi\left|m_{\tilde{Z}_{i}}\right|} \tilde{\lambda}^{1 / 2}\left(m_{\tilde{Z}_{i} j}, m_{h}, m_{\tilde{Z}_{j}}\right)\left[m_{\tilde{Z}_{i}}^{2}+m_{\tilde{Z}_{j}}^{2}-m_{h}^{2}+2 m_{\tilde{Z}_{i}} m_{\tilde{Z}_{j}}\right]
\end{gathered}
$$

where

$$
X_{i j}^{h}=\frac{1}{2}\left[N_{3 i} \sin \alpha+N_{4 i} \cos \alpha\right]\left(-g N_{2 j}+g^{\prime} N_{1 j}\right),
$$

$X_{j i}^{h}$ is the same but with $i \leftrightarrow j$. For $\tilde{Z}_{i} \rightarrow H \tilde{Z}_{j}$ the formula is the same except one must change $\sin \alpha \rightarrow-\cos \alpha$, $\cos \alpha \rightarrow \sin \alpha$ and $m_{h} \rightarrow m_{H}$.

$$
\Gamma\left(\tilde{Z}_{i} \rightarrow A \tilde{Z}_{j}\right)=\frac{\left(X_{i j}^{A}+X_{j i}^{A}\right)^{2}}{16 \pi\left|m_{\tilde{Z}_{i}}\right|} \tilde{\lambda}^{1 / 2}\left(m_{\tilde{Z}_{i j}}, m_{A}, m_{\tilde{Z}_{j}}\right)\left[m_{\tilde{Z}_{i}}^{2}+m_{\tilde{Z}_{j}}^{2}-m_{A}^{2}-2 m_{\tilde{Z}_{i}} m_{\tilde{Z}_{j}}\right]
$$

where

$$
X_{i j}^{A}=\frac{1}{2}\left[N_{3 i} \sin \beta-N_{4 i} \cos \beta\right]\left(-g N_{2 j}+g^{\prime} N_{1 j}\right),
$$

and $X_{j i}^{A}$ is the same but with $i \leftrightarrow j$.

\section{Appendix F.6. Higgs Sector}

Once more, the partial widths for all of the Higgs decays incorporated into SOFTSUSY were rederived, including for the three-body and 1-loop decays, however the majority of them can also be found in "The Higgs Hunter's Guide" [32].

$$
\Gamma(h \rightarrow q \bar{q})=\frac{3 g^{2} m_{h}}{32 \pi}\left(\frac{m_{q}}{m_{W}}\right)^{2}\left(1-4 \frac{m_{q}^{2}}{m_{h}^{2}}\right)^{\frac{3}{2}} \mathcal{J}^{2},
$$

where

$$
\mathcal{J}= \begin{cases}\frac{\cos \alpha}{\sin \beta}, & \text { for up type quarks }(\mathrm{u}, \mathrm{c}, \mathrm{t}), \\ \frac{\sin \alpha}{\cos \beta}, & \text { for down type quarks }(\mathrm{d}, \mathrm{s}, \mathrm{b}) .\end{cases}
$$

The same formulae apply for the decays to leptons, however without the factor of 3 which arises due to colour. This is similar for $H \rightarrow q q$ except we must make the replacements $\sin \alpha \rightarrow-\cos \alpha, \cos \alpha \rightarrow \sin \alpha$ and $m_{h} \rightarrow m_{H}$.

With regards to the SOFTSUSY spectrum generator, when the mixing parameter is set to -1 it considers only third family Yukawa couplings to be non zero. This would mean no $h \rightarrow \mu \mu$ decay, which may be important phenomenologically in spite of its small branching ratio. In this case, for the decay, we use the pole muon mass to calculate the branching ratio.

$$
\Gamma(A \rightarrow q \bar{q})=\frac{3 g^{2} \mathcal{J}_{A}^{2}}{32 \pi}\left(\frac{m_{q}}{m_{W}}\right)^{2} m_{A} \sqrt{\left.1-4\left(\frac{m_{q}}{m_{A}}\right)^{2}\right)},
$$

where

$$
\mathcal{J}_{A}= \begin{cases}1 /(\tan \beta), & \text { for up type quarks (u,c,t), } \\ \tan \beta, & \text { for down type quarks }(\mathrm{d}, \mathrm{s}, \mathrm{b}) .\end{cases}
$$

Again, the same formulae apply for the decays to leptons, however without the factor of 3 which arises due to colour.

$$
\Gamma\left(h \rightarrow \tilde{Z}_{i} \tilde{Z}_{j}\right)=\frac{\left|m_{h}\right|}{8 \pi}\left(X_{i j}^{h}+X_{j i}^{h}\right)^{2} \tilde{\lambda}^{\frac{1}{2}}\left(m_{h}, m_{\tilde{Z}_{i}}, m_{\tilde{Z}_{j}}\right)\left(1-\left(\frac{m_{\tilde{Z}_{i}}+m_{\tilde{Z}_{j}}}{m_{h}}\right)^{2}\right),
$$


with an extra pre-factor of $\frac{1}{2}$ if $i=j$ (as the above formula includes a pre-factor of 2 from $\tilde{Z}_{i} \tilde{Z}_{j}$ being indistinguishable from $\tilde{Z}_{j} \tilde{Z}_{i}$ ). Here $X_{i j}^{h}$ is as in Eq. (F.90) and $X_{j i}^{h}$ is the same but with $i \leftrightarrow j$. Again similar formulae exist for $H \rightarrow \tilde{Z}_{i} \tilde{Z}_{j}$ except we transform $\sin \alpha \rightarrow-\cos \alpha, \cos \alpha \rightarrow \sin \alpha$ and $m_{h} \rightarrow m_{H}$.

$$
\Gamma\left(A \rightarrow \tilde{Z}_{i} \tilde{Z}_{j}\right)=\frac{\left|m_{A}\right|}{8 \pi}\left(X_{i j}^{A}+X_{j i}^{A}\right)^{2} \tilde{\lambda}^{\frac{1}{2}}\left(m_{A}, m_{\tilde{Z}_{i}}, m_{\tilde{Z}_{j}}\right)\left(1-\left(\frac{m_{\tilde{Z}_{i}}+m_{\tilde{Z}_{j}}}{m_{A}}\right)^{2}\right),
$$

here $X_{i j}^{A}$ is as given in Eq. F.92 and $X_{j i}^{A}$ is the same but with $i \leftrightarrow j$.

$$
\Gamma\left(\phi \rightarrow \tilde{W}_{i}^{+} \tilde{W}_{i}^{-}\right)=\frac{g^{2}\left|m_{\phi}\right|}{4 \pi} S^{2} \tilde{\lambda}^{a}\left(m_{\phi}, m_{\tilde{W}_{i}}, m_{\tilde{W}_{i}}\right),
$$

where $a=3 / 2$ for $\phi=h, H$ or $a=1 / 2$ for $A$ and $S$ is given by:

$$
\begin{gathered}
S= \begin{cases}\frac{1}{2}\left(-\sin \alpha \sin \theta_{R} \cos \theta_{L}+\cos \alpha \sin \theta_{L} \cos \theta_{R}\right), & \text { for: } h \rightarrow \tilde{W}_{1} \tilde{W}_{1}, \\
\frac{1}{2}\left(\sin \alpha \cos \theta_{R} \sin \theta_{L}-\cos \alpha \cos \theta_{L} \sin \theta_{R}\right), & \text { for: } h \rightarrow \tilde{W}_{2} \tilde{W}_{2}, \\
\frac{1}{2}\left(\cos \alpha \sin \theta_{R} \cos \theta_{L}+\sin \alpha \sin \theta_{L} \cos \theta_{R}\right), & \text { for: } H \rightarrow \tilde{W}_{1} \tilde{W}_{1}, \\
-\frac{1}{2}\left(\cos \alpha \cos \theta_{R} \sin \theta_{L}+\sin \alpha \cos \theta_{L} \sin \theta_{R}\right), & \text { for: } H \rightarrow \tilde{W}_{2} \tilde{W}_{2}, \\
\frac{1}{2}\left(\sin \beta \sin \theta_{R} \cos \theta_{L}+\cos \beta \sin \theta_{L} \cos \theta_{R}\right), & \text { for: } A \rightarrow \tilde{W}_{1} \tilde{W}_{1}, \\
-\frac{1}{2}\left(\sin \beta \cos \theta_{R} \sin \theta_{L}+\cos \beta \cos \theta_{L} \sin \theta_{R}\right), & \text { for: } A \rightarrow \tilde{W}_{2} \tilde{W}_{2} .\end{cases} \\
\Gamma\left(\phi \rightarrow \tilde{W}_{i}^{+} \tilde{W}_{j}^{-}\right)=\frac{g^{2}}{16 \pi\left|m_{\phi}\right|} \tilde{\lambda}^{\frac{1}{2}}\left(m_{\phi}, m_{\tilde{W}_{i}}, m_{\tilde{W}_{j}}\right)\left[S^{2}\left(1-\left(\frac{m_{\tilde{W}_{i}}+m_{\tilde{W}_{j}}}{m_{\phi}}\right)^{2}\right)+P^{2}\left(1-\left(\frac{m_{\tilde{W}_{i}}-m_{\tilde{W}_{j}}}{m_{\phi}}\right)^{2}\right)\right],
\end{gathered}
$$

where

$$
S= \begin{cases}\frac{1}{2}\left(\sin \alpha \sin \theta_{R} \sin \theta_{L}+\cos \alpha \cos \theta_{L} \cos \theta_{R}-\sin \theta_{L} \sin \theta_{R} \cos \alpha-\cos \theta_{L} \cos \theta_{R} \sin \alpha\right), & \text { for: } \phi=h, \\ \frac{1}{2}\left(-\cos \alpha \sin \theta_{R} \sin \theta_{L}+\sin \alpha \cos \theta_{L} \cos \theta_{R}-\sin \theta_{L} \sin \theta_{R} \sin \alpha+\cos \theta_{L} \cos \theta_{R} \cos \alpha\right), & \text { for: } \phi=H, \\ \frac{1}{2}\left(-\sin \beta \sin \theta_{R} \sin \theta_{L}+\cos \beta \cos \theta_{L} \cos \theta_{R}+\sin \theta_{L} \sin \theta_{R} \cos \beta-\cos \theta_{L} \cos \theta_{R} \sin \beta\right), & \text { for: } \phi=A .\end{cases}
$$

$P$ is the same as $S$ except the signs of the first two terms are reversed.

$$
\begin{aligned}
\Gamma(h \rightarrow A A) & =\frac{g^{2} m_{W}^{2}}{128 \pi\left|m_{h}\right| \cos ^{4}\left(\theta_{W}\right)} \tilde{\lambda}^{\frac{1}{2}}\left(m_{h}, m_{A}, m_{A}\right) \sin ^{2}(\alpha+\beta) \cos ^{2} 2 \beta . \\
\Gamma(H \rightarrow h h) & =\frac{g^{2} m_{W}^{2}}{128 \pi\left|m_{H}\right| \cos ^{4}\left(\theta_{W}\right)} \tilde{\lambda}^{\frac{1}{2}}\left(m_{H}, m_{h}, m_{h}\right)[\cos 2 \alpha \cos (\alpha+\beta)-2 \sin 2 \alpha \sin (\alpha+\beta)]^{2} . \\
\Gamma(H \rightarrow A A) & =\frac{g^{2} m_{W}^{2}}{128 \pi\left|m_{H}\right| \cos ^{4}\left(\theta_{W}\right)} \tilde{\lambda}^{\frac{1}{2}}\left(m_{H}, m_{A}, m_{A}\right) \cos ^{2} 2 \beta \cos ^{2}(\alpha+\beta) . \\
\Gamma\left(H \rightarrow H^{+} H^{-}\right) & =\frac{g^{2} m_{W}^{2}}{16 \pi\left|m_{H}\right|} \tilde{\lambda}^{\frac{1}{2}}\left(m_{H}, m_{H^{+}}, m_{H^{-}}\right)\left[\cos (\beta-\alpha)-\frac{\cos (\alpha+\beta) \cos 2 \beta}{2 \cos ^{2} \theta_{W}}\right]^{2} . \\
\Gamma(h \rightarrow A Z) & =\frac{g^{2}\left|m_{h}^{3}\right| \cos ^{2}(\beta-\alpha)}{64 \pi \cos ^{2} \theta_{w} m_{Z}^{2}} \tilde{\lambda}^{\frac{3}{2}}\left(m_{h}, m_{Z}, m_{A}\right) .
\end{aligned}
$$

The decay $H \rightarrow A Z$ follows the same formula but with the changes $\cos (\beta-\alpha) \rightarrow \sin (\beta-\alpha)$ and $m_{h} \rightarrow m_{H}$.

$$
\Gamma(A \rightarrow h Z)=\frac{g^{2}\left|m_{A}^{3}\right| \cos ^{2}(\beta-\alpha)}{64 \pi \cos ^{2} \theta_{w} m_{Z}^{2}} \tilde{\lambda}^{\frac{3}{2}}\left(m_{A}, m_{Z}, m_{h}\right) .
$$

The decay $A \rightarrow H Z$ is not included as it's largely ruled out by SUSY mass constraints.

$$
\Gamma\left(h \rightarrow \tilde{q}_{L / R} \tilde{q}_{L / R}^{*}\right)=\frac{3}{16 \pi m_{h}} \tilde{\lambda}^{1 / 2}\left(m_{h}, m_{\tilde{q}_{L / R}}, m_{\tilde{q}_{L / R}}\right) C_{h \tilde{q}_{L / R} \tilde{q}_{L / R}}^{2}
$$


where

$$
\begin{gathered}
C_{h \tilde{q}_{L / R} \tilde{q}_{L / R}}= \begin{cases}g\left[m_{W}\left(\frac{1}{2}-\frac{1}{6} \frac{g^{\prime 2}}{g^{2}}\right) \sin (\beta+\alpha)-\frac{m_{u}^{2} \cos \alpha}{m_{W} \sin \beta}\right], & \text { for: } \tilde{u}_{L} \overline{\tilde{u}}_{L}, \\
g\left[m_{W}\left(-\frac{1}{2}-\frac{1}{6} \frac{g^{\prime 2}}{g^{2}}\right) \sin (\beta+\alpha)+\frac{m_{d}^{2} \sin \alpha}{m_{W} \cos \beta}\right], & \text { for: } \tilde{d}_{L} \overline{\tilde{d}}_{L}, \\
g\left[\frac{2}{3} m_{W} \frac{g^{\prime 2}}{g^{2}} \sin (\beta+\alpha)-\frac{m_{u}^{2} \cos \alpha}{m_{W} \sin \beta}\right], & \text { for: } \tilde{u}_{R} \overline{\tilde{u}}_{R}, \\
g\left[\frac{-m_{W}}{3} \frac{g^{\prime 2}}{g^{2}} \sin (\beta+\alpha)+\frac{m_{d}^{2} \sin \alpha}{m_{W} \cos \beta}\right], & \text { for: } \tilde{d}_{R} \overline{\tilde{d}}_{R}, \\
\frac{g m_{u}}{2 m_{W} \sin \beta}\left(\mu \sin \alpha+A_{u} \cos \alpha\right), & \text { for: } \tilde{u}_{L} \overline{\tilde{u}}_{R} \text { or } \tilde{u}_{R} \overline{\tilde{u}}_{L}, \\
\frac{g m_{d}}{2 m_{W} \cos \beta}\left(-\mu \cos \alpha-A_{d} \sin \alpha\right), & \text { for: } \tilde{d}_{L} \overline{\tilde{d}}_{R} \text { or } \tilde{d}_{R} \overline{\tilde{d}}_{L} .\end{cases} \\
\Gamma\left(H \rightarrow \tilde{q}_{L / R} \tilde{q}_{L / R}^{*}\right)=\frac{3}{16 \pi m_{H}} \tilde{\lambda}^{1 / 2}\left(m_{H}, m_{\tilde{q}_{L / R}}, m_{\tilde{q}_{L / R}}\right) C_{H \tilde{q}_{L / R} \tilde{q}_{L / R}}^{2},
\end{gathered}
$$

where

$$
C_{H \tilde{q}_{L / R} \tilde{q}_{L / R}}= \begin{cases}g\left[-m_{W}\left(\frac{1}{2}-\frac{1}{6} \frac{g^{\prime 2}}{g^{2}}\right) \cos (\beta+\alpha)-\frac{m_{u}^{2} \sin \alpha}{m_{W} \sin \beta}\right], & \text { for: } \tilde{u}_{L} \overline{\tilde{u}}_{L}, \\ g\left[m_{W}\left(\frac{1}{2}+\frac{1}{6} \frac{g^{2}}{g^{2}}\right) \cos (\beta+\alpha)-\frac{m_{d}^{2} \cos \alpha}{m_{W} \cos \beta}\right], & \text { for: } \tilde{d}_{L} \overline{\tilde{d}}_{L}, \\ g\left[\frac{-2 m_{W}}{3} \frac{g^{\prime 2}}{g^{2}} \cos (\beta+\alpha)-\frac{m_{u}^{2} \sin \alpha}{m_{W} \cos \beta}\right], & \text { for: } \tilde{u}_{R} \overline{\tilde{u}}_{R}, \\ g\left[\frac{m_{W}}{3} \frac{g^{\prime 2}}{g^{2}} \cos (\beta+\alpha)-\frac{m_{d}^{2} \cos \alpha}{m_{W} \cos \beta}\right], & \text { for: } \tilde{d}_{R} \overline{\tilde{d}}_{R}, \\ \frac{g m_{u}}{2 m_{W} \sin \beta}\left(-\mu \cos \alpha+A_{u} \sin \alpha\right), & \text { for: } \tilde{u}_{L} \overline{\tilde{u}}_{R} \text { or } \tilde{u}_{R} \overline{\tilde{u}}_{L}, \\ \frac{g m_{d}}{2 m_{W} \cos \beta}\left(-\mu \sin \alpha+A_{d} \cos \alpha\right), & \text { for: } \tilde{d}_{L} \tilde{\tilde{d}}_{R} \text { or } \tilde{d}_{R} \tilde{\tilde{d}}_{L} .\end{cases}
$$$$
\Gamma\left(h \rightarrow \tilde{l}_{L / R} \overline{\tilde{l}}_{L / R}\right)=\frac{1}{16 \pi m_{h}} \tilde{\lambda}^{1 / 2}\left(m_{h}, m_{\tilde{l}_{L / R}}, m_{\tilde{l}_{L / R}}\right) C_{h \tilde{h}_{L / R} \tilde{l}_{L / R}}^{2},
$$

where

$$
C_{h \tilde{l}_{L / R} \tilde{I}_{L / R}}= \begin{cases}g\left[m_{W}\left(\frac{1}{2}+\frac{1}{2} \frac{g^{\prime 2}}{g^{2}}\right)\right] \sin (\beta+\alpha), & \text { for } \tilde{v}_{L} \tilde{v}_{L}^{*}, \\ g\left[m_{W}\left(-\frac{1}{2}+\frac{1}{2} \frac{g^{\prime 2}}{g^{2}}\right) \sin (\alpha+\beta)+\frac{m_{\tilde{e}_{L}}^{2} \sin \alpha}{m_{W} \cos \beta}\right], & \text { for } \tilde{e}_{L} \tilde{e}_{L}^{*}, \\ g\left[-m_{W} \frac{g^{\prime 2}}{g^{2}} \sin (\alpha+\beta)+\frac{m_{\tilde{e}_{L}}^{2} \sin \alpha}{m_{W} \cos \beta}\right], & \text { for } \tilde{e}_{R} \tilde{e}_{R}^{*}, \\ \frac{g m_{\tilde{e}_{L}}}{2 m_{W} \cos \beta}\left(-\mu \cos \alpha-A_{e} \sin \alpha\right), & \text { for } \tilde{e}_{L} \tilde{e}_{R}^{*} \text { or } \tilde{e}_{R} \tilde{e}_{L}^{*} .\end{cases}
$$

For third generation sfermions, the formulae are more complicated as a result of sfermion mixing and Yukawa coupling effects:

$$
\Gamma\left(h \rightarrow \tilde{t}_{i} \tilde{t}_{j}^{*}\right)=\frac{3}{16 \pi m_{h}} \tilde{\lambda}^{1 / 2}\left(m_{h}, m_{\tilde{t}_{i}}, m_{\tilde{t}_{j}}\right) C_{h \tilde{t}_{i} \tilde{t}_{j}}^{2},
$$

where here $i$ and $j$ can each be 1 or 2 independently of each other. The coupling depends on $i$ and $j$, for $\tilde{t}_{1} \tilde{t}_{1}^{*}$ (i.e. $i=j=1)$ :

$$
C_{h \tilde{t}_{1} \tilde{t}_{1}}=\cos ^{2} \theta_{t} C_{h \tilde{t}_{L} \tilde{t}_{L}}+\sin ^{2} \theta_{t} C_{h \tilde{t}_{R} \tilde{t}_{R}}-2 \sin \theta_{t} \cos \theta_{t} C_{h \tilde{t}_{L} \tilde{t}_{R}}
$$

where $C_{h \tilde{t}_{L} \tilde{t}_{L}}, C_{h \tilde{t}_{R} \tilde{t}_{R}}$ and $C_{h \tilde{t}_{L} \tilde{t}_{R}}$ are the corresponding couplings of $\tilde{u}_{L} \overline{\tilde{u}}_{L}, \tilde{u}_{R} \overline{\tilde{u}}_{R}$ and $\tilde{u}_{L} \overline{\tilde{u}}_{R}$, respectively with the changes $m_{u} \rightarrow m_{t}$ and $A_{u} \rightarrow A_{t}$. For $\tilde{t}_{2} \tilde{t}_{2}^{*}$ make the replacements $\cos \theta_{t} \rightarrow \sin \theta_{t}, \sin \theta_{t} \rightarrow-\cos \theta_{t}, m_{\tilde{t}_{1}} \rightarrow m_{\tilde{t}_{2}}$. For $\tilde{t}_{1} \tilde{t}_{2}^{*}$ or $\tilde{t}_{2} \tilde{t}_{1}^{*}:$

$$
C_{h \tilde{t}_{1} \tilde{t}_{2}}=\left(C_{h \tilde{t}_{L} \tilde{t}_{L}}-C_{h \tilde{t}_{R} \tilde{t}_{R}}\right) \cos \theta_{t} \sin \theta_{t}+C_{h \tilde{t}_{L} \tilde{t}_{R}} \cos 2 \theta_{t}
$$

For $H \rightarrow \tilde{t}_{i} \tilde{t}_{j}^{*}$ everything is as above but one must transform $\sin \alpha \rightarrow-\cos \alpha$ and $\cos \alpha \rightarrow \sin \alpha$ and $m_{h} \rightarrow m_{H}$.

$$
\Gamma\left(h \rightarrow \tilde{b}_{i} \tilde{b}_{j}^{*}\right)=\frac{3}{16 \pi m_{h}} \tilde{\lambda}^{1 / 2}\left(m_{h}, m_{\tilde{b}_{i}}, m_{\tilde{b}_{j}}\right) C_{h \tilde{b}_{i} \tilde{b}_{j}}^{2}
$$

For $\tilde{b}_{1} \tilde{b}_{1}^{*}$, i.e. $i=j=1$ :

$$
C_{h \tilde{b}_{1} \tilde{b}_{1}}=C_{h \tilde{b}_{L} \tilde{b}_{L}} \cos ^{2} \theta_{b}+C_{h \tilde{b}_{R} \tilde{b}_{R}} \sin ^{2} \theta_{b}-2 \cos \theta_{b} \sin \theta_{b} C_{h \tilde{b}_{L} \tilde{b}_{R}}
$$


where $C_{h \tilde{b}_{L} \tilde{b}_{L}}, C_{h \tilde{E}_{R} \tilde{b}_{R}}$ and $C_{h \tilde{b}_{L} \tilde{b}_{R}}$ correspond to the couplings given for $\tilde{d}_{L} \overline{\tilde{d}}_{L}, \tilde{d}_{R} \overline{\tilde{d}}_{R}$ and $\tilde{d}_{L} \overline{\tilde{d}}_{R}$ with the changes $m_{d} \rightarrow m_{b}$ and $A_{d} \rightarrow A_{b}$. For $\tilde{b}_{2} \tilde{b}_{2}^{*}$ it's the same except one must change $\cos \theta_{b} \rightarrow \sin \theta_{b}, \sin \theta_{b} \rightarrow-\cos \theta_{b}, m_{\tilde{b}_{1}} \rightarrow m_{\tilde{b}_{2}}$. For $\tilde{b}_{1} \tilde{b}_{2}^{*}$ or $\tilde{b}_{2} \overline{\tilde{b}}_{1}$ :

$$
C_{h \tilde{b}_{1} \tilde{b}_{2}}=\left(C_{h \tilde{h}_{L} \tilde{b}_{L}}-C_{h \tilde{b}_{R} \tilde{b}_{R}}\right) \sin \theta_{b} \cos \theta_{b}+C_{h \tilde{b}_{L} \tilde{b}_{R}} \cos 2 \theta_{b}
$$

For $H \rightarrow \tilde{b}_{i} \tilde{b}_{j}^{*}$ everything is as above with the replacements $\sin \alpha \rightarrow-\cos \alpha$ and $\cos \alpha \rightarrow \sin \alpha$ and $m_{h} \rightarrow m_{H}$.

$$
\Gamma\left(h \rightarrow \tilde{\tau}_{1} \tilde{\tau}_{1}^{*}\right)=\frac{1}{16 \pi m_{h}} \tilde{\lambda}^{\frac{1}{2}} C_{h \tilde{\tau}_{1} \tilde{1}_{1}}^{2},
$$

where

$$
C_{h \tilde{T}_{1} \tilde{\tau}_{1}}=C_{h \tilde{L}_{L} \tilde{\tau}_{L}} \sin ^{2} \theta_{\tau}+C_{h \tilde{T}_{R} \tilde{\tau}_{R}} \cos ^{2} \theta_{\tau}+2 \cos \theta_{\tau} \sin \theta_{\tau} C_{h \tilde{T}_{L} \tilde{\tau}_{R}}
$$

$h \rightarrow \tilde{\tau}_{2} \tilde{\tau}_{2}^{*}$ is the same with the replacements $\cos \theta_{\tau} \rightarrow \sin \theta_{\tau}, \sin \theta_{\tau} \rightarrow-\cos \theta_{\tau}$ and $m_{\tilde{\tau}_{1}} \rightarrow m_{\tilde{\tau}_{2}}$. For $h \rightarrow \tilde{\tau}_{1} \tilde{\tau}_{2}^{*}$ or $\tilde{\tau}_{2} \tilde{\tau}_{1}^{*}$ the coupling is instead given by:

$$
C_{h \tilde{\tau}_{1} \tilde{\tau}_{2}}=\left(C_{h \tilde{\tau}_{R} \tilde{\tau}_{R}}-C_{h \tilde{\tau}_{L} \tilde{\tau}_{L}}\right) \cos \theta_{\tau} \sin \theta_{\tau}+C_{h \tilde{\tau}_{L} \tilde{\tau}_{R}} \cos 2 \theta_{\tau} .
$$

$C_{h \tilde{\tau}_{L} \tilde{\tau}_{L}}, C_{h \tilde{\tau}_{R} \tilde{\tau}_{R}}$ and $C_{h \tilde{\tau}_{L} \tilde{\tau}_{R}}$ are identical to the corresponding couplings of $\tilde{e}_{L} \tilde{e}_{L}^{*}, \tilde{e}_{R} \tilde{e}_{R}^{*}$ and $\tilde{e}_{L} \tilde{e}_{R}^{*}$ respectively, with the expected replacements. For $H \rightarrow \tilde{\tau}_{i} \tilde{\tau}_{j}^{*}$ everything is as above with the changes $\sin \alpha \rightarrow-\cos \alpha, \cos \alpha \rightarrow \sin \alpha$ and $m_{h} \rightarrow m_{H}$.

$$
\Gamma\left(A \rightarrow \tilde{f}_{i} \tilde{f}_{j}^{*}\right)=\frac{N_{c}}{16 \pi m_{A}} \tilde{\lambda}^{1 / 2} C_{A \tilde{f}_{i} \tilde{f}_{j}}^{2}
$$

note $i \neq j$ by CP conservation, and $N_{c}$ is 3 for squarks and 1 for sleptons. The coupling is given by:

$$
\begin{gathered}
C_{A \tilde{f}_{i} \tilde{f}_{j}}=\left\{\begin{array}{l}
\frac{g m_{f}}{2 m_{W}}\left(\mu+A_{f} \cot \beta\right), \text { for } \quad u \text {-type sfermions } \tilde{u}, \tilde{c}, \tilde{t} \tilde{v}, \\
\frac{g m_{f}}{2 m_{W}}\left(\mu+A_{f} \tan \beta\right), \text { for } \quad d \text {-type sfermions } \tilde{d}, \tilde{s}, \tilde{b}, \tilde{l} .
\end{array}\right. \\
\Gamma\left(H^{+} \rightarrow q \bar{q}^{\prime}\right)=\frac{3 g^{2} C K M^{2}}{32 \pi m_{W}^{2} m_{H^{+}}} \tilde{\lambda}^{1 / 2}\left(m_{H^{+}}, m_{q_{1}}, m_{q_{2}}\right)\left\{\left[m_{q_{1}}^{2} \tan ^{2} \beta+\frac{m_{q_{2}}^{2}}{\tan ^{2} \beta}\right]\left(m_{H^{+}}^{2}-m_{q_{1}}^{2}-m_{q_{2}}^{2}\right)-4 m_{q_{1}}^{2} m_{q_{2}}^{2}\right\},
\end{gathered}
$$

here $m_{q_{1}}$ is the mass of the $u$-type quark and $m_{q_{2}}$ is the mass of the $d$-type quark.

$$
\Gamma\left(H^{+} \rightarrow \tilde{Z}_{i} \tilde{W}_{j}\right)=\frac{1}{8 \pi m_{H^{+}}} \tilde{\lambda}^{1 / 2}\left(m_{H^{+}}, m_{\tilde{Z}_{i}}, m_{\tilde{W}_{j}}\right)\left[\left(a^{2}+b^{2}\right)\left(m_{H^{+}}^{2}-m_{\tilde{Z}_{i}}^{2}-m_{\tilde{W}_{j}}^{2}\right)-2\left(a^{2}-b^{2}\right) m_{\tilde{Z}_{i}} m_{\tilde{W}_{j}}\right],
$$

where for $j=1$ i.e. $\tilde{W}_{1}$ :

$$
a=\frac{1}{2}\left(-\cos \beta A_{2}+\sin \beta A_{4}\right), \quad b=\frac{1}{2}\left(-\cos \beta A_{2}-\sin \beta A_{4}\right),
$$

for $j=2$ i.e. $\tilde{W}_{2}$ :

$$
a=\frac{1}{2}\left(-\cos \beta A_{1}+\sin \beta A_{3}\right), \quad b=\frac{1}{2}\left(-\cos \beta A_{1}-\sin \beta A_{3}\right) .
$$

The $A_{i}$ are:

$$
\begin{aligned}
& A_{1}=\frac{1}{\sqrt{2}}\left[g N_{2 i}+g^{\prime} N_{1 i}\right] \sin \theta_{R}-g N_{4 i} \cos \theta_{R}, \\
& A_{2}=\frac{1}{\sqrt{2}}\left[-g N_{2 i}-g^{\prime} N_{1 i}\right] \cos \theta_{R}-g N_{4 i} \sin \theta_{R}, \\
& A_{3}=\frac{1}{\sqrt{2}}\left[g N_{2 i}+g^{\prime} N_{1 i}\right] \sin \theta_{L}+g N_{3 i} \cos \theta_{L}, \\
& A_{4}=\frac{1}{\sqrt{2}}\left[-g N_{2 i}-g^{\prime} N_{1 i}\right] \cos \theta_{L}+g N_{3 i} \sin \theta_{L} .
\end{aligned}
$$




$$
\begin{gathered}
\Gamma\left(H^{+} \rightarrow W^{+} h\right)=\frac{g^{2} \cos ^{2}(\beta-\alpha) m_{H^{+}}^{3}}{64 \pi m_{W}^{2}} \tilde{\lambda}^{3 / 2}\left(m_{H^{+}}, m_{W}, m_{h}\right) . \\
\Gamma\left(H^{+} \rightarrow \tilde{q}_{L / R} \tilde{q}_{L / R}^{\prime}\right)=\frac{3 B}{16 \pi m_{H^{+}}} \tilde{\lambda}^{1 / 2}\left(m_{H^{+}}, m_{\tilde{q}_{L / R}}, m_{\tilde{q}_{L / R}^{\prime}}\right),
\end{gathered}
$$

where $B$ is the coupling and is given by:

$$
\begin{gathered}
B= \begin{cases}\frac{g}{\sqrt{2}}\left[-m_{W} \sin 2 \beta+\frac{1}{m_{W}}\left(m_{d}^{2} \tan \beta+m_{u} \cot \beta\right)\right], & \text { for } \tilde{u}_{L} \tilde{d}_{L}, \\
g m_{u} m_{d}(\tan \beta+\cot \beta) \frac{1}{\sqrt{2} m_{W}}, & \text { for } \tilde{u}_{R} \tilde{d}_{R}, \\
\frac{-g m_{d}}{\sqrt{2} m_{W}}\left(A_{d} \tan \beta+\mu\right), & \text { for } \tilde{u}_{L} \tilde{d}_{R}, \\
\frac{-g m_{u}}{\sqrt{2} m_{W}}\left(A_{u} \cot \beta+\mu\right) & \text { for } \tilde{u}_{R} \tilde{d}_{L} .\end{cases} \\
\Gamma\left(H^{+} \rightarrow \tilde{q}_{i} \tilde{q}_{j}{ }_{j}\right)=\frac{3}{16 \pi m_{H^{+}}} \tilde{\lambda}^{1 / 2}\left(m_{H^{+}}, m_{\tilde{q}_{i}}, m_{\tilde{q}_{j}}\right) C^{2},
\end{gathered}
$$

note $q$ is the top squark and $q^{\prime}$ the bottom squark; for $i=j=1$ we have $\tilde{t}_{1} \tilde{b}_{1}$ and:

$$
C=\cos \theta_{t} \cos \theta_{b} B_{\tilde{u}_{L} \tilde{d}_{L}}+\sin \theta_{t} \sin \theta_{b} B_{\tilde{u}_{R} \tilde{d}_{R}}-\cos \theta_{t} \sin \theta_{b} B_{\tilde{u}_{L} \tilde{d}_{R}}-\sin \theta_{t} \cos \theta_{b} B_{\tilde{u}_{R} \tilde{d}_{L}},
$$

for a $\tilde{b}_{2}$ we take $\cos \theta_{b} \rightarrow \sin \theta_{b}, \sin \theta_{b} \rightarrow-\cos \theta_{b}$; for a $\tilde{t}_{2}$ we take $\cos \theta_{t} \rightarrow \sin \theta_{t}, \sin \theta_{t} \rightarrow-\cos \theta_{t}$. Note, the same formulae as in Eq.s (F.135) and (F.137) can be used for decays of $H^{ \pm}$to sleptons, however for staus, because of the conventions used, one must use $\theta_{\tau}-\pi / 2$ which means the replacements $\cos \theta_{\tau} \rightarrow \sin \theta_{\tau}$ and $\sin \theta_{\tau} \rightarrow-\cos \theta_{\tau}$ are necessary in $C$ in Eq. (F.138).

Decays to two vector bosons are somewhat more complicated. Included in SOFTSUSY are the cases both where the Higgs has mass $m_{h / H}>2 m_{V}$, and so decays to two on-shell vector bosons, and also the case where the Higgs has mass $m_{V}<m_{h / H}<2 m_{V}$, so that it may only undergo a decay to one on-shell vector boson and one off-shell vector boson, which then decays into a fermion anti-fermion pair, i.e. $h / H \rightarrow Z Z^{*} \rightarrow Z f \bar{f}$ or $h / H \rightarrow W W^{*} \rightarrow W f^{\prime} \bar{f}$. This is technically a $1 \rightarrow 3$ decay but is included here as it is computed exactly without the need for numerical integration, unlike the $1 \rightarrow 3$ decays listed later. To obtain the formulae for $h / H \rightarrow V V^{*}$, one therefore sums over all possible outgoing $f^{\left({ }^{\prime}\right)} \bar{f}$ into which the $V^{*}$ may decay. First consider the case where $m_{V}<m_{h / H}<2 m_{V}$ so we have decays $h / H \rightarrow W W^{*} \rightarrow W f^{\prime} \bar{f}$ and $h / H \rightarrow Z Z^{*} \rightarrow Z f \bar{f}$, this is how the SM-like lightest Higgs, $h$, will decay:

$$
\begin{gathered}
\Gamma\left(h / H \rightarrow Z Z^{*}\right)=\frac{G_{F}^{2} m_{h / H} m_{W}^{4} c_{h / H V V}^{2}}{64 \pi^{3} \cos ^{4} \theta_{W}} F\left(\epsilon_{Z}\right)\left[7-\frac{40}{3} \sin ^{2} \theta_{W}+\frac{160}{9} \sin ^{4} \theta_{W}\right], \\
\Gamma\left(h / H \rightarrow W W^{*}\right)=\frac{3 G_{F}^{2} m_{W}^{4} m_{h / H} c_{h / H V V}^{2}}{16 \pi^{3}} F\left(\epsilon_{W}\right),
\end{gathered}
$$

here

$$
\epsilon_{V}=\frac{m_{V}}{m_{h / H}}, \quad c_{h V V}=\sin (\beta-\alpha), \quad c_{H V V}=\cos (\beta-\alpha),
$$

and

$$
F\left(\epsilon_{V}\right)=\frac{3\left(1-8 \epsilon_{V}^{2}+20 \epsilon_{V}^{4}\right)}{\sqrt{4 \epsilon_{V}^{2}-1}} \cos ^{-1}\left[\frac{3 \epsilon_{V}^{2}-1}{2 \epsilon_{V}^{3}}\right]-\left(1-\epsilon_{V}^{2}\right)\left(\frac{47}{2} \epsilon_{V}^{2}-\frac{13}{2}+\frac{1}{\epsilon_{V}^{2}}\right)-3\left(1-6 \epsilon_{V}^{2}+4 \epsilon_{V}^{4}\right) \log \left(\epsilon_{V}\right) .
$$

If however $m_{h / H}>2 m_{V}$ then the decay to two on-shell vector bosons occurs instead and the formulae are:

$$
\begin{gathered}
\Gamma(h / H \rightarrow W W)=\frac{G_{F} m_{h / H}^{3}}{8 \pi \sqrt{2}} \tilde{\lambda}^{\frac{1}{2}}\left(m_{h / H}, m_{W}, m_{W}\right)\left(1-r^{2}+\frac{3}{4} r^{4}\right) c_{h / H W W}^{2}, \\
\Gamma(h / H \rightarrow Z Z)=\frac{G_{F} m_{h / H}^{3}}{16 \pi \sqrt{2}} \tilde{\lambda}^{\frac{1}{2}}\left(m_{h / H}, m_{Z}, m_{Z}\right)\left(1-r^{2}+\frac{3}{4} r^{4}\right) c_{h / H Z Z}^{2},
\end{gathered}
$$


where $r=2 \frac{m_{V}}{m_{h / H}}$.

Note throughout many of the decay formulae there is some ambiguity at tree-level about whether one should use $\frac{g^{2}}{8 m_{W}^{2}}$ or $\frac{G_{F}}{\sqrt{2}}$, whilst these are formally equivalent they are not at a given order of calculation as $\frac{G_{F}}{\sqrt{2}}$ is a measured value, thereby containing many higher order vertex corrections. In general throughout the program we use $\frac{G_{F}}{\sqrt{2}}$ as this is found to give better agreement where higher order calculations are available. For example in the di-boson decays comparing with HDECAY-3.4, which includes higher orders and finite width effects, we find that our agreement is improved using $\frac{G_{F}}{\sqrt{2}}$.

One loop decays to $\gamma \gamma$ :

The function $f(\tau)$ appears, it is given previously in Eq. E.2p,

$$
\Gamma(\phi \rightarrow \gamma \gamma)=\frac{G_{F} \alpha_{e m}^{2}\left(m_{\phi}\right) m_{\phi}^{3}}{128 \pi^{3} \sqrt{2}} \|\left.\Sigma I_{l o o p}^{\phi}\right|^{2},
$$

where the $I_{\text {loop }}^{\phi}$ are the amplitudes of the contributions of different particles in the loop to the decay $\phi \rightarrow \gamma \gamma$. The top contributions are:

$$
\begin{gathered}
I_{t}^{h}=\frac{4}{3} \frac{\cos \alpha}{\sin \beta}[-2 \tau\{1+(1-\tau) f(\tau)\}], \\
I_{t}^{H}=\frac{4}{3} \frac{\sin \alpha}{\sin \beta}[-2 \tau\{1+(1-\tau) f(\tau)\}], \\
I_{t}^{A}=-\frac{8}{3} \tau f(\tau) \cot \beta .
\end{gathered}
$$

The stop contributions for $h \rightarrow \gamma \gamma$ are:

$$
\begin{aligned}
& I_{\tilde{t}_{1}}^{h}=\frac{4}{3} \tau(1-\tau f(\tau))\left[R_{\tilde{t}_{L} \tilde{t}_{L}}^{1} \cos ^{2} \theta_{t}+R_{\tilde{t}_{R} \tilde{\tau}_{R}}^{1} \sin ^{2} \theta_{t}-2 R_{\tilde{t}_{L} \tilde{T}_{R}}^{1} \cos \theta_{t} \sin \theta_{t}\right], \\
& I_{\tilde{t}_{2}}^{h}=\frac{4}{3} \tau(1-\tau f(\tau))\left[R_{\tilde{t}_{L} \tilde{\tau}_{L}}^{2} \sin ^{2} \theta_{t}+R_{\tilde{t}_{\tau_{R}} \tilde{T}_{R}}^{2} \cos ^{2} \theta_{t}+2 R_{\tilde{t}_{L} \tilde{t}_{R}}^{2} \cos \theta_{t} \sin \theta_{t}\right],
\end{aligned}
$$

where

$$
\begin{aligned}
& R_{\tilde{t}_{L} \tilde{t}_{L}}^{i}=R_{\tilde{t}_{L}} \frac{m_{W}}{g m_{\tilde{t}_{i}}}=\left[m_{W}\left(\frac{1}{2}-\frac{1}{6} \tan ^{2} \theta_{W}\right) \sin (\alpha+\beta)-\frac{m_{t}^{2} \cos \alpha}{m_{W} \sin \beta}\right] \frac{m_{W}}{m_{\tilde{t}_{i}}}, \\
& R_{\tilde{t}_{R} \tilde{t}_{R}}^{i}=R_{\tilde{t}_{R}} \frac{m_{W}}{g m_{\tilde{t}_{i}}}=\left[m_{W} \frac{2}{3} \tan ^{2} \theta_{W} \sin (\alpha+\beta)-\frac{m_{t}^{2} \cos \alpha}{m_{W} \sin \beta}\right] \frac{m_{W}}{m_{\tilde{t}_{i}}}, \\
& R_{\tilde{t}_{L} \tilde{t}_{R}}^{i}=R_{\tilde{t}_{L} \tilde{t}_{R}} \frac{m_{W}}{g m_{\tilde{t}_{i}}}=\frac{m_{t}}{2 m_{W} \sin \beta}\left(\mu \sin \alpha+A_{t} \cos \alpha\right) \frac{m_{W}}{m_{\tilde{t}_{i}}}
\end{aligned}
$$

The stop contributions for $H$ are the same but the $R_{\tilde{t}_{L} \tilde{t}_{L}}^{i}, R_{\tilde{R}_{R} \tilde{t}_{R}}^{i}, R_{\tilde{t}_{L} \tilde{t}_{R}}^{i}$ (note $i=1,2$ it represents whether the loop is a $\tilde{t}_{1}$ or a $\tilde{t}_{2}$ loop) are different:

$$
\begin{aligned}
& R_{\tilde{t}_{L} \tilde{t}_{L}}^{i}=\frac{m_{W}}{m_{\tilde{t}_{i}}}\left[-m_{W}\left(\frac{1}{2}-\frac{1}{6} \tan ^{2} \theta_{W}\right) \cos (\alpha+\beta)-\frac{m_{t}^{2} \sin \alpha}{m_{W} \sin \beta}\right], \\
& R_{\tilde{t}_{R} \tilde{\tau}_{R}}^{i}=\frac{m_{W}}{m_{\tilde{t}_{i}}}\left[-m_{W} \frac{2}{3} \tan ^{2} \theta_{W} \cos (\alpha+\beta)-\frac{m_{t}^{2} \sin \alpha}{m_{W} \sin \beta}\right], \\
& R_{\tilde{t}_{L} \tilde{\tau}_{R}}^{i}=\frac{m_{W}}{m_{\tilde{t}_{i}}} \frac{m_{t}}{2 m_{W} \sin \beta}\left(-\mu \cos \alpha+A_{t} \sin \alpha\right) .
\end{aligned}
$$

$A \rightarrow \gamma \gamma$ has no stop loop contribution because of CP conservation, i.e. $I_{\tilde{t}_{1 / 2}}^{A}=0$. The bottom contributions are:

$$
I_{b}^{h}=-\frac{1}{3}[-2 \tau\{1+(1-\tau) f(\tau)\}] \frac{\sin \alpha}{\cos \beta},
$$




$$
\begin{gathered}
I_{b}^{H}=\frac{1}{3}[-2 \tau\{1+(1-\tau) f(\tau)\}] \frac{\cos \alpha}{\cos \beta}, \\
I_{b}^{A}=-\frac{1}{3}\{2 \tau f(\tau)\} \tan \beta .
\end{gathered}
$$

The sbottom contributions to $h \rightarrow \gamma \gamma$ are:

$$
\begin{aligned}
& I_{\tilde{b}_{1}}^{h}=\frac{1}{3} \tau\{1-\tau f(\tau)\}\left[R_{\tilde{b}_{L} \tilde{b}_{L}}^{1} \cos ^{2} \theta_{b}+R_{\tilde{b}_{R} \tilde{b}_{R}}^{1} \sin ^{2} \theta_{b}-2 \sin \theta_{b} \cos \theta_{b} R_{\tilde{b}_{L} \tilde{b}_{R}}^{1}\right], \\
& I_{\tilde{b}_{2}}^{h}=\frac{1}{3} \tau\{1-\tau f(\tau)\}\left[R_{\tilde{b}_{L} \tilde{b}_{L}}^{2} \sin ^{2} \theta_{b}+R_{\tilde{b}_{R} \tilde{b}_{R}}^{2} \cos ^{2} \theta_{b}+2 \sin \theta_{b} \cos \theta_{b} R_{\tilde{b}_{L} \tilde{b}_{R}}^{2}\right],
\end{aligned}
$$

where here

$$
\begin{aligned}
& R_{\tilde{b}_{L} \tilde{b}_{L}}^{i}=\frac{m_{W}}{m_{\tilde{b}_{i}}}\left[m_{W}\left(-\frac{1}{2}-\frac{1}{6} \tan ^{2} \theta_{W}\right) \sin (\alpha+\beta)+\frac{m_{b}^{2} \sin \alpha}{m_{W} \cos \beta}\right], \\
& R_{\tilde{b}_{R} \tilde{b}_{R}}^{i}=\frac{m_{W}}{m_{\tilde{b}_{i}}}\left[-\frac{1}{3} m_{W} \tan ^{2} \theta_{W} \sin (\alpha+\beta)+\frac{m_{b}^{2} \sin \alpha}{m_{W} \cos \beta}\right], \\
& R_{\tilde{b}_{L} \tilde{b}_{R}}^{i}=\frac{m_{W}}{m_{\tilde{b}_{i}}} \frac{m_{b}}{2 m_{W} \cos \beta}\left(-\mu \cos \alpha-A_{b} \sin \alpha\right) .
\end{aligned}
$$

For $H \rightarrow \gamma \gamma$ the sbottom contributions are the same except the $R_{\tilde{b}_{L} \tilde{b}_{L}}^{i}, R_{\tilde{b}_{R} \tilde{b}_{R}}^{i}, R_{\tilde{b}_{L} \tilde{b}_{R}}^{i}$ change:

$$
\begin{aligned}
& R_{\tilde{b}_{L} \tilde{b}_{L}}^{i}=\frac{m_{W}}{g m_{\tilde{b}_{i}}} g\left[m_{W}\left(\frac{1}{2}+\frac{1}{6} \tan ^{2} \theta_{W}\right) \cos (\alpha+\beta)-\frac{m_{b}^{2} \cos \alpha}{m_{W} \cos \beta}\right], \\
& R_{\tilde{b}_{R} \tilde{b}_{R}}^{i}=\frac{m_{W}}{g m_{\tilde{b}_{i}}} g\left[m_{W}\left(\frac{1}{3} \tan ^{2} \theta_{W}\right) \cos (\alpha+\beta)-\frac{m_{b}^{2} \cos \alpha}{m_{W} \cos \beta}\right], \\
& R_{\tilde{b}_{L} \tilde{b}_{R}}^{i}=\frac{m_{W}}{g m_{\tilde{b}_{i}}} g \frac{m_{b}}{2 m_{W} \cos \beta}\left[-\mu \sin \alpha+A_{b} \cos \alpha\right] .
\end{aligned}
$$

$A \rightarrow \gamma \gamma$ has no sbottom loop contribution because of CP conservation, i.e. $I_{\tilde{b}_{1 / 2}}^{A}=0$. The charm loop contributions are given by:

$$
\begin{gathered}
I_{c}^{h}=\frac{4}{3}[-2 \tau\{1+(1-\tau) f(\tau)\}] \frac{\cos \alpha}{\sin \beta}, \\
I_{c}^{H}=\frac{4}{3}[-2 \tau\{1+(1-\tau) f(\tau)\}] \frac{\sin \alpha}{\sin \beta}, \\
I_{c}^{A}=-\frac{4}{3}(2 \tau f(\tau)) \cot \beta .
\end{gathered}
$$

$\tau$ loop contributions are given by:

$$
\begin{gathered}
I_{\tau}^{h}=2 \tau[1+(1-\tau) f(\tau)] \frac{\sin \alpha}{\cos \beta}, \\
I_{\tau}^{H}=-2 \tau[1+(1-\tau) f(\tau)] \frac{\cos \alpha}{\cos \beta}, \\
I_{\tau}^{A}=-2 \tau f(\tau) \tan \beta .
\end{gathered}
$$

$\tilde{\tau}_{i}$ contributions to $h \rightarrow \gamma \gamma$ are:

$$
\begin{aligned}
& I_{\tilde{\tau}_{1}}=\tau\{1-\tau f(\tau)\}\left[R_{\tilde{\tau}_{L} \tilde{\tau}_{L}}^{1} \sin ^{2} \theta_{\tau}+R_{\tilde{\tau}_{R} \tilde{\tau}_{R}}^{1} \cos ^{2} \theta_{\tau}+2 \sin \theta_{\tau} \cos \theta_{\tau} R_{\tilde{\tau}_{L} \tilde{\tau}_{R}}^{1}\right], \\
& I_{\tilde{\tau}_{2}}=\tau\{1-\tau f(\tau)\}\left[R_{\tilde{\tau}_{L} \tilde{\tau}_{L}}^{2} \cos ^{2} \theta_{\tau}+R_{\tilde{\tau}_{R} \tilde{\tau}_{R}}^{2} \sin ^{2} \theta_{\tau}-2 \sin \theta_{\tau} \cos \theta_{\tau} R_{\tilde{\tau}_{L} \tilde{\tau}_{R}}^{2}\right],
\end{aligned}
$$


here

$$
\begin{array}{r}
R_{\tilde{\tau}_{L} \tilde{\tau}_{L}}^{i}=\frac{m_{W}}{m_{\tilde{\tau}_{i}}}\left[m_{W}\left(-\frac{1}{2}+\frac{1}{2} \tan ^{2} \theta_{W}\right) \sin (\alpha+\beta)+\frac{m_{\tau}^{2} \sin \alpha}{m_{W} \cos \beta}\right], \\
R_{\tilde{\tau}_{R} \tilde{\tau}_{R}}^{i}=\frac{m_{W}}{m_{\tilde{\tau}_{i}}}\left[-m_{W} \tan ^{2} \theta_{W} \sin (\alpha+\beta)+\frac{m_{\tau}^{2} \sin \alpha}{m_{W} \cos \beta}\right], \\
R_{\tilde{\tau}_{L} \tilde{\tau}_{R}}^{i}=\frac{m_{W}}{m_{\tilde{\tau}_{i}}} \frac{m_{\tau}}{2 m_{W} \cos \beta}\left(-\mu \cos \alpha-A_{\tau} \sin \alpha\right) .
\end{array}
$$

For $H \rightarrow \gamma \gamma$ via $\tilde{\tau}_{i}$ it's the same except the $R_{\tilde{\tau}_{L} \tilde{\tau}_{L}}^{i}, R_{\tilde{\tau}_{R} \tilde{\tau}_{R}}^{i}, R_{\tilde{\tau}_{L} \tilde{\tau}_{R}}^{i}$ differ:

$$
\begin{aligned}
R_{\tilde{\tau}_{L} \tilde{\tau}_{L}}^{i} & =\frac{m_{W}}{m_{\tilde{\tau}_{i}}}\left[m_{W}\left(\frac{1}{2}-\frac{1}{2} \tan ^{2} \theta_{W}\right) \cos (\alpha+\beta)-\frac{m_{\tau}^{2} \cos \alpha}{m_{W} \cos \beta}\right], \\
R_{\tilde{\tau}_{R} \tilde{\tau}_{R}}^{i} & =\frac{m_{W}}{m_{\tilde{\tau}_{i}}}\left[m_{W} \tan ^{2} \theta_{W} \cos (\alpha+\beta)-\frac{m_{\tau}^{2} \cos \alpha}{m_{W} \cos \beta}\right], \\
R_{\tilde{\tau}_{L} \tilde{\tau}_{R}}^{i} & =\frac{m_{W}}{m_{\tilde{\tau}_{i}}} \frac{m_{\tau}}{2 m_{W} \cos \beta}\left(-\mu \sin \alpha+A_{\tau} \cos \alpha\right) .
\end{aligned}
$$

$A \rightarrow \gamma \gamma$ has no stau loop contribution because of CP conservation, i.e. $I_{\tilde{\tau}_{1 / 2}}^{A}=0$. The $W$ loop contributions are:

$$
\begin{aligned}
& I_{W}^{h}=[2+3 \tau+3 \tau(2-\tau) f(\tau)] \sin (\beta-\alpha), \\
& I_{W}^{H}=[2+3 \tau+3 \tau(2-\tau) f(\tau)] \cos (\beta-\alpha) .
\end{aligned}
$$

$A \rightarrow \gamma \gamma$ has no $W$ loop contribution because of CP conservation, i.e. $I_{W}^{A}=0 . H^{+}$loop contributions are:

$$
\begin{aligned}
I_{H^{+}}^{h} & =\tau\{1-\tau f(\tau)\} \frac{m_{W}^{2}}{m_{H^{+}}^{2}}\left[\sin (\beta-\alpha)+\frac{\cos 2 \beta \sin (\beta+\alpha)}{2 \cos ^{2} \theta_{W}}\right], \\
I_{H^{+}}^{H} & =\tau\{1-\tau f(\tau)\} \frac{m_{W}^{2}}{m_{H^{+}}^{2}}\left[\cos (\beta-\alpha)+\frac{\cos 2 \beta \cos (\beta+\alpha)}{2 \cos ^{2} \theta_{W}}\right] .
\end{aligned}
$$

$A \rightarrow \gamma \gamma$ has no $H^{+}$loop contribution because of CP conservation, i.e. $I_{H^{+}}^{A}=0 . \tilde{W}_{i}^{+}$loop contributions are:

$$
\begin{aligned}
I_{\tilde{W}_{1}^{+}}^{h} & =[-2 \tau\{1+(1-\tau) f(\tau)\}] \frac{m_{W}}{m_{\tilde{W}_{1}^{+}}} \sqrt{2}\left(-\sin \alpha \sin \theta_{R} \cos \theta_{L}+\cos \alpha \sin \theta_{L} \cos \theta_{R}\right), \\
I_{\tilde{W}_{2}^{+}}^{h} & =[-2 \tau\{1+(1-\tau) f(\tau)\}] \frac{m_{W}}{m_{\tilde{W}_{2}^{+}}} \sqrt{2}\left(\sin \alpha \cos \theta_{R} \sin \theta_{L}-\cos \alpha \cos \theta_{L} \sin \theta_{R}\right) .
\end{aligned}
$$

For $\tilde{W}_{i}^{+}$contributions to $H$ everything is the same except the replacements $\cos \alpha \rightarrow \sin \alpha$ and $\sin \alpha \rightarrow-\cos \alpha$ are required. For $A$ the $\tilde{W}_{i}^{+}$contributions have:

$$
\begin{gathered}
I_{\tilde{W}_{1}^{+}}^{A}=-2 \tau f(\tau) \frac{m_{W}}{m_{\tilde{W}_{1}^{+}}} \sqrt{2}\left(\sin \theta_{R} \cos \theta_{L} \sin \beta+\sin \theta_{L} \cos \theta_{R} \cos \beta\right), \\
I_{\tilde{W}_{2}^{+}}^{A}=2 \tau f(\tau) \frac{m_{W}}{m_{\tilde{W}_{2}^{+}}} \sqrt{2}\left(\cos \theta_{R} \sin \theta_{L} \sin \beta+\cos \theta_{L} \sin \theta_{R} \cos \beta\right) .
\end{gathered}
$$

$\phi \rightarrow g g$

The coloured particle loop contribution for $\phi \rightarrow g g$ are exactly the same, except the pre-factor changes and the bottom and sbottom contributions get multiplied by 4 in their amplitudes. There can be no uncoloured particles in the loop so there are no W, charged Higgs, chargino, lepton or slepton contributions; only quark and squark loop contributions: 


$$
\Gamma(\phi \rightarrow g g)=\frac{\alpha_{s}^{2}\left(m_{\phi}\right) G_{F} m_{\phi}^{3}}{128 \pi^{3} \sqrt{2}} \frac{9}{8} \Sigma\left|I_{l o o p}^{\phi}\right|^{2},
$$

with the $I_{b}^{\phi} \rightarrow 4 I_{b}^{\phi}, I_{\tilde{b}_{i}}^{\phi} \rightarrow 4 I_{\tilde{b}_{i}}^{\phi}$ and the remaining $I_{\text {loop }}^{\phi}$ as in the $\phi \rightarrow \gamma \gamma$ decays.

Loop decays to $Z \gamma$ : Throughout the function $g(\tau)$ appears, it is given previously in Eq. (E.3),

$$
\Gamma(\phi \rightarrow Z \gamma)=\frac{m_{\phi}^{3} \alpha_{e m}^{2}\left(m_{\phi}\right)}{64 \pi^{3}} \frac{G_{F}}{\sqrt{2}}\left(1-\frac{m_{Z}}{m_{\phi}}\right)^{3} \Sigma\left|I_{l o o p}^{\phi}\right|^{2} .
$$

$I_{1}\left(\tau_{a}, \tau_{a Z}\right)$ and $I_{2}\left(\tau_{a}, \tau_{a Z}\right)$ also occur frequently, where $\tau_{a Z}=4\left(\frac{m_{a}}{m_{Z}}\right)^{2}$ cf $\tau_{a}=4\left(\frac{m_{a}}{m_{h_{i}}}\right)^{2}$, they are as follows:

$$
\begin{gathered}
I_{1}\left(\tau_{a}, \tau_{a Z}\right)=\frac{\tau_{a} \tau_{a Z}}{2\left(\tau_{a}-\tau_{a Z}\right)}+\frac{\tau_{a}^{2} \tau_{a Z}^{2}}{2\left(\tau_{a}-\tau_{a Z}\right)^{2}}\left[f\left(\tau_{a}\right)-f\left(\tau_{a Z}\right)\right]+\frac{\tau_{a}^{2} \tau_{a Z}}{\left(\tau_{a}-\tau_{a Z}\right)^{2}}\left[g\left(\tau_{a}\right)-g\left(\tau_{a Z}\right)\right], \\
I_{2}\left(\tau_{a}, \tau_{a Z}\right)=-\frac{\tau_{a} \tau_{a Z}}{2\left(\tau_{a}-\tau_{a Z}\right)}\left[f\left(\tau_{a}\right)-f\left(\tau_{a Z}\right)\right] .
\end{gathered}
$$

The fermion loop contributions are:

$$
I_{t}^{h}=3 \frac{\cos \alpha}{\sin \beta} \frac{-\frac{4}{3}\left(\frac{1}{2}-\frac{4}{3} \sin ^{2} \theta_{W}\right)}{\sin \theta_{W} \cos \theta_{W}}\left(I_{1}\left(\tau_{t}, \tau_{t Z}\right)-I_{2}\left(\tau_{t}, \tau_{t Z}\right)\right),
$$

The expression for $I_{c}^{h}$ is analogous.

$$
I_{b}^{h}=-3 \frac{\sin \alpha}{\cos \beta} \frac{\frac{2}{3}\left(-\frac{1}{2}+\frac{2}{3} \sin ^{2} \theta_{W}\right)}{\sin \theta_{w} \cos \theta_{W}}\left(I_{1}\left(\tau_{b}, \tau_{b Z}\right)-I_{2}\left(\tau_{b}, \tau_{b Z}\right)\right),
$$

A similar expression gives $I_{s}^{h}$. For the $I_{f}^{H}$ transform $\cos \alpha \rightarrow \sin \alpha, \sin \alpha \rightarrow-\cos \alpha$.

$$
I_{t}^{A}=3 \cot \beta \frac{\frac{4}{3}\left(\frac{1}{2}-\frac{4}{3} \sin ^{2} \theta_{W}\right)}{\sin \theta_{W} \cos \theta_{W}} I_{2}\left(\tau_{t}, \tau_{t Z}\right),
$$

Again an analogous expression gives $I_{c}^{A}$.

$$
I_{b}^{A}=-3 \tan \beta \frac{\frac{2}{3}\left(-\frac{1}{2}+\frac{2}{3} \sin ^{2} \theta_{W}\right)}{\sin \theta_{w} \cos \theta_{W}} I_{2}\left(\tau_{b}, \tau_{b Z}\right) .
$$

$I_{s}^{A}$ is given similarly. The $W$ loop contributions are given by:

$$
I_{W}^{h}=-\frac{\sin (\beta-\alpha)}{\tan \theta_{W}}\left[4\left(3-\tan ^{2} \theta_{W}\right) I_{2}\left(\tau_{W}, \tau_{W Z}\right)+\left\{\left(1+\frac{2}{\tau_{W}}\right) \tan ^{2} \theta_{W}-\left(5+\frac{2}{\tau_{W}}\right)\right\} I_{1}\left(\tau_{W}, \tau_{W Z}\right)\right] .
$$

$I_{W}^{H}$ is the same but with the change $\sin (\beta-\alpha) \rightarrow \cos (\beta-\alpha) . I_{W}^{A}=0$ by CP conservation. $H^{+}$contributions are:

$$
I_{H^{+}}^{h}=\left[\sin (\beta-\alpha)+\frac{\cos 2 \beta \sin (\beta+\alpha)}{2 \cos ^{2} \theta_{W}}\right] \frac{\left(1-2 \sin ^{2} \theta_{W}\right)}{\cos \theta_{W} \sin \theta_{W}} I_{1}\left(\tau_{H^{+}}, \tau_{H^{+} Z}\right) \frac{m_{W}^{2}}{m_{H^{+}}^{2}} .
$$

For $H$, the $I_{H^{+}}^{H}$ are the same except the replacements $\sin (\beta-\alpha) \rightarrow \cos (\beta-\alpha)$ and $\sin (\beta+\alpha) \rightarrow-\cos (\beta+\alpha)$ which occur because of the transformations $\cos \alpha \rightarrow \sin \alpha$ and $\sin \alpha \rightarrow-\cos \alpha$. Meanwhile $I_{H^{+}}^{A}=0$ by CP conservation. 


\section{Appendix G. MSSM Three Body Decay Formulae}

The following decay modes are included in SOFTSUSY:

1. $h \rightarrow W W^{*} \rightarrow W f^{\prime} \bar{f}$

2. $h \rightarrow Z Z^{*} \rightarrow Z f \bar{f}$

3. $\tilde{g} \rightarrow \tilde{Z}_{i} q \bar{q}$

4. $\tilde{g} \rightarrow \tilde{W}_{i} q \bar{q}^{\prime}$

5. $\tilde{Z}_{i} \rightarrow \tilde{Z}_{j} f \bar{f}$ where $i>j$

6. $\tilde{Z}_{i} \rightarrow \tilde{W}_{j} f \bar{f}^{\prime}$

7. $\tilde{W}_{j} \rightarrow \tilde{Z}_{i} f \bar{f}^{\prime}$

The modes included are the most phenomenologically relevant modes, the formulae used were not rederived although are written in our notation and are restructured to match the calculations performed in SOFTSUSY. The formulae are as provided in sPHENO-3.3.8 [11, 35], which were based on the calculations in [34, 48].

$h \rightarrow V V * \rightarrow V f \bar{f}$ Detailed previously, see Eqs. F.140, F.139.

Appendix G.1. Gluino $1 \rightarrow 3$ Decays

$\tilde{g} \rightarrow \tilde{Z}_{i} q \bar{q}$

First the formulae for the decays to a neutralino and a quark-anti-quark pair of the first two generations; in this formula the Yukawa coupling contributions, squark mixing effects and final state quark masses have been neglected as they are negligible. The formulae after this for the third generation quarks include all such effects.

$$
\Gamma\left(\tilde{g} \rightarrow q \bar{q} \tilde{Z}_{i}\right)=\frac{\alpha_{s}}{8 \pi^{2}}\left[\left|A_{\tilde{Z}_{i}}^{q}\right|^{2}\left(\psi_{L} \pm \phi_{L}\right)+\left|B_{\tilde{Z}_{i}}^{q}\right|^{2}\left(\psi_{R} \pm \phi_{R}\right)\right]
$$

Here which of the \pm signs to take depends on the signs of the neutralino and gluino masses; the ' + ' sign applies for the case when both masses have the same sign so $m_{\tilde{Z}_{i}}>0$ and $m_{\tilde{g}}>0$ (or when they are both less than 0 ) and the '-' sign applies when one (but not both) of $m_{\tilde{Z}_{i}}$ and $m_{\tilde{g}}$ are negative. The signs essentially account for the fact that the couplings should become complex as the masses become negative. Here the $\psi_{L / R}$ and $\phi_{L / R}$ are integrals related to the $\tilde{\psi}$ and $\tilde{\phi}$ integrals given later in Eq.s G.74 and G.78) by:

$$
\begin{aligned}
& \psi_{L / R}=\frac{1}{\pi^{2} m_{\tilde{g}}} \tilde{\psi}\left(m_{\tilde{g}}, m_{\tilde{q}_{L / R}}, m_{\tilde{q}_{L / R}}, m_{\tilde{Z}_{i}}\right) . \\
& \phi_{L / R}=\frac{1}{\pi^{2} m_{\tilde{g}}} \tilde{\psi}\left(m_{\tilde{g}}, m_{\tilde{q}_{L / R}}, m_{\tilde{q}_{L / R}}, m_{\tilde{Z}_{i}}\right) .
\end{aligned}
$$

The $A_{\tilde{Z}_{i}}^{q}$ and $B_{\tilde{Z}_{i}}^{q}$ are couplings which depend upon if the quarks are "up-type" or "down-type" in $S U(2)_{L}$ and are:

$$
\begin{gathered}
A_{\tilde{Z}_{i}}^{q}=\left\{\begin{array}{l}
\frac{1}{\sqrt{2}}\left(-g N_{2 i}-\frac{g^{\prime}}{3} N_{1 i}\right), \text { for "up-type" quarks, } \\
\frac{1}{\sqrt{2}}\left(g N_{2 i}-\frac{g^{\prime}}{3} N_{1 i}\right), \text { for "down-type" quarks, }
\end{array}\right. \\
B_{\tilde{Z}_{i}}^{q}=\left\{\begin{array}{l}
\frac{-4}{3 \sqrt{2}} g^{\prime} N_{1 i}, \text { for "up-type" quarks, } \\
\frac{2}{3 \sqrt{2}} g^{\prime} N_{1 i}, \text { for "down-type" quarks. }
\end{array}\right.
\end{gathered}
$$

For the more complicated case of decays to third generation quarks; the Yukawa coupling contributions, squarkmixing effects and final state quark masses are all included as they can have significant effects. The decay is mediated by either $\tilde{t}_{1}$ or $\tilde{t}_{2}$ in the $\mathrm{t}$ or $\mathrm{u}$ channel, giving 4 Feynman diagrams $(2$ shown below as $j=1,2)$ and 6 (i.e. $\left.{ }^{4} C_{2}\right)$ interferences. The six interferences can be split into 2 "diagonal" contributions $\left(\tilde{t}_{1} \mathrm{t}-\tilde{t}_{1} \mathrm{u}\right.$ interference and $\tilde{t}_{2} \mathrm{t}-\tilde{t}_{2}$ $\mathrm{u}$ interference) and 4 "non-diagonal" contributions ( $\tilde{t}_{1} \mathrm{t}-\tilde{t}_{2} \mathrm{t}, \tilde{t}_{1} \mathrm{t}-\tilde{t}_{2} \mathrm{u}, \tilde{t}_{1} \mathrm{u}-\tilde{t}_{2} \mathrm{t}$ and $\tilde{t}_{1} \mathrm{u}-\tilde{t}_{2} \mathrm{u}$ interferences). The possibility of negative neutralino masses (which can be absorbed into imaginary couplings) is also included. The formulae are adopted from sPHENO, it should be noted that differences exit between these formulae and those present in Baer and Tata's book [41]. 


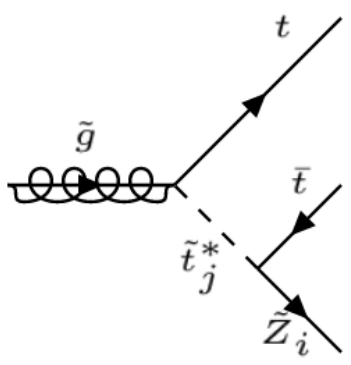

(a) channel a

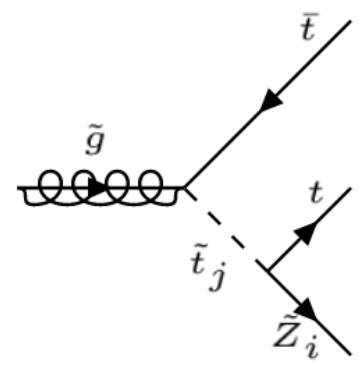

(b) channel b

Figure G.6: Feynman diagrams for the 3 body decay of a gluino into a neutralino and a top anti-top pair, as mediated by stops $\tilde{t}_{1 / 2} . i=1,2,3,4$ and $j=1,2$.

The formulae for the case of $\tilde{g} \rightarrow \tilde{Z}_{i} t \bar{t}$ are given, from this decays to other quarks can be obtained by making the appropriate replacements.

$$
\Gamma\left(\tilde{g} \rightarrow t \bar{t} \tilde{Z}_{i}\right)=\frac{\alpha_{s}}{8 \pi^{4} m_{\tilde{g}}}\left[\Gamma_{\tilde{t}_{1}}+\Gamma_{\tilde{t}_{2}}+\Gamma_{\tilde{t}_{1} \tilde{t}_{2}}\right] .
$$

The $\Gamma_{\tilde{t}_{1}}, \Gamma_{\tilde{t}_{2}}, \Gamma_{\tilde{t}_{1} \tilde{t}_{2}}$ can be split up into different contributions:

$$
\begin{gathered}
\Gamma_{\tilde{t}_{1}}=\Gamma_{L L}\left(\tilde{t}_{1}\right) \cos ^{2} \theta_{t}+\Gamma_{R R}\left(\tilde{t}_{1}\right) \sin ^{2} \theta_{t}-\sin \theta_{t} \cos \theta_{t}\left[\Gamma_{L_{1} R_{1}}\left(\tilde{t}_{1}\right)+\Gamma_{L_{1} R_{2}}\left(\tilde{t}_{1}\right)+\Gamma_{L_{2} R_{1}}\left(\tilde{t}_{1}\right)+\Gamma_{L_{2} R_{2}}\left(\tilde{t}_{1}\right)\right] . \\
\Gamma_{\tilde{t}_{2}}=\Gamma_{L L}\left(\tilde{t}_{2}\right) \sin ^{2} \theta_{t}+\Gamma_{R R}\left(\tilde{t}_{2}\right) \cos ^{2} \theta_{t}+\sin \theta_{t} \cos \theta_{t}\left[\Gamma_{L_{1} R_{1}}\left(\tilde{t}_{2}\right)+\Gamma_{L_{1} R_{2}}\left(\tilde{t}_{2}\right)+\Gamma_{L_{2} R_{1}}\left(\tilde{t}_{2}\right)+\Gamma_{L_{2} R_{2}}\left(\tilde{t}_{2}\right)\right] . \\
\Gamma_{\tilde{t}_{1} \tilde{t}_{2}}=\left[\Gamma_{L L}\left(\tilde{t}_{1}, \tilde{t}_{2}\right)+\Gamma_{R R}\left(\tilde{t}_{1}, \tilde{t}_{2}\right)\right] \sin \theta_{t} \cos \theta_{t}+\Gamma_{L R}\left(\tilde{t}_{1}, \tilde{t}_{2}\right) \cos ^{2} \theta_{t}+\Gamma_{R L}\left(\tilde{t}_{1}, \tilde{t}_{2}\right) \sin ^{2} \theta_{t} .
\end{gathered}
$$

The moduli of the complex couplings are as follows:

$$
\begin{array}{ll}
\left|\alpha_{1}^{\tilde{t}_{1}}\right|=\tilde{A}_{\tilde{Z}_{i}}^{t} \cos \theta_{t}-f_{t} N_{4 i} \sin \theta_{t}, \quad & \left|\beta_{1}^{\tilde{t}_{1}}\right|=f_{t} N_{4 i} \cos \theta_{t}+\tilde{B}_{\tilde{Z}_{i}}^{t} \sin \theta_{t}, \\
\left|\alpha_{1}^{\tilde{\tau}_{2}}\right|=\tilde{A}_{\tilde{Z}_{i}}^{t} \sin \theta_{t}+f_{t} N_{4 i} \cos \theta_{t}, & \left|\beta_{1}^{\tilde{\tau}_{2}}\right|=f_{t} N_{4 i} \sin \theta_{t}-\tilde{B}_{\tilde{Z}_{i}}^{t} \cos \theta_{t},
\end{array}
$$

where

$$
\begin{gathered}
\tilde{A}_{\tilde{Z}_{i}}^{t}=-\frac{g}{\sqrt{2}} N_{2 i}-\frac{g^{\prime}}{3 \sqrt{2}} N_{1 i}, \\
\tilde{B}_{\tilde{Z}_{i}}^{t}=-\frac{4}{3} \frac{g^{\prime}}{\sqrt{2}} N_{1 i}, \\
f_{t}=\frac{g m_{t}^{r u n}}{\sqrt{2} M_{W} \sin \beta} .
\end{gathered}
$$

The couplings themselves are then complex and depend upon the sign of the corresponding neutralinos mass. They are of the form $(a, b)$, where this represents the complex number $a+b i$. For positive masses the couplings are just:

$$
a^{\tilde{t}_{1}}=\left(\left|\alpha_{1}^{\tilde{t}_{1}}\right|, 0\right), \quad b^{\tilde{t}_{1}}=\left(\left|\beta_{1}^{\tilde{t}_{1}}\right|, 0\right), \quad a^{\tilde{t}_{2}}=\left(\left|\alpha_{1}^{\tilde{t}_{2}}\right|, 0\right), \quad b^{\tilde{t}_{2}}=\left(\left|\beta_{1}^{\tilde{t}_{1}}\right|, 0\right) .
$$

Meanwhile, for negative neutralino masses the effect of our field redefinition is to multiply the corresponding row of the neutralino mixing matrix by $-i$ therefore the couplings are then:

$$
a^{\tilde{t}_{1}}=\left(0,-\left|\alpha_{1}^{\tilde{t}_{1}}\right|\right), \quad b^{\tilde{t}_{1}}=\left(0,-\left|\beta_{1}^{\tilde{t}_{1}}\right|\right), \quad a^{\tilde{t}_{2}}=\left(0,-\left|\alpha_{1}^{\tilde{t}_{1}}\right|\right), \quad b^{\tilde{t}_{2}}=\left(0,-\left|\beta_{1}^{\tilde{t}_{2}}\right|\right) .
$$


In addition to account for differences in interactions for positive and negative neutralino masses as a result of this coupling difference and the extra associated $\gamma_{5}$ matrices we must also include factors of:

$$
(-1)^{\theta_{i}}=\left\{\begin{array}{l}
+1, \text { for positive neutralino masses, } \\
-1, \text { for negative neutralino masses }
\end{array}\right.
$$

The formula we use for $\Gamma_{\tilde{t}_{1}}$ is:

$$
\begin{aligned}
\Gamma_{\tilde{t}_{1}}= & -1)^{\theta_{i}}\left[\left(a^{\tilde{t}_{1}}{ }^{2}+b^{\tilde{t}_{1}}\right) \tilde{\psi}\left(m_{\tilde{g}}, m_{\tilde{t}_{1}}, m_{\tilde{t}_{1}}, m_{\tilde{Z}_{i}}\right)+4 a^{\tilde{t}_{1}} b^{\tilde{t}_{1}} m_{t} m_{\tilde{Z}_{i}} \tilde{\chi}\left(m_{\tilde{g}}, m_{\tilde{t}_{1}}, m_{\tilde{t}_{1}}, m_{\tilde{Z}_{i}}\right)\right. \\
& -4 \sin \theta_{t} \cos \theta_{t}\left(a^{\tilde{t}_{1}}{ }^{2}+b^{\tilde{t}_{1}}{ }^{2}\right) m_{\tilde{g}} m_{t} X\left(m_{\tilde{g}}, m_{\tilde{t}_{1}}, m_{\tilde{t}_{1}}, m_{\tilde{Z}_{i}}\right) \\
& -8 \sin \theta_{t} \cos \theta_{t} a^{\tilde{t}_{1}} b^{\tilde{t}_{1}} m_{\tilde{g}} m_{t}^{2} m_{\tilde{Z}_{i}} \zeta\left(m_{\tilde{g}}, m_{\tilde{t}_{1}}, m_{\tilde{t}_{1}}, m_{\tilde{Z}_{i}}\right) \\
& -2 \sin \theta_{t} \cos \theta_{t} a^{\tilde{t}_{1}} b^{\tilde{t}_{1}} Y\left(m_{\tilde{g}}, m_{\tilde{t}_{1}}, m_{\tilde{t}_{1}}, m_{\tilde{Z}_{i}}\right)+\left\{a^{\tilde{t}_{1}}{ }^{2} \cos ^{2} \theta_{t}+b^{\tilde{t}_{1}}{ }^{2} \sin ^{2} \theta_{t}\right\} \tilde{\phi}\left(m_{\tilde{g}}, m_{\tilde{t}_{1}}, m_{\tilde{t}_{1}}, m_{\tilde{Z}_{i}}\right) \\
& -2 m_{t}^{2} \sin \theta_{t} \cos \theta_{t} a^{\tilde{t}_{1}} b^{\tilde{t}_{1}} \xi\left(m_{\tilde{g}}, m_{\tilde{t}_{1}}, m_{\tilde{t}_{1}}, m_{\tilde{Z}_{i}}\right)+m_{\tilde{g}} m_{t} a^{\tilde{t}_{1}} b^{\tilde{t}_{1}} \xi\left(m_{\tilde{g}}, m_{\tilde{t}_{1}}, m_{\tilde{t}_{1}}, m_{\tilde{Z}_{i}}\right) \\
& -m_{\tilde{g}} m_{t} a^{\tilde{t}_{1}} b^{\tilde{t}_{1}} m_{\tilde{Z}_{i}}^{2} \tilde{\rho}\left(m_{\tilde{g}}, m_{\tilde{t}_{1}}, m_{\tilde{t}_{1}}, m_{\tilde{Z}_{i}}\right)+m_{\tilde{g}} m_{t}^{2} m_{\tilde{Z}_{i}}\left\{a^{\tilde{t}_{1}}{ }^{2} \sin ^{2} \theta_{t}+b^{\tilde{t}_{1}}{ }^{2} \cos ^{2} \theta_{t}\right\} \tilde{\rho}\left(m_{\tilde{g}}, m_{\tilde{t}_{1}}, m_{\tilde{t}_{1}}, m_{\tilde{Z}_{i}}\right) \\
& -m_{\tilde{Z}_{i}} m_{t} \sin \theta_{t} \cos \theta_{t}\left(a^{\tilde{t}_{1}}{ }^{2}+b^{\tilde{t}_{1}}{ }^{2}\right) m_{\tilde{g}}^{2} \tilde{\rho}\left(m_{\tilde{g}}, m_{\tilde{t}_{1}}, m_{\tilde{t}_{1}}, m_{\tilde{Z}_{i}}\right) \\
& \left.+m_{\tilde{Z}_{i}} m_{t} \sin \theta_{t} \cos \theta_{t}\left(a^{\tilde{t}_{1}}{ }^{2}+b^{\tilde{t}_{1}}{ }^{2}\right) \xi\left(m_{\tilde{g}}, m_{\tilde{t}_{1}}, m_{\tilde{t}_{1}}, m_{\tilde{Z}_{i}}\right)\right] .
\end{aligned}
$$

The $\Gamma_{\tilde{t}_{2}}$ formula we use is:

$$
\begin{aligned}
\Gamma_{\tilde{t}_{2}}= & (-1)^{\theta_{i}}\left[\left(a^{\tilde{t}_{2}}{ }^{2}+b^{\tilde{t}_{2}}{ }^{2}\right) \tilde{\psi}\left(m_{\tilde{g}}, m_{\tilde{t}_{2}}, m_{\tilde{t}_{2}}, m_{\tilde{Z}_{i}}\right)+4 a^{\tilde{t}_{2}} b^{\tilde{t}_{2}} m_{t} m_{\tilde{Z}_{i}} \tilde{\chi}\left(m_{\tilde{g}}, m_{\tilde{t}_{2}}, m_{\tilde{t}_{2}}, m_{\tilde{Z}_{i}}\right)\right. \\
& +4 m_{\tilde{g}} m_{t} \sin \theta_{t} \cos \theta_{t}\left(a^{\tilde{t}_{2}}{ }^{2}+b^{\tilde{t}_{2}}{ }^{2}\right) X\left(m_{\tilde{g}}, m_{\tilde{t}_{2}}, m_{\tilde{t}_{2}}, m_{\tilde{Z}_{i}}\right) \\
& +8 \sin \theta_{t} \cos \theta_{t} a^{\tilde{t}_{2}} b^{\tilde{t}_{2}} m_{\tilde{g}} m_{t}^{2} m_{\tilde{Z}_{i}} \zeta\left(m_{\tilde{g}}, m_{\tilde{t}_{2}}, m_{\tilde{t}_{2}}, m_{\tilde{Z}_{i}}\right)+2 \sin \theta_{t} \cos \theta_{t} a^{\tilde{t}_{2}} b^{\tilde{t}_{2}} Y\left(m_{\tilde{g}}, m_{\tilde{t}_{2}}, m_{\tilde{t}_{2}}, m_{\tilde{Z}_{i}}\right) \\
& +\left\{a^{\tilde{t}_{2}}{ }^{2} \sin ^{2} \theta_{t}+\cos ^{2} \theta_{t} b^{\tilde{t}_{2}}{ }^{2} \tilde{\phi}\left(m_{\tilde{g}}, m_{\tilde{t}_{2}}, m_{\tilde{t}_{2}}, m_{\tilde{Z}_{i}}\right)+2 m_{t}^{2} \sin \theta_{t} \cos \theta_{t} a^{\tilde{t}_{2}} b^{\tilde{t}_{2}} \xi\left(m_{\tilde{g}}, m_{\tilde{t}_{2}}, m_{\tilde{t}_{2}}, m_{\tilde{Z}_{i}}\right)\right. \\
& +m_{\tilde{g}} m_{t} a^{\tilde{t}_{2}} b^{\tilde{t}_{2}} \xi\left(m_{\tilde{g}}, m_{\tilde{t}_{2}}, m_{\tilde{t}_{2}}, m_{\tilde{Z}_{i}}\right)-m_{\tilde{g}} m_{t} m_{\tilde{Z}_{i}}^{2} a^{\tilde{t}_{2}} b^{\tilde{t}_{2}} \tilde{\rho}\left(m_{\tilde{g}}, m_{\tilde{t}_{2}}, m_{\tilde{t}_{2}}, m_{\tilde{Z}_{i}}\right) \\
& +m_{\tilde{g}} m_{\tilde{Z}_{i}} m_{t}^{2}\left\{a^{\tilde{t}_{2}}{ }^{2} \cos ^{2} \theta_{t}+b^{\tilde{t}_{2}}{ }^{2} \sin ^{2} \theta_{t}\right\} \tilde{\rho}\left(m_{\tilde{g}}, m_{\tilde{t}_{2}}, m_{\tilde{t}_{2}}, m_{\tilde{Z}_{i}}\right) \\
& -m_{\tilde{Z}_{i}} m_{t} \sin \theta_{t} \cos \theta_{t}\left(a^{\tilde{t}_{2}}{ }^{2}+b^{\tilde{t}_{2}}{ }^{2}\right) \xi\left(m_{\tilde{g}}, m_{\tilde{t}_{2}}, m_{\tilde{t}_{2}}, m_{\tilde{Z}_{i}}\right) \\
& \left.+m_{\tilde{g}}^{2} m_{\tilde{Z}_{i}} m_{t} \sin \theta_{t} \cos \theta_{t}\left(a^{\tilde{t}_{2}}{ }^{2}+b^{\tilde{t}^{2}}\right) \tilde{\rho}\left(m_{\tilde{g}}, m_{\tilde{t}_{2}}, m_{\tilde{t}_{2}}, m_{\tilde{Z}_{i}}\right)\right] .
\end{aligned}
$$

For $\Gamma_{\tilde{t}_{1} \tilde{t}_{2}}$, our formula, again extracted from sPHENO-3.3.8, is:

$$
\begin{aligned}
& \Gamma \tilde{t}_{1} \tilde{t}_{2}=(-1)^{\theta_{i}}\left[4 m_{\tilde{g}} m_{t}\left(\cos ^{2} \theta_{t}-\sin ^{2} \theta_{t}\right)\left(a^{\tilde{t}_{1}} a^{\tilde{t}_{2}}+b^{\tilde{t}_{1}} b^{\tilde{t}_{2}}\right) X\left(m_{\tilde{g}}, m_{\tilde{t}_{1}}, m_{\tilde{t}_{2}}, m_{\tilde{Z}_{i}}\right)\right. \\
& +4 m_{\tilde{g}} m_{t}^{2} m_{\tilde{Z}_{i}}\left(a^{\tilde{t}_{1}} b^{\tilde{t}_{2}}+b^{\tilde{t}_{1}} a^{\tilde{t}_{2}}\right)\left(\cos ^{2} \theta_{t}-\sin ^{2} \theta_{t}\right) \zeta\left(m_{\tilde{g}}, m_{\tilde{t}_{1}}, m_{\tilde{t}_{2}}, m_{\tilde{Z}_{i}}\right) \\
& +2\left\{b^{\tilde{t}_{1}} a^{\tilde{t}_{1}} \cos ^{2} \theta_{t}-\sin ^{2} \theta_{t} b^{\tilde{t}_{2}} a^{\tilde{t}_{1}}\right\} Y\left(m_{\tilde{g}}, m_{\tilde{t}_{1}}, m_{\tilde{t}_{2}}, m_{\tilde{Z}_{i}}\right) \\
& +2 \sin \theta_{t} \cos \theta_{t}\left(a^{\tilde{t}_{1}} a^{\tilde{t}_{2}}-b^{\tilde{t}_{1}} b^{\tilde{t}_{2}}\right) \tilde{\phi}\left(m_{\tilde{g}}, m_{\tilde{t}_{1}}, m_{\tilde{t}_{2}}, m_{\tilde{Z}_{i}}\right) \\
& +2 m_{t} m_{\tilde{Z}_{i}}\left(a^{\tilde{t}_{1}} a^{\tilde{t}_{2}}-b^{\tilde{t}_{1}} b^{\tilde{t}_{2}}\right) \chi^{\prime}\left(m_{\tilde{g}}, m_{\tilde{t}_{1}}, m_{\tilde{t}_{2}}, m_{\tilde{Z}_{i}}\right) \\
& +2 m_{t}^{2}\left\{\cos ^{2} \theta_{t} a^{\tilde{t}_{1}} b^{\tilde{t}_{2}}-\sin ^{2} \theta_{t} b^{\tilde{t}_{1}} a^{\tilde{t}_{2}}\right\} \xi\left(m_{\tilde{g}}, m_{\tilde{t}_{1}}, m_{\tilde{t}_{2}}, m_{\tilde{Z}_{i}}\right) \\
& +2 m_{\tilde{g}} m_{t}^{2} m_{\tilde{Z}_{i}}\left(b^{\tilde{t}_{1}} b^{\tilde{t}_{2}}-a^{\tilde{t}_{1}} a^{\tilde{t}_{2}}\right) \sin \theta_{t} \cos \theta_{t} \tilde{\rho}\left(m_{\tilde{g}}, m_{\tilde{t}_{1}}, m_{\tilde{t}_{2}}, m_{\tilde{Z}_{i}}\right) \\
& -4 \sin \theta_{t} \cos \theta_{t} m_{\tilde{g}} m_{t}\left(a^{\tilde{t}_{1}} b^{\tilde{t}_{2}}-b^{\tilde{t}_{1}} a^{\tilde{t}_{2}}\right) \chi^{\prime}\left(m_{\tilde{g}}, m_{\tilde{t}_{1}}, m_{\tilde{t}_{2}}, m_{\tilde{Z}_{i}}\right) \\
& -2 \sin \theta_{t} \cos \theta_{t} m_{\tilde{g}} m_{t}\left(a^{\tilde{t}_{1}} b^{\tilde{t}_{2}}-b^{\tilde{t}_{1}} a^{\tilde{t}_{2}}\right) \xi\left(m_{\tilde{g}}, m_{\tilde{t}_{1}}, m_{\tilde{t}_{2}}, m_{\tilde{Z}_{i}}\right) \\
& +2 m_{t} m_{\tilde{Z}_{i}}\left\{\sin ^{2} \theta_{t} a^{\tilde{t}_{1}} a^{\tilde{t}_{2}}-\cos ^{2} \theta_{t} b^{\tilde{t}_{1}} b^{\tilde{t}_{2}}\right\} \xi\left(m_{\tilde{g}}, m_{\tilde{t}_{1}}, m_{\tilde{t}_{2}}, m_{\tilde{Z}_{i}}\right) \\
& +2 m_{\tilde{g}}^{3} m_{t}\left(a^{\tilde{t}_{1}} b_{1}^{\tilde{t}_{2}}-b^{\tilde{t}_{2}} a^{\tilde{t}_{2}}\right) \sin \theta_{t} \cos \theta_{t} \tilde{\rho}\left(m_{\tilde{g}}, m_{\tilde{t}_{1}}, m_{\tilde{t}_{2}}, m_{\tilde{Z}_{i}}\right) \\
& \left.-2 m_{\tilde{g}}^{2} m_{t} m_{\tilde{Z}_{i}}\left\{\sin ^{2} \theta_{t} a^{\tilde{t}_{1}} a^{\tilde{t}_{2}}-\cos ^{2} \theta_{t} b^{\tilde{t}_{1}} b^{\tilde{t}_{2}}\right\} \tilde{\rho}\left(m_{\tilde{g}}, m_{\tilde{t}_{1}}, m_{\tilde{t}_{2}}, m_{\tilde{Z}_{i}}\right)\right] \text {. }
\end{aligned}
$$




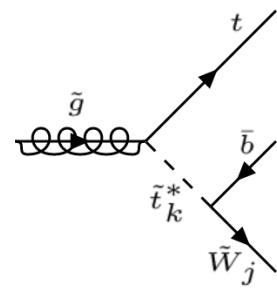

(a)

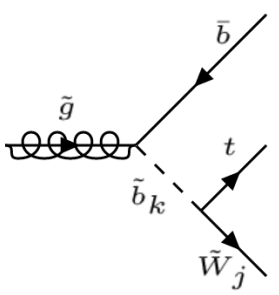

(b)

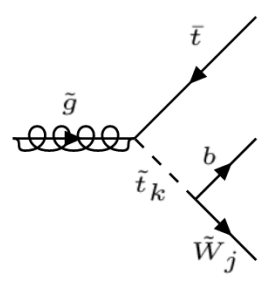

(c)

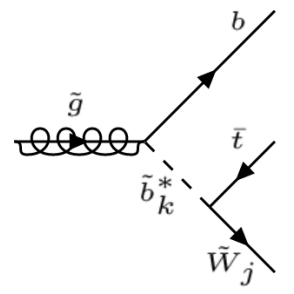

(d)

Figure G.7: Feynman diagrams for the 3 body decay of a gluino into a chargino and a top-bottom pair, as mediated by stops $\tilde{t}_{1 / 2}$ and sbottoms $\tilde{t}_{1 / 2}$. $j, k=1,2$.

For the 3 body decay of $\tilde{g} \rightarrow \tilde{Z}_{i} b \bar{b}$ then the formulae are exactly as above but with the replacements: $m_{\tilde{t}_{i}} \rightarrow m_{\tilde{b}_{i}}$,

$$
\begin{aligned}
\tilde{A}_{\tilde{Z}_{i}}^{t} & \rightarrow \tilde{A}_{\tilde{Z}_{i},}^{b} \\
\tilde{B}_{\tilde{Z}_{i}}^{t} & \rightarrow \tilde{B}_{\tilde{Z}_{i}}^{b}, \\
f_{t} & \rightarrow f_{b}, \\
N_{4 i} & \rightarrow N_{3 i}, \\
\theta_{t} & \rightarrow \theta_{b}, \\
m_{t} & \rightarrow m_{b} .
\end{aligned}
$$

In our program both the $\tilde{g} \rightarrow \tilde{Z}_{i} t \bar{t}$ and $\tilde{g} \rightarrow \tilde{Z}_{i} b \bar{b}$ decays are implemented in the same function, just depending on whether its decaying to tops or bottoms different couplings are used as described above.

$$
\begin{gathered}
\tilde{A}_{\tilde{Z}_{i}}^{b}=\frac{g}{\sqrt{2}} N_{2 i}-\frac{g^{\prime}}{3 \sqrt{2}} N_{1 i}, \\
\tilde{B}_{\tilde{Z}_{i}}^{b}=\frac{2}{3} \frac{g^{\prime}}{\sqrt{2}} N_{1 i}, \\
f_{b}=\frac{g m_{b}^{r u n}}{\sqrt{2} M_{W} \cos \beta} .
\end{gathered}
$$

Note that the integrals used in these equations, $\tilde{\psi}, \tilde{\chi}, X, \zeta, \tilde{\phi}, \xi, \tilde{\rho}, Y, \chi^{\prime}$ are given below in Eq: G.74, (G.75, G.76, G.77, G.78), G.79, G.80 (G.81, G.82) respectively, when the formulae for $\tilde{Z}_{i} \rightarrow \tilde{Z}_{j} f \bar{f}$ are given.

\section{$\tilde{g} \rightarrow \tilde{W}_{i} q \bar{q}^{\prime}$}

The decay of a gluino into a chargino, quark $(q)$ and anti-quark $\left(\overline{q^{\prime}}\right)$ can occur via intermediate squarks of either the $q$ or $q^{\prime}$, therefore there are four possible intermediates in the case where intra-generational squark mixing effects are included. For example, $\tilde{g} \rightarrow \tilde{W}_{j} t \bar{b}$ may proceed via $\tilde{t}_{1}, \tilde{t}_{2}, \tilde{b}_{1}$ or $\tilde{b}_{2}$. Again there are both $\mathrm{t}$ and u channel contributions which may contribute to $\tilde{g} \rightarrow \tilde{W}_{j} t \bar{b}$ or $\tilde{g} \rightarrow \tilde{W}_{j} b \bar{t}$. The 8 diagrams are therefore shown as a set of $4(k=1,2$ for each intermediate shown) in Fig G.7. There are therefore 4 squared contributions to each of $t \bar{b}$ and $b \bar{t}$ as well as $\tilde{t}_{1} \tilde{t}_{1}, \tilde{t}_{1} \tilde{t}_{2}, \tilde{t}_{2} \tilde{t}_{2}, \tilde{b}_{1} \tilde{b}_{1}, \tilde{b}_{1} \tilde{b}_{2}, \tilde{b}_{2} \tilde{b}_{2}, \tilde{t}_{1} b_{1}, \tilde{t}_{1} \tilde{b}_{2}, \tilde{t}_{2} \tilde{b}_{1}$ and $\tilde{t}_{2} \tilde{b}_{2}$ interferences. Note "diagonal" interferences such as $\tilde{t}_{1} \tilde{t}_{1}$ are included with non-interference squared terms into the $\Gamma_{\tilde{t}_{1}}$ type contributions. In the formulae, the Yukawa couplings, intra-generational squark mixing and final state fermion masses are all accounted for; however whilst the bottom quark mass $m_{b}$ is included in the phase space, it is neglected from the squared matrix element, this drops any $\tilde{b}_{k} \tilde{b}_{l}$ interferences as these are proportional to $m_{b}$. The approach used follows that in Baer and Tata's book 'Weak Scale Supersymmetry' [41] with the formulae we use taken from sPHENO [11, 35], based on the calculations in reference [34]. The formulae used, as in sPHENO, differ in a few places from those in Baer and Tata.

$$
\Gamma\left(\tilde{g} \rightarrow t \bar{b} \tilde{W}_{i}^{-}\right)=\frac{\alpha_{s}}{16 \pi^{2} m_{\tilde{g}}}\left(\Gamma_{\tilde{t}_{1}}+\Gamma_{\tilde{t}_{2}}+\Gamma_{\tilde{t}_{1} \tilde{t}_{2}}+\Gamma_{\tilde{b}_{1}}+\Gamma_{\tilde{b}_{2}}+\Gamma_{\tilde{t}_{1} \tilde{b}_{1}}+\Gamma_{\tilde{t}_{1} \tilde{b}_{2}}+\Gamma_{\tilde{t}_{2} \tilde{b}_{1}}+\Gamma_{\tilde{t}_{2} \tilde{b}_{2}}\right) .
$$

The chargino - quark - squark couplings are given below, remember we have the chargino mixing angle $\theta_{L}$ and $\theta_{R}$ pre-transformed so that $\theta_{L / R} \rightarrow-\theta_{L / R}+\pi / 2$ in order to use the convention where the lightest mass chargino eigenstate 
$\tilde{W}_{1}$ appears first in the multiplet:

$$
\begin{aligned}
& \alpha_{\tilde{W}_{1}}^{\tilde{t}_{1}}=-g \sin \theta_{L} \cos \theta_{t}+f_{t} \cos \theta_{R} \sin \theta_{t}, \\
& \beta_{\tilde{W}_{1}}^{\tilde{f}_{1}}=-f_{b} \cos \theta_{L} \cos \theta_{t}, \\
& \alpha_{\tilde{W}_{1}}^{\tilde{b}_{1}}=-g \sin \theta_{L} \cos \theta_{b}+f_{b} \cos \theta_{L} \sin \theta_{b}, \\
& \beta_{\tilde{W}_{1}}^{\tilde{b}_{1}}=-f_{t} \cos \theta_{R} \cos \theta_{b}, \\
& \alpha_{\tilde{W}_{2}}^{\tilde{\tau}_{1}}=-g \cos \theta_{L} \cos \theta_{t}-f_{t} \sin \theta_{R} \sin \theta_{t}, \\
& \beta_{\tilde{W}_{\tilde{W}_{2}}}^{\tilde{\tilde{t}}_{1}}=f_{b} \sin \theta_{L} \cos \theta_{t}, \\
& \alpha_{\tilde{W}_{1}}^{\tilde{b}_{1}}=-g \cos \theta_{L} \cos \theta_{b}-f_{b} \sin \theta_{L} \sin \theta_{b}, \\
& \beta_{\tilde{W}_{2}}^{\tilde{b}_{1}}=f_{t} \sin \theta_{R} \cos \theta_{b} .
\end{aligned}
$$

We obtain the couplings for $\tilde{t}_{2}$ and $\tilde{b}_{2}$ by changing $\cos \theta_{q} \rightarrow \sin \theta_{q}$ and $\sin \theta_{q} \rightarrow-\cos \theta_{q}$. The $\tilde{W}_{2}$ couplings are obtained from those of $\tilde{W}_{1}$ by making the replacements $\cos \theta_{L / R} \rightarrow-\sin \theta_{L / R}$ and $\sin \theta_{L / R} \rightarrow \cos \theta_{L / R}$.

The contributions in (G.24) are as follows:

$$
\begin{aligned}
& \Gamma_{\tilde{t}_{1}}=\left[\left(\alpha_{\tilde{W}_{i}}^{\tilde{t}_{1}}\right)^{2}+\left(\beta_{\tilde{W}_{i}}^{\tilde{t}_{1}}\right)^{2}\right]\left[G_{1}\left(m_{\tilde{g}}, m_{\tilde{t}_{1}}, m_{\tilde{W}_{i}}\right)-\sin 2 \theta_{t} G_{8}\left(m_{\tilde{g}}, m_{\tilde{t}_{1}}, m_{\tilde{t}_{1}}, m_{\tilde{W}_{i}}\right)\right], \\
& \Gamma_{\tilde{t}_{2}}=\left[\left(\alpha_{\tilde{W}_{i}}^{\tilde{\tau}_{2}}\right)^{2}+\left(\beta_{\tilde{W}_{i}}^{\tilde{t}_{2}}\right)^{2}\right]\left[G_{1}\left(m_{\tilde{g}}, m_{\tilde{t}_{2}}, m_{\tilde{W}_{i}}\right)+\sin 2 \theta_{t} G_{8}\left(m_{\tilde{g}}, m_{\tilde{t}_{2}}, m_{\tilde{t}_{2}}, m_{\tilde{W}_{i}}\right)\right], \\
& \Gamma_{\tilde{b}_{1}}=\left[\left(\alpha_{\tilde{W}_{i}}^{\tilde{b}_{1}}\right)^{2}+\left(\beta_{\tilde{W}_{i}}^{\tilde{b}_{1}}\right)^{2}\right] G_{2}\left(m_{\tilde{g}}, m_{\tilde{b}_{1}}, m_{\tilde{W}_{i}}\right)+\alpha_{\tilde{W}_{i}}^{\tilde{b}_{1}} \beta_{\tilde{W}_{i}}^{\tilde{b}_{1}} G_{3}\left(m_{\tilde{g}}, m_{\tilde{b}_{1}}, m_{\tilde{W}_{i}}\right), \\
& \Gamma_{\tilde{b}_{2}}=\left[\left(\alpha_{\tilde{W}_{i}}^{\tilde{b}_{2}}\right)^{2}+\left(\beta_{\tilde{W}_{i}}^{\tilde{b}_{2}}\right)^{2}\right] G_{2}\left(m_{\tilde{g}}, m_{\tilde{b}_{2}}, m_{\tilde{W}_{i}}\right)+\alpha_{\tilde{W}_{i}}^{\tilde{b}_{2}} \beta_{\tilde{W}_{i}}^{\tilde{w}_{2}} G_{3}\left(m_{\tilde{g}}, m_{\tilde{b}_{2}}, m_{\tilde{W}_{i}}\right), \\
& \Gamma_{\tilde{t}_{1} \tilde{t}_{2}}=2\left(\alpha_{\tilde{W}_{i}}^{\tilde{t}_{1}} \alpha_{\tilde{W}_{i}}^{\tilde{\tau}_{2}}+\beta_{\tilde{W}_{i}}^{\tilde{t}_{1}} \beta_{\tilde{W}_{i}}^{\tilde{t}_{2}}\right) \cos 2 \theta_{t} G_{8}\left(m_{\tilde{g}}, m_{\tilde{t}_{1}}, m_{\tilde{t}_{2}}, m_{\tilde{W}_{i}}\right), \\
& \Gamma_{\tilde{t}_{1} \tilde{b}_{1}}=\left(\cos \theta_{t} \sin \theta_{b} \alpha_{\tilde{W}_{i}}^{\tilde{b}_{1}} \beta_{\tilde{W}_{i}}^{\tilde{t}_{1}}+\sin \theta_{t} \cos \theta_{b} \beta_{\tilde{W}_{i}}^{\tilde{b}_{1}} \alpha_{\tilde{W}_{i}}^{\tilde{t}_{1}}\right) G_{6}\left(m_{\tilde{g}}, m_{\tilde{t}_{1}}, m_{\tilde{b}_{1}}, m_{\tilde{W}_{i}}\right) \\
& -\left(\cos \theta_{t} \cos \theta_{b} \alpha_{\tilde{W}_{i}}^{\tilde{o}_{1}} \alpha_{\tilde{W}_{i}}^{\tilde{t}_{1}}+\sin \theta_{t} \sin \theta_{b} \beta_{\tilde{W}_{i}}^{\tilde{b}_{1}} \beta_{\tilde{W}_{i}}^{\tilde{t}_{1}}\right) G_{4}\left(m_{\tilde{g}}, m_{\tilde{t}_{1}}, m_{\tilde{b}_{1}}, m_{\tilde{W}_{i}}\right) \\
& -\left(\cos \theta_{t} \cos \theta_{b} \beta_{\tilde{W}_{i}}^{\tilde{b}_{1}} \alpha_{\tilde{W}_{i}}^{\tilde{t}_{1}}+\sin \theta_{t} \sin \theta_{b} \alpha_{\tilde{W}_{i}}^{\tilde{b}_{1}} \beta_{\tilde{W}_{i}}^{\tilde{t}_{1}}\right) G_{5}\left(m_{\tilde{g}}, m_{\tilde{t}_{1}}, m_{\tilde{b}_{1}}, m_{\tilde{W}_{i}}\right) \\
& -\left(\cos \theta_{t} \sin \theta_{b} \beta_{\tilde{W}_{i}}^{\tilde{b}_{1}} \beta_{\tilde{W}_{i}}^{\tilde{t}_{1}}+\sin \theta_{t} \cos \theta_{b} \alpha_{\tilde{W}_{i}}^{\tilde{b}_{1}} \alpha_{\tilde{W}_{i}}^{\tilde{t}_{1}}\right) G_{7}\left(m_{\tilde{g}}, m_{\tilde{t}_{1}}, m_{\tilde{b}_{1}}, m_{\tilde{W}_{i}}\right), \\
& \Gamma_{\tilde{t}_{1} \tilde{b}_{2}}=\left(-\cos \theta_{t} \cos \theta_{b} \alpha_{\tilde{W}_{i}}^{\tilde{b}_{1}} \beta_{\tilde{W}_{i}}^{\tilde{t}_{1}}+\sin \theta_{t} \sin \theta_{b} \beta_{\tilde{W}_{i}}^{\tilde{b}_{1}} \alpha_{\tilde{W}_{i}}^{\tilde{\tau}_{1}}\right) G_{6}\left(m_{\tilde{g}}, m_{\tilde{t}_{1}}, m_{\tilde{b}_{1}}, m_{\tilde{W}_{i}}\right) \\
& -\left(\cos \theta_{t} \sin \theta_{b} \alpha_{\tilde{W}_{i}}^{\tilde{b}_{1}} \alpha_{\tilde{W}_{i}}^{\tilde{t}_{1}}-\sin \theta_{t} \cos \theta_{b} \beta_{\tilde{W}_{i}}^{\tilde{b}_{1}} \beta_{\tilde{W}_{i}}^{\tilde{t}_{1}}\right) G_{4}\left(m_{\tilde{g}}, m_{\tilde{t}_{1}}, m_{\tilde{b}_{1}}, m_{\tilde{W}_{i}}\right) \\
& -\left(\cos \theta_{t} \sin \theta_{b} \beta_{\tilde{W}_{i}}^{\tilde{b}_{1}} \alpha_{\tilde{W}_{i}}^{\tilde{t}_{1}}-\sin \theta_{t} \cos \theta_{b} \alpha_{\tilde{W}_{i}}^{\tilde{W}_{1}} \beta_{\tilde{W}_{i}}^{\tilde{t}_{1}}\right) G_{5}\left(m_{\tilde{g}}, m_{\tilde{t}_{1}}, m_{\tilde{b}_{1}}, m_{\tilde{W}_{i}}\right) \\
& -\left(-\cos \theta_{t} \cos \theta_{b} \beta_{\tilde{W}_{i}}^{\tilde{c}_{1}} \beta_{\tilde{W}_{i}}^{\tilde{1}_{1}}+\sin \theta_{t} \sin \theta_{b} \alpha_{\tilde{W}_{i}}^{\tilde{b}_{1}} \alpha_{\tilde{W}_{i}}^{\tilde{c}_{1}}\right) G_{7}\left(m_{\tilde{g}}, m_{\tilde{t}_{1}}, m_{\tilde{b}_{1}}, m_{\tilde{W}_{i}}\right), \\
& \Gamma_{\tilde{t}_{2} \tilde{b}_{1}}=\left(\sin \theta_{t} \sin \theta_{b} \alpha_{\tilde{W}_{i}}^{\tilde{b}_{1}} \beta_{\tilde{W}_{i}}^{\tilde{t}_{1}}-\cos \theta_{t} \cos \theta_{b} \beta_{\tilde{W}_{i}}^{\tilde{b}_{1}} \alpha_{\tilde{W}_{i}}^{\tilde{t}_{1}}\right) G_{6}\left(m_{\tilde{g}}, m_{\tilde{t}_{1}}, m_{\tilde{b}_{1}}, m_{\tilde{W}_{i}}\right) \\
& -\left(\sin \theta_{t} \cos \theta_{b} \alpha_{\tilde{W}_{i}}^{\tilde{b}_{1}} \alpha_{\tilde{W}_{i}}^{\tilde{t}_{1}}-\cos \theta_{t} \sin \theta_{b} \beta_{\tilde{W}_{i}}^{\tilde{b}_{1}} \beta_{\tilde{W}_{i}}^{\tilde{t}_{1}}\right) G_{4}\left(m_{\tilde{g}}, m_{\tilde{t}_{1}}, m_{\tilde{b}_{1}}, m_{\tilde{W}_{i}}\right) \\
& -\left(\sin \theta_{t} \cos \theta_{b} \beta_{\tilde{W}_{i}}^{\tilde{b}_{1}} \alpha_{\tilde{W}_{i}}^{\tilde{t}_{1}}-\cos \theta_{t} \sin \theta_{b} \alpha_{\tilde{W}_{i}}^{\tilde{w}_{1}} \beta_{\tilde{W}_{i}}^{\tilde{t}_{1}}\right) G_{5}\left(m_{\tilde{g}}, m_{\tilde{t}_{1}}, m_{\tilde{b}_{1}}, m_{\tilde{W}_{i}}\right) \\
& -\left(\sin \theta_{t} \sin \theta_{b} \beta_{\tilde{W}_{i}}^{\tilde{b}_{1}} \beta_{\tilde{W}_{i}}^{\tilde{t}_{1}}-\cos \theta_{t} \cos \theta_{b} \alpha_{\tilde{W}_{i}}^{\tilde{b}_{1}} \alpha_{\tilde{W}_{i}}^{\tilde{t}_{1}}\right) G_{7}\left(m_{\tilde{g}}, m_{\tilde{t}_{1}}, m_{\tilde{b}_{1}}, m_{\tilde{W}_{i}}\right),
\end{aligned}
$$




$$
\begin{aligned}
\Gamma_{\tilde{t}_{2} \tilde{b}_{2}}= & -\left(\sin \theta_{t} \cos \theta_{b} \alpha_{\tilde{W}_{i}}^{\tilde{b}_{1}} \beta_{\tilde{W}_{i}}^{\tilde{t}_{1}}+\cos \theta_{t} \sin \theta_{b} \beta_{\tilde{W}_{i}}^{\tilde{b}_{1}} \alpha_{\tilde{W}_{i}}^{\tilde{t}_{1}}\right) G_{6}\left(m_{\tilde{g}}, m_{\tilde{t}_{1}}, m_{\tilde{b}_{1}}, m_{\tilde{W}_{i}}\right) \\
& -\left(\sin \theta_{t} \sin \theta_{b} \alpha_{\tilde{W}_{i}}^{\tilde{b}_{1}} \alpha_{\tilde{W}_{i}}^{\tilde{t}_{1}}+\cos \theta_{t} \cos \theta_{b} \beta_{\tilde{W}_{i}}^{\tilde{b}_{1}} \beta_{\tilde{W}_{i}}^{\tilde{t}_{1}}\right) G_{4}\left(m_{\tilde{g}}, m_{\tilde{t}_{1}}, m_{\tilde{b}_{1}}, m_{\tilde{W}_{i}}\right) \\
& -\left(\sin \theta_{t} \sin \theta_{b} \beta_{\tilde{W}_{i}}^{\tilde{b}_{1}} \alpha_{\tilde{W}_{i}}^{\tilde{t}_{1}}+\cos \theta_{t} \cos \theta_{b} \alpha_{\tilde{W}_{i}}^{\tilde{b}_{1}} \beta_{\tilde{W}_{i}}^{\tilde{t}_{1}}\right) G_{5}\left(m_{\tilde{g}}, m_{\tilde{t}_{1}}, m_{\tilde{b}_{1}}, m_{\tilde{W}_{i}}\right) \\
& +\left(\sin \theta_{t} \cos \theta_{b} \beta_{\tilde{W}_{i}}^{\tilde{b}_{1}} \beta_{\tilde{W}_{i}}^{\tilde{t}_{1}}+\cos \theta_{t} \sin \theta_{b} \alpha_{\tilde{W}_{i}}^{\tilde{b}_{1}} \alpha_{\tilde{W}_{i}}^{\tilde{t}_{1}}\right) G_{7}\left(m_{\tilde{g}}, m_{\tilde{t}_{1}}, m_{\tilde{b}_{1}}, m_{\tilde{W}_{i}}\right) .
\end{aligned}
$$

The integrals $G_{1}$ to $G_{8}$ are given by the following, where $s_{t}=m_{\tilde{g}}^{2}+m_{t}^{2}-2 E_{t} m_{\tilde{g}}$ and $s_{b}=m_{\tilde{g}}^{2}+m_{b}^{2}-2 E_{b} m_{\tilde{g}}$ :

$$
\begin{aligned}
& G_{1}\left(m_{\tilde{g}}, m_{\tilde{t}_{k}}, m_{\tilde{W}_{i}}\right)=m_{\tilde{g}} \int \frac{d E_{t} p_{t} E_{t}\left(s_{t}-m_{\tilde{W}_{i}}^{2}\right)^{2}}{\left(s_{t}-m_{\tilde{t}_{k}}^{2}\right)^{2} s_{t}}, \\
& G_{2}\left(m_{\tilde{g}}, m_{\tilde{b}_{k}}, m_{\tilde{W}_{i}}\right)=m_{\tilde{g}} \int d E_{b} E_{b}^{2} \lambda^{\frac{1}{2}}\left(s_{b}, m_{\tilde{W}_{i}}^{2}, m_{t}^{2}\right) \frac{s_{b}-m_{t}^{2}-m_{\tilde{W}_{i}}^{2}}{\left(s_{b}^{2}-m_{\tilde{b}_{k}}^{2}\right)^{2} s_{b}}, \\
& G_{3}\left(m_{\tilde{g}}, m_{\tilde{b}_{k}}, m_{\tilde{W}_{i}}\right)=\int d E_{b} E_{b}^{2} \lambda^{\frac{1}{2}}\left(s_{b}, m_{\tilde{W}_{i}}^{2}, m_{t}^{2}\right) \frac{4 m_{\tilde{g}} m_{\tilde{W}_{i}} m_{t}}{\left(s_{b}^{2}-m_{\tilde{b}_{k}}^{2}\right)^{2} s_{b}}, \\
& G_{4}\left(m_{\tilde{g}}, m_{\tilde{t}_{j}}, m_{\tilde{b}_{k}}, m_{\tilde{W}_{i}}\right)=m_{\tilde{g}} m_{\tilde{W}_{i}} \int \frac{d E_{t}}{s_{t}-m_{\tilde{t}_{j}}^{2}}\left[E_{b}(\max )-E_{b}(\min )-\frac{m_{\tilde{b}_{j}}^{2}+m_{t}^{2}-2 E_{t} m_{\tilde{g}}-m_{\tilde{W}_{i}}^{2}}{2 m_{\tilde{g}}} \log X\right], \\
& G_{5}\left(m_{\tilde{g}}, m_{\tilde{t}_{j}}, m_{\tilde{b}_{k}}, m_{\tilde{W}_{i}}\right)=\frac{m_{t}}{2} \int d E_{t} \frac{s_{t}-m_{\tilde{W}_{i}}^{2}}{s_{t}-m_{\tilde{t}_{j}}^{2}} \log X, \\
& G_{6}\left(m_{\tilde{g}}, m_{\tilde{t}_{j}}, m_{\tilde{b}_{k}}, m_{\tilde{W}_{i}}\right)=\frac{1}{2} \int \frac{d E_{t}}{s_{t}-m_{\tilde{t}_{j}}^{2}}\left\{\left[m_{\tilde{g}}\left(s_{t}-m_{\tilde{W}_{i}}^{2}\right)+\frac{m_{\tilde{b}_{k}}^{2}-m_{\tilde{g}}^{2}}{m_{\tilde{g}}} s_{t}\right] \log X-2 s_{t}\left[E_{b}(\max )-E_{b}(\min )\right]\right\}, \\
& G_{7}\left(m_{\tilde{g}}, m_{\tilde{t}_{j}}, m_{\tilde{b}_{k}}, m_{\tilde{W}_{i}}\right)=\frac{1}{2} m_{\tilde{W}_{i}} m_{t} \int \frac{d E_{t}}{s_{t}-m_{\tilde{t}_{j}}^{2}}\left\{2\left[E_{b}(\max )-E_{b}(\min )\right]-\frac{m_{\tilde{b}_{k}}^{2}-m_{\tilde{g}}^{2}}{m_{\tilde{g}}} \log X\right\}, \\
& G_{8}\left(m_{\tilde{g}}, m_{\tilde{t}_{1}}, m_{\tilde{t}_{2}}, m_{\tilde{W}_{i}}\right)=m_{\tilde{g}} m_{t} \int d E_{t} \frac{\left(s_{t}-m_{\tilde{W}_{i}}^{2}\right)\left[E_{b}(\max )-E_{b}(\min )\right]}{\left(s_{t}-m_{\tilde{t}_{1}}^{2}\right)\left(s_{t}-m_{\tilde{t}_{2}}^{2}\right)} .
\end{aligned}
$$

The limits of integration here are $m_{t}$ to $\left(m_{\tilde{g}}^{2}+m_{t}^{2}-\left(m_{\tilde{W}_{i}}+m_{b}\right)^{2}\right) /\left(2 m_{\tilde{g}}\right)$ for the $E_{t}$ integrals, and $m_{b}$ to $\left(m_{\tilde{g}}^{2}-\left(m_{t}+\right.\right.$ $\left.\left.m_{\tilde{W}_{i}}\right)^{2}\right) /\left(2 m_{\tilde{g}}\right)$ for the $E_{b}$ integrals. Here $p_{t}=\sqrt{E_{t}^{2}-m_{t}^{2}}, E_{b}(\max / \min )$ and $X$ are given by:

$$
\begin{gathered}
E_{b}(\max / \min )=\frac{\left(s_{t}+m_{b}^{2}-m_{\tilde{W}_{i}}^{2}\right)\left(m_{\tilde{g}}-E_{t}\right) \pm \lambda^{\frac{1}{2}}\left(s_{t}, m_{b}^{2}, m_{\tilde{W}_{i}}^{2}\right)}{2 s_{t}}, \\
X=\frac{m_{\tilde{b}_{j}}^{2}+2 E_{b}(\max ) m_{\tilde{g}}-m_{\tilde{g}}^{2}}{m_{\tilde{b}_{j}}^{2}+2 E_{b}(\min ) m_{\tilde{g}}-m_{\tilde{g}}^{2}}
\end{gathered}
$$

The formulae for the first and second generation quarks can be obtained from those for the third generation straightforwardly, in fact they are simpler as the Yukawa coupling can often be neglected. 


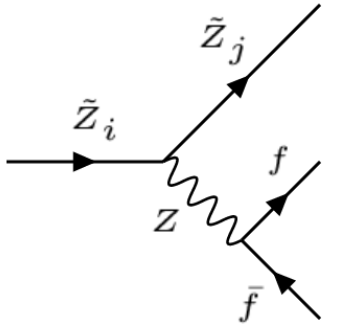

(a)

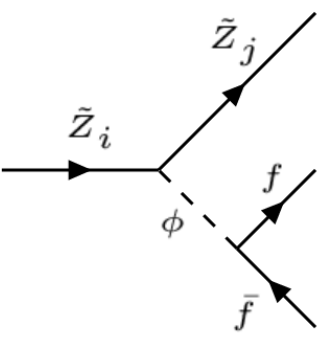

(b)

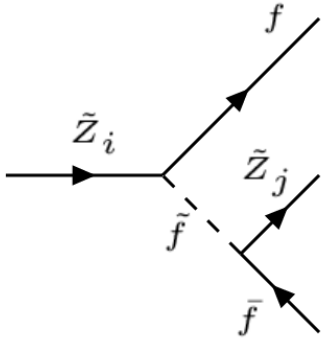

(c)

Figure G.8: Feynman diagrams for the 3 body decay of a neutralino into a lighter neutralino and a fermion anti-fermion pair, as mediated by $Z$ bosons, Higgs bosons $\phi=h, H, A$, or sfermions $\tilde{f}_{1 / 2} . i>j$ and $i, j \in 1,2,3,4$.

\section{Appendix G.2. Neutralino $1 \rightarrow 3$ Decays}

$$
\tilde{Z}_{i} \rightarrow \tilde{Z}_{j} f \bar{f}
$$

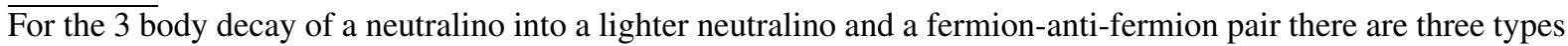
of contribution, as illustrated in the Feynman diagrams in Fig G.8, Z boson exchange, neutral Higgs boson exchange and sfermion exchange. The effects of Yukawa couplings, sfermion mixing and finite non-zero quark masses in the final state have been included. Similarly, the effects of negative neutralino masses in initial or final states are included. However, we have taken the approach of Baer et al in references [34, 41]; whilst we have included the effects of the quark mass in the phase space, the quark mass has been approximated as zero in the squared matrix element. This approximation is justified on the basis that the fermion-anti-fermion pair may not be a $t \bar{t}$ pair as the decay calculator only evaluates the 3 body decays when 2 body decays are absent, given the dominance of 2 body modes over 3 body modes in branching ratios. Whenever the 3 body mode $\tilde{Z}_{i} \rightarrow \tilde{Z}_{j} t \bar{t}$ is available then so are the 2 body modes $\tilde{Z}_{i} \rightarrow \tilde{Z}_{j} Z$ and $\tilde{Z}_{i} \rightarrow \tilde{Z}_{j} h$, which will make the 3 body modes negligible. It is however crucial to include the effects of the nonzero quark masses in the phase space, as has been done, as often the phase space available to these decays is limited (e.g. for compressed spectra) and so the reduction in phase space caused by the finite quark masses is important. Similarly, with non-zero quark masses the Higgs intermediate contributions are allowed. Nonetheless the effect of the approximation is just to remove the Higgs boson - $Z$ interferences and CP even - CP odd Higgs boson interferences, which are generally necessarily small compared to other contributions. The formulae for the included contributions themselves are all taken from sPHENO, as for the other 3 body modes. It should also be noted that the calculation in sPHENO was done in the Feynman gauge so a goldstone contribution, corresponding to the longitudinal component of the $Z$ boson, is required. The included contributions are therefore the squared $Z$ (including goldstone), $\phi$ and $\tilde{f}$ contributions as well as $h H, Z \tilde{f}, \phi \tilde{f}$ and $Z A$ interferences. ${ }^{5}$

$$
\begin{aligned}
\Gamma\left(\tilde{Z}_{i} \rightarrow \tilde{Z}_{j} f \bar{f}\right)=\frac{N_{c}}{512 \pi^{3}\left|m_{\tilde{Z}_{i}}\right|^{3}}\left(\Gamma_{Z}+\Gamma_{h}+\Gamma_{H}+\Gamma_{A}+\Gamma_{h H}+\Gamma_{\tilde{f}}-4 \Gamma_{h \tilde{f}_{1}}-4 \Gamma_{h \tilde{f}_{2}}-4 \Gamma_{H \tilde{f}_{1}}\right. \\
-4 \Gamma_{H \tilde{f}_{2}}-4 \Gamma_{A \tilde{f}_{1}}-4 \Gamma_{A \tilde{f}_{2}}+4 \Gamma_{Z \tilde{f}_{1}}-4 \Gamma_{Z \tilde{f}_{2}}-4 \Gamma_{Z A}+\Gamma_{G} \\
\left.+2 \Gamma_{G A}-4 \Gamma_{Z G}-4 \Gamma_{G \tilde{f}_{1}}-4 \Gamma_{G \tilde{f}_{2}}\right) .
\end{aligned}
$$

$G$ indicates the goldstone contribution. In the following contributions we again account for negative neutralino masses via factors of -1 corresponding to the effects of absorbing factors of $-i \gamma_{5}$ into the couplings for negative mass neutralinos:

The contributions are as follows:

$$
(-1)^{\theta_{i}}=\left\{\begin{array}{l}
1, \text { for } m_{\tilde{Z}_{i}}>0, \\
-1, \text { for } m_{\tilde{Z}_{i}}<0 .
\end{array}\right.
$$

$$
\Gamma_{h}=2\left(X_{i j}^{h}+X_{j i}^{h}\right)^{2} f_{q}^{2} t_{\alpha_{h}}^{2}\left[I_{4}^{h}-2 m_{f}^{2} I_{3}^{h}+2(-1)^{\theta_{j}}\left|m_{\tilde{Z}_{i}}\left\|m_{\tilde{Z}_{j}}\left|I_{2}^{h}-4(-1)^{\theta_{j}}\right| m_{\tilde{Z}_{i}}\right\| m_{\tilde{Z}_{j}}\right| m_{f}^{2} I_{1}^{h}\right],
$$

\footnotetext{
${ }^{5}$ Calculations of the differential decay rates for this mode are also available, using a different approach to that used here, in reference [33].
} 
where

$$
\begin{aligned}
& s=m_{\tilde{Z}_{i}}^{2}+m_{\tilde{Z}_{j}}^{2}-2\left|m_{\tilde{Z}_{i}}\right| E, \\
& E_{\text {max }}=\frac{m_{\tilde{Z}_{i}}^{2}+m_{\tilde{Z}_{j}}^{2}-4 m_{f}^{2}}{2\left|m_{\tilde{Z}_{i}}\right|}, \\
& I_{1}^{h}=\int_{\left|m_{\tilde{Z}_{j}}\right|}^{E_{\max }} d E \frac{2\left|m_{\tilde{Z}_{i}}\right| \sqrt{s-4 m_{f}^{2}} \sqrt{E^{2}-m_{\tilde{Z}_{j}}^{2}} 2\left|m_{\tilde{Z}_{i}}\right|}{\sqrt{s}\left(s-m_{h}^{2}\right)^{2}}, \\
& I_{2}^{h}=\int_{\left|m_{\tilde{Z}_{j}}\right|}^{E_{\max }} d E \frac{2\left|m_{\tilde{Z}_{i}}\right| \sqrt{s-4 m_{f}^{2}} \sqrt{E^{2}-m_{\tilde{Z}_{j}}^{2}} 2\left|m_{\tilde{Z}_{i}}\right|\left(s-2 m_{f}^{2}\right)}{\sqrt{s}\left(s-m_{h}^{2}\right)^{2}}, \\
& I_{3}^{h}=\int_{\left|m_{\tilde{Z}_{j}}\right|}^{E_{\max }} d E \frac{2\left|m_{\tilde{Z}_{i}}\right| \sqrt{s-4 m_{f}^{2}} \sqrt{E^{2}-m_{\tilde{Z}_{j}}^{2}} 2\left|m_{\tilde{Z}_{i}}\right| 2\left|m_{\tilde{Z}_{i}}\right| E}{\sqrt{s}\left(s-m_{h}^{2}\right)^{2}}, \\
& I_{4}^{h}=\int_{\left|m_{\tilde{Z}_{j}}\right|}^{E_{\max }} d E \frac{2\left|m_{\tilde{Z}_{i}}\right| \sqrt{s-4 m_{f}^{2}} \sqrt{E^{2}-m_{\tilde{Z}_{j}}^{2}} 2\left|m_{\tilde{Z}_{i}}\right|\left(s-2 m_{f}^{2}\right) 2\left|m_{\tilde{Z}_{i}}\right| E}{\sqrt{s}\left(s-m_{h}^{2}\right)^{2}} . \\
& \Gamma_{H}=2\left(X_{i j}^{H}+X_{j i}^{H}\right)^{2} f_{q}^{2} t_{\alpha_{H}}^{2}\left[I_{4}^{H}-2 m_{f}^{2} I_{3}^{H}+2(-1)^{\theta_{j}}\left|m_{\tilde{Z}_{i}}\right|\left|m_{\tilde{Z}_{j}}\right| I_{2}^{H}-4(-1)^{\theta_{j}}\left|m_{\tilde{Z}_{i}}\right|\left|m_{\tilde{Z}_{j}}\right| m_{f}^{2} I_{1}^{H}\right] .
\end{aligned}
$$

where the $I_{1,2,3,4}^{H}$ are exactly the same as the $I_{1,2,3,4}^{h}$ with the change $m_{h} \rightarrow m_{H}$.

$$
\begin{aligned}
\Gamma_{Z}=64 g^{2} \sin ^{2} \theta_{W} W_{i j}^{2}[ & 4(-1)^{\theta_{j}}\left|m_{\tilde{Z}_{i}}\right|\left|m_{\tilde{Z}_{j}}\right| m_{f}^{2}\left(\alpha_{f}^{2}-\beta_{f}^{2}\right) I_{4}^{Z}+m_{f}^{2}\left(\alpha_{f}^{2}-\beta_{f}^{2}\right) I_{3}^{Z} \\
& \left.+(-1)^{\theta_{i}}(-1)^{\theta_{j}}\left|m_{\tilde{Z}_{i}}\right|\left|m_{\tilde{Z}_{j}}\right|\left(\alpha_{f}^{2}+\beta_{f}^{2}\right) I_{2}^{Z}+\frac{1}{2}\left(\alpha_{f}^{2}+\beta_{f}^{2}\right) I_{1}^{Z}\right],
\end{aligned}
$$

where the integrals $I_{i}^{Z}$ are:

$$
\begin{gathered}
I_{1}^{Z}=\int_{4 m_{f}^{2}}^{\left(\left|m_{\tilde{z}_{i}}\right|-\mid m_{\tilde{Z}_{j}}\right)^{2}} d s\left[\frac { 1 } { 3 s ^ { 2 } ( s - m _ { Z } ^ { 2 } ) ^ { 2 } } \left\{-2 s^{4}+\left(m_{\tilde{Z}_{i}}^{2}+m_{\tilde{Z}_{j}}^{2}+2 m_{f}^{2}\right) s^{3}+\left[\left(m_{\tilde{Z}_{i}}^{2}-m_{\tilde{Z}_{j}}^{2}\right)^{2}\right.\right.\right. \\
\left.\left.-2\left(m_{\tilde{Z}_{i}}^{2}+m_{\tilde{Z}_{j}}^{2}\right) 2 m_{f}^{2}\right] s^{2}+2 m_{f}^{2}\left(m_{\tilde{Z}_{i}}^{2}-m_{\tilde{Z}_{j}}^{2}\right)^{2} s\right\} \frac{1}{s} \\
\left.\quad \times \sqrt{\left(s-\left(\left|m_{\tilde{Z}_{i}}\right|-\left|m_{\tilde{Z}_{j}}\right|\right)^{2}\right)\left(\left(s-\left(\left|m_{\tilde{Z}_{i}}\right|+\left|m_{\tilde{Z}_{j}}\right|\right)^{2}\right)\right.} \sqrt{s\left(s-4 m_{f}^{2}\right)}\right], \\
I_{2}^{Z}=\int_{4 m_{f}^{2}}^{\left(\left|m_{\tilde{Z}_{i}}\right|-\mid m_{\tilde{Z}_{j}}\right)^{2}} d s\left[\frac{1}{s\left(s-m_{Z}^{2}\right)^{2}}\left(s-2 m_{f}^{2}\right) \sqrt{\left(s-\left(\left|m_{\tilde{Z}_{i}}\right|-\left|m_{\tilde{Z}_{j}}\right|\right)^{2}\right)\left(\left(s-\left(\left|m_{\tilde{Z}_{i}}\right|+\left|m_{\tilde{Z}_{j}}\right|\right)^{2}\right)\right.}\right. \\
\left.\quad \times \sqrt{s\left(s-4 m_{f}^{2}\right)}\right], \\
\int_{4 m_{f}^{2}}^{\left(\left|m_{\tilde{Z}_{i}}\right|-\left|m_{\tilde{Z}_{j}}\right|\right)^{2}} d s\left[\frac{1}{s\left(s-m_{Z}^{2}\right)^{2}}\left(m_{\tilde{Z}_{i}}^{2}+m_{\tilde{Z}_{j}}^{2}-s\right) \sqrt{\left(s-\left(\left|m_{\tilde{Z}_{i}}\right|-\left|m_{\tilde{Z}_{j}}\right|\right)^{2}\right)\left(\left(s-\left(\left|m_{\tilde{Z}_{i}}\right|+\left|m_{\tilde{Z}_{j}}\right|\right)^{2}\right)\right.}\right. \\
\left.\times \sqrt{s\left(s-4 m_{f}^{2}\right)}\right], \\
I_{4}^{Z}=\int_{4 m_{f}^{2}}^{\left(\left|m_{\tilde{Z}_{i}}\right|-\left|m_{\tilde{Z}_{j}}\right|\right)^{2}} d s\left[\frac{1}{s\left(s-m_{Z}^{2}\right)^{2}} \sqrt{\left(s-\left(\left|m_{\tilde{Z}_{i}}\right|-\left|m_{\tilde{Z}_{j}}\right|\right)^{2}\right)\left(\left(s-\left(\left|m_{\tilde{Z}_{i}}\right|+\left|m_{\tilde{Z}_{j}}\right|\right)^{2}\right)\right.} \sqrt{s\left(s-4 m_{f}^{2}\right)}\right] .
\end{gathered}
$$




$$
\Gamma_{A}=\left(X_{i j}^{A}+X_{j i}^{A}\right)^{2} A_{q}^{2}\left[I_{4}^{A}+2 m_{f}^{2} I_{3}^{A}-2(-1)^{\theta_{i}}(-1)^{\theta_{j}}\left|m_{\tilde{Z}_{i}}\left\|m_{\tilde{Z}_{j}}\left|I_{2}^{A}-4(-1)^{\theta_{i}}(-1)^{\theta_{j}} m_{f}^{2}\right| m_{\tilde{Z}_{i}}\right\| m_{\tilde{Z}_{j}}\right| I_{1}^{A}\right],
$$

where the integrals $I_{i}^{A}$ are:

$$
\begin{gathered}
I_{1}^{A}=\int_{\left|m_{\tilde{z}_{j}}\right|}^{E_{\max }} d E \sqrt{E^{2}-m_{\tilde{Z}_{j}}^{2}} \sqrt{s-4 m_{f}^{2}} \frac{4 m_{\tilde{Z}_{i}}^{2}}{\sqrt{s}\left(s-m_{A}^{2}\right)^{2}}, \\
I_{2}^{A}=\int_{\left|m_{\tilde{z}_{j}}\right|}^{E_{\max }} d E \sqrt{E^{2}-m_{\tilde{Z}_{j}}^{2}} \sqrt{s-4 m_{f}^{2}} \frac{4 m_{\tilde{Z}_{i}}^{2}\left(s-2 m_{f}^{2}\right)}{\sqrt{s}\left(s-m_{A}^{2}\right)^{2}}, \\
I_{3}^{A}=\int_{\left|m_{\tilde{z}_{j}}\right|}^{E_{\max }} d E \sqrt{E^{2}-m_{\tilde{Z}_{j}}^{2}} \sqrt{s-4 m_{f}^{2}} \frac{4 m_{\tilde{Z}_{i}}^{2} 2\left|m_{\tilde{Z}_{i}}\right| E}{\sqrt{s}\left(s-m_{A}^{2}\right)^{2}}, \\
I_{4}^{A}=\int_{\left|m_{\tilde{z}_{j}}\right|}^{E_{\max }} d E \sqrt{E^{2}-m_{\tilde{Z}_{j}}^{2}} \sqrt{s-4 m_{f}^{2}} \frac{4 m_{\tilde{Z}_{i}}^{2} 2\left|m_{\tilde{Z}_{i}}\right| E\left(s-2 m_{f}^{2}\right)}{\sqrt{s}\left(s-m_{A}^{2}\right)^{2}}, \\
\Gamma_{h H}=4\left(X_{i j}^{h}+X_{j i}^{h}\right)\left(X_{i j}^{H}+X_{j i}^{H}\right) f_{q}^{2} t_{\alpha_{h}} t_{\alpha_{H}}\left[I_{4}^{h H}-2 m_{f}^{2} I_{3}^{h H}+2(-1)^{\theta_{i}}(-1)^{\theta_{j}}\left|m_{\tilde{Z}_{i}}\right|\left|m_{\tilde{Z}_{j}}\right| I_{2}^{h H}\right. \\
\left.-4(-1)^{\theta_{i}}(-1)^{\theta_{j}}\left|m_{\tilde{Z}_{i}}\right|\left|m_{\tilde{Z}_{j}}\right| m_{f}^{2} I_{1}^{h H}\right],
\end{gathered}
$$

where:

$$
\begin{aligned}
I_{1}^{h H}= & \int_{\left|m_{\tilde{z}_{j}}\right|}^{E_{\max }} d E\left[2\left|m_{\tilde{Z}_{i}}\right| \sqrt{s-4 m_{f}^{2}} \sqrt{E^{2}-m_{\tilde{Z}_{j}}^{2}} \frac{2\left|m_{\tilde{z}_{j}}\right|}{\sqrt{s}\left(s-m_{h}^{2}\right)\left(s-m_{H}^{2}\right)}\right], \\
I_{2}^{h H}= & \int_{\left|m_{\tilde{z}_{j}}\right|}^{E_{\max }} d E\left[2\left|m_{\tilde{Z}_{i}}\right| \sqrt{s-4 m_{f}^{2}} \sqrt{E^{2}-m_{\tilde{Z}_{j}}^{2}} \frac{2\left|m_{\tilde{Z}_{i}}\right|\left(s-2 m_{f}^{2}\right)}{\sqrt{s}\left(s-m_{h}^{2}\right)\left(s-m_{H}^{2}\right)}\right], \\
I_{3}^{h H}= & \int_{\left|m_{\tilde{z}_{j}}\right|}^{E_{\max }} d E\left[2\left|m_{\tilde{Z}_{i}}\right| \sqrt{s-4 m_{f}^{2}} \sqrt{E^{2}-m_{\tilde{Z}_{j}}^{2}} \frac{4 m_{\tilde{Z}_{i}}^{2} E}{\sqrt{s}\left(s-m_{h}^{2}\right)\left(s-m_{H}^{2}\right)}\right], \\
I_{4}^{h H}= & \int_{\left|m_{\tilde{z}_{j}}\right|}^{E_{\max }} d E\left[2\left|m_{\tilde{Z}_{i}}\right| \sqrt{s-4 m_{f}^{2}} \sqrt{E^{2}-m_{\tilde{Z}_{j}}^{2}} \frac{4 m_{\tilde{Z}_{i}}^{2} E\left(s-2 m_{f}^{2}\right)}{\sqrt{s}\left(s-m_{h}^{2}\right)\left(s-m_{H}^{2}\right)}\right] . \\
& \Gamma_{\tilde{f}}=2 \Gamma_{\tilde{f}_{1} \tilde{f}_{1}}^{\text {diag }}+2 \Gamma_{\tilde{f}_{2} \tilde{z}_{2}}^{\text {diag }}+2 \Gamma_{\tilde{f} \tilde{f}}^{\text {nondiag }}+\Gamma_{\tilde{f}_{1} \tilde{f}_{1}}^{t u}+\Gamma_{\tilde{f}_{2} \tilde{z}_{2}}^{t u}+2 \Gamma_{\tilde{f}_{1} \tilde{f}_{2}}^{t u},
\end{aligned}
$$

There are many sfermion contributions included here, they are as follows:

$$
\begin{aligned}
& \Gamma_{\tilde{1}_{1} \tilde{f}_{1}}^{\text {diag }}=\frac{8 m_{\tilde{Z}_{i}}^{2}}{\pi^{2}}\left(\alpha_{\tilde{f}_{1}}^{\tilde{Z}_{i}{ }^{2}}+\beta_{\tilde{f}_{1}}{\tilde{Z_{i}}}^{2}\right)\left(\alpha_{\tilde{f}_{1}}^{\tilde{Z}_{j}{ }^{2}}+\beta_{\tilde{f}_{1}}^{\tilde{Z}_{j}}\right) \tilde{\psi}^{2}\left(\tilde{Z}_{i}, \tilde{f}_{1}, \tilde{f}_{1}, \tilde{Z}_{j}\right) \\
& +\frac{32 m_{\tilde{Z}_{i}}^{2}}{\pi^{2}}(-1)^{\theta_{j}}\left(\alpha_{\tilde{f}_{1}}^{\tilde{Z}_{i}}+\beta_{\tilde{f}_{1}}^{2}\right) \alpha_{\tilde{f}_{1}}^{2} \beta_{\tilde{f}_{1}}^{\tilde{Z}_{j}} m_{f} \mid m_{\tilde{Z}_{j}} \tilde{\tilde{\chi}_{1}}\left(\tilde{Z}_{i}, \tilde{f}_{1}, \tilde{f}_{1}, \tilde{Z}_{j}\right) \\
& -\frac{32 m_{\tilde{Z}_{i}}^{2}}{\pi^{2}}(-1)^{\theta_{i}}\left(\alpha_{\tilde{f}_{1}}^{\tilde{Z}_{i}^{2}}+\beta_{\tilde{f}_{1}}^{\tilde{Z}_{j}}\right) \alpha_{\tilde{f}_{1}}^{2} \beta_{\tilde{z}_{1}}^{\tilde{Z}_{i}} m_{f}\left|m_{\tilde{Z}_{i}}\right| X\left(\tilde{Z}_{i}, \tilde{f}_{1}, \tilde{f}_{1}, \tilde{Z}_{j}\right) \\
& -\frac{64 m_{\tilde{Z}_{i}}^{2}}{\pi^{2}}(-1)^{\theta_{i}}(-1)^{\theta_{j}} \alpha_{\tilde{f}_{1}}^{\tilde{Z}_{i}} \beta_{\tilde{f}_{1}}^{\tilde{z}_{i}} \alpha_{\tilde{f}_{1}}^{\tilde{z}_{j}} \beta_{\tilde{f}_{1}}^{\tilde{Z}_{j}}\left|m_{\tilde{Z}_{i}}\right| m_{f}^{2}\left|m_{\tilde{Z}_{j}}\right| \zeta\left(\tilde{Z}_{i}, \tilde{f}_{1}, \tilde{f}_{1}, \tilde{Z}_{j}\right) \text {, }
\end{aligned}
$$




$$
\begin{aligned}
& \Gamma_{\tilde{f}_{2} \tilde{f}_{2}}^{\text {diag }}=\frac{8 m_{\tilde{Z}_{i}}^{2}}{\pi^{2}}\left(\alpha_{\tilde{f}_{2}}^{\tilde{Z}_{i}{ }^{2}}+\beta_{\tilde{f}_{2}}^{\tilde{Z}_{i_{2}}{ }^{2}}\right)\left(\alpha_{\tilde{f}_{2}}^{\tilde{Z}_{j}{ }^{2}}+\beta_{\tilde{f}_{2}}^{\tilde{Z}_{j}}{ }^{2}\right) \tilde{\psi}\left(\tilde{Z}_{i}, \tilde{f}_{2}, \tilde{f}_{2}, \tilde{Z}_{j}\right) \\
& +\frac{32 m_{\tilde{Z}_{i}}^{2}}{\pi^{2}}(-1)^{\theta_{j}}\left(\alpha_{\tilde{f}_{2}}^{\tilde{Z}_{i}}+\beta_{\tilde{f}_{2}}^{\tilde{Z}_{2}}\right) \alpha_{\tilde{f}_{2}}^{2_{2}} \beta_{\tilde{Z}_{2}}^{\tilde{Z}_{j}} m_{f} \mid m_{\tilde{Z}_{j}} \tilde{\tilde{\chi}_{i}}\left(\tilde{Z}_{i}, \tilde{f}_{2}, \tilde{f}_{2}, \tilde{Z}_{j}\right) \\
& -\frac{32 m_{\tilde{Z}_{i}}^{2}}{\pi^{2}}(-1)^{\theta_{i}}\left(\alpha_{\tilde{f}_{2}}^{\tilde{Z}_{j}^{2}}+\beta_{\tilde{z}_{2}}^{\tilde{Z}_{j}}\right) \alpha_{\tilde{f}_{2}}^{2} \beta_{\tilde{z}_{2}}^{\tilde{Z}_{i}} m_{f}\left|m_{\tilde{Z}_{i}}\right| X\left(\tilde{Z}_{i}, \tilde{f}_{2}, \tilde{f}_{2}, \tilde{Z}_{j}\right) \\
& -\frac{64 m_{\tilde{Z}_{i}}^{2}}{\pi^{2}}(-1)^{\theta_{i}}(-1)^{\theta_{j}} \alpha_{\tilde{f}_{2}}^{\tilde{Z}_{i}} \beta_{\tilde{f}_{2}}^{\tilde{z}_{i}} \tilde{\alpha}_{\tilde{f}_{2}} \beta_{\tilde{f}_{2}} \tilde{Z}_{j}\left|m_{\tilde{Z}_{i}}\right| m_{f}^{2}\left|m_{\tilde{Z}_{j}}\right| \zeta\left(\tilde{Z}_{i}, \tilde{f}_{2}, \tilde{f}_{2}, \tilde{Z}_{j}\right),
\end{aligned}
$$

where the $\tilde{\psi}, \tilde{\chi}, X, \zeta$ integrals have been used before for the gluino 3 body decays and are:

$$
\begin{gathered}
E_{f}^{\max }=\frac{m_{\tilde{Z}_{i}}^{2}-2 m_{f} \mid m_{\tilde{Z}_{j}}-m_{\tilde{Z}_{j}}^{2}}{2\left|m_{\tilde{Z}_{i}}\right|}, \\
\tilde{\psi}\left(m_{\tilde{Z}_{i}}, m_{\tilde{f}_{1}}, m_{\tilde{f}_{2}}, m_{\tilde{Z}_{j}}\right)=\pi^{2} m_{\tilde{Z}_{i}} \int_{m_{f}}^{E_{f}^{\max }} d E_{f} \sqrt{E_{f}^{2}-m_{f}^{2}} E_{f} \frac{\lambda^{\frac{1}{2}}\left(s, m_{f}^{2}, m_{\tilde{Z}_{j}}^{2}\right)}{s} \frac{m_{\tilde{Z}_{i}}^{2}-m_{\tilde{Z}_{j}}^{2}-2 m_{\tilde{Z}_{i}} E_{f}}{\left(s-m_{\tilde{f}_{1}}^{2}\right)\left(s-m_{\tilde{f}_{2}}^{2}\right)}, \\
\tilde{\chi}\left(m_{\tilde{Z}_{i}}, m_{\tilde{f}_{1}}, m_{\tilde{f}_{2}}, m_{\tilde{Z}_{j}}\right)=\pi^{2} m_{\tilde{Z}_{i}} \int_{m_{f}}^{E_{f}^{m a x}} d E_{f} \sqrt{E_{f}^{2}-m_{f}^{2}} E_{f} \frac{\lambda^{\frac{1}{2}}\left(s, m_{f}^{2}, m_{\tilde{Z}_{j}}^{2}\right)}{s} \frac{1}{\left(s-m_{\tilde{f}_{1}}^{2}\right)\left(s-m_{\tilde{f}_{2}}^{2}\right.}, \\
X\left(m_{\tilde{Z}_{i}}, m_{\tilde{f}_{1}}, m_{\tilde{f}_{2}}, m_{\tilde{Z}_{j}}\right)=\frac{\pi^{2}}{2} \int_{m_{f}}^{E_{f}^{m a x}} d E_{f} \sqrt{E_{f}^{2}-m_{f}^{2}} \frac{m_{\tilde{Z}_{i}}^{2}-m_{\tilde{Z}_{j}}^{2}-2 m_{\tilde{Z}_{i}} E_{f}}{m_{\tilde{Z}_{i}}^{2}+m_{f}^{2}-2 m_{\tilde{Z}_{i}} E_{f}} \frac{\lambda^{\frac{1}{2}}\left(s, m_{f}^{2}, m_{\tilde{Z}_{j}}^{2}\right)}{\left(s-m_{\tilde{f}_{1}}^{2}\right)\left(s-m_{\tilde{f}_{2}}^{2}\right)}, \\
\zeta\left(m_{\tilde{Z}_{i}}, m_{\tilde{f}_{1}}, m_{\tilde{f}_{2}}, m_{\tilde{Z}_{j}}\right)=\pi^{2} \int_{m_{f}}^{E_{f}^{m a x}} d E_{f} \frac{\left[E_{\tilde{f}}(m a x)-E_{\tilde{f}}(m i n)\right]}{\left(s-m_{\tilde{f}_{1}}^{2}\right)\left(s-m_{\tilde{f}_{2}}^{2}\right)} .
\end{gathered}
$$

Later the following integrals will also be required:

$$
\begin{aligned}
\tilde{\phi}\left(m_{\tilde{Z}_{i}}, m_{\tilde{f}_{1}}, m_{\tilde{f}_{2}}, m_{\tilde{Z}_{j}}\right)=\frac{1}{2} \pi^{2}\left|m_{\tilde{Z}_{i}}\right|\left|m_{\tilde{Z}_{j}}\right| \int_{m_{f}}^{E_{f}^{\max }} d E_{f} \frac{1}{s-m_{\tilde{f}_{1}}^{2}}\left[-\left[E_{\tilde{f}}(\max )-E_{\tilde{f}}(\min )\right]-\right. \\
\left.\frac{m_{\tilde{Z}_{j}}^{2}-m_{f}^{2}+2\left|m_{\tilde{Z}_{i}}\right| E_{f}-m_{\tilde{f}_{2}}^{2}}{2\left|m_{\tilde{Z}_{i}}\right|} \log Z\left(m_{\tilde{f}_{2}}\right)\right],
\end{aligned}
$$

where $Z\left(m_{\tilde{f}_{2}}\right)=\frac{m_{\bar{Z}_{i}}^{2}+m_{f}^{2}-2\left|m_{\tilde{z}_{i}}\right| E_{\tilde{f}}(\max )-m_{\tilde{f}_{2}}^{2}}{m_{\tilde{Z}_{i}}^{2}+m_{f}^{2}-2\left|m_{\tilde{z}_{i}}\right| E_{\tilde{f}}(\min )-m_{\tilde{f}_{2}}^{2}}$

$$
\begin{gathered}
\xi\left(m_{\tilde{Z}_{i}}, m_{\tilde{f}_{1}}, m_{\tilde{f}_{2}}, m_{\tilde{Z}_{j}}\right)=\frac{1}{2} \pi^{2} \int_{m_{f}}^{E_{f}^{\max } d E_{f} \frac{1}{s-m_{\tilde{f}_{1}}^{2}}[}\left[E_{\tilde{f}_{f}}(\max )-E_{\bar{f}_{f}}(\min )\right] \\
\left.-\frac{m_{\tilde{Z}_{i}}^{2}-m_{f}^{2}-2\left|m_{\tilde{Z}_{i}}\right| E_{f}+m_{\tilde{f}_{2}}^{2}}{2\left|m_{\tilde{Z}_{i}}\right|} \log Z\left(m_{\tilde{f}_{2}}\right)\right], \\
\tilde{\rho}\left(m_{\tilde{Z}_{i}}, m_{\tilde{f}_{1}}, m_{\tilde{f}_{2}}, m_{\tilde{Z}_{j}}\right)=-\frac{\pi^{2}}{2\left|m_{\tilde{Z}_{i}}\right|} \int_{m_{f}}^{E_{f}^{\max }} d E_{f} \frac{1}{s-m_{\tilde{f}_{1}}^{2}} \log Z\left(m_{\tilde{f}_{2}}\right),
\end{gathered}
$$




$$
\begin{array}{rl}
Y\left(m_{\tilde{Z}_{i}}, m_{\tilde{f}_{1}}, m_{\tilde{f}_{2}}, m_{\tilde{Z}_{j}}\right)=\frac{\pi^{2}}{2} \int_{m_{f}}^{E_{f}^{m a x}} & d E_{f} \frac{1}{s-m_{\tilde{f}_{1}}^{2}}\left[E_{\tilde{f}^{\prime}}(\max )-E_{\tilde{f}}(\min )\right] s \\
& +\frac{1}{2\left|m_{\tilde{Z}_{i}}\right|}\left(m_{\tilde{Z}_{i}}^{2} m_{\tilde{Z}_{j}}^{2}-m_{\tilde{Z}_{i}}^{2} m_{\tilde{f}_{2}}^{2}+m_{f}^{4}+2\left|m_{\tilde{Z}_{i}}\right| E_{f} m_{\tilde{f}_{2}}^{2}-m_{\tilde{f}_{2}}^{2} m_{f}^{2}\right) \\
& \left.\times \log Z\left(m_{\tilde{f}_{2}}\right)\right] \\
\chi^{\prime}\left(m_{\tilde{Z}_{i}}, m_{\tilde{f}_{1}}, m_{\tilde{f}_{2}}, m_{\tilde{Z}_{j}}\right) & =\frac{-\pi^{2}}{2} \int_{m_{f}}^{E_{f}^{m a x}} \frac{d E_{f} E_{f}}{s-m_{\tilde{f}_{2}}^{2}} \log Z\left(m_{\tilde{f}_{1}}\right),
\end{array}
$$

where here

$$
\begin{aligned}
& E_{\bar{f}}(\max / \min )=\frac{\left(s+m_{f}^{2}-m_{\tilde{Z}_{j}}^{2}\right)\left(\left|m_{\tilde{Z}_{i}}\right|-E_{f}\right) \pm \sqrt{\left(E_{f}^{2}-m_{f}^{2}\right)\left(s+m_{f}^{2}-m_{\tilde{Z}_{j}}^{2}\right)^{2}-4\left(E_{f}^{2}-m_{f}^{2}\right) m_{f}^{2} s}}{2 s} . \\
& \Gamma_{\tilde{f} \tilde{f}}^{\text {nondiag }}=\frac{16 m_{\tilde{Z}_{i}}^{2}}{\pi^{2}}\left[\beta_{\tilde{f}_{1}}^{\tilde{z}_{i}} \beta_{\tilde{f}_{2}}^{\tilde{Z}_{i}}+\alpha_{\tilde{f}_{1}}^{\tilde{Z}_{i}} \alpha_{\tilde{f}_{2}}^{\tilde{Z}_{i}}\right)\left(\alpha_{\tilde{f}_{1}}^{\tilde{z}_{j}} \alpha_{\tilde{f}_{2}}^{\tilde{Z}_{j}}+\beta_{\tilde{f}_{2}}^{\tilde{Z}_{j}} \beta_{\tilde{f}_{2}}^{\tilde{Z}_{j}}\right) \tilde{\psi}_{(}\left(\tilde{Z}_{i}, \tilde{f}_{1}, \tilde{f}_{2}, \tilde{Z}_{j}\right) \\
& +2(-1)^{\theta_{j}}\left(\beta_{\tilde{f}_{1}}^{\tilde{z}_{i}} \beta_{\tilde{f}_{2}}^{\tilde{Z}_{i}}+\alpha_{\tilde{f}_{1}}^{\tilde{Z}_{i}} \alpha_{\tilde{f}_{2}}^{\tilde{Z}_{i}}\right)\left(\alpha_{\tilde{f}_{2}}^{\tilde{Z}_{j}} \beta_{\tilde{f}_{1}}^{\tilde{Z}_{j}}+\alpha_{\tilde{f}_{1}}^{\tilde{Z}_{j}} \beta_{\tilde{f}_{2}}^{\tilde{Z}_{j}}\right) m_{f} \mid m_{\tilde{Z}_{j}} \tilde{\chi}\left(\tilde{Z}_{i}, \tilde{f}_{1}, \tilde{f}_{2}, \tilde{Z}_{j}\right) \\
& -2(-1)^{\theta_{i}}\left(\alpha_{\tilde{f}_{1}}^{\tilde{Z}_{i}} \beta_{\tilde{f}_{2}}^{\tilde{z}_{i}}+\alpha_{\tilde{f}_{2}}^{\tilde{z}_{i}} \beta_{\tilde{f}_{1}}^{\tilde{Z}_{i}}\right)\left(\alpha_{\tilde{f}_{1}}^{\tilde{Z}_{j}} \alpha_{\tilde{f}_{2}}^{\tilde{Z}_{j}}+\beta_{\tilde{f}_{2}}^{\tilde{Z}_{j}} \beta_{\tilde{f}_{1}}^{\tilde{z}_{j}}\right)\left|m_{\tilde{Z}_{i}}\right| m_{f} X\left(\tilde{Z}_{i}, \tilde{f}_{1}, \tilde{f}_{2}, \tilde{Z}_{j}\right) \\
& \left.-2(-1)^{\theta_{i}}(-1)^{\theta_{j}}\left(\alpha_{\tilde{f}_{1}}^{\tilde{Z}_{i}} \beta_{\tilde{f}_{2}}^{\tilde{z}_{i}}+\alpha_{\tilde{f}_{2}}^{\tilde{Z}_{i}} \beta_{\tilde{f}_{1}}^{\tilde{Z}_{i}}\right)\left(\alpha_{\tilde{f}_{2}}^{\tilde{Z}_{j}} \beta_{\tilde{f}_{1}}^{\tilde{Z}_{j}}+\alpha_{\tilde{f}_{1}}^{\tilde{z}_{j}} \beta_{\tilde{f}_{2}}^{\tilde{Z}_{j}}\right) m_{f}^{2}\left|m_{\tilde{Z}_{i}}\right|\left|m_{\tilde{Z}_{j}}\right| \zeta\left(\tilde{Z}_{i}, \tilde{f}_{1}, \tilde{f}_{2}, \tilde{Z}_{j}\right)\right], \\
& \Gamma_{\tilde{f}_{1} \tilde{f}_{1}}^{t u}=-2\left\{8\left(\alpha_{\tilde{f}_{1}}^{\tilde{Z}_{i}} \beta_{\tilde{f}_{1}}^{\tilde{Z}_{i}} \beta_{\tilde{f}_{1}}^{\tilde{z}_{j}} \alpha_{\tilde{f}_{1}}^{\tilde{Z}_{j}}+\beta_{\tilde{f}_{1}}^{\tilde{Z}_{i}} \alpha_{\tilde{f}_{1}}^{\tilde{Z}_{i}} \alpha_{\tilde{f}_{1}}^{\tilde{f}_{j}} \beta_{\tilde{f}_{1}}^{\tilde{Z}_{j}}\right) m_{\tilde{Z}_{i}}^{2} \pi^{2}(-1)^{\theta_{i}} Y\left(\tilde{Z}_{i}, \tilde{f}_{1}, \tilde{f}_{1}, \tilde{Z}_{j}\right)\right. \\
& \left.-\left(\alpha_{\tilde{f}_{1}}^{\tilde{Z}_{i}} \alpha_{\tilde{f}_{1}}^{\tilde{Z}_{j}}+\beta_{\tilde{f}_{1}}^{\tilde{Z}_{i_{1}}^{2}} \beta_{\tilde{f}_{1}}^{\tilde{Z}_{j}}\right)^{2}\right) m_{\tilde{Z}_{i}}^{2} \pi^{2}(-1)^{\theta_{i}}(-1)^{\theta_{j}} \tilde{\phi}\left(\tilde{Z}_{i}, \tilde{f}_{1}, \tilde{f}_{1}, \tilde{Z}_{j}\right) \\
& +\left(\alpha_{\tilde{f}_{1}}^{\tilde{Z}_{i}} \beta_{\tilde{f}_{1}}^{\tilde{z}_{i}} \alpha_{\tilde{z}_{1}}^{\tilde{Z}_{j}} \beta_{\tilde{f}_{1}}^{\tilde{Z}_{j}}+\alpha_{\tilde{f}_{1}}^{\tilde{Z}_{i}} \beta_{\tilde{f}_{1}}^{\tilde{Z}_{i}} \alpha_{\tilde{f}_{1}}^{\tilde{Z}_{j}} \beta_{\tilde{z}_{1}}^{\tilde{Z}_{j}}\right) m_{f}^{2} \frac{8 m_{\tilde{Z}_{i}}^{2}}{\pi^{2}}(-1)^{\theta_{i}} \xi\left(\tilde{Z}_{i}, \tilde{f}_{1}, \tilde{f}_{1}, \tilde{Z}_{j}\right) \\
& -\left\{\left(\alpha_{\tilde{f}_{1}}^{\tilde{Z}_{i}} \alpha_{\tilde{f}_{1}}^{2} \beta_{\tilde{f}_{1}}^{\tilde{Z}_{j}}+\beta_{\tilde{f}_{1}}^{\tilde{Z}_{i}} \alpha_{\tilde{f}_{1}}^{2} \tilde{z}_{\tilde{f}_{1}}^{\tilde{Z}_{j}}\right)\left|m_{\tilde{Z}_{i}}\right| m_{f}\right\}\left[\frac{8 m_{\tilde{Z}_{i}}^{2}}{\pi^{2}} \xi\left(\tilde{Z}_{i}, \tilde{f}_{1}, \tilde{f}_{1}, \tilde{Z}_{j}\right)\right. \\
& \left.-\frac{4 m_{\tilde{Z}_{i}}^{2}}{\pi^{2}}\left(m_{\tilde{Z}_{i}}^{2}+m_{\tilde{Z}_{j}}^{2}\right)(-1)^{\theta_{i}} \tilde{\rho}\left(\tilde{Z}_{i}, \tilde{f}_{1}, \tilde{f}_{1}, \tilde{Z}_{j}\right)+\frac{8 m_{\tilde{Z}_{i}}^{2}}{\pi^{2}} \chi^{\prime}\left(\tilde{Z}_{i}, \tilde{f}_{1}, \tilde{f}_{1}, \tilde{Z}_{j}\right)\right] \\
& +\left|m_{\tilde{Z}_{j}}\right|(-1)^{\theta_{j}} m_{f}\left(\alpha_{\tilde{f}_{1}}^{\tilde{Z}_{i}} \tilde{z}_{\tilde{f}_{1}}^{\tilde{z}_{i}} \alpha_{\tilde{f}_{1}}^{\tilde{Z}_{j}{ }^{2}}+\alpha_{\tilde{f}_{1}}^{\tilde{z}_{i}} \beta_{\tilde{f}_{1}}^{\tilde{Z}_{i}} \beta_{\tilde{f}_{1}}^{\tilde{Z}_{j}{ }^{2}}\right)\left[\frac{-8 m_{\tilde{Z}_{i}}^{2}}{\pi^{2}} \xi\left(\tilde{Z}_{i}, \tilde{f}_{1}, \tilde{f}_{1}, \tilde{Z}_{j}\right)\right.
\end{aligned}
$$

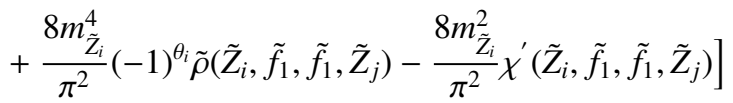

$$
\begin{aligned}
& -\left(\beta_{\tilde{f}_{1}}^{\tilde{Z}_{1}} \beta_{\tilde{f}_{1}}^{2} \alpha_{\tilde{f}_{1}}^{\tilde{Z}_{j}}+\alpha_{\tilde{f}_{1}}^{\tilde{Z}_{\tilde{Z}_{1}}{ }^{2}} \alpha_{\tilde{f}_{1}}^{\tilde{z}_{j}} \beta_{\tilde{f}_{1}}^{\tilde{Z}_{j}}\right)\left|m_{\tilde{Z}_{i}}\right|(-1)^{\theta_{i}} m_{f}\left[\frac{4 m_{\tilde{Z}_{i}}^{2}}{\pi^{2}}\left(m_{\tilde{Z}_{i}}^{2}-m_{\tilde{Z}_{j}}^{2}\right) \tilde{\rho}\left(\tilde{Z}_{i}, \tilde{f}_{1}, \tilde{f}_{1}, \tilde{Z}_{j}\right)\right. \\
& \left.-\frac{8 m_{\tilde{Z}_{i}}^{2}}{\pi^{2}} \chi^{\prime}\left(\tilde{Z}_{i}, \tilde{f}_{1}, \tilde{f}_{1}, \tilde{Z}_{j}\right)\right] \\
& +\left(\alpha_{\tilde{f}_{1}}^{\tilde{Z}_{i}} \beta_{\tilde{f}_{1}}^{\tilde{Z}_{i}} \alpha_{\tilde{f}_{1}}^{\tilde{z}_{j}{ }^{2}}+\alpha_{\tilde{f}_{1}}^{\tilde{Z}_{i}} \beta_{\tilde{z}_{1}}^{\tilde{Z}_{i}} \beta_{\tilde{f}_{1}}^{\tilde{Z}_{j}{ }^{2}}\right) m_{f}\left|m_{\tilde{Z}_{j}}\right| \frac{8 m_{\tilde{Z}_{i}}^{2}}{\pi^{2}}(-1)^{\theta_{i}}(-1)^{\theta_{j}} \chi^{\prime}\left(\tilde{Z}_{i}, \tilde{f}_{1}, \tilde{f}_{1}, \tilde{Z}_{j}\right) \\
& \left.-2\left(\beta_{\tilde{f}_{1}}^{\tilde{Z}_{i}}{ }^{2} \alpha_{\tilde{f}_{1}}^{\tilde{Z}_{j}{ }^{2}}+\alpha_{\tilde{f}_{1}}^{\tilde{Z}_{i}{ }^{2}} \beta_{\tilde{f}_{1}}^{\tilde{Z}_{j}}\right) m_{f}^{2}(-1)^{\theta_{i}}(-1)^{\theta_{j}}\left|m_{\tilde{Z}_{i}}\right|\left|m_{\tilde{Z}_{j}}\right| \frac{4 m_{\tilde{Z}_{i}}^{2}}{\pi^{2}} \tilde{\rho}\left(\tilde{Z}_{i}, \tilde{f}_{1}, \tilde{f}_{1}, \tilde{Z}_{j}\right)\right\} \text {. }
\end{aligned}
$$


$\Gamma_{\tilde{f}_{2} \tilde{f}_{2}}^{t u}$ is as above but with $\tilde{f}_{1} \rightarrow \tilde{f}_{2}$ everywhere including in the masses and integrals.

$$
\begin{aligned}
& \Gamma_{\tilde{f}_{1} \tilde{f}_{2}}^{t u}=-2\left\{8\left(\alpha_{\tilde{f}_{1}}^{\tilde{Z}_{i}} \beta_{\tilde{f}_{2}}^{\tilde{z}_{i}} \beta_{\tilde{f}_{1}}^{\tilde{f}_{j}} \alpha_{\tilde{f}_{2}}^{\tilde{Z}_{j}}+\beta_{\tilde{f}_{1}}^{\tilde{Z}_{i}} \alpha_{\tilde{f}_{2}}^{\tilde{Z}_{i}} \alpha_{\tilde{f}_{1}}^{\tilde{z}_{j}} \beta_{\tilde{f}_{2}}^{\tilde{Z}_{j}}\right) m_{\tilde{Z}_{i}}^{2} \pi^{2} Y\left(\tilde{Z}_{i}, \tilde{f}_{1}, \tilde{f}_{2}, \tilde{Z}_{j}\right)\right. \\
& -\left(\alpha_{\tilde{f}_{1}}^{\tilde{Z}_{i}} \alpha_{\tilde{f}_{2}}^{\tilde{z}_{i}} \alpha_{\tilde{f}_{1}}^{\tilde{Z}_{j}} \alpha_{\tilde{f}_{2}}^{\tilde{z}_{j}}+\beta_{\tilde{f}_{1}}^{\tilde{Z}_{i}} \beta_{\tilde{f}_{2}}^{\tilde{Z}_{i}} \beta_{\tilde{f}_{1}}^{\tilde{f}_{j}} \beta_{\tilde{f}_{2}}^{\tilde{Z}_{j}}\right) 8 m_{\tilde{Z}_{i}}^{2} \pi^{2}(-1)^{\theta_{i}}(-1)^{\theta_{j}} \tilde{\phi}\left(\tilde{Z}_{i}, \tilde{f}_{1}, \tilde{f}_{2}, \tilde{Z}_{j}\right) \\
& +\left(\alpha_{\tilde{f}_{2}}^{\tilde{Z}_{i}} \beta_{\tilde{z}_{1}}^{\tilde{Z}_{i}} \alpha_{\tilde{f}_{2}}^{\tilde{Z}_{j}} \beta_{\tilde{f}_{1}}^{\tilde{Z}_{j}}+\alpha_{\tilde{f}_{1}}^{\text {tilde }} \beta_{\tilde{f}_{2}}^{\tilde{Z}_{i}} \alpha_{\tilde{f}_{1}}^{\tilde{Z}_{j}} \beta_{\tilde{f}_{j}}^{\tilde{Z}_{j}}\right) m_{f}^{2} \frac{8 m_{\tilde{Z}_{i}}^{2}}{\pi^{2}}(-1)^{\theta_{i}} \xi\left(\tilde{Z}_{i}, \tilde{f}_{1}, \tilde{f}_{2}, \tilde{Z}_{j}\right) \\
& -\left\{\left(\alpha_{\tilde{f}_{1}}^{\tilde{Z}_{i}} \alpha_{\tilde{f}_{2}}^{\tilde{Z}_{i}} \alpha_{\tilde{f}_{2}}^{\tilde{Z}_{j}} \beta_{\tilde{f}_{1}}^{\tilde{Z}_{j}}+\beta_{\tilde{f}_{1}}^{\tilde{Z}_{i_{1}}} \beta_{\tilde{f}_{2}}^{\tilde{Z}_{i}} \alpha_{\tilde{f}_{1}}^{\tilde{Z}_{j}} \beta_{\tilde{f}_{2}}^{\tilde{Z}_{j}}\right)\left|m_{\tilde{Z}_{i}}\right| m_{f}\right\}\left[\frac{8 m_{\tilde{Z}_{i}}^{2}}{\pi^{2}} \xi\left(\tilde{Z}_{i}, \tilde{f}_{1}, \tilde{f}_{2}, \tilde{Z}_{j}\right)\right. \\
& \left.-\frac{4 m_{\tilde{Z}_{i}}^{2}}{\pi^{2}}\left(m_{\tilde{Z}_{i}}^{2}+m_{\tilde{Z}_{j}}^{2}\right)(-1)^{\theta_{i}} \tilde{\rho}\left(\tilde{Z}_{i}, \tilde{f}_{1}, \tilde{f}_{2}, \tilde{Z}_{j}\right)+\frac{8 m_{\tilde{Z}_{i}}^{2}}{\pi^{2}} \chi^{\prime}\left(\tilde{Z}_{i}, \tilde{f}_{1}, \tilde{f}_{2}, \tilde{Z}_{j}\right)\right] \\
& +\left|m_{\tilde{Z}_{j}}\right|(-1)^{\theta_{j}} m_{f}\left(\alpha_{\tilde{f}_{2}}^{\tilde{z}_{i}} \beta_{\tilde{f}_{1}}^{\tilde{z}_{i}} \alpha_{\tilde{f}_{1}}^{\tilde{Z}_{j}} \alpha_{\tilde{f}_{2}}^{\tilde{Z}_{j}}+\alpha_{\tilde{f}_{1}}^{\tilde{Z}_{i}} \beta_{\tilde{f}_{2}}^{\tilde{z}_{i}} \beta_{\tilde{f}_{1}}^{\tilde{Z}_{j}} \beta_{\tilde{f}_{2}}^{\tilde{z}_{j}}\right)\left[\frac{-8 m_{\tilde{Z}_{i}}^{2}}{\pi^{2}} \xi\left(\tilde{Z}_{i}, \tilde{f}_{1}, \tilde{f}_{2}, \tilde{Z}_{j}\right)\right. \\
& \left.+\frac{8 m_{\tilde{Z}_{i}}^{4}}{\pi^{2}}(-1)^{\theta_{i}} \tilde{\rho}\left(\tilde{Z}_{i}, \tilde{f}_{1}, \tilde{f}_{2}, \tilde{Z}_{j}\right)-\frac{8 m_{\tilde{Z}_{i}}^{2}}{\pi^{2}} \chi^{\prime}\left(\tilde{Z}_{i}, \tilde{f}_{1}, \tilde{f}_{2}, \tilde{Z}_{j}\right)\right] \\
& -\left(\beta_{\tilde{f}_{1}}^{\tilde{Z}_{i}} \beta_{\tilde{z}_{2}}^{\tilde{Z}_{i}} \beta_{\tilde{f}_{1}}^{\tilde{z}_{j}} \alpha_{\tilde{f}_{j}}^{\tilde{Z}_{j}}+\alpha_{\tilde{f}_{1}}^{\tilde{Z}_{i_{1}}} \alpha_{\tilde{f}_{i}}^{\tilde{Z}_{i}} \alpha_{\tilde{f}_{1}}^{\tilde{Z}_{j}} \beta_{\tilde{f}_{2}}^{\tilde{z}_{\tilde{z}_{2}}}\right)\left|m_{\tilde{Z}_{i}}\right|(-1)^{\theta_{i}} m_{f}\left[\frac{4 m_{\tilde{Z}_{i}}^{2}}{\pi^{2}}\left(m_{\tilde{Z}_{i}}^{2}-m_{\tilde{Z}_{j}}^{2}\right) \tilde{\rho}\left(\tilde{Z}_{i}, \tilde{f}_{1}, \tilde{f}_{2}, \tilde{Z}_{j}\right)\right. \\
& \left.\left.-\frac{8 m_{\tilde{Z}_{i}}^{2}}{\pi^{2}} \chi^{\prime}\left(\tilde{Z}_{i}, \tilde{f}_{1}, \tilde{f}_{2}, \tilde{Z}_{j}\right)\right)\right] \\
& +\left(\alpha_{\tilde{f}_{1}}^{\tilde{Z}_{i_{1}}} \beta_{\tilde{f}_{2}}^{\tilde{z}_{i}} \alpha_{\tilde{f}_{1}}^{\tilde{Z}_{j}} \alpha_{\tilde{f}_{2}}^{\tilde{z}_{j}}+\alpha_{\tilde{f}_{2}}^{\tilde{Z}_{i}} \beta_{\tilde{f}_{1}}^{\tilde{Z}_{i}} \beta_{\tilde{f}_{1}}^{\tilde{z}_{j}} \beta_{\tilde{f}_{2}}^{\tilde{z}_{i}} m_{f}(-1)^{\theta_{j}} \mid m_{\tilde{Z}_{j}}\right) \mid \frac{8 m_{\tilde{Z}_{i}}^{2}}{\pi^{2}} \chi^{\prime}\left(\tilde{Z}_{i}, \tilde{f}_{1}, \tilde{f}_{2}, \tilde{Z}_{j}\right) \\
& \left.-2\left(\beta_{\tilde{f}_{1}}^{\tilde{Z}_{i}} \beta_{\tilde{f}_{2}}^{\tilde{Z}_{i}} \alpha_{\tilde{f}_{1}}^{\tilde{Z}_{j}} \alpha_{\tilde{z}_{j}}^{\tilde{Z}_{j}}+\alpha_{\tilde{f}_{1}}^{\tilde{Z}_{i}} \alpha_{\tilde{f}_{2}}^{\tilde{Z}_{i}} \beta_{\tilde{f}_{1}}^{\tilde{Z}_{j}} \beta_{\tilde{f}_{2}}^{\tilde{Z}_{j_{2}}}\right) m_{f}^{2}(-1)^{\theta_{i}}(-1)^{\theta_{j}}\left|m_{\tilde{Z}_{i}}\right|\left|m_{\tilde{Z}_{j}}\right| \frac{4 m_{\tilde{Z}_{i}}^{2}}{\pi^{2}} \tilde{\rho}\left(\tilde{Z}_{i}, \tilde{f}_{1}, \tilde{f}_{2}, \tilde{Z}_{j}\right)\right\} .
\end{aligned}
$$

As for the $Z$ sfermion interferences:

$$
\Gamma_{Z \tilde{f}_{1}}=(-1)^{\theta_{i}}\left(C_{1}^{Z \tilde{f}_{1}} I_{1}^{Z \tilde{f}_{1}}+C_{2}^{Z \tilde{f}_{1}} Z_{2}^{Z \tilde{f}_{1}}+C_{3}^{Z \tilde{f}_{1}} I_{3}^{Z \tilde{f}_{1}}+C_{4}^{Z \tilde{f}_{1}} I_{4}^{Z \tilde{f}_{1}}+C_{5}^{Z \tilde{f}_{1}} I_{5}^{Z \tilde{f}_{1}}+C_{6}^{Z \tilde{f}_{1}} I_{6}^{Z \tilde{f}_{1}}+C_{7}^{Z \tilde{f}_{1}} I_{7}^{Z \tilde{f}_{1}}+C_{8}^{Z \tilde{f}_{1}} I_{8}^{Z \tilde{f_{1}}}\right),
$$

where

$$
\begin{aligned}
& C_{1}^{Z \tilde{f}_{1}}=-4 W_{i j} g \sin \theta_{W}\left[-\alpha_{\tilde{f}_{1}}^{\tilde{Z}_{i}}\left(\alpha_{f}-\beta_{f}\right) \beta_{\tilde{f}_{1}}^{\tilde{Z}_{j}}+\beta_{\tilde{f}_{1}}^{\tilde{z}_{i_{1}}}\left(\alpha_{f}+\beta_{f}\right) \alpha_{\tilde{f}_{1}}^{\tilde{Z}_{j}}\right] m_{f}\left|m_{\tilde{Z}_{i}}\right|, \\
& C_{2}^{Z \tilde{f}_{1}}=-4(-1)^{\theta_{i}}(-1)^{\theta_{j}} W_{i j} g \sin \theta_{W}\left[-\alpha_{\tilde{f}_{1}}^{\tilde{Z}_{i}}\left(\alpha_{f}+\beta_{f}\right) \beta_{\tilde{f}_{1}}^{\tilde{Z}_{j}}+\beta_{\tilde{f}_{1}}^{\tilde{Z}_{i}}\left(\alpha_{f}-\beta_{f}\right) \alpha_{\tilde{f}_{1}}^{\tilde{z}_{j}}\right] m_{f}\left|m_{\tilde{Z}_{j}}\right|, \\
& C_{3}^{Z \tilde{f_{1}}}=-4(-1)^{\theta_{i}} W_{i j} g \sin \theta_{W}\left[\beta_{\tilde{f}_{1}}^{\tilde{Z}_{i}}\left(\alpha_{f}+\beta_{f}\right) \beta_{\tilde{f}_{1}}^{\tilde{Z}_{j}}-\alpha_{\tilde{f}_{1}}^{\tilde{Z}_{i}}\left(\alpha_{f}-\beta_{f}\right) \alpha_{\tilde{f}_{1}}^{\tilde{Z}_{j}}\right], \\
& C_{4}^{Z \tilde{f_{1}}}=-8 W_{i j} g \sin \theta_{W}\left[-\alpha_{\tilde{f}_{1}}^{\tilde{Z}_{i}}\left(\alpha_{f}+\beta_{f}\right) \beta_{\tilde{f}_{1}}^{\tilde{Z}_{j}}+\beta_{\tilde{f}_{1}}^{\tilde{z}_{i_{1}}}\left(\alpha_{f}-\beta_{f}\right) \alpha_{\tilde{f}_{1}}^{\tilde{Z}_{j}}\right]\left|m_{\tilde{Z}_{i}}\right| m_{f}, \\
& C_{5}^{Z \tilde{f}_{1}}=-8(-1)^{\theta_{i}}(-1)^{\theta_{j}} W_{i j} g \sin \theta_{W}\left[-\alpha_{\tilde{f}_{1}}^{\tilde{Z}_{i_{1}}}\left(\alpha_{f}-\beta_{f}\right) \beta_{\tilde{f}_{1}}^{\tilde{Z}_{j}}+\beta_{\tilde{f}_{1}}^{\tilde{Z}_{i}}\left(\alpha_{f}+\beta_{f}\right) \alpha_{\tilde{f}_{1}}^{\tilde{Z}_{j}}\right]\left|m_{\tilde{Z}_{j}}\right| m_{f}, \\
& C_{6}^{Z \tilde{f}_{1}}=-4(-1)^{\theta_{j}} W_{i j} g \sin \theta_{W}\left[\beta_{\tilde{f}_{1}}^{\tilde{Z}_{i}}\left(\alpha_{f}+\beta_{f}\right) \beta_{\tilde{f}_{1}}^{\tilde{Z}_{j}}-\alpha_{\tilde{f}_{1}}^{\tilde{z}_{i}}\left(\alpha_{f}-\beta_{f}\right) \alpha_{\tilde{f}_{1}}^{\tilde{Z}_{j}}\right]\left|m_{\tilde{Z}_{i}}\right|\left|m_{\tilde{Z}_{j}}\right|, \\
& C_{7}^{Z \tilde{f}_{1}}=-4(-1)^{\theta_{i}} W_{i j} g \sin \theta_{W}\left[\beta_{\tilde{f}_{1}}^{\tilde{Z}_{i}}\left(\alpha_{f}-\beta_{f}\right) \beta_{\tilde{f}_{1}}^{\tilde{Z}_{j}}-\alpha_{\tilde{f}_{1}}^{\tilde{Z}_{i}}\left(\alpha_{f}+\beta_{f}\right) \alpha_{\tilde{f}_{1}}^{\tilde{q} t i l d e Z_{j}}\right] m_{f}^{2}, \\
& C_{8}^{Z \tilde{f}_{1}}=-16(-1)^{\theta_{j}} W_{i j} g \sin \theta_{W}\left[\beta_{\tilde{f}_{1}}^{\tilde{Z}_{i}}\left(\alpha_{f}-\beta_{f}\right) \beta_{\tilde{f}_{1}}^{\tilde{z}_{j}}-\alpha_{\tilde{f}_{1}}^{\tilde{Z}_{i}}\left(\alpha_{f}+\beta_{f}\right) \alpha_{\tilde{f}_{1}}^{\tilde{z}_{j}}\right] m_{f}^{2}\left|m_{\tilde{Z}_{i}}\right|\left|m_{\tilde{Z}_{j}}\right| .
\end{aligned}
$$

The upper limit for the integrals here is $E_{\text {upper }}=\frac{\left(m_{\bar{Z}_{i}}^{2}+m_{\bar{Z}_{j}}^{2}-4 m_{f}^{2}\right)}{2\left|m_{\bar{z}_{i}}\right|}$. The argument of the logarithm in these integrals is as follows: 


$$
L=\left[\left|m_{\tilde{Z}_{i}}\right|\left(E_{Q}+Q^{\prime}\right)-\mu^{2}\right] /\left[\left|m_{\tilde{Z}_{i}}\right|\left(E_{Q}-Q^{\prime}\right)-\mu^{2}\right],
$$

In these expressions, $E_{Q}=\frac{s+m_{\tilde{Z}_{i}}^{2}-m_{\tilde{Z}_{j}}^{2}}{\left|m_{\tilde{Z}_{i}}\right|}, Q^{\prime}=\sqrt{E_{Q}^{2}-s} \sqrt{1-4 m_{f}^{2} / s}$ and $\mu^{2}=s+m_{\tilde{f}_{1}}^{2}-m_{\tilde{Z}_{j}}^{2}-m_{q}^{2}$, where $s=$ $m_{\tilde{Z}_{i}}^{2}+m_{\tilde{Z}_{j}}^{2}-2\left|m_{\tilde{Z}_{i}}\right| E$. The necessary integrals are given by:

$$
\begin{aligned}
& I_{1}^{Z \tilde{f}_{1}}=2\left|m_{\tilde{Z}_{i}}\right| \int_{\left|m_{\tilde{Z}_{j}}\right|}^{E_{u p p e r}} d E \frac{1}{s-m_{Z}^{2}}\left[-2\left|m_{\tilde{Z}_{i}}\right| \sqrt{1-4 m_{q}^{2} / s} \sqrt{E^{2}-m_{\tilde{Z}_{j}}^{2}}-\left(m_{\tilde{f}_{1}}^{2}-m_{f}^{2}+m_{\tilde{Z}_{j}}^{2}-2\left|m_{\tilde{Z}_{i}}\right| E\right) \log L\right], \\
& I_{2}^{Z \tilde{f}_{1}}=2\left|m_{\tilde{Z}_{i}}\right| \int_{\left|m_{\tilde{Z}_{j}}\right|}^{E_{u p p e r}} d E \frac{1}{s-m_{Z}^{2}}\left[2\left|m_{\tilde{Z}_{i}}\right| \sqrt{1-4 m_{q}^{2} / s} \sqrt{E^{2}-m_{\tilde{Z}_{j}}^{2}}+\left(m_{\tilde{f}_{1}}^{2}+m_{\tilde{Z}_{i}}^{2}-2\left|m_{\tilde{Z}_{i}}\right| E-m_{f}^{2}\right) \log L\right], \\
& I_{3}^{Z \tilde{f}_{1}}=2\left|m_{\tilde{Z}_{i}}\right| \int_{\left|m_{\tilde{Z}_{j}}\right|}^{E_{\text {upper }}} d E \frac{1}{s-m_{Z}^{2}}\left[\left\{m_{\tilde{Z}_{i}}^{2}+2 m_{f}^{2}+m_{\tilde{Z}_{j}}^{2}-\frac{3}{2} m_{\tilde{f}_{1}}^{2}-\frac{1}{2}\left(m_{f}^{2}+\left|m_{\tilde{Z}_{i}}\right| E\right.\right.\right. \\
& \left.\left.+\left|m_{\tilde{Z}_{i}}\right| \sqrt{1-4 m_{f}^{2} / s} \sqrt{E^{2}-m_{\tilde{Z}_{j}}^{2}}\right)\right\}\left(m_{f}^{2}+\left|m_{\tilde{Z}_{i}}\right| E+\left|m_{\tilde{Z}_{i}}\right| \sqrt{1-4 m_{f}^{2} / s} \sqrt{E^{2}-m_{\tilde{Z}_{j}}^{2}}-m_{\tilde{f}_{1}}^{2}\right) \\
& -\left\{m_{\tilde{Z}_{i}}^{2}+2 m_{f}^{2}+m_{\tilde{Z}_{j}}^{2}-\frac{3}{2} m_{\tilde{f}_{1}}^{2}-\frac{1}{2}\left(m_{f}^{2}+\left|m_{\tilde{Z}_{i}}\right| E-\left|m_{\tilde{Z}_{i}}\right| \sqrt{1-4 m_{f}^{2} / s} \sqrt{E^{2}-m_{\tilde{Z}_{j}}^{2}}\right)\right. \\
& \left.\times\left(m_{f}^{2}+\left|m_{\tilde{Z}_{i}}\right| E-\left|m_{\tilde{Z}_{i}}\right| \sqrt{1-4 m_{f}^{2} / s} \sqrt{E^{2}-m_{\tilde{Z}_{j}}^{2}}\right)-m_{\tilde{f}_{1}}^{2}\right\} \\
& \left.+\left(m_{\tilde{Z}_{i}}^{2}+m_{f}^{2}-m_{\tilde{f}_{1}}^{2}\right)\left(m_{\tilde{f}_{1}}^{2}-m_{f}^{2}-m_{\tilde{Z}_{j}}^{2}\right) \log L\right] \text {, } \\
& I_{4}^{Z \tilde{f}_{1}}=2\left|m_{\tilde{Z}_{i}}\right| \int_{\left|m_{\tilde{z}_{j}}\right|}^{E_{u p p e r}} d E \frac{1}{s-m_{Z}^{2}}\left[2\left|m_{\tilde{Z}_{i}}\right| \sqrt{1-4 m_{f}^{2} / s} \sqrt{E^{2}-m_{\tilde{Z}_{j}}^{2}}+\left(m_{\tilde{f}_{1}}^{2}-m_{f}^{2}-m_{\tilde{Z}_{j}}^{2}\right) \log L\right], \\
& I_{5}^{Z \tilde{f}_{1}}=2\left|m_{\tilde{Z}_{i}}\right| \int_{\left|m_{\tilde{Z}_{j}}\right|}^{E_{u p p e r}} d E \frac{-1}{s-m_{Z}^{2}}\left[2\left|m_{\tilde{Z}_{i}}\right| \sqrt{1-4 m_{f}^{2} / s} \sqrt{E^{2}-m_{\tilde{Z}_{j}}^{2}}+\left(m_{\tilde{f}_{1}}^{2}-m_{f}^{2}-m_{\tilde{Z}_{i}}^{2}\right) \log L\right] \text {, } \\
& I_{6}^{Z \tilde{f}_{1}}=2\left|m_{\tilde{Z}_{i}}\right| \int_{\left|m_{\tilde{Z}_{j}}\right|}^{E_{u p p e r}} d E \frac{1}{s-m_{Z}^{2}}\left(s-2 m_{f}^{2}\right) \log L \\
& I_{7}^{Z \tilde{f}_{1}}=2\left|m_{\tilde{Z}_{i}}\right| \int_{\left|m_{\tilde{Z}_{j}}\right|}^{E_{u p p e r}} d E \frac{1}{s-m_{Z}^{2}} 2\left|m_{\tilde{Z}_{i}}\right| E \log L, \\
& I_{8}^{Z \tilde{f}_{1}}=2\left|m_{\tilde{Z}_{i}}\right| \int_{\left|\tilde{z}_{\tilde{z}_{j}}\right|}^{E_{u p p e r}} d E \frac{1}{s-m_{Z}^{2}} \log L
\end{aligned}
$$

$\Gamma_{Z \tilde{f}_{2}}$ is exactly the same as $\Gamma_{Z \tilde{f}_{1}}$ but with the change $\tilde{f}_{1} \rightarrow \tilde{f}_{2}$ throughout to get the couplings $C_{1, \ldots, 8}^{Z \tilde{f}_{2}}$ and the integrals $I_{1, \ldots, 8}^{Z \tilde{f}_{2}}$.

$$
\Gamma_{h \tilde{f}_{1}}=C_{1}^{h \tilde{f}_{1}} I_{1}^{h \tilde{f}_{1}}+C_{2}^{h \tilde{f}_{1}} I_{2}^{h \tilde{f}_{1}}+C_{3}^{h \tilde{f}_{1}} I_{3}^{h \tilde{f}_{1}}+C_{4}^{h \tilde{f}_{1}} I_{4}^{h \tilde{f}_{1}}+C_{5}^{h \tilde{f}_{1}} I_{5}^{h \tilde{f}_{1}}+C_{6}^{h \tilde{f}_{1}} I_{6}^{h \tilde{f}_{1}}+C_{7}^{h \tilde{f}_{1}} I_{7}^{h \tilde{f}_{1}}+C_{8}^{h \tilde{f}_{1}} I_{8}^{h \tilde{f}_{1}},
$$


where here the couplings are:

$$
\begin{aligned}
& C_{1}^{h \tilde{f}_{1}}=-\frac{1}{2}(-1)^{\theta_{i}}(-1)^{\theta_{j}}\left(X_{i j}^{h}+X_{j i}^{h}\right) \frac{f_{q}}{\sqrt{2}} t_{\alpha_{h}}\left(\alpha_{\tilde{f}_{1}}^{\tilde{Z}_{i}} \beta_{\tilde{f}_{1}}^{\tilde{Z}_{j}}+\beta_{\tilde{f}_{1}}^{\tilde{Z}_{i}} \alpha_{\tilde{f}_{1}}^{\tilde{Z}_{j}}\right), \\
& C_{2}^{h \tilde{f}_{1}}=-(-1)^{\theta_{j}}\left(X_{i j}^{h}+X_{j i}^{h}\right) \frac{f_{q}}{\sqrt{2}} t_{\alpha_{h}}\left(\beta_{\tilde{f}_{1}}^{\tilde{Z}_{i}} \beta_{\tilde{f}_{1}}^{\tilde{Z}_{j}}+\alpha_{\tilde{f}_{1}}^{\tilde{Z}_{i}} \alpha_{\tilde{f}_{1}}^{\tilde{Z}_{j}}\right)\left|m_{\tilde{Z}_{i}}\right| m_{f}, \\
& C_{3}^{h \tilde{f}_{1}}=(-1)^{\theta_{i}}\left(X_{i j}^{h}+X_{j i}^{h}\right) \frac{f_{q}}{\sqrt{2}} t_{\alpha_{h}}\left(\beta_{\tilde{f}_{1}}^{\tilde{Z}_{i}} \beta_{\tilde{f}_{1}}^{\tilde{Z}_{j}}+\alpha_{\tilde{f}_{1}}^{\tilde{Z}_{i}} \alpha_{\tilde{f}_{1}}^{\tilde{Z}_{j}}\right)\left|m_{\tilde{Z}_{j}}\right| m_{f}, \\
& C_{4}^{h \tilde{f}_{1}}=-(-1)^{\theta_{j}}\left(X_{i j}^{h}+X_{j i}^{h}\right) \frac{f_{q}}{\sqrt{2}} t_{\alpha_{h}}\left(-\beta_{\tilde{f}_{1}}^{\tilde{Z}_{i}} \beta_{\tilde{f}_{1}}^{\tilde{Z}_{j}}-\alpha_{\tilde{f}_{1}}^{\tilde{Z}_{i}} \alpha_{\tilde{f}_{1}}^{\tilde{Z}_{j}}\right)\left|m_{\tilde{Z}_{i}}\right| m_{f}, \\
& C_{5}^{h \tilde{f}_{1}}=-(-1)^{\theta_{i}}\left(X_{i j}^{h}+X_{j i}^{h}\right) \frac{f_{q}}{\sqrt{2}} t_{\alpha_{h}}\left(\beta_{\tilde{f}_{1}}^{\tilde{Z}_{i}} \beta_{\tilde{f}_{1}}^{\tilde{Z}_{j}}+\alpha_{\tilde{f}_{1}}^{\tilde{Z}_{i}} \alpha_{\tilde{f}_{1}}^{\tilde{Z}_{j}}\right)\left|m_{\tilde{Z}_{j}}\right| m_{f}, \\
& C_{6}^{h \tilde{f}_{1}}=\left(X_{i j}^{h}+X_{j i}^{h}\right) \frac{f_{q}}{\sqrt{2}} t_{\alpha_{h}}\left(-\alpha_{\tilde{f}_{1}}^{\tilde{Z}_{i}} \beta_{\tilde{f}_{1}}^{\tilde{Z}_{j}}-\beta_{\tilde{f}_{1}}^{\tilde{Z}_{i}} \alpha_{\tilde{f}_{1}}^{\tilde{Z}_{j}}\right)\left|m_{\tilde{Z}_{i}}\right|\left|m_{\tilde{Z}_{j}}\right|, \\
& C_{7}^{h \tilde{f}_{1}}=(-1)^{\theta_{i}}(-1)^{\theta_{j}}\left(X_{i j}^{h}+X_{j i}^{h}\right) \frac{f_{q}}{\sqrt{2}} t_{\alpha_{h}}\left(\alpha_{\tilde{f}_{1}}^{\tilde{Z}_{i}} \beta_{\tilde{f}_{1}}^{\tilde{z}_{j}}+\beta_{\tilde{f}_{1}}^{\tilde{Z}_{i}} \alpha_{\tilde{f}_{1}}^{\tilde{Z}_{j}}\right) m_{f}^{2}, \\
& C_{8}^{h \tilde{f}_{1}}=2\left(X_{i j}^{h}+X_{j i}^{h}\right) \frac{f_{q}}{\sqrt{2}} t_{\alpha_{h}}\left(\alpha_{\tilde{f}_{1}}^{\tilde{Z}_{i}} \beta_{\tilde{f}_{1}}^{\tilde{Z}_{j}}+\beta_{\tilde{f}_{1}}^{\tilde{Z}_{i}} \alpha_{\tilde{f}_{1}}^{\tilde{Z}_{j}}\right)\left|m_{\tilde{Z}_{i}}\right| m_{f}^{2}\left|m_{\tilde{Z}_{j}}\right| .
\end{aligned}
$$

The necessary integrals are:

$$
\begin{aligned}
& I_{1}^{h \tilde{f}_{1}}=2\left|m_{\tilde{Z}_{i}}\right| \int_{\left|m_{\tilde{Z}_{j}}\right|}^{E_{u p p e r}} d E \frac{2}{s-m_{h}^{2}}\left[2 s\left|m_{\tilde{Z}_{i}}\right| \sqrt{E^{2}-m_{\tilde{Z}_{j}}^{2}} \sqrt{1-4 m_{f}^{2} / s}+\left\{m_{\tilde{f}_{1}}^{2} s-m_{f}^{2}\left(m_{\tilde{Z}_{i}}^{2}+m_{\tilde{Z}_{j}}^{2}\right)\right\} \log L\right], \\
& I_{2}^{h \tilde{f}_{1}}=-2\left|m_{\tilde{Z}_{i}}\right| \int_{\left|m_{\tilde{Z}_{j}}\right|}^{E_{\text {upper }}} d E \frac{1}{s-m_{h}^{2}}\left[2\left|m_{\tilde{Z}_{i}}\right| \sqrt{E^{2}-m_{\tilde{Z}_{j}}^{2}} \sqrt{1-4 m_{f}^{2} / s}+\left(m_{\tilde{f}_{1}}^{2}-m_{f}^{2}+m_{\tilde{Z}_{j}}^{2}-2 m_{\tilde{Z}_{i}} E\right) \log L\right], \\
& I_{3}^{h \tilde{f}_{1}}=2\left|m_{\tilde{Z}_{i}}\right| \int_{\left|m_{\tilde{Z}_{j}}\right|}^{E_{\text {upper }}} d E \frac{1}{s-m_{h}^{2}}\left[2\left|m_{\tilde{Z}_{i}}\right| \sqrt{E^{2}-m_{\tilde{Z}_{j}}^{2}} \sqrt{1-4 m_{f}^{2} / s}+\left(m_{\tilde{f}_{1}}^{2}-m_{f}^{2}+m_{\tilde{Z}_{i}}^{2}-2 m_{\tilde{Z}_{i}} E\right) \log L\right], \\
& I_{4}^{h \tilde{f}_{1}}=2\left|m_{\tilde{Z}_{i}}\right| \int_{\left|m_{\tilde{Z}_{j}}\right|}^{E_{\text {upper }}} d E \frac{1}{s-m_{h}^{2}}\left[2\left|m_{\tilde{Z}_{i}}\right| \sqrt{E^{2}-m_{\tilde{Z}_{j}}^{2}} \sqrt{1-4 m_{f}^{2} / s}+\left(m_{\tilde{f}_{1}}^{2}-m_{f}^{2}+m_{\tilde{Z}_{j}}^{2}\right) \log L\right], \\
& I_{5}^{h \tilde{f}_{1}}=-2\left|m_{\tilde{Z}_{i}}\right| \int_{\left|m_{\tilde{Z}_{j}}\right|}^{E_{\text {upper }}} d E \frac{1}{s-m_{h}^{2}}\left[2\left|m_{\tilde{Z}_{i}}\right| \sqrt{E^{2}-m_{\tilde{Z}_{j}}^{2}} \sqrt{1-4 m_{f}^{2} / s}+\left(m_{\tilde{f}_{1}}^{2}-m_{f}^{2}+m_{\tilde{Z}_{i}}^{2}\right) \log L\right] \text {, } \\
& I_{6}^{h \tilde{f}_{1}}=-2\left|m_{\tilde{Z}_{i}}\right| \int_{\left|m_{\tilde{Z}_{j}}\right|}^{E_{\text {upper }}} d E \frac{1}{s-m_{h}^{2}}\left(s-2 m_{f}^{2}\right) \log L \\
& I_{7}^{h \tilde{f}_{1}}=-2\left|m_{\tilde{Z}_{i}}\right| \int_{\left|m_{\tilde{Z}_{j}}\right|}^{E_{\text {upper }}} d E \frac{1}{s-m_{h}^{2}}\left(2\left|m_{\tilde{Z}_{i}}\right| E\right) \log L \\
& I_{8}^{h \tilde{f}_{1}}=-2\left|m_{\tilde{Z}_{i}}\right| \int_{\left|m_{\tilde{Z}_{j}}\right|}^{E_{\text {upper }}} d E \frac{1}{s-m_{h}^{2}} \log L
\end{aligned}
$$

Note $\Gamma_{h \tilde{f}_{2}}$ is exactly the same as $\Gamma_{h \tilde{f}_{1}}$ but with the replacement $\tilde{f}_{1} \rightarrow \tilde{f}_{2}$ throughout to get the couplings $C_{1, \ldots, 8}^{h \tilde{f}_{2}}$ and the integrals $I_{1, \ldots, 8}^{h \tilde{f}_{2}}$. Similarly, one can obtain the $\Gamma_{H \tilde{f}_{1}}$ from $\Gamma_{h \tilde{f}_{1}}$ by replacing $\mathrm{h}$ by $\mathrm{H}$ throughout all the couplings, 
masses and integrals; therefore the changes $X_{i j}^{h}+X_{j i}^{h} \rightarrow X_{i j}^{H}+X_{j i}^{H}$ and $t_{\alpha_{h}} \rightarrow t_{\alpha_{H}}$ are made. One can then obtain $\Gamma_{H \tilde{f}_{2}}$ again by changing $\tilde{f}_{1} \rightarrow \tilde{f}_{2}$ throughout the couplings, masses and integrals.

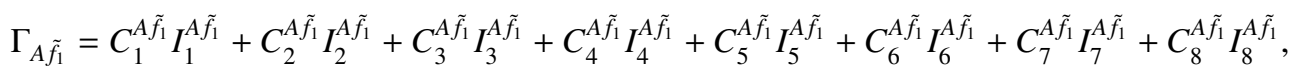

here we have:

$$
\begin{aligned}
& C_{1}^{A \tilde{f}_{1}}=\frac{1}{2}(-1)^{\theta_{i}}\left(X_{i j}^{A}+X_{j i}^{A}\right) \frac{A_{q}}{2}\left(\alpha_{\tilde{f}_{1}}^{\tilde{z}_{i}} \beta_{\tilde{f}_{1}}^{\tilde{Z}_{j}}+\beta_{\tilde{f}_{1}}^{\tilde{Z}_{i}} \tilde{z}_{\tilde{f}_{1}}^{\tilde{Z}_{j}}\right), \\
& C_{2}^{A \tilde{f}_{1}}=-\left(X_{i j}^{A}+X_{j i}^{A}\right) \frac{A_{q}}{2}\left|m_{\tilde{Z}_{i}}\right| m_{f}\left(\beta_{\tilde{f}_{1}}^{\tilde{Z}_{i}} \beta_{\tilde{f}_{1}}^{\tilde{Z}_{j}}+\alpha_{\tilde{f}_{1}}^{\tilde{Z}_{i}} \alpha_{\tilde{f}_{1}}^{\tilde{Z}_{j}}\right), \\
& C_{3}^{A \tilde{f}_{1}}=(-1)^{\theta_{i}}(-1)^{\theta_{j}}\left(X_{i j}^{A}+X_{j i}^{A}\right) \frac{A_{q}}{2}\left|m_{\tilde{Z}_{j}}\right| m_{f}\left(\beta_{\tilde{f}_{1}}^{\tilde{z}_{i}} \beta_{\tilde{f}_{1}}^{\tilde{Z}_{j}}+\alpha_{\tilde{f}_{1}}^{\tilde{Z}_{i}} \alpha_{\tilde{f}_{1}}^{\tilde{Z}_{j}}\right) \text {, } \\
& C_{4}^{A \tilde{f}_{1}}=-\left(X_{i j}^{A}+X_{j i}^{A}\right) \frac{A_{q}}{2}\left|m_{\tilde{Z}_{i}}\right| m_{f}\left(\beta_{\tilde{f}_{1}}^{\tilde{Z}_{i}} \beta_{\tilde{f}_{1}}^{\tilde{Z}_{j}}+\alpha_{\tilde{f}_{1}}^{\tilde{Z}_{i}} \alpha_{\tilde{f}_{1}}^{\tilde{Z}_{j}}\right), \\
& C_{5}^{A \tilde{f}_{1}}=(-1)^{\theta_{i}}(-1)^{\theta_{j}}\left(X_{i j}^{A}+X_{j i}^{A}\right) \frac{A_{q}}{2}\left|m_{\tilde{Z}_{j}}\right| m_{f}\left(\beta_{\tilde{f}_{1}}^{\tilde{Z}_{i}} \beta_{\tilde{f}_{1}}^{\tilde{Z}_{j}}+\alpha_{\tilde{f}_{1}}^{\tilde{Z}_{i}} \alpha_{\tilde{f}_{1}}^{\tilde{Z}_{j}}\right) \text {, } \\
& C_{6}^{A \tilde{f}_{1}}=-(-1)^{\theta_{j}}\left(X_{i j}^{A}+X_{j i}^{A}\right) \frac{A_{q}}{2}\left|m_{\tilde{Z}_{i}}\right|\left|m_{\tilde{Z}_{j}}\right|\left(\alpha_{\tilde{f}_{1}}^{\tilde{Z}_{i}} \beta_{\tilde{f}_{1}}^{\tilde{z}_{j}}+\beta_{\tilde{f}_{1}}^{\tilde{Z}_{i}} \alpha_{\tilde{f}_{1}}^{\tilde{Z}_{j}}\right), \\
& C_{7}^{A \tilde{f}_{1}}=(-1)^{\theta_{i}}\left(X_{i j}^{A}+X_{j i}^{A}\right) \frac{A_{q}}{2} m_{f}^{2}\left(\alpha_{\tilde{f}_{1}}^{\tilde{Z}_{i}} \beta_{\tilde{f}_{1}}^{\tilde{Z}_{j}}+\beta_{\tilde{f}_{1}}^{\tilde{Z}_{i}} \alpha_{\tilde{f}_{1}}^{\tilde{Z}_{j}}\right), \\
& C_{8}^{A \tilde{f}_{1}}=-(-1)^{\theta_{j}}\left(X_{i j}^{A}+X_{j i}^{A}\right) A_{q} m_{f}^{2}\left|m_{\tilde{Z}_{i}} \| m_{\tilde{Z}_{j}}\right|\left(\alpha_{\tilde{f}_{1}}^{\tilde{Z}_{i}} \beta_{\tilde{f}_{1}}^{\tilde{Z}_{j}}+\beta_{\tilde{f}_{1}}^{\tilde{z}_{i_{1}}} \alpha_{\tilde{f}_{1}}^{\tilde{Z}_{j}}\right) \text {. }
\end{aligned}
$$

The $I_{i}^{A \tilde{f}_{1}}$ are exactly as the $I_{i}^{h \tilde{f}_{1}}$ but with the change $m_{h} \rightarrow m_{A}$.

$$
\Gamma_{Z A}=2 C_{1}^{Z A} I_{1}^{Z A}+2 C_{2}^{Z A} I_{2}^{Z A}
$$

where

$$
\begin{aligned}
& C_{1}^{Z A}=-4(-1)^{\theta_{i}}(-1)^{\theta_{j}} W_{i j}\left(X_{i j}^{A}+X_{j i}^{A}\right) A_{q} g \sin \theta_{W} \beta_{f}\left|m_{\tilde{Z}_{j}}\right| m_{f}, \\
& C_{2}^{Z A}=4 W_{i j}\left(X_{i j}^{A}+X_{j i}^{A}\right) A_{q} g \sin \theta_{W} \beta_{f}\left|m_{\tilde{Z}_{i}}\right| m_{f} .
\end{aligned}
$$

The integrals included here are:

$$
\begin{gathered}
I_{1}^{Z A}=2\left|m_{\tilde{Z}_{i}}\right| \int_{\left|m_{\tilde{Z}_{j}}\right|}^{E_{u p p e r}} d_{E} \frac{1}{\left(s-m_{z}^{2}\right)\left(s-m_{A}^{2}\right)} 2\left|m_{\tilde{Z}_{i}}\right| \sqrt{E^{2}-m_{\tilde{Z}_{j}}^{2}} \sqrt{1-4 m_{f}^{2} / s}\left\{m_{\tilde{Z}_{i}}^{2}-\left|m_{\tilde{Z}_{i}}\right| E\right\}, \\
I_{2}^{Z A}=-2\left|m_{\tilde{Z}_{i}}\right| \int_{\left|m_{\tilde{Z}_{j}}\right|}^{E_{u p p e r}} d_{E} \frac{1}{\left(s-m_{z}^{2}\right)\left(s-m_{A}^{2}\right)} 2\left|m_{\tilde{Z}_{i}}\right| \sqrt{E^{2}-m_{\tilde{Z}_{j}}^{2}} \sqrt{1-4 m_{f}^{2} / s}\left\{m_{\tilde{Z}_{j}}^{2}-\left|m_{\tilde{Z}_{i}}\right| E\right\} .
\end{gathered}
$$

The goldstone contribution is:

$$
\Gamma_{G}=4 c_{G \tilde{Z}_{i} \tilde{Z}_{j}}^{2} c_{G f f}^{2}\left[I_{4}^{G}+2 m_{f}^{2} I_{3}^{G}-2(-1)^{\theta_{i}}(-1)^{\theta_{j}}\left|m_{\tilde{Z}_{i}} \| m_{\tilde{z}_{j}}\right| I_{2}^{G}-4(-1)^{\theta_{i}}(-1)^{\theta_{j}} m_{f}^{2}\left|m_{\tilde{Z}_{i}}\right|\left|m_{\tilde{Z}_{j}}\right| I_{1}^{G}\right] .
$$

The goldstone interferences with fermions are given by:

$$
\Gamma_{G \tilde{f_{1}}}=C_{1}^{G \tilde{f_{1}}} I_{1}^{G \tilde{f_{1}}}+C_{2}^{G \tilde{f_{1}}} I_{2}^{G \tilde{f_{1}}}+C_{3}^{G \tilde{f_{1}}} I_{3}^{G \tilde{f_{1}}}+C_{4}^{G \tilde{f_{1}}} I_{4}^{G \tilde{f_{1}}}+C_{5}^{G \tilde{f_{1}}} I_{5}^{G \tilde{f_{1}}}+C_{6}^{G \tilde{f} \tilde{f}_{1}} I_{6}^{G \tilde{f_{1}}}+C_{7}^{G \tilde{f_{1}}} I_{7}^{G \tilde{f_{1}}}+C_{8}^{G \tilde{f_{1}}} I_{8}^{G \tilde{\tilde{f}_{1}}},
$$


where

$$
\begin{aligned}
& C_{1}^{G \tilde{f}_{1}}=\frac{1}{2} c_{G \tilde{Z}_{i} \tilde{z}_{j}} c_{G f f}\left(\alpha_{\tilde{f}_{1}}^{\tilde{Z}_{i}} \beta_{\tilde{f}_{1}}^{\tilde{Z}_{j}}+\beta_{\tilde{f}_{1}}^{\tilde{Z}_{i}} \alpha_{\tilde{f}_{1}}^{\tilde{Z}_{j}}\right), \\
& C_{2}^{G \tilde{f_{1}}}=-(-1)^{\theta_{i}} m_{f}\left|m_{\tilde{Z}_{i}}\right| c_{G \tilde{Z}_{i} \tilde{Z}_{j}} c_{G f f}\left(\beta_{\tilde{f}_{1}}^{\tilde{Z}_{i}} \tilde{\tilde{f}}_{\tilde{f}_{1}}^{\tilde{z}_{j}}+\alpha_{\tilde{f}_{1}}^{\tilde{Z}_{i}} \alpha_{\tilde{f}_{1}}^{\tilde{Z}_{j}}\right), \\
& C_{3}^{G \tilde{f}_{1}}=(-1)^{\theta_{j}} m_{f}\left|m_{\tilde{Z}_{j}}\right| c_{G \tilde{Z}_{i} \tilde{Z}_{j}} c_{G f f}\left(\alpha_{\tilde{f}_{1}}^{\tilde{Z}_{i}} \alpha_{\tilde{f}_{1}}^{\tilde{Z}_{j}}+\beta_{\tilde{f}_{1}}^{\tilde{Z}_{i}} \beta_{\tilde{f}_{1}}^{\tilde{Z}_{j}}\right), \\
& C_{4}^{G \tilde{f_{1}}}=-(-1)^{\theta_{i}} m_{f}\left|m_{\tilde{Z}_{i}}\right| c_{G \tilde{Z}_{i} \tilde{Z}_{j}} c_{G f f}\left(\alpha_{\tilde{z}_{1}}^{\tilde{Z}_{i}} \alpha_{\tilde{f}_{1}}^{\tilde{Z}_{j}}+\beta_{\tilde{f}_{1}}^{\tilde{z}_{i}} \beta_{\tilde{f}_{1}}^{\tilde{Z}_{j}}\right), \\
& C_{5}^{G \tilde{f}_{1}}=(-1)^{\theta_{j}}\left|m_{\tilde{Z}_{i}}\right| m_{f} c_{G \tilde{Z}_{i} \tilde{z}_{j}} c_{G f f}\left(\alpha_{\tilde{f}_{1}}^{\tilde{Z}_{i}} \alpha_{\tilde{f}_{1}}^{\tilde{z}_{j}}+\beta_{\tilde{f}_{1}}^{\tilde{Z}_{i}} \beta_{\tilde{f}_{1}}^{\tilde{Z}_{j}}\right), \\
& C_{6}^{G \tilde{f}_{1}}=-(-1)^{\theta_{i}}(-1)^{\theta_{j}}\left|m_{\tilde{Z}_{i}} \| m_{\tilde{Z}_{j}}\right| c_{G \tilde{Z}_{i} \tilde{Z}_{j}} c_{G f f}\left(\alpha_{\tilde{f}_{1}}^{\tilde{z}_{i}} \beta_{\tilde{f}_{1}}^{\tilde{Z}_{j}}+\beta_{\tilde{f}_{1}}^{\tilde{Z}_{i}} \alpha_{\tilde{f}_{1}}^{\tilde{Z}_{j}}\right) \text {, } \\
& C_{7}^{G \tilde{f}_{1}}=m_{f}^{2} c_{G \tilde{z}_{i} \tilde{Z}_{j}} c_{G f f}\left(\alpha_{\tilde{f}_{1}}^{\tilde{z}_{i}} \beta_{\tilde{f}_{1}}^{\tilde{Z}_{j}}+\beta_{\tilde{f}_{1}}^{\tilde{z}_{i}} \alpha_{\tilde{f}_{1}}^{\tilde{Z}_{j}}\right), \\
& C_{8}^{G \tilde{f_{1}}}=-2(-1)^{\theta_{i}}(-1)^{\theta_{j}} m_{f}^{2}\left|m_{\tilde{Z}_{i}} \| m_{\tilde{Z}_{j}}\right| c_{G \tilde{Z}_{i} \tilde{z}_{j}} c_{G f f}\left(\alpha_{\tilde{f}_{1}}^{\tilde{Z}_{i}} \beta_{\tilde{f}_{1}}^{\tilde{Z}_{j}}+\beta_{\tilde{f}_{1}}^{\tilde{z}_{i}} \alpha_{\tilde{f}_{1}}^{\tilde{z}_{j}}\right) \text {. }
\end{aligned}
$$

The $I_{1, \ldots, 8}^{G \tilde{f}_{1}}$ integrals are the same as the $I_{1, \ldots, 8}^{h \tilde{f}_{1}}$ but with the replacement $m_{h} \rightarrow m_{Z}$ as the mass of the goldstone is the $Z$ mass as it represents the longitudinal component of the $Z$ boson. Similar changes apply to the $I_{1, \ldots, 8}^{G \tilde{f}_{2}}$, whilst in the couplings we apply the replacement $\tilde{f}_{1} \rightarrow \tilde{f}_{2}$ throughout. The $Z$-goldstone interference contribution is:

$$
\Gamma_{Z G}=2 C_{1}^{Z G} I_{1}^{Z G}+2 C_{2}^{Z G} I_{2}^{Z G},
$$

where

$$
\begin{gathered}
C_{1}^{Z G}=-8 W_{i j}(-1)^{\theta_{j}} m_{f}\left|m_{\tilde{Z}_{j}}\right| c_{G f f} c_{G \tilde{Z}_{i} \tilde{z}_{j}} g \sin \theta_{W} \beta_{f}, \\
C_{2}^{Z G}=8 W_{i j}(-1)^{\theta_{i}} m_{f}\left|m_{\tilde{Z}_{i}}\right| c_{G f f} c_{G \tilde{Z}_{i} \tilde{Z}_{j}} g \sin \theta_{W} \beta_{f} .
\end{gathered}
$$

The $I_{1,2}^{Z G}$ are the same as the $I_{1,2}^{Z A}$ but with the expected change $m_{A} \rightarrow m_{Z}$.

$$
\Gamma_{G A}=C_{1}^{G A} C_{3}^{G A} I_{4}^{G A}-2 C_{1}^{G A} C_{4}^{G A} m_{f}^{2} I_{3}^{G A}+2 C_{2}^{G A} C_{3}^{G A}\left|m_{\tilde{Z}_{i}}\right|\left|m_{\tilde{Z}_{j}}\right| I_{2}^{G A}-4 C_{2}^{G A} C_{4}^{G A} m_{f}^{2}\left|m_{\tilde{Z}_{i}}\right|\left|m_{\tilde{Z}_{j}}\right| I_{1}^{G A},
$$

where here

$$
\begin{gathered}
C_{1}^{G A}=-2(-1)^{\theta_{i}} c_{G \tilde{Z}_{i} \tilde{Z}_{j}}\left(X_{i j}^{A}+X_{j i}^{A}\right), \\
C_{2}^{G A}=2(-1)^{\theta_{j}} c_{G \tilde{Z}_{i} \tilde{Z}_{j}}\left(X_{i j}^{A}+X_{j i}^{A}\right), \\
C_{3}^{G A}=-A_{q} c_{G f f}, \\
C_{4}^{G A}=A_{q} c_{G f f} .
\end{gathered}
$$

The integrals are:

$$
\begin{aligned}
& I_{1}^{G A}=2\left|m_{\tilde{Z}_{i}}\right| \int_{\left|m_{\tilde{z}_{j}}\right|}^{E_{\max }} d E \frac{2\left|m_{\tilde{Z}_{i}}\right| \sqrt{E^{2}-m_{\tilde{Z}_{j}}^{2}} \sqrt{1-4 m_{f}^{2} / s}}{\left(s-m_{Z}^{2}\right)\left(s-m_{A}^{2}\right)}, \\
& I_{2}^{G A}=2\left|m_{\tilde{Z}_{i}}\right| \int_{\left|m_{\tilde{z}_{j}}\right|}^{E_{\max }} d E \frac{2\left|m_{\tilde{Z}_{i}}\right|\left(s-2 m_{f}^{2}\right) \sqrt{E^{2}-m_{\tilde{Z}_{j}}^{2}} \sqrt{1-4 m_{f}^{2} / s}}{\left(s-m_{Z}^{2}\right)\left(s-m_{A}^{2}\right)}, \\
& I_{3}^{G A}=2\left|m_{\tilde{Z}_{i}}\right| \int_{\left|m_{\tilde{z}_{j}}\right|}^{E_{\max }} d E \frac{4\left|m_{\tilde{Z}_{i}}\right|^{2} E \sqrt{E^{2}-m_{\tilde{Z}_{j}}^{2}} \sqrt{1-4 m_{f}^{2} / s}}{\left(s-m_{Z}^{2}\right)\left(s-m_{A}^{2}\right)}, \\
& I_{4}^{G A}=2\left|m_{\tilde{Z}_{i}}\right| \int_{\left|m_{\tilde{Z}_{j}}\right|}^{E_{\max }} d E \frac{4\left|m_{\tilde{Z}_{i}}\right|^{2} E\left(s-2 m_{f}^{2}\right) \sqrt{E^{2}-m_{\tilde{Z}_{j}}^{2}} \sqrt{1-4 m_{f}^{2} / s}}{\left(s-m_{Z}^{2}\right)\left(s-m_{A}^{2}\right)} .
\end{aligned}
$$


Now the list of the couplings used is:

$$
W_{i j}=0.25 \sqrt{g^{2}+g^{\prime 2}}\left(N_{4 i} N_{4 j}-N_{3 i} N_{3 j}\right) .
$$

The $X_{i j}^{\phi}$ couplings are:

$$
\begin{aligned}
& X_{i j}^{h}=-\frac{1}{2}(-1)^{\theta_{i}}(-1)^{\theta_{j}}\left[-N_{3 i} \sin \alpha-N_{4 i} \cos \alpha\right]\left(-g N_{2 j}+g^{\prime} N_{1 j}\right), \\
& X_{i j}^{H}=-\frac{1}{2}(-1)^{\theta_{i}}(-1)^{\theta_{j}}\left[N_{3 i} \cos \alpha-N_{4 i} \sin \alpha\right]\left(-g N_{2 j}+g^{\prime} N_{1 j}\right), \\
& X_{i j}^{A}=\frac{1}{2}(-1)^{\theta_{i}}(-1)^{\theta_{j}}\left[N_{3 i} \sin \beta-N_{4 i} \cos \beta\right]\left(-g N_{2 j}+g^{\prime} N_{1 j}\right) \text {. } \\
& f_{q}=\left\{\begin{array}{l}
\frac{g m_{q}^{\text {run }}}{\sqrt{2} m_{W_{\min } \sin \beta}}, \text { for } u \text {-type quarks, } \\
\frac{g m_{q}^{\text {run }}}{\sqrt{2} m_{W} \cos \beta}, \text { for } d \text {-type quarks, } \\
0, \text { for neutrinos } v, \\
\frac{g m_{l}^{\text {run }}}{\sqrt{2} m_{W} \cos \beta}, \text { for charged leptons. }
\end{array}\right. \\
& A_{q}=\left\{\begin{array}{l}
\frac{g m_{q}^{r u n}}{m_{W^{\prime} \tan \beta} \beta}, \text { for } u \text {-type quarks, } \\
\frac{g m_{q}^{r u n} \tan \beta}{m_{W}}, \text { for } d \text {-type quarks, } \\
0, \text { for neutrinos } v, \\
\frac{g m_{l}^{r u n} \tan \beta}{m_{W}}, \text { for charged leptons. }
\end{array}\right. \\
& t_{\alpha_{h}}=\left\{\begin{array}{l}
\cos \alpha, \text { for } u \text {-type quarks, } \\
-\sin \alpha, \text { for } d \text {-type quarks, } \\
\cos \alpha, \text { for neutrinos } v, \\
-\sin \alpha, \text { for charged leptons. }
\end{array}\right. \\
& t_{\alpha_{H}}=\left\{\begin{array}{l}
\sin \alpha, \text { for } u \text {-type quarks, } \\
\cos \alpha, \text { for } d \text {-type quarks, } \\
\sin \alpha, \text { for neutrinos } v, \\
\cos \alpha, \text { for charged leptons. }
\end{array}\right. \\
& \alpha_{f}=\left\{\begin{array}{l}
-\frac{5 g^{\prime} p}{12 g}+\frac{g}{4 g^{\prime}}, \text { for } u \text {-type quarks, } \\
\frac{g^{\prime} p}{12 g}-\frac{g}{4 g^{\prime}}, \text { for } d \text {-type quarks, } \\
\frac{1}{4}\left(\frac{g^{\prime}}{g}+\frac{g}{g^{\prime}}\right), \text { for neutrinos } v, \\
\frac{3}{4} \frac{g^{\prime}}{g}-\frac{g}{4 g^{\prime}}, \text { for charged leptons. }
\end{array}\right. \\
& \beta_{f}=\left\{\begin{array}{l}
-\frac{1}{4}\left(\frac{g^{\prime}}{g}+\frac{g}{g^{\prime}}\right), \text { for } u \text {-type quarks, } \\
\frac{1}{4}\left(\frac{g^{\prime}}{g}+\frac{g}{g^{\prime}}\right), \text { for } d \text {-type quarks, } \\
-\frac{1}{4}\left(\frac{g^{\prime}}{g}+\frac{g}{g^{\prime}}\right), \text { for neutrinos } v, \\
\frac{1}{4}\left(\frac{g^{\prime}}{g}+\frac{g}{g^{\prime}}\right), \text { for charged leptons. }
\end{array}\right. \\
& \alpha_{\tilde{f}_{1}}^{\tilde{Z}_{i}}=\left\{\begin{array}{l}
A_{\tilde{Z}_{i}} \cos \theta_{q}-f_{q} N_{4 i} \sin \theta_{q}, \text { for } u \text {-type quarks, } \\
A_{\tilde{Z}_{i}} \cos \theta_{q}-f_{q} N_{3 i} \sin \theta_{q}, \text { for } d \text {-type quarks, } \\
A_{\tilde{Z}_{i}} \cos \theta_{q}, \text { for neutrinos } v, \\
A_{\tilde{Z}_{i}} \sin \theta_{q}+f_{q} N_{3 i} \cos \theta_{q}, \text { for charged leptons. }
\end{array}\right.
\end{aligned}
$$




$$
\begin{aligned}
& \alpha_{\tilde{f}_{2}}^{\tilde{Z}_{i}}=\left\{\begin{array}{l}
A_{\tilde{Z}_{i}} \sin \theta_{q}+f_{q} N_{4 i} \cos \theta_{q}, \text { for } u \text {-type quarks, } \\
A_{\tilde{Z}_{i}} \sin \theta_{q}+f_{q} N_{3 i} \cos \theta_{q}, \text { for } d \text {-type quarks, } \\
A_{\tilde{Z}_{i}} \sin \theta_{q}, \text { for neutrinos } v, \\
-A_{\tilde{Z}_{i}} \cos \theta_{q}+f_{q} N_{3 i} \sin \theta_{q}, \text { for charged leptons. }
\end{array}\right. \\
& \beta_{\tilde{f}_{1}}^{\tilde{Z}_{i}}=\left\{\begin{array}{l}
f_{q} N_{4 i} \cos \theta_{q}+B_{\tilde{Z}_{i}} \sin \theta_{q}, \text { for } u \text {-type quarks, } \\
f_{q} N_{3 i} \cos \theta_{q}+B_{\tilde{Z}_{i}} \sin \theta_{q}, \text { for } d \text {-type quarks, } \\
0, \text { for neutrinos } v, \\
f_{q} N_{3 i} \sin \theta_{q}-B_{\tilde{Z}_{i}} \cos \theta_{q}, \text { for charged leptons. }
\end{array}\right. \\
& \beta_{\tilde{f_{2}}}^{\tilde{Z}_{i}}=\left\{\begin{array}{l}
f_{q} N_{4 i} \sin \theta_{q}-B_{\tilde{Z}_{i}} \cos \theta_{q}, \text { for } u \text {-type quarks, } \\
f_{q} N_{3 i} \sin \theta_{q}-B_{\tilde{Z}_{i}} \cos \theta_{q}, \text { for } d \text {-type quarks, } \\
0, \text { for neutrinos } v, \\
-f_{q} N_{3 i} \cos \theta_{q}-B_{\tilde{Z}_{i}} \sin \theta_{q}, \text { for charged leptons. }
\end{array}\right. \\
& A_{\tilde{Z}_{i}}=\left\{\begin{array}{l}
-\frac{g}{\sqrt{2}} N_{2 i}-\frac{g^{\prime}}{3 \sqrt{2}} N_{1 i}, \text { for } u \text {-type quarks } \\
\frac{g}{\sqrt{2}} N_{2 i}-\frac{g^{\prime}}{3 \sqrt{2}} N_{1 i}, \text { for } d \text {-type quarks, } \\
-\frac{g}{\sqrt{2}} N_{2 i}+\frac{g^{\prime}}{3 \sqrt{2}} N_{1 i}, \text { for neutrinos } v \\
\frac{g}{\sqrt{2}} N_{2 i}+\frac{g^{\prime}}{3 \sqrt{2}} N_{1 i}, \text { for charged leptons. }
\end{array}\right. \\
& B_{\tilde{Z}_{i}}=\left\{\begin{array}{l}
-\frac{4 g^{\prime}}{3 \sqrt{2}} N_{1 i}, \text { for } u \text {-type quarks, } \\
\frac{2 g^{\prime}}{3 \sqrt{2}} N_{1 i}, \text { for } d \text {-type quarks, } \\
0, \text { for neutrinos } v, \\
\sqrt{2} g^{\prime} N_{1 i}, \text { for charged leptons. }
\end{array}\right. \\
& c_{G f f}=\left\{\begin{array}{l}
\frac{-f_{q} \sin \beta}{\sqrt{2}}, \text { for } u \text {-type quarks, } \\
\frac{f_{q} \cos \beta}{\sqrt{2}}, \text { for } d \text {-type quarks, } \\
0, \text { for neutrinos } v \\
\frac{f_{q} \cos \beta}{\sqrt{2}}, \text { for charged leptons. }
\end{array}\right.
\end{aligned}
$$

$\tilde{Z}_{i} \rightarrow \tilde{W}_{j} f^{\prime} \bar{f}$

We turn now onto the 3 body decays of a neutralino into a chargino, fermion and anti-fermion. As for all the other 3 body modes included, this mode is only calculated if no 2 body modes are kinematically accessible. There are 4 main contributions to these decays, with $W$ boson, $H^{ \pm}, \tilde{f}^{\prime}{ }_{k}$ and $\tilde{f}_{k}$ intermediates, the Feynman diagrams for these are shown in Figure G.9 Therefore there are nominally 6 squared contributions and 15 interferences; however, as the calculation is again done in Feynman gauge, the goldstone boson corresponding to the longitudinal components of the $W$ boson must be added, adding a further squared contribution and its 6 interferences.

For this decay mode, and the "reverse" decay mode $\tilde{W}_{j} \rightarrow \tilde{Z}_{i} f^{\prime} \bar{f}$, the formulae used are extracted from the sPHENO code, based on the work in references [34, 41]. Note that $f^{\prime}, f$ are fermions with third components of weak isospin $\frac{1}{2}$ and $-\frac{1}{2}$ respectively. A difference relative to these references is that, following the formulae of sPHENO, the expressions given do not neglect $m_{f}$ in the Dirac algebra of the squared matrix element (whereas in [34, 41] it is neglected here, but of course included in the phase space). As a result there is also $W H^{ \pm}$interference which is not present if $m_{f}$ is neglected in the Dirac algebra. The possibilities of positive and negative neutralino and chargino masses are included via $(-1)^{\theta_{i}}$ and $(-1)^{\theta_{j}}$ factors. Similarly the fermion Yukawa couplings are included and the formulae themselves allow for mixing of the fermions. However in our program, mixing is only considered for the third generation of sfermions and here this 3 body mode $\tilde{Z}_{i} \rightarrow \tilde{W}_{j} t \bar{b}$ is not calculated as the 2 body modes $\tilde{Z}_{i} \rightarrow W \tilde{W}_{j}$ 


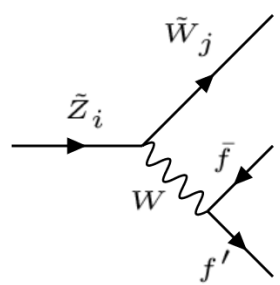

(a) $W$

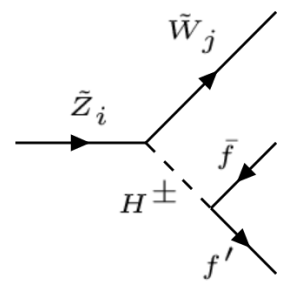

(b) $H^{ \pm}$

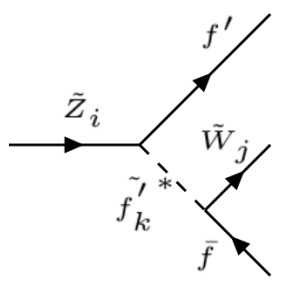

(c) $\tilde{f}_{k}{ }_{k}$

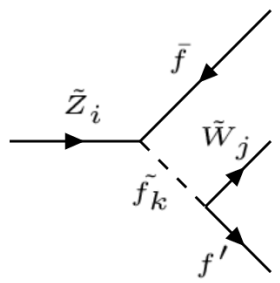

(d) $\tilde{f}_{k}$

Figure G.9: $W, H^{ \pm}, \tilde{f}^{\prime}{ }_{k}, \tilde{f}_{k}$ contributions to the $\tilde{Z}_{i} \rightarrow \tilde{W}_{j} f^{\prime} \bar{f}$ decay. $i=1,2,3,4, j=1,2, k=1,2$. There are then also interferences between all these contributions.

and $\tilde{Z}_{i} \rightarrow h \tilde{W}_{j}$ are then kinematically available and will dominate the branching ratios. The overall expression for the partial width is therefore given by:

$$
\begin{aligned}
& \Gamma=\frac{N_{c}}{512 \pi^{3}\left|m_{Z_{i}}\right|^{3}}\left[\Gamma_{W}+\Gamma_{\tilde{f}_{1}}+\Gamma_{\tilde{f}_{2}}+\Gamma_{\tilde{f}^{\prime}{ }_{1}}+\Gamma_{\tilde{f}^{\prime}{ }_{2}}-2 \Gamma_{\tilde{f}^{\prime}{ }_{1} \tilde{f}_{1}}-2 \Gamma_{\tilde{f}^{\prime}{ }_{1} \tilde{f}_{2}}-2 \Gamma_{\tilde{f}^{\prime}{ }_{2} \tilde{f}_{1}}-2 \Gamma_{\tilde{f}^{\prime}{ }_{2} \tilde{f}_{2}}+2 \Gamma_{W H^{ \pm}}\right. \\
& +2 \Gamma_{W G}+\Gamma_{H^{ \pm}}+\Gamma_{G}-2 \Gamma_{W \tilde{f}_{1}^{\prime}}-2 \Gamma_{W \tilde{f}_{2}^{\prime}}-2 \Gamma_{W \tilde{f}_{1}}-2 \Gamma_{W \tilde{f}_{2}}+2 \Gamma_{H^{ \pm} G}-2 \Gamma_{G \tilde{f}_{1}^{\prime}} \\
& \left.-2 \Gamma_{G \tilde{f}_{2}^{\prime}}-2 \Gamma_{G \tilde{f}_{1}}-2 \Gamma_{G \tilde{f}_{2}}-2 \Gamma_{H^{ \pm} \tilde{f}_{1}^{\prime}}-2 \Gamma_{H^{ \pm} \tilde{f}_{2}^{\prime}}-2 \Gamma_{H^{ \pm} \tilde{f}_{1}}-2 \Gamma_{H^{ \pm} \tilde{f}_{2}}+2 \Gamma_{\tilde{f}_{1}^{\prime} \tilde{f}_{2}^{\prime}{ }_{2}}+2 \Gamma_{\tilde{f}_{1} \tilde{f}_{2}}\right] \text {. }
\end{aligned}
$$

Note here $G$ refers to the goldstone contribution which is the longitudinal component of the $W$ and so has mass equal to the $W$ boson mass. Here the following variables and couplings are used:

$$
N_{c}=\left\{\begin{array}{l}
3, \text { for } f^{\prime} \bar{f} \text { quarks, } \\
1, \text { for } f^{\prime} \bar{f} \text { charged leptons or neutrinos. }
\end{array}\right.
$$

There are several factors of (-1) depending on whether the neutralino or chargino have negative masses, and also there are factors of (-1) if the decay chargino $\rightarrow$ neutralino $f^{\prime} \bar{f}$ is being considered rather than neutralino $\rightarrow$ chargino $f^{\prime} \bar{f}$.

$$
\begin{gathered}
(-1)^{\theta_{i}}=\left\{\begin{array}{l}
1, \text { for } m_{\tilde{Z}_{i}}>0, \\
-1, \text { for } m_{\tilde{Z}_{i}}<0 .
\end{array}\right. \\
(-1)^{\theta_{j}}=\left\{\begin{array}{l}
1, \text { for } m_{\tilde{W}_{j}}>0, \\
-1, \text { for } m_{\tilde{W}_{j}}<0 .
\end{array}\right. \\
(-1)^{\theta_{c}}=\left\{\begin{array}{l}
1, \text { for neutralino decaying to chargino, } \\
-1, \text { for chargino decaying to neutralino. }
\end{array}\right.
\end{gathered}
$$

The following couplings are used:

$$
\begin{aligned}
& f_{u}=\frac{g m_{q^{\prime}}^{r u n}}{\sqrt{2} \sin \beta m_{W}}, \\
& f_{d}=\frac{g m_{q}^{r u n}}{\sqrt{2} \cos \beta m_{W}} .
\end{aligned}
$$

For $\tilde{W}_{1}^{+}$, i.e. the lightest chargino $(j=1)$, and where $i$ is the index of the neutralino:

$$
\begin{aligned}
& C_{\tilde{W} \tilde{Z} W}^{L}=g \sin \theta_{L} N_{2 i}+\frac{\cos \theta_{L} N_{3 i}}{\sqrt{2}}, \\
& C_{\tilde{W} \tilde{Z} W}^{R}=g \sin \theta_{R} N_{2 i}-\frac{\cos \theta_{R} N_{4 i}}{\sqrt{2}},
\end{aligned}
$$




$$
\begin{aligned}
& C_{\tilde{W}_{\tilde{Z} H_{+}}}^{L}=\left(g \sin \theta_{R} N_{4 i}+\frac{\cos \theta_{R}}{\sqrt{2}}\right)\left(g^{\prime} N_{1 i}+g N_{2 i}\right), \\
& C_{\tilde{W} \tilde{Z}_{H+}}^{R}=\left(g \sin \theta_{L} N_{3 i}-\frac{\left.\cos \theta_{L}\right)}{\sqrt{2}}\right)\left(g^{\prime} N_{1 i}+g N_{2 i}\right), \\
& \alpha_{\tilde{f}_{1} \tilde{W}_{1}}^{\tilde{W}_{1}}=-g \sin \theta_{R} \cos \theta_{q^{\prime}}+f_{u} \cos \theta_{R} \sin \theta_{q^{\prime}}, \\
& \beta_{\tilde{f}_{1} \tilde{W}_{1}}^{\tilde{W}_{1}}=-f_{d} \cos \theta_{L} \cos \theta_{q^{\prime}}(-1)^{\theta_{c}}, \\
& \alpha_{\tilde{f}_{1}}^{\tilde{W}_{1}}=-g \sin \theta_{L} \cos \theta_{q}+f_{d} \cos \theta_{L} \sin \theta_{q}(-1)^{\theta_{c}}, \\
& \beta_{\tilde{f}_{1}}^{\tilde{W}_{1}}=-f_{u} \cos \theta_{R} \cos \theta_{q}, \\
& \alpha_{\tilde{f}_{2}}^{\tilde{W}_{1}}=g \sin \theta_{R} \sin \theta_{q^{\prime}}(-1)^{\theta_{c}}-f_{u} \cos \theta_{R} \cos \theta_{q^{\prime}}, \\
& \beta_{\tilde{f}_{2}{ }_{2}}^{\tilde{W}_{1}}=-f_{d} \cos \theta_{L} \sin \theta_{q^{\prime}}(-1)^{\theta_{c}}, \\
& \alpha_{\tilde{f}_{2}}^{\tilde{W}_{1}}=-f_{d} \cos \theta_{L} \cos \theta_{q}+g \sin \theta_{L} \sin \theta_{q}, \\
& \beta_{\tilde{f}_{2}}^{\tilde{W}_{1}}=-f_{u} \cos \theta_{R} \sin \theta_{q} .
\end{aligned}
$$

Note that because of the conventions adopted, if the fermions considered are $\tau$ and $v_{\tau}$, so that the intermediates are $\tilde{\tau}_{1}$ and $\tilde{\tau}_{2}$, then the mixing angles in the formulae for this 3 body decay must be rotated so that one must take $\cos \theta_{\tau} \rightarrow \sin \theta_{\tau}$ and $\sin \theta_{\tau} \rightarrow-\cos \theta_{\tau}$ in the formulae listed for the $\tilde{Z}_{i} \rightarrow \tilde{W}_{j} f^{\prime} \bar{f}$ and for the reverse decay $\tilde{W}_{j} \rightarrow \tilde{Z}_{i} f^{\prime} \bar{f}$. Note that in this case where the fermions are $\tau$ and $v_{\tau}$, then $\theta_{q}$ would be the mixing angle for the $\tilde{\tau}$, whilst $\theta_{q^{\prime}}=0$ as the is no mixing for $\tilde{v_{\tau}}$.

For $\tilde{W}_{2}^{+}$, i.e. the heaviest chargino $(j=2)$, where $i$ is the index of the neutralino:

$$
\begin{aligned}
& C_{\tilde{W} \tilde{Z} W}^{L}=g \cos \theta_{L} N_{2 i}-\frac{\sin \theta_{L} N_{3 i}}{\sqrt{2}}, \\
& C_{\tilde{W} \tilde{Z} W}^{R}=g \cos \theta_{R} N_{2 i}+\frac{\sin \theta_{R} N_{4 i}}{\sqrt{2}}, \\
& C_{\tilde{W_{Z}} \tilde{L}_{+}}^{L}=\left[g \cos \theta_{R} N_{4 i}-\frac{\sin \theta_{R}}{\sqrt{2}}\right]\left(g^{\prime} N_{1 i}+g N_{2 i}\right), \\
& C_{\tilde{W} \tilde{Z} H_{+}}^{R}=\left[g \cos \theta_{L} N_{3 i}+\frac{\left.\sin \theta_{L}\right)}{\sqrt{2}}\right]\left(g^{\prime} N_{1 i}+g N_{2 i}\right), \\
& \alpha_{\tilde{f}^{\prime} 1}^{\tilde{W}_{2}}=-g \cos \theta_{R} \cos \theta_{q^{\prime}}-f_{u} \sin \theta_{R} \sin \theta_{q^{\prime}}, \\
& \beta_{\tilde{f}^{\prime} 1}^{\tilde{W}_{2}}=f_{d} \sin \theta_{L} \cos \theta_{q^{\prime}}(-1)^{\theta_{c}}, \\
& \alpha_{\tilde{f}_{1}}^{\tilde{W}_{2}}=-g \cos \theta_{L} \cos \theta_{q}-f_{d} \sin \theta_{L} \sin \theta_{q}(-1)^{\theta_{c}}, \\
& \beta_{\tilde{f}_{1}}^{\tilde{W}_{2}}=f_{u} \sin \theta_{R} \cos \theta_{q}, \\
& \alpha_{\tilde{f}_{2}^{\prime}}^{\tilde{W}_{2}}=g \cos \theta_{R} \sin \theta_{q^{\prime}}(-1)^{\theta_{c}}+f_{u} \sin \theta_{R} \cos \theta_{q^{\prime}}, \\
& \beta_{\tilde{f}_{\tilde{f}_{2}}}^{\tilde{W}_{2}}=f_{d} \sin \theta_{L} \sin \theta_{q^{\prime}}(-1)^{\theta_{c}}, \\
& \alpha_{\tilde{f}_{2}}^{\tilde{W}_{2}}=f_{d} \sin \theta_{L} \cos \theta_{q}+g \cos \theta_{L} \sin \theta_{q}, \\
& \beta_{\tilde{f}_{2}}^{\tilde{W}_{2}}=f_{u} \sin \theta_{R} \sin \theta_{q} .
\end{aligned}
$$


There are also the neutralino couplings to the $f^{\prime} \bar{f}$ pair and these depend upon whether they are quarks(q) or leptons(l) and whether they are " $u$-type" or " $d$-type" (i.e. third component of weak isospin being $+\frac{1}{2}$ or $-\frac{1}{2}$ respectively). For quarks:

$$
\begin{gathered}
A_{\tilde{Z}_{i}}^{u}=-\frac{g}{\sqrt{2}} N_{2 i}-\frac{g^{\prime}}{3 \sqrt{2}} N_{1 i}, \\
B_{\tilde{Z}_{i}}^{u}=-\frac{4 g^{\prime}}{3 \sqrt{2}} N_{1 i}, \\
\alpha_{\tilde{Z}_{i} \tilde{f}_{1}}^{u}=-A_{\tilde{Z}_{i}}^{u} \cos \theta_{q^{\prime}}(-1)^{\theta_{j}}(-1)^{\theta_{i}}(-1)^{\theta_{c}}-f_{u} N_{4 i} \sin \theta_{q^{\prime}}, \\
\beta_{\tilde{Z}_{i} \tilde{f}_{1}}^{u}=f_{u} N_{4 i} \cos \theta_{q^{\prime}}(-1)^{\theta_{c}}-B_{\tilde{Z}_{i}}^{u} \sin \theta_{q^{\prime}}, \\
\alpha_{\tilde{Z}_{i} \tilde{z}_{2}}^{u}=-f_{u} N_{4 i} \cos \theta_{q^{\prime}}(-1)^{\theta_{i}}+A_{\tilde{Z}_{i}}^{u} \sin \theta_{q^{\prime}}(-1)^{\theta_{c}}, \\
\beta_{\tilde{Z}_{i} \tilde{f}_{2}}^{u}=B_{\tilde{Z}_{i}}^{u} \cos \theta_{q^{\prime}}(-1)^{\theta_{j}}(-1)^{\theta_{i}}(-1)^{\theta_{c}}+f_{u} N_{4 i} \sin \theta_{q^{\prime}}, \\
A_{\tilde{Z}_{i}}^{d}=\frac{g}{\sqrt{2}} N_{2 i}-\frac{g^{\prime}}{3 \sqrt{2}} N_{1 i}, \\
B_{\tilde{Z}_{i}}^{d}=\frac{2 g^{\prime}}{3 \sqrt{2}} N_{1 i}, \\
\alpha_{\tilde{Z}_{i} \tilde{f}_{i}}^{d}=-A_{\tilde{Z}_{i}}^{d} \cos \theta_{q}(-1)^{\theta_{j}}(-1)^{\theta_{i}}(-1)^{\theta_{c}}-f_{d} N_{4 i} \sin \theta_{q}, \\
\beta_{\tilde{Z}_{\tilde{Z}_{1}} \tilde{f}_{1}}^{d}=f_{d} N_{3 i} \cos \theta_{q}(-1)^{\theta_{c}}-B_{\tilde{Z}_{i}}^{d} \sin \theta_{q}(-1)^{\theta_{i}}, \\
\alpha_{\tilde{Z}_{i} \tilde{f}_{2}}^{d}=f_{d} N_{3 i} \cos \theta_{q}(-1)^{\theta_{c}}-(-1)^{\theta_{c}}(-1)^{\theta_{i}} A_{\tilde{Z}_{i}}^{d} \sin \theta_{q}, \\
\beta_{\tilde{Z}_{i} \tilde{f}_{2}}^{d}=B_{\tilde{Z}_{i}}^{d} \cos \theta_{q}(-1)^{\theta_{j}}(-1)^{\theta_{i}}(-1)^{\theta_{c}}+f_{d} N_{4 i} \sin \theta_{q} .
\end{gathered}
$$

Again, remember for the case of $\tau$ and $v_{\tau}$ as $f$ and $f^{\prime}$ respectively then one must take $\cos \theta_{\tau} \rightarrow \sin \theta_{\tau}$ and $\sin \theta_{\tau} \rightarrow$ $-\cos \theta_{\tau}$ in the formulae listed for this decay mode.

For leptons instead the neutralino couplings are:

$$
\begin{gathered}
\left.A_{\tilde{Z}_{i}}^{u}=-\frac{g}{\sqrt{2}} N_{2 i}\right)+\frac{g^{\prime}}{\sqrt{2}} N_{1 i}, \\
B_{\tilde{Z}_{i}}^{u}=0, \\
A_{\tilde{Z}_{i}}^{d}=\frac{g}{\sqrt{2}} N_{2 i}+\frac{g^{\prime}}{\sqrt{2}} N_{1 i}, \\
B_{\tilde{Z}_{i}}^{d}=\sqrt{2} g^{\prime} N_{1 i} .
\end{gathered}
$$

The $\alpha$ and $\beta$ couplings are as before except $\alpha_{\tilde{Z}_{i} \tilde{f}_{2}}^{u}=0$ and $\beta_{\tilde{Z}_{i} \tilde{f}_{2}}^{u}=0$ as there are no RH sneutrinos. Note in SOFTSUSY we use the same function for a neutralino decaying to a chargino as a chargino decaying to a neutralino, in general the changes required are $m_{\tilde{Z}_{i}} \leftrightarrow m_{\tilde{W}_{j}}, m_{f^{\prime}} \leftrightarrow m_{f}$ and $m_{\tilde{f}^{\prime}} \leftrightarrow m_{\tilde{f}}$, in some places there are further effects on the integrals or couplings, where this occurs it's listed in the following formulae.

Contribution by contribution, the couplings are:

$\Gamma_{W}$

The upper limit of integration here is:

$$
\mathcal{T}=\frac{1}{2\left|m_{\tilde{Z}_{i}}\right|}\left(m_{\tilde{Z}_{i}}^{2}+m_{\tilde{W}_{j}}^{2}-m_{f}^{2}-m_{f^{\prime}}^{2}-2 m_{f} m_{f^{\prime}}\right),
$$

We also use $s$ and $\lambda$ given by:

$$
s=m_{\tilde{Z}_{i}}^{2}+m_{\tilde{Z}_{j}}^{2}-2\left|m_{\tilde{Z}_{i}}\right| E
$$




$$
\lambda=\sqrt{\left(s-\left(m_{f}+m_{f^{\prime}}\right)^{2}\right)\left(s-\left(m_{f}-m_{f^{\prime}}\right)^{2}\right)} .
$$

The necessary integrals are:

$$
\begin{aligned}
& I_{W}^{1}=2\left|m_{\tilde{Z}_{i}}\right| \int_{\left|m_{\tilde{w}_{j}}\right|}^{\mathcal{T}} d E \frac{2\left|m_{\tilde{Z}_{i}}\right|}{s} \lambda \sqrt{E^{2}-m_{\tilde{W}_{j}}^{2}}\left[-2 s^{4}+\left(\left(m_{\tilde{Z}_{i}}^{2}+m_{\tilde{W}_{j}}^{2}+m_{f}^{2}+m_{f^{\prime}}^{2}\right)\right) s^{3}\right. \\
&+\left(\left(m_{\tilde{Z}_{i}}^{2}-m_{\tilde{W}_{j}}^{2}\right)^{2}+\left(m_{f}^{2}-m_{f^{\prime}}^{2}\right)^{2}-2\left(m_{\tilde{Z}_{i}}^{2}+m_{\tilde{W}_{j}}^{2}\right)^{2}\left(m_{f}^{2}+m_{f^{\prime}}^{2}\right) s^{2}\right. \\
&+\left(\left(m_{\tilde{Z}_{i}}^{2}+m_{\tilde{W}_{j}}^{2}\right)\left(m_{f}^{2}-m_{f^{\prime}}^{2}\right)^{2}+\left(m_{f}^{2}+m_{f^{\prime}}^{2}\right)\left(m_{\tilde{Z}_{i}}^{2}-m_{\tilde{W}_{j}}^{2}\right)^{2}\right) s \\
&\left.-2\left(m_{\tilde{Z}_{i}}^{2}-m_{\tilde{W}_{j}}^{2}\right)^{2}\left(m_{f}^{2}+m_{f^{\prime}}^{2}\right)^{2}\right] \frac{1}{3 s^{2}} \frac{1}{\left(s-m_{W}^{2}\right)^{2}}, \\
& I_{W}^{2}=2\left|m_{\tilde{Z}_{i}}\right| \int_{\left|m_{\tilde{W}_{j}}\right|}^{\mathcal{T}} d E \frac{2\left|m_{\tilde{Z}_{i}}\right|}{s} \lambda \sqrt{E^{2}-m_{\tilde{W}_{j}}^{2}}\left(s-m_{f}^{2}-m_{f^{\prime}}^{2}\right) \frac{1}{\left(s-m_{W}^{2}\right)^{2}} .
\end{aligned}
$$

Then

$$
\Gamma_{W}=-8 C_{\tilde{W} \tilde{Z} W}^{L} C_{\tilde{W} \tilde{Z} W}^{R} \frac{g^{2}}{2}\left|m_{\tilde{Z}_{i}}\right|\left|m_{\tilde{W}_{j}}\right| I_{W}^{2} \mid(-1)^{\theta_{i}}(-1)^{\theta_{j}}+2\left(C_{\tilde{W} \tilde{Z} W}^{L}{ }^{2}+C_{\tilde{W} \tilde{Z} W}^{R}{ }^{2}\right) \frac{g^{2}}{2} I_{W}^{1} .
$$

$\underline{\Gamma_{H^{ \pm}}}$

$$
\begin{aligned}
\omega_{H^{+} \tilde{W}+\tilde{Z}}^{L} & =C_{\tilde{W} \tilde{Z} H_{+}}^{L} \cos \beta, \\
\omega_{H^{+} \tilde{W}+\tilde{Z}}^{R} & =C_{\tilde{W} \tilde{Z} H_{+}}^{R} \sin \beta, \\
C_{H^{+} f f^{\prime}}^{u} & =f_{u} \cos \beta, \\
C_{H^{+} f f^{\prime}}^{d} & =f_{d} \sin \beta,
\end{aligned}
$$

The relevant combinations of these couplings for this contribution are:

$$
\begin{aligned}
& \mathcal{V}_{H+}^{(1)}=\omega_{H^{+} \tilde{W}+\tilde{Z}}^{L}{ }^{2}+\omega_{H^{+} \tilde{W}+\tilde{Z}}^{R}, \\
& \mathcal{V}_{H+}^{(2)}=\omega_{H^{+} \tilde{W}+\tilde{Z}}^{L} \omega_{H^{+} \tilde{W}+\tilde{Z}}^{R}(-1)^{\theta_{i}}, \\
& \mathcal{V}_{H+}^{(3)}=C_{H^{+} f f^{\prime}}^{u}{ }^{2}+C_{H^{+} f f^{\prime}}^{d}{ }^{2} \text {, } \\
& \mathcal{V}_{H+}^{(4)}=C_{H^{+} f f^{\prime}}^{u} C_{H^{+} f f^{\prime}}^{d} .
\end{aligned}
$$

The integrals are:

$$
\begin{gathered}
I_{H^{ \pm}}^{1}=2\left|m_{\tilde{Z}_{i}}\right| \int_{\left|m_{\tilde{W}_{j}}\right|}^{\mathcal{T}} d E \frac{2\left|m_{\tilde{Z}_{i}}\right|}{s} \sqrt{\left(s-\left(m_{f^{\prime}}+m_{f}\right)^{2}\right)\left(s-\left(m_{f^{\prime}}-m_{f}\right)^{2}\right)} \sqrt{E^{2}-m_{\tilde{W}_{j}}^{2}} \frac{1}{\left(s-m_{H^{ \pm}}^{2}\right)^{2}}, \\
I_{H^{ \pm}}^{2}=2\left|m_{\tilde{Z}_{i}}\right| \int_{\left|m_{\tilde{W}_{j}}\right|}^{\mathcal{T}} d E\left[\frac{2\left|m_{\tilde{Z}_{i}}\right|}{s} \sqrt{\left(s-\left(m_{f^{\prime}}+m_{f}\right)^{2}\right)\left(s-\left(m_{f^{\prime}}-m_{f}\right)^{2}\right)} \sqrt{E^{2}-m_{\tilde{W}_{j}}^{2}}\right. \\
\left.\times\left(s-m_{f}^{2}-m_{f^{\prime}}^{2}\right) \frac{1}{\left(s-m_{H^{ \pm}}^{2}\right)^{2}}\right], \\
I_{H^{ \pm}}^{3}=2\left|m_{\tilde{Z}_{i}}\right| \int_{\left|m_{\tilde{j}_{j}}\right|}^{\mathcal{T}} d E\left[\frac{2\left|m_{\tilde{Z}_{i}}\right|}{s} \sqrt{\left(s-\left(m_{f^{\prime}}+m_{f}\right)^{2}\right)\left(s-\left(m_{f^{\prime}}-m_{f}\right)^{2}\right)} \sqrt{E^{2}-m_{\tilde{W}_{j}}^{2}}\right. \\
\left.\times 2\left|m_{\tilde{Z}_{i}}\right| E \frac{1}{\left(s-m_{H^{ \pm}}^{2}\right)^{2}}\right],
\end{gathered}
$$




$$
\begin{aligned}
I_{H^{ \pm}}^{4}=2\left|m_{\tilde{Z}_{i}}\right| \int_{\left|m_{\tilde{W}_{j}}\right|}^{\mathcal{T}} d E\left[\frac{2\left|m_{\tilde{Z}_{i}}\right|}{s} \sqrt{\left(s-\left(m_{f^{\prime}}+m_{f}\right)^{2}\right)\left(s-\left(m_{f^{\prime}}-m_{f}\right)^{2}\right)} \sqrt{E^{2}-m_{\tilde{W}_{j}}^{2}}\right. \\
\left.\times 2\left|m_{\tilde{Z}_{i}}\right| E\left(s-m_{f}^{2}-m_{f^{\prime}}^{2}\right) \frac{1}{\left(s-m_{H^{ \pm}}^{2}\right)^{2}}\right]
\end{aligned}
$$

The overall contribution is then:

$$
\begin{aligned}
\Gamma_{H^{ \pm}}= & \mathcal{V}_{H+}^{(1)} \mathcal{V}_{H+}^{(3)} I_{H^{ \pm}}^{4}-4 \mathcal{V}_{H+}^{(1)} \mathcal{V}_{H+}^{(4)} I_{H^{ \pm}}^{3} m_{f^{\prime}} m_{f^{\prime}}+4 \mathcal{V}_{H+}^{(2)} \mathcal{V}_{H+}^{(3)} I_{H^{ \pm}}^{2}\left|m_{\tilde{Z}_{i}}\right|\left|m_{\tilde{W}_{j}}\right|(-1)^{\theta_{j}} \\
& -16 \mathcal{V}_{H+}^{(2)} \mathcal{V}_{H+}^{(4)} I_{H^{ \pm}}^{1} m_{f} m_{f^{\prime}}\left|m_{\tilde{Z}_{i}}\right|\left|m_{\tilde{W}_{j}}\right|(-1)^{\theta_{j}} .
\end{aligned}
$$

$\frac{\Gamma_{G}}{N_{0}}$

Note here $G$ refers to the goldstone contribution which is the longitudinal component of the W and so has mass equal to the $W$ boson mass. The couplings used are:

$$
\begin{gathered}
\omega_{G \tilde{W} \tilde{Z}}^{L}=C_{\tilde{W} \tilde{Z} H+}^{L} \sin \beta, \\
\omega_{G \tilde{W} \tilde{Z}}^{R}=-C_{\tilde{W} \tilde{Z} H+}^{R} \cos \beta, \\
C_{G f f^{\prime}}^{u}=f_{u} \sin \beta, \\
C_{G f f^{\prime}}^{d}=-f_{d} \cos \beta, \\
\mathcal{V}_{G}^{(1)}=\omega_{G \tilde{W} \tilde{Z}}^{L}+\omega_{G \tilde{W} \tilde{Z}}^{R}, \\
\mathcal{V}_{G}^{(2)}=\omega_{G \tilde{W} \tilde{Z}}^{L} \omega_{G \tilde{W} \tilde{Z}}^{R}(-1)^{\theta_{i}}, \\
\mathcal{V}_{G}^{(3)}=C_{G f f^{\prime}}^{u}+C_{G f f^{\prime}}^{d}, \\
\mathcal{V}_{G}^{(4)}=C_{G f f^{\prime}}^{u} C_{G f f^{\prime}}^{d} .
\end{gathered}
$$

The integrals here $I_{G}^{1}$ etc are exactly the same as those for $H^{ \pm}$but with the change $m_{H^{ \pm}} \rightarrow m_{W}$.

$$
\begin{aligned}
\Gamma_{G}= & \mathcal{V}_{G}^{(1)} \mathcal{V}_{G}^{(3)} I_{G}^{4}-4 \mathcal{V}_{G}^{(1)} \mathcal{V}_{G}^{(4)} I_{G}^{3} m_{f} m_{f^{\prime}}+4 \mathcal{V}_{G}^{(2)} \mathcal{V}_{G}^{(3)} I_{G}^{2}\left|m_{\tilde{Z}_{i}} \| m_{\tilde{W}_{j}}\right|(-1)^{\theta_{j}} \\
& -16 \mathcal{V}_{G}^{(2)} \mathcal{V}_{G}^{(4)} I_{G}^{1} m_{f} m_{f^{\prime}}\left|m_{\tilde{Z}_{i}} \| m_{\tilde{W}_{j}}\right|(-1)^{\theta_{j}} .
\end{aligned}
$$

$\underline{\Gamma_{\tilde{f}^{\prime}}}$

$$
\begin{aligned}
& \mathcal{V}_{\tilde{f}^{\prime} 1}^{(1)}=\alpha_{\tilde{Z}_{i} \tilde{f}_{1}}^{u}+\beta_{\tilde{Z}_{i} \tilde{f}_{1}}^{u}, \\
& \mathcal{V}_{\tilde{f}^{\prime}{ }_{1}}^{(2)}=-\alpha_{\tilde{Z}_{i} \tilde{f}_{1}}^{u}{ }^{2} \beta_{\tilde{Z}_{i} \tilde{f}_{1}}^{u}{ }^{2}(-1)^{\theta_{i}}, \\
& \mathcal{V}_{\tilde{f}^{\prime}{ }_{1}}^{(3)}=\alpha_{\tilde{f}^{\prime}{ }_{1}}^{\tilde{W}^{2}}+\beta_{\tilde{f}^{\prime}{ }_{1}}^{\tilde{W}^{2}}, \\
& \mathcal{V}_{\tilde{f}^{\prime} 1}^{(4)}=-\alpha_{\tilde{f}^{\prime} 1}^{\tilde{W}} \beta_{\tilde{f}^{\prime} 1}^{\tilde{W}} .
\end{aligned}
$$

Now the integrals $I_{\tilde{f}^{\prime},}^{1,2,4}$ are exactly as the $I_{H^{ \pm}}^{1,2,3,4}$ integrals in G.237) to G.240 but with lower limit $m_{f^{\prime}}$, upper limit of integration $E_{\text {upper }}=\frac{1}{2\left|m_{\tilde{z}_{1}}\right|}\left(m_{\tilde{Z}_{i}}^{2}+m_{f^{\prime}}^{2}-m_{f}^{2}-m_{\tilde{Z}_{j}}^{2}-2 m_{f}\left|m_{\tilde{W}_{j}}\right|\right)$ and the replacements $m_{H^{ \pm}} \rightarrow m_{\tilde{f}^{\prime} 1},\left|m_{\tilde{W}_{j}}\right| \rightarrow m_{f^{\prime}}$ and $m_{f^{\prime}} \rightarrow\left|m_{\tilde{W}_{j}}\right|$ Then:

$$
\begin{aligned}
& \Gamma_{\tilde{f}^{\prime} 1}=\mathcal{V}_{\tilde{f}^{\prime} 1}^{(1)} \mathcal{V}_{\tilde{f}^{\prime} I_{1}}^{(3)} I_{\tilde{f}^{\prime} 1}^{4}-4 \mathcal{V}_{\tilde{f}^{\prime} 1}^{(1)} \mathcal{V}_{\tilde{f}^{\prime} 1}^{(4)} m_{f}\left|m_{\tilde{W}_{j}}\right| I_{\tilde{f}^{\prime} 1}^{3}+4 \mathcal{V}_{\tilde{f}^{\prime} 1}^{(2)} \mathcal{V}_{\tilde{f}^{\prime}, 1}^{(3)}\left|m_{\tilde{Z}_{i}}\right| m_{f^{\prime}} I_{\tilde{f}^{\prime} 1}^{2} \\
& -16 \mathcal{V}_{\tilde{f}^{\prime} 1}^{(2)} \mathcal{V}_{\tilde{f}^{\prime} 1}^{(4)}\left|m_{\tilde{Z}_{i}} \| m_{\tilde{W}_{j}}\right| m_{f^{\prime}} m_{f} I_{\tilde{f}^{\prime} 1}^{1} \text {. }
\end{aligned}
$$

$\underline{\Gamma_{\tilde{f}_{2}^{\prime}}}$ 
Everything for $\Gamma_{\tilde{f}^{\prime}{ }_{2}}$ is exactly as for $\Gamma_{\tilde{f}_{1}^{\prime}}$ but with the change $m_{\tilde{f}_{1}^{\prime}} \rightarrow m_{\tilde{f}^{\prime}{ }_{2}}$ and the coupling combinations:

$$
\begin{aligned}
& \mathcal{V}_{\tilde{f}^{\prime}{ }_{2}}^{(1)}=\alpha_{\tilde{Z}_{i} \tilde{f}_{2}}^{u}{ }^{2}+\beta_{\tilde{Z}_{i} \tilde{f}_{2}}^{u}{ }^{2}, \\
& \mathcal{V}_{\tilde{f}^{\prime}{ }_{2}}^{(2)}=\alpha_{\tilde{Z}_{i} \tilde{f}_{2}}^{u} \beta_{\tilde{Z}_{i} \tilde{f}_{2}}^{2}{ }^{2}, \\
& \mathcal{V}_{\tilde{f}^{\prime}{ }_{2}}^{(3)}=\alpha_{\tilde{f}^{\prime}{ }_{2}}^{\tilde{W}^{2}}+\beta_{\tilde{f}^{\prime}{ }_{2}}^{\tilde{W}^{2}} \text {, } \\
& \mathcal{V}_{\tilde{f}^{\prime}{ }_{2}}^{(4)}=\alpha_{\tilde{f}_{2}^{\prime}{ }_{2}}^{\tilde{W}} \beta_{\tilde{f}^{\prime}{ }_{2}}^{\tilde{W}} .
\end{aligned}
$$

The contribution is then:

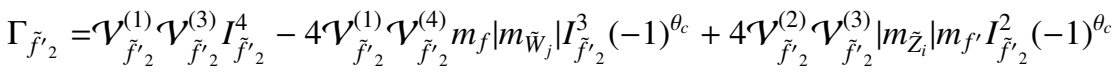

$$
\begin{aligned}
& -16 \mathcal{V}_{\tilde{f}_{2}^{\prime}}^{(2)} \mathcal{V}_{\tilde{f}_{2}^{\prime}}^{(4)}\left|m_{\tilde{Z}_{i}} \| m_{\tilde{W}_{j}}\right| m_{f^{\prime}} m_{f} I_{\tilde{f}_{2}^{\prime}} \text {. }
\end{aligned}
$$

$\Gamma_{\tilde{f}_{1}}$

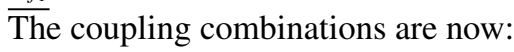

$$
\begin{aligned}
& \mathcal{V}_{\tilde{f}_{1}}^{(1)}=\alpha_{\tilde{Z}_{i} \tilde{f}_{1}}^{d}{ }^{2}+\beta_{\tilde{Z}_{i} \tilde{f}_{1}}^{d}{ }^{2}, \\
& \mathcal{V}_{\tilde{f}_{1}}^{(2)}=-(-1)^{\theta_{i}} \alpha_{\tilde{Z}_{i} \tilde{f}_{1}}^{d} \beta_{\tilde{Z}_{i} \tilde{f}_{1}}^{d}, \\
& \mathcal{V}_{\tilde{f}_{1}}^{(3)}=\alpha_{\tilde{f}_{1}}^{\tilde{W}^{2}}+\beta_{\tilde{f}_{1}}^{\tilde{W}^{2}}, \\
& \mathcal{V}_{\tilde{f}_{1}}^{(4)}=-\alpha_{\tilde{f}_{1}}^{\tilde{W}} \beta_{\tilde{f}_{1}}^{\tilde{W}_{1}}(-1)^{\theta_{j}} .
\end{aligned}
$$

The integrals are are exactly as the $I_{H^{ \pm}}^{1,2,3,4}$ integrals in (G.237) to G.240) but the lower limit is now $m_{f}$, the upper limit is $E_{\text {upper } 2}=\frac{1}{2\left|m_{\tilde{Z}_{i}}\right|}\left[m_{\tilde{Z}_{i}}^{2}+m_{f}^{2}-m_{\tilde{W}_{j}}^{2}-m_{f^{\prime}}^{2}-2 m_{f^{\prime}}\left|m_{\tilde{W}_{j}}\right|\right]$ and in general relative to the $H^{ \pm}$integrals we must make the changes $m_{H^{ \pm}} \rightarrow m_{\tilde{f}_{1}},\left|m_{\tilde{W}_{j}}\right| \rightarrow m_{f}$ and $m_{f} \rightarrow\left|m_{\tilde{W}_{j}}\right|$.

$$
\begin{aligned}
\Gamma_{\tilde{f}_{1}}= & \mathcal{V}_{\tilde{f}_{1}}^{(1)} \mathcal{V}_{\tilde{f}_{1}}^{(3)} I_{\tilde{f}_{1}}^{4}-4 \mathcal{V}_{\tilde{f}_{1}}^{(1)} \mathcal{V}_{\tilde{f}_{1}}^{(4)} m_{f^{\prime}}\left|m_{\tilde{W}_{j}}\right| I_{\tilde{f}_{1}}^{3}(-1)^{\theta_{c}}(-1)^{\theta_{j}}+4 \mathcal{V}_{\tilde{f}_{1}}^{(2)} \mathcal{V}_{\tilde{f}_{1}}^{(3)}\left|m_{\tilde{Z}_{i}}\right| m_{f} I_{\tilde{f}_{1}}^{2}(-1)^{\theta_{c}} \\
& -16 \mathcal{V}_{\tilde{f}_{1}}^{(2)} \mathcal{V}_{\tilde{f}_{1}}^{(4)}\left|m_{\tilde{Z}_{i}}\right|\left|m_{\tilde{W}_{j}}\right| m_{f} m_{f^{\prime}}(-1)^{\theta_{j}} I_{\tilde{f}_{1}}^{1} .
\end{aligned}
$$

\section{$\underline{\Gamma_{\tilde{f}}}$}

$\overline{\text { Nominally }} \Gamma_{\tilde{f}_{2}}$ has the same expression as $\Gamma_{\tilde{f}_{1}}$ with the replacement $\tilde{f}_{1} \rightarrow \tilde{f}_{2}$, however differences in expressions for couplings mean we have slight differences; the coupling combinations are now:

$$
\begin{aligned}
& \mathcal{V}_{\tilde{f}_{2}}^{(1)}=\alpha_{\tilde{Z}_{i} \tilde{f}_{2}}^{d}+\beta_{\tilde{Z}_{i} \tilde{f}_{2}}^{d}, \\
& \mathcal{V}_{\tilde{f}_{2}}^{(2)}=\alpha_{\tilde{Z}_{i} \tilde{f}_{2}}^{d} \beta_{\tilde{Z}_{i} \tilde{f}_{2}}^{d}, \\
& \mathcal{V}_{\tilde{f}_{2}}^{(3)}=\alpha_{\tilde{f}_{2}}^{\tilde{W}^{2}}+\beta_{\tilde{f}_{2}}^{\tilde{W}^{2}}, \\
& \boldsymbol{V}_{\tilde{f_{2}}}^{(4)}=\alpha_{\tilde{f_{2}}}^{\tilde{W}_{\tilde{f}}} \beta_{\tilde{f}_{2}}^{\tilde{W}_{2}}(-1)^{\theta_{c}} .
\end{aligned}
$$

Therefore the contribution is given by:

$$
\begin{aligned}
\Gamma_{\tilde{f}_{2}}= & \mathcal{V}_{\tilde{f}_{2}}^{(1)} \mathcal{V}_{\tilde{f}_{2}}^{(3)} I_{\tilde{f}_{2}}^{4}-4 \mathcal{V}_{\tilde{f}_{2}}^{(1)} \mathcal{V}_{\tilde{f}_{2}}^{(4)} m_{f^{\prime}}\left|m_{\tilde{W}_{j}}\right| I_{\tilde{f}_{2}}^{3}(-1)^{\theta_{c}}+4 \mathcal{V}_{\tilde{f}_{2}}^{(2)} \mathcal{V}_{\tilde{f}_{2}}^{(3)}\left|m_{\tilde{Z}_{i}}\right| m_{f} I_{\tilde{f}_{2}}^{2}(-1)^{\theta_{c}} \\
& -16 \mathcal{V}_{\tilde{f}_{2}}^{(2)} \mathcal{V}_{\tilde{f}_{2}}^{(4)}\left|m_{\tilde{Z}_{i}}\right|\left|m_{\tilde{W}_{j}}\right| m_{f} m_{f^{\prime}} I_{\tilde{f}_{2}}^{1} .
\end{aligned}
$$

$\underline{\Gamma_{\tilde{f}^{\prime}{ }_{1} \tilde{f}_{1}}}$ 
Here the coupling combinations differ significantly depending on which way around the decay is being considered, i.e. neutralino to chargino or chargino to neutralino. The following are fixed regardless of this:

$$
\begin{aligned}
& \mathcal{V}_{\tilde{f}_{1}, \tilde{f}_{1}}^{(1)}=\frac{1}{2}\left[\alpha_{\tilde{Z}_{i} \tilde{f}_{1}}^{u} \beta_{\tilde{Z}_{i} \tilde{f}_{1}}^{d} \beta_{\tilde{f}_{1}^{\prime} 1}^{\tilde{W}} \alpha_{\tilde{f}_{1}}^{\tilde{W}}(-1)^{\theta_{i}}+\beta_{\tilde{Z}_{i} \tilde{f}_{1}}^{u} \alpha_{\tilde{Z}_{i} \tilde{f}_{1}}^{d} \alpha_{\tilde{f}_{1}^{\prime}}^{\tilde{W}} \beta_{\tilde{f}_{1}}^{\tilde{W}}\right](-1)^{\theta_{j}}, \\
& \mathcal{V}_{\tilde{f}_{1}, \tilde{f}_{1}}^{(2)}=-\left|m_{\tilde{Z}_{i}}\right|\left|m_{\tilde{W}_{j}}\right|\left[(-1)^{\theta_{i}} \alpha_{\tilde{Z}_{i} \tilde{f}_{1}}^{u} \alpha_{\tilde{Z}_{i} \tilde{f}_{1}}^{d} \alpha_{\tilde{f}_{1}^{\prime}, 1}^{\tilde{W}} \alpha_{\tilde{f}_{1}}^{\tilde{W}}+\beta_{\tilde{Z}_{i} \tilde{f}_{1}}^{u} \beta_{\tilde{Z}_{i} \tilde{f}_{1}}^{d} \beta_{\tilde{f}^{\prime}, 1}^{\tilde{W}} \beta_{\tilde{f}_{1}}^{\tilde{W}}\right](-1)^{\theta_{j}}, \\
& \mathcal{V}_{\tilde{f}^{\prime}, \tilde{f}_{1}}^{(3)}=-m_{f} m_{f^{\prime}}\left[\beta_{\tilde{z}_{i} \tilde{f}_{1}}^{u} \alpha_{\tilde{z}_{i} \tilde{f}_{1}}^{d} \beta_{\tilde{f}^{\prime}{ }_{1}}^{\tilde{W}} \alpha_{\tilde{f}_{1}}^{\tilde{W}}+(-1)^{\theta_{i}} \alpha_{\tilde{Z}_{i} \tilde{f}_{1}}^{u} \beta_{\tilde{z}_{i} \tilde{f}_{1}}^{d} \alpha_{\tilde{f}_{1}}^{\tilde{W}} \beta_{\tilde{f}_{1}}^{\tilde{W}}\right](-1)^{\theta_{j}}(-1)^{\theta_{c}}, \\
& \mathcal{V}_{\tilde{f}^{\prime}, \tilde{f}_{1}}^{(8)}=-2\left|m_{\tilde{Z}_{i}} \| m_{\tilde{W}_{j}}\right| m_{f} m_{f^{\prime}}\left[\beta_{\tilde{Z}_{i} \tilde{f}_{1}}^{u} \beta_{\tilde{Z}_{i} \tilde{f}_{1}}^{d} \alpha_{\tilde{f}^{\prime}}^{\tilde{W}} \alpha_{\tilde{f}_{1}}^{\tilde{W}}+(-1)^{\theta_{i}} \alpha_{\tilde{Z}_{i} \tilde{f}_{1}}^{u} \alpha_{\tilde{Z}_{i} \tilde{f}_{1}}^{d} \beta_{\tilde{f}^{\prime}}^{\tilde{W}} \beta_{\tilde{f}_{1}}^{\tilde{W}}\right](-1)^{\theta_{j}}(-1)^{\theta_{c}} .
\end{aligned}
$$

Meanwhile, if the decay is neutralino to chargino:

$$
\begin{aligned}
& \mathcal{V}_{\tilde{f}_{1}, \tilde{f}_{1}}^{(4)}=-\left|m_{\tilde{Z}_{i}}\right| m_{f}\left[(-1)^{\theta_{i}} \alpha_{\tilde{Z}_{i} \tilde{f}_{1}}^{u} \alpha_{\tilde{Z}_{i} \tilde{f}_{1}}^{d} \beta_{\tilde{f}^{\prime}}^{\tilde{W}} \alpha_{\tilde{f}_{1}}^{\tilde{W}}+\beta_{\tilde{Z}_{i} \tilde{f}_{1}}^{u} \beta_{\tilde{Z}_{i} \tilde{f}_{1}}^{d} \alpha_{\tilde{f}^{\prime}}^{\tilde{W}} \beta_{\tilde{f}_{1}}^{\tilde{W}}\right], \\
& \mathcal{V}_{\tilde{f}^{\prime}, 1 \tilde{f}_{1}}^{(5)}=m_{f^{\prime}} \mid m_{\tilde{W}_{j}}\left[\beta_{\tilde{Z}_{i} \tilde{f}_{1}}^{u} \alpha_{\tilde{Z}_{i} \tilde{f}_{1}}^{d} \alpha_{\tilde{f}^{\prime} 1}^{\tilde{W}} \alpha_{\tilde{f}_{1}}^{\tilde{W}}+(-1)^{\theta_{i}} \alpha_{\tilde{Z}_{i} \tilde{f}_{1}}^{u} \beta_{\tilde{z}_{i} \tilde{f}_{1}}^{d} \beta_{\tilde{f}^{\prime} 1}^{\tilde{W}} \beta_{\tilde{f}_{1}}^{\tilde{W}}\right](-1)^{\theta_{j}}, \\
& \mathcal{V}_{\tilde{f}^{\prime}, \tilde{f}_{1}}^{(6)}=-\left|m_{\tilde{Z}_{i}}\right| m_{f^{\prime}}\left[\beta_{\tilde{Z}_{i} \tilde{f}_{1}}^{u} \beta_{\tilde{Z}_{i} \tilde{f}_{1}}^{d} \beta_{\tilde{f}^{\prime}}^{\tilde{W}} \alpha_{\tilde{f}_{1}}^{\tilde{W}}+(-1)^{\theta_{i}} \alpha_{\tilde{Z}_{i} \tilde{f}_{1}}^{u} \alpha_{\tilde{Z}_{i} \tilde{f}_{1}}^{d} \alpha_{\tilde{f}_{1}^{\prime}}^{\tilde{W}} \beta_{\tilde{f}_{1}}^{\tilde{W}}\right] \text {, } \\
& \mathcal{V}_{\tilde{f}_{1}^{\prime}, \tilde{f}_{1}}^{(7)}=\left|m_{\tilde{W}_{j}}\right| m_{f}\left[(-1)^{\theta_{i}} \alpha_{\tilde{Z}_{i} \tilde{f}_{1}}^{u} \tilde{z}_{\tilde{Z}_{i} \tilde{f}_{1}}^{d} \alpha_{\tilde{f}_{1}^{\prime} 1}^{\tilde{W}} \alpha_{\tilde{f}_{1}}^{\tilde{W}}+\beta_{\tilde{Z}_{i} \tilde{f}_{1}}^{u} \alpha_{\tilde{Z}_{i} \tilde{f}_{1}}^{d} \beta_{\tilde{f}_{1}^{\prime} 1}^{\tilde{W}} \beta_{\tilde{f}_{1}}^{\tilde{W}}\right](-1)^{\theta_{j}} \text {. }
\end{aligned}
$$

Whilst if the decay is instead chargino to neutralino:

$$
\begin{aligned}
& \mathcal{V}_{\tilde{f}^{\prime}, \tilde{f}_{1}}^{(4)}=\left|m_{\tilde{Z}_{i}}\right| m_{f}\left[\alpha_{\tilde{f}_{1}{ }_{1}}^{\tilde{W}} \alpha_{\tilde{f}_{1}}^{\tilde{W}} \beta_{\tilde{Z}_{i} \tilde{f}_{1}}^{u} \alpha_{\tilde{Z}_{i} \tilde{f}_{1}}^{d}+\beta_{\tilde{f}_{1}^{\prime}}^{\tilde{W}} \beta_{\tilde{f}_{1}}^{\tilde{W}} \alpha_{\tilde{Z}_{i} \tilde{f}_{1}}^{u} \beta_{\tilde{Z}_{i} \tilde{f}_{1}}^{d}\right](-1)^{\theta_{j}}, \\
& \mathcal{V}_{\tilde{f}^{\prime} 1 \tilde{f}_{1}}^{(5)}=-\left|m_{\tilde{W}_{j}}\right| m_{f^{\prime}}\left[\alpha_{\tilde{f}_{1}}^{\tilde{W}} \beta_{\tilde{f}^{\prime} 1}^{\tilde{W}} \alpha_{\tilde{Z}_{i} \tilde{f}_{1}}^{u} \alpha_{\tilde{Z}_{i} \tilde{f}_{1}}^{d}+\alpha_{\tilde{f}_{1}^{\prime}}^{\tilde{W}} \beta_{\tilde{f}_{1}}^{\tilde{W}} \beta_{\tilde{z}_{i} \tilde{f}_{1}}^{u} \beta_{\tilde{Z}_{i} \tilde{f}_{1}}^{d}\right](-1)^{\theta_{j}}, \\
& \mathcal{V}_{\tilde{f}_{1}^{\prime} \tilde{f}_{1}}^{(6)}=-\left|m_{\tilde{Z}_{i}}\right| m_{f^{\prime}}\left[\beta_{\tilde{f}_{1}^{\prime} 1}^{\tilde{W}} \beta_{\tilde{f}_{1}}^{\tilde{W}} \beta_{\tilde{Z}_{i} \tilde{f}_{1}}^{u} \alpha_{\tilde{Z}_{i} \tilde{f}_{1}}^{d}+\alpha_{\tilde{f}_{1}^{\prime} 1}^{\tilde{W}} \alpha_{\tilde{f}_{1}}^{\tilde{W}_{1}} \alpha_{\tilde{Z}_{i} \tilde{f}_{1}}^{u} \beta_{\tilde{z}_{i} \tilde{f}_{1}}^{d}\right](-1)^{\theta_{j}}, \\
& \mathcal{V}_{\tilde{f}^{\prime}, \tilde{f}_{1}}^{(7)}=\left|m_{\tilde{W}_{j}}\right| m_{f}\left[\alpha_{\tilde{f}_{1}^{\prime} 1}^{\tilde{W}} \beta_{\tilde{f}_{1}}^{\tilde{W}} \alpha_{\tilde{Z}_{i} \tilde{f}_{1}}^{u} \alpha_{\tilde{Z}_{i} \tilde{f}_{1}}^{d}+\beta_{\tilde{f}_{1}^{\prime} 1}^{\tilde{W}} \alpha_{\tilde{f}_{1}}^{\tilde{W}} \beta_{\tilde{Z}_{i} \tilde{f}_{1}}^{u} \beta_{\tilde{Z}_{i} \tilde{f}_{1}}^{d}\right](-1)^{\theta_{j}} \text {. }
\end{aligned}
$$

We also need the following integrals, note $s=m_{\tilde{Z}_{i}}^{2}+m_{f^{\prime}}^{2}-2\left|m_{\tilde{Z}_{i}}\right| E, \lambda=\sqrt{\left(s-\left(m_{f}+m_{\tilde{W}}\right)^{2}\right)\left(s-\left(m_{f}-m_{\tilde{W}}\right)^{2}\right)}$, $A=\left[m_{f}^{2}+m_{\tilde{W}_{j}}^{2}+2\left|m_{\tilde{Z}_{i}}\right| E+\left(m_{\tilde{Z}_{i}}^{2}-m_{f^{\prime}}^{2}\right)\left(m_{\tilde{W}}^{2}-m_{f}^{2}\right) \frac{1}{s}+2\left|m_{\tilde{Z}_{i}}\right| \lambda \frac{1}{s} \sqrt{E^{2}-m_{f^{\prime}}^{2}}-2 m_{\tilde{f}_{1}}^{2}\right]$ and $B=\left[m_{f}^{2}+m_{\tilde{W}_{j}}^{2}+2\left|m_{\tilde{Z}_{i}}\right| E+\right.$ $\left.\left(m_{\tilde{Z}_{i}}^{2}-m_{f^{\prime}}^{2}\right)\left(m_{\tilde{W}}^{2}-m_{f}^{2}\right) \frac{1}{s}-2\left|m_{\tilde{Z}_{i}}\right| \lambda \frac{1}{s} \sqrt{E^{2}-m_{f^{\prime}}^{2}}-2 m_{\tilde{f}_{1}}^{2}\right]$ :

$$
\begin{aligned}
& I_{\tilde{f}^{\prime}, \tilde{f}_{1}}^{1}=4\left|m_{\tilde{Z}_{i}}\right| \int_{m_{f^{\prime}}}^{E_{u p p e r}} d E \frac{\left[2\left|m_{\tilde{z}_{i}}\right| \lambda \sqrt{E^{2}-m_{f^{\prime}}^{2}}+\left(m_{\tilde{f}_{1}}^{2} s-m_{\tilde{Z}_{i}}^{2} m_{\tilde{W}_{j}}^{2}-m_{f}^{2} m_{f^{\prime}}^{2}\right) \log (A / B)\right]}{s-m_{\tilde{f}_{1}^{\prime}}^{2}}, \\
& I_{\tilde{f}^{\prime}, \tilde{f}_{1}}^{2}=-2\left|m_{\tilde{Z}_{i}}\right| \int_{m_{f^{\prime}}}^{E_{u p p e r}} d E \frac{\left[2\left|m_{\tilde{Z}_{i}}\right| \lambda \frac{1}{s} \sqrt{E^{2}-m_{f^{\prime}}^{2}}+\left(m_{\tilde{f}_{1}}^{2}-2 \mid m_{\tilde{Z}_{i}} E+m_{f^{\prime}}^{2}-m_{\tilde{W}_{j}}^{2}\right) \log (A / B)\right]}{s-m_{\tilde{f}_{1}^{\prime}}^{2}}, \\
& I_{\tilde{f}_{1}, \tilde{f}_{1}}^{3}=2\left|m_{\tilde{Z}_{i}}\right| \int_{m_{f^{\prime}}}^{E_{u p p e r}} d E \frac{\left[2\left|m_{\tilde{Z}_{i}}\right| \lambda \frac{1}{s} \sqrt{E^{2}-m_{f^{\prime}}^{2}}+\left(m_{\tilde{f}_{1}}^{2}-2 \mid m_{\tilde{z}_{i}} E+m_{\tilde{Z}_{i}}^{2}-m_{f}^{2}\right) \log (A / B)\right]}{s-m_{\tilde{f}_{1}^{\prime}}^{2}}, \\
& I_{\tilde{f}^{\prime}, \tilde{f}_{1}}^{4}=2\left|m_{\tilde{Z}_{i}}\right| \int_{m_{f^{\prime}}}^{E_{u p p e r}} d E \frac{\left[2\left|m_{\tilde{Z}_{i}}\right| \lambda \frac{1}{s} \sqrt{E^{2}-m_{f^{\prime}}^{2}}+\left(m_{\tilde{f}_{1}}^{2}-m_{\tilde{W}_{j}}^{2}-m_{f^{\prime}}^{2}\right) \log (A / B)\right]}{s-m_{\tilde{f}_{1}^{\prime}}^{2}},
\end{aligned}
$$




$$
\begin{aligned}
& I_{\tilde{f}_{1}, \tilde{f}_{1}}^{5}=2\left|m_{\tilde{Z}_{i}}\right| \int_{m_{f^{\prime}}}^{E_{u p p e r}} d E \frac{\left[2\left|m_{\tilde{z}_{i}}\right| \lambda \frac{1}{s} \sqrt{E^{2}-m_{f^{\prime}}^{2}}+\left(m_{\tilde{f}_{1}}^{2}-m_{\tilde{Z}_{i}}^{2}-m_{f}^{2}\right) \log (A / B)\right]}{s-m_{\tilde{f}_{1}}^{2}}, \\
& I_{\tilde{f}_{1}, \tilde{f}_{\mathrm{i}}}^{6}=2\left|m_{\tilde{Z}_{i}}\right| \int_{m_{f^{\prime}}}^{E_{u p p e r}} d E \frac{\log (A / B)\left(s-m_{f}^{2}-m_{\tilde{W}_{j}}^{2}\right)}{s-m_{\tilde{f}_{1}}^{2}}, \\
& I_{\tilde{f}_{1}, \tilde{f}_{1}}^{7}=2\left|m_{\tilde{Z}_{i}}\right| \int_{m_{f^{\prime}}}^{E_{u p p e r}} d E \frac{\log (A / B) 2\left|m_{\tilde{Z}_{i}}\right| E}{s-m_{\tilde{f}_{1}}^{2}}, \\
& I_{\tilde{f}_{1}^{\prime} \tilde{f}_{1}}^{8}=2\left|m_{\tilde{Z}_{i}}\right| \int_{m_{f^{\prime}}}^{E_{\text {upper }}} d E \frac{\log (A / B)}{s-m_{\tilde{f}^{\prime} 1}^{2}} .
\end{aligned}
$$

The $\Gamma_{\tilde{f}^{\prime} 1} \tilde{f}_{1}$ contribution is then given by:

$$
\begin{aligned}
& \Gamma_{\tilde{f}^{\prime} 1, \tilde{f}_{1}}=\mathcal{V}_{\tilde{f}^{\prime} 1 \tilde{f}_{1}}^{(1)} I_{\tilde{f}^{\prime} 1 \tilde{f}_{1}}^{1}+\mathcal{V}_{\tilde{f}^{\prime} 1 \tilde{f}_{1}}^{(2)} I_{\tilde{f}^{\prime} 1 \tilde{f}_{1}}^{2}+\mathcal{V}_{\tilde{f}^{\prime} 1 \tilde{f}_{1}}^{(3)} I_{\tilde{f}^{\prime} 1 \tilde{f}_{1}}^{3}+\mathcal{V}_{\tilde{f}^{\prime} 1 \tilde{f}_{1}}^{(4)} I_{\tilde{f}^{\prime} 1 \tilde{f}_{1}}^{4} \\
& +\mathcal{V}_{\tilde{f}^{\prime} 1 \tilde{f}_{1}}^{(5)} I_{\tilde{f}^{\prime} 1 \tilde{f}_{1}}^{5}+\mathcal{V}_{\tilde{f}_{1}, \tilde{f}_{1}}^{(\tilde{f})} I_{\tilde{f}^{\prime} 1 \tilde{f}_{1}}^{6}+\mathcal{V}_{\tilde{f}^{\prime}, \tilde{f}_{1}}^{(7)} I_{\tilde{f}^{\prime}, \tilde{f}_{1}}^{7}+\mathcal{V}_{\tilde{f}_{1}, \tilde{f}_{1}}^{(8)} I_{\tilde{f}^{\prime}, \tilde{f}_{1}}^{8} .
\end{aligned}
$$

The $\Gamma_{\tilde{f}_{1}^{\prime} \tilde{f}_{2}}, \Gamma_{\tilde{f}_{2}^{\prime} \tilde{f}_{1}}$ and $\Gamma_{\tilde{f}_{2}^{\prime}} \tilde{f}_{2}$ contributions follow analogously, they are given here as slight differences in the expressions for couplings complicated the expressions.

$\underline{\Gamma_{\tilde{f}_{1}} \tilde{f}_{2}}$

$$
\begin{aligned}
& \mathcal{V}_{\tilde{f}^{\prime} 1 \tilde{f}_{2}}^{(1)}=-\frac{1}{2}\left[\alpha_{\tilde{Z}_{i} \tilde{f}_{1}}^{u} \beta_{\tilde{Z}_{i} \tilde{z}_{2}}^{d} \beta_{\tilde{f}_{1}^{\prime} 1}^{\tilde{W}} \alpha_{\tilde{f}_{2}}^{\tilde{W}}+\beta_{\tilde{z}_{i} \tilde{f}_{1}}^{u} \alpha_{\tilde{Z}_{i} \tilde{z}_{2}}^{d} \alpha_{\tilde{f}_{1}^{\prime} 1}^{\tilde{W}} \beta_{\tilde{f}_{2}}^{\tilde{W}}\right](-1)^{\theta_{i}}, \\
& \mathcal{V}_{\tilde{f}^{\prime} 1 \tilde{f}_{2}}^{(2)}=\left|m_{\tilde{Z}_{i}}\right|\left|m_{\tilde{W}_{j}}\right|\left[(-1)^{\theta_{i}} \alpha_{\tilde{Z}_{i} \tilde{f}_{1}}^{u} \alpha_{\tilde{Z}_{i} \tilde{f}_{2}}^{d} \alpha_{\tilde{f}^{\prime} 1}^{\tilde{W}} \alpha_{\tilde{f}_{2}}^{\tilde{W}}+\beta_{\tilde{Z}_{i} \tilde{f}_{1}}^{u} \beta_{\tilde{Z}_{i} \tilde{f}_{2}}^{d} \beta_{\tilde{f}_{1}^{\prime} 1}^{\tilde{W}} \beta_{\tilde{f}_{2}}^{\tilde{W}}\right], \\
& \mathcal{V}_{\tilde{f}_{1}, \tilde{f}_{2}}^{(3)}=(-1)^{\theta_{i}} m_{f} m_{f^{\prime}}\left[\beta_{\tilde{z}_{i} \tilde{f}_{1}}^{u} \alpha_{\tilde{z}_{i} \tilde{f}_{2}}^{d} \beta_{\tilde{f}^{\prime}, 1}^{\tilde{W}} \alpha_{\tilde{f}_{2}}^{\tilde{W}}-(-1)^{\theta_{c}} \alpha_{\tilde{z}_{i} \tilde{f}_{1}}^{u} \beta_{\tilde{z}_{i} \tilde{f}_{2}}^{d} \alpha_{\tilde{f}_{1}}^{\tilde{W}} \beta_{\tilde{f}_{2}}^{\tilde{W}}\right] \text {, } \\
& \mathcal{V}_{\tilde{f}_{1}, \tilde{f}_{2}}^{(8)}=2\left|m_{\tilde{Z}_{i}} \| m_{\tilde{W}_{j}}\right| m_{f} m_{f^{\prime}}\left[(-1)^{\theta_{i}} \beta_{\tilde{Z}_{i} \tilde{f}_{1}}^{u} \beta_{\tilde{Z}_{i} \tilde{f}_{2}}^{d} \alpha_{\tilde{f}^{\prime}, 1}^{\tilde{W}} \alpha_{\tilde{f}_{2}}^{\tilde{W}}+\alpha_{\tilde{Z}_{i} \tilde{f}_{1}}^{u} \alpha_{\tilde{Z}_{i} \tilde{f}_{2}}^{d} \beta_{\tilde{f}_{1}^{\prime} 1}^{\tilde{W}} \beta_{\tilde{f}_{2}}^{\tilde{W}}\right] .
\end{aligned}
$$

If the decay is neutralino to chargino:

$$
\begin{aligned}
& \mathcal{V}_{\tilde{f}^{\prime}, \tilde{f}_{2}}^{(4)}=(-1)^{\theta_{i}}\left|m_{\tilde{Z}_{i}}\right| m_{f}\left[\alpha_{\tilde{Z}_{i} \tilde{f}_{1}}^{u} \alpha_{\tilde{Z}_{i} \tilde{f}_{2}}^{d} \beta_{\tilde{f}^{\prime}}^{\tilde{W}} \alpha_{\tilde{f}_{2}}^{\tilde{W}}-\beta_{\tilde{Z}_{i} \tilde{f}_{1}}^{u} \beta_{\tilde{Z}_{i} \tilde{f}_{2}}^{d} \alpha_{\tilde{f}^{\prime},}^{\tilde{W}} \beta_{\tilde{f}_{2}}^{\tilde{W}}\right] \text {, } \\
& \mathcal{V}_{\tilde{f}^{\prime} 1 \tilde{z}_{2}}^{(5)}=m_{f^{\prime}} \mid m_{\tilde{W}_{j}}\left[(-1)^{\theta_{i}} \beta_{\tilde{i}_{i} \tilde{f}_{1}}^{u} \alpha_{\tilde{Z}_{i} \tilde{f}_{2}}^{d} \alpha_{\tilde{f}_{1}^{\prime} 1}^{\tilde{W}} \alpha_{\tilde{z}_{2}}^{\tilde{W}}+\alpha_{\tilde{z}_{i} \tilde{f}_{1}}^{u} \beta_{\tilde{Z}_{i} \tilde{f}_{2}}^{d} \beta_{\tilde{f}^{\prime} 1}^{\tilde{W}} \beta_{\tilde{f}_{2}}^{\tilde{W}}\right](-1)^{\theta_{j}}, \\
& \mathcal{V}_{\tilde{f}_{1}{ }_{1} \tilde{f}_{2}}^{(6)}=-(-1)^{\theta_{i}}\left|m_{\tilde{Z}_{i}}\right| m_{f^{\prime}}\left[-\beta_{\tilde{z}_{i} f_{1}}^{u} \beta_{\tilde{z}_{i} \tilde{f}_{2}}^{d} \beta_{\tilde{f}_{1}^{\prime} 1}^{\tilde{W}} \alpha_{\tilde{f}_{2}}^{\tilde{W}}+\alpha_{\tilde{Z}_{i} \tilde{f}_{1}}^{u} \alpha_{\tilde{z}_{i} \tilde{f}_{2}}^{d} \alpha_{\tilde{f}^{\prime} 1}^{\tilde{W}} \beta_{\tilde{f}_{2}}^{\tilde{W}}\right] \text {, } \\
& \mathcal{V}_{\tilde{f}_{1}^{\prime}, \tilde{f}_{2}}^{(7)}=\left|m_{\tilde{W}_{j}}\right| m_{f}\left[(-1)^{\theta_{i}} \alpha_{\tilde{z}_{i} \tilde{f}_{1}}^{u} \beta_{\tilde{z}_{i} \tilde{f}_{2}}^{d} \alpha_{\tilde{f}_{1}^{\prime} 1}^{\tilde{W}} \alpha_{\tilde{f}_{2}}^{\tilde{W}}+\beta_{\tilde{z}_{i} \tilde{f}_{1}}^{u} \alpha_{\tilde{Z}_{i} \tilde{z}_{2}}^{d} \beta_{\tilde{f}_{1}^{\prime} 1}^{\tilde{W}} \beta_{\tilde{f}_{2}}^{\tilde{W}}\right](-1)^{\theta_{j}} .
\end{aligned}
$$

Whilst if the decay is instead chargino to neutralino:

$$
\begin{aligned}
& \mathcal{V}_{\tilde{f}^{\prime}, \tilde{f}_{2}}^{(4)}=\left|m_{\tilde{Z}_{i}}\right| m_{f}\left[(-1)^{\theta_{j}} \alpha_{\tilde{f}_{1}^{\prime} 1}^{\tilde{W}} \alpha_{\tilde{f}_{2}}^{\tilde{W}} \beta_{\tilde{Z}_{i} \tilde{f}_{1}}^{u} \alpha_{\tilde{Z}_{i} \tilde{f}_{2}}^{d}-\beta_{\tilde{f}_{1}^{\prime} 1}^{\tilde{W}} \beta_{\tilde{f}_{2}}^{\tilde{W}} \alpha_{\tilde{Z}_{i} \tilde{f}_{1}}^{u} \beta_{\tilde{Z}_{i} \tilde{f}_{2}}^{d}\right], \\
& \mathcal{V}_{\tilde{f}_{1}^{\prime}, \tilde{f}_{2}}^{(5)}=-\left|m_{\tilde{W}_{j}}\right| m_{f^{\prime}}\left[\alpha_{\tilde{f}_{2}}^{\tilde{W}} \beta_{\tilde{f}_{1}^{\prime} 1}^{\tilde{W}} \alpha_{\tilde{Z}_{i} \tilde{f}_{1}}^{u} \alpha_{\tilde{Z}_{i} \tilde{f}_{2}}^{d}-(-1)^{\theta_{j}} \alpha_{\tilde{f}_{1}^{\prime}{ }_{1}}^{\tilde{W}} \beta_{\tilde{f}_{2}}^{\tilde{W}} \beta_{\tilde{Z}_{i} \tilde{f}_{1}}^{u} \beta_{\tilde{Z}_{i} \tilde{f}_{2}}^{d}\right] \text {, } \\
& \mathcal{V}_{\tilde{f}_{1}^{\prime} \tilde{f}_{2}}^{(6)}=\left|m_{\tilde{Z}_{i}}\right| m_{f^{\prime}}\left[-\beta_{\tilde{f}_{1}^{\prime} 1}^{\tilde{W}} \beta_{\tilde{f}_{2}}^{\tilde{W}} \beta_{\tilde{Z}_{i} \tilde{f}_{1}}^{u} \alpha_{\tilde{Z}_{i} \tilde{f}_{2}}^{d}-\alpha_{\tilde{f}_{1}^{\prime} 1}^{\tilde{W}} \alpha_{\tilde{f}_{2}}^{\tilde{W}_{1}} \alpha_{\tilde{Z}_{i} \tilde{f}_{1}}^{u} \beta_{\tilde{Z}_{i} \tilde{f}_{2}}^{d}\right] \text {, } \\
& \mathcal{V}_{\tilde{f}_{1}^{\prime} \tilde{f}_{2}}^{(7)}=\left|m_{\tilde{W}_{j}}\right| m_{f}\left[-\alpha_{\tilde{f}_{1}^{\prime} 1}^{\tilde{W}} \beta_{\tilde{f}_{2}}^{\tilde{W}} \alpha_{\tilde{Z}_{i} \tilde{f}_{1}}^{u} \alpha_{\tilde{Z}_{i} \tilde{f}_{2}}^{d}+(-1)^{\theta_{j}} \beta_{\tilde{f}_{1}^{\prime} 1}^{\tilde{W}} \alpha_{\tilde{f}_{2}}^{\tilde{W}} \beta_{\tilde{z}_{i} \tilde{f}_{1}}^{u} \beta_{\tilde{z}_{i} \tilde{f}_{2}}^{d}\right] .
\end{aligned}
$$


The integrals are as in the $\tilde{f}^{\prime}{ }_{1} \tilde{f}_{1}$ case, with the appropriate mass replacements. Similarly, $\Gamma_{\tilde{f}_{1} \tilde{f}_{2}}$ is just the product of each coupling combination $\mathcal{V}_{\tilde{f}^{\prime}, \tilde{f}_{2}}^{(k)}$ with each corresponding integral $I_{\tilde{f}^{\prime} 1, \tilde{f}_{2}}^{k}$.

$\underline{\Gamma_{\tilde{f}_{2}{ }_{2} \tilde{f}_{1}}}$

$$
\mathcal{V}_{\tilde{f}^{\prime} 2 \tilde{f}_{1}}^{(8)}=\left|m_{\tilde{Z}_{i}}\right|\left|m_{\tilde{W}_{j}}\right| m_{f} m_{f^{\prime}}(-1)^{\theta_{i}}(-1)^{\theta_{c}}\left[\beta_{\tilde{Z}_{i} \tilde{f}_{2}}^{u} \beta_{\tilde{Z}_{i} \tilde{f}_{1}}^{d} \alpha_{\tilde{f}_{2}^{\prime}}^{\tilde{W}} \alpha_{\tilde{f}_{1}}^{\tilde{W}}-\alpha_{\tilde{Z}_{i} \tilde{f}_{2}}^{u} \alpha_{\tilde{Z}_{i} \tilde{f}_{1}}^{d} \beta_{\tilde{f}_{2}^{\prime} 2}^{\tilde{W}} \beta_{\tilde{f}_{1}}^{\tilde{W}}\right] .
$$

If the decay is neutralino to chargino:

$$
\begin{aligned}
& \mathcal{V}_{\tilde{f}_{2} \tilde{f}_{1}}^{(1)}=\frac{1}{2}(-1)^{\theta_{i}}\left[-\alpha_{\tilde{Z}_{i} \tilde{f}_{2}}^{u} \beta_{\tilde{Z}_{i} \tilde{f}_{1}}^{d} \beta_{\tilde{f}^{\prime}{ }_{2}}^{\tilde{W}} \alpha_{\tilde{f}_{1}}^{\tilde{W}}+\beta_{\tilde{Z}_{i} \tilde{f}_{2}}^{u} \alpha_{\tilde{Z}_{i} \tilde{f}_{1}}^{d} \alpha_{\tilde{f}_{2}^{\prime}{ }_{2}}^{\tilde{W}} \beta_{\tilde{f}_{1}}^{\tilde{W}}\right], \\
& \mathcal{V}_{\tilde{f}_{2}^{\prime} \tilde{f}_{1}}^{(2)}=-\left|m_{\tilde{Z}_{i}}\right|\left|m_{\tilde{W}_{j}}\right|\left[\alpha_{\tilde{z}_{i} \tilde{f}_{2}}^{u} \alpha_{\tilde{Z}_{i} \tilde{f}_{1}}^{d} \alpha_{\tilde{f}^{\prime}{ }_{2}}^{\tilde{W}} \alpha_{\tilde{f}_{1}}^{\tilde{W}}-\beta_{\tilde{Z}_{i} \tilde{f}_{2}}^{u} \beta_{\tilde{Z}_{i} \tilde{f}_{1}}^{d} \beta_{\tilde{f}_{2}^{\prime} 2}^{\tilde{W}} \beta_{\tilde{f}_{1}}^{\tilde{W}}\right], \\
& \mathcal{V}_{\tilde{f}_{2}^{\prime} \tilde{f}_{1}}^{(3)}=(-1)^{\theta_{i}} m_{f} m_{f^{\prime}}\left[-\beta_{\tilde{Z}_{i} \tilde{f}_{2}}^{u} \alpha_{\tilde{Z}_{i} \tilde{f}_{1}}^{d} \beta_{\tilde{f}^{\prime}, 2}^{\tilde{W}} \alpha_{\tilde{f}_{1}}^{\tilde{W}}+\alpha_{\tilde{Z}_{i} \tilde{f}_{2}}^{u} \beta_{\tilde{Z}_{i} \tilde{f}_{1}}^{d} \alpha_{\tilde{f}_{2}}^{\tilde{W}} \beta_{\tilde{f}_{1}}^{\tilde{W}}\right], \\
& \mathcal{V}_{\tilde{f}_{2}^{\prime} \tilde{f}_{1}}^{(4)}=\left|m_{\tilde{Z}_{i}}\right| m_{f}\left[\alpha_{\tilde{Z}_{i} \tilde{z}_{2}}^{u} \alpha_{\tilde{Z}_{i} \tilde{f}_{1}}^{d} \beta_{\tilde{f}^{\prime}{ }_{2}}^{\tilde{W}} \alpha_{\tilde{f}_{1}}^{\tilde{W}}+(-1)^{\theta_{i}} \beta_{\tilde{Z}_{i} \tilde{f}_{2}}^{u} \beta_{\tilde{Z}_{i} \tilde{f}_{1}}^{d} \alpha_{\tilde{f}^{\prime} 2}^{\tilde{W}} \beta_{\tilde{f}_{1}}^{\tilde{W}}\right], \\
& \mathcal{V}_{\tilde{f}_{2}^{\prime} 2 \tilde{f}_{1}}^{(5)}=(-1)^{\theta_{i}} m_{f^{\prime}}\left|m_{\tilde{W}_{j}}\right|\left[\beta_{\tilde{z}_{i} \tilde{f}_{2}}^{u} \alpha_{\tilde{Z}_{i} \tilde{f}_{1}}^{d} \alpha_{\tilde{f}^{\prime}{ }_{2}}^{\tilde{W}} \alpha_{\tilde{f}_{1}}^{\tilde{W}}-\alpha_{\tilde{Z}_{i} \tilde{f}_{2}}^{u} \beta_{\tilde{Z}_{i} \tilde{f}_{1}}^{d} \beta_{\tilde{f}_{2}^{\prime} 2}^{\tilde{W}} \beta_{\tilde{f}_{1}}^{\tilde{W}}\right](-1)^{\theta_{j}} \text {, } \\
& \mathcal{V}_{\tilde{f}_{2}^{\prime} \tilde{f}_{1}}^{(6)}=-(-1)^{\theta_{i}}\left|m_{\tilde{Z}_{i}}\right| m_{f^{\prime}}\left[-\beta_{\tilde{Z}_{i} \tilde{f}_{2}}^{u} \beta_{\tilde{Z}_{i} \tilde{f}_{1}}^{d} \beta_{\tilde{f}_{2}^{\prime} 2}^{\tilde{W}} \alpha_{\tilde{f}_{1}}^{\tilde{W}}+(-1)^{\theta_{i}} \alpha_{\tilde{Z}_{i} \tilde{f}_{2}}^{u} \alpha_{\tilde{Z}_{i} \tilde{f}_{1}}^{d} \alpha_{\tilde{f}^{\prime}{ }_{2}}^{\tilde{W}} \beta_{\tilde{f}_{1}}^{\tilde{W}}\right], \\
& \mathcal{V}_{\tilde{f}_{2}^{\prime}, \tilde{f}_{1}}^{(7)}=-\left|m_{\tilde{W}_{j}}\right| m_{f}\left[\alpha_{\tilde{z}_{i} \tilde{f}_{2}}^{u} \beta_{\tilde{Z}_{i} \tilde{f}_{1}}^{d} \alpha_{\tilde{f}_{2}^{\prime} 2}^{\tilde{W}} \alpha_{\tilde{f}_{1}}^{\tilde{W}}+(-1)^{\theta_{i}} \beta_{\tilde{Z}_{i} \tilde{f}_{2}}^{u} \alpha_{\tilde{Z}_{i} \tilde{f}_{1}}^{d} \beta_{\tilde{f}_{2}^{\prime} 2}^{\tilde{W}} \beta_{\tilde{f}_{1}}^{\tilde{W}}\right](-1)^{\theta_{j}} .
\end{aligned}
$$

Whilst if the decay is instead chargino to neutralino:

$$
\begin{aligned}
& \mathcal{V}_{\tilde{f}_{2}^{\prime} \tilde{f}_{1}}^{(1)}=\frac{1}{2}\left[\alpha_{\tilde{Z}_{i} \tilde{z}_{2}}^{u} \beta_{\tilde{Z}_{i} \tilde{f}_{1}}^{d} \beta_{\tilde{f}^{\prime} 2}^{\tilde{W}} \alpha_{\tilde{f}_{1}}^{\tilde{W}}+\beta_{\tilde{Z}_{i} \tilde{z}_{2}}^{u} \alpha_{\tilde{Z}_{i} \tilde{f}_{1}}^{d} \alpha_{\tilde{f}_{2}^{\prime}{ }_{2}}^{\tilde{W}} \beta_{\tilde{f}_{1}}^{\tilde{W}}\right](-1)^{\theta_{i}}, \\
& \mathcal{V}_{\tilde{f}_{2} \tilde{f}_{1}}^{(2)}=\left|m_{\tilde{Z}_{i}} \| m_{\tilde{W}_{j}}\right|(-1)^{\theta_{i}}\left[\alpha_{\tilde{Z}_{i} \tilde{f}_{2}}^{u} \alpha_{\tilde{Z}_{i} \tilde{f}_{1}}^{d} \alpha_{\tilde{f}^{\prime} 2}^{\tilde{W}} \alpha_{\tilde{f}_{1}}^{\tilde{W}}+\beta_{\tilde{Z}_{i} \tilde{f}_{2}}^{u} \int_{\tilde{Z}_{i} \tilde{f}_{1}}^{d} \beta_{\tilde{f}_{2}^{\prime}}^{\tilde{W}} \tilde{\tilde{f}}_{\tilde{f}_{1}}^{\tilde{W}}\right] \text {, } \\
& \mathcal{V}_{\tilde{f}_{2} \tilde{f}_{1}}^{(3)}=-m_{f} m_{f^{\prime}}\left[\beta_{\tilde{Z}_{i} \tilde{f}_{2}}^{u} \alpha_{\tilde{Z}_{i} \tilde{f}_{1}}^{d} \beta_{\tilde{f}^{\prime}, 2}^{\tilde{W}} \alpha_{\tilde{f}_{1}}^{\tilde{W}}+\alpha_{\tilde{Z}_{i} \tilde{f}_{2}}^{u} \beta_{\tilde{Z}_{i} \tilde{f}_{1}}^{d} \alpha_{\tilde{f}_{2}}^{\tilde{W}} \beta_{\tilde{f}_{1}}^{\tilde{W}}\right] \text {, } \\
& \mathcal{V}_{\tilde{f}^{\prime} 2 \tilde{f}_{1}}^{(4)}=-\left|m_{\tilde{Z}_{i}}^{\left(\tilde{Z}_{i}\right.}\right| m_{f}\left[\alpha_{\tilde{f}^{\prime} 2}^{\tilde{W}} \alpha_{\tilde{f}_{1}}^{\tilde{W}} \beta_{\tilde{Z}_{i} \tilde{f}_{2}}^{u} \alpha_{\tilde{Z}_{i} \tilde{f}_{1}}^{d}+\beta_{\tilde{f}^{\prime}}^{\tilde{W}} \beta_{\tilde{f}_{1}}^{\tilde{W}} \alpha_{\tilde{Z}_{i} \tilde{f}_{2}}^{u} \beta_{\tilde{Z}_{i} \tilde{f}_{1}}^{d}\right] \text {, } \\
& \mathcal{V}_{\tilde{f}_{2}^{\prime} \tilde{f}_{1}}^{(5)}=\left|m_{\tilde{W}_{j}}\right| m_{f^{\prime}}\left[\alpha_{\tilde{f}_{1}}^{\tilde{W}} \beta_{\tilde{f}_{2}^{\prime} 2}^{\tilde{W}} \alpha_{\tilde{Z}_{i} \tilde{f}_{2}}^{u} \alpha_{\tilde{Z}_{i} \tilde{f}_{1}}^{d}+\alpha_{\tilde{f}_{2}^{\prime} 2}^{\tilde{W}} \beta_{\tilde{f}_{1}}^{\tilde{W}} \beta_{\tilde{Z}_{i} \tilde{f}_{2}}^{u} \beta_{\tilde{Z}_{i} \tilde{f}_{1}}^{d}\right] \text {, } \\
& \mathcal{V}_{\tilde{f}^{\prime} 2 \tilde{f}_{1}}^{(6)}=\left|m_{\tilde{Z}_{i}}\right| m_{f^{\prime}}\left[\beta_{\tilde{f}_{2}^{\prime}}^{\tilde{W}} \beta_{\tilde{f}_{1}}^{\tilde{W}} \beta_{\tilde{Z}_{i} \tilde{f}_{2}}^{u} \alpha_{\tilde{Z}_{i} \tilde{f}_{1}}^{d}+\alpha_{\tilde{f}_{2}^{\prime} 2}^{\tilde{W}} \alpha_{\tilde{f}_{1}}^{\tilde{W}_{1}} \alpha_{\tilde{Z}_{i} \tilde{f}_{2}}^{u} \beta_{\tilde{z}_{i} \tilde{f}_{1}}^{d}\right] \text {, } \\
& \mathcal{V}_{\tilde{f}_{2}, \tilde{f}_{1}}^{(7)}=-\left|m_{\tilde{W}_{j}}\right| m_{f}\left[\alpha_{\tilde{f}_{2}^{\prime}{ }_{2}}^{\tilde{W}} \beta_{\tilde{f}_{1}}^{\tilde{W}} \alpha_{\tilde{Z}_{i} \tilde{f}_{2}}^{u} \alpha_{\tilde{Z}_{i} \tilde{f}_{1}}^{d}+\beta_{\tilde{f}^{\prime}{ }_{2}}^{\tilde{W}} \alpha_{\tilde{f}_{1}}^{\tilde{W}} \beta_{\tilde{Z}_{i} \tilde{f}_{2}}^{u} \beta_{\tilde{Z}_{i} \tilde{f}_{1}}^{d}\right] .
\end{aligned}
$$

Again, the integrals are as in the $\tilde{f}_{1}^{\prime}{ }_{1} \tilde{f}_{1}$ case with the obvious mass replacements. $\Gamma_{\tilde{f}^{\prime}} \tilde{f}_{1}$ is just the product of each coupling combination $\mathcal{V}_{\tilde{f}^{\prime} f_{1} \tilde{f}_{1}}^{(k)}$ with each corresponding integral $I_{\tilde{f}_{2}^{\prime} \tilde{f}_{1}}^{k}$.

$\underline{\Gamma_{\tilde{f}^{\prime}, \tilde{f}_{2}}}$

$$
\mathcal{V}_{\tilde{f}_{2}^{\prime} \tilde{f}_{2}}^{(8)}=-\left|m_{\tilde{Z}_{i}} \| m_{\tilde{W}_{j}}\right| m_{f} m_{f^{\prime}}(-1)^{\theta_{i}}(-1)^{\theta_{j}}\left[\beta_{\tilde{Z}_{i} \tilde{f}_{2}}^{u} \beta_{\tilde{Z}_{i} \tilde{z}_{2}}^{d} \alpha_{\tilde{f}_{2}^{\prime} 2}^{\tilde{W}} \alpha_{\tilde{f}_{2}}^{\tilde{W}}-\alpha_{\tilde{Z}_{i} \tilde{f}_{2}}^{u} \alpha_{\tilde{Z}_{i} \tilde{f}_{2}}^{d} \beta_{\tilde{f}_{2}^{\prime} 2}^{\tilde{W}} \beta_{\tilde{f}_{2}}^{\tilde{W}}\right] .
$$


If the decay is neutralino to chargino:

$$
\begin{aligned}
& \mathcal{V}_{\tilde{f}_{2}^{\prime} \tilde{f}_{2}}^{(1)}=\frac{1}{2}(-1)^{\theta_{j}}\left[-\alpha_{\tilde{Z}_{i} \tilde{f}_{2}}^{u} \beta_{\tilde{Z}_{i} \tilde{f}_{2}}^{d} \beta_{\tilde{f}^{\prime}{ }_{2}}^{\tilde{W}} \alpha_{\tilde{f}_{2}}^{\tilde{W}}+(-1)^{\theta_{i}} \beta_{\tilde{Z}_{i} \tilde{f}_{2}}^{u} \alpha_{\tilde{Z}_{i} \tilde{f}_{2}}^{d} \alpha_{\tilde{f}^{\prime}{ }_{2}}^{\tilde{W}} \beta_{\tilde{f}_{2}}^{\tilde{W}}\right], \\
& \mathcal{V}_{\tilde{f}^{\prime}{ }_{2} \tilde{f}_{2}}^{(2)}=\left|m_{\tilde{Z}_{i}} \| m_{\tilde{W}_{j}}\right|(-1)^{\theta_{j}}\left[\alpha_{\tilde{Z}_{i} \tilde{f}_{2}}^{u} \alpha_{\tilde{Z}_{i} \tilde{f}_{2}}^{d} \alpha_{\tilde{f}_{2}^{\prime}{ }_{2}}^{\tilde{W}} \alpha_{\tilde{f}_{2}}^{\tilde{W}}-\beta_{\tilde{Z}_{i} \tilde{f}_{2} \tilde{Z}_{\tilde{Z}_{i} \tilde{f}_{2}}^{u}}^{d} \beta_{\tilde{f}^{\prime}{ }_{2}}^{\tilde{W}} \beta_{\tilde{f}_{2}}^{\tilde{W}}\right], \\
& \mathcal{V}_{\tilde{f}^{\prime}{ }_{2} \tilde{f}_{2}}^{(3)}=-m_{f} m_{f^{\prime}}\left[-(-1)^{\theta_{i}} \beta_{\tilde{Z}_{i} \tilde{f}_{2}}^{u} \alpha_{\tilde{Z}_{i} \tilde{f}_{2} \tilde{f}_{2}}^{d} \beta_{\tilde{f}^{\prime}{ }_{2}}^{\tilde{W}} \alpha_{\tilde{f}_{2}}^{\tilde{W}}+\alpha_{\tilde{Z}_{i} \tilde{f}_{2}{ }_{\tilde{Z}_{i} \tilde{f}_{2}}^{u}}^{u} \alpha_{\tilde{f}_{2}}^{\tilde{W}} \beta_{\tilde{f}_{2}}^{\tilde{W}}\right] \text {, } \\
& \mathcal{V}_{\tilde{f}^{\prime}{ }_{2} \tilde{f}_{2}}^{(4)}=-\left|m_{\tilde{Z}_{i}}\right| m_{f}(-1)^{\theta_{j}}\left[\alpha_{\tilde{Z}_{i} \tilde{f}_{2}}^{u} \alpha_{\tilde{Z}_{i} \tilde{f}_{2}}^{d} \beta_{\tilde{f}_{2}^{\prime}{ }_{2}}^{\tilde{W}} \alpha_{\tilde{f}_{2}}^{\tilde{W}}-(-1)^{\theta_{i}} \beta_{\tilde{Z}_{i} \tilde{f}_{2}}^{u} \beta_{\tilde{Z}_{i} \tilde{f}_{2}}^{d} \alpha_{\tilde{f}_{2}^{\prime}{ }_{2}}^{\tilde{W}} \beta_{\tilde{f}_{2}}^{\tilde{W}}\right], \\
& \mathcal{V}_{\tilde{f}^{\prime}{ }_{2} \tilde{f}_{2}}^{(5)}=-m_{f^{\prime}}\left|m_{\tilde{W}_{j}}\right|\left[(-1)^{\theta_{i}}(-1)^{\theta_{j}} \beta_{\tilde{Z}_{i} \tilde{f}_{2}}^{u} \alpha_{\tilde{Z}_{i} \tilde{f}_{2}}^{d} \alpha_{\tilde{f}^{\prime}{ }_{2}}^{\tilde{W}} \alpha_{\tilde{f}_{2}}^{\tilde{W}}+\alpha_{\tilde{Z}_{i} \tilde{f}_{2}}^{u} \beta_{\tilde{Z}_{i} \tilde{f}_{2}}^{d} \beta_{\tilde{f}_{2}^{\prime}{ }_{2}}^{\tilde{W}} \beta_{\tilde{f}_{2}}^{\tilde{W}}\right] \text {, } \\
& \mathcal{V}_{\tilde{f}_{2}{ }_{2} \tilde{f}_{2}}^{(6)}=\left|m_{\tilde{Z}_{i}}\right| m_{f^{\prime}}\left[(-1)^{\theta_{i}} \beta_{\tilde{Z}_{i} \tilde{f}_{2}}^{u} \beta_{\tilde{Z}_{i} \tilde{f}_{2}}^{d} \beta_{\tilde{f}_{2}^{\prime}{ }_{2}}^{\tilde{W}} \alpha_{\tilde{f}_{2}}^{\tilde{W}}-\alpha_{\tilde{Z}_{i} \tilde{f}_{2}}^{u} \alpha_{\tilde{Z}_{i} \tilde{f}_{2}}^{d} \alpha_{\tilde{f}_{2}^{\prime}{ }_{2}}^{\tilde{W}} \beta_{\tilde{f}_{2}}^{\tilde{W}}\right], \\
& \mathcal{V}_{\tilde{f}^{\prime} \tilde{f}_{2}}^{(7)}=\left|m_{\tilde{W}_{j}}\right| m_{f}\left[\alpha_{\tilde{Z}_{i} \tilde{f}_{2}}^{u} \beta_{\tilde{Z}_{i} \tilde{f}_{2}}^{d} \alpha_{\tilde{f}^{\prime}{ }_{2}}^{\tilde{W}} \alpha_{\tilde{f}_{2}}^{\tilde{W}}-\beta_{\tilde{Z}_{i} \tilde{f}_{2}}^{u} \alpha_{\tilde{Z}_{i} \tilde{f}_{2} \tilde{f}_{\tilde{f}^{\prime}}}^{d} \beta_{\tilde{f}_{2}}^{\tilde{W}} \beta_{\tilde{f}_{2}}^{\tilde{W}}\right](-1)^{\theta_{j}} \text {. }
\end{aligned}
$$

Whilst if the decay is instead chargino to neutralino:

$$
\begin{aligned}
& \mathcal{V}_{\tilde{f}^{\prime}{ }_{2} \tilde{f}_{2}}^{(1)}=-\frac{1}{2}\left[(-1)^{\theta_{j}} \alpha_{\tilde{Z}_{i} \tilde{f}_{2}}^{u} \beta_{\tilde{Z}_{i} \tilde{f}_{2} \tilde{f}^{\prime}{ }^{\prime}{ }_{2}}^{d} \beta_{\tilde{f}_{2}}^{\tilde{W}}-\beta_{\tilde{Z}_{i} \tilde{f}_{2}}^{u} \alpha_{\tilde{Z}_{i} \tilde{f}_{2}}^{d} \alpha_{\tilde{f}^{\prime}{ }_{2}}^{\tilde{W}} \beta_{\tilde{f}_{2}}^{\tilde{W}}\right], \\
& \mathcal{V}_{\tilde{f}^{\prime}{ }_{2} \tilde{f}_{2}}^{(2)}=-\left|m_{\tilde{Z}_{i}}\right|\left|m_{\tilde{W}_{j}}(-1)^{\theta_{i}}\right|\left[(-1)^{\theta_{j}} \alpha_{\tilde{Z}_{i} \tilde{f}_{2}}^{u} \alpha_{\tilde{Z}_{i} \tilde{f}_{2}}^{d} \alpha_{\tilde{f}^{\prime}{ }_{2}}^{\tilde{W}} \alpha_{\tilde{f}_{2}}^{\tilde{W}}-\beta_{\tilde{Z}_{i} \tilde{f}_{2}}^{u} \beta_{\tilde{Z}_{i} \tilde{f}_{2}}^{d} \beta_{\tilde{f}^{\prime}{ }_{2}}^{\tilde{W}} \beta_{\tilde{f}_{2}}^{\tilde{W}}\right] \text {, } \\
& \mathcal{V}_{\tilde{f}_{2}{ }_{2} \tilde{f}_{2}}^{(3)}=m_{f} m_{f^{\prime}}\left[-(-1)^{\theta_{j}} \beta_{\tilde{Z}_{i} \tilde{f}_{2}}^{u} \alpha_{\tilde{Z}_{i} \tilde{f}_{2}}^{d} \beta_{\tilde{f}^{\prime}{ }_{2}}^{\tilde{W}} \alpha_{\tilde{f}_{2}}^{\tilde{W}}-\alpha_{\tilde{Z}_{i} \tilde{f}_{2}}^{u} \beta_{\tilde{Z}_{i} \tilde{f}_{2}}^{d} \alpha_{\tilde{f}_{2}}^{\tilde{W}} \beta_{\tilde{f}_{2}}^{\tilde{W}}\right] \text {, } \\
& \mathcal{V}_{\tilde{f}_{2}^{\prime}{ }_{2} \tilde{f}_{2}}^{(4)}=\left|m_{\tilde{Z}_{i}}\right| m_{f}(-1)^{\theta_{j}}\left[\alpha_{\tilde{f}^{\prime}{ }_{2}}^{\tilde{W}} \alpha_{\tilde{f}_{2}}^{\tilde{W}} \beta_{\tilde{Z}_{i} \tilde{f}_{2}}^{u} \alpha_{\tilde{Z}_{i} \tilde{f}_{2}}^{d}+\beta_{\tilde{f}^{\prime}{ }_{2}}^{\tilde{W}} \beta_{\tilde{f}_{2}}^{\tilde{W}} \alpha_{\tilde{Z}_{i} \tilde{f}_{2}}^{u} \beta_{\tilde{Z}_{i} \tilde{f}_{2}}^{d}\right] \text {, } \\
& \mathcal{V}_{\tilde{f}^{\prime}{ }_{2} \tilde{f}_{2}}^{(5)}=\left|m_{\tilde{W}_{j}}\right| m_{f^{\prime}}\left[\alpha_{\tilde{f}_{2}}^{\tilde{W}} \beta_{\tilde{f}^{\prime}{ }_{2}}^{\tilde{W}} \alpha_{\tilde{Z}_{i} \tilde{f}_{2}}^{u} \alpha_{\tilde{Z}_{i} \tilde{f}_{2}}^{d}-\alpha_{\tilde{f}^{\prime}{ }_{2}}^{\tilde{W}} \beta_{\tilde{f}_{2}}^{\tilde{W}} \beta_{\tilde{Z}_{i} \tilde{f}_{2}}^{u} \beta_{\tilde{Z}_{i} \tilde{f}_{2}}^{d}\right], \\
& \mathcal{V}_{\tilde{f}^{\prime}{ }_{2} \tilde{f}_{2}}^{(6)}=(-1)^{\theta_{j}}\left|m_{\tilde{Z}_{i}}\right| m_{f^{\prime}}\left[\beta_{\tilde{f}^{\prime}{ }_{2}}^{\tilde{W}} \beta_{\tilde{f}_{2}}^{\tilde{W}} \beta_{\tilde{Z}_{i} \tilde{f}_{2}}^{u} \alpha_{\tilde{Z}_{i} \tilde{f}_{2}}^{d}+\alpha_{\tilde{f}^{\prime}{ }_{2}}^{\tilde{W}} \alpha_{\tilde{f}_{2}}^{\tilde{W}_{1}} \alpha_{\tilde{Z}_{i} \tilde{f}_{2}}^{u} \beta_{\tilde{Z}_{i} \tilde{f}_{2}}^{d}\right] \text {, } \\
& \mathcal{V}_{\tilde{f}^{\prime}{ }_{2} \tilde{f}_{2}}^{(7)}=\left|m_{\tilde{W}_{j}}\right| m_{f}\left[-\alpha_{\tilde{f}^{\prime}{ }_{2}}^{\tilde{W}} \beta_{\tilde{f}_{2}}^{\tilde{W}} \alpha_{\tilde{Z}_{i} \tilde{f}_{2}}^{u} \alpha_{\tilde{Z}_{i} \tilde{f}_{2}}^{d}+\beta_{\tilde{f}^{\prime}{ }_{2}}^{\tilde{W}} \alpha_{\tilde{f}_{2}}^{\tilde{W}} \beta_{\tilde{Z}_{i} \tilde{f}_{2}}^{u} \beta_{\tilde{Z}_{i} \tilde{f}_{2}}^{d}\right] .
\end{aligned}
$$

The integrals are again as in the $\tilde{f}^{\prime}{ }_{1} \tilde{f}_{1}$ case with the obvious mass replacements and $\Gamma_{\tilde{f}^{\prime}{ }_{2} \tilde{f}_{2}}$ is just the product of each coupling combination $\mathcal{V}_{\tilde{f}_{2}{ }_{2} \tilde{f}_{2}}^{(k)}$ with each corresponding integral $I_{\tilde{f}^{\prime}{ }_{2} \tilde{f}_{2}}^{k}$.

$\Gamma_{W H^{ \pm}}$

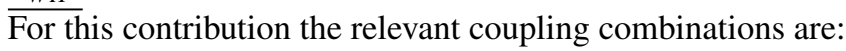

$$
\begin{aligned}
& \mathcal{V}_{W H^{ \pm}}^{(1)}=-\left(C_{\tilde{W} \tilde{Z} W}^{R} \omega_{H^{+} \tilde{W}^{+} \tilde{Z}}^{R}+C_{\tilde{W} \tilde{Z} W}^{L} \omega_{H^{+} \tilde{W}^{+} \tilde{Z}}^{L}\right) \frac{g}{\sqrt{2}} C_{H^{+} f f^{\prime}}^{u}\left|m_{\tilde{W}_{j}}\right| m_{f^{\prime}}(-1)^{\theta_{c}}, \\
& \mathcal{V}_{W H^{ \pm}}^{(2)}=\left(C_{\tilde{W} \tilde{Z} W}^{L} \omega_{H^{+} \tilde{W}^{+} \tilde{Z}}^{R}+C_{\tilde{W} \tilde{Z} W^{2}}^{R} \omega_{H^{+} \tilde{W}^{+} \tilde{Z}}^{L}\right) \frac{g}{\sqrt{2}} C_{H^{+} f f^{\prime}}^{d}\left|m_{\tilde{Z}_{i}}\right| m_{f}(-1)^{\theta_{i}}(-1)^{\theta_{j}}(-1)^{\theta_{c}}, \\
& \mathcal{V}_{W H^{ \pm}}^{(3)}=\left(C_{\tilde{W} \tilde{Z} W^{2}}^{R} \omega_{H^{+} \tilde{W}^{+} \tilde{Z}}^{R}+C_{\tilde{W} \tilde{Z} W}^{L} \omega_{H^{+} \tilde{W}^{+} \tilde{Z}}^{L}\right) \frac{g}{\sqrt{2}} C_{H^{+} f f^{\prime}}^{d}\left|m_{\tilde{W}_{j}}\right| m_{f}(-1)^{\theta_{c}}, \\
& \mathcal{V}_{W H^{ \pm}}^{(4)}=-\left(C_{\tilde{W} \tilde{Z} W^{L}}^{L} \omega_{H^{+} \tilde{W}^{+} \tilde{Z}}^{R}+C_{\tilde{W} \tilde{Z} W^{\prime}}^{R} \omega_{H^{+} \tilde{W}^{+} \tilde{Z}}^{L}\right) \frac{g}{\sqrt{2}} C_{H^{+} f f^{\prime}}^{u}\left|m_{\tilde{Z}_{i}}\right| m_{f^{\prime}}(-1)^{\theta_{i}}(-1)^{\theta_{j}}(-1)^{\theta_{c}} .
\end{aligned}
$$

The integrals are as follows, with upper limit $E_{\text {upper } 3}=\frac{1}{2\left|m_{\tilde{Z}_{i}}\right|}\left(m_{\tilde{Z}_{i}}^{2}+m_{\tilde{W}_{j}}^{2}-m_{f}^{2}-m_{f^{\prime}}^{2}-2 m_{f} m_{f^{\prime}}\right), s=m_{\tilde{Z}_{i}}^{2}+m_{\tilde{W}_{j}}^{2}-2\left|m_{\tilde{Z}_{i}}\right| E$, 
$\lambda=\sqrt{\left(s-\left(m_{f}+m_{f^{\prime}}\right)^{2}\right)\left(s-\left(m_{f}-m_{f^{\prime}}\right)^{2}\right)}, \mathcal{A}=2 \mid m_{\tilde{Z}_{i}} E+m_{f}^{2}+m_{f^{\prime}}^{2}-\left(m_{\tilde{Z}_{i}}^{2}-m_{\tilde{W}_{j}}^{2}\right)\left(m_{f}^{2}-m_{f^{\prime}}^{2}\right) / s$ and $\mathcal{B}=\frac{2\left|m_{\tilde{z}_{i}}\right|}{s} \lambda \sqrt{E^{2}-m_{\tilde{W}_{j}}^{2}}$ :

$$
\begin{aligned}
& I_{W H^{ \pm}}^{1}=2\left|m_{\tilde{Z}_{i}}\right| \int_{\left|m_{\tilde{W}_{j}}\right|}^{E_{\text {upper } 3}} d E \frac{-\frac{1}{2} \mathcal{A} \mathcal{B}+\left(m_{\tilde{Z}_{i}}^{2}+m_{f}^{2}\right) \mathcal{B}}{\left(s-m_{W}^{2}\right)\left(s-m_{H^{ \pm}}^{2}\right)}, \\
& I_{W H^{ \pm}}^{2}=2\left|m_{\tilde{Z}_{i}}\right| \int_{\left|m_{\tilde{W}_{j}}\right|}^{E_{\text {upper } 3}} d E \frac{\frac{1}{2} \mathcal{A} \mathcal{B}-\left(m_{\tilde{W}_{j}}^{2}+m_{f^{\prime}}^{2}\right) \mathcal{B}}{\left(s-m_{W}^{2}\right)\left(s-m_{H^{ \pm}}^{2}\right)}, \\
& I_{W H^{ \pm}}^{3}=2\left|m_{\tilde{Z}_{i}}\right| \int_{\left|m_{\tilde{W}_{j}}\right|}^{E_{u p p e r 3}} d E \frac{-\frac{1}{2} \mathcal{A} \mathcal{B}+\left(m_{\tilde{Z}_{i}}^{2}-2\left|m_{\tilde{Z}_{i}}\right| E-m_{f}^{2}\right) \mathcal{B}}{\left(s-m_{W}^{2}\right)\left(s-m_{H^{ \pm}}^{2}\right)}, \\
& I_{W H^{ \pm}}^{4}=2\left|m_{\tilde{Z}_{i}}\right| \int_{\left|m_{\tilde{W}_{j}}\right|}^{E_{u p p e r 3}} d E \frac{-\frac{1}{2} \mathcal{A} \mathcal{B}-\left(m_{\tilde{Z}_{j}}^{2}-2\left|m_{\tilde{Z}_{i}}\right|-m_{f^{\prime}}^{2}\right) \mathcal{B}}{\left(s-m_{W}^{2}\right)\left(s-m_{H^{ \pm}}^{2}\right)},
\end{aligned}
$$

So overall:

$$
\Gamma_{W H^{ \pm}}=\mathcal{V}_{W H^{ \pm}}^{(1)} I_{W H^{ \pm}}^{1}(-1)^{\theta_{c}}(-1)^{\theta_{j}}+\mathcal{V}_{W H^{ \pm}}^{(2)} I_{W H^{ \pm}}^{2}(-1)^{\theta_{c}}(-1)^{\theta_{j}}+\mathcal{V}_{W H^{ \pm}}^{(3)} I_{W H^{ \pm}}^{3}+\mathcal{V}_{W H^{ \pm}}^{(4)} I_{W H^{ \pm}}^{4} .
$$

$\underline{\Gamma_{W G}}$

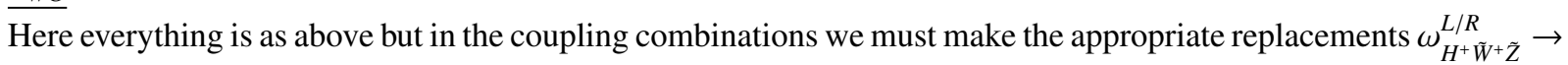
$\omega_{G \tilde{W} \tilde{Z}}^{L / R}$, whilst in the integrals we make the change $m_{H^{ \pm}} \rightarrow m_{\text {goldstone }}=m_{W}$. However because of subtle differences in the definitions of the couplings, the overall contribution here is given by:

$$
\Gamma_{W G}=\mathcal{V}_{W G}^{(1)} I_{W G}^{1}+\mathcal{V}_{W G}^{(2)} I_{W G}^{2}+\mathcal{V}_{W G}^{(3)} I_{W G}^{3}+\mathcal{V}_{W G}^{(4)} I_{W G}^{4} .
$$

$\Gamma_{W \tilde{f}_{1} 1}$

The coupling combinations are:

$$
\begin{aligned}
& \mathcal{V}_{W \tilde{f}^{\prime}, 1}^{(1)}=-2 C_{\tilde{W} \tilde{Z} W}^{L} \alpha_{\tilde{Z}_{i} \tilde{f}_{1}}^{u} \frac{g}{\sqrt{2}} \beta_{\tilde{f}^{\prime}, 1}^{\tilde{W}_{j}}\left|m_{\tilde{Z}_{i}}\right| m_{f}(-1)^{\theta_{j}}, \\
& \mathcal{V}_{W \tilde{f}_{1}^{\prime \prime}}^{(2)}=-2 C_{\tilde{W} \tilde{Z} W}^{L} \beta_{\tilde{Z}_{i} \tilde{f}_{1}} \frac{g}{\sqrt{2}} \alpha_{\tilde{f}_{1}}^{\tilde{W}_{j}} m_{f^{\prime}}\left|m_{\tilde{W}_{j}}\right|(-1)^{\theta_{c}}, \\
& \mathcal{V}_{W \tilde{f}_{1}^{\prime}{ }_{1}}^{(3)}=2 C_{\tilde{W} \tilde{Z} W}^{R} \alpha_{\tilde{Z}_{i} \tilde{f}_{1}}^{u} \frac{g}{\sqrt{2}} \alpha_{\tilde{f}^{\prime}{ }_{1}}^{\tilde{W}_{j}}(-1)^{\theta_{i}}(-1)^{\theta_{j}}(-1)^{\theta_{c}}, \\
& \mathcal{V}_{W \tilde{f}_{1}^{\prime} 1}^{(4)}=(-1)^{\theta_{i}} 4 C_{\tilde{W} \tilde{Z} W}^{R} \beta_{\tilde{Z}_{i} \tilde{f}_{1}}^{u} \frac{g}{\sqrt{2}} \alpha_{\tilde{f}^{\prime} 1}^{\tilde{W}_{j}}\left|m_{\tilde{Z}_{i}}\right| m_{f^{\prime}}(-1)^{\theta_{c}}, \\
& \mathcal{V}_{W \tilde{f}^{\prime} 1}^{(5)}=4 C_{\tilde{W} \tilde{Z} W^{\prime}}^{R} \alpha_{\tilde{Z}_{i} \tilde{f}_{1}}^{u} \frac{g}{\sqrt{2}} \beta_{\tilde{f}^{\prime} 1}^{\tilde{W}_{j}} m_{f}\left|m_{\tilde{W}_{j}}\right|(-1)^{\theta_{i}}, \\
& \mathcal{V}_{W \tilde{f}_{1}^{\prime} 1}^{(6)}=-2 C_{\tilde{W} \tilde{Z} W}^{L} \alpha_{\tilde{Z}_{i} \tilde{f}_{1}}^{u} \frac{g}{\sqrt{2}} \alpha_{\tilde{f}^{\prime}{ }_{1}}^{\tilde{W}_{j}}\left|m_{\tilde{Z}_{i}}\right|\left|m_{\tilde{W}_{j}}\right|(-1)^{\theta_{c}}, \\
& \mathcal{V}_{W \tilde{f}_{1}^{\prime}{ }_{1}}^{(7)}=-2 C_{\tilde{W} \tilde{Z} W}^{L} \beta_{\tilde{Z}_{i} \tilde{f}_{1}}^{u} \frac{g}{\sqrt{2}} \beta_{\tilde{f}^{\prime}{ }_{1}}^{\tilde{W}_{f}} m_{f^{\prime}} m_{f}, \\
& \mathcal{V}_{W \tilde{f}_{1}{ }_{1}}^{(8)}=8 C_{\tilde{W} \tilde{Z} W}^{R} \beta_{\tilde{Z}_{i} \tilde{f}_{1}}^{u} \frac{g}{\sqrt{2}} \beta_{\tilde{f}_{1}^{\prime} 1}^{\tilde{W}_{1}}\left|m_{\tilde{Z}_{i}}\right| m_{f^{\prime}} m_{f}\left|m_{\tilde{W}_{j}}\right|(-1)^{\theta_{i}}(-1)^{\theta_{j}} .
\end{aligned}
$$

The integrals are as follows with $s=m_{\tilde{Z}_{i}}^{2}+m_{\tilde{W}_{j}}^{2}-2\left|m_{\tilde{Z}_{i}}\right| E, \lambda=\sqrt{\left(s-\left(m_{f^{\prime}}+m_{f}\right)^{2}\right)\left(s-\left(m_{f^{\prime}}-m_{f}\right)^{2}\right)}, A=m_{f^{\prime}}^{2}+m_{f}^{2}+$ $2\left|m_{\tilde{Z}_{i}}\right| E+\left(m_{\tilde{Z}_{i}}^{2}-m_{\tilde{W}_{j}}^{2}\right)\left(m_{f}^{2}-m_{f^{\prime}}^{2}\right) / s, B=2\left|m_{\tilde{Z}_{i}}\right| \lambda \sqrt{E^{2}-m_{\tilde{W}_{j}}^{2}}, Z=\frac{A+B-2 m_{\tilde{f}^{\prime} 1}^{2}}{A-B-2 m_{\tilde{f}^{\prime}}^{2}}$ :

$$
I_{W \tilde{f}^{\prime} 1}^{1}=-2\left|m_{\tilde{Z}_{i}}\right| \int_{\left|m_{\tilde{W}_{j}}\right|}^{E_{\text {upper3 }} \mid} d E \frac{B+\left(m_{\tilde{f}_{1}^{\prime}}^{2}+m_{\tilde{W}_{j}}^{2}-2\left|m_{\tilde{Z}_{i}}\right| E-m_{f}^{2}\right) \log (Z)}{s-m_{W}^{2}},
$$




$$
\begin{aligned}
& I_{W \tilde{f}^{\prime} 1}^{2}=2\left|m_{\tilde{Z}_{i}}\right| \int_{\left|m_{\tilde{w}_{j}}\right|}^{E_{u p p e r 3}} d E \frac{B+\left(m_{\tilde{f}_{1}^{\prime} 1}^{2}+m_{\tilde{Z}_{i}}^{2}-2\left|m_{\tilde{i}_{i^{\prime}}}\right| E-m_{f^{\prime}}^{2}\right) \log (Z)}{s-m_{W}^{2}}, \\
& I_{W \tilde{f}^{\prime} 1}^{3}=2\left|m_{\tilde{Z}_{i}}\right| \int_{\left|m_{\tilde{W}_{j}}\right|}^{E_{\text {upper } 3}} d E\left[\{ m _ { \tilde { Z } _ { i } } ^ { 2 } + m _ { f } ^ { 2 } + m _ { f ^ { \prime } } ^ { 2 } + m _ { \tilde { W } _ { j } } ^ { 2 } - 1 . 5 m _ { \tilde { f } ^ { \prime } { } _ { 1 } } ^ { 2 } - 0 . 2 5 ( A + B ) \} \left(\frac{1}{2}(A+B)-m_{\tilde{f}^{\prime}{ }_{1}}^{2}\right.\right. \\
& -\left(m_{\tilde{Z}_{i}}^{2}+m_{f}^{2}+m_{f^{\prime}}^{2}+m_{\tilde{W}_{j}}^{2}-1.5 m_{\tilde{f}_{1}^{\prime} 1}^{2}-0.25(A-B)\right)\left(\frac{1}{2}(A-B)-m_{\tilde{f}^{\prime} 1}^{2}\right) \\
& \left.+\left(m_{\tilde{Z}_{i}}^{2}+m_{f^{\prime}}^{2}-m_{\tilde{f}^{\prime} 1}^{2}\right)\left(m_{\tilde{f}^{\prime} 1}^{2}-m_{f}^{2}-m_{\tilde{W}_{j}}^{2}\right) \log (Z)\right] \frac{1}{s-m_{W}^{2}}, \\
& I_{W \tilde{f}^{\prime},}^{4}=2\left|m_{\tilde{z}_{i}}\right| \int_{\left|m_{\tilde{W}_{j}}\right|}^{E_{\text {upper3 }}} d E \frac{B+\left(m_{\tilde{f}^{\prime}}^{2}-m_{f}^{2}-m_{\tilde{W}_{j}}^{2}\right) \log (Z)}{s-m_{W}^{2}}, \\
& I_{W \tilde{f}_{1},}^{5}=-2\left|m_{\tilde{\tilde{Z}}_{i}}\right| \int_{\left|m_{\tilde{W}_{j}}\right|}^{E_{\text {upper3 }}} d E \frac{B+\left(m_{\tilde{f}_{1},}^{2}-m_{\tilde{Z}_{i}}^{2}-m_{f^{\prime}}^{2}\right) \log (Z)}{s-m_{W}^{2}}, \\
& I_{W \tilde{f}^{\prime},}^{6}=2\left|m_{\tilde{Z}_{i}}\right| \int_{\left|m_{\tilde{W}_{j}}\right|}^{E_{u p p e r 3}} d E \frac{\left(s-m_{f^{\prime}}^{2}-m_{f}^{2}\right) \log (Z)}{s-m_{W}^{2}}, \\
& I_{W \tilde{f}_{1}^{\prime} 1}^{7}=2\left|m_{\tilde{Z}_{i}}\right| \int_{\left|m_{\tilde{W}_{j}}\right|}^{E_{u p p e r 3}} d E \frac{2\left|m_{\tilde{Z}_{i}}\right| E \log (Z)}{s-m_{W}^{2}}, \\
& I_{W \tilde{f}^{\prime}, 1}^{8}=2\left|m_{\tilde{Z}_{i}}\right| \int_{\left|m_{\tilde{j}_{j}}\right|}^{E_{\text {upper } 3}} d E \frac{\log (Z)}{s-m_{W}^{2}} \text {. }
\end{aligned}
$$

Therefore the overall contribution is:

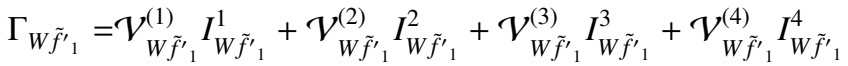

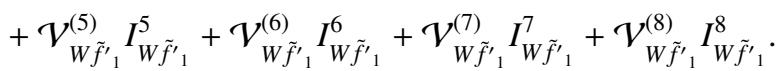

$\Gamma_{W \tilde{f}_{2}}$

The coupling combinations here are:

$$
\begin{aligned}
& \mathcal{V}_{W \tilde{f}_{2} 2}^{(1)}=2 C_{\tilde{W} \tilde{Z} W}^{L} \alpha_{\tilde{Z}_{i} \tilde{f}_{2}}^{u} \frac{g}{\sqrt{2}} \beta_{\tilde{f}^{\prime}, 2}^{\tilde{W}_{j}}\left|m_{\tilde{Z}_{i}}\right| m_{f}(-1)^{\theta_{j}}(-1)^{\theta_{c}}, \\
& \mathcal{V}_{W \tilde{f}_{2} 2}^{(2)}=2 C_{\tilde{W} \tilde{Z} W}^{L} \beta_{\tilde{Z}_{i} \tilde{f}_{2}}^{u} \frac{g}{\sqrt{2}} \alpha_{\tilde{f}_{2}^{\prime} 2}^{\tilde{W}_{j}} m_{f^{\prime}}\left|m_{\tilde{W}_{j}}\right|(-1)^{\theta_{i}}(-1)^{\theta_{j}}, \\
& \mathcal{V}_{W \tilde{f}^{\prime}{ }_{2}}^{(3)}=2 C_{\tilde{W} \tilde{Z} W}^{R} \alpha_{\tilde{Z}_{i} \tilde{f}_{2}}^{u} \frac{g}{\sqrt{2}} \alpha_{\tilde{f}^{\prime}{ }_{2}}^{\tilde{W}_{j}}(-1)^{\theta_{i}}, \\
& \mathcal{V}_{W \tilde{f}^{\prime} 2}^{(4)}=-4 C_{\tilde{W} \tilde{Z} W}^{R} \beta_{\tilde{i}_{i} \tilde{f}_{2}}^{u} \frac{g}{\sqrt{2}} \alpha_{\tilde{f}^{\prime}{ }_{2}}^{\tilde{W}_{j}}\left|m_{\tilde{Z}_{i}}\right| m_{f^{\prime}}, \\
& \mathcal{V}_{W \tilde{f}^{\prime} 2}^{(5)}=-4 C_{\tilde{W} \tilde{Z} W}^{R} \alpha_{\tilde{Z}_{i} \tilde{f}_{2}}^{u} \frac{g}{\sqrt{2}} \beta_{\tilde{f}^{\prime} 2}^{\tilde{W}_{j}} m_{f}\left|m_{\tilde{W}_{j}}\right|(-1)^{\theta_{i}}(-1)^{\theta_{c}}, \\
& \mathcal{V}_{W \tilde{f}_{2}{ }_{2}}^{(6)}=-2 C_{\tilde{W} \tilde{Z} W^{2}}^{L} \alpha_{\tilde{Z}_{i} \tilde{f}_{2}}^{u} \frac{g}{\sqrt{2}} \alpha_{\tilde{f}^{\prime}{ }_{2}}^{\tilde{W}_{j}}\left|m_{\tilde{Z}_{i}} \| m_{\tilde{W}_{j}}\right|(-1)^{\theta_{j}}, \\
& \mathcal{V}_{W \tilde{f}_{2}{ }_{2}}^{(7)}=-2 C_{\tilde{W} \tilde{Z} W}^{L} \beta_{\tilde{Z}_{i} \tilde{f}_{2}}^{u} \frac{g}{\sqrt{2}} \beta_{\tilde{f}^{\prime}{ }_{2}}^{\tilde{W}_{j}} m_{f^{\prime}} m_{f}(-1)^{\theta_{i}}(-1)^{\theta_{j}}(-1)^{\theta_{c}} \text {, } \\
& \mathcal{V}_{W \tilde{f}_{2}{ }_{2}}^{(8)}=8 C_{\tilde{W} \tilde{Z} W}^{R} \beta_{\tilde{Z}_{i} \tilde{f}_{2}}^{u} \frac{g}{\sqrt{2}} \beta_{\tilde{f}^{\prime}{ }_{2}}^{\tilde{W}_{j}}\left|m_{\tilde{Z}_{i}}\right| m_{f^{\prime}} m_{f}\left|m_{\tilde{W}_{j}}\right|(-1)^{\theta_{c}} .
\end{aligned}
$$


The integrals here are exactly as for $W \tilde{f}^{\prime}{ }_{1}$ with the change $m_{\tilde{f}^{\prime} 1} \rightarrow m_{\tilde{f}_{2}^{\prime}}$. As above $\Gamma_{W \tilde{f}_{2}{ }_{2}}$ is then the sum of the products of coupling combinations, $\mathcal{V}^{(i)}$ and integrals, $I_{W \tilde{f}^{\prime} 2}^{i}$.

$\Gamma_{W \tilde{f}_{1}}$

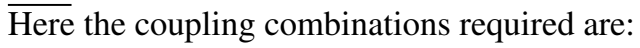

$$
\begin{aligned}
& \mathcal{V}_{W \tilde{f}_{1}}^{(6)}=-2 C_{\tilde{W} \tilde{Z} W}^{R} \alpha_{\tilde{Z}_{i} \tilde{f}_{1}}^{d} \frac{g}{\sqrt{2}} \alpha_{\tilde{f}_{1}}^{\tilde{W}_{j}}\left|m_{\tilde{Z}_{i}}\right|\left|m_{\tilde{W}_{j}}\right|(-1)^{\theta_{c}}, \\
& \mathcal{V}_{W \tilde{f}_{1}}^{(7)}=-2 C_{\tilde{W} \tilde{Z} W}^{R} \beta_{\tilde{Z}_{i} \tilde{f}_{1}}^{d} \frac{g}{\sqrt{2}} \beta_{\tilde{f}_{1}}^{\tilde{W}_{j}} m_{f} m_{f^{\prime}}, \\
& \mathcal{V}_{W \tilde{f}_{1}}^{(8)}=8 C_{\tilde{W} \tilde{Z} W}^{L} \beta_{\tilde{Z}_{i} \tilde{f}_{1}}^{d} \frac{g}{\sqrt{2}} \beta_{\tilde{f}_{1}}^{\tilde{W}_{j}}\left|m_{\tilde{Z}_{i}}\right| m_{f} m_{f^{\prime}}\left|m_{\tilde{W}_{j}}\right|(-1)^{\theta_{i}}(-1)^{\theta_{j}} .
\end{aligned}
$$

The other coupling combinations depend upon if it is a neutralino decaying into a chargino or a chargino decaying into a neutralino, for a neutralino decaying:

$$
\begin{aligned}
& \mathcal{V}_{W \tilde{f}_{1}}^{(1)}=-2 C_{\tilde{W} \tilde{Z} \tilde{Z}^{2}}^{R} \alpha_{\tilde{Z}_{i} \tilde{f}_{1}}^{d} \frac{g}{\sqrt{2}} \beta_{\tilde{f}_{1}}^{\tilde{W}_{j}}\left|m_{\tilde{Z}_{i}}\right| m_{f^{\prime}}, \\
& \mathcal{V}_{W \tilde{f}_{1}}^{(2)}=-2 C_{\tilde{W} \tilde{Z} \tilde{Z}^{2}}^{R} \beta_{\tilde{Z}_{i} \tilde{f}_{1}}^{d} \frac{g}{\sqrt{2}} \alpha_{\tilde{f}_{1}}^{\tilde{W}_{j}} m_{f}\left|m_{\tilde{W}_{j}}\right|(-1)^{\theta_{j}}, \\
& \mathcal{V}_{W \tilde{f}_{1}}^{(3)}=2 C_{\tilde{W} \tilde{Z} W}^{R} \alpha_{\tilde{Z}_{i} \tilde{f}_{1}}^{d} \frac{g}{\sqrt{2}} \alpha_{\tilde{f}_{1}}^{\tilde{W}_{j}}, \\
& \mathcal{V}_{W \tilde{f}_{1}}^{(4)}=4 C_{\tilde{W} \tilde{Z} W}^{L} \beta_{\tilde{Z}_{i} \tilde{f}_{1}}^{d} \frac{g}{\sqrt{2}} \alpha_{\tilde{f}_{1}}^{\tilde{W}_{j}}\left|m_{\tilde{Z}_{i}}\right| m_{f}(-1)^{\theta_{i}}, \\
& \mathcal{V}_{W \tilde{f}_{1}}^{(5)}=4 C_{\tilde{W} \tilde{Z} W}^{L} \alpha_{\tilde{Z}_{i} \tilde{f}_{1}}^{d} \frac{g}{\sqrt{2}} \beta_{\tilde{f}_{1}}^{\tilde{W}_{j}} m_{f^{\prime}}\left|m_{\tilde{W}_{j}}\right|(-1)^{\theta_{i}}(-1)^{\theta_{j}},
\end{aligned}
$$

whilst if it's a chargino decaying:

$$
\begin{aligned}
& \mathcal{V}_{W \tilde{f}_{1}}^{(1)}=2 C_{\tilde{W} \tilde{Z} W}^{R} \beta_{\tilde{Z}_{i} \tilde{f}_{1}}^{d} \alpha_{\tilde{f}_{1}}^{\tilde{W}_{j}} \frac{g}{\sqrt{2}}\left|m_{\tilde{Z}_{i}}\right| m_{f^{\prime}}(-1)^{\theta_{i}}, \\
& \mathcal{V}_{W \tilde{f}_{1}}^{(2)}=-2 C_{\tilde{W} \tilde{Z} \tilde{Z}^{2}}^{R} \alpha_{\tilde{Z}_{i} \tilde{f}_{1}}^{d} \beta_{\tilde{f}_{1}} \beta_{\tilde{W}_{j}} \frac{g}{\sqrt{2}} m_{f}\left|m_{\tilde{W}_{j}}\right|, \\
& \mathcal{V}_{W \tilde{f}_{1}}^{(3)}=-2 C_{\tilde{W} \tilde{Z} W}^{L} \alpha_{\tilde{Z}_{i} \tilde{f}_{1}}^{d} \alpha_{\tilde{f}_{1}}^{\tilde{W}_{j}} \frac{g}{\sqrt{2}}(-1)^{\theta_{i}}(-1)^{\theta_{j}}, \\
& \mathcal{V}_{W \tilde{f}_{1}}^{(4)}=4 C_{\tilde{W} \tilde{Z} W}^{L} \alpha_{\tilde{Z}_{i} \tilde{f}_{1}}^{d} \beta_{\tilde{f}_{1}}^{\tilde{W}_{j}} \frac{g}{\sqrt{2}}\left|m_{\tilde{Z}_{i}}\right| m_{f}(-1)^{\theta_{i}}(-1)^{\theta_{j}}, \\
& \mathcal{V}_{W \tilde{f}_{1}}^{(5)}=-4 C_{\tilde{W} \tilde{Z} W}^{L} \beta_{\tilde{Z}_{i} \tilde{f}_{1}}^{d} \alpha_{\tilde{f}_{1}}^{\tilde{W}_{j}} \frac{g}{\sqrt{2}} m_{f^{\prime}}\left|m_{\tilde{W}_{j}}\right|(-1)^{\theta_{j}} .
\end{aligned}
$$

Then the integrals are exactly as for $W \tilde{f}^{\prime}{ }_{1}$ but with the changes $m_{f^{\prime}} \leftrightarrow m_{f}, m_{\tilde{f}_{1}^{\prime} 1} \rightarrow m_{\tilde{f}_{1}}$. $\Gamma_{W \tilde{f}_{1}}$ is, as above, just the sum of the products of coupling combinations and corresponding integrals.

$\Gamma_{W \tilde{f}_{2}}$

The coupling combinations now are:

$$
\begin{aligned}
& \mathcal{V}_{W \tilde{f}_{2}}^{(6)}=2 C_{\tilde{W} \tilde{Z} W}^{R} \alpha_{\tilde{Z}_{i} \tilde{f}_{2}}^{d} \frac{g}{\sqrt{2}} \alpha_{\tilde{f}_{2}}^{\tilde{W}_{j}}\left|m_{\tilde{Z}_{i}} \| m_{\tilde{W}_{j}}\right|(-1)^{\theta_{j}}(-1)^{\theta_{c}}, \\
& \mathcal{V}_{W \tilde{f}_{2}}^{(7)}=-2 C_{\tilde{W} \tilde{Z} W}^{R} \beta_{\tilde{Z}_{i} \tilde{f}_{2}}^{d} \frac{g}{\sqrt{2}} \beta_{\tilde{f}_{2}}^{\tilde{W}_{j}} m_{f} m_{f^{\prime}}(-1)^{\theta_{c}}, \\
& \mathcal{V}_{W \tilde{f}_{2}}^{(8)}=8 C_{\tilde{W} \tilde{Z} W}^{L} \beta_{\tilde{Z}_{i} \tilde{f}_{2}}^{d} \frac{g}{\sqrt{2}} \beta_{\tilde{f}_{2}}\left|m_{\tilde{Z}_{i}}\right| m_{f} m_{f^{\prime}}\left|m_{\tilde{W}_{j}}\right|(-1)^{\theta_{i}}(-1)^{\theta_{j}}(-1)^{\theta_{c}} .
\end{aligned}
$$


Again, here some of the coupling combinations depend upon which way around the decay occurs, i.e. neutralino to chargino or chargino to neutralino, for neutralino decaying:

$$
\begin{aligned}
& \mathcal{V}_{W \tilde{f}_{2}}^{(1)}=-2 C_{\tilde{W} \tilde{Z} W}^{R} \alpha_{\tilde{Z}_{i} \tilde{f}_{2}}^{d} \frac{g}{\sqrt{2}} \beta_{\tilde{f}_{2}}^{\tilde{W}_{j}}\left|m_{\tilde{Z}_{i}}\right| m_{f^{\prime}}, \\
& \mathcal{V}_{W \tilde{f}_{2}}^{(2)}=2 C_{\tilde{W} \tilde{Z} W}^{R} \beta_{\tilde{Z}_{i} \tilde{f}_{2}}^{d} \frac{g}{\sqrt{2}} \alpha_{\tilde{f}_{2}}^{\tilde{W}_{j}} m_{f}\left|m_{\tilde{W}_{j}}\right|(-1)^{\theta_{j}}, \\
& \mathcal{V}_{W \tilde{f}_{2}}^{(3)}=-2 C_{\tilde{W} \tilde{Z} W}^{R} \alpha_{\tilde{Z}_{i} \tilde{f}_{2}}^{d} \frac{g}{\sqrt{2}} \alpha_{\tilde{f}_{2}}^{\tilde{W}_{j}}, \\
& \mathcal{V}_{W \tilde{f}_{2}}^{(4)}=-4 C_{\tilde{W} \tilde{Z} W}^{L} \beta_{\tilde{Z}_{i} \tilde{f}_{2}}^{d} \frac{g}{\sqrt{2}} \alpha_{\tilde{f}_{2}}^{\tilde{W}_{j}}\left|m_{\tilde{Z}_{i}}\right| m_{f}(-1)^{\theta_{i}}, \\
& \mathcal{V}_{W \tilde{f}_{2}}^{(5)}=4 C_{\tilde{W} \tilde{Z} W}^{L} \alpha_{\tilde{Z}_{i} \tilde{f}_{2}}^{d} \frac{g}{\sqrt{2}} \beta_{\tilde{f}_{2}}^{\tilde{W}_{j}} m_{f^{\prime}}\left|m_{\tilde{W}_{j}}\right|(-1)^{\theta_{i}}(-1)^{\theta_{j}},
\end{aligned}
$$

whilst if it's a chargino decaying:

$$
\begin{aligned}
& \mathcal{V}_{W \tilde{f}_{2}}^{(1)}=-2 C_{\tilde{W} \tilde{Z} W}^{R} \beta_{\tilde{Z}_{i} \tilde{f}_{2}}^{d} \alpha_{\tilde{f}_{2}}^{\tilde{W}_{j}} \frac{g}{\sqrt{2}}\left|m_{\tilde{Z}_{i}}\right| m_{f^{\prime}}(-1)^{\theta_{i}}(-1)^{\theta_{j}}(-1)^{\theta_{c}}, \\
& \mathcal{V}_{W \tilde{f}_{2}}^{(2)}=2 C_{\tilde{W} \tilde{Z} W}^{R} \alpha_{\tilde{Z}_{i} \tilde{f}_{2}}^{d} \beta_{\tilde{f}_{2}}^{\theta W_{j}} \frac{g}{\sqrt{2}} m_{f}\left|m_{\tilde{W}_{j}}\right|(-1)^{\theta_{c}}, \\
& \mathcal{V}_{W \tilde{f}_{2}}^{(3)}=-2 C_{\tilde{W} \tilde{Z} W}^{L} \alpha_{\tilde{Z}_{i} \tilde{f}_{2}}^{d} \alpha_{\tilde{f}_{j}}^{\tilde{W}_{j}} \frac{g}{\sqrt{2}}(-1)^{\theta_{i}}(-1)^{\theta_{c}}, \\
& \mathcal{V}_{W \tilde{f}_{2}}^{(4)}=-4 C_{\tilde{W} \tilde{Z} W}^{L} \alpha_{\tilde{Z}_{i} \tilde{f}_{2}}^{d} \beta_{\tilde{f}_{2}}^{\tilde{W}_{j}} \frac{g}{\sqrt{2}}\left|m_{\tilde{Z}_{i}}\right| m_{f}(-1)^{\theta_{i}}(-1)^{\theta_{j}}(-1)^{\theta_{c}}, \\
& \mathcal{V}_{W \tilde{f}_{2}}^{(5)}=4 C_{\tilde{W} \tilde{Z} W}^{L} \beta_{\tilde{Z}_{i} \tilde{f}_{2}}^{d} \alpha_{\tilde{f}_{2}}^{\tilde{W}_{j}} \frac{g}{\sqrt{2}} m_{f^{\prime}}\left|m_{\tilde{W}_{j}}\right|(-1)^{\theta_{c}} .
\end{aligned}
$$

Then the integrals, and indeed the overall expression for $\Gamma_{W \tilde{f}_{2}}$, are just like that for $W \tilde{f}_{1}$ but with the expected replacement $m_{\tilde{f}_{1}} \rightarrow m_{\tilde{f}_{2}}$.

$\Gamma_{H^{ \pm} G}$

The coupling combinations are:

$$
\begin{aligned}
& \mathcal{V}_{H^{ \pm} G}^{(1)}=\omega_{G \tilde{W} \tilde{Z}}^{L} \omega_{H^{+} \tilde{W}^{+} \tilde{Z}}^{L}+\omega_{G \tilde{W} \tilde{Z}}^{R} \omega_{H^{+} \tilde{W}+\tilde{Z}}^{R}, \\
& \mathcal{V}_{H^{ \pm} G}^{(2)}=\left(\omega_{G \tilde{W} \tilde{Z}}^{R} \omega_{H^{+} \tilde{W}^{+} \tilde{Z}}^{L}+\omega_{G \tilde{W} \tilde{Z}}^{L} \omega_{H^{+} \tilde{W}^{+} \tilde{Z}}^{R}\right)(-1)^{\theta_{i}}(-1)^{\theta_{j}}, \\
& \mathcal{V}_{H^{ \pm} G}^{(3)}=C_{G f f^{\prime}}^{u} C_{H^{+} f f^{\prime}}^{u}+C_{G f f^{\prime}}^{d} C_{H^{+} f f^{\prime}}^{d}, \\
& \mathcal{V}_{H^{ \pm} G}^{(4)}=C_{G f f^{\prime}}^{d} C_{H^{+} f f^{\prime}}^{u}+C_{G f f^{\prime}}^{u} C_{H^{+} f f^{\prime}}^{d},
\end{aligned}
$$

and the integrals with $s=m_{\tilde{Z}_{i}}^{2}+m_{\tilde{W}_{j}}^{2}-2\left|m_{\tilde{Z}_{i}}\right| E, \lambda=\sqrt{\left(s-\left(m_{f}+m_{f^{\prime}}\right)^{2}\right)\left(s-\left(m_{f}-m_{f^{\prime}}\right)^{2}\right)}$ are:

$$
\begin{gathered}
I_{H^{ \pm} G}^{1}=2\left|m_{\tilde{Z}_{i}}\right| \int_{\left|m_{\tilde{W}_{j}}\right|}^{E_{\text {upper } 3}} d E \frac{\lambda \sqrt{E^{2}-m_{\tilde{W}_{j}}^{2}}}{s\left(s-m_{W}^{2}\right)\left(s-m_{H^{ \pm}}^{2}\right)}, \\
I_{H^{ \pm} G}^{2}=2\left|m_{\tilde{Z}_{i}}\right| \int_{\left|m_{\tilde{W}_{j}}\right|}^{E_{u p p e r 3}} d E \frac{2\left|m_{\tilde{Z}_{i}}\right| \lambda \sqrt{E^{2}-m_{\tilde{W}_{j}}^{2}}\left(s-m_{f}^{2}-m_{f^{\prime}}^{2}\right)}{s\left(s-m_{W}^{2}\right)\left(s-m_{H^{ \pm}}^{2}\right)}, \\
I_{H^{ \pm} G}^{3}=2\left|m_{\tilde{Z}_{i} \mid}\right| \int_{\left|m_{\tilde{w}_{j}}\right|}^{E_{u p p e r 3}} d E \frac{2\left|m_{\tilde{Z}_{i}}\right| \lambda \sqrt{E^{2}-m_{\tilde{W}_{j}}^{2}} 2\left|m_{\tilde{Z}_{i}}\right| E}{s\left(s-m_{W}^{2}\right)\left(s-m_{H^{ \pm}}^{2}\right)},
\end{gathered}
$$




$$
I_{H^{ \pm} G}^{4}=2\left|m_{\tilde{Z}_{i}}\right| \int_{\left|m_{\tilde{W}_{j}}\right|}^{E_{\text {upper3 }} \mid} d E \frac{2\left|m_{\tilde{Z}_{i}}\right| \lambda \sqrt{E^{2}-m_{\tilde{W}_{j}}^{2}}\left(s-m_{f}^{2}-m_{f^{\prime}}^{2}\right) 2\left|m_{\tilde{Z}_{i}}\right| E}{s\left(s-m_{W}^{2}\right)\left(s-m_{H^{ \pm}}^{2}\right)} .
$$

The overall contribution is then:

$$
\begin{aligned}
\Gamma_{H^{ \pm} G}= & \mathcal{V}_{H^{ \pm} G}^{(1)} \mathcal{V}_{H^{ \pm} G}^{(3)} I_{H^{ \pm} G}^{4}-2 \mathcal{V}_{H^{ \pm} G}^{(1)} \mathcal{V}_{H^{ \pm} G}^{(4)} m_{f} m_{f^{\prime}} I_{H^{ \pm} G}^{3}+2 \mathcal{V}_{H^{ \pm} G}^{(2)} \mathcal{V}_{H^{ \pm} G}^{(3)}\left|m_{\tilde{Z}_{i}} \| m_{\tilde{W}_{j}}\right| I_{H^{ \pm} G}^{2} \\
& -4 \mathcal{V}_{H^{ \pm} G}^{(2)} \mathcal{V}_{H^{ \pm} G}^{(4)}\left|m_{\tilde{Z}_{i}} \| m_{\tilde{W}_{j}}\right| m_{f} m_{f^{\prime}} I_{H^{ \pm} G}^{1}(-1)^{\theta_{j}} .
\end{aligned}
$$

$\Gamma_{G \tilde{f} \tilde{f}^{\prime}}$

Here the required coupling combinations are dependent on whether it's neutralino to chargino or chargino to neutralino. For a neutralino decaying:

$$
\begin{aligned}
& \mathcal{V}_{G \tilde{f}^{\prime} 1}^{(1)}=\frac{1}{2}\left(\omega_{G \tilde{W} \tilde{Z}}^{R} \alpha_{\tilde{Z}_{i} \tilde{f}_{1}}^{u} C_{G f f^{\prime}}^{d} \beta_{\tilde{f}^{\prime} 1}^{\tilde{W}_{j}}+\omega_{G \tilde{W} \tilde{Z}}^{L} \beta_{\tilde{Z}_{i} \tilde{f}_{1}}^{u} C_{G f f^{\prime}}^{u} \alpha_{\tilde{f}^{\prime}{ }_{1}}^{\tilde{W}_{j}}\right), \\
& \mathcal{V}_{G \tilde{f}^{\prime}{ }_{1}}^{(2)}=-\left(\omega_{G \tilde{W} \tilde{Z}^{\prime}}^{R} \beta_{\tilde{Z}_{i} \tilde{f}_{1}}^{u} C_{G f f^{\prime}}^{u}, \beta_{\tilde{f}^{\prime}{ }_{1}}^{\tilde{W}_{j}}+\omega_{G \tilde{W} \tilde{Z}}^{L} \alpha_{\tilde{Z}_{i} \tilde{f}_{1}}^{u} C_{G f f^{\prime}}^{d} \alpha_{\tilde{f}^{\prime}{ }_{1}}^{\tilde{W}_{j}}\right) m_{f}\left|m_{\tilde{Z}_{i}}\right|, \\
& \mathcal{V}_{G \tilde{f}_{1}^{\prime}{ }_{1}^{(3)}}^{(3)}=\left(\omega_{G \tilde{W} \tilde{Z}}^{L} \beta_{\tilde{Z}_{i} \tilde{f}_{1}}^{u} C_{G f f^{\prime}}^{d}, \beta_{\tilde{f}^{\prime}{ }_{1}}^{\tilde{W}_{j}}+\omega_{G \tilde{W} \tilde{Z}}^{R} \alpha_{\tilde{Z}_{i} \tilde{f}_{1}}^{u} C_{G f f^{\prime}}^{u}, \alpha_{\tilde{f}^{\prime}{ }_{1}}^{\tilde{W}_{j}}\right)(-1)^{\theta_{i}} m_{f^{\prime}}\left|m_{\tilde{W}_{j}}\right|,
\end{aligned}
$$

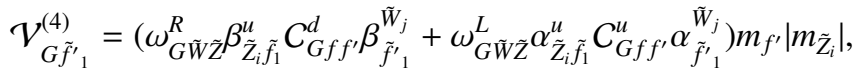

$$
\begin{aligned}
& \mathcal{V}_{G \tilde{f}_{1} \tilde{f}_{1}}^{(5)}=\left(\omega_{G \tilde{W} \tilde{Z}}^{L} \beta_{\tilde{Z}_{i} \tilde{f}_{1}}^{u} C_{G f f^{\prime}}^{u}, \beta_{\tilde{f}^{\prime} 1}^{\tilde{W}_{j}}-(-1)^{\theta_{i}} \omega_{G \tilde{W} \tilde{Z}}^{R} \alpha_{\tilde{Z}_{i} \tilde{f}_{1}}^{u} C_{G f f^{\prime}}^{d} \alpha_{\tilde{f}^{\prime},}^{\tilde{W}_{1}}\right) m_{f}\left|m_{\tilde{W}_{j}}\right| \text {, } \\
& \mathcal{V}_{G \tilde{f}_{1}^{\prime}}^{(6)}=(-1)^{\theta_{i}}\left(\omega_{G \tilde{W} \tilde{Z}^{2}}^{L} \alpha_{\tilde{Z}_{i} \tilde{f}_{1}}^{u} C_{G f f^{\prime}}^{d} \beta_{\tilde{f}^{\prime}{ }_{1}}^{\tilde{W}_{j}}+\omega_{G \tilde{W} \tilde{Z}^{\prime}}^{R} \beta_{\tilde{Z}_{i} \tilde{f}_{1}}^{u} C_{G f f^{\prime}}^{u}, \alpha_{\tilde{f}^{\prime}{ }_{1}}^{\tilde{W}_{j}}\right)\left|m_{\tilde{Z}_{i}} \| m_{\tilde{W}_{j}}\right|,
\end{aligned}
$$

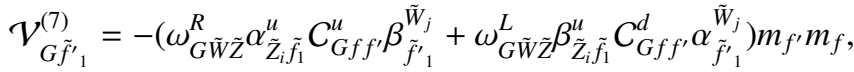

$$
\begin{aligned}
& \mathcal{V}_{G \tilde{f}_{1}^{\prime}}^{(8)}=2\left(\omega_{G \tilde{W} \tilde{Z}}^{L} \alpha_{\tilde{Z}_{i} \tilde{f}_{1}}^{u} C_{G f f^{\prime}}^{u} \beta_{\tilde{f}^{\prime}{ }_{1}}^{\tilde{W}_{j}}+(-1)^{\theta_{i}} \omega_{G \tilde{W} \tilde{Z}}^{R} \beta_{\tilde{Z}_{i} \tilde{f}_{1}}^{u} C_{G f f^{\prime}}^{d} \alpha_{\tilde{f}^{\prime} 1}^{\tilde{W}_{j}}\right)\left|m_{\tilde{Z}_{i}}\right| m_{f^{\prime}} m_{f}\left|m_{\tilde{W}_{j}}\right| .
\end{aligned}
$$

For a chargino decaying:

$$
\begin{aligned}
& \mathcal{V}_{G \tilde{f}^{\prime}{ }_{1}}^{(1)}=-\frac{1}{2}\left(\omega_{G \tilde{W} \tilde{Z}}^{L} \alpha_{\tilde{Z}_{i} \tilde{f}_{1}}^{u} C_{G f f^{\prime}}^{u} \beta_{\tilde{f}^{\prime}{ }_{1}}^{\tilde{W}_{j}}+(-1)^{\theta_{f}} \omega_{G \tilde{W} \tilde{Z}^{\prime}}^{R} \beta_{\tilde{Z}_{i} \tilde{f}_{1}}^{u} C_{G f f^{\prime}}^{d} \alpha_{\tilde{f}^{\prime}{ }_{1}}^{\tilde{W}_{j}}\right), \\
& \mathcal{V}_{G \tilde{f}_{1},}^{(2)}=\left(\omega_{G \tilde{W} \tilde{Z}}^{L} \alpha_{\tilde{Z}_{i} \tilde{f}_{1}}^{u} C_{G f f^{\prime}}^{d} \alpha_{\tilde{f}^{\prime}, 1}^{\tilde{W}_{j}}+(-1)^{\theta_{j}} \omega_{G \tilde{W} \tilde{Z}}^{R} \beta_{\tilde{Z}_{i} \tilde{f}_{1}}^{u} C_{G f f^{\prime}}^{u}, \beta_{\tilde{f}^{\prime}, 1}^{\tilde{W}_{j}}\right) m_{f^{\prime}}\left|m_{\tilde{Z}_{i}}\right|, \\
& \mathcal{V}_{G \tilde{f}^{\prime} 1}^{(3)}=-\left(\omega_{G \tilde{W} \tilde{Z}^{2}}^{R} \alpha_{\tilde{Z}_{i} \tilde{f}_{1}}^{u} C_{G f f^{\prime}}^{u} \alpha_{\tilde{f}^{\prime}{ }_{1}}^{\tilde{W}_{j}}+(-1)^{\theta_{j}} \omega_{G \tilde{W} \tilde{Z}^{\prime}}^{L} \beta_{\tilde{Z}_{i} \tilde{f}_{1}}^{u} C_{G f f^{\prime}}^{d}, \beta_{\tilde{f}^{\prime}{ }_{1}}^{\tilde{W}_{j}}\right)(-1)^{\theta_{j}} m_{f}\left|m_{\tilde{W}_{j}}\right| \text {, } \\
& \mathcal{V}_{G \tilde{f}_{1}}^{(4)}=-\left(\omega_{G \tilde{W} \tilde{Z}}^{L} \alpha_{\tilde{Z}_{i} \tilde{f}_{1}}^{u} C_{G f f^{\prime}}^{u} \alpha_{\tilde{f}^{\prime} 1}^{\tilde{W}_{j}}+(-1)^{\theta_{j}} \omega_{G \tilde{W} \tilde{Z}^{2}}^{R} \beta_{\tilde{Z}_{i} \tilde{f}_{1}}^{u} C_{G f f^{\prime}}^{d} \beta_{\tilde{f}^{\prime}{ }_{1}}^{\tilde{W}_{j}}\right) m_{f}\left|m_{\tilde{Z}_{i}}\right|, \\
& \mathcal{V}_{G \tilde{f}_{1}^{\prime}{ }_{1}}^{(5)}=\left((-1)^{\theta_{j}} \omega_{G \tilde{W} \tilde{Z}^{2}}^{R} \alpha_{\tilde{Z}_{i} \tilde{f}_{1}}^{u} C_{G f f^{\prime}}^{d} \alpha_{\tilde{f}^{\prime}{ }_{1}}^{\tilde{W}_{j}}+\omega_{G \tilde{W} \tilde{Z}}^{L} \beta_{\tilde{Z}_{i} \tilde{f}_{1}}^{u} C_{G f f^{\prime}}^{u} \beta_{\tilde{f}^{\prime}{ }_{1}}^{\tilde{W}_{j}}\right) m_{f^{\prime}}\left|m_{\tilde{W}_{j}}\right|, \\
& \mathcal{V}_{G \tilde{f}_{1}^{\prime}}^{(6)}=-\left((-1)^{\theta_{j}} \omega_{G \tilde{W} \tilde{Z}}^{R} \alpha_{\tilde{Z}_{i} \tilde{f}_{1}}^{u} C_{G f f^{\prime}}^{u} \beta_{\tilde{f}^{\prime}{ }_{1}}^{\tilde{W}_{j}}+\omega_{G \tilde{W} \tilde{Z}}^{L} \beta_{\tilde{Z}_{i} \tilde{f}_{1}}^{u} C_{G f f^{\prime}}^{d} \alpha_{\tilde{f}^{\prime}{ }_{1}}^{\tilde{W}_{j}}\right)\left|m_{\tilde{Z}_{i}} \| m_{\tilde{W}_{j}}\right|, \\
& \mathcal{V}_{G \tilde{f}_{1}^{\prime}}^{(7)}=\left(\omega_{G \tilde{W} \tilde{Z}}^{L} \alpha_{\tilde{Z}_{i} \tilde{f}_{1}}^{u} C_{G f f}^{d} C_{\tilde{f}^{\prime}{ }_{1}}^{\tilde{W}_{j}}+(-1)^{\theta_{j}} \omega_{G \tilde{W} \tilde{Z}^{\prime}}^{R} \beta_{\tilde{Z}_{i} \tilde{f}_{1}}^{u} C_{G f f^{\prime}}^{u} \alpha_{\tilde{f}^{\prime}{ }_{1}}^{\tilde{W}_{j}}\right) m_{f^{\prime}} m_{f}, \\
& \mathcal{V}_{G \tilde{f}_{1}^{\prime}}^{(8)}=2\left((-1)^{\theta_{j}} \omega_{G \tilde{W} \tilde{Z}}^{R} \alpha_{\tilde{Z}_{i} \tilde{f}_{1}}^{u} C_{G f f^{\prime}}^{d}, \beta_{\tilde{f}^{\prime}{ }_{1}}^{\tilde{W}_{j}}+\omega_{G \tilde{W} \tilde{Z}}^{L} \beta_{\tilde{Z}_{i} \tilde{f}_{1}}^{u} C_{G f f^{\prime}}^{u} \alpha_{\tilde{f}^{\prime},}^{\tilde{W}_{j}}\right)\left|m_{\tilde{Z}_{i}}\right| m_{f^{\prime}} m_{f}\left|m_{\tilde{W}_{j}}\right| .
\end{aligned}
$$

The integrals necessary are, for neutralino decaying with $s=m_{\tilde{Z}_{i}}^{2}+m_{\tilde{W}_{j}}^{2}-2\left|m_{\tilde{Z}_{i}}\right| E, \lambda=\sqrt{\left(s-\left(m_{f^{\prime}}+m_{f}\right)^{2}\right)\left(s-\left(m_{f^{\prime}}-m_{f}\right)^{2}\right)}, A=m_{f}^{2}+m_{f^{\prime}}^{2}+2\left|m_{\tilde{Z}_{i}}\right| E+\left(m_{\tilde{Z}_{i}}^{2}-m_{\tilde{W}_{j}}^{2}\right)\left(m_{f}^{2}-m_{f^{\prime}}^{2}\right) / s$, 
$\left.B=2\left|m_{\tilde{Z}_{i}}\right| \lambda / s \sqrt{E^{2}-m_{\tilde{W}_{j}}^{2}}, Z=\frac{\left(\frac{1}{2}(A+B)-m_{\tilde{f}^{\prime}}^{2}\right)}{\left(\frac{1}{2}(A-B)-m_{\tilde{f}^{\prime} 1}^{2}\right.}\right)$, given by:

$$
\begin{aligned}
& I_{G \tilde{f}_{1}^{\prime}}^{1}=2\left|m_{\tilde{Z}_{i}}\right| \int_{\left|m_{\tilde{W}_{j}}\right|}^{E_{\text {upper } 3}} d E \frac{2\left[s B+\left(m_{\tilde{f}_{1}^{\prime}}^{2} s-m_{\tilde{Z}_{i}} m_{f}^{2}-m_{f}{ }^{2} m_{\tilde{W}_{j}}^{2}\right) \log (Z)\right]}{s-m_{W}^{2}}, \\
& I_{G \tilde{f}^{\prime}{ }_{1}}^{2}=-2\left|m_{\tilde{Z}_{i}}\right| \int_{\left|m_{\tilde{W}_{j}}\right|}^{E_{\text {upper } 3}} d E \frac{\left[B+\left(m_{\tilde{f}^{\prime}{ }_{1}}^{2}+m_{\tilde{W}_{j}}^{2}-2\left|m_{\tilde{Z}_{i}}\right| E-m_{f}^{2}\right) \log (Z)\right]}{s-m_{W}^{2}}, \\
& I_{G \tilde{f}_{1}^{\prime}{ }_{1}}^{3}=2\left|m_{\tilde{Z}_{i}}\right| \int_{\left|m_{\tilde{W}_{j}}\right|}^{E_{\text {upper } 3}} d E \frac{\left[B+\left(m_{\tilde{f}_{1}^{\prime}}+m_{\tilde{Z}_{i}}^{2}-2\left|m_{\tilde{Z}_{i}}\right| E-m_{f^{\prime}}^{2}\right) \log (Z)\right]}{s-m_{W}^{2}}, \\
& I_{G \tilde{f}^{\prime}{ }_{1}}^{4}=2\left|m_{\tilde{Z}_{i}}\right| \int_{\left|m_{\tilde{W}_{j}}\right|}^{E_{\text {upper } 3}} d E \frac{\left[B+\left(m_{\tilde{f}_{1}^{\prime}}^{2}-m_{f}^{2}-m_{\tilde{W}_{j}}^{2}\right) \log (Z)\right]}{s-m_{W}^{2}}, \\
& I_{G \tilde{f}^{\prime}{ }_{1}}^{5}=-2\left|m_{\tilde{Z}_{i}}\right| \int_{\left|m_{\tilde{W}_{j}}\right|}^{E_{\text {upper } 3}} d E \frac{\left[B+\left(m_{\tilde{f}^{\prime}{ }_{1}}^{2}-m_{\tilde{Z}_{i}}^{2}-m_{f^{\prime}}^{2}\right) \log (Z)\right]}{s-m_{W}^{2}}, \\
& I_{G \tilde{f}_{1}^{\prime}}^{6}=2\left|m_{\tilde{Z}_{i}}\right| \int_{\left|m_{\tilde{W}_{j}}\right|}^{E_{\text {upper } 3}} d E \frac{\left(s-m_{f^{\prime}}^{2}-m_{f}^{2}\right) \log (Z)}{s-m_{W}^{2}}, \\
& I_{G \tilde{f}^{\prime}{ }_{1}}^{7}=2\left|m_{\tilde{Z}_{i}}\right| \int_{\left|m_{\tilde{w}_{j}}\right|}^{E_{\text {upper3 } 3}} d E \frac{2\left|m_{\tilde{Z}_{i}}\right| E \log (Z)}{s-m_{W}^{2}}, \\
& I_{G \tilde{f}^{\prime}{ }_{1}}^{8}=2\left|m_{\tilde{Z}_{i}}\right| \int_{\left|m_{\tilde{w}_{j}}\right|}^{E_{\text {upper } 3}} d E \frac{\log (Z)}{s-m_{W}^{2}} .
\end{aligned}
$$

For a chargino decaying the integrals have the same expressions but one must swap integrals 2 and 4 and integrals 3 and 5. The overall contribution is the product of each coupling combination with the corresponding integral:

$$
\begin{aligned}
& \Gamma_{G \tilde{f}^{\prime}{ }_{1}}=\mathcal{V}_{G \tilde{f}_{1}{ }_{1}}^{(1)} I_{G \tilde{f}^{\prime}{ }_{1}}^{1}+\mathcal{V}_{G \tilde{f}^{\prime}{ }_{1}}^{(2)} I_{G \tilde{f}^{\prime}{ }_{1}}^{2}+\mathcal{V}_{G \tilde{f}^{\prime}{ }_{1}}^{(3)} I_{G \tilde{f}^{\prime}{ }_{1}}^{3}+\mathcal{V}_{G \tilde{f}_{1}{ }_{1}}^{(4)} I_{G \tilde{f}^{\prime}{ }_{1}}^{4}
\end{aligned}
$$

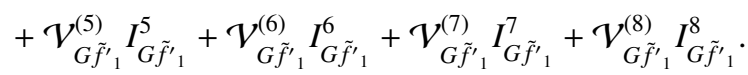

$\Gamma_{G \tilde{f}^{\prime}{ }_{2}}$

$\overline{\text { Again }}$ here the coupling combinations depend upon if we are considering neutralino to chargino or chargino to neutralino, for neutralino decaying:

$$
\begin{aligned}
& \mathcal{V}_{G \tilde{f}_{2}^{\prime}}^{(1)}=-\frac{1}{2}(-1)^{\theta_{i}}\left[-(-1)^{\theta_{i}} \omega_{G \tilde{W} \tilde{Z}^{\prime}}^{R} \alpha_{\tilde{Z}_{i} \tilde{f}_{2}}^{u} C_{G f f^{\prime}}^{d} \beta_{\tilde{f}^{\prime}{ }_{2}}^{\tilde{W}_{j}}+\omega_{G \tilde{W} \tilde{Z}^{\prime}}^{L} \beta_{\tilde{Z}_{i} \tilde{f}_{2}}^{u} C_{G f f^{\prime}}^{u} \alpha_{\tilde{f}^{\prime}{ }_{2}}^{\tilde{W}_{j}}\right], \\
& \mathcal{V}_{G \tilde{f}_{2}^{\prime}}^{(2)}=(-1)^{\theta_{i}}\left(\omega_{G \tilde{W} \tilde{Z}^{2}}^{R} \beta_{\tilde{Z}_{i} \tilde{f}_{2}}^{u} C_{G f f^{\prime}}^{u} \beta_{\tilde{f}^{\prime}{ }_{2}}^{\tilde{W}_{j}}-\omega_{G \tilde{W} \tilde{Z}}^{L} \alpha_{\tilde{Z}_{i} \tilde{f}_{2}}^{u} C_{G f f^{\prime}}^{d} \alpha_{\tilde{f}^{\prime}{ }_{2}}^{\tilde{W}_{j}}\right)\left|m_{\tilde{Z}_{i}}\right| m_{f}, \\
& \mathcal{V}_{G \tilde{f}^{\prime}{ }_{2}}^{(3)}=-\left(\omega_{G \tilde{W} \tilde{Z}}^{L} \beta_{\tilde{Z}_{i} \tilde{f}_{2}}^{u} C_{G f f^{\prime}}^{d} \beta_{\tilde{f}^{\prime}{ }_{2}}^{\tilde{W}_{j}}-\omega_{G \tilde{W} \tilde{Z}^{2}}^{R} \alpha_{\tilde{Z}_{i} \tilde{f}_{2}}^{u} C_{G f f^{\prime}}^{u} \alpha_{\tilde{f}^{\prime}{ }_{2}}^{\tilde{W}_{j}}\right) m_{f^{\prime}}\left|m_{\tilde{W}_{j}}\right|(-1)^{\theta_{j}}, \\
& \mathcal{V}_{G \tilde{f}^{\prime}{ }_{2}}^{(4)}=-\left(\omega_{G \tilde{W} \tilde{Z}}^{R} \beta_{\tilde{Z}_{i} \tilde{f}_{2}}^{u} C_{G f f^{\prime}}^{d} \beta_{\tilde{f}^{\prime}{ }_{2}}^{\tilde{W}_{j}}-(-1)^{\theta_{i}} \omega_{G \tilde{W} \tilde{Z}^{2}}^{L} \alpha_{\tilde{Z}_{i} \tilde{f}_{2}}^{u} C_{G f f^{\prime}}^{u} \alpha_{\tilde{f}^{\prime}{ }_{2}}^{\tilde{W}_{j}}\right)\left|m_{\tilde{Z}_{i}}\right| m_{f^{\prime}}, \\
& \mathcal{V}_{G \tilde{f}_{2}^{\prime}}^{(5)}=\left(\omega_{G \tilde{W} \tilde{Z}^{2}}^{L} \beta_{\tilde{Z}_{i} \tilde{f}_{2}}^{u} C_{G f f^{\prime}}^{u} \beta_{\tilde{f}^{\prime}{ }_{2}}^{\tilde{W}_{j}}+\omega_{G \tilde{W} \tilde{Z}^{\prime}}^{R} \alpha_{\tilde{Z}_{i} \tilde{f}_{2}}^{u} C_{G f f^{\prime}}^{d} \alpha_{\tilde{f}_{2}^{\prime}}^{\tilde{W}_{j}}\right) m_{f}\left|m_{\tilde{W}_{j}}\right|(-1)^{\theta_{j}}, \\
& \mathcal{V}_{G \tilde{f}_{1}^{\prime}{ }_{1}}^{(6)}=\left[\omega_{G \tilde{W} \tilde{Z}}^{L} \alpha_{\tilde{Z}_{i} \tilde{f}_{2}}^{u} C_{G f f^{\prime}}^{d} \beta_{\tilde{f}^{\prime}{ }_{2}}^{\tilde{W}_{j}}-(-1)^{\theta_{i}} \omega_{G \tilde{W} \tilde{Z}}^{R} \beta_{\tilde{Z}_{i} \tilde{f}_{2}}^{u} C_{G f f^{\prime}}^{u} \alpha_{\tilde{f}^{\prime}{ }_{2}}^{\tilde{W}_{j}}\right]\left|m_{\tilde{Z}_{i}}\right|\left|m_{\tilde{W}_{j}}\right|, \\
& \mathcal{V}_{G \tilde{f}_{2}^{\prime}}^{(7)}=\left[-(-1)^{\theta_{i}} \omega_{G \tilde{W} \tilde{Z}}^{R} \alpha_{\tilde{Z}_{i} \tilde{f}_{2}}^{u} C_{G f f^{\prime}}^{u} \beta_{\tilde{f}^{\prime}{ }_{2}}^{\tilde{W}_{j}}+\omega_{G \tilde{W} \tilde{Z}^{2}}^{L} \beta_{\tilde{Z}_{i} \tilde{f}_{2}}^{u} C_{G f f^{\prime}}^{d} \alpha_{\tilde{f}_{2}^{\prime}}^{\tilde{W}_{j}}\right] m_{f^{\prime}} m_{f}, \\
& \mathcal{V}_{G \tilde{f}_{2}^{\prime}{ }_{2}}^{(8)}=2\left[-\omega_{G \tilde{W} \tilde{Z}^{2}}^{L} \alpha_{\tilde{Z}_{i} \tilde{f}_{2}}^{u} C_{G f f^{\prime}}^{u} \beta_{\tilde{f}^{\prime}{ }_{2}}^{\tilde{W}_{j}}+(-1)^{\theta_{i}} \omega_{G \tilde{W} \tilde{Z}}^{R} \beta_{\tilde{Z}_{i} \tilde{f}_{2}}^{u} C_{G f f^{\prime}}^{d} \alpha_{\tilde{f}^{\prime}{ }_{2}}^{\tilde{W}_{j}}\right]\left|m_{\tilde{Z}_{i}}\right| m_{f^{\prime}} m_{f}\left|m_{\tilde{W}_{j}}\right| \text {, }
\end{aligned}
$$


whilst if it's a chargino decaying into a neutralino:

$$
\begin{aligned}
& \mathcal{V}_{G \tilde{f}^{\prime} 2}^{(1)}=\frac{1}{2}\left[\omega_{G \tilde{W} \tilde{Z}^{2}}^{L} \beta_{\tilde{f}_{2}^{\prime}}^{\tilde{W}_{j}} C_{G f f^{\prime}}^{u} \alpha_{\tilde{Z}_{i} \tilde{f}_{2}}^{u}(-1)^{\theta_{j}}+\omega_{G \tilde{W} \tilde{Z}}^{R} \tilde{\alpha}_{\tilde{f}^{\prime}, 2}^{\tilde{W}_{j}} C_{G f f^{\prime}}^{d} \beta_{\tilde{Z}_{i} \tilde{f}_{2}}^{u}\right], \\
& \mathcal{V}_{G \tilde{f}_{2}^{\prime} 2}^{(2)}=-\left((-1)^{\theta_{j}} \omega_{G \tilde{W} \tilde{Z}^{\prime}}^{L} \alpha_{\tilde{f}^{\prime}{ }_{2}}^{\tilde{W}_{j}} C_{G f f^{\prime}}^{d} \alpha_{\tilde{Z}_{i} \tilde{z}_{2}}^{u}+\omega_{G \tilde{W} \tilde{Z}^{\prime} \tilde{f}_{2}}^{R} \beta_{\tilde{W}_{j}}^{\tilde{W}_{j}} C_{G f f^{\prime}}^{u} \beta_{\tilde{Z}_{i} \tilde{f}_{2}}^{u}\right)\left|m_{\tilde{Z}_{i}}\right| m_{f^{\prime}}, \\
& \mathcal{V}_{G \tilde{f}_{2}^{\prime}{ }_{2}}^{(3)}=\left(\omega_{G \tilde{W} \tilde{Z}^{2}}^{R} \alpha_{\tilde{f}^{\prime}{ }_{2}}^{\tilde{W}_{j}} C_{G f f^{\prime}}^{u} \alpha_{\tilde{Z}_{i} \tilde{f}_{2}}^{u}+(-1)^{\theta_{j}} \omega_{G \tilde{W} \tilde{Z}_{2}}^{L} \beta_{\tilde{f}^{\prime}{ }_{2}}^{\tilde{W}_{j}} C_{G f f}^{d} \beta_{\tilde{Z}_{i} \tilde{z}_{2}}^{u}\right) m_{f}\left|m_{\tilde{W}_{j}}\right|, \\
& \mathcal{V}_{G \tilde{f}^{\prime} 2}^{(4)}=\left((-1)^{\theta_{j}} \omega_{G \tilde{W} \tilde{Z}}^{L} \alpha_{\tilde{f}^{\prime}{ }_{2}}^{\tilde{W}_{j}} \mathcal{C}_{G f f^{\prime}}^{u} \alpha_{\tilde{Z}_{i} \tilde{z}_{2}}^{u}+\omega_{G \tilde{W} \tilde{Z}^{\prime}}^{R} \beta_{\tilde{f}^{\prime}{ }_{2}}^{\tilde{W}_{j}} C_{G f f^{\prime}}^{d} \beta_{\tilde{Z}_{i} \tilde{f}_{2}}^{u}\right)\left|m_{\tilde{Z}_{i}}\right| m_{f},
\end{aligned}
$$

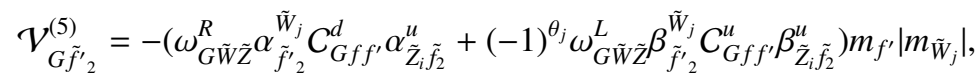

$$
\begin{aligned}
& \mathcal{V}_{G \tilde{f}_{2} 2}^{(6)}=\left[\omega_{G \tilde{W} \tilde{Z}^{\prime}}^{R} \beta_{\tilde{f}^{\prime}{ }_{2}}^{\tilde{W}_{j}} C_{G f f^{\prime}}^{u} \alpha_{\tilde{Z}_{i} \tilde{z}_{2}}^{u}+(-1)^{\theta_{j}} \omega_{G \tilde{W} \tilde{Z}^{2}}^{L} \alpha_{\tilde{f}^{\prime}{ }_{2}}^{\tilde{W}_{j}} C_{G f f}^{d} \beta_{\tilde{Z}_{i} \tilde{z}_{2}}^{u}\right]\left|m_{\tilde{W}_{j}}\right|\left|m_{\tilde{Z}_{i}}\right|, \\
& \mathcal{V}_{G \tilde{f}_{2}^{\prime}}^{(7)}=-\left[(-1)^{\theta_{j}} \omega_{G \tilde{W} \tilde{Z}^{\prime}}^{L} \beta_{\tilde{f}_{2}^{\prime} 2}^{\tilde{W}_{j}} C_{G f f^{\prime}}^{d} \alpha_{\tilde{Z}_{i} \tilde{f}_{2}}^{u}+\omega_{G \tilde{W} \tilde{Z}}^{R}{\tilde{f_{\tilde{f}}^{\prime}}}_{\tilde{W}_{j}}^{\tilde{W}_{j}} C_{G f f^{\prime}}^{u} \beta_{\tilde{Z}_{i} \tilde{f}_{2}}^{u}\right] m_{f} m_{f^{\prime}}, \\
& \mathcal{V}_{G \tilde{f}_{2}^{\prime}}^{(8)}=-2\left[\omega_{G \tilde{W} \tilde{Z}^{\prime}}^{R} \beta_{\tilde{f}^{\prime}{ }_{2}}^{\tilde{W}_{j}} C_{G f f^{\prime}}^{d} \alpha_{\tilde{Z}_{i} \tilde{z}_{2}}^{u}+(-1)^{\theta_{j}} \omega_{G \tilde{W} \tilde{Z}^{2}}^{L} \alpha_{\tilde{f}^{\prime}{ }_{2}}^{\tilde{W}_{j}} C_{G f f^{\prime}}^{u} \beta_{\tilde{Z}_{i} \tilde{f}_{2}}^{u}\right] m_{f} m_{f^{\prime}}\left|m_{\tilde{W}_{j}}\right| m_{\tilde{Z}_{i}} \mid .
\end{aligned}
$$

The integrals are exactly as for $G \tilde{f}^{\prime}{ }_{1}$ but with the change $m_{\tilde{f}^{\prime} 1} \rightarrow m_{\tilde{f}^{\prime} 2}$, and similar changes produce the overall expression for $\Gamma_{G \tilde{f}^{\prime}}$.

$\Gamma_{H^{ \pm} \tilde{f}^{\prime} 1}$

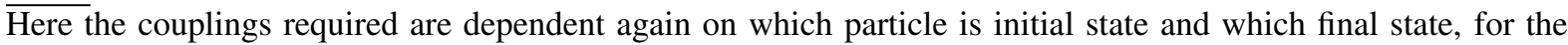
neutralino as the decaying (i.e. initial state) particle:

$$
\begin{aligned}
& \mathcal{V}_{H^{ \pm} \tilde{f}_{1}^{\prime \prime}}^{(1)}=-\frac{1}{2}\left[(-1)^{\theta_{i}} \omega_{H^{+} \tilde{W}^{+}}^{R} \tilde{Z}_{\tilde{Z}_{i} \tilde{f}_{1}}^{u} C_{H^{+} f f^{\prime}}^{d} \beta_{\tilde{f}^{\prime}{ }_{1}}^{\tilde{W}_{j}}+\omega_{H^{+} \tilde{W}^{+} \tilde{Z}^{2}}^{L} \beta_{\tilde{Z}_{i} \tilde{f}_{1}}^{u} C_{H^{+} f f^{\prime}}^{u} \alpha_{\tilde{f}^{\prime}{ }_{1}}^{\tilde{W}_{j}}\right], \\
& \mathcal{V}_{H^{ \pm} \tilde{f}_{1}^{\prime}}^{(2)}=-\left(\omega_{H^{+} \tilde{W}^{+} \tilde{Z}^{2}}^{R} \beta_{\tilde{Z}_{i} \tilde{f}_{1}}^{u} C_{H^{+} f f^{\prime}}^{u}, \beta_{\tilde{f}^{\prime}{ }_{1}}^{\tilde{W}_{j}}+\omega_{H^{+}+\tilde{W}^{+} \tilde{Z}^{L}}^{L} \alpha_{\tilde{Z}_{i} \tilde{f}_{1}}^{u} C_{H^{+} f f^{\prime}}^{d} \alpha_{\tilde{f}^{\prime}{ }_{1}}^{\tilde{W}_{j}}\right)\left|m_{\tilde{Z}_{i}}\right| m_{f}, \\
& \mathcal{V}_{H^{ \pm} \tilde{f}_{1}^{\prime}}^{(3)}=\left(\omega_{H^{+} \tilde{W^{+}+} \tilde{Z}_{\tilde{Z}_{i} \tilde{f}_{1}}^{L}}^{u} C_{H^{+} f f^{\prime}}^{d}, \beta_{\tilde{f}^{\prime} 1}^{\tilde{W}_{j}}+\omega_{H^{+} \tilde{W}^{+} \tilde{Z}^{2}}^{R} \alpha_{\tilde{z}_{i} \tilde{f}_{1}}^{u} C_{H^{+} f f^{\prime}}^{u} \alpha_{\tilde{f}^{\prime}{ }_{1}}^{\tilde{W}_{j}}\right) m_{f^{\prime}}\left|m_{\tilde{W}_{j}}\right|(-1)^{\theta_{i}}, \\
& \mathcal{V}_{H^{ \pm} \tilde{f}^{\prime} 1}^{(4)}=-\left[(-1)^{\theta_{i}} \omega_{H^{+} \tilde{W}^{+}+\tilde{Z}^{\prime}}^{R} \beta_{\tilde{Z}_{i} \tilde{f}_{1}}^{u} C_{H^{+} f f^{\prime}}^{d}, \beta_{\tilde{f}^{\prime}{ }_{1}^{\prime}}^{\tilde{W}_{j}}-\omega_{H^{+} \tilde{W}^{+}+\alpha^{2}}^{L} \alpha_{\tilde{Z}_{i} \tilde{f}_{1}}^{u} C_{H^{+} f f^{\prime}}^{u} \alpha_{\tilde{f}^{\prime}, 1}^{\tilde{W}_{j}}\right]\left|m_{\tilde{Z}_{i}}\right| m_{f^{\prime}}, \\
& \mathcal{V}_{H^{ \pm} \tilde{f}_{1}^{\prime}}^{(5)}=-\left(\omega_{H^{+} \tilde{W}+\tilde{Z}}^{L} \beta_{\tilde{Z}_{i} \tilde{f}_{1}}^{u} C_{H^{+} f f^{\prime}}^{u} \beta_{\tilde{f}_{1}^{\prime}}^{\tilde{W}_{1}}+\omega_{H^{+} \tilde{W}^{+}+\tilde{Z}^{2}}^{R} \alpha_{\tilde{Z}_{i} \tilde{f}_{1}}^{u} C_{H^{+} f f^{\prime}}^{d} \alpha_{\tilde{f}^{\prime},}^{\tilde{W}_{j}}\right) m_{f}\left|m_{\tilde{W}_{j}}\right|(-1)^{\theta_{i}}, \\
& \mathcal{V}_{H^{ \pm} \tilde{f}_{1}^{\prime}}^{(6)}=(-1)^{\theta_{i}}\left[\omega_{H^{+} \tilde{W}^{+} \tilde{Z}^{2}}^{L} \alpha_{\tilde{Z}_{i} \tilde{f}_{1}}^{u} C_{H^{+} f f^{\prime}}^{d} \beta_{\tilde{f}^{\prime} 1}^{\tilde{W}_{j}}+\omega_{H^{+} \tilde{W}^{+} \tilde{Z}^{\prime}}^{R} \beta_{\tilde{Z}_{i} \tilde{f}_{1}}^{u} C_{H^{+} f f^{\prime}}^{u} \alpha_{\tilde{f}^{\prime} 1}^{\tilde{W}_{j}}\right]\left|m_{\tilde{Z}_{i}} \| m_{\tilde{W}_{j}}\right|, \\
& \mathcal{V}_{H^{ \pm} \tilde{f}_{1}^{\prime}}^{(7)}=-\left(\omega_{H^{+} \tilde{W}^{+}+\tilde{Z}^{\prime}}^{R} \alpha_{\tilde{Z}_{i} \tilde{f}_{1}}^{u} C_{H^{+} f f^{\prime}}^{u} \beta_{\tilde{f}^{\prime}{ }_{1}}^{\tilde{W}_{j}}+\omega_{H^{+} \tilde{W}^{+}+\tilde{Z}^{2}}^{L} \beta_{\tilde{Z}_{i} \tilde{f}_{1}}^{u} C_{H^{+} f f^{\prime}}^{d} \alpha_{\tilde{f}^{\prime}{ }_{1}}^{\tilde{W}_{j}}\right) m_{f^{\prime}} m_{f}, \\
& \mathcal{V}_{H^{+} \tilde{f}^{\prime}{ }_{1}}^{(8)}=-2(-1)^{\theta_{i}}\left[\omega_{H^{+} \tilde{W^{+}}+\tilde{Z}^{2}}^{L} \alpha_{\tilde{Z}_{i} \tilde{f}_{1}}^{u} C_{H^{+} f f^{\prime}}^{u} \beta_{\tilde{f}^{\prime}{ }_{1}}^{\tilde{W}_{j}}+\omega_{H^{+} \tilde{W}^{+}+\tilde{Z}^{\prime}}^{R} \beta_{\tilde{Z}_{i} \tilde{f}_{1}}^{u} C_{H^{+} f f^{\prime}}^{d} \alpha_{\tilde{f}^{\prime}{ }_{1}}^{\tilde{W}_{j}}\right]\left|m_{\tilde{Z}_{i}} \| m_{\tilde{W}_{j}}\right| m_{f^{\prime}} m_{f} .
\end{aligned}
$$


If the initial state is a chargino:

$$
\begin{aligned}
& \mathcal{V}_{H^{ \pm} \tilde{f}^{\prime}{ }_{1}}^{(1)}=\frac{1}{2}(-1)^{\theta_{j}}\left[\omega_{H^{+} \tilde{W}^{+} \tilde{Z}^{L}}^{L} \beta_{{\tilde{f^{\prime}}}_{1}}^{\tilde{W}_{j}} C_{H^{+} f f^{\prime}}^{u} \alpha_{\tilde{Z}_{i} \tilde{f}_{1}}^{u}+(-1)^{\theta_{j}} \omega_{H^{+} \tilde{W}^{+} \tilde{Z}^{2}}^{R} \alpha_{\tilde{f}^{\prime}{ }_{1}}^{\tilde{W}_{j}} C_{H^{+} f f^{\prime}}^{d} \beta_{\tilde{Z}_{i} \tilde{f}_{1}}^{u}\right], \\
& \mathcal{V}_{H^{ \pm} \tilde{f}^{\prime}{ }_{1}}^{(2)}=\left[\omega_{H^{+} \tilde{W}^{+} \tilde{Z}^{L}}^{L} \alpha_{\tilde{f}^{\prime}{ }_{1}}^{\tilde{W}_{j}} C_{H^{+} f f^{\prime}}^{d} \alpha_{\tilde{Z}_{i} \tilde{f}_{1}}^{u}+(-1)^{\theta_{j}} \omega_{H^{+} \tilde{W}^{+} \tilde{Z}^{\prime}}^{R} \beta_{\tilde{f}_{1}^{\prime}{ }_{1}}^{\tilde{W}_{j}} C_{H^{+} f f^{\prime}}^{u} \beta_{\tilde{Z}_{i} \tilde{f}_{1}}^{u}\right]\left|m_{\tilde{Z}_{i}}\right| m_{f^{\prime}}, \\
& \mathcal{V}_{H^{ \pm} \tilde{f}_{1}^{\prime}{ }_{1}}^{(3)}=-\left[(-1)^{\theta_{j}} \omega_{H^{+} \tilde{W}^{+} \tilde{Z}^{R}}^{R} \alpha_{\tilde{f}^{\prime}{ }_{1}}^{\tilde{W}_{j}} C_{H^{+} f f^{\prime}}^{u} \alpha_{\tilde{Z}_{i} \tilde{f}_{1}}^{u}+\omega_{H^{+} \tilde{W}^{+} \tilde{Z}^{2}}^{L} \beta_{\tilde{f}^{\prime}{ }_{1}}^{\tilde{W}_{j}} C_{H^{+} f f^{\prime}}^{d} \beta_{\tilde{Z}_{i} \tilde{f}_{1}}^{u}\right] m_{f}\left|m_{\tilde{W}_{j}}\right|, \\
& \mathcal{V}_{H^{ \pm} \tilde{f}^{\prime}{ }_{1}}^{(4)}=-\left[\omega_{H^{+} \tilde{W}^{+} \tilde{Z}^{L}}^{L} \alpha_{\tilde{f}^{\prime}{ }_{1}}^{\tilde{W}_{j}} C_{H^{+} f f^{\prime}}^{u} \alpha_{\tilde{Z}_{i} \tilde{f}_{1}}^{u}+(-1)^{\theta_{j}} \omega_{H^{+} \tilde{W}^{+} \tilde{Z}^{R}}^{R} \beta_{\tilde{f}^{\prime}{ }_{1}}^{\tilde{W}_{j}} C_{H^{+} f f^{\prime}}^{d} \beta_{\tilde{Z}_{i} \tilde{f}_{1}}^{u}\right]\left|m_{\tilde{Z}_{i}}\right| m_{f}, \\
& \mathcal{V}_{H^{ \pm} \tilde{f}_{1}^{\prime}}^{(5)}=\left[(-1)^{\theta_{j}} \omega_{H^{+} \tilde{W}^{+} \tilde{Z}^{R}}^{\alpha_{\tilde{f}^{\prime}{ }_{1}}^{\tilde{W}_{j}}} C_{H^{+} f f^{\prime}}^{d} \alpha_{\tilde{Z}_{i} \tilde{f}_{1}}^{u}+\omega_{H^{+} \tilde{W}^{+} \tilde{Z}^{L}}^{L} \beta_{\tilde{f}^{\prime}{ }_{1}}^{\tilde{W}_{j}} C_{H^{+} f f^{\prime}}^{u} \beta_{\tilde{Z}_{i} \tilde{f}_{1}}^{u}\right] m_{f^{\prime}}\left|m_{\tilde{W}_{j}}\right|, \\
& \mathcal{V}_{H^{ \pm} \tilde{f}^{\prime}{ }_{1}}^{(6)}=\left[\omega_{H^{+} \tilde{W}^{+} \tilde{Z}^{2}}^{R} \beta_{\tilde{f}^{\prime}{ }_{1}}^{\tilde{W}_{j}} C_{H^{+} f f^{\prime}}^{u} \alpha_{\tilde{Z}_{i} \tilde{f}_{1}}^{u}+(-1)^{\theta_{j}} \omega_{H^{+} \tilde{W}^{+} \tilde{Z}^{L}}^{L} \alpha_{\tilde{f}^{\prime}{ }_{1}}^{\tilde{W}_{j}} C_{H^{+} f f^{\prime}}^{d} \beta_{\tilde{Z}_{i} \tilde{f}_{1}}^{u}\right]\left|m_{\tilde{W}_{j}} \| m_{\tilde{Z}_{i}}\right|, \\
& \mathcal{V}_{H^{ \pm} \tilde{f}^{\prime}{ }_{1}}^{(7)}=-\left[(-1)^{\theta_{j}} \omega_{H^{+} \tilde{W}^{+} \tilde{Z}^{L}}^{L} \beta_{\tilde{f}^{\prime}{ }_{1}}^{\tilde{W}_{j}} C_{H^{+} f f^{\prime}}^{d} \alpha_{\tilde{Z}_{i} \tilde{f}_{1}}^{u}+\omega_{H^{+} \tilde{W}^{+} \tilde{Z}^{2}}^{R} \alpha_{\tilde{f}^{\prime}{ }_{1}}^{\tilde{W}_{j}} C_{H^{+} f f^{\prime}}^{u} \beta_{\tilde{Z}_{i} \tilde{f}_{1}}^{u}\right] m_{f} m_{f^{\prime}}, \\
& \mathcal{V}_{H^{ \pm} \tilde{f}^{\prime}{ }_{1}}^{(8)}=-2\left[\omega_{H^{+} \tilde{W}^{+} \tilde{Z}^{2}}^{R} \beta_{\tilde{f}^{\prime}{ }_{1}}^{\tilde{W}_{j}} C_{H^{+} f f^{\prime}}^{d} \alpha_{\tilde{Z}_{i} \tilde{f}_{1}}^{u}+(-1)^{\theta_{j}} \omega_{H^{+} \tilde{W}^{+} \tilde{Z}^{L}}^{L} \alpha_{\tilde{f}^{\prime}{ }_{1}}^{\tilde{W}_{j}} C_{H^{+} f f^{\prime}}^{u} \beta_{\tilde{Z}_{i} \tilde{f}_{1}}^{u}\right] m_{f} m_{f^{\prime}}\left|m_{\tilde{W}_{j}} \| m_{\tilde{Z}_{i}}\right| .
\end{aligned}
$$

The integrals required are exactly as in the $G \tilde{f}^{\prime}{ }_{1}$ but with the expected change $m_{W} \rightarrow m_{H^{ \pm}} . \Gamma_{H^{ \pm} \tilde{f}_{1}^{\prime}}$ is then given exactly as $\Gamma_{G \tilde{f}^{\prime}}$.

$\Gamma_{H^{ \pm} \tilde{f}_{2}^{\prime}}$

The coupling combinations now are, if it's a neutralino decaying:

$$
\begin{aligned}
& \mathcal{V}_{H^{ \pm} \tilde{f}^{\prime}{ }_{2}}^{(1)}=-\frac{1}{2}\left[(-1)^{\theta_{i}} \omega_{H^{+} \tilde{W}^{+} \tilde{Z}^{R}}^{R} \alpha_{\tilde{Z}_{i} \tilde{f}_{2}}^{u} C_{H^{+} f f^{\prime}}^{d} \beta_{\tilde{f}^{\prime}{ }_{2}}^{\tilde{W}_{j}}+\omega_{H^{+} \tilde{W}^{+} \tilde{Z}^{L}}^{L} \beta_{\tilde{Z}_{i} \tilde{f}_{2}}^{u} C_{H^{+} f f^{\prime}}^{u} \alpha_{\tilde{f}^{\prime}{ }_{2}}^{\tilde{W}_{j}}\right], \\
& \mathcal{V}_{H^{ \pm} \tilde{f}^{\prime}{ }_{2}}^{(2)}=\left(\omega_{H^{+} \tilde{W}^{+} \tilde{Z}^{2}}^{R} \beta_{\tilde{Z}_{i} \tilde{f}_{2}}^{u} C_{H^{+} f f^{\prime}}^{u} \beta_{\tilde{f}^{\prime}{ }_{2}}^{\tilde{W}_{j}}-\omega_{H^{+} \tilde{W}^{+} \tilde{Z}^{L}}^{L} \alpha_{\tilde{Z}_{i} \tilde{f}_{2}}^{u} C_{H^{+} f f^{\prime}}^{d} \alpha_{\tilde{f}^{\prime}{ }_{2}}^{\tilde{W}_{j}}\right) m_{f}\left|m_{\tilde{Z}_{i}}\right|(-1)^{\theta_{i}}, \\
& \mathcal{V}_{H^{ \pm} \tilde{f}_{2}^{\prime}}^{(3)}=-\left[(-1)^{\theta_{i}} \omega_{H^{+} \tilde{W}^{+} \tilde{Z}^{L}}^{L} \beta_{\tilde{Z}_{i} \tilde{f}_{2}}^{u} C_{H^{+} f f^{\prime}}^{d} \beta_{\tilde{f}^{\prime}{ }_{2}}^{\tilde{W}_{j}}+\omega_{H^{+} \tilde{W}^{+} \tilde{Z}^{2}}^{R} \alpha_{\tilde{Z}_{i} \tilde{f}_{2}}^{u} C_{H^{+} f f^{\prime}}^{u} \alpha_{\tilde{f}^{\prime}{ }_{2}}^{\tilde{W}_{j}}\right] m_{f^{\prime}}\left|m_{\tilde{W}_{j}}\right| \text {, } \\
& \mathcal{V}_{H^{+} \tilde{f}_{2}^{\prime}}^{(4)}=-\left[(-1)^{\theta_{i}} \omega_{H^{+} \tilde{W}^{+} \tilde{Z}^{2}}^{R} \beta_{\tilde{Z}_{i} \tilde{f}_{2}}^{u} C_{H^{+} f f^{\prime}}^{d} \beta_{\tilde{f}^{\prime}{ }_{2}}^{\tilde{W}_{j}}+\omega_{H^{+} \tilde{W}^{+} \tilde{Z}^{L}}^{L} \alpha_{\tilde{Z}_{i} \tilde{f}_{2}}^{u} C_{H^{+} f f^{\prime}}^{u} \alpha_{\tilde{f}_{2}^{\prime}}^{\tilde{W}_{j}}\right]\left|m_{\tilde{Z}_{i}}\right| m_{f^{\prime}}, \\
& \mathcal{V}_{H^{ \pm} \tilde{f}^{\prime}{ }_{2}}^{(5)}=\left(\omega_{H^{+} \tilde{W}^{+} \tilde{Z}^{2}}^{L} \beta_{\tilde{Z}_{i} \tilde{f}_{2}}^{u} C_{H^{+} f f^{\prime}}^{u} \beta_{\tilde{f}^{\prime}{ }_{2}}^{\tilde{W}_{j}}-\omega_{H^{+} \tilde{W}^{+} \tilde{Z}^{2}}^{R} \alpha_{\tilde{Z}_{i} \tilde{f}_{2}}^{u} C_{H^{+} f f^{\prime}}^{d} \alpha_{\tilde{f}^{\prime}{ }_{2}}^{\tilde{W}_{j}}\right) m_{f}\left|m_{\tilde{W}_{j}}\right|(-1)^{\theta_{j}}, \\
& \mathcal{V}_{H^{ \pm} \tilde{f}^{\prime}{ }_{2}}^{(6)}=\left[\omega_{H^{+} \tilde{W}^{+} \tilde{Z}}^{L} \alpha_{\tilde{Z}_{i} \tilde{f}_{2}}^{u} C_{H^{+} f f^{\prime}}^{d} \beta_{\tilde{f}^{\prime}{ }_{2}}^{\tilde{W}_{j}}-(-1)^{\theta_{i}} \omega_{H^{+} \tilde{W}^{+} \tilde{Z}^{2}}^{R} \beta_{\tilde{Z}_{i} \tilde{f}_{2}}^{u} C_{H^{+} f f^{\prime}}^{u} \alpha_{\tilde{f}^{\prime}{ }_{2}}^{\tilde{W}_{j}}\right]\left|m_{\tilde{Z}_{i}} \| m_{\tilde{W}_{j}}\right|, \\
& \mathcal{V}_{H^{ \pm} \tilde{f}_{2}^{\prime}}^{(7)}=\left[-(-1)^{\theta_{i}} \omega_{H^{+} \tilde{W}^{+} \tilde{Z}^{R}}^{R} \alpha_{\tilde{Z}_{i} \tilde{f}_{2}}^{u} C_{H^{+} f f^{\prime}}^{u} \beta_{\tilde{f}^{\prime}}^{\tilde{W}_{j}}+\omega_{H^{+} \tilde{W}^{+} \tilde{Z}^{L}}^{L} \beta_{\tilde{Z}_{i} \tilde{f}_{2}}^{u} C_{H^{+} f f^{\prime}}^{d} \alpha_{\tilde{f}^{\prime}{ }_{2}}^{\tilde{W}_{j}}\right] m_{f^{\prime}} m_{f}, \\
& \mathcal{V}_{H^{ \pm} \tilde{f}^{\prime}{ }_{2}}^{(8)}=2(-1)^{\theta_{i}}\left[-\omega_{H^{+} \tilde{W}^{+} \tilde{Z}^{L}}^{L} \alpha_{\tilde{Z}_{i} \tilde{f}_{2}}^{u} C_{H^{+} f f^{\prime}}^{u} \beta_{\tilde{f}^{\prime}{ }_{2}}^{\tilde{W}_{j}}+\omega_{H^{+} \tilde{W}^{+} \tilde{Z}^{2}}^{R} \beta_{\tilde{Z}_{i} \tilde{f}_{2}}^{u} C_{H^{+} f f^{\prime}}^{d} \alpha_{\tilde{f}^{\prime}{ }_{2}}^{\tilde{W}_{j}}\right]\left|m_{\tilde{Z}_{i}} \| m_{\tilde{W}_{j}}\right| m_{f^{\prime}} m_{f} \text {. }
\end{aligned}
$$

Whilst if it's a chargino decaying:

$$
\begin{aligned}
& \mathcal{V}_{H^{ \pm} \tilde{f}^{\prime}{ }_{2}}^{(1)}=\frac{1}{2}\left[(-1)^{\theta_{j}} \omega_{H^{+} \tilde{W}^{+} \tilde{Z}^{L}}^{L} \beta_{\tilde{f}^{\prime}{ }_{2}}^{\tilde{W}_{j}} C_{H^{+} f f^{\prime}}^{u} \alpha_{\tilde{Z}_{i} \tilde{f}_{2}}^{u}+\omega_{H^{+} \tilde{W}^{+} \tilde{Z}^{2}}^{R} \alpha_{\tilde{f}^{\prime}{ }_{2}}^{\tilde{W}_{j}} C_{H^{+} f f^{\prime}}^{d} \beta_{\tilde{Z}_{i} \tilde{f}_{2}}^{u}\right], \\
& \mathcal{V}_{H^{ \pm} \tilde{f}_{2}{ }_{2}}^{(2)}=-\left[(-1)^{\theta_{j}} \omega_{H^{+} \tilde{W}^{+} \tilde{Z}^{L}}^{L} \alpha_{\tilde{f}^{\prime}{ }_{2}}^{\tilde{W}_{j}} C_{H^{+} f f^{\prime}}^{d} \alpha_{\tilde{Z}_{i} \tilde{f}_{2}}^{u}+\omega_{H^{+} \tilde{W}^{+} \tilde{Z}^{2}}^{R} \beta_{\tilde{f}^{\prime}{ }_{2}}^{\tilde{W}_{j}} C_{H^{+} f f^{\prime}}^{u} \beta_{\tilde{Z}_{i} \tilde{f}_{2}}^{u}\right]\left|m_{\tilde{Z}_{i}}\right| m_{f^{\prime}}, \\
& \mathcal{V}_{H^{ \pm} \tilde{f}^{\prime}{ }_{2}}^{(3)}=\left(\omega_{H^{+} \tilde{W}^{+} \tilde{Z}^{2}}^{R} \alpha_{\tilde{f}^{\prime}{ }_{2}}^{\tilde{W}_{j}} C_{H^{+} f f^{\prime}}^{u} \alpha_{\tilde{Z}_{i} \tilde{f}_{2}}^{u}+(-1)^{\theta_{j}} \omega_{H^{+} \tilde{W}^{+} \tilde{Z}^{L}}^{L} \beta_{\tilde{f}^{\prime}{ }_{2}}^{\tilde{W}_{j}} C_{H^{+} f f^{\prime}}^{d} \beta_{\tilde{Z}_{i} \tilde{f}_{2}}^{u}\right) m_{f}\left|m_{\tilde{W}_{j}}\right| \text {, } \\
& \mathcal{V}_{H^{ \pm} \tilde{f}^{\prime}{ }_{2}}^{(4)}=\left[(-1)^{\theta_{j}} \omega_{H^{+} \tilde{W}^{+} \tilde{Z}^{L}}^{L} \alpha_{\tilde{f}^{\prime}{ }_{2}}^{\tilde{W}_{j}} C_{H^{+} f f^{\prime}}^{u} \alpha_{\tilde{Z}_{i} \tilde{f}_{2}}^{u}+\omega_{H^{+} \tilde{W}^{+} \tilde{Z}^{\prime}}^{R} \beta_{\tilde{f}^{\prime}{ }_{2}}^{\tilde{W}_{j}} C_{H^{+} f f^{\prime}}^{d} \beta_{\tilde{Z}_{i} \tilde{f}_{2}}^{u}\right]\left|m_{\tilde{Z}_{i}}\right| m_{f}, \\
& \mathcal{V}_{H^{ \pm} \tilde{f}_{2}^{\prime}{ }_{2}}^{(5)}=-\left[\omega_{H^{+} \tilde{W}^{+} \tilde{Z}^{2}}^{R} \alpha_{\tilde{f}^{\prime}{ }_{2}}^{\tilde{W}_{j}} C_{H^{+} f f^{\prime}}^{d} \alpha_{\tilde{Z}_{i} \tilde{f}_{2}}^{u}+(-1)^{\theta_{j}} \omega_{H^{+} \tilde{W}^{+} \tilde{Z}^{L}}^{L} \beta_{\tilde{f}^{\prime}{ }_{2}}^{\tilde{W}_{j}} C_{H^{+} f f^{\prime}}^{u} \beta_{\tilde{Z}_{i} \tilde{f}_{2}}^{u}\right] m_{f^{\prime}}\left|m_{\tilde{W}_{j}}\right| \text {, } \\
& \mathcal{V}_{H^{ \pm} \tilde{f}_{2}^{\prime}}^{(6)}=\left(\omega_{H^{+} \tilde{W}^{+} \tilde{Z}^{R}}^{R} \beta_{\tilde{f}^{\prime}{ }_{2}}^{\tilde{W}_{j}} C_{H^{+} f f^{\prime}}^{u} \alpha_{\tilde{Z}_{i} \tilde{f}_{2}}^{u}+(-1)^{\theta_{j}} \omega_{H^{+} \tilde{W}^{+} \tilde{Z}^{L}}^{L} \alpha_{\tilde{f}^{\prime}{ }_{2}}^{\tilde{W}_{j}} C_{H^{+} f f^{\prime}}^{d} \beta_{\tilde{Z}_{i} \tilde{f}_{2}}^{u}\right)\left|m_{\tilde{W}_{j}} \| m_{\tilde{Z}_{i}}\right|, \\
& \mathcal{V}_{H^{ \pm} \tilde{f}^{\prime}{ }_{2}}^{(7)}=-\left[(-1)^{\theta_{j}} \omega_{H^{+} \tilde{W}^{+} \tilde{Z}^{L}}^{L} \beta_{\tilde{f}^{\prime}{ }_{2}}^{\tilde{W}_{j}} C_{H^{+} f f^{\prime}}^{d} \alpha_{\tilde{Z}_{i} \tilde{f}_{2}}^{u}+\omega_{H^{+} \tilde{W}^{+} \tilde{Z}^{2}}^{R} \alpha_{\tilde{f}^{\prime}{ }_{2}}^{\tilde{W}_{j}} C_{H^{+} f f^{\prime}}^{u} \beta_{\tilde{Z}_{i} \tilde{f}_{2}}^{u}\right] m_{f} m_{f^{\prime}}, \\
& \mathcal{V}_{H^{ \pm} \tilde{f}^{\prime}{ }_{2}}^{(8)}=-2\left[\omega_{H^{+} \tilde{W}^{+} \tilde{Z}^{2}}^{R} \beta_{\tilde{f}_{2}^{\prime}}^{\tilde{W}_{j}} C_{H^{+} f f^{\prime}}^{d} \alpha_{\tilde{Z}_{i} \tilde{f}_{2}}^{u}+(-1)^{\theta_{j}} \omega_{H^{+} \tilde{W}^{+} \tilde{Z}^{L}}^{L} \alpha_{\tilde{f}^{\prime}{ }_{2}}^{\tilde{W}_{j}} C_{H^{+} f f^{\prime}}^{u} \beta_{\tilde{Z}_{i} \tilde{f}_{2}}^{u}\right] m_{f} m_{f^{\prime}}\left|m_{\tilde{W}_{j}}\right|\left|m_{\tilde{Z}_{i}}\right| \text {. }
\end{aligned}
$$


The required integrals are as for $G \tilde{f}_{2}^{\prime}$ but with with the mass change $m_{W} \rightarrow m_{H^{ \pm}}$. Similarly, $\Gamma_{H^{ \pm} \tilde{f}_{2}}$ is given analogously.

$\Gamma_{G \tilde{f_{1}}}$

$\overline{T h e}$ coupling combinations here again depend upon if it's a neutralino decaying or chargino decaying, if it's a neutralino decaying:

$$
\begin{aligned}
& \mathcal{V}_{G \tilde{f}_{1}}^{(1)}=-\frac{1}{2}\left[\omega_{G \tilde{W} \tilde{Z}}^{R} \beta_{\tilde{f}_{1} \tilde{Z}_{i}}^{d} C_{G f f^{\prime}}^{d} \alpha_{\tilde{f}_{1}}^{\tilde{W}_{j}}+(-1)^{\theta_{i}} \omega_{G \tilde{W} \tilde{Z}}^{L} \alpha_{\tilde{f}_{1} \tilde{Z}_{i}}^{d} C_{G f f^{\prime}}^{u} \beta_{\tilde{f}_{1}}^{\tilde{W}_{j}}\right], \\
& \mathcal{V}_{G \tilde{f}_{1}}^{(2)}=-\left(\omega_{G \tilde{W} \tilde{Z}}^{R} \alpha_{\tilde{f}_{1} \tilde{Z}_{i}}^{d} C_{G f f^{\prime}}^{u} \alpha_{\tilde{f}_{1}}^{\tilde{W}_{j}}+(-1)^{\theta_{i}} \omega_{G \tilde{W} \tilde{Z}^{L}}^{L} \beta_{\tilde{f}_{1} \tilde{Z}_{i}}^{d} C_{G f f^{\prime}}^{d} \beta_{\tilde{f}_{1}}^{\tilde{W}_{j}}\right)\left|m_{\tilde{Z}_{i}}\right| m_{f^{\prime}}, \\
& \mathcal{V}_{G \tilde{f}_{1}}^{(3)}=\left[(-1)^{\theta_{i}} \omega_{G \tilde{W} \tilde{Z}^{L}}^{L} \alpha_{\tilde{f}_{1} \tilde{Z}_{i}}^{d} C_{G f f^{\prime}}^{d} \alpha_{\tilde{f}_{1}}^{\tilde{W}_{j}}+\omega_{G \tilde{W} \tilde{Z}}^{R} \beta_{\tilde{f}_{1} \tilde{Z}_{i}}^{d} C_{G f f^{\prime}}^{u} \beta_{\tilde{f}_{1}}^{\tilde{W}_{j}}\right] m_{f}\left|m_{\tilde{W}_{j}}\right|, \\
& \mathcal{V}_{G \tilde{f}_{1}}^{(4)}=\left(\omega_{G \tilde{W} \tilde{Z}}^{R} \alpha_{\tilde{f}_{1} \tilde{Z}_{i}}^{d} C_{G f f^{\prime}}^{d} \alpha_{\tilde{f}_{1}}^{\tilde{W}_{j}}-(-1)^{\theta_{i}} \omega_{G \tilde{W} \tilde{Z}}^{L} \beta_{\tilde{f}_{1} \tilde{Z}_{i}}^{d} C_{G f f^{\prime}}^{u} \beta_{\tilde{f}_{1}}^{\tilde{W}_{j}}\right)\left|m_{\tilde{Z}_{i}}\right| m_{f}, \\
& \mathcal{V}_{G \tilde{f}_{1}}^{(5)}=-\left[(-1)^{\theta_{i}} \omega_{G \tilde{W} \tilde{Z}}^{L} \alpha_{\tilde{f}_{1} \tilde{Z}_{i}}^{d} C_{G f f^{\prime}}^{u} \alpha_{\tilde{f}_{1}}^{\tilde{W}_{j}}+\omega_{G \tilde{W} \tilde{Z}}^{R} \beta_{\tilde{f}_{1} \tilde{Z}_{i}}^{d} C_{G f f^{\prime}}^{d} \beta_{\tilde{f}_{1}}^{\tilde{W}_{j}}\right] m_{f^{\prime}}\left|m_{\tilde{W}_{j}}\right|, \\
& \mathcal{V}_{G \tilde{f}_{1}}^{(6)}=-\left[(-1)^{\theta_{i}} \omega_{G \tilde{W} \tilde{Z}}^{L} \beta_{\tilde{f}_{1} \tilde{Z}_{i}}^{d} C_{G f f^{\prime}}^{d} \alpha_{\tilde{f}_{1}}^{\tilde{W}_{j}}-\omega_{G \tilde{W} \tilde{Z}}^{R} \alpha_{\tilde{f}_{1} \tilde{Z}_{i}}^{d} C_{G f f^{\prime}}^{u} \beta_{\tilde{f}_{1}}^{\tilde{W}_{j}}\right]\left|m_{\tilde{Z}_{i}}\right|\left|m_{\tilde{W}_{j}}\right| \text {, } \\
& \mathcal{V}_{G \tilde{f}_{1}}^{(7)}=-\left(\omega_{G \tilde{W} \tilde{Z}}^{R} \beta_{\tilde{f}_{1} \tilde{Z}_{i}}^{d} C_{G f f^{\prime}}^{u} \alpha_{\tilde{f}_{1}}^{\tilde{W}_{j}}-(-1)^{\theta_{i}} \omega_{G \tilde{W} \tilde{Z}}^{L} \alpha_{\tilde{f}_{1} \tilde{Z}_{i}}^{d} C_{G f f^{\prime}}^{d} \beta_{\tilde{f}_{1}}^{\tilde{W}_{j}}\right) m_{f^{\prime}} m_{f}, \\
& \mathcal{V}_{G \tilde{f}_{1}}^{(8)}=-2\left[(-1)^{\theta_{i}} \omega_{G \tilde{W} \tilde{Z}}^{L} \beta_{\tilde{f}_{1} \tilde{Z}_{i}}^{d} C_{G f f^{\prime}}^{u} \alpha_{\tilde{f}_{1}}^{\tilde{W}_{j}}+\omega_{G \tilde{W} \tilde{Z}}^{R} \alpha_{\tilde{f}_{1} \tilde{Z}_{i}}^{d} C_{G f f^{\prime}}^{d} \beta_{\tilde{f}_{1}}^{\tilde{W}_{j}}\right]\left|m_{\tilde{Z}_{i}} \| m_{\tilde{W}_{j}}\right| m_{f^{\prime}} m_{f} .
\end{aligned}
$$

On the other hand, if it's instead a chargino decaying to neutralino:

$$
\begin{aligned}
& \mathcal{V}_{G \tilde{f}_{1}}^{(1)}=\frac{1}{2}\left[(-1)^{\theta_{j}} \omega_{G \tilde{W} \tilde{Z}^{L}}^{L} \beta_{\tilde{f}_{1}}^{\tilde{W}_{j}} C_{G f f^{\prime}}^{u} \alpha_{\tilde{f}_{1} \tilde{Z}_{i}}^{d}+\omega_{G \tilde{W} \tilde{Z}}^{R} \alpha_{\tilde{f}_{1}}^{\tilde{W}_{j}} C_{G f f^{\prime}}^{d} \beta_{\tilde{f}_{1} \tilde{Z}_{i}}^{d}\right], \\
& \mathcal{V}_{G \tilde{f}_{1}}^{(2)}=\left[(-1)^{\theta_{j}} \omega_{G \tilde{W} \tilde{Z}}^{L} \alpha_{\tilde{f}_{1}}^{\tilde{W}_{j}} C_{G f f^{\prime}}^{d} \alpha_{\tilde{f}_{1} \tilde{Z}_{i}}^{d}+\omega_{G \tilde{W} \tilde{Z}^{2}}^{R} \beta_{\tilde{f}_{1}}^{\tilde{W}_{j}} C_{G f f^{\prime}}^{u} \beta_{\tilde{f}_{1} \tilde{Z}_{i}}^{d}\right]\left|m_{\tilde{Z}_{i}}\right| m_{f}, \\
& \mathcal{V}_{G \tilde{f}_{1}}^{(3)}=-\left(\omega_{G \tilde{W} \tilde{Z}^{2}}^{R} \alpha_{\tilde{f}_{1}}^{\tilde{W}_{j}} C_{G f f^{\prime}}^{u} \alpha_{\tilde{f}_{1} \tilde{Z}_{i}}^{d}+(-1)^{\theta_{j}} \omega_{G \tilde{W} \tilde{Z}}^{L} \beta_{\tilde{f}_{1}}^{\tilde{W}_{j}} C_{G f f^{\prime}}^{d} \beta_{\tilde{f}_{1} \tilde{Z}_{i}}^{d}\right) m_{f^{\prime}}\left|m_{\tilde{W}_{j}}\right|, \\
& \mathcal{V}_{G \tilde{f}_{1}}^{(4)}=-\left[(-1)^{\theta_{j}} \omega_{G \tilde{W} \tilde{Z}^{2}}^{L} \alpha_{\tilde{f}_{1}}^{\tilde{W}_{j}} C_{G f f^{\prime}}^{u} \alpha_{\tilde{f}_{1} \tilde{Z}_{i}}^{d}+\omega_{G \tilde{W} \tilde{Z}^{2}}^{R} \beta_{\tilde{f}_{1}}^{\tilde{W}_{j}} C_{G f f^{\prime}}^{d} \beta_{\tilde{f}_{1} \tilde{Z}_{i}}^{d}\right]\left|m_{\tilde{Z}_{i}}\right| m_{f^{\prime}}, \\
& \mathcal{V}_{G \tilde{f}_{1}}^{(5)}=\left(\omega_{G \tilde{W} \tilde{Z}^{R}}^{R} \alpha_{\tilde{f}_{1}}^{\tilde{W}_{j}} C_{G f f^{\prime}}^{d}+(-1)^{\theta_{j}} \omega_{G \tilde{W} \tilde{Z}^{2}}^{L} \beta_{\tilde{f}_{1}}^{\tilde{W}_{j}} C_{G f f^{\prime}}^{u} \beta_{\tilde{f}_{1} \tilde{Z}_{i}}^{d}\right) m_{f}\left|m_{\tilde{W}_{j}}\right|, \\
& \mathcal{V}_{G \tilde{f}_{1}}^{(6)}=\left(\omega_{G \tilde{W} \tilde{Z}^{2}}^{R} \beta_{\tilde{f}_{1}}^{\tilde{W}_{j}} C_{G f f^{\prime}}^{u} \alpha_{\tilde{f}_{1} \tilde{Z}_{i}}^{d}+(-1)^{\theta_{j}} \omega_{G \tilde{W} \tilde{Z}^{L}}^{L} \alpha_{\tilde{f}_{1}}^{\tilde{W}_{j}} C_{G f f^{\prime}}^{d} \beta_{\tilde{f}_{1} \tilde{Z}_{i}}^{d}\right)\left|m_{\tilde{W}_{j}} \| m_{\tilde{Z}_{i}}\right|, \\
& \mathcal{V}_{G \tilde{f}_{1}}^{(7)}=-\left[(-1)^{\theta_{j}} \omega_{G \tilde{W} \tilde{Z}^{L}}^{L} \beta_{\tilde{f}_{1}}^{\tilde{W}_{j}} C_{G f f^{\prime}}^{d} \alpha_{\tilde{f}_{1} \tilde{Z}_{i}}^{d}+\omega_{G \tilde{W} \tilde{Z}^{2}}^{R} \alpha_{\tilde{f}_{1}}^{\tilde{W}_{j}} C_{G f f^{\prime}}^{u} \beta_{\tilde{f}_{1} \tilde{Z}_{i}}^{d}\right] m_{f} m_{f^{\prime}} \text {, } \\
& \mathcal{V}_{G \tilde{f}_{1}}^{(8)}=-2\left[\omega_{G \tilde{W} \tilde{Z}^{R}}^{R} \beta_{\tilde{f}_{1}}^{\tilde{W}_{j}} C_{G f f^{\prime}}^{d} \alpha_{\tilde{f}_{1} \tilde{Z}_{i}}^{d}+(-1)^{\theta_{j}} \omega_{G \tilde{W} \tilde{Z}}^{L} \alpha_{\tilde{f}_{1}}^{\tilde{W}_{j}} C_{G f f^{\prime}}^{u} \beta_{\tilde{f}_{1} \tilde{Z}_{i}}^{d}\right] m_{f} m_{f^{\prime}}\left|m_{\tilde{W}_{j}} \| m_{\tilde{Z}_{i}}\right| .
\end{aligned}
$$

The integrals are exactly as for $G \tilde{f}_{1}^{\prime}{ }_{1}$ except you must swap $m_{f^{\prime}} \leftrightarrow m_{f}$ and $m_{\tilde{f}_{1}} \leftrightarrow m_{\tilde{f}_{1}}$. As for $G \tilde{f}^{\prime}$, for the case of a chargino decaying to a neutralino you must relabel integrals such that integrals 2 and 4 are swapped as are integrals 3 and 5. The $\Gamma_{G \tilde{f}_{1}}$ is then given analogously to $\Gamma_{G \tilde{f}_{1}^{\prime}}$ as the sum of the products of the coupling combinations with corresponding integrals.

$\underline{\Gamma_{G \tilde{f}}}$ 
Here the coupling combinations are, if a neutralino is decaying:

$$
\begin{aligned}
& \mathcal{V}_{G \tilde{f}_{2}}^{(1)}=-\frac{1}{2}\left[\omega_{G \tilde{W} \tilde{Z}}^{R} \beta_{\tilde{f}_{2} \tilde{Z}_{i}}^{d} C_{G f f^{\prime}}^{d} \alpha_{\tilde{f}_{2}}^{\tilde{W}_{j}}+(-1)^{\theta_{i}} \omega_{G \tilde{W} \tilde{Z}}^{L} \alpha_{\tilde{f_{2}} \tilde{Z}_{i}}^{d} C_{G f f^{\prime}}^{u} \beta_{\tilde{f}_{2}}^{\tilde{W}_{j}}\right], \\
& \mathcal{V}_{G \tilde{f}_{2}}^{(2)}=\left[\omega_{G \tilde{W} \tilde{Z}^{2}}^{R} \alpha_{\tilde{f}_{2} \tilde{Z}_{i}}^{d} C_{G f f^{\prime}}^{u} \alpha_{\tilde{f}_{2}}^{\tilde{W}_{j}}+(-1)^{\theta_{i}} \omega_{G \tilde{W} \tilde{Z}}^{L} \beta_{\tilde{f}_{2} \tilde{Z}_{i}}^{d} C_{G f f^{\prime}}^{d} \beta_{\tilde{f}_{2}}^{\tilde{W}_{j}}\right]\left|m_{\tilde{Z}_{i}}\right| m_{f^{\prime}}, \\
& \mathcal{V}_{G \tilde{f}_{2}}^{(3)}=-\left((-1)^{\theta_{i}} \omega_{G \tilde{W} \tilde{Z}}^{L} \alpha_{\tilde{f}_{2} \tilde{Z}_{i}}^{d} C_{G f f^{\prime}}^{d} \alpha_{\tilde{f}_{2}}^{\tilde{W}_{j}}+\omega_{G \tilde{W} \tilde{Z}}^{R} \beta_{\tilde{f}_{2} \tilde{Z}_{i}}^{d} C_{G f f^{\prime}}^{u} \beta_{\tilde{f}_{2}}^{\tilde{W}_{j}}\right) m_{f}\left|m_{\tilde{W}_{j}}\right| \text {, } \\
& \mathcal{V}_{G \tilde{f}_{2}}^{(4)}=-\left[\omega_{G \tilde{W} \tilde{Z}}^{R} \alpha_{\tilde{f}_{2} \tilde{Z}_{i}}^{d} C_{G f f^{\prime}}^{d} \alpha_{\tilde{f}_{2}}^{\tilde{W}_{j}}+(-1)^{\theta_{i}} \omega_{G \tilde{W} \tilde{Z}}^{L} \beta_{\tilde{f}_{2} \tilde{Z}_{i}}^{d} C_{G f f^{\prime}}^{u} \beta_{\tilde{f}_{2}}^{\tilde{W}_{j}}\right]\left|m_{\tilde{Z}_{i}}\right| m_{f}, \\
& \mathcal{V}_{G \tilde{f}_{2}}^{(5)}=\left[(-1)^{\theta_{i}} \omega_{G \tilde{W} \tilde{Z}^{L}}^{L} \alpha_{\tilde{f}_{2} \tilde{Z}_{i}}^{d} C_{G f f^{\prime}}^{u} \alpha_{\tilde{f}_{2}}^{\tilde{W}_{j}}+\omega_{G \tilde{W} \tilde{Z}}^{R} \beta_{\tilde{f}_{2} \tilde{Z}_{i}}^{d} C_{G f f^{\prime}}^{d} \beta_{\tilde{f}_{2}}^{\tilde{W}_{j}}\right] m_{f^{\prime}}\left|m_{\tilde{W}_{j}}\right|, \\
& \mathcal{V}_{G \tilde{f}_{2}}^{(6)}=-\left((-1)^{\theta_{i}} \omega_{G \tilde{W} \tilde{Z}^{2}}^{L} \beta_{\tilde{f}_{2} \tilde{Z}_{i}}^{d} C_{G f f^{\prime}}^{d} \alpha_{\tilde{f}_{2}}^{\tilde{W}_{j}}+\omega_{G \tilde{W} \tilde{Z}}^{R} \alpha_{\tilde{f}_{2} \tilde{Z}_{i}}^{d} C_{G f f^{\prime}}^{u} \beta_{\tilde{f}_{2}}^{\tilde{W}_{j}}\right)\left|m_{\tilde{Z}_{i}} \| m_{\tilde{W}_{j}}\right|(-1)^{\theta_{j}}, \\
& \mathcal{V}_{G \tilde{f}_{2}}^{(7)}=\left[\omega_{G \tilde{W} \tilde{Z}}^{R} \beta_{\tilde{f}_{2} \tilde{Z}_{i}}^{d} C_{G f f^{\prime}}^{u} \alpha_{\tilde{f}_{2}}^{\tilde{W}_{j}}+(-1)^{\theta_{i}} \omega_{G \tilde{W} \tilde{Z}}^{L} \alpha_{\tilde{f}_{2} \tilde{Z}_{i}}^{d} C_{G f f^{\prime}}^{d} \beta_{\tilde{f}_{2}}^{\tilde{W}_{j}}\right] m_{f^{\prime}} m_{f}, \\
& \mathcal{V}_{G \tilde{f}_{2}}^{(8)}=2\left[(-1)^{\theta_{i}} \omega_{G \tilde{W} \tilde{Z}}^{L} \beta_{\tilde{f}_{2} \tilde{Z}_{i}}^{d} C_{G f f^{\prime}}^{u} \alpha_{\tilde{f}_{2}}^{\tilde{W}_{j}}+\omega_{G \tilde{W} \tilde{Z}}^{R} \alpha_{\tilde{f}_{2} \tilde{Z}_{i}}^{d} C_{G f f^{\prime}}^{d} \beta_{\tilde{f}_{2}}^{\tilde{W}_{j}}\right]\left|m_{\tilde{Z}_{i}}\right| m_{f^{\prime}} m_{f}\left|m_{\tilde{W}_{j}}\right|(-1)^{\theta_{j}} .
\end{aligned}
$$

If it's a chargino decaying:

$$
\begin{aligned}
& \mathcal{V}_{G \tilde{f}_{2}}^{(1)}=-\frac{1}{2}(-1)^{\theta_{j}}\left[\omega_{G \tilde{W} \tilde{Z}^{2}}^{L} \beta_{\tilde{f}_{2}}^{\tilde{W}_{j}} C_{G f f^{\prime}}^{u} \alpha_{\tilde{f}_{2} \tilde{Z}_{i}}^{d}-\omega_{G \tilde{W} \tilde{Z}}^{R} \alpha_{\tilde{f}_{2}}^{\tilde{W}_{j}} C_{G f f^{\prime}}^{d} \beta_{\tilde{f}_{2} \tilde{Z}_{i}}^{d}\right], \\
& \mathcal{V}_{G \tilde{f}_{2}}^{(2)}=-\left[\omega_{G \tilde{W} \tilde{Z}}^{L} \alpha_{\tilde{f}_{2}}^{\tilde{W}_{j}} \mathcal{C}_{G f f^{\prime}}^{d} \alpha_{\tilde{f}_{2} \tilde{Z}_{i}}^{d}-(-1)^{\theta_{j}} \omega_{G \tilde{W} \tilde{Z}^{R}}^{R} \beta_{\tilde{f}_{2}}^{\tilde{W}_{j}} \mathcal{C}_{G f f^{\prime}}^{u} \beta_{\tilde{f}_{2} \tilde{Z}_{i}}^{d}\right]\left|m_{\tilde{Z}_{i}}\right| m_{f}, \\
& \mathcal{V}_{G \tilde{f}_{2}}^{(3)}=\left[(-1)^{\theta_{j}} \omega_{G \tilde{W} \tilde{Z}^{R}}^{R} \alpha_{\tilde{f}_{2}}^{\tilde{W}_{j}} C_{G f f^{\prime}}^{u} \alpha_{\tilde{f}_{2} \tilde{Z}_{i}}^{d}-\omega_{G \tilde{W} \tilde{Z}^{2}}^{L} \beta_{\tilde{f}_{2}}^{\tilde{W}_{j}} C_{G f f^{\prime}}^{d} \beta_{\tilde{f}_{2} \tilde{Z}_{i}}^{d}\right] m_{f^{\prime}}\left|m_{\tilde{W}_{j}}\right| \text {, } \\
& \mathcal{V}_{G \tilde{f}_{2}}^{(4)}=\left(\omega_{G \tilde{W} \tilde{Z}^{2}}^{L} \alpha_{\tilde{f}_{2}}^{\tilde{W}_{j}} C_{G f f^{\prime}}^{u} \alpha_{\tilde{f}_{2} \tilde{Z}_{i}}^{d}+(-1)^{\theta_{j}} \omega_{G \tilde{W} \tilde{Z}^{R}}^{R} \beta_{\tilde{f}_{2}}^{\tilde{W}_{j}} C_{G f f^{\prime}}^{d} \beta_{\tilde{f}_{2} \tilde{Z}_{i}}^{d}\right)\left|m_{\tilde{Z}_{i}}\right| m_{f^{\prime}}, \\
& \mathcal{V}_{G \tilde{f}_{2}}^{(5)}=-\left[(-1)^{\theta_{j}} \omega_{G \tilde{W} \tilde{Z}}^{R} \alpha_{\tilde{f}_{2}}^{\tilde{W}_{j}} C_{G f f^{\prime}}^{d} \alpha_{\tilde{f}_{2} \tilde{Z}_{i}}^{d}+\omega_{G \tilde{W} \tilde{Z}}^{L} \beta_{\tilde{f}_{2}}^{\tilde{W}_{j}} C_{G f f^{\prime}}^{u} \beta_{\tilde{f}_{2} \tilde{Z}_{i}}^{d}\right] m_{f}\left|m_{\tilde{W}_{j}}\right| \text {, } \\
& \mathcal{V}_{G \tilde{f}_{2}}^{(6)}=\left[\omega_{G \tilde{W} \tilde{Z}^{2}}^{R} \beta_{\tilde{f}_{2}}^{\tilde{W}_{j}} C_{G f f^{\prime}}^{u} \alpha_{\tilde{f}_{2} \tilde{Z}_{i}}^{d}+\omega_{G \tilde{W} \tilde{Z}}^{L} \alpha_{\tilde{f}_{2}}^{\tilde{W}_{j}} C_{G f f^{\prime}}^{d} \beta_{\tilde{f}_{2} \tilde{Z}_{i}}^{d}\right]\left|m_{\tilde{W}_{j}}\right|\left|m_{\tilde{Z}_{i}}\right|, \\
& \mathcal{V}_{G \tilde{f}_{2}}^{(7)}=-(-1)^{\theta_{j}}\left[\omega_{G \tilde{W} \tilde{Z}^{2}}^{L} \beta_{\tilde{f}_{2}}^{\tilde{W}_{j}} C_{G f f^{\prime}}^{d} \alpha_{\tilde{f}_{2} \tilde{Z}_{i}}^{d}+\omega_{G \tilde{W} \tilde{Z}}^{R} \alpha_{\tilde{f}_{2}}^{\tilde{W}_{j}} C_{G f f^{\prime}}^{u} \beta_{\tilde{f}_{2} \tilde{Z}_{i}}^{d}\right] m_{f} m_{f^{\prime}} \text {, } \\
& \mathcal{V}_{G \tilde{f}_{2}}^{(8)}=2\left[(-1)^{\theta_{j}} \omega_{G \tilde{W} \tilde{Z}}^{R} \beta_{\tilde{f}_{2}}^{\tilde{W}_{j}} C_{G f f^{\prime}}^{d} \alpha_{\tilde{f}_{2} \tilde{Z}_{i}}^{d}+\omega_{G \tilde{W} \tilde{Z}}^{L} \alpha_{\tilde{f}_{2}}^{\tilde{W}_{j}} C_{G f f^{\prime}}^{u} \beta_{\tilde{f}_{2} \tilde{Z}_{i}}^{d}\right] m_{f} m_{f^{\prime}}\left|m_{\tilde{W}_{j}}\right|\left|m_{\tilde{Z}_{i}}\right| .
\end{aligned}
$$

The integrals are exactly as for $G \tilde{f}_{2}^{\prime}{ }_{2}$ except you must swap $m_{f^{\prime}} \leftrightarrow m_{f}$ and $m_{\tilde{f}_{2}} \leftrightarrow m_{\tilde{f}_{2}}$. As for $G \tilde{f}_{2}^{\prime}$, for the case of a chargino decaying to a neutralino you must relabel integrals such that integrals 2 and 4 are swapped as are integrals 3 and 5. The $\Gamma_{G \tilde{f}_{2}}$ is then given analogously to $\Gamma_{G \tilde{f}_{2}^{\prime}}$ as the sum of the products of the coupling combinations with corresponding integrals.

$\Gamma_{H^{ \pm} \tilde{f}_{1}}$ 
Again couplings depend upon which direction the decay occurs, if it is a neutralino decaying the couplings are:

$$
\begin{aligned}
& \mathcal{V}_{H^{ \pm} \tilde{f}_{1}}^{(1)}=\frac{1}{2}\left[\omega_{H^{+} \tilde{W}^{+}}^{R} \tilde{Z}_{\tilde{Z}_{i} \tilde{f}_{1}}^{d} C_{H^{+} f f^{\prime}}^{d} \alpha_{\tilde{f}_{1}}^{\tilde{W}_{j}}+\omega_{H^{+} \tilde{W}^{+} \tilde{Z}^{L}}^{L} \alpha_{\tilde{Z}_{i} \tilde{f}_{1}}^{d} C_{H^{+} f f^{\prime}}^{u} \beta_{\tilde{f}_{1}}^{\tilde{W}_{j}}\right], \\
& \mathcal{V}_{H^{ \pm} \tilde{f}_{1}}^{(2)}=-\left[\omega_{H^{+} \tilde{W}^{+} \tilde{Z}}^{R} \alpha_{\tilde{Z}_{i} \tilde{f}_{1}}^{d} C_{H^{+} f f^{\prime}}^{u} \alpha_{\tilde{f}_{1}}^{\tilde{W}_{j}}+\omega_{H^{+} \tilde{W}^{+}}^{L} \tilde{Z}_{\tilde{Z}_{i} \tilde{f}_{1}}^{d} C_{H^{+} f f^{\prime}}^{d} \beta_{\tilde{f}_{1}}^{\tilde{W}_{j}}\right]\left|m_{\tilde{Z}_{i}}\right| m_{f^{\prime}}, \\
& \mathcal{V}_{H^{ \pm} \tilde{f}_{1}}^{(3)}=\left(\omega_{H^{+} \tilde{W}^{+} \tilde{Z}^{2}}^{L} \alpha_{\tilde{Z}_{i} \tilde{f}_{1}}^{d} C_{H^{+} f f^{\prime}}^{d} \alpha_{\tilde{f}_{1}}^{\tilde{W}_{j}}+\omega_{H^{+} \tilde{W}^{+} \tilde{Z}}^{R} \beta_{\tilde{Z}_{i} \tilde{f}_{1}}^{d} C_{H^{+} f f^{\prime}}^{u} \beta_{\tilde{f}_{1}}^{\tilde{W}_{j}}\right) m_{f}\left|m_{\tilde{W}_{j}}\right|(-1)^{\theta_{i}}, \\
& \mathcal{V}_{H^{ \pm} \tilde{f}_{1}}^{(4)}=\left(\omega_{H^{+} \tilde{W}^{+} \tilde{Z}}^{R} \alpha_{\tilde{Z}_{i} \tilde{f}_{1}}^{d} C_{H^{+} f f^{\prime}}^{d} \alpha_{\tilde{f}_{1}}^{\tilde{W}_{j}}+\omega_{H^{+} \tilde{W}^{+} \tilde{Z}^{L}}^{L} \beta_{\tilde{Z}_{i} \tilde{f}_{1}}^{d} C_{H^{+} f f^{\prime}}^{u} \beta_{\tilde{f}_{1}}^{\tilde{W}_{j}}\right)\left|m_{\tilde{Z}_{i}}\right| m_{f}, \\
& \mathcal{V}_{H^{ \pm} \tilde{f}_{1}}^{(5)}=-\left[(-1)^{\theta_{i}} \omega_{H^{+} \tilde{W}^{+} \tilde{Z}}^{L} \alpha_{\tilde{Z}_{i} \tilde{f}_{1}}^{d} C_{H^{+} f f^{\prime}}^{u} \alpha_{\tilde{f}_{1}}^{\tilde{W}_{j}}+\omega_{H^{+} \tilde{W}^{+} \tilde{Z}^{2}}^{R} \beta_{\tilde{Z}_{i} \tilde{f}_{1}}^{d} C_{H^{+} f f^{\prime}}^{d} \beta_{\tilde{f}_{1}}^{\tilde{W}_{j}}\right] m_{f^{\prime}}\left|m_{\tilde{W}_{j}}\right| \text {, } \\
& \mathcal{V}_{H^{ \pm} \tilde{f}_{1}}^{(6)}=\left[(-1)^{\theta_{i}} \omega_{H^{+} \tilde{W}^{+} \tilde{Z}^{L}}^{L} \beta_{\tilde{Z}_{i} \tilde{f}_{1}}^{d} C_{H^{+} f f^{\prime}}^{d} \alpha_{\tilde{f}_{1}}^{\tilde{W}_{j}}-\omega_{H^{+} \tilde{W}^{+} \tilde{Z}^{2}}^{R} \alpha_{\tilde{Z}_{i} \tilde{f}_{1}}^{d} C_{H^{+} f f^{\prime}}^{u} \beta_{\tilde{f}_{1}}^{\tilde{W}_{j}}\right]\left|m_{\tilde{Z}_{i}} \| m_{\tilde{W}_{j}}\right|, \\
& \mathcal{V}_{H^{ \pm} \tilde{f}_{1}}^{(7)}=-\left[\omega_{H^{+} \tilde{W}^{+} \tilde{Z}}^{R} \beta_{\tilde{Z}_{i} \tilde{f}_{1}}^{d} C_{H^{+} f f^{\prime}}^{u} \alpha_{\tilde{f}_{1}}^{\tilde{W}_{j}}-(-1)^{\theta_{i}} \omega_{H^{+} \tilde{W}^{+} \tilde{Z}^{L}}^{L} \alpha_{\tilde{Z}_{i} \tilde{f}_{1}}^{d} C_{H^{+} f f^{\prime}}^{d} \beta_{\tilde{f}_{1}}^{\tilde{W}_{j}}\right] m_{f^{\prime}} m_{f}, \\
& \mathcal{V}_{H^{ \pm} \tilde{f}_{1}}^{(8)}=-2\left[(-1)^{\theta_{i}} \omega_{H^{+} \tilde{W}^{+} \tilde{Z}^{L}}^{L} \beta_{\tilde{Z}_{i} \tilde{f}_{1}}^{d} C_{H^{+} f f^{\prime}}^{u} \alpha_{\tilde{f}_{1}}^{\tilde{W}_{j}}-\omega_{H^{+} \tilde{W}^{+} \tilde{Z}^{R}}^{R} \alpha_{\tilde{Z}_{i} \tilde{f}_{1}}^{d} C_{H^{+} f f^{\prime}}^{d} \beta_{\tilde{f}_{1}}^{\tilde{W}_{j}}\right]\left|m_{\tilde{Z}_{i}} \| m_{\tilde{W}_{j}}\right| m_{f^{\prime}} m_{f} \text {. }
\end{aligned}
$$

Whilst if it's a chargino decaying:

$$
\begin{aligned}
& \mathcal{V}_{H^{ \pm} \tilde{f}_{1}}^{(1)}=\frac{1}{2}\left[(-1)^{\theta_{j}} \omega_{H^{+} \tilde{W}^{+} \tilde{Z}^{L}}^{L} \beta_{\tilde{f}_{1}}^{\tilde{W}_{j}} C_{H^{+} f f^{\prime}}^{u} \alpha_{\tilde{Z}_{i} \tilde{f}_{1}}^{d}+\omega_{H^{+} \tilde{W}^{+} \tilde{Z}^{R}}^{\alpha_{\tilde{f}_{1}}^{\tilde{W}_{j}}} C_{H^{+} f f^{\prime}}^{d} \beta_{\tilde{Z}_{i} \tilde{f}_{1}}^{d}\right], \\
& \mathcal{V}_{H^{ \pm} \tilde{f}_{1}}^{(2)}=\left[(-1)^{\theta_{j}} \omega_{H^{+} \tilde{W}^{+} \tilde{Z}^{L}}^{L} \alpha_{\tilde{f}_{1}}^{\tilde{W}_{j}} C_{H^{+} f f^{\prime}}^{d} \alpha_{\tilde{Z}_{i} \tilde{f}_{1}}^{d}+\omega_{H^{+} \tilde{W}^{+} \tilde{Z}^{2}}^{R} \beta_{\tilde{f}_{1}}^{\tilde{W}_{j}} C_{H^{+} f f^{\prime}}^{u} \beta_{\tilde{Z}_{i} \tilde{f}_{1}}^{d}\right]\left|m_{\tilde{Z}_{i}}\right| m_{f}, \\
& \mathcal{V}_{H^{ \pm} \tilde{f}_{1}}^{(3)}=-\left[\omega_{H^{+} \tilde{W}^{+} \tilde{Z}^{2}}^{\alpha_{\tilde{f}_{1}}^{\tilde{W}_{j}}} C_{H^{+} f f^{\prime}}^{u} \alpha_{\tilde{Z}_{i} \tilde{f}_{1}}^{d}+(-1)^{\theta_{j}} \omega_{H^{+} \tilde{W}^{+} \tilde{Z}^{L}}^{L} \beta_{\tilde{f}_{1}}^{\tilde{W}_{j}} C_{H^{+} f f^{\prime}}^{d} \beta_{\tilde{Z}_{i} \tilde{f}_{1}}^{d}\right] m_{f^{\prime}}\left|m_{\tilde{W}_{j}}\right| \text {, } \\
& \mathcal{V}_{H^{ \pm} \tilde{f}_{1}}^{(4)}=-\left[(-1)^{\theta_{j}} \omega_{H^{+} \tilde{W}^{+} \tilde{Z}^{L}}^{L} \alpha_{\tilde{f}_{1}}^{\tilde{W}_{j}} C_{H^{+} f f^{\prime}}^{u} \alpha_{\tilde{Z}_{i} \tilde{f}_{1}}^{d}+\omega_{H^{+} \tilde{W}^{+} \tilde{Z}}^{R} \beta_{\tilde{f}_{1}}^{\tilde{W}_{j}} C_{H^{+} f f^{\prime}}^{d} \beta_{\tilde{Z}_{i} \tilde{f}_{1}}^{d}\right]\left|m_{\tilde{Z}_{i}}\right| m_{f^{\prime}}, \\
& \mathcal{V}_{H^{ \pm} \tilde{f}_{1}}^{(5)}=\left(\omega_{H^{+} \tilde{W}^{+} \tilde{Z}^{2}}^{R} \alpha_{\tilde{f}_{1}}^{\tilde{W}_{j}} C_{H^{+} f f^{\prime}}^{d} \alpha_{\tilde{Z}_{i} \tilde{f}_{1}}^{d}+(-1)^{\theta_{j}} \omega_{H^{+} \tilde{W}^{+} \tilde{Z}^{L}}^{L} \beta_{\tilde{f}_{1}}^{\tilde{W}_{j}} C_{H^{+} f f^{\prime}}^{u} \beta_{\tilde{Z}_{i} \tilde{f}_{1}}^{d}\right) m_{f}\left|m_{\tilde{W}_{j}}\right| \text {, } \\
& \mathcal{V}_{H^{ \pm} \tilde{f}_{1}}^{(6)}=\left(\omega_{H^{+} \tilde{W}^{+} \tilde{Z}^{2}}^{R} \beta_{\tilde{f}_{1}}^{\tilde{W}_{j}} C_{H^{+} f f^{\prime}}^{u} \alpha_{\tilde{Z}_{i} \tilde{f}_{1}}^{d}+(-1)^{\theta_{j}} \omega_{H^{+} \tilde{W}^{+} \tilde{Z}^{L}}^{L} \alpha_{\tilde{f}_{1}}^{\tilde{W}_{j}} C_{H^{+} f f^{\prime}}^{d} \beta_{\tilde{Z}_{i} \tilde{f}_{1}}^{d}\right)\left|m_{\tilde{W}_{j}}\right|\left|m_{\tilde{Z}_{i}}\right|, \\
& \mathcal{V}_{H^{ \pm} \tilde{f}_{1}}^{(7)}=-\left[(-1)^{\theta_{j}} \omega_{H^{+} \tilde{W}^{+} \tilde{Z}}^{L} \beta_{\tilde{f}_{1}}^{\tilde{W}_{j}} C_{H^{+} f f^{\prime}}^{d} \alpha_{\tilde{Z}_{i} \tilde{f}_{1}}^{d}+\omega_{H^{+} \tilde{W}^{+} \tilde{Z}^{2}}^{\alpha_{\tilde{f}_{1}}^{\tilde{W}_{j}}} C_{H^{+} f f^{\prime}}^{u} \beta_{\tilde{Z}_{i} \tilde{f}_{1}}^{d}\right] m_{f} m_{f^{\prime}} \text {, } \\
& \mathcal{V}_{H^{ \pm} \tilde{f}_{1}}^{(8)}=-2\left[\omega_{H^{+} \tilde{W}^{+} \tilde{Z}^{2}}^{R} \beta_{\tilde{f}_{1}}^{\tilde{W}_{j}} C_{H^{+} f f^{\prime}}^{d} \alpha_{\tilde{Z}_{i} \tilde{f}_{1}}^{d}+(-1)^{\theta_{j}} \omega_{H^{+} \tilde{W}^{+} \tilde{Z}^{L}}^{L} \alpha_{\tilde{f}_{1}}^{\tilde{W}_{j}} C_{H^{+} f f^{\prime}}^{u} \beta_{\tilde{Z}_{i} \tilde{f}_{1}}^{d}\right] m_{f} m_{f^{\prime}}\left|m_{\tilde{W}_{j}} \| m_{\tilde{Z}_{i}}\right| .
\end{aligned}
$$

The integrals and overall contribution are given exactly as for $H^{ \pm} \tilde{f}^{\prime}{ }_{1}$ but $m_{\tilde{f}_{1}} \rightarrow m_{\tilde{f}_{1}}$. Remember the integrals' labelling depends on whether it's a neutralino decaying (to a chargino) or a chargino decaying (to a neutralino).

$\Gamma_{H^{ \pm} \tilde{f}_{2}}$

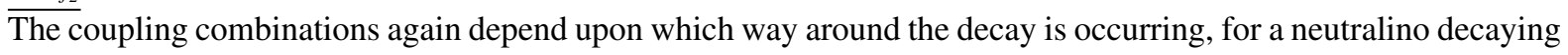


the coupling combinations are:

$$
\begin{aligned}
& \mathcal{V}_{H^{ \pm} \tilde{f}_{2}}^{(1)}=-\frac{1}{2}\left[\omega_{H^{+} \tilde{W}^{+} \tilde{Z}^{2}}^{R} \beta_{\tilde{f}_{2} \tilde{Z}_{i}}^{d} C_{H^{+} f f^{\prime}}^{d} \alpha_{\tilde{f}_{2}}^{\tilde{W}_{j}}+\omega_{H^{+} \tilde{W}^{+} \tilde{Z}^{L}}^{L} \alpha_{\tilde{f}_{2} \tilde{Z}_{i}}^{d} C_{H^{+} f f^{\prime}}^{u} \beta_{\tilde{f}_{2}}^{\tilde{W}_{j}}\right], \\
& \mathcal{V}_{H^{ \pm} \tilde{f}_{2}}^{(2)}=\left(\omega_{H^{+} \tilde{W}^{+} \tilde{Z}}^{R} \alpha_{\tilde{f}_{2} \tilde{Z}_{i}}^{d} C_{H^{+} f f^{\prime}}^{u} \alpha_{\tilde{f}_{2}}^{\tilde{W}_{j}}+\omega_{H^{+} \tilde{W}^{+} \tilde{Z}^{L}}^{L} \beta_{\tilde{f}_{2} \tilde{Z}_{i}}^{d} C_{H^{+} f f^{\prime}}^{d} \beta_{\tilde{f}_{2}}^{\tilde{W}_{j}}\right]\left|m_{\tilde{Z}_{i}}\right| m_{f^{\prime}}, \\
& \mathcal{V}_{H^{ \pm} \tilde{f}_{2}}^{(3)}=-\left[\omega_{H^{+} \tilde{W}^{+} \tilde{Z}}^{L} L \alpha_{\tilde{f}_{2} \tilde{Z}_{i}}^{d} C_{H^{+} f f^{\prime}}^{d} \alpha_{\tilde{f}_{2}}^{\tilde{W}_{j}}+\omega_{H^{+} \tilde{W}^{+} \tilde{Z}^{2}}^{R} \beta_{\tilde{f}_{2} \tilde{Z}_{i}}^{d} C_{H^{+} f f^{\prime}}^{u} \beta_{\tilde{f}_{2}}^{\tilde{W}_{j}}\right] m_{f}\left|m_{\tilde{W}_{j}}\right|(-1)^{\theta_{i}}, \\
& \mathcal{V}_{H^{+} \tilde{f}_{2}}^{(4)}=-\left[\omega_{H^{+} \tilde{W}^{+} \tilde{Z}^{2}}^{R} \alpha_{\tilde{f}_{2} \tilde{Z}_{i}}^{d} C_{H^{+} f f^{\prime}}^{d} \alpha_{\tilde{f}_{2}}^{\tilde{W}_{j}}+\omega_{H^{+} \tilde{W}^{+} \tilde{Z}}^{L} \beta_{\tilde{f}_{2} \tilde{Z}_{i}}^{d} C_{H^{+} f f^{\prime}}^{u} \beta_{\tilde{f}_{2}}^{\tilde{W}_{j}}\right]\left|m_{\tilde{Z}_{i}}\right| m_{f}, \\
& \mathcal{V}_{H^{ \pm} \tilde{f}_{2}}^{(5)}=\left[(-1)^{\theta_{i}} \omega_{H^{+} \tilde{W}^{+} \tilde{Z}^{L}}^{L} \alpha_{\tilde{f}_{2} \tilde{Z}_{i}}^{d} C_{H^{+} f f^{\prime}}^{u} \alpha_{\tilde{f}_{2}}^{\tilde{W}_{j}}+\omega_{H^{+} \tilde{W}^{+} \tilde{Z}}^{R} \beta_{\tilde{f}_{2} \tilde{Z}_{i}}^{d} C_{H^{+} f f^{\prime}}^{d} \beta_{\tilde{f}_{2}}^{\tilde{W}_{j}}\right] m_{f^{\prime}}\left|m_{\tilde{W}_{j}}\right|, \\
& \mathcal{V}_{H^{+} \tilde{f}_{2}}^{(6)}=-\left[\omega_{H^{+} \tilde{W}^{+} \tilde{Z}}^{L} \beta_{\tilde{f}_{2} \tilde{Z}_{i}}^{d} C_{H^{+} f f^{\prime}}^{d} \alpha_{\tilde{f}_{2}}^{\tilde{W}_{j}}+\omega_{H^{+} \tilde{W}^{+} \tilde{Z}}^{R} \alpha_{\tilde{f}_{2} \tilde{Z}_{i}}^{d} C_{H^{+} f f^{\prime}}^{u} \beta_{\tilde{f}_{2}}^{\tilde{W}_{j}}\right]\left|m_{\tilde{Z}_{i}} \| m_{\tilde{W}_{j}}\right|(-1)^{\theta_{i}}(-1)^{\theta_{j}}, \\
& \mathcal{V}_{H^{ \pm} \tilde{f}_{2}}^{(7)}=\left[\omega_{H^{+} \tilde{W}^{+} \tilde{Z}^{2}}^{R} \beta_{\tilde{f}_{2} \tilde{Z}_{i}}^{d} C_{H^{+} f f^{\prime}}^{u} \alpha_{\tilde{f}_{2}}^{\tilde{W}_{j}}+\omega_{H^{+} \tilde{W}^{+} \tilde{Z}^{L}}^{L} \alpha_{\tilde{f}_{2} \tilde{Z}_{i}}^{d} C_{H^{+} f f^{\prime}}^{d} \beta_{\tilde{f}_{2}}^{\tilde{W}_{j}}\right] m_{f^{\prime}} m_{f}, \\
& \mathcal{V}_{H^{ \pm} \tilde{f}_{2}}^{(8)}=2\left[(-1)^{\theta_{i}} \omega_{H^{+} \tilde{W}^{+} \tilde{Z}}^{L} \beta_{\tilde{f}_{2} \tilde{Z}_{i}}^{d} C_{H^{+} f f^{\prime}}^{u} \alpha_{\tilde{f}_{2}}^{\tilde{W}_{j}}+\omega_{H^{+} \tilde{W}^{+} \tilde{Z}^{R}}^{R} \alpha_{\tilde{f}_{2} \tilde{Z}_{i}}^{d} C_{H^{+} f f^{\prime}}^{d} \beta_{\tilde{f}_{2}}^{\tilde{W}_{j}}\right]\left|m_{\tilde{Z}_{i}}\right| m_{f^{\prime}} m_{f}\left|m_{\tilde{W}_{j}}\right|(-1)^{\theta_{j}} \text {. }
\end{aligned}
$$

If it's a chargino decaying:

$$
\begin{aligned}
& \mathcal{V}_{H^{ \pm} \tilde{f}_{2}}^{(1)}=\frac{1}{2}\left[\omega_{H^{+} \tilde{W}^{+} \tilde{Z}^{2}}^{L} \beta_{\tilde{f}_{2}}^{\tilde{W}_{j}} C_{H^{+} f f^{\prime}}^{u} \alpha_{\tilde{f}_{2} \tilde{Z}_{i}}^{d}+(-1)^{\theta_{j}} \omega_{H^{+} \tilde{W}^{+} \tilde{Z}^{R}}^{R} \alpha_{\tilde{f}_{2}}^{\tilde{W}_{j}} C_{H^{+} f f^{\prime}}^{d} \beta_{\tilde{f}_{2} \tilde{Z}_{i}}^{d}\right], \\
& \left.\mathcal{V}_{H^{ \pm} \tilde{f}_{2}}^{(2)}=-\left[\omega_{H^{+} \tilde{W}^{+} \tilde{Z}^{L}}^{L} \alpha_{\tilde{f}_{2}}^{\tilde{W}_{j}} C_{H^{+} f f^{\prime}}^{d} \alpha_{\tilde{f}_{2} \tilde{Z}_{i}}^{d}-(-1)^{\theta_{j}} \omega_{H^{+} \tilde{W}^{+} \tilde{Z}^{R}}^{R} \beta_{\tilde{f}_{2}}^{\tilde{W}_{j}} C_{H^{+} f f^{\prime}}^{u} \beta_{\tilde{f}_{2} \tilde{Z}_{i}}^{d}\right]\left|m_{\tilde{Z}_{i}}\right| m_{f}\right] \text {, } \\
& \mathcal{V}_{H^{ \pm} \tilde{f}_{2}}^{(3)}=\left[(-1)^{\theta_{j}} \omega_{H^{+} \tilde{W}^{+} \tilde{Z}^{R}}^{R} \alpha_{\tilde{f}_{2}}^{\tilde{W}_{j}} C_{H^{+} f f^{\prime}}^{u} \alpha_{\tilde{f}_{2} \tilde{Z}_{i}}^{d}-\omega_{H^{+} \tilde{W}^{+} \tilde{Z}^{L}}^{L} \beta_{\tilde{f}_{2}}^{\tilde{W}_{j}} C_{H^{+} f f^{\prime}}^{d} \beta_{\tilde{f}_{2} \tilde{Z}_{i}}^{d}\right] m_{f^{\prime}}\left|m_{\tilde{W}_{j}}\right|, \\
& \mathcal{V}_{H^{ \pm} \tilde{f}_{2}}^{(4)}=\left(\omega_{H^{+} \tilde{W}^{+} \tilde{Z}^{L}}^{L} \alpha_{\tilde{f}_{2}}^{\tilde{W}_{j}} C_{H^{+} f f^{\prime}}^{u} \alpha_{\tilde{f}_{2} \tilde{Z}_{i}}^{d}-(-1)^{\theta_{j}} \omega_{H^{+} \tilde{W}^{+} \tilde{Z}^{R}}^{R} \beta_{\tilde{f}_{2}}^{\tilde{W}_{j}} C_{H^{+} f f^{\prime}}^{d} \beta_{\tilde{f}_{2} \tilde{Z}_{i}}^{d}\right)\left|m_{\tilde{Z}_{i}}\right| m_{f^{\prime}},
\end{aligned}
$$

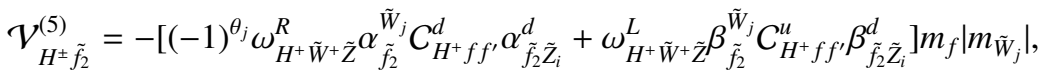

$$
\begin{aligned}
& \mathcal{V}_{H^{ \pm} \tilde{f}_{2}}^{(6)}=\left(\omega_{H^{+} \tilde{W}^{+} \tilde{Z}^{\tilde{f}_{2}}}^{\beta_{\tilde{W}_{j}}^{\tilde{W}_{j}}} C_{H^{+} f f^{\prime}}^{u} \alpha_{\tilde{f}_{2} \tilde{Z}_{i}}^{d}+\omega_{H^{+} \tilde{W}^{+} \tilde{Z}^{L}}^{L} \alpha_{\tilde{f}_{2}}^{\tilde{W}_{j}} C_{H^{+} f f^{\prime}}^{d} \beta_{\tilde{f}_{2} \tilde{Z}_{i}}^{d}\right)\left|m_{\tilde{W}_{j}}\right|\left|m_{\tilde{Z}_{i}}\right|, \\
& \mathcal{V}_{H^{ \pm} \tilde{f}_{2}}^{(7)}=-(-1)^{\theta_{j}}\left[\omega_{H^{+} \tilde{W}^{+} \tilde{Z}}^{L} \beta_{\tilde{f}_{2}}^{\tilde{W}_{j}} C_{H^{+} f f^{\prime}}^{d} \alpha_{\tilde{f}_{2} \tilde{Z}_{i}}^{d}+\omega_{H^{+} \tilde{W}^{+} \tilde{Z}}^{R} \alpha_{\tilde{f}_{2}}^{\tilde{W}_{j}} C_{H^{+} f f^{\prime}}^{u} \beta_{\tilde{f}_{2} \tilde{Z}_{i}}^{d}\right] m_{f} m_{f^{\prime}} \text {, } \\
& \mathcal{V}_{H^{+} \tilde{f}_{2}}^{(8)}=2\left[(-1)^{\theta_{j}} \omega_{H^{+} \tilde{W}^{+} \tilde{Z}^{2}}^{R} \beta_{\tilde{f}_{2}}^{\tilde{W}_{j}} C_{H^{+} f f^{\prime}}^{d} \alpha_{\tilde{f}_{2} \tilde{Z}_{i}}^{d}-\omega_{H^{+} \tilde{W}^{+} \tilde{Z}^{2}}^{L} \alpha_{\tilde{f}_{2}}^{\tilde{W}_{j}} C_{H^{+} f f^{\prime}}^{u} \beta_{\tilde{f}_{2} \tilde{Z}_{i}}^{d}\right] m_{f} m_{f^{\prime}}\left|m_{\tilde{W}_{j}}\right|\left|m_{\tilde{Z}_{i}}\right| .
\end{aligned}
$$

The integrals and overall contribution are given exactly as for $H^{ \pm} \tilde{f}_{2}^{\prime}$ but $m_{\tilde{f}_{2}} \rightarrow m_{\tilde{f}_{2}}$. Remember the integrals labelling depends on whether it's a neutralino decaying (to a chargino) or a chargino decaying (to a neutralino).

$\Gamma_{\tilde{f}_{1}{ }_{1} \tilde{f}_{2}{ }_{2}}$

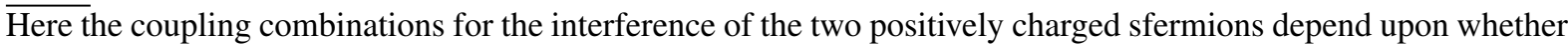
the decay is neutralino to chargino or chargino to neutralino. For neutralino to chargino:

$$
\begin{aligned}
& \mathcal{V}_{\tilde{f}^{\prime}{ }_{2} \tilde{f}^{\prime}{ }_{2}}^{(1)}=\left(\beta_{\tilde{f}_{1} \tilde{Z}_{i}}^{u} \beta_{\tilde{f}_{2} \tilde{Z}_{i}}^{u}+\alpha_{\tilde{f}_{1} \tilde{Z}_{i}}^{u} \alpha_{\tilde{f}_{2} \tilde{Z}_{i}}^{u}\right)(-1)^{\theta_{i}}, \\
& \mathcal{V}_{\tilde{f}_{2}{ }_{2} \tilde{f}_{2}^{\prime}{ }_{2}}^{(2)}=\left(\alpha_{\tilde{f}_{1} \tilde{z}_{i}}^{u} \beta_{\tilde{f}_{2} \tilde{Z}_{i}}^{u}+\beta_{\tilde{f}_{1} \tilde{z}_{i}}^{u} \alpha_{\tilde{f}_{2} \tilde{Z}_{i}}^{u}\right)(-1)^{\theta_{i}}, \\
& \mathcal{V}_{\tilde{f}^{\prime}{ }_{2} \tilde{f}^{\prime}{ }_{2}}^{(3)}=\left(-\alpha_{\tilde{f}_{1}^{\prime}{ }_{1}}^{\tilde{W}_{j}} \alpha_{\tilde{f}^{\prime}{ }_{2}}^{\tilde{W}_{j}}+\beta_{\tilde{f}^{\prime}{ }_{1}}^{\tilde{W}_{j}} \beta_{\tilde{f}^{\prime}{ }_{2}}^{\tilde{W}_{j}}\right)(-1)^{\theta_{i}},
\end{aligned}
$$

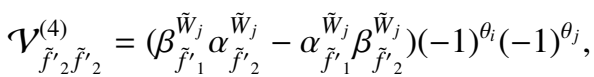


whilst for chargino to neutralino:

$$
\begin{aligned}
& \mathcal{V}_{\tilde{f}^{\prime}{ }_{2} \tilde{f}^{\prime}{ }_{2}}^{(1)}=-\beta_{\tilde{f}^{\prime}{ }_{1}}^{\tilde{W}_{j}} \beta_{\tilde{f}^{\prime}{ }_{2}}^{\tilde{W}_{j}}+\alpha_{\tilde{f}^{\prime}{ }_{1}}^{\tilde{W}_{j}} \alpha_{\tilde{f}^{\prime}{ }_{2}{ }^{\tilde{W}_{j}}}^{\tilde{W}_{j}}(-1)^{\theta_{j}}, \\
& \mathcal{V}_{\tilde{f}^{\prime}{ }_{2} \tilde{f}^{\prime}{ }_{2}}^{(2)}=(-1)^{\theta_{j}} \alpha_{\tilde{f}^{\prime}{ }_{1}}^{\tilde{W}_{j}} \beta_{\tilde{f}^{\prime}{ }_{2}}^{\tilde{W}_{j}}-\alpha_{\tilde{f}^{\prime}{ }_{2}}^{\tilde{W}_{j}} \beta_{\tilde{f}^{\prime}{ }_{2}}^{\tilde{W}_{j}}, \\
& \mathcal{V}_{\tilde{f}_{2}{ }_{2} \tilde{f}^{\prime}{ }_{2}}^{(3)}=\left(-\alpha_{\tilde{f}_{1} \tilde{Z}_{i}}^{u} \alpha_{\tilde{f}_{2} \tilde{Z}_{i}}^{u}+(-1)^{\theta_{j}} \beta_{\tilde{f}_{1} \tilde{Z}_{i}}^{u} \beta_{\tilde{f}_{2} \tilde{Z}_{i}}^{u}\right) \text {, } \\
& \mathcal{V}_{\tilde{f}^{\prime}{ }_{2} \tilde{f}_{2}^{\prime}{ }_{2}}^{(4)}=\left(-\alpha_{\tilde{f}_{1} \tilde{Z}_{i}}^{u} \beta_{\tilde{z}_{2} \tilde{Z}_{i}}^{u}+(-1)^{\theta_{j}} \alpha_{\tilde{f}_{2} \tilde{Z}_{i}}^{u} \beta_{\tilde{f}_{1} \tilde{Z}_{i}}^{u}\right) \text {. }
\end{aligned}
$$

The integrals are as follows with $s=m_{\tilde{Z}_{i}}^{2}+m_{f^{\prime}}^{2}-2\left|m_{\tilde{Z}_{i}}\right| E$ and $\lambda=\sqrt{\left(s-\left(m_{f}+m_{\tilde{W}_{j}}\right)^{2}\right)\left(s-\left(m_{f}-m_{\tilde{W}_{j}}\right)^{2}\right.}$ :

$$
\begin{aligned}
& I_{\tilde{f}_{1} \tilde{f}_{f_{2}}}^{1}=2\left|m_{\tilde{Z}_{i}}\right| \int_{m_{f^{\prime}}}^{E_{\text {upper }}} 2\left|m_{\tilde{Z}_{i}}\right| \sqrt{E^{2}-m_{f^{\prime}}^{2}} \frac{\lambda}{\left.s\left(s-m_{\tilde{f}_{1}}^{2}\right)\left(s-m_{\tilde{f}^{\prime}{ }_{2}}\right)^{2}\right)}, \\
& I_{\tilde{f}_{1} \tilde{f}_{f_{2}}}^{2}=2\left|m_{\tilde{Z}_{i}}\right| \int_{m_{f^{\prime}}}^{E_{\text {upper }}} 2\left|m_{\tilde{Z}_{i}}\right| \sqrt{E^{2}-m_{f^{\prime}}^{2}} \frac{\left(s-m_{f}^{2}-m_{\tilde{W}_{j}}^{2}\right) \lambda}{\left.s\left(s-m_{\tilde{f}_{1}^{\prime}}^{2}\right)\left(s-m_{\tilde{f}^{\prime}{ }_{2}}\right)^{2}\right)}, \\
& I_{\tilde{f}^{\prime}{ }_{1} \tilde{f}^{\prime}{ }_{2}}^{3}=2\left|m_{\tilde{Z}_{i}}\right| \int_{m_{f^{\prime}}}^{E_{\text {upper }}} 2\left|m_{\tilde{Z}_{i}}\right| \sqrt{E^{2}-m_{f^{\prime}}^{2}} \frac{2\left|m_{\tilde{Z}_{i}}\right| E \lambda}{\left.s\left(s-m_{\tilde{f}^{\prime}{ }_{1}}^{2}\right)\left(s-m_{\tilde{f}^{\prime}{ }_{2}}\right)^{2}\right)}, \\
& I_{\tilde{f}^{\prime}{ }_{1}^{\prime} \tilde{f}_{2}^{\prime}}^{4}=2\left|m_{\tilde{Z}_{i}}\right| \int_{m_{f^{\prime}}}^{E_{\text {upper }}} 2\left|m_{\tilde{Z}_{i}}\right| \sqrt{E^{2}-m_{f^{\prime}}^{2}} \frac{\left(s-m_{f}^{2}-m_{\tilde{W}_{j}}^{2}\right) 2\left|m_{\tilde{Z}_{i}}\right| E \lambda}{\left.s\left(s-m_{\tilde{f}^{\prime}{ }_{1}}^{2}\right)\left(s-m_{\tilde{f}_{2}^{\prime}}\right)^{2}\right)} .
\end{aligned}
$$

Now if it's instead a chargino decaying, as described before, swap the chargino and neutralino masses throughout, but also here you must relabel the integrals $I_{\tilde{f}^{\prime}{ }_{1} \tilde{f}^{\prime}{ }_{2}}^{2} \leftrightarrow I_{\tilde{f}^{\prime}{ }_{1} \tilde{f}_{2}{ }_{2}}^{3}$. Also for a chargino decaying one must interchange $m_{f}$ and $m_{f^{\prime}}$. For a neutralino decaying:

$$
\begin{aligned}
& \Gamma_{\tilde{f}_{1}{ }_{1} \tilde{f}^{\prime}{ }_{2}}=-4 \mathcal{V}_{\tilde{f}^{\prime}{ }_{2} \tilde{f}^{\prime}{ }_{2}}^{(2)} \mathcal{V}_{\tilde{f}^{\prime}{ }_{2} \tilde{f}^{\prime}{ }_{2}}^{(4)}\left|m_{\tilde{Z}_{i}}\right|\left|m_{\tilde{W}_{j}}\right| m_{f^{\prime}} m_{f} I_{\tilde{f}^{\prime}{ }_{1} \tilde{f}^{\prime}{ }_{2}}^{1}+2 \mathcal{V}_{\tilde{f}^{\prime}{ }_{2} \tilde{f}^{\prime}{ }_{2}}^{(2)} \mathcal{V}_{\tilde{f}^{\prime}{ }_{2} \tilde{f}^{\prime}{ }_{2}}^{(3)}\left|m_{\tilde{Z}_{i}}\right| m_{f^{\prime}} I_{\tilde{f}^{\prime}{ }_{1} \tilde{f}^{\prime}{ }_{2}}^{2}
\end{aligned}
$$

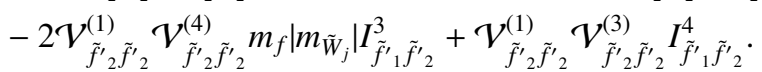

$\Gamma_{\tilde{f}_{1} \tilde{f}_{2}}$

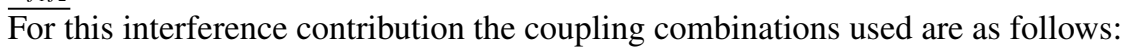

$$
\begin{aligned}
& \mathcal{V}_{\tilde{f}_{1} \tilde{f}_{2}}^{(1)}=-\left[(-1)^{\theta_{c}} \alpha_{\tilde{Z}_{i} \tilde{f}_{1}}^{d} \alpha_{\tilde{Z}_{i} \tilde{f}_{2}}^{d}+\beta_{\tilde{Z}_{i} \tilde{f}_{1}}^{d} \beta_{\tilde{Z}_{i} \tilde{f}_{2}}^{d}\right](-1)^{\theta_{j}}, \\
& \mathcal{V}_{\tilde{f}_{1} \tilde{f}_{2}}^{(2)}=\left[(-1)^{\theta_{i}} \beta_{\tilde{Z}_{i} \tilde{f}_{1}}^{d} \alpha_{\tilde{Z}_{i} \tilde{f}_{2}}^{d}-\alpha_{\tilde{Z}_{i} \tilde{f}_{1}}^{d} \beta_{\tilde{Z}_{i} \tilde{f}_{2}}^{d}\right](-1)^{\theta_{j}}(-1)^{\theta_{c},}, \\
& \mathcal{V}_{\tilde{f}_{1} \tilde{f}_{2}}^{(3)}=\left[\beta_{\tilde{f}_{1}}^{\tilde{W}} \beta_{\tilde{f}_{2}}^{\tilde{W}}-\alpha_{\tilde{f}_{1}}^{\tilde{W}} \alpha_{\tilde{f}_{2}}^{\tilde{W}_{2}}\right](-1)^{\theta_{i}}, \\
& \mathcal{V}_{\tilde{f}_{1} \tilde{f}_{2}}^{(4)}=\left(\alpha_{\tilde{f}_{1}}^{\tilde{W}} \beta_{\tilde{f}_{2}}^{\tilde{W}}-\beta_{\tilde{f}_{1}}^{\tilde{W}} \alpha_{\tilde{f}_{2}}^{\tilde{W}}\right)(-1)^{\theta_{c}} .
\end{aligned}
$$

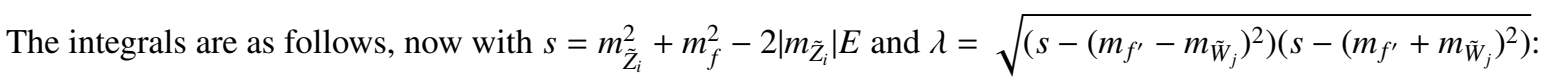

$$
\begin{gathered}
I_{\tilde{f}_{1} \tilde{f}_{2}}^{1}=2\left|m_{\tilde{Z}_{i}}\right| \int_{m_{f}}^{E_{\text {upper } 2}} d E \frac{2\left|m_{\tilde{Z}_{i}}\right|}{s} \lambda \sqrt{E^{2}-m_{f}^{2}} \frac{1}{\left(s-m_{\tilde{f}_{1}}^{2}\right)\left(s-m_{\tilde{f}_{2}}^{2}\right)}, \\
I_{\tilde{f}_{1} \tilde{f}_{2}}^{2}=2\left|m_{\tilde{Z}_{i}}\right| \int_{m_{f}}^{E_{\text {upper } 2}} d E \frac{2\left|m_{\tilde{Z}_{i}}\right|}{s} \lambda \sqrt{E^{2}-m_{f}^{2}} \frac{s-m_{\tilde{W}_{j}}^{2}-m_{f^{\prime}}^{2}}{\left(s-m_{\tilde{f}_{1}}^{2}\right)\left(s-m_{\tilde{f}_{2}}^{2}\right)},
\end{gathered}
$$




$$
\begin{gathered}
I_{\tilde{f}_{1} \tilde{f}_{2}}^{3}=2\left|m_{\tilde{Z}_{i}}\right| \int_{m_{f}}^{E_{\text {upper } 2}} d E \frac{2\left|m_{\tilde{Z}_{i}}\right|}{s} \lambda \sqrt{E^{2}-m_{f}^{2}} \frac{2\left|m_{\tilde{Z}_{i}}\right| E}{\left(s-m_{\tilde{f}_{1}}^{2}\right)\left(s-m_{\tilde{f}_{2}}^{2}\right)}, \\
I_{\tilde{f}_{1} \tilde{f}_{2}}^{4}=2\left|m_{\tilde{Z}_{i}}\right| \int_{m_{f}}^{E_{\text {upper } 2}} d E \frac{2\left|m_{\tilde{Z}_{i}}\right|}{s} \lambda \sqrt{E^{2}-m_{f}^{2}} \frac{2\left|m_{\tilde{Z}_{i}}\right| E\left(s-m_{\tilde{W}_{j}}^{2}-m_{f^{\prime}}^{2}\right)}{\left(s-m_{\tilde{f}_{1}}^{2}\right)\left(s-m_{\tilde{f}_{2}}^{2}\right)},
\end{gathered}
$$

The expression for the overall contribution as a product of these couplings and integrals is:

$$
\begin{aligned}
\Gamma_{\tilde{f}_{1} \tilde{f}_{2}}=-[ & (-1)^{\theta_{i}}(-1)^{\theta_{c}} \mathcal{V}_{\tilde{f}_{1} \tilde{f}_{2}}^{(1)} \mathcal{V}_{\tilde{f}_{1} \tilde{f}_{2}}^{(3)} I_{\tilde{f}_{1} \tilde{f}_{2}}^{4}+2(-1)^{\theta_{i}} \mathcal{V}_{\tilde{f}_{1} \tilde{f}_{2}}^{(1)} \mathcal{V}_{\tilde{f}_{1} \tilde{f}_{2}}^{(4)} m_{f}\left|m_{\tilde{W}_{j}}\right| I_{\tilde{f}_{1} \tilde{f}_{2}}^{2} \\
& \left.\left.+2(-1)^{\theta_{c}} \mathcal{V}_{\tilde{f}_{1} \tilde{f}_{2}}^{(2)} \mathcal{V}_{\tilde{f}_{1} \tilde{f}_{2}}^{(3)}\left|m_{\tilde{Z}_{i}}\right| m_{f^{\prime}} I_{\tilde{f}_{1} \tilde{f}_{2}}^{3}+4 \mathcal{V}_{\tilde{f}_{1} \tilde{f}_{2}}^{(2)} \mathcal{V}_{\tilde{f}_{1} \tilde{f}_{2}}^{(4)}\left|m_{\tilde{Z}_{i}}\right|\left|m_{\tilde{W}_{j}}\right| m_{f} m_{f^{\prime}} I_{\tilde{f}_{1} \tilde{f}_{2}}^{1}\right)\right] .
\end{aligned}
$$

It should be noted the partial widths of the decays $\tilde{Z}_{i} \rightarrow \tilde{W}_{j} f^{\prime} \bar{f}$ and the "reverse" decay $\tilde{W}_{j} \rightarrow \tilde{Z}_{i} f^{\prime} \bar{f}$ may show strong dependence on the quark masses taken for kinematic masses and for the running masses (which is used to set Yukawa couplings), of course depending on the details of the exact spectrum considered. These mass choice effects, along with the fact that SPHENO allows (small) mixing in the first two generations of sfermions whereas it is neglected in SOFTSUSY, can cause larger differences between SOFTSUSY and SPHENO of around $25 \%$. If the same mass choices are made and SPHENO's small mixing angles inserted by hand into the SOFTSUSY code then these differences are reduced to around $10 \%$.

Appendix G.3. Chargino $1 \rightarrow 3$ Decays

$\underline{\tilde{W}_{j} \rightarrow \tilde{Z}_{i} f^{\prime} \bar{f}}$

It is detailed above in the formulae for $\tilde{Z}_{i} \rightarrow \tilde{W}_{j} f^{\prime} \bar{f}$ how to adapt the formula for the chargino decaying into the neutralino rather than the neutralino decaying into the chargino.

\section{Appendix H. Decays to Gravitinos}

In certain SUSY-breaking scenarios, particularly gauge-mediated SUSY breaking (GMSB), the gravitino can be very light and therefore may be the Lightest Supersymmetric Particle (LSP). Moreover, the gravitino has longitudinal components from the goldstino which couple much more strongly than gravitational strength, this therefore provides interactions relevant to collider phenomenology, resulting in gravitino-SUSY-SM couplings that affect collider signatures, when the gravitino is the LSP. Consequently Next-to-Lightest Supersymmetric Particle (NLSP) decays to gravitino LSPs may be of interest and are included within our decay calculator program SOFTSUSY. The following decay modes are relevant when the initial SUSY particle is the NLSP:

1. $\tilde{g} \rightarrow g \tilde{G}$

2. $\tilde{q}_{i} \rightarrow q \tilde{G}$

3. $\tilde{l} \rightarrow l \tilde{G}$

4. $\tilde{Z}_{i} \rightarrow \gamma \tilde{G}$

5. $\tilde{Z}_{i} \rightarrow Z \tilde{G}$

6. $\tilde{Z}_{i} \rightarrow \phi \tilde{G}$

Decays of Higgs bosons to the gravitino are not relevant as there are always other decays available to the Higgs (whether $h$ or $A$ ) which dominate its branching ratio, for example even a Higgs boson were the NLSP then decays to $\gamma \gamma$ would be available and occur much more quickly than the Planck suppressed decays to gravitinos. The formulae used were rederived but nonetheless are as provided in [41]:

$$
\begin{gathered}
\Gamma(\tilde{g} \rightarrow g \tilde{G})=\frac{m_{\tilde{g}}^{5}}{48 \pi m_{\tilde{G}}^{2} M_{P}^{r e d^{2}}} . \\
\Gamma(\tilde{q} \rightarrow q \tilde{G})=\frac{\left(m_{\tilde{q}}^{2}-m_{q}^{2}\right)^{4}}{48 \pi m_{\tilde{q}}^{3} m_{\tilde{G}}^{2} M_{P}^{r e d}{ }^{2}} .
\end{gathered}
$$




$$
\begin{gathered}
\Gamma\left(\tilde{Z}_{i} \rightarrow \gamma \tilde{G}\right)=\frac{\left|m_{\tilde{Z}_{i}}\right|^{5}}{48 \pi m_{\tilde{G}}^{2} M_{P}^{r e d}{ }^{2}}\left[N_{1 i} \cos \theta_{W}+N_{2 i} \sin \theta_{W}\right]^{2} . \\
\Gamma\left(\tilde{Z}_{i} \rightarrow Z \tilde{G}\right)=\frac{\left(m_{\tilde{Z}_{i}}^{2}-m_{Z}^{2}\right)^{4}}{96 \pi m_{\tilde{G}}^{2} M_{P}^{r e d}{ }^{2} \mid m_{\tilde{Z}_{i}}^{3}}\left[2\left(N_{1 i} \sin \theta_{W}-N_{2 i} \cos \theta_{W}\right)^{2}+\left(N_{4 i} \sin \beta-N_{3 i} \cos \beta\right)^{2}\right] . \\
\Gamma\left(\tilde{Z}_{i} \rightarrow \phi \tilde{G}\right)=\frac{\left(m_{\tilde{Z}_{i}}^{2}-m_{\phi}^{2}\right)^{4}}{96 \pi m_{\tilde{G}}^{2} M_{P}^{r e d}\left|m_{\tilde{Z}_{i}}\right|^{3}} C_{h / H / A}^{2},
\end{gathered}
$$

where

$$
C_{h / H / A}=\left\{\begin{array}{l}
N_{4 i} \cos \alpha-N_{3 i} \sin \alpha, \text { for } \mathrm{h}, \\
N_{4 i} \sin \alpha+N_{3 i} \cos \alpha, \text { for } \mathrm{H}, \\
N_{4 i} \cos \beta+N_{3 i} \sin \beta, \text { for } \mathrm{A} .
\end{array}\right.
$$

Note that $M_{P}^{r e d}=\frac{M_{P}}{\sqrt{8 \pi}} \approx 2.4 \times 10^{18} \mathrm{GeV}$.

\section{Appendix I. NMSSM Decays}

Only 2 body decays have been included in the NMSSM, this does however include the important loop decays of the neutral Higgs bosons to $\gamma \gamma, Z \gamma$ and $g g$, as well as QCD corrections to the neutral Higgs decays to $q \bar{q}$ and $g g$. We do however include the decays $\phi \rightarrow W W^{*} \rightarrow W f^{\prime} \bar{f}$ and $\phi \rightarrow Z Z^{*} \rightarrow Z f \bar{f}^{6}$ Note that throughout $S(A, B)$ is the now $3 \times 3 \mathrm{CP}$ even Higgs mixing matrix and $P(A, B)$ is the $2 \times 3 \mathrm{CP}$ odd Higgs mixing matrix (with goldstone excluded). The additional NMSSM variables include $\lambda$ (distinct from the $\tilde{\lambda}(A, B, C)$ used for kinematic part of decay widths given above), $\kappa$ and $\mu_{e f f}=\frac{\lambda\langle S\rangle}{\sqrt{2}}$. The conventions used for the NMSSM decay formulae were detailed earlier in section 3.2, with differences with respect to the SOFTSUSY NMSSM manual [3] noted. The conventions are those of NMSSMTOols [13, 14, 27, 28], against which the formulae were checked for consistency and which provided a useful guide.

The NMSSM simply involves the addition of a gauge singlet chiral superfield to the MSSM, therefore the NMSSM has an additional neutralino, additional CP even neutral Higgs and additional CP odd neutral Higgs, therefore any decays not involving the extended neutralino or extended Higgs sectors are as in the MSSM.

\section{Appendix I.1. CP Even Higgs Decays}

First the decay to a fermion and anti-fermion, with no QCD corrections (QCD corrected formulae given later in Appendix J.

$$
\Gamma\left(h_{i} \rightarrow f \bar{f}\right)=\frac{N_{c} G_{F} m_{h_{i}}}{\sqrt{2} 4 \pi} m_{q}^{2}\left(1-4 \frac{m_{q}^{2}}{m_{h_{i}}^{2}}\right)^{\frac{3}{2}} \mathcal{A}_{h_{i} f f}^{N M S S M},
$$

where

$$
\mathcal{A}_{h_{i} f f}^{N M S S M}=\left\{\begin{array}{l}
\frac{S(i, 1)}{\sin \beta}, \text { for ' } \mathrm{u} \text { '-type fermions, } \\
\frac{S(i, 2)}{\cos \beta}, \text { for 'd'-type fermions. }
\end{array}\right.
$$

For squarks of the same handedness of the first two generations (so no mixing and negligible quark masses) the decay widths for the $\mathrm{CP}$ even Higgs i ( $\mathrm{i}=1,2,3$ are mass ordered $\mathrm{CP}$ even neutral Higgs bosons) are

$$
\Gamma\left(h_{i} \rightarrow \tilde{q}_{L / R} \tilde{q}_{L / R}\right)=\frac{N_{c}}{16 \pi m_{h_{i}}} \tilde{\lambda}^{\frac{1}{2}}\left(m_{h_{i}}, m_{\tilde{q}_{L / R}}, m_{\tilde{q}_{L / R}}\right) C^{2},
$$

\footnotetext{
${ }^{6}$ The decays $\phi \rightarrow W W^{*} \rightarrow W f^{\prime} \bar{f}$ and $\phi \rightarrow Z Z^{*} \rightarrow Z f \bar{f}$, whilst strictly being 3 body, are classified as having NDA (Number of Daughters) of 2 according to SLHA conventions.
} 
where

$$
C=\left\{\begin{array}{l}
g\left(m_{W}\left(\frac{1}{2}-\frac{\tan ^{2} \theta_{W}}{6}\right)[\sin \beta S(i, 1)-\cos \beta S(i, 2)]-\frac{m_{q}^{2} S(i, 1)}{m_{W} \sin \beta}\right), \text { for up-type LH squarks, } \\
g\left(m_{W}\left(-\frac{1}{2}-\frac{\tan ^{2} \theta_{W}}{6}\right)[\sin \beta S(i, 1)-\cos \beta S(i, 2)]-\frac{m_{q}^{2} S(i, 2)}{m_{W} \cos \beta}\right), \text { for down-type LH squarks, } \\
g\left(\frac{2}{3} m_{W} \tan ^{2} \theta_{W}[\sin \beta S(i, 1)-\cos \beta S(i, 2)]-\frac{m_{q}^{2} S(i, 1)}{m_{W} \sin \beta}\right), \text { for up-type RH squarks, } \\
g\left(\frac{-1}{3} m_{W} \tan ^{2} \theta_{W}[\sin \beta S(i, 1)-\cos \beta S(i, 2)]-\frac{m_{q}^{2} S(i, 2)}{m_{W} \cos \beta}\right), \text { for down-type RH squarks. }
\end{array}\right.
$$

For sleptons the same formulae apply but without the factor of 3 in the pre-factor from $N_{c}$ and with the coupling $C$ now (note "down type" here means the charged sleptons (i.e. sleptons of the electron, muon, tau)):

$$
C=\left\{\begin{array}{l}
g\left(m_{W}\left(\frac{1}{2}+\frac{\tan ^{2} \theta_{W}}{2}\right)[\sin \beta S(i, 1)-\cos \beta S(i, 2)]\right), \text { for sneutrinos (i.e. equivalent of up-type LH), } \\
g\left(m_{W}\left(\frac{1}{2}-\frac{\tan ^{2} \theta_{W}}{6}\right)[\sin \beta S(i, 1)-\cos \beta S(i, 2)]-\frac{m_{q}^{2} S(i, 2)}{m_{W} \cos \beta}\right), \text { for down-type LH sleptons, } \\
g\left(m_{W}\left(\tan ^{2} \theta_{W}[\sin \beta S(i, 1)-\cos \beta S(i, 2)]-\frac{m_{q}^{2} S(i, 1)}{m_{W} \cos \beta}\right),\right. \text { for down-type RH sleptons. }
\end{array}\right.
$$

For squarks of opposite handedness:

$$
\Gamma\left(h_{i} \rightarrow \tilde{q}_{L / R} \tilde{q}_{R / L}\right)=\frac{N_{c}}{16 \pi m_{h_{i}}} \tilde{\lambda}^{\frac{1}{2}}\left(m_{h_{i}}, m_{\tilde{q}_{L}}, m_{\tilde{q}_{R}}\right) \mathcal{D}^{2},
$$

where

$$
\mathcal{D}=\left\{\begin{array}{l}
\frac{g m_{q}}{2 m_{W} \sin \beta}\left[A_{q} S(i, 1)-\mu_{e f f} S(i, 2)-\lambda \frac{\sqrt{2} m_{W} \cos \beta}{g S(i, 3)}\right], \text { for up-type squarks of different handedness, } \\
\frac{g m_{q}}{2 m_{W} \cos \beta}\left[A_{q} S(i, 2)-\mu_{e f f} S(i, 1)-\lambda \frac{\sqrt{2} m_{W} \sin \beta}{g S(i, 3)}\right], \text { for down-type squarks of different handedness. }
\end{array}\right.
$$

For the decay to two sleptons with different handedness then we can use the same formulae as for two squarks of opposite handedness above but dividing by 3 to account for the fact sleptons aren't coloured (i.e. no factor $N_{c}$ ). Note as only LH sneutrinos exist, only decays to charged sleptons of opposite handedness are possible, i.e. only "downtype sleptons" of different handedness. For third generation squarks and sleptons the formulae are more complicated $\left(j=1,2\right.$ indicates $\tilde{t}_{1}$ and $\left.\tilde{t}_{2}\right)$ :

$$
\Gamma\left(h_{i} \rightarrow \tilde{t}_{j} \tilde{t}_{j}\right)=\frac{3}{16 \pi m_{h_{i}}} \tilde{\lambda}^{\frac{1}{2}}\left(m_{h_{i}}, m_{\tilde{t}_{j}}, m_{\tilde{t}_{j}}\right) C_{t_{j} t_{j}}^{2},
$$

where

$$
\begin{aligned}
C_{t_{1} t_{1}}= & \cos ^{2} \theta_{t} \sqrt{2}\left[h_{u}^{2}\left\langle h_{1}\right\rangle S(i, 1)+\left(\frac{g^{\prime 2}}{12}-\frac{g^{2}}{4}\right)\left\{\left\langle h_{1}\right\rangle S(i, 1)-\left\langle h_{2}\right\rangle S(i, 2)\right\}\right] \\
& +\sin ^{2} \theta_{t} \sqrt{2}\left[h_{u}^{2}\left\langle h_{1}\right\rangle S(i, 1)-\frac{g^{\prime 2}}{3}\left\{\left\langle h_{1}\right\rangle S(i, 1)-\left\langle h_{2}\right\rangle S(i, 2)\right\}\right] \\
& +2 \cos \theta_{t} \sin \theta_{t} \frac{h_{u}}{\sqrt{2}}\left(A_{t} S(i, 1)-\mu_{e f f} S(i, 2)-\lambda\left\langle h_{2}\right\rangle S(i, 3)\right) .
\end{aligned}
$$

This was for $j=1$ i.e. for $\tilde{t}_{1} \tilde{t}_{1}$. For $j=2$, make the replacements $\cos \theta_{t} \rightarrow-\sin \theta_{t}$ and $\sin \theta_{t} \rightarrow \cos \theta_{t}$.

For different stops:

$$
\Gamma\left(h_{i} \rightarrow \tilde{t}_{1} \tilde{t}_{2}\right)=\frac{3}{16 \pi m_{h_{i}}} \tilde{\lambda}^{\frac{1}{2}}\left(m_{h_{i}}, m_{\tilde{t}_{j}}, m_{\tilde{t}_{j}}\right) C_{t_{1} t_{2}}^{2},
$$

where

$$
\begin{aligned}
C_{t_{1} t_{2}}= & \cos \theta_{t} \sin \theta_{t}\left[\sqrt{2}\left(h_{u}^{2}\left\langle h_{1}\right\rangle S(i, 1)-\frac{g^{\prime 2}}{3}\left\{\left\langle h_{1}\right\rangle S(i, 1)-\left\langle h_{2}\right\rangle S(i, 2)\right\}\right)\right. \\
& \left.-\sqrt{2}\left(h_{u}^{2}\left\langle h_{1}\right\rangle S(i, 1)+\left(\frac{g^{\prime 2}}{12}-\frac{g^{2}}{4}\right)\left\{\left\langle h_{1}\right\rangle S(i, 1)-\left\langle h_{2}\right\rangle S(i, 2)\right\}\right)\right] \\
& +\left(\cos ^{2} \theta_{t}-\sin ^{2} \theta_{t}\right) \frac{h_{u}}{\sqrt{2}}\left(A_{t} S(i, 1)-\mu_{e f f} S(i, 2)-\lambda\left\langle h_{2}\right\rangle S(i, 3)\right) .
\end{aligned}
$$


Note $\left\langle h_{1}\right\rangle=\frac{\sqrt{2} m_{W} \sin \beta}{g}$ and $\left\langle h_{2}\right\rangle=\frac{\sqrt{2} m_{W} \cos \beta}{g}$, whilst $h_{u}=\frac{m_{t}^{r u n}}{\left\langle h_{1}\right\rangle}$. For decays to sbottoms the decay formulae are the same (with the expected mass changes) except:

$$
\begin{aligned}
C_{b_{1} b_{1}}= & \cos ^{2} \theta_{b} \sqrt{2}\left[h_{d}^{2}\left\langle h_{2}\right\rangle S(i, 2)+\left(\frac{g^{\prime 2}}{12}+\frac{g^{2}}{4}\right)\left\{\left\langle h_{1}\right\rangle S(i, 1)-\left\langle h_{2}\right\rangle S(i, 2)\right\}\right] \\
& +\sin ^{2} \theta_{b} \sqrt{2}\left[h_{d}^{2}\left\langle h_{2}\right\rangle S(i, 2)+\frac{g^{\prime 2}}{6}\left\{\left\langle h_{1}\right\rangle S(i, 1)-\left\langle h_{2}\right\rangle S(i, 2)\right\}\right] \\
& +2 \cos \theta_{b} \sin \theta_{b} \frac{h_{d}}{\sqrt{2}}\left(A_{b} S(i, 2)-\mu_{e f f} S(i, 1)-\lambda\left\langle h_{1}\right\rangle S(i, 3)\right),
\end{aligned}
$$

and

$$
\begin{aligned}
C_{b_{1} b_{2}}= & \cos \theta_{b} \sin \theta_{b}\left[\sqrt{2}\left(h_{d}^{2}\left\langle h_{2}\right\rangle S(i, 2)+\frac{g^{\prime 2}}{6}\left\{\left\langle h_{1}\right\rangle S(i, 1)-\left\langle h_{2}\right\rangle S(i, 2)\right\}\right)\right. \\
& \left.-\sqrt{2}\left(h_{d}^{2}\left\langle h_{2}\right\rangle S(i, 2)+\left(\frac{g^{\prime 2}}{12}+\frac{g^{2}}{4}\right)\left\{\left\langle h_{1}\right\rangle S(i, 1)-\left\langle h_{2}\right\rangle S(i, 2)\right\}\right)\right] \\
& +\left(\cos ^{2} \theta_{b}-\sin ^{2} \theta_{b}\right) \frac{h_{d}}{\sqrt{2}}\left(A_{b} S(i, 2)-\mu_{e f f} S(i, 1)-\lambda\left\langle h_{1}\right\rangle S(i, 3)\right) .
\end{aligned}
$$

Note $h_{d}=\frac{m_{b}^{r u n}}{\left\langle h_{2}\right\rangle}$. Again to get the $\tilde{b}_{2} \tilde{b}_{2}$ coupling from the $\tilde{b}_{1} \tilde{b}_{1}$, make the changes $\cos \theta_{b} \rightarrow-\sin \theta_{b}$ and $\sin \theta_{b} \rightarrow \cos \theta_{b}$. For staus:

$$
\begin{gathered}
\Gamma\left(h_{i} \rightarrow \tilde{\tau}_{j} \tilde{\tau}_{k}\right)=\frac{1}{16 \pi m_{h_{i}}} \tilde{\lambda}^{\frac{1}{2}}\left(m_{h_{i}}, m_{\tilde{\tau}_{j}}, m_{\tilde{\tau}_{k}}\right) C_{\tau_{j} \tau_{k}}^{2} . \\
C_{\tau_{1} \tau_{1}}=\sin ^{2} \theta_{\tau} \sqrt{2}\left[h_{\tau}^{2}\left\langle h_{2}\right\rangle S(i, 2)+\left(-\frac{g^{\prime 2}}{4}+\frac{g^{2}}{4}\right)\left\{\left\langle h_{1}\right\rangle S(i, 1)-\left\langle h_{2}\right\rangle S(i, 2)\right\}\right] \\
+\cos ^{2} \theta_{\tau} \sqrt{2}\left[h_{\tau}^{2}\left\langle h_{2}\right\rangle S(i, 2)+\frac{g^{\prime 2}}{2}\left\{\left\langle h_{1}\right\rangle S(i, 1)-\left\langle h_{2}\right\rangle S(i, 2)\right\}\right] \\
+2 \cos \theta_{\tau} \sin \theta_{\tau} \frac{h_{\tau}}{\sqrt{2}}\left(A_{\tau} S(i, 2)-\mu_{e f f} S(i, 1)-\lambda\left\langle h_{1}\right\rangle S(i, 3)\right), \\
C_{\tau_{2} \tau_{2}=} \cos ^{2} \theta_{\tau} \sqrt{2}\left[h_{\tau}^{2}\left\langle h_{2}\right\rangle S(i, 2)+\left(-\frac{g^{\prime 2}}{4}+\frac{g^{2}}{4}\right)\left\{\left\langle h_{1}\right\rangle S(i, 1)-\left\langle h_{2}\right\rangle S(i, 2)\right\}\right] \\
+\sin ^{2} \theta_{\tau} \sqrt{2}\left[h_{\tau}^{2}\left\langle h_{2}\right\rangle S(i, 2)+\frac{g^{\prime 2}}{2}\left\{\left\langle h_{1}\right\rangle S(i, 1)-\left\langle h_{2}\right\rangle S(i, 2)\right\}\right] \\
-2 \cos \theta_{\tau} \sin \theta_{\tau} \frac{h_{\tau}}{\sqrt{2}}\left(A_{\tau} S(i, 2)-\mu_{e f f} S(i, 1)-\lambda\left\langle h_{1}\right\rangle S(i, 3)\right),
\end{gathered}
$$

and

$$
\begin{aligned}
\mathcal{C}_{\tau_{1} \tau_{2}}= & -\cos \theta_{\tau} \sin \theta_{\tau}\left[\sqrt{2}\left(h_{\tau}^{2}\left\langle h_{2}\right\rangle S(i, 2)+\frac{g^{\prime 2}}{2}\left\{\left\langle h_{1}\right\rangle S(i, 1)-\left\langle h_{2}\right\rangle S(i, 2)\right\}\right)\right. \\
& \left.-\sqrt{2}\left(h_{\tau}^{2}\left\langle h_{2}\right\rangle S(i, 2)+\left(-\frac{g^{\prime 2}}{4}+\frac{g^{2}}{4}\right)\left\{\left\langle h_{1}\right\rangle S(i, 1)-\left\langle h_{2}\right\rangle S(i, 2)\right\}\right)\right] \\
& +\left(\sin ^{2} \theta_{\tau}-\cos ^{2} \theta_{\tau}\right) \frac{h_{\tau}}{\sqrt{2}}\left(A_{\tau} S(i, 2)-\mu_{e f f} S(i, 1)-\lambda\left\langle h_{1}\right\rangle S(i, 3)\right) .
\end{aligned}
$$

Now the decays to charginos, first of all decays to the same chargino:

$$
\Gamma\left(h_{i} \rightarrow \tilde{W}_{j} \tilde{W}_{j}\right)=\frac{m_{h_{i}}}{8 \pi} \tilde{\lambda}^{\frac{3}{2}}\left(m_{h_{i}}, m_{\tilde{W}_{j}}, m_{\tilde{W}_{j}}\right) \mathcal{F}_{j j}^{2}
$$

where

$$
\mathcal{F}_{j j}=\left\{\begin{array}{l}
\frac{\lambda}{\sqrt{2}} S(i, 3) \cos \theta_{L} \cos \theta_{R}+\frac{g}{\sqrt{2}}\left[S(i, 1) \sin \theta_{L} \cos \theta_{R}+S(i, 2) \cos \theta_{L} \sin \theta_{R}\right], \text { for } j=1, \\
\frac{\lambda}{\sqrt{2}} S(i, 3) \sin \theta_{L} \sin \theta_{R}-\frac{g}{\sqrt{2}}\left[S(i, 1) \cos \theta_{L} \sin \theta_{R}+S(i, 2) \sin \theta_{L} \cos \theta_{R}\right], \text { for } j=2
\end{array}\right.
$$


For decays to different charginos:

$$
\Gamma\left(h_{i} \rightarrow \tilde{W}_{1} \tilde{W}_{2}\right)=\frac{m_{h_{i}}}{16 \pi} \tilde{\lambda}^{\frac{1}{2}}\left(m_{h_{i}}, m_{\tilde{W}_{1}}, m_{\tilde{W}_{2}}\right)\left[\left(c_{1}^{2}+c_{2}^{2}\right) \frac{1}{m_{h_{i}}^{2}}\left(m_{h_{i}}^{2}-m_{\tilde{W}_{1}}^{2}-m_{\tilde{W}_{2}}^{2}\right)-4 c_{1} c_{2} \frac{m_{\tilde{W}_{1}} m_{\tilde{W}_{2}}}{m_{h_{i}}^{2}}\right],
$$

where

$$
\begin{aligned}
& c_{1}=\frac{\lambda}{\sqrt{2}} S(i, 3) \cos \theta_{L} \sin \theta_{R}+\frac{g}{\sqrt{2}}\left(S(i, 1) \sin \theta_{L} \sin \theta_{R}-S(i, 2) \cos \theta_{L} \cos \theta_{R}\right), \\
& c_{2}=\frac{\lambda}{\sqrt{2}} S(i, 3) \sin \theta_{L} \cos \theta_{R}-\frac{g}{\sqrt{2}}\left(S(i, 1) \cos \theta_{L} \cos \theta_{R}-S(i, 2) \sin \theta_{L} \sin \theta_{R}\right) .
\end{aligned}
$$

Now the decays to neutralinos:

$$
\Gamma\left(h_{i} \rightarrow \tilde{Z}_{j} \tilde{Z}_{k}\right)=\frac{\alpha_{j k} m_{h_{i}}}{16 \pi}\left(1-\left(\frac{m_{\tilde{Z}_{j}}+m_{\tilde{Z}_{k}}}{m_{h_{i}}}\right)^{2}\right) \tilde{\lambda}^{\frac{1}{2}}\left(m_{h_{i}}, m_{\tilde{Z}_{j}}, m_{\tilde{Z}_{k}}\right) \mathcal{G}_{i j k}^{2}
$$

where

$$
\begin{aligned}
\mathcal{G}_{i j k}= & \frac{\lambda}{\sqrt{2}}\left[S(i, 1)\left(N_{3 j} N_{5 k}+N_{5 j} N_{3 k}\right)+S(i, 2)\left(N_{4 j} N_{5 k}+N_{5 j} N_{4 k}\right)\right. \\
& \left.+S(i, 3)\left(N_{3 j} N_{4 k}+N_{4 j} N_{3 k}\right)\right]-\sqrt{2} \kappa S(i, 3) N_{5 j} N_{5 k} \\
& +\frac{g^{\prime}}{2}\left[-S(i, 1)\left(N_{1 j} N_{4 k}+N_{1 k} N_{4 j}\right)\right. \\
& \left.+S(i, 2)\left(N_{1 j} N_{3 k}+N_{3 j} N_{1 k}\right)\right]+\frac{g}{2}\left[S(i, 1)\left(N_{2 j} N_{4 k}+N_{4 j} N_{2 k}\right)\right. \\
& \left.-S(i, 2)\left(N_{2 j} N_{3 k}+N_{3 j} N_{2 k}\right)\right] .
\end{aligned}
$$

Here $N_{a b}$ is the neutralino mixing matrix which is now $5 \times 5$ as the singlino mixes with the four original neutralinos. The neutralinos here are in order of increasing mass. The conventions for the NMSSM were detailed previously in section 3.2. Note the $\alpha_{j k}$ is 2 if $j \neq k$ and 1 if $j=k$ in order to account for indistinguishability of particles.

The neutral Higgs decays to CP odd neutral Higgs bosons are given by:

$$
\Gamma\left(h_{i} \rightarrow A_{j} A_{k}\right)=\frac{1}{16 \pi m_{h_{i}}} \tilde{\lambda}^{\frac{1}{2}}\left(m_{h_{i}}, m_{A_{j}}, m_{A_{k}}\right) Q_{j k}^{2},
$$

where

$$
\begin{aligned}
Q_{j k}= & \frac{g^{2}+g^{\prime 2}}{4 \sqrt{2}}\left[\left\langle h_{1}\right\rangle\{C(i, j, k, 1,1,1)-C(i, j, k, 1,2,2)\}+\left\langle h_{2}\right\rangle\{C(i, j, k, 2,2,2)-C(i, j, k, 2,1,1)\}\right] \\
& +\frac{\lambda A_{\lambda}}{\sqrt{2}}\{C(i, j, k, 1,2,3)+C(i, j, k, 2,1,3)+C(i, j, k, 3,1,2)\}-\frac{\kappa A_{k}}{\sqrt{2}} C(i, j, k, 3,3,3) \\
& +\frac{\lambda^{2}}{\sqrt{2}}\left[\left\langle h_{1}\right\rangle\{C(i, j, k, 1,2,2)+C(i, j, k, 1,3,3)\}+\left\langle h_{2}\right\rangle\{C(i, j, k, 2,1,1)+C(i, j, k, 2,3,3)\}\right. \\
& \left.+\frac{\mu_{e f f}}{\lambda}\{C(i, j, k, 3,1,1)+C(i, j, k, 3,2,2)\}\right]+\frac{\kappa^{2} \sqrt{2} \mu_{e f f}}{\lambda} C(i, j, k, 3,3,3) \\
& +\frac{\lambda \kappa}{\sqrt{2}}\left[\left\langle h_{1}\right\rangle\{C(i, j, k, 2,3,3)-2 C(i, j, k, 3,2,3)\}+\left\langle h_{2}\right\rangle\{C(i, j, k, 1,3,3)-2 C(i, j, k, 3,1,3)\}\right. \\
& \left.+2 \frac{\mu_{e f f}}{\lambda}\{C(i, j, k, 3,1,2)-C(i, j, k, 1,2,3)-C(i, j, k, 2,1,3)\}\right] .
\end{aligned}
$$

The $C(i, j, k, x, y, z)$ is the same coupling which appears later in (I.111).

$$
\Gamma\left(h_{i} \rightarrow A_{j} Z\right)=\frac{\left(g^{2}+g^{\prime 2}\right) m_{h_{i}}^{3}}{64 \pi m_{Z}^{2}} \tilde{\lambda}^{\frac{3}{2}}\left(m_{h_{i}}, m_{A_{j}}, m_{Z}\right) \mathcal{R}_{i j}^{2},
$$


where

$$
\begin{gathered}
\mathcal{R}_{i j}=(S(i, 1) \cos \beta-S(i, 2) \sin \beta)(P(j, 1) \cos \beta-P(j, 2) \sin \beta) . \\
\Gamma\left(h_{i} \rightarrow H^{+} H^{-}\right)=\frac{1}{16 \pi m_{h_{i}}} \tilde{\lambda}^{\frac{1}{2}}\left(m_{h_{i}}, m_{H^{ \pm}}, m_{H^{ \pm}}\right) \mathcal{S}_{i}^{2},
\end{gathered}
$$

where

$$
\begin{aligned}
\mathcal{S}_{i}= & \frac{\lambda \mu_{e f f}}{\sqrt{2}}\left[2 S(i, 3) \cos ^{2} \beta+2 S(i, 3) \sin ^{2} \beta\right]-\frac{\lambda^{2} m_{W} \sin \beta}{g}(2 S(i, 2)+2 S(i, 1)) \cos \beta \sin \beta \\
& +\mu_{e f f} \kappa 2 \sqrt{2} S(i, 3) \cos \beta \sin \beta+\lambda A_{\lambda} \sqrt{2} S(i, 3) \cos \beta \sin \beta \\
& +\frac{g^{\prime 2}}{4} \frac{m_{W}}{g}\left[\sin \beta\left(2 S(i, 1) \cos ^{2} \beta-2 S(i, 1) \sin ^{2} \beta\right)+\cos \beta\left(2 S(i, 2) \sin ^{2} \beta-2 S(i, 2) \cos ^{2} \beta\right)\right] \\
& +\frac{g m_{W}}{4}\left[\sin \beta\left(2 S(i, 1) \sin ^{2} \beta-2 S(i, 2) \cos ^{2} \beta+4 S(i, 2) \sin \beta \cos \beta\right)\right. \\
& \left.+\cos \beta\left(2 S(i, 2) \cos ^{2} \beta+2 S(i, 2) \sin ^{2} \beta+4 S(i, 1) \sin \beta \cos \beta\right)\right] . \\
& \quad \Gamma\left(h_{i} \rightarrow W^{ \pm} H^{ \pm}\right)=\frac{G_{F} m_{h_{i}}^{3}}{8 \pi}[S(i, 1) \cos \beta-S(i, 2) \sin \beta]^{2} \tilde{\lambda}^{\frac{3}{2}}\left(m_{h_{i}}, m_{W^{ \pm}}, m_{H^{ \pm}}\right) .
\end{aligned}
$$

In this equation a factor of 2 has been included as it could be either $W^{+} H^{-}$or $W^{-} H^{+}$

$$
\Gamma\left(h_{i} \rightarrow h_{j} h_{k}\right)=\frac{\alpha_{j k}}{32 \pi m_{h_{i}}} \tilde{\lambda}^{\frac{1}{2}}\left(m_{h_{i}}, m_{h_{j}}, m_{h_{k}}\right)\left[C_{h_{i} h_{j} h_{k}}^{N M S S M}\right]^{2},
$$

where again $\alpha_{j k}=1$ if $j=k$ and 2 otherwise. Also remember that $\mu_{e f f}=\frac{\lambda\langle s\rangle}{\sqrt{2}},\left\langle h_{1}\right\rangle=\frac{\sqrt{2} m_{W} \sin \beta}{g}$ and $\left\langle h_{2}\right\rangle=\frac{\sqrt{2} m_{W} \cos \beta}{g}$, where $\lambda$ is a parameter of the NMSSM which couples the singlino to higgsinos. Here $C_{h_{i} h_{j} h_{k}}^{N M S S M}$ is given by:

$$
\begin{aligned}
C_{h_{i} h_{j} h_{k}}^{N M S S M}= & \frac{g^{2}+g^{\prime 2}}{4 \sqrt{2}}\left[\left\langle h_{1}\right\rangle\left[S_{i j k}(1,1,1)-S_{i j k}(1,2,2)\right]+\left\langle h_{2}\right\rangle\left[S_{i j k}(2,2,2)-S_{i j k}(2,1,1)\right]\right]-\frac{\lambda A_{\lambda}}{\sqrt{2}} S_{i j k}(1,2,3)+\frac{\kappa A_{\kappa}}{3 \sqrt{2}} S_{i j k}(3,3,3) \\
& +\frac{\lambda^{2}}{\sqrt{2}}\left[\left\langle h_{1}\right\rangle\left[S_{i j k}(1,2,2)+S_{i j k}(1,3,3)\right]+\left\langle h_{2}\right\rangle\left[S_{i j k}(2,1,1)+S_{i j k}(2,3,3)\right]+\frac{\mu_{e f f}}{\lambda}\left[S_{i j k}(3,1,1)+S_{i j k}(3,2,2)\right]\right] \\
& +\kappa^{2} \sqrt{2} \frac{\mu_{e f f}}{\lambda} S_{i j k}(3,3,3)-\frac{\lambda \kappa}{\sqrt{2}}\left[\left\langle h_{1}\right\rangle S_{i j k}(3,2,3)+\left\langle h_{2}\right\rangle S_{i j k}(3,1,3)+2 \frac{\mu_{e f f}}{\lambda} S_{i j k}(1,2,3)\right],
\end{aligned}
$$

where $S_{i j k}(x, y, z)$ is just the symmetric combination of triples of $S$ matrix elements with each of $i, j, k$ with each of $x, y, z$, i.e.:

$$
\begin{aligned}
S_{i j k}(x, y, z)= & S(i, x) S(j, y) S(k, z)+S(i, x) S(k, y) S(j, z)+S(j, x) S(i, y) S(k, z) \\
& +S(j, x) S(i, z) S(k, y)+S(k, x) S(i, y) S(j, z)+S(k, x) S(j, y) S(i, z) .
\end{aligned}
$$

Decays to two vector bosons are complicated by the consideration of whether the Higgs boson mass is greater than twice the mass of the vector boson, just as they were complicated in the MSSM. Included in SOFTSUSY for the NMSSM are the cases both where the Higgs has mass $m_{h / H / H 3}>2 m_{V}$, and so decays to two on-shell vector bosons, and also the case where the Higgs has mass $m_{V}<m_{h / H / H 3}<2 m_{V}$ so that it may only undergo a decay to one on-shell vector boson and one off-shell vector boson, which then decays into a fermion anti-fermion pair, i.e. $h / H / H 3 \rightarrow$ $W W^{*} \rightarrow W f^{\prime} \bar{f}$ and $h / H / H 3 \rightarrow Z Z^{*} \rightarrow Z f \bar{f}$, exactly as were included for the MSSM.

As in the MSSM, first consider $m_{V}<m_{h / H / H 3}<2 m_{V}$. The only difference compared with the MSSM formulae is in the couplings $c_{h / H / H 3 V V}$.

$$
\begin{aligned}
\Gamma\left(h / H / H 3 \rightarrow Z Z^{*}\right) & =\frac{G_{F}^{2} m_{h / H / H 3} m_{W}^{4} c_{h / H / H 3 V V}^{2}}{64 \pi^{3} \cos ^{4} \theta_{W}} F\left(\epsilon_{Z}\right)\left[7-\frac{40}{3} \sin ^{2} \theta_{W}+\frac{160}{9} \sin ^{4} \theta_{W}\right], \\
\Gamma\left(h / H / H 3 \rightarrow W W^{*}\right) & =\frac{3 G_{F}^{2} m_{W}^{4} m_{h / H / H 3} c_{h / H / H 3 V V}^{2}}{16 \pi^{3}} F\left(\epsilon_{W}\right),
\end{aligned}
$$


where here

$$
\begin{gathered}
\epsilon_{V}=\frac{m_{V}}{m_{h / H / H 3}}, \\
c_{h V V}=S(1,1) \sin \beta+S(1,2) \cos \beta, \\
c_{H V V}=S(2,1) \sin \beta+S(2,2) \cos \beta, \\
c_{H 3 V V}=S(3,1) \sin \beta+S(3,2) \cos \beta,
\end{gathered}
$$

and as before

$$
F\left(\epsilon_{V}\right)=\frac{3\left(1-8 \epsilon_{V}^{2}+20 \epsilon_{V}^{4}\right)}{\sqrt{4 \epsilon_{V}^{2}-1}} \cos ^{-1}\left[\frac{3 \epsilon_{V}^{2}-1}{2 \epsilon_{V}^{3}}\right]-\left(1-\epsilon_{V}^{2}\right)\left(\frac{47}{2} \epsilon_{V}^{2}-\frac{13}{2}+\frac{1}{\epsilon_{V}^{2}}\right)-3\left(1-6 \epsilon_{V}^{2}+4 \epsilon_{V}^{4}\right) \log \left(\epsilon_{V}\right) .
$$

If however $m_{h / H / H 3}>2 m_{V}$ then the decay to two on-shell vector bosons occurs instead and the formulae are then:

$$
\begin{aligned}
\Gamma(h / H / H 3 \rightarrow W W) & =\frac{G_{F} m_{h / H / H 3}^{3}}{8 \pi \sqrt{2}} \tilde{\lambda}^{\frac{1}{2}}\left(m_{h / H / H 3}, m_{W}, m_{W}\right)\left(1-r^{2}+\frac{3}{4} r^{4}\right) c_{h / H / H 3 W W}^{2}, \\
\Gamma(h / H / H 3 \rightarrow Z Z) & =\frac{G_{F} m_{h / H / H 3}^{3}}{16 \pi \sqrt{2}} \tilde{\lambda}^{\frac{1}{2}}\left(m_{h / H / H 3}, m_{Z}, m_{Z}\right)\left(1-r^{2}+\frac{3}{4} r^{4}\right) c_{h / H / H 3 Z Z}^{2} .
\end{aligned}
$$

Remember $r=2 \frac{m_{V}}{m_{h / H / H 3}}$.

Now onto the loop decays of the neutral Higgs bosons in the NMSSM:

$$
\Gamma\left(h_{i} \rightarrow \gamma \gamma\right)=\frac{G_{F}}{\sqrt{2}} \frac{m_{h_{i}}^{3} \alpha_{e m}^{2}\left(m_{h_{i}}\right)}{32 \pi^{2}}\left|M_{\gamma \gamma}\right|^{2}
$$

where

$$
\begin{aligned}
\left|M_{\gamma \gamma}\right|^{2}= & {\left[I_{t}^{r}+I_{b}^{r}+I_{c}^{r}+I_{\tau}^{r}+I_{\tilde{W}_{1}}^{r}+I_{\tilde{W}_{2}}^{r}+I_{W}^{r}+I_{H^{ \pm}}^{r}+I_{\tilde{c}_{L}}^{r}+I_{\tilde{c}_{R}}^{r}+I_{\tilde{s}_{L}}^{r}+I_{\tilde{S}_{R}}^{r}+I_{\tilde{\mu}_{L}}^{r}+I_{\tilde{\mu}_{R}}^{r}+I_{\tilde{t}_{1}}^{r}+I_{\tilde{t}_{2}}^{r}\right.} \\
& \left.+I_{\tilde{b}_{1}}^{r}+I_{\tilde{b}_{2}}^{r}+I_{\tilde{\tau}_{1}}^{r}+I_{\tilde{\tau}_{2}}^{r}\right]^{2}+\left[I_{t}^{i}+I_{b}^{i}+I_{c}^{i}+I_{\tau}^{i}+I_{\tilde{W}_{1}}^{i}+I_{\tilde{W}_{2}}^{i}+I_{W}^{i}+I_{H^{ \pm}}^{i}+I_{\tilde{c}_{L}}^{i}+I_{\tilde{c}_{R}}^{i}+I_{\tilde{s}_{L}}^{i}\right. \\
& \left.+I_{\tilde{s}_{R}}^{i}+I_{\tilde{\mu}_{L}}^{i}+I_{\tilde{\mu}_{R}}^{i}+I_{\tilde{t}_{1}}^{i}+I_{\tilde{t}_{2}}^{i}+I_{\tilde{b}_{1}}^{i}+I_{\tilde{b}_{2}}^{i}+I_{\tilde{\tau}_{1}}^{i}+I_{\tilde{\tau}_{2}}^{i}\right]^{2} .
\end{aligned}
$$

The $I_{a}^{r / i}$ are given below and are the real (r) and imaginary (i) parts.

$$
I_{a}=c_{a} k_{a},
$$

where the $c_{a}$ is the coupling for particle $a$ and the $k_{a}$ is the kinetic part for particle $a$. The coupling is real whilst the kinetic part may be complex, it is from the kinetic part therefore that we get real and imaginary contributions. The couplings of the various loop particles are:

$$
\begin{aligned}
c_{t} & =\frac{4}{3} \frac{S(i, 1)}{\sin \beta}, \\
c_{c} & =\frac{4}{3} \frac{S(i, 1)}{\sin \beta}, \\
c_{b} & =\frac{1}{3} \frac{S(i, 2)}{\cos \beta}, \\
c_{\tau} & =\frac{S(i, 2)}{\cos \beta}, \\
c_{W}=S(i, 1) & \sin \beta+S(i, 2) \cos \beta,
\end{aligned}
$$




$$
\begin{aligned}
& c_{\tilde{W}_{1}}=\frac{1}{\sqrt{\sqrt{2} G_{F}} m_{\tilde{W}_{1}}} \frac{\lambda}{\sqrt{2}} S(i, 3) \cos \theta_{L} \cos \theta_{R}+\frac{g}{\sqrt{2}}\left[S(i, 1) \sin \theta_{L} \cos \theta_{R}+S(i, 2) \cos \theta_{L} \sin \theta_{R}\right], \\
& c_{\tilde{W}_{2}}=\frac{1}{\sqrt{\sqrt{2} G_{F}} m_{\tilde{W}_{2}}} \frac{\lambda}{\sqrt{2}} S(i, 3) \sin \theta_{L} \sin \theta_{R}-\frac{g}{\sqrt{2}}\left[S(i, 1) \cos \theta_{L} \sin \theta_{R}+S(i, 2) \sin \theta_{L} \cos \theta_{R}\right], \\
& c_{\tilde{c}_{L}}=\frac{4}{3} \frac{2 m_{W}}{g m_{\tilde{c}_{L}}^{2}}\left[\frac{g^{\prime 2}}{12}+\frac{g^{2}}{4}\right] \frac{2 m_{W}}{g}(\sin \beta S(i, 1)-\cos \beta S(i, 2)), \\
& c_{\tilde{c}_{R}}=\frac{4}{3} \frac{2 m_{W}}{g m_{\tilde{c}_{R}}^{2}} \frac{g^{\prime 2}}{6} \frac{2 m_{W}}{g}(\sin \beta S(i, 1)-\cos \beta S(i, 2)), \\
& c_{\tilde{s}_{L}}=\frac{1}{3} \frac{2 m_{W}}{g m_{\tilde{s}_{L}}^{2}}\left[\frac{g^{\prime 2}}{12}+\frac{g^{2}}{4}\right] \frac{2 m_{W}}{g}(\sin \beta S(i, 1)-\cos \beta S(i, 2)), \\
& c_{\tilde{S}_{R}}=\frac{1}{3} \frac{2 m_{W}}{g m_{\tilde{s}_{R}}^{2}} \frac{g^{\prime 2}}{6} \frac{2 m_{W}}{g}(\sin \beta S(i, 1)-\cos \beta S(i, 2)), \\
& c_{\tilde{\mu}_{L}}=\frac{2 m_{W}}{g m_{\tilde{\mu}_{L}}^{2}}\left[\frac{-g^{\prime 2}}{4}+\frac{g^{2}}{4}\right] \frac{2 m_{W}}{g}(\sin \beta S(i, 1)-\cos \beta S(i, 2)), \\
& c_{\tilde{\mu}_{R}}=\frac{2 m_{W}}{g m_{\tilde{\mu}_{R}}^{2}} \frac{g^{\prime 2}}{2} \frac{2 m_{W}}{g}(\sin \beta S(i, 1)-\cos \beta S(i, 2)), \\
& c_{\tilde{t}_{1}}=\frac{1}{2 \sqrt{\sqrt{2} G_{F}} m_{\tilde{t}_{1}}^{2}}\left\{\operatorname { c o s } ^ { 2 } \theta _ { t } \sqrt { 2 } \left[f_{t}^{2} \sqrt{2} \frac{m_{W} \sin \beta}{g} S(i, 1)+\left(\frac{g^{\prime 2}}{12}-\frac{g^{2}}{4}\right)\left\{\frac{\sqrt{2} m_{W} \sin \beta}{g} S(i, 1)\right.\right.\right. \\
& \left.\left.-\frac{\sqrt{2} m_{W} \cos \beta}{g} S(i, 2)\right\}\right]+\sin ^{2} \theta_{t} \sqrt{2}\left[f_{t}^{2} \frac{\sqrt{2} m_{W} \sin \beta}{g} S(i, 1)\right. \\
& \left.-\frac{g^{\prime 2}}{3}\left\{\frac{\sqrt{2} m_{W} \sin \beta}{g} S(i, 1)-\frac{\sqrt{2} m_{W} \cos \beta}{g} S(i, 2)\right\}\right] \\
& \left.+2 \sin \theta_{t} \cos \theta_{t} \frac{f_{t}}{\sqrt{2}}\left[A_{t} S(i, 1)-\mu_{e f f} S(i, 2)-\lambda \frac{\sqrt{2} m_{W} \cos \beta}{g} S(i, 3)\right]\right\}, \\
& c_{\tilde{t}_{2}}=\frac{1}{2 \sqrt{\sqrt{2} G_{F}} m_{\tilde{t}_{2}}^{2}}\left\{\operatorname { s i n } ^ { 2 } \theta _ { t } \sqrt { 2 } \left[f_{t}^{2} \sqrt{2} \frac{m_{W} \sin \beta}{g} S(i, 1)+\left(\frac{g^{\prime 2}}{12}-\frac{g^{2}}{4}\right)\left\{\frac{\sqrt{2} m_{W} \sin \beta}{g} S(i, 1)\right.\right.\right. \\
& \left.\left.-\frac{\sqrt{2} m_{W} \cos \beta}{g} S(i, 2)\right\}\right]+\cos ^{2} \theta_{t} \sqrt{2}\left[f_{t}^{2} \frac{\sqrt{2} m_{W} \sin \beta}{g} S(i, 1)\right. \\
& \left.-\frac{g^{\prime 2}}{3}\left\{\frac{\sqrt{2} m_{W} \sin \beta}{g} S(i, 1)-\frac{\sqrt{2} m_{W} \cos \beta}{g} S(i, 2)\right\}\right] \\
& \left.-2 \sin \theta_{t} \cos \theta_{t} \frac{f_{t}}{\sqrt{2}}\left[A_{t} S(i, 1)-\mu_{e f f} S(i, 2)-\lambda \frac{\sqrt{2} m_{W} \cos \beta}{g} S(i, 3)\right]\right\}, \\
& c_{\tilde{b}_{1}}=\frac{1}{2 \sqrt{\sqrt{2} G_{F}} m_{\tilde{b}_{1}}^{2}}\left\{\operatorname { c o s } ^ { 2 } \theta _ { b } \sqrt { 2 } \left[f_{b}^{2} \sqrt{2} \frac{m_{W} \cos \beta}{g} S(i, 2)+\left(\frac{g^{\prime 2}}{12}+\frac{g^{2}}{4}\right)\left\{\frac{\sqrt{2} m_{W} \sin \beta}{g} S(i, 1)\right.\right.\right. \\
& \left.\left.-\frac{\sqrt{2} m_{W} \cos \beta}{g} S(i, 2)\right\}\right]+\sin ^{2} \theta_{b} \sqrt{2}\left[f_{b}^{2} \frac{\sqrt{2} m_{W} \cos \beta}{g} S(i, 2)+\frac{g^{\prime 2}}{6}\left\{\frac{\sqrt{2} m_{W} \sin \beta}{g} S(i, 1)\right.\right. \\
& \left.\left.\left.-\frac{\sqrt{2} m_{W} \cos \beta}{g} S(i, 2)\right\}\right]+2 \sin \theta_{b} \cos \theta_{b} \frac{f_{b}}{\sqrt{2}}\left[A_{b} S(i, 2)-\mu_{e f f} S(i, 1)-\lambda \frac{\sqrt{2} m_{W} \sin \beta}{g} S(i, 3)\right]\right\} \text {, }
\end{aligned}
$$




$$
\begin{aligned}
& c_{\tilde{b}_{2}}=\frac{1}{2 \sqrt{\sqrt{2} G_{F}} m_{\tilde{b}_{2}}^{2}}\left\{\operatorname { s i n } ^ { 2 } \theta _ { b } \sqrt { 2 } \left[f_{b}^{2} \sqrt{2} \frac{m_{W} \cos \beta}{g} S(i, 2)+\left(\frac{g^{\prime 2}}{12}+\frac{g^{2}}{4}\right)\left\{\frac{\sqrt{2} m_{W} \sin \beta}{g} S(i, 1)\right.\right.\right. \\
& \left.\left.-\frac{\sqrt{2} m_{W} \cos \beta}{g} S(i, 2)\right\}\right]+\cos ^{2} \theta_{b} \sqrt{2}\left[f_{b}^{2} \frac{\sqrt{2} m_{W} \cos \beta}{g} S(i, 2)+\frac{g^{\prime 2}}{6}\left\{\frac{\sqrt{2} m_{W} \sin \beta}{g} S(i, 1)\right.\right. \\
& \left.\left.\left.-\frac{\sqrt{2} m_{W} \cos \beta}{g} S(i, 2)\right\}\right]-2 \sin \theta_{b} \cos \theta_{b} \frac{f_{b}}{\sqrt{2}}\left[A_{b} S(i, 2)-\mu_{e f f} S(i, 1)-\lambda \frac{\sqrt{2} m_{W} \sin \beta}{g} S(i, 3)\right]\right\}, \\
& c_{\tilde{\tau}_{1}}=\frac{1}{2 \sqrt{\sqrt{2} G_{F}} m_{\tilde{\tau}_{1}}^{2}}\left\{\operatorname { c o s } ^ { 2 } \theta _ { \tau } \sqrt { 2 } \left[f_{\tau}^{2} \sqrt{2} \frac{m_{W} \cos \beta}{g} S(i, 2)+\left(-\frac{g^{\prime 2}}{4}+\frac{g^{2}}{4}\right)\left\{\frac{\sqrt{2} m_{W} \sin \beta}{g} S(i, 1)\right.\right.\right. \\
& \left.\left.-\frac{\sqrt{2} m_{W} \cos \beta}{g} S(i, 2)\right\}\right]+\sin ^{2} \theta_{\tau} \sqrt{2}\left[f_{\tau}^{2} \frac{\sqrt{2} m_{W} \cos \beta}{g} S(i, 2)+\frac{g^{\prime 2}}{2}\left\{\frac{\sqrt{2} m_{W} \sin \beta}{g} S(i, 1)\right.\right. \\
& \left.\left.\left.-\frac{\sqrt{2} m_{W} \cos \beta}{g} S(i, 2)\right\}\right]+2 \sin \theta_{\tau} \cos \theta_{\tau} \frac{f_{\tau}}{\sqrt{2}}\left[A_{\tau} S(i, 2)-\mu_{e f f} S(i, 1)-\lambda \frac{\sqrt{2} m_{W} \sin \beta}{g} S(i, 3)\right]\right\}, \\
& c_{\tilde{\tau}_{2}}=\frac{1}{2 \sqrt{\sqrt{2} G_{F}} m_{\tilde{\tau}_{2}}^{2}}\left\{\operatorname { s i n } ^ { 2 } \theta _ { \tau } \sqrt { 2 } \left[f_{\tau}^{2} \sqrt{2} \frac{m_{W} \cos \beta}{g} S(i, 2)+\left(-\frac{g^{\prime 2}}{4}+\frac{g^{2}}{4}\right)\left\{\frac{\sqrt{2} m_{W} \sin \beta}{g} S(i, 1)\right.\right.\right. \\
& \left.\left.-\frac{\sqrt{2} m_{W} \cos \beta}{g} S(i, 2)\right\}\right]+\cos ^{2} \theta_{\tau} \sqrt{2}\left[f_{\tau}^{2} \frac{\sqrt{2} m_{W} \cos \beta}{g} S(i, 2)+\frac{g^{\prime 2}}{2}\left\{\frac{\sqrt{2} m_{W} \sin \beta}{g} S(i, 1)\right.\right. \\
& \left.\left.\left.-\frac{\sqrt{2} m_{W} \cos \beta}{g} S(i, 2)\right\}\right]-2 \sin \theta_{\tau} \cos \theta_{\tau} \frac{f_{\tau}}{\sqrt{2}}\left[A_{\tau} S(i, 2)-\mu_{e f f} S(i, 1)-\lambda \frac{\sqrt{2} m_{W} \sin \beta}{g} S(i, 3)\right]\right\}, \\
& c_{H^{ \pm}}=\frac{\lambda \mu_{e f f}}{\sqrt{2}}\left[2 S(i, 3) \cos ^{2} \beta+2 S(i, 3) \sin ^{2} \beta\right]-\lambda^{2} \frac{m_{W} \sin \beta}{g} 2 S(i, 2) \cos \beta \sin \beta \\
& -\frac{m_{W} \sin \beta}{g} 2 S(i, 1) \cos \beta \sin \beta+\mu_{e f f} \kappa 2 \sqrt{2} S(i, 3) \cos \beta \sin \beta+\frac{\lambda A_{\lambda}}{\sqrt{2}} 2 S(i, 3) \cos \beta \sin \beta \\
& +\frac{g^{\prime 2}}{4} \frac{m_{W}}{g}\left[\sin \beta\left\{2 S(i, 1) \cos ^{2} \beta-2 S(i, 1) \sin ^{2} \beta\right\}+\cos \beta\left\{2 S(i, 2) \sin ^{2} \beta-2 S(i, 2) \cos ^{2} \beta\right\}\right] \\
& +\frac{g m_{W}}{4}\left[\sin \beta\left\{2 S(i, 1) \cos ^{2} \beta+2 S(i, 1) \sin ^{2} \beta+4 S(i, 2) \sin \beta \cos \beta\right\}\right. \\
& \left.+\cos \beta\left\{2 S(i, 2) \cos ^{2} \beta+2 S(i, 2) \sin ^{2} \beta+4 S(i, 1) \sin \beta \cos \beta\right\}\right],
\end{aligned}
$$

where we remember that

$$
f_{t}=\frac{g m_{t}}{\sqrt{2} m_{W} \sin \beta}, \quad f_{b}=\frac{g m_{b}}{\sqrt{2} m_{W} \cos \beta}, \quad f_{\tau}=\frac{g m_{\tau}}{\sqrt{2} m_{W} \cos \beta} .
$$

The kinetic parts meanwhile are as follows, they depend upon the $f(\tau)$ given in Eq. E.2).

For fermions ( $\operatorname{spin} \frac{1}{2}$ ), i.e. the quarks and the charginos:

$$
k_{a}=2 \tau_{a}\left[1-\tau_{a} f\left(\tau_{a}\right)\right] .
$$

For scalars (e.g. sfermions and $H^{ \pm}$):

$$
k_{a}=\tau_{a}\left(\tau_{a} f\left(\tau_{a}\right)-1\right)
$$

For spin 1 (i.e $W^{ \pm}$bosons):

$$
k_{a}=-\left[2+3 \tau_{a}+3 \tau_{a}\left(2-\tau_{a}\right) f\left(\tau_{a}\right)\right] .
$$

That's all the information needed for $h_{i} \rightarrow \gamma \gamma$. 


$$
\Gamma\left(h_{i} \rightarrow Z \gamma\right)=\frac{G_{F}}{\sqrt{2}} \frac{m_{h_{i}}^{3} \alpha_{e m}^{2}\left(m_{h_{i}}\right)}{64 \pi^{3}}\left(1-\left(\frac{m_{Z}}{m_{h_{i}}}\right)^{2}\right)^{3}\left|M_{Z \gamma}\right|^{2},
$$

where

$$
\left|M_{Z \gamma}\right|^{2}=\left(I_{t}^{r}+I_{b}^{r}+I_{c}^{r}+I_{\tilde{W}_{1}}^{r}+I_{\tilde{W}_{2}}^{r}+I_{W}^{r}+\mathcal{I}_{H^{ \pm}}^{r}\right)^{2}+\left(I_{t}^{i}+I_{b}^{i}+I_{c}^{i}+I_{\tilde{W}_{1}}^{i}+I_{\tilde{W}_{2}}^{i}+I_{W}^{i}+I_{H^{ \pm}}^{i}\right)^{2} .
$$

Again $\mathcal{I}_{a}^{r / i}$ are the real and imaginary parts of each contribution and $\mathcal{I}_{a}=c_{a} k_{a}$, where now the $c_{a}$ and $k_{a}$ are different to above as this is now for decays to $Z \gamma$. Now the couplings are:

$$
\begin{aligned}
& c_{t}=-2\left(1-\frac{8}{3} \sin ^{2} \theta_{W}\right) \frac{1}{\sin \theta_{W} \cos \theta_{W}} \frac{S(i, 1)}{\sin \beta}, \\
& c_{c}=-2\left(1-\frac{8}{3} \sin ^{2} \theta_{W}\right) \frac{1}{\sin \theta_{W} \cos \theta_{W}} \frac{S(i, 1)}{\sin \beta}, \\
& c_{b}=\left(-1+\frac{4}{3} \sin ^{2} \theta_{W}\right) \frac{1}{\sin \theta_{W} \cos \theta_{W}} \frac{S(i, 2)}{\cos \beta}, \\
& c_{W}=-\frac{g}{g^{\prime}}[S(i, 1) \sin \beta+S(i, 2) \cos \beta], \\
& c_{\tilde{W}_{1}}=\frac{4 m_{W}}{m_{\tilde{W}_{1}} g \sin \theta_{W} \cos \theta_{W}}\left[\frac{\lambda}{\sqrt{2}} S(i, 3) \cos \theta_{L} \cos \theta_{R}+\frac{g}{\sqrt{2}}\left\{S(i, 1) \sin \theta_{L} \cos \theta_{R}+S(i, 2) \cos \theta_{L} \sin \theta_{R}\right\}\right] \\
& \times\left[-\sin ^{2} \theta_{R}-\frac{1}{2} \cos ^{2} \theta_{R}+2 \sin ^{2} \theta_{W}-\sin ^{2} \theta_{L}-\frac{1}{2} \cos ^{2} \theta_{L}\right], \\
& c_{\tilde{W}_{2}}=\frac{4 m_{W}}{m_{\tilde{W}_{2}} g \sin \theta_{W} \cos \theta_{W}}\left[\frac{\lambda}{\sqrt{2}} S(i, 3) \sin \theta_{L} \sin \theta_{R}-\frac{g}{\sqrt{2}}\left\{S(i, 1) \cos \theta_{L} \sin \theta_{R}+S(i, 2) \sin \theta_{L} \cos \theta_{R}\right\}\right] \\
& \times\left[-\cos ^{2} \theta_{R}-\frac{1}{2} \sin ^{2} \theta_{R}+2 \sin ^{2} \theta_{W}-\cos ^{2} \theta_{L}-\frac{1}{2} \sin ^{2} \theta_{L}\right], \\
& c_{H^{ \pm}}=\left(1-2 \sin ^{2} \theta_{W}\right) \frac{1}{2 \sin \theta_{W} \cos \theta_{W} m_{H^{ \pm}}^{2}} \frac{1}{\sqrt{\sqrt{2} G_{F}}}\left\{\frac{\lambda \mu_{e f f}}{\sqrt{2}}\left[2 S(i, 3) \cos ^{2} \beta+2 S(i, 3) \sin ^{2} \beta\right]\right. \\
& -\frac{\lambda^{2} m_{W} \sin \beta}{g} 2 S(i, 2) \cos \beta \sin \beta-\frac{\lambda^{2} m_{W} \sin \beta}{g} 2 S(i, 1) \cos \beta \sin \beta+\mu_{e f f} \kappa 2 \sqrt{2} S(i, 3) \cos \beta \sin \beta \\
& +\lambda A_{\lambda} \sqrt{2} S(i, 3) \cos \beta \sin \beta+\frac{g^{\prime 2}}{4} \frac{m_{W}}{g}\left[\sin \beta\left(2 S(i, 1) \cos ^{2} \beta-2 S(i, 1) \sin ^{2} \beta\right]+\cos \beta\left[2 S(i, 2) \sin ^{2} \beta\right.\right. \\
& \left.-2 S(i, 2) \cos ^{2} \beta\right]+\frac{g m_{W}}{4}\left(\sin \beta\left[2 S(i, 1) \cos ^{2} \beta+2 S(i, 1) \sin ^{2} \beta+4 S(i, 2) \sin \beta \cos \beta\right]\right. \\
& \left.\left.+\cos \beta\left[2 S(i, 2) \cos ^{2} \beta+2 S(i, 2) \sin ^{2} \beta+4 S(i, 1) \sin \beta \cos \beta\right]\right)\right\} .
\end{aligned}
$$

Now the kinetic parts depend upon both $f\left(\tau_{a}\right)$ in Eq. E.2), $g\left(\tau_{a}\right)$ in Eq. (E.3) and $f\left(\tau_{a Z}\right), g\left(\tau_{a Z}\right)$ where $\tau_{a Z}=4\left(\frac{m_{a}}{m_{Z}}\right)^{2}$ cf $\tau_{a}=4\left(\frac{m_{a}}{m_{h_{i}}}\right)^{2}$.

For the spin $\frac{1}{2}$ particles (quarks or charginos):

$$
k_{a}=\frac{\tau_{a} \tau_{a Z}}{2\left(\tau_{a}-\tau_{a Z}\right)}+\frac{\left(\tau_{a} \tau_{a Z}\right)^{2}}{2\left(\tau_{a}-\tau_{a Z}\right)^{2}}\left[f\left(\tau_{a}\right)-f\left(\tau_{a Z}\right)\right]+\frac{\tau_{a}^{2} \tau_{a Z}}{\left(\tau_{a}-\tau_{a Z}\right)^{2}}\left[g\left(\tau_{a}\right)-g\left(\tau_{a Z}\right)\right]+\frac{\tau_{a} \tau_{a Z}}{2\left(\tau_{a}-\tau_{a Z}\right)}\left[f\left(\tau_{a}\right)-f\left(\tau_{a Z}\right)\right] .
$$

For scalars (charged Higgs bosons):

$$
k_{a}=\frac{\tau_{a} \tau_{a Z}}{2\left(\tau_{a}-\tau_{a Z}\right)}+\frac{\left(\tau_{a} \tau_{a Z}\right)^{2}}{2\left(\tau_{a}-\tau_{a Z}\right)^{2}}\left[f\left(\tau_{a}\right)-f\left(\tau_{a Z}\right)\right]+\frac{\tau_{a}^{2} \tau_{a Z}}{\left(\tau_{a}-\tau_{a Z}\right)^{2}}\left[g\left(\tau_{a}\right)-g\left(\tau_{a Z}\right)\right] .
$$


For the spin 1 bosons (W bosons):

$$
\begin{aligned}
k_{a}= & -4\left(3-\tan ^{2} \theta_{W}\right) \frac{\tau_{a} \tau_{a Z}}{2\left(\tau_{a}-\tau_{a Z}\right)}\left[f\left(\tau_{a}\right)-f\left(\tau_{a Z}\right)\right]+\left\{\left(1+\frac{2}{\tau_{a}}\right) \tan ^{2} \theta_{W}-\left(5+\frac{2}{\tau_{a}}\right)\right\}\left[\frac{\tau_{a} \tau_{a Z}}{2\left(\tau_{a}-\tau_{a Z}\right)}\right. \\
& \left.+\frac{\left(\tau_{a} \tau_{a Z}\right)^{2}}{2\left(\tau_{a}-\tau_{a Z}\right)^{2}}\left[f\left(\tau_{a}\right)-f\left(\tau_{a Z}\right)\right]+\frac{\tau_{a}^{2} \tau_{a Z}}{\left(\tau_{a}-\tau_{a Z}\right)^{2}}\left[g\left(\tau_{a}\right)-g\left(\tau_{a Z}\right)\right]\right] .
\end{aligned}
$$

That's all the information required for $h_{i} \rightarrow Z \gamma$.

Next consider gluon gluon:

$$
\Gamma\left(h_{i} \rightarrow g g\right)=\frac{G_{F} m_{h_{i}}^{3} \alpha_{s}^{2}\left(m_{h_{i}}\right)}{64 \sqrt{2} \pi^{3}}\left|M_{g g}\right|^{2},
$$

where

$$
\begin{aligned}
\left|M_{g g}\right|^{2}= & {\left[J_{t}^{r}+J_{b}^{r}+J_{c}^{r}+J_{\tilde{c}_{L}}^{r}+J_{\tilde{c}_{R}}^{r}+J_{\tilde{s}_{L}}^{r}+J_{\tilde{s}_{R}}^{r}+J_{\tilde{t}_{1}}^{r}+J_{\tilde{t}_{2}}^{r}+J_{\tilde{b}_{1}}^{r}+J_{\tilde{b}_{2}}^{r}\right]^{2} } \\
& +\left[J_{t}^{i}+J_{b}^{i}+J_{c}^{i}+J_{\tilde{c}_{L}}^{i}+J_{\tilde{c}_{R}}^{i}+J_{\tilde{s}_{L}}^{i}+J_{\tilde{s}_{R}}^{i}+J_{\tilde{t}_{1}}^{i}+J_{\tilde{t}_{2}}^{i}+J_{\tilde{b}_{1}}^{i}+J_{\tilde{b}_{2}}^{i}\right]^{2} .
\end{aligned}
$$

As usual each of the $J_{a}$ are given by $J_{a}=c_{a} k_{a}$, where the coupling parts $c_{a}$ and kinetic parts $k_{a}$ are given below:

$$
\begin{aligned}
& c_{t}=\frac{S(i, 1)}{\sin \beta} \quad c_{c}=\frac{S(i, 1)}{\sin \beta} \quad c_{b}=\frac{S(i, 2)}{\cos \beta}, \\
& c_{\tilde{c}_{L}}=\frac{2 m_{W}}{g m_{\tilde{c}_{L}}^{2}}\left(\frac{g^{\prime 2}}{12}+\frac{g^{2}}{4}\right) \frac{2 m_{W}}{g}[\sin \beta S(i, 1)-\cos \beta S(i, 2)], \\
& c_{\tilde{c}_{R}}=\frac{2 m_{W}}{g m_{\tilde{c}_{R}}^{2}} \frac{g^{\prime 2}}{6} \frac{2 m_{W}}{g}[\sin \beta S(i, 1)-\cos \beta S(i, 2)], \\
& c_{\tilde{s}_{L}}=\frac{2 m_{W}}{g m_{\tilde{s}_{L}}^{2}}\left(\frac{g^{\prime 2}}{12}+\frac{g^{2}}{4}\right) \frac{2 m_{W}}{g}[\sin \beta S(i, 1)-\cos \beta S(i, 2)], \\
& c_{\tilde{S}_{R}}=\frac{2 m_{W}}{g m_{\tilde{S}_{R}}^{2}} \frac{g^{\prime 2}}{6} \frac{2 m_{W}}{g}[\sin \beta S(i, 1)-\cos \beta S(i, 2)], \\
& c_{\tilde{t}_{1}}=\frac{m_{W}}{g m_{\tilde{t}_{1}}^{2}}\left[\cos ^{2} \theta_{t} \sqrt{2}\left(f_{t}^{2} \sqrt{2} \frac{m_{W} \sin \beta}{g} S(i, 1)+\left(\frac{g^{\prime 2}}{12}-\frac{g^{2}}{4}\right)\left\{\sqrt{2} \frac{m_{W}}{g}(S(i, 1) \sin \beta-\cos \beta S(i, 2))\right\}\right)\right. \\
& +\sin ^{2} \theta_{t} \sqrt{2}\left(f_{t}^{2} \sqrt{2} \frac{m_{W} \sin \beta}{g} S(i, 1)-\frac{g^{\prime 2}}{3}\left\{\sqrt{2} \frac{m_{W}}{g}(\sin \beta S(i, 1)-\cos \beta S(i, 2))\right\}\right) \\
& \left.+2 \sin \theta_{t} \cos \theta_{t} \frac{f_{t}}{\sqrt{2}}\left(A_{t} S(i, 1)-\mu_{e f f} S(i, 2)-\lambda \sqrt{2} \frac{m_{W} \cos \beta}{g} S(i, 3)\right)\right], \\
& c_{\tilde{t}_{2}}=\frac{m_{W}}{g m_{\tilde{t}_{2}}^{2}}\left[\sin ^{2} \theta_{t} \sqrt{2}\left(f_{t}^{2} \sqrt{2} \frac{m_{W} \sin \beta}{g} S(i, 1)+\left(\frac{g^{\prime 2}}{12}-\frac{g^{2}}{4}\right)\left\{\sqrt{2} \frac{m_{W}}{g}(S(i, 1) \sin \beta-\cos \beta S(i, 2))\right\}\right)\right. \\
& +\cos ^{2} \theta_{t} \sqrt{2}\left(f_{t}^{2} \sqrt{2} \frac{m_{W} \sin \beta}{g} S(i, 1)-\frac{g^{\prime 2}}{3}\left\{\sqrt{2} \frac{m_{W}}{g}(\sin \beta S(i, 1)-\cos \beta S(i, 2))\right\}\right) \\
& \left.-2 \sin \theta_{t} \cos \theta_{t} \frac{f_{t}}{\sqrt{2}}\left(A_{t} S(i, 1)-\mu_{e f f} S(i, 2)-\lambda \sqrt{2} \frac{m_{W} \cos \beta}{g} S(i, 3)\right)\right], \\
& c_{\tilde{b}_{1}}=\frac{m_{W}}{g m_{\tilde{b}_{1}}^{2}}\left[\cos ^{2} \theta_{b} \sqrt{2}\left(f_{b}^{2} \sqrt{2} \frac{m_{W} \cos \beta}{g} S(i, 2)+\left(\frac{g^{\prime 2}}{12}+\frac{g^{2}}{4}\right)\left\{\sqrt{2} \frac{m_{W}}{g}(S(i, 1) \sin \beta-\cos \beta S(i, 2))\right\}\right)\right. \\
& +\sin ^{2} \theta_{b} \sqrt{2}\left(f_{b}^{2} \sqrt{2} \frac{m_{W} \cos \beta}{g} S(i, 2)+\frac{g^{\prime 2}}{6}\left\{\sqrt{2} \frac{m_{W}}{g}(\sin \beta S(i, 1)-\cos \beta S(i, 2))\right\}\right) \\
& \left.+2 \sin \theta_{b} \cos \theta_{b} \frac{f_{b}}{\sqrt{2}}\left(A_{b} S(i, 2)-\mu_{e f f} S(i, 1)-\lambda \sqrt{2} \frac{m_{W} \sin \beta}{g} S(i, 3)\right)\right],
\end{aligned}
$$




$$
\begin{aligned}
c_{\tilde{b}_{2}}= & \frac{m_{W}}{g m_{\tilde{b}_{2}}^{2}}\left[\sin ^{2} \theta_{B} \sqrt{2}\left(f_{b}^{2} \sqrt{2} \frac{m_{W} \cos \beta}{g} S(i, 2)+\left(\frac{g^{\prime 2}}{12}+\frac{g^{2}}{4}\right)\left\{\sqrt{2} \frac{m_{W}}{g}(S(i, 1) \sin \beta-\cos \beta S(i, 2))\right\}\right)\right. \\
& +\cos ^{2} \theta_{b} \sqrt{2}\left(f_{b}^{2} \sqrt{2} \frac{m_{W} \cos \beta}{g} S(i, 2)+\frac{g^{\prime 2}}{6}\left\{\sqrt{2} \frac{m_{W}}{g}(\sin \beta S(i, 1)-\cos \beta S(i, 2))\right\}\right) \\
& \left.-2 \sin \theta_{b} \cos \theta_{b} \frac{f_{b}}{\sqrt{2}}\left(A_{b} S(i, 2)-\mu_{e f f} S(i, 1)-\lambda \sqrt{2} \frac{m_{W} \sin \beta}{g} S(i, 3)\right)\right] .
\end{aligned}
$$

The kinetic parts are then exactly as in the $h_{i} \rightarrow \gamma \gamma$ case.

\section{Appendix I.2. CP Odd Higgs Decays}

First of all decays to a quark and an anti-quark:

$$
\Gamma\left(A_{i} \rightarrow q \bar{q}\right)=\frac{3 G_{F}}{4 \pi \sqrt{2}} m_{q}^{2} m_{A_{i}} \sqrt{1-\frac{4 m_{q}^{2}}{m_{A_{i}}^{2}}} \mathcal{A},
$$

where

$$
\mathcal{A}=\left\{\begin{array}{l}
{\left[\frac{S(i, 1)}{\sin \beta}\right]^{2}, \text { for up-type quarks }(\mathrm{u}, \mathrm{c}, \mathrm{t}),} \\
{\left[\frac{S(i, 2)}{\cos \beta}\right]^{2}, \text { for down-type quarks }(\mathrm{d}, \mathrm{s}, \mathrm{b}) .}
\end{array}\right.
$$

Use the same formulae for decays to leptons but divide by 3 as the 3 in the pre-factor is $N_{c}$. Now decays to sfermions, because of $\mathrm{CP}$ conservation, decays can only go to sfermions of different handedness.

$$
\Gamma\left(A_{i} \rightarrow \tilde{q}_{L} \tilde{q}_{R}^{*}\right)=\frac{1}{16 \pi m_{A_{i}}} \tilde{\lambda}^{\frac{1}{2}}\left(m_{A_{i}}, m_{\tilde{q}_{L}}, m_{\tilde{q}_{R}}\right) C_{A_{i} \tilde{q} \tilde{q}}^{2},
$$

where

$$
C_{A_{i} \tilde{q} \tilde{q}}=\left\{\begin{array}{l}
\frac{f_{q}}{\sqrt{2}}\left[A_{\tilde{q}} P(i, 1)+\mu_{e f f} P(i, 2)+\lambda \sqrt{2} \frac{m_{W} \cos \beta}{g} P(i, 3)\right], \text { for } u \text {-type squarks, } \\
\frac{f_{q}}{\sqrt{2}}\left[A_{\tilde{q}} P(i, 2)+\mu_{e f f} P(i, 1)+\lambda \sqrt{2} \frac{m_{W} \sin \beta}{g} P(i, 3)\right], \text { for } d \text {-type squarks. }
\end{array}\right.
$$

Where you must remember that the expression for $f_{q}$ differs for up type and down type quarks, for example see (F.8).

Note, [I.977 holds even for third generation squarks; as we see in the MSSM, in the NMSSM the decays of CP odd Higgs bosons to squarks are independent of the sfermion mixing angles. The formulae for the decays to squarks also hold for decays to sleptons, but again one must divide by 3 .

Decays to neutralinos:

$$
\Gamma\left(A_{i} \rightarrow \tilde{Z}_{j} \tilde{Z}_{k}\right)=\frac{m_{A_{i}}}{16 \pi}\left[1-\left(\frac{m_{\tilde{Z}_{j}}-m_{\tilde{Z}_{k}}}{m_{A_{i}}}\right)^{2}\right] \tilde{\lambda}^{\frac{1}{2}}\left(m_{A_{i}}, m_{\tilde{Z}_{j}}, m_{\tilde{Z}_{k}}\right) \alpha_{i j} C_{A_{i} \tilde{Z}_{j} \tilde{Z}_{k}}^{2},
$$

where, as for the $\mathrm{CP}$ even decays to neutralinos, the $\alpha_{j k}$ factor accounts for indistinguishability and so is 1 if $j=k$ (i.e. decay to two of the same neutralinos) and 2 if $j \neq k$ (i.e. decays to two different neutralino mass eigenstates). The coupling is given by:

$$
\begin{aligned}
C_{A_{i} \tilde{Z}_{j} \tilde{z}_{k}}= & \frac{\lambda}{\sqrt{2}}\left[P(i, 1)\left(N_{3 j} N_{5 k}+N_{5 j} N_{3 k}\right)+P(i, 2)\left(N_{4 j} N_{5 k}+N_{5 j} N_{4 k}\right)\right. \\
& \left.+P(i, 3)\left(N_{3 j} N_{4 k}+N_{4 j} N_{3 k}\right)\right]-\sqrt{2} \kappa P(i, 3) N_{5 j} N_{5 k} \\
& -\tan \theta_{W} \frac{g}{2}\left[-P(i, 1)\left(N_{1 j} N_{4 k}+N_{4 j} N_{1 k}\right)+P(i, 2)\left(N_{1 j} N_{3 k}\right.\right. \\
& \left.\left.+N_{3 j} N_{1 k}\right)\right]-\frac{g}{2}\left[P(i, 1)\left(N_{2 j} N_{4 k}+N_{4 j} N_{2 k}\right)-P(i, 2)\left(N_{2 j} N_{3 k}\right.\right. \\
& \left.\left.+N_{3 j} N_{2 k}\right)\right] .
\end{aligned}
$$

Decays to charginos, first consider decays to the two of the same chargino:

$$
\Gamma\left(A_{i} \rightarrow \tilde{W}_{j} \tilde{W}_{j}\right)=\frac{m_{A_{i}}}{8 \pi} \tilde{\lambda}^{\frac{1}{2}}\left(m_{A_{i}}, m_{\tilde{W}_{j}}, m_{\tilde{W}_{j}}\right) S^{2},
$$


here

$$
S=\left\{\begin{array}{l}
\frac{\lambda}{\sqrt{2}} P(i, 3) \cos \theta_{L} \cos \theta_{R}-\frac{g}{\sqrt{2}}\left[P(i, 1) \sin \theta_{L} \cos \theta_{R}+P(i, 2) \cos \theta_{L} \sin \theta_{R}\right], \text { for } j=1, \\
\frac{\lambda}{\sqrt{2}} P(i, 3) \sin \theta_{L} \sin \theta_{R}+\frac{g}{\sqrt{2}}\left[P(i, 1) \cos \theta_{L} \sin \theta_{R}+P(i, 2) \sin \theta_{L} \cos \theta_{R}\right], \text { for } j=2 .
\end{array}\right.
$$

Meanwhile for decays to different charginos:

$$
\Gamma\left(A_{i} \rightarrow \tilde{W}_{1} \tilde{W}_{2}\right)=\frac{m_{A_{i}}}{8 \pi} \tilde{\lambda}^{\frac{1}{2}}\left(m_{A_{i}}, m_{\tilde{W}_{1}}, m_{\tilde{W}_{2}}\right)\left[\left(c_{1}^{2}+c_{2}^{2}\right) \frac{1}{m_{A_{i}}^{2}}\left(m_{A_{i}}^{2}-m_{\tilde{W}_{1}}^{2}-m_{\tilde{W}_{2}}^{2}\right)+4 c_{1} c_{2} \frac{m_{\tilde{W}_{1}} m_{\tilde{W}_{2}}}{m_{A_{i}}^{2}}\right],
$$

Here,

$$
\begin{aligned}
& c_{1}=\frac{\lambda}{\sqrt{2}} P(i, 3) \cos \theta_{L} \sin \theta_{R}-\frac{g}{\sqrt{2}}\left[P(i, 1) \sin \theta_{L} \sin \theta_{R}-P(i, 2) \cos \theta_{L} \cos \theta_{R}\right], \\
& c_{2}=\frac{\lambda}{\sqrt{2}} P(i, 3) \sin \theta_{L} \cos \theta_{R}+\frac{g}{\sqrt{2}}\left[P(i, 1) \cos \theta_{L} \cos \theta_{R}-P(i, 2) \sin \theta_{L} \sin \theta_{R}\right] .
\end{aligned}
$$

Decays to $\mathrm{CP}$ even neutral Higgs bosons and a $Z$ boson:

$$
\Gamma\left(A_{i} \rightarrow h_{j} Z\right)=\frac{G_{F} m_{A_{i}}^{3}}{8 \pi \sqrt{2}} \tilde{\lambda}^{\frac{3}{2}}\left(m_{A_{i}}, m_{h_{j}}, m_{Z}\right) C_{A_{i} h_{j} Z}^{2},
$$

where

$$
C_{A_{i} h_{j} Z}=\left\{\begin{array}{l}
{[S(1,1) \cos \beta-S(1,2) \sin \beta] \cos \theta_{A}, \text { for } i=j=1,} \\
{[S(1,1) \cos \beta-S(1,2) \sin \beta] \sin \theta_{A}, \text { for } i=2, j=1,} \\
{[S(2,1) \cos \beta-S(2,2) \sin \beta] \cos \theta_{A}, \text { for } i=1, j=2,} \\
{[S(2,1) \cos \beta-S(2,2) \sin \beta] \sin \theta_{A}, \text { for } i=j=2,} \\
{[S(3,1) \cos \beta-S(3,2) \sin \beta] \cos \theta_{A}, \text { for } i=1, j=3,} \\
{[S(3,1) \cos \beta-S(3,2) \sin \beta] \sin \theta_{A}, \text { for } i=2, j=3 .}
\end{array}\right.
$$

The decay of a CP odd neutral Higgs to a charged Higgs and a $W$ boson in the NMSSM is given by:

$$
\Gamma\left(A \rightarrow H^{ \pm} W^{ \pm}\right)=\frac{G_{F} m_{A}^{3}}{8 \pi \sqrt{2}} \tilde{\lambda}^{\frac{3}{2}}\left(m_{A}, m_{H^{ \pm}}, m_{W}\right) \cos ^{2} \theta_{A},
$$

for $A_{2}$ undergoing the same decay transform $\cos \theta_{A} \rightarrow \sin \theta_{A}$ and $m_{A} \rightarrow m_{A_{2}}$.

$$
\Gamma\left(A_{2} \rightarrow A h_{i}\right)=\frac{1}{16 \pi m_{h_{i}}} \tilde{\lambda}^{\frac{1}{2}}\left(m_{A_{2}}, m_{A}, m_{h_{i}}\right)\left[C_{A A 2 h_{i}}^{N M S S}\right]^{2},
$$

where the coupling $C_{A A 2 h_{i}}^{N M S S M}$ is:

$$
\begin{aligned}
C_{A A 2 h_{i}}^{N M S S M}= & \frac{g^{2}+g^{\prime 2}}{4 \sqrt{2}}\left[\left\langle h_{1}\right\rangle[C(i, 1,2,1,1,1)-C(i, 1,2,1,2,2)]+\left\langle h_{2}\right\rangle[C(i, 1,2,2,2,2)-C(i, 1,2,2,1,1)]\right] \\
& +\frac{\lambda A_{\lambda}}{\sqrt{2}}[C(i, 1,2,1,2,3)+C(i, 1,2,2,1,3)+C(i, 1,2,3,1,2)]-\frac{\kappa A_{\kappa}}{\sqrt{2}} C(i, 1,2,3,3,3) \\
& +\frac{\lambda^{2}}{\sqrt{2}}\left[\left\langle h_{1}\right\rangle[C(i, 1,2,1,2,2)+C(i, 1,2,1,3,3)]+\left\langle h_{2}\right\rangle[C(i, 1,2,2,1,1)+C(i, 1,2,2,3,3)]\right. \\
& \left.+\mu_{e f f} \lambda[C(i, 1,2,3,1,1)+C(i, 1,2,3,2,2)]\right]+\frac{\kappa^{2} \sqrt{2} \mu_{e f f}}{\lambda} C(i, 1,2,3,3,3) \\
& +\frac{\lambda \kappa}{\sqrt{2}}\left[\left\langle h_{1}\right\rangle[C(i, 1,2,2,3,3)-2 C(i, 1,2,3,2,3)]+\left\langle h_{2}\right\rangle[C(i, 1,2,1,3,3)-2 C(i, 1,2,3,1,3)]\right. \\
& \left.+2 \frac{\mu_{e f f}}{\lambda}[C(i, 1,2,3,1,2)-C(i, 1,2,1,2,3)-C(i, 1,2,2,1,3)]\right],
\end{aligned}
$$


where here $C(i, 1,2, x, y, z)$ is notation for

$$
C(i, 1,2, x, y, z)=S(i, x)[P(1, y) P(2, z)+P(1, z) P(2, y)] .
$$

Now the loop decays of the CP odd Higgs bosons. First consider decays to $\gamma \gamma$ :

$$
\Gamma\left(A_{i} \rightarrow \gamma \gamma\right)=\frac{G_{F} m_{A_{i}}^{3} \alpha_{e m}^{2}\left(m_{A_{i}}\right)}{32 \pi^{3} \sqrt{2}}\left|M_{A_{i} \gamma \gamma}\right|^{2},
$$

where

$$
\left|M_{A_{i} \gamma \gamma}\right|^{2}=\left(\mathcal{J}_{t}^{r}+\mathcal{J}_{b}^{r}+\mathcal{J}_{c}^{r}+\mathcal{J}_{\tau}^{r}+\mathcal{J}_{\tilde{W}_{1}}^{r}+\mathcal{J}_{\tilde{W}_{2}}^{r}\right)^{2}+\left(\mathcal{J}_{t}^{i}+\mathcal{J}_{b}^{i}+\mathcal{J}_{c}^{i}+\mathcal{J}_{\tau}^{i}+\mathcal{J}_{\tilde{W}_{1}}^{i}+\mathcal{J}_{\tilde{W}_{2}}^{i}\right)^{2} .
$$

The $\mathcal{J}_{a}^{r / i}$ here are the real and imaginary parts respectively of $c_{a} k_{a}$ where the $c_{a}$ and $k_{a}$ for this decay mode are given below:

$$
\begin{gathered}
c_{t}=\frac{4}{3} \frac{P(i, 1)}{\sin \beta}, \quad c_{b}=\frac{1}{3} \frac{P(i, 2)}{\cos \beta}, \quad c_{c}=\frac{4}{3} \frac{P(i, 1)}{\sin \beta}, \quad c_{\tau}=\frac{P(i, 2)}{\cos \beta}, \\
c_{\tilde{W}_{1}}=\frac{2 m_{W}}{g m_{\tilde{W}_{1}}}\left[\frac{\lambda}{\sqrt{2}} P(i, 3) \cos \theta_{L} \cos \theta_{R}-\frac{g}{\sqrt{2}}\left(P(i, 1) \sin \theta_{L} \cos \theta_{R}+P(i, 2) \cos \theta_{L} \sin \theta_{R}\right)\right], \\
c_{\tilde{W}_{2}}=\frac{2 m_{W}}{g m_{\tilde{W}_{2}}}\left[\frac{\lambda}{\sqrt{2}} P(i, 3) \sin \theta_{L} \sin \theta_{R}+\frac{g}{\sqrt{2}}\left(P(i, 1) \cos \theta_{L} \sin \theta_{R}+P(i, 2) \sin \theta_{L} \cos \theta_{R}\right)\right] .
\end{gathered}
$$

Meanwhile the kinetic parts are, for the quarks or the charginos (as both are spin $\frac{1}{2}$ ):

$$
k_{a}=\tau_{a} f\left(\tau_{a}\right) .
$$

The next loop decay is to $Z \gamma$ :

$$
\Gamma\left(A_{i} \rightarrow Z \gamma\right)=\frac{G_{F}}{\sqrt{2}} \frac{m_{A_{i}}^{3} \alpha_{e m}^{2}\left(m_{A_{i}}\right)}{64 \pi^{3}}\left(1-\left(\frac{m_{Z}}{m_{A_{i}}}\right)^{2}\right)\left|M_{A_{i} Z \gamma}\right|^{2},
$$

where

$$
\left|M_{A_{i} Z \gamma}\right|^{2}=\left(\mathcal{K}_{t}^{r}+\mathcal{K}_{b}^{r}+\mathcal{K}_{c}^{r}+\mathcal{K}_{\tilde{W}_{1}}^{r}+\mathcal{K}_{\tilde{W}_{2}}^{r}\right)^{2}+\left(\mathcal{K}_{t}^{i}+\mathcal{K}_{b}^{i}+\mathcal{K}_{c}^{i}+\mathcal{K}_{\tilde{W}_{1}}^{i}+\mathcal{K}_{\tilde{W}_{2}}^{i}\right)^{2} .
$$

As usual each $\mathcal{K}_{a}^{r / i}$ is the real/imaginary part of $c_{a} k_{a}$ where the $c_{a}$ and $k_{a}$ for this mode are given below:

$$
\begin{aligned}
& c_{t}=-2(1-\left.\frac{8}{3} \sin ^{2} \theta_{W}\right) \frac{1}{\sin \theta_{W} \cos \theta_{W}} \frac{P(i, 1)}{\sin \beta}, \quad c_{c}=-2\left(1-\frac{8}{3} \sin ^{2} \theta_{W}\right) \frac{1}{\sin \theta_{W} \cos \theta_{W}} \frac{P(i, 1)}{\sin \beta}, \\
& c_{b}=\left(-1+\frac{4}{3} \sin ^{2} \theta_{W}\right) \frac{1}{\sin \theta_{W} \cos \theta_{W}} \frac{P(i, 2)}{\cos \beta}, \\
& c_{\tilde{W}_{1}=}=\frac{m_{W}}{m_{\tilde{W}_{1}} g \sin \theta_{W} \cos \theta_{W}}\left[-\sin ^{2} \theta_{R}-\frac{1}{2} \cos ^{2} \theta_{R}-\sin ^{2} \theta_{L}-\frac{1}{2} \cos ^{2} \theta_{L}+2 \sin ^{2} \theta_{W}\right] \\
& \times\left(\frac{\lambda}{\sqrt{2}} P(i, 3) \cos \theta_{L} \cos \theta_{R}-\frac{g}{\sqrt{2}}\left[P(i, 1) \sin \theta_{L} \cos \theta_{R}+P(i, 2) \cos \theta_{L} \sin \theta_{R}\right]\right), \\
& c_{\tilde{W}_{2}=}= \frac{m_{W}}{m_{\tilde{W}_{2}} g \sin \theta_{W} \cos \theta_{W}}\left[-\cos ^{2} \theta_{R}-\frac{1}{2} \sin ^{2} \theta_{R}-\cos ^{2} \theta_{L}-\frac{1}{2} \sin ^{2} \theta_{L}+2 \sin ^{2} \theta_{W}\right] \\
& \times\left(\frac{\lambda}{\sqrt{2}} P(i, 3) \sin \theta_{L} \sin \theta_{R}+\frac{g}{\sqrt{2}}\left[P(i, 1) \cos \theta_{L} \sin \theta_{R}+P(i, 2) \sin \theta_{L} \cos \theta_{R}\right]\right),
\end{aligned}
$$

The kinetic parts are all of the following form, where remember $\tau_{a Z}=4\left(\frac{m_{a}}{m_{Z}}\right)^{2} \operatorname{cf} \tau_{a}=4\left(\frac{m_{a}}{m_{\phi}}\right)^{2}$ :

$$
k_{a}=\frac{\tau_{a} \tau_{a Z}}{2\left(\tau_{a}-\tau_{a Z}\right)}\left[f\left(\tau_{a}\right)-f\left(\tau_{a Z}\right)\right]
$$


Finally, for the loop decay to $g g$ :

$$
\Gamma\left(A_{i} \rightarrow g g\right)=\frac{G_{F}}{\sqrt{2}} \frac{m_{A_{i}}^{3} \alpha_{s}^{2}\left(m_{A_{i}}\right)}{16 \pi^{3}}\left|M_{A_{i} g g}\right|^{2},
$$

where

$$
\left|M_{A_{i} g g}\right|^{2}=\left(\mathcal{R}_{t}^{r}+\mathcal{R}_{b}^{r}+\mathcal{R}_{c}^{r}\right)^{2}+\left(\mathcal{R}_{t}^{i}+\mathcal{R}_{b}^{i}+\mathcal{R}_{c}^{i}\right)^{2} .
$$

Again the $\mathcal{R}_{a}^{r / i}$ are the real and imaginary parts of $c_{a} k_{a}$, where for this mode they are:

$$
c_{t}=\frac{P(i, 1)}{\sin \beta}, \quad c_{b}=\frac{P(i, 2)}{\cos \beta}, \quad c_{c}=\frac{P(i, 1)}{\sin \beta} .
$$

The kinetic parts are just:

$$
k_{a}=\tau_{a} f\left(\tau_{a}\right) .
$$

Appendix I.3. Decays into Higgs Bosons

$$
\Gamma\left(\tilde{b}_{2} \rightarrow \tilde{b}_{1} h_{i}\right)=\frac{1}{16 \pi m_{\tilde{b}_{2}}} \tilde{\lambda}^{\frac{1}{2}}\left(m_{\tilde{b}_{2}}, m_{\tilde{b}_{1}}, m_{h_{i}}\right)\left[\cos \theta_{b} \sin \theta_{b}\left(c_{R}-c_{L}\right)+\left(\cos ^{2} \theta_{b}-\sin ^{2} \theta_{b}\right) c_{L R}\right]^{2},
$$

where

$$
\begin{aligned}
c_{L} & =-\sqrt{2}\left[f_{b}^{2}\left\langle h_{2}\right\rangle S(i, 2)+\left(\frac{g^{\prime 2}}{12}+\frac{g^{2}}{4}\right)\left[\left\langle h_{1}\right\rangle S(i, 1)-\left\langle h_{2}\right\rangle S(i, 2)\right],\right. \\
c_{R} & =-\sqrt{2}\left[f_{b}^{2}\left\langle h_{2}\right\rangle S(i, 2)+\left(\frac{g^{\prime 2}}{6}\right)\left[\left\langle h_{1}\right\rangle S(i, 1)-\left\langle h_{2}\right\rangle S(i, 2)\right],\right. \\
c_{L R} & =-\frac{f_{b}}{\sqrt{2}}\left[A_{b} S(i, 2)-\mu_{e f f} S(i, 1)-\lambda\left\langle h_{1}\right\rangle S(i, 3)\right] . \\
\Gamma\left(\tilde{t}_{2} \rightarrow \tilde{t}_{1} h_{i}\right) & =\frac{1}{16 \pi m_{\tilde{t}_{2}}} \tilde{\lambda}^{\frac{1}{2}}\left(m_{\tilde{t}_{2}}, m_{\tilde{t}_{1}}, m_{h_{i}}\right)\left[\cos \theta_{t} \sin \theta_{t}\left(c_{R}-c_{L}\right)+\left(\cos ^{2} \theta_{t}-\sin ^{2} \theta_{t}\right) c_{L R}\right]^{2},
\end{aligned}
$$

where

$$
\begin{aligned}
c_{L} & =-\sqrt{2}\left[f_{t}^{2}\left\langle h_{1}\right\rangle S(i, 1)+\left(\frac{g^{\prime 2}}{12}-\frac{g^{2}}{4}\right)\left[\left\langle h_{1}\right\rangle S(i, 1)-\left\langle h_{2}\right\rangle S(i, 2)\right],\right. \\
c_{R} & =-\sqrt{2}\left[f_{t}^{2}\left\langle h_{1}\right\rangle S(i, 1)-\left(\frac{g^{\prime 2}}{3}\right)\left[\left\langle h_{1}\right\rangle S(i, 1)-\left\langle h_{2}\right\rangle S(i, 2)\right],\right. \\
c_{L R} & =-\frac{f_{t}}{\sqrt{2}}\left[A_{t} S(i, 1)-\mu_{e f f} S(i, 2)-\lambda\left\langle h_{2}\right\rangle S(i, 3)\right] . \\
\Gamma\left(\tilde{\tau}_{2} \rightarrow \tilde{\tau}_{1} h_{i}\right) & =\frac{1}{16 \pi m_{\tilde{\tau}_{2}}} \tilde{\lambda}^{\frac{1}{2}}\left(m_{\tilde{\tau}_{2}}, m_{\tilde{\tau}_{1}}, m_{h_{i}}\right)\left[\cos \theta_{\tau} \sin \theta_{\tau}\left(c_{R}-c_{L}\right)+\left(\cos ^{2} \theta_{\tau}-\sin ^{2} \theta_{\tau}\right) c_{L R}\right]^{2},
\end{aligned}
$$

where

$$
\begin{aligned}
& c_{L}=-\sqrt{2}\left[f_{\tau}^{2}\left\langle h_{2}\right\rangle S(i, 2)+\left(\frac{-g^{\prime 2}}{4}+\frac{g^{2}}{4}\right)\left[\left\langle h_{1}\right\rangle S(i, 1)-\left\langle h_{2}\right\rangle S(i, 2)\right],\right. \\
& c_{R}=-\sqrt{2}\left[f_{\tau}^{2}\left\langle h_{2}\right\rangle S(i, 2)+\left(\frac{g^{\prime 2}}{2}\right)\left[\left\langle h_{1}\right\rangle S(i, 1)-\left\langle h_{2}\right\rangle S(i, 2)\right]\right], \\
& c_{L R}=-\frac{f_{\tau}}{\sqrt{2}}\left[A_{\tau} S(i, 2)-\mu_{e f f} S(i, 1)-\lambda\left\langle h_{1}\right\rangle S(i, 3)\right] . \\
& \Gamma\left(\tilde{b}_{2} \rightarrow \tilde{b}_{1} A_{i}\right)=\frac{1}{16 \pi m_{\tilde{b}_{2}}} \tilde{\lambda}^{\frac{1}{2}}\left(m_{\tilde{b}_{2}}, m_{\tilde{b}_{1}}, m_{\tilde{A}_{i}}\right)\left[\cos ^{2} \theta_{b}-\sin ^{2} \theta_{b}\right]^{2} A_{L R}^{2},
\end{aligned}
$$


where

$$
A_{L R}=\frac{f_{b}}{\sqrt{2}}\left[A_{b} P(i, 2)+\mu_{e f f} P(i, 1)+\lambda\left\langle h_{1}\right\rangle P(i, 3)\right] .
$$

For $\tilde{\tau}_{2} \rightarrow \tilde{\tau}_{1} A_{i}$ the formulae are the same except the changes $\theta_{b} \rightarrow \theta_{\tau}, m_{b} \rightarrow m_{\tau}$ and now $A_{L R}$ is given by:

$$
A_{L R}=\frac{f_{\tau}}{\sqrt{2}}\left[A_{\tau} P(i, 2)+\mu_{e f f} P(i, 1)+\lambda\left\langle h_{1}\right\rangle P(i, 3)\right] .
$$

For $\tilde{t}_{2} \rightarrow \tilde{t}_{1} A_{i}$ the formulae are the same except the changes $\theta_{b} \rightarrow \theta_{t}, m_{b} \rightarrow m_{t}$ and now $A_{L R}$ is given by:

$$
A_{L R}=\frac{f_{t}}{\sqrt{2}}\left[A_{t} P(i, 1)+\mu_{e f f} P(i, 2)+\lambda\left\langle h_{2}\right\rangle P(i, 3)\right]
$$

The chargino decays to lighter charginos and a CP even neutral Higgs:

$$
\Gamma\left(\tilde{W}_{2} \rightarrow \tilde{W}_{1} h_{i}\right)=\frac{1}{32 \pi\left|m_{\tilde{W}_{2}}\right|} \tilde{\lambda}^{1 / 2}\left(m_{\tilde{W}_{2}}, m_{\tilde{W}_{1}}, m_{h_{i}}\right)\left[\left(c_{1}^{2}+c_{2}^{2}\right)\left(m_{\tilde{W}_{1}}^{2}+m_{\tilde{W}_{2}}^{2}-m_{h_{i}}^{2}\right)+4 c_{1} c_{2} m_{\tilde{W}_{1}} m_{\tilde{W}_{2}}\right]^{2},
$$

here the $c_{1}$ and $c_{2}$ are:

$$
\begin{aligned}
& c_{1}=\frac{\lambda}{\sqrt{2}} S(i, 3) \cos \theta_{L} \sin \theta_{R}+\frac{g}{\sqrt{2}}\left[S(i, 1) \sin \theta_{L} \sin \theta_{R}-S(i, 2) \cos \theta_{L} \cos \theta_{R}\right], \\
& c_{2}=\frac{\lambda}{\sqrt{2}} S(i, 3) \sin \theta_{L} \cos \theta_{R}-\frac{g}{\sqrt{2}}\left[S(i, 1) \cos \theta_{L} \cos \theta_{R}-S(i, 2) \sin \theta_{L} \sin \theta_{R}\right] .
\end{aligned}
$$

The chargino decays to lighter charginos and a CP odd neutral Higgs:

$$
\Gamma\left(\tilde{W}_{2} \rightarrow \tilde{W}_{1} A_{i}\right)=\frac{1}{32 \pi\left|m_{\tilde{W}_{2}}\right|} \tilde{\lambda}^{1 / 2}\left(m_{\tilde{W}_{2}}, m_{\tilde{W}_{1}}, m_{A_{i}}\right)\left[\left(C_{1}^{2}+C_{2}^{2}\right)\left(m_{\tilde{W}_{1}}^{2}+m_{\tilde{W}_{2}}^{2}-m_{h_{i}}^{2}\right)+4 C_{1} C_{2} m_{\tilde{W}_{1}} m_{\tilde{W}_{2}}\right]^{2},
$$

here the $C_{1}$ and $C_{2}$ are:

$$
\begin{aligned}
& C_{1}=\frac{\lambda}{\sqrt{2}} P(i, 3) \cos \theta_{L} \sin \theta_{R}-\frac{g}{\sqrt{2}}\left[P(i, 1) \sin \theta_{L} \sin \theta_{R}-P(i, 2) \cos \theta_{L} \cos \theta_{R}\right], \\
& C_{2}=\frac{\lambda}{\sqrt{2}} P(i, 3) \sin \theta_{L} \cos \theta_{R}+\frac{g}{\sqrt{2}}\left[P(i, 1) \cos \theta_{L} \cos \theta_{R}-P(i, 2) \sin \theta_{L} \sin \theta_{R}\right] .
\end{aligned}
$$

The formulae for $\Gamma\left(H^{ \pm} \rightarrow W h_{i}\right)$ are just as above for $\Gamma\left(h_{i} \rightarrow W H^{ \pm}\right)$in (I.31) but with $m_{h_{i}} \leftrightarrow m_{H^{ \pm}}$. Similarly the formulae for $\Gamma\left(H^{ \pm} \rightarrow W A_{i}\right)$ are just as above for $\Gamma\left(A_{i} \rightarrow W H^{ \pm}\right)$in $(I .108)$ but with the replacement $m_{A_{i}} \leftrightarrow m_{H^{ \pm}}$.

\section{Appendix I.4. Neutralino Decays}

$$
\Gamma\left(\tilde{Z}_{i} \rightarrow \tilde{q}_{L / R} \bar{q}\right)=\frac{N_{c} g^{2}}{32 \pi\left|m_{\tilde{Z}_{i}}\right|} \tilde{\lambda}^{\frac{1}{2}}\left(m_{\tilde{Z}_{i}}, m_{\tilde{q}_{L / R}}, m_{q}\right) C_{\mathcal{L} / \mathcal{R}}^{2}\left(m_{\tilde{Z}_{i}}^{2}-m_{\tilde{q}_{L / R}}^{2}+m_{q}^{2}\right),
$$

where

$$
\begin{aligned}
& C_{L}=\left\{\begin{array}{l}
-\sqrt{2}\left[\frac{2}{3} c(1) \sin \theta_{W}+\left(\frac{1}{2}-\frac{2}{3} \sin ^{2} \theta_{W}\right) \frac{c(2)}{\cos \theta_{W}}\right], \text { for } \tilde{u}_{L}, \\
\sqrt{2}\left[\frac{1}{3} c(1) \sin \theta_{W}+\left(\frac{1}{2}-\frac{1}{3} \sin ^{2} \theta_{W}\right) \frac{c(2)}{\cos \theta_{W}}\right], \text { for } \tilde{d}_{L}, \\
\sqrt{2}\left[-c(1) \sin \theta_{W}+\left(\frac{1}{2}-\sin ^{2} \theta_{W}\right) \frac{c(2)}{\cos \theta_{W}}\right], \text { for } \tilde{l}_{L}, \\
\frac{-c(2)}{\sqrt{2}} \cos \theta_{W}, \text { for } \tilde{v}_{L},
\end{array}\right. \\
& C_{R}=\left\{\begin{array}{l}
-\sqrt{2} \frac{2}{3} \sin \theta_{W}\left[c(2) \tan \theta_{W}-c(1)\right], \text { for } \tilde{u}_{R}, \\
\sqrt{2} \frac{1}{3} \sin \theta_{W}\left[c(2) \tan \theta_{W}-c(1)\right], \text { for } \tilde{d}_{R}, \\
\sqrt{2} \sin \theta_{W}\left[c(2) \tan \theta_{W}-c(1)\right], \text { for } \tilde{l}_{R}, \\
0, \text { for } \tilde{v}_{R} .
\end{array}\right.
\end{aligned}
$$


Here $N_{c}=3$ for quarks and 1 for leptons and $c(1)$ and $c(2)$ are given by:

$$
c(1)=N_{1 i} \cos \theta_{W}+N_{2 i} \sin \theta_{W}, \quad c(2)=-N_{1 i} \sin \theta_{W}+N_{2 i} \cos \theta_{W} .
$$

For the third generation, the generalisation is as expected but with extra Yukawa interactions:

$$
\Gamma\left(\tilde{Z}_{i} \rightarrow \tilde{t}_{1 / 2} \bar{t}\right)=\frac{3 g^{2}}{32 \pi\left|m_{\tilde{Z}_{i}}\right|} \tilde{\lambda}^{\frac{1}{2}}\left(m_{\tilde{Z}_{i}}, m_{\tilde{t}_{1 / 2}}, m_{t}\right)\left[\left(c_{1}^{2}+c_{2}^{2}\right)\left(m_{\tilde{Z}_{i}}^{2}-m_{\tilde{t}_{1 / 2}}^{2}+m_{t}^{2}\right)+4 m_{t} m_{\tilde{Z}_{i}} c_{1} c_{2}\right],
$$

where:

$$
\begin{aligned}
& c_{1}=\left\{\begin{array}{l}
\sqrt{2} \cos \theta_{t}\left[-\frac{2}{3} c(1) \sin \theta_{W}+\left(-\frac{1}{2}+\frac{2}{3} \sin ^{2} \theta_{W}\right) \frac{c(2)}{\cos \theta_{W}}\right]-\sin \theta_{t} \frac{f_{t}}{g} N_{4 i}, \text { for } \tilde{t}_{1}, \\
-\sqrt{2} \sin \theta_{t}\left[-\frac{2}{3} c(1) \sin \theta_{W}+\left(-\frac{1}{2}+\frac{2}{3} \sin ^{2} \theta_{W}\right) \frac{c(2)}{\cos \theta_{W}}\right]-\cos \theta_{t} \frac{f_{t}}{g} N_{4 i}, \text { for } \tilde{t}_{2},
\end{array}\right. \\
& c_{2}=\left\{\begin{array}{l}
-\sqrt{2} \sin \theta_{t} \frac{2}{3} \sin \theta_{W}\left[c(2) \tan \theta_{W}-c(1)\right]-\cos \theta_{t} \frac{f_{t}}{g} N_{4 i}, \text { for } \tilde{t}_{1}, \\
-\sqrt{2} \cos \theta_{t} \frac{2}{3} \sin \theta_{W}\left[c(2) \tan \theta_{W}-c(1)\right]+\sin \theta_{t} \frac{f_{t}}{g} N_{4 i}, \text { for } \tilde{t}_{2},
\end{array}\right. \\
& \Gamma\left(\tilde{Z}_{i} \rightarrow \tilde{b}_{1 / 2} \bar{b}\right)=\frac{3 g^{2}}{32 \pi\left|m_{\tilde{Z}_{i}}\right|} \tilde{\lambda}^{\frac{1}{2}}\left(m_{\tilde{Z}_{i}}, m_{\tilde{b}_{1 / 2}}, m_{b}\right)\left[\left(c_{1}^{2}+c_{2}^{2}\right)\left(m_{\tilde{Z}_{i}}^{2}-m_{\tilde{b}_{1 / 2}}^{2}+m_{b}^{2}\right)+4 m_{b} m_{\tilde{Z}_{i}} c_{1} c_{2}\right],
\end{aligned}
$$

where:

$$
\begin{gathered}
c_{1}=\left\{\begin{array}{l}
\sqrt{2} \cos \theta_{b}\left[\frac{1}{3} c(1) \sin \theta_{W}+\left(\frac{1}{2}-\frac{1}{3} \sin ^{2} \theta_{W}\right) \frac{c(2)}{\cos \theta_{W}}\right]-\sin \theta_{b} \frac{f_{b}}{g} N_{3 i}, \text { for } \tilde{b}_{1}, \\
-\sqrt{2} \sin \theta_{b}\left[\frac{1}{3} c(1) \sin \theta_{W}+\left(\frac{1}{2}-\frac{1}{3} \sin ^{2} \theta_{W}\right) \frac{c(2)}{\cos \theta_{W}}\right]-\cos \theta_{b} \frac{f_{b}}{g} N_{3 i}, \text { for } \tilde{b}_{2},
\end{array}\right. \\
c_{2}=\left\{\begin{array}{l}
\sqrt{2} \sin \theta_{b} \frac{1}{3} \sin \theta_{W}\left[c(2) \tan \theta_{W}-c(1)\right]-\cos \theta_{b} \frac{f_{b}}{g} N_{3 i}, \text { for } \tilde{b}_{1}, \\
\sqrt{2} \cos \theta_{b} \frac{1}{3} \sin \theta_{W}\left[c(2) \tan \theta_{W}-c(1)\right]+\sin \theta_{b} \frac{f_{b}}{g} N_{3 i}, \text { for } \tilde{b}_{2} .
\end{array}\right. \\
\Gamma\left(\tilde{Z}_{i} \rightarrow \tilde{\tau}_{1 / 2} \bar{\tau}\right)=\frac{g^{2}}{32 \pi\left|m_{\tilde{Z}_{i}}\right|} \tilde{\lambda}^{\frac{1}{2}}\left(m_{\tilde{Z}_{i}}, m_{\tilde{\tau}_{1 / 2}}, m_{\tau}\right)\left[\left(c_{1}^{2}+c_{2}^{2}\right)\left(m_{\tilde{Z}_{i}}^{2}-m_{\tilde{\tau}_{1 / 2}}^{2}+m_{\tau}^{2}\right)+4 m_{\tau} m_{\tilde{Z}_{i}} c_{1} c_{2}\right],
\end{gathered}
$$

where:

$$
\begin{aligned}
& c_{1}=\left\{\begin{array}{l}
\sqrt{2} \cos \theta_{\tau}\left[c(1) \sin \theta_{W}+\left(\frac{1}{2}-\sin ^{2} \theta_{W}\right) \frac{c(2)}{\cos \theta_{W}}\right]-\sin \theta_{\tau} \frac{f_{\tau}}{g} N_{3 i}, \text { for } \tilde{\tau}_{1}, \\
-\sqrt{2} \sin \theta_{\tau}\left[c(1) \sin \theta_{W}+\left(\frac{1}{2}-\sin ^{2} \theta_{W}\right) \frac{c(2)}{\cos \theta_{W}}\right]-\cos \theta_{\tau} \frac{f_{\tau}}{g} N_{3 i}, \text { for } \tilde{\tau}_{2},
\end{array}\right. \\
& c_{2}=\left\{\begin{array}{l}
\sqrt{2} \sin \theta_{\tau} \sin \theta_{W}\left[c(2) \tan \theta_{W}-c(1)\right]-\cos \theta_{\tau} \frac{f_{\tau}}{g} N_{3 i}, \text { for } \tilde{\tau}_{1}, \\
\sqrt{2} \cos \theta_{\tau} \sin \theta_{W}\left[c(2) \tan \theta_{W}-c(1)\right]+\sin \theta_{\tau} \frac{f_{\tau}}{g} N_{3 i}, \text { for } \tilde{\tau}_{2} .
\end{array}\right.
\end{aligned}
$$

Neutralino decays to a chargino and $W$ boson:

$$
\begin{aligned}
\Gamma\left(\tilde{Z}_{i} \rightarrow W \tilde{W}_{1}\right)=\frac{g^{2}}{32 \pi\left|m_{\tilde{Z}_{i}}\right|} \tilde{\lambda}^{1 / 2}\left(m_{\tilde{Z}_{i}}, m_{\tilde{W}_{1}}, m_{W}\right)[ & -12 m_{\tilde{Z}_{i}} m_{\tilde{W}_{j}} c_{L} c_{R}+\left(c_{L}^{2}+c_{R}^{2}\right)\left\{\left(m_{\tilde{W}_{1}}^{2}+m_{\tilde{Z}_{i}}^{2}-m_{W}^{2}\right)\right. \\
& \left.\left.+\left(m_{\tilde{Z}_{i}}^{2}+m_{W}^{2}-m_{\tilde{W}_{j}}^{2}\right)\left(m_{\tilde{Z}_{i}}^{2}-m_{W}^{2}-m_{\tilde{W}_{j}}^{2}\right) \frac{1}{m_{W}^{2}}\right\}\right]
\end{aligned}
$$

where:

$$
\begin{aligned}
& c_{L}=\frac{-1}{\sqrt{2}} N_{4 i} \cos \theta_{R}+N_{2 i} \sin \theta_{R}, \\
& c_{R}=\frac{1}{\sqrt{2}} N_{3 i} \cos \theta_{L}+N_{2 i} \sin \theta_{L} .
\end{aligned}
$$

For $\tilde{W}_{2}$ just take $m_{\tilde{W}_{1}} \rightarrow m_{\tilde{W}_{2}}, \cos \theta_{R} \rightarrow \sin \theta_{R}, \cos \theta_{L} \rightarrow \sin \theta_{L}, \sin \theta_{R} \rightarrow-\cos \theta_{R}$ and $\sin \theta_{L} \rightarrow-\cos \theta_{L}$. 
Neutralino decays to a chargino and charged Higgs boson:

$$
\Gamma\left(\tilde{Z}_{i} \rightarrow H^{ \pm} \tilde{W}_{1}\right)=\frac{1}{32 \pi\left|m_{\tilde{Z}_{i}}\right|} \tilde{\lambda}^{1 / 2}\left(m_{\tilde{Z}_{i}}, m_{\tilde{W}_{1}}, m_{H^{ \pm}}\right)\left[\left(C_{L}^{2}+C_{R}^{2}\right)\left\{\left(m_{\tilde{W}_{1}}^{2}+m_{\tilde{Z}_{i}}^{2}-m_{H^{ \pm}}^{2}\right)+4 C_{L} C_{R} m_{\tilde{Z}_{i}} m_{\tilde{W}_{1}}\right\}\right],
$$

where:

$$
\begin{aligned}
& C_{L}=\lambda \cos \beta N_{5 i} \cos \theta_{L}-\frac{\sin \beta}{\sqrt{2}}\left[g^{\prime} N_{1 i}+g N_{2 i}\right] \cos \theta_{L}+g \sin \beta N_{3 i} \sin \theta_{L} \\
& C_{R}=\lambda \sin \beta N_{5 i} \cos \theta_{R}+\frac{\cos \beta}{\sqrt{2}}\left[g^{\prime} N_{1 i}+g N_{2 i}\right] \cos \theta_{R}+g \cos \beta N_{4 i} \sin \theta_{R}
\end{aligned}
$$

Again for $\tilde{W}_{2}$ just take $m_{\tilde{W}_{1}} \rightarrow m_{\tilde{W}_{2}}, \cos \theta_{R} \rightarrow \sin \theta_{R}, \cos \theta_{L} \rightarrow \sin \theta_{L}, \sin \theta_{R} \rightarrow-\cos \theta_{R}$ and $\sin \theta_{L} \rightarrow-\cos \theta_{L}$.

Neutralino decays to a lighter neutralino and $Z$ boson:

$$
\begin{aligned}
\Gamma\left(\tilde{Z}_{i} \rightarrow Z \tilde{Z}_{j}\right)=\frac{g^{2}}{32 \pi\left|m_{\tilde{Z}_{i}}\right|} \tilde{\lambda}^{\frac{1}{2}}\left(m_{\tilde{Z}_{i}}, m_{\tilde{Z}_{j}}, m_{Z}\right)[ & -12 m_{\tilde{Z}_{i}} m_{\tilde{Z}_{j}} c_{L Z} c_{R Z}+\left(c_{L Z}^{2}+c_{R Z}^{2}\right)\left\{\left(m_{\tilde{Z}_{i}}^{2}+m_{\tilde{Z}_{j}}^{2}-m_{Z}^{2}\right)\right. \\
& \left.\left.+\left(m_{\tilde{Z}_{i}}^{2}-m_{\tilde{Z}_{j}}^{2}+m_{Z}^{2}\right)\left(m_{\tilde{Z}_{i}}^{2}-m_{\tilde{Z}_{j}}^{2}-m_{Z}^{2}\right) \frac{1}{m_{Z}^{2}}\right\}\right],
\end{aligned}
$$

here we have:

$$
c_{L Z}=-c_{R Z}=\frac{1}{2 \cos \theta_{W}}\left[N_{3 i} N_{3 j}-N_{4 i} N_{4 j}\right] .
$$

Neutralino decays to a lighter neutralino and CP even neutral Higgs boson:

$$
\Gamma\left(\tilde{Z}_{i} \rightarrow h_{k} \tilde{Z}_{j}\right)=\frac{1}{4 \pi\left|m_{\tilde{Z}_{i}}\right|} \tilde{\lambda}^{\frac{1}{2}}\left(m_{\tilde{Z}_{i}}, m_{h_{k}}, m_{\tilde{Z}_{j}}\right) C_{\tilde{Z}_{i} \tilde{Z}_{j} h_{k}}^{2}\left[m_{\tilde{Z}_{i}}^{2}+m_{\tilde{Z}_{j}}^{2}-m_{h_{k}}^{2}+2 m_{\tilde{Z}_{i}} m_{\tilde{Z}_{j}}\right],
$$

where

$$
\begin{aligned}
C_{\tilde{Z}_{i} \tilde{Z}_{j} h_{k}}= & \frac{\lambda}{2 \sqrt{2}}\left[S(k, 1)\left(N_{3 i} N_{5 j}+N_{3 j} N_{5 i}\right)+S(k, 2)\left(N_{4 i} N_{5 j}+N_{4 j} N_{5 i}\right)\right. \\
& \left.+S(k, 3)\left(N_{3 i} N_{4 j}+N_{4 i} N_{3 j}\right)\right]-\sqrt{2} \kappa S(k, 3) N_{5 i} N_{5 j} \\
& +\frac{g^{\prime}}{2}\left[-S(k, 1)\left(N_{1 i} N_{4 j}+N_{1 j} N_{4 i}\right)+S(k, 2)\left(N_{1 i} N_{3 j}+N_{1 j} N_{3 i}\right)\right] \\
& +\frac{g}{2}\left[S(k, 1)\left(N_{2 i} N_{4 j}+N_{2 j} N_{4 i}\right)-S(k, 2)\left(N_{2 i} N_{3 j}+N_{2 j} N_{3 i}\right)\right] .
\end{aligned}
$$

Neutralino decays to a lighter neutralino and CP odd neutral Higgs boson:

$$
\Gamma\left(\tilde{Z}_{i} \rightarrow A_{k} \tilde{Z}_{j}\right)=\frac{1}{4 \pi\left|m_{\tilde{Z}_{i}}\right|} \tilde{\lambda}^{\frac{1}{2}}\left(m_{\tilde{Z}_{i}}, m_{A_{k}}, m_{\tilde{Z}_{j}}\right)\left[\mathcal{G}_{\tilde{Z} \tilde{Z} A_{k}}^{2}\right]\left[m_{\tilde{Z}_{i}}^{2}+m_{\tilde{Z}_{j}}^{2}-m_{A_{k}}^{2}-2 m_{\tilde{Z}_{i}} m_{\tilde{Z}_{j}}\right],
$$

where

$$
\begin{aligned}
\mathcal{G}_{\tilde{Z}_{i} \tilde{Z}_{j} A_{k}}= & \frac{-\lambda}{2 \sqrt{2}}\left[P(k, 1)\left(N_{3 i} N_{5 j}+N_{3 j} N_{5 i}\right)+P(k, 2)\left(N_{4 i} N_{5 j}+N_{4 j} N_{5 i}\right)\right. \\
& \left.+P(k, 3)\left(N_{3 i} N_{4 j}+N_{4 i} N_{3 j}\right)\right]-\sqrt{2} \kappa P(k, 3) N_{5 i} N_{5 j} \\
& -\frac{g^{\prime}}{2}\left[-P(k, 1)\left(N_{1 i} N_{4 j}+N_{1 j} N_{4 i}\right)+P(k, 2)\left(N_{1 i} N_{3 j}+N_{1 j} N_{3 i}\right)\right] \\
& -\frac{g}{2}\left[P(k, 1)\left(N_{2 i} N_{4 j}+N_{2 j} N_{4 i}\right)-P(k, 2)\left(N_{2 i} N_{3 j}+N_{2 j} N_{3 i}\right)\right] .
\end{aligned}
$$

Note the $C$ and $G$ couplings here are similar to those given for the reverse decays of Higgs bosons to neutralinos earlier, with $i, j, k$ permuted accordingly. 


\section{Appendix I.5. Decays into Neutralinos}

For the first two generations of quarks and squarks:

$$
\Gamma\left(\tilde{q}_{L / R} \rightarrow q \tilde{Z}_{i}\right)=\frac{g^{2}}{16 \pi m_{\tilde{q}_{L / R}}} B_{\tilde{q}_{L / R}}^{2} \tilde{\lambda}^{\frac{1}{2}}\left(m_{\tilde{q}_{L / R}}, m_{\tilde{Z}_{i}}, m_{q}\right)\left[m_{\tilde{q}_{L / R}}^{2}-m_{\tilde{Z}_{i}}^{2}-m_{q}^{2}\right]
$$

where:

$$
B_{\tilde{q}_{L / R}}=\left\{\begin{array}{l}
-\sqrt{2}\left[\frac{2}{3} c(1) \sin \theta_{W}+\left(\frac{1}{2}-\frac{2}{3} \sin ^{2} \theta_{W}\right) \frac{c(2)}{\cos \theta_{W}}\right], \text { for } \tilde{u}_{L} \text { type } \\
-\sqrt{2} \frac{2}{3} \sin \theta_{W}\left[c(2) \tan \theta_{W}-c(1)\right], \text { for } \tilde{u}_{R} \text { type, } \\
\sqrt{2}\left[c(1) \frac{1}{3} \sin \theta_{W}+\left(\frac{1}{2}-\frac{1}{3} \sin ^{2} \theta_{W}\right) \frac{c(2)}{\cos \theta_{W}}\right], \text { for } \tilde{d}_{L} \text { type } \\
\sqrt{2} \frac{1}{3} \sin \theta_{W}\left[c(2) \tan \theta_{W}-c(1)\right], \text { for } \tilde{d}_{R} \text { type. }
\end{array}\right.
$$

The decay formulae for decays of the first two generations of sleptons to leptons and neutralinos are the same as for the squarks here but with the coupling change $B_{\tilde{q}_{L / R}} \rightarrow B_{\tilde{l}_{L / R}}$ and squark masses exchanged for slepton masses and quark masses for lepton masses:

$$
B_{\tilde{l}_{L / R}}=\left\{\begin{array}{l}
-\frac{c(2)}{\sqrt{2} \cos \theta_{W}}, \text { for } \tilde{v}_{L} \text { type } \\
0, \text { for } \tilde{v}_{R} \text { type (as no RH sneutrinos) } \\
\sqrt{2}\left[c(1) \sin \theta_{W}+\left(\frac{1}{2}-\sin ^{2} \theta_{W}\right) \frac{c(2)}{\cos \theta_{W}}\right], \text { for } \tilde{l}_{L} \text { type } \\
\sqrt{2} \sin \theta_{W}\left[c(2) \tan \theta_{W}-c(1)\right], \text { for } \tilde{l}_{R} \text { type. }
\end{array}\right.
$$

For the third generation:

$$
\Gamma\left(\tilde{t}_{1 / 2} \rightarrow t \tilde{Z}_{i}\right)=\frac{g^{2}}{16 \pi m_{\tilde{t}_{1 / 2}}}\left[\left(d_{1}^{2}+d_{2}^{2}\right)\left(m_{\tilde{t}_{1 / 2}}^{2}-m_{t}^{2}-m_{\tilde{Z}_{i}}^{2}\right)-4 d_{1} d_{2} m_{t} m_{\tilde{Z}_{i}}\right] \tilde{\lambda}^{\frac{1}{2}}\left(m_{\tilde{t}_{1 / 2}}, m_{\tilde{Z}_{i}}, m_{t}\right),
$$

where

$$
\begin{aligned}
& d_{1}=\left\{\begin{array}{l}
\cos \theta_{t} \sqrt{2}\left[-\frac{2}{3} c(1) \sin \theta_{W}+\left(-\frac{1}{2}+\frac{2}{3} \sin ^{2} \theta_{W}\right) \frac{c(2)}{\cos \theta_{W}}\right]-\sin \theta_{t} \frac{f_{t}}{g} N_{4 i}, \text { for } \tilde{t}_{1}, \\
-\sin \theta_{t} \sqrt{2}\left[-\frac{2}{3} c(1) \sin \theta_{W}+\left(-\frac{1}{2}+\frac{2}{3} \sin ^{2} \theta_{W}\right) \frac{c(2)}{\cos \theta_{W}}\right]-\cos \theta_{t} \frac{f_{t}}{g} N_{4 i}, \text { for } \tilde{t}_{2},
\end{array}\right. \\
& d_{2}=\left\{\begin{array}{l}
-\frac{2}{3} \sin \theta_{t} \sqrt{2} \sin \theta_{W}\left[c(2) \tan \theta_{W}-c(1)\right]-\cos \theta_{t} \frac{f_{t}}{g} N_{4 i}, \text { for } \tilde{t}_{1}, \\
-\frac{2}{3} \cos \theta_{t} \sqrt{2} \sin \theta_{W}\left[c(2) \tan \theta_{W}-c(1)\right]+\sin \theta_{t} \frac{f_{t}}{g} N_{4 i}, \text { for } \tilde{t}_{2} .
\end{array}\right. \\
& \Gamma\left(\tilde{b}_{1 / 2} \rightarrow b \tilde{Z}_{i}\right)=\frac{g^{2}}{16 \pi m_{\tilde{b}_{1 / 2}}}\left[\left(f_{1}^{2}+f_{2}^{2}\right)\left(m_{\tilde{b}_{1 / 2}}^{2}-m_{b}^{2}-m_{\tilde{Z}_{i}}^{2}\right)-4 f_{1} f_{2} m_{b} m_{\tilde{Z}_{i}}\right] \tilde{\lambda}^{\frac{1}{2}}\left(m_{\tilde{b}_{1 / 2}}, m_{\tilde{Z}_{i}}, m_{b}\right),
\end{aligned}
$$

where

$$
\begin{aligned}
& f_{1}=\left\{\begin{array}{l}
\cos \theta_{b} \sqrt{2}\left[\frac{1}{3} c(1) \sin \theta_{W}+\left(\frac{1}{2}-\frac{1}{3} \sin ^{2} \theta_{W}\right) \frac{c(2)}{\cos \theta_{W}}\right]-\sin \theta_{b} \frac{f_{b}}{g} N_{3 i}, \text { for } \tilde{b}_{1}, \\
-\sin \theta_{b} \sqrt{2}\left[\frac{1}{3} c(1) \sin \theta_{W}+\left(\frac{1}{2}-\frac{1}{3} \sin ^{2} \theta_{W}\right) \frac{c(2)}{\cos \theta_{W}}\right]-\cos \theta_{b} \frac{f_{b}}{g} N_{3 i}, \text { for } \tilde{b}_{2},
\end{array}\right. \\
& f_{2}=\left\{\begin{array}{l}
\frac{1}{3} \sin \theta_{b} \sqrt{2} \sin \theta_{W}\left[c(2) \tan \theta_{W}-c(1)\right]-\cos \theta_{b} \frac{f_{b}}{g} N_{3 i}, \text { for } \tilde{b}_{1}, \\
\frac{1}{3} \cos \theta_{b} \sqrt{2} \sin \theta_{W}\left[c(2) \tan \theta_{W}-c(1)\right]+\sin \theta_{b} \frac{f_{b}}{g} N_{3 i}, \text { for } \tilde{b}_{2} .
\end{array}\right. \\
& \Gamma\left(\tilde{\tau}_{1 / 2} \rightarrow \tau \tilde{Z}_{i}\right)=\frac{g^{2}}{16 \pi m_{\tilde{\tau}_{1 / 2}}}\left[\left(g_{1}^{2}+g_{2}^{2}\right)\left(m_{\tilde{\tau}_{1 / 2}}^{2}-m_{\tau}^{2}-m_{\tilde{Z}_{i}}^{2}\right)-4 g_{1} g_{2} m_{\tau} m_{\tilde{Z}_{i}}\right] \tilde{\lambda}^{\frac{1}{2}}\left(m_{\tilde{\tau}_{1 / 2}}, m_{\tilde{Z}_{i}}, m_{\tau}\right),
\end{aligned}
$$

where

$$
\begin{aligned}
& g_{1}=\left\{\begin{array}{l}
\cos \theta_{\tau} \sqrt{2}\left[c(1) \sin \theta_{W}+\left(\frac{1}{2}-\sin ^{2} \theta_{W}\right) \frac{c(2)}{\cos \theta_{W}}\right]-\sin \theta_{\tau} \frac{f_{\tau}}{g} N_{3 i}, \text { for } \tilde{\tau}_{1}, \\
-\sin \theta_{\tau} \sqrt{2}\left[c(1) \sin \theta_{W}+\left(\frac{1}{2}-\sin ^{2} \theta_{W}\right) \frac{c(2)}{\cos \theta_{W}}\right]-\cos \theta_{\tau} \frac{f_{\tau}}{g} N_{3 i}, \text { for } \tilde{\tau}_{2},
\end{array}\right. \\
& g_{2}=\left\{\begin{array}{l}
\sin \theta_{\tau} \sqrt{2} \sin \theta_{W}\left[c(2) \tan \theta_{W}-c(1)\right]-\cos \theta_{\tau} \frac{f_{\tau}}{g} N_{3 i}, \text { for } \tilde{\tau}_{1}, \\
\cos \theta_{\tau} \sqrt{2} \sin \theta_{W}\left[c(2) \tan \theta_{W}-c(1)\right]+\sin \theta_{\tau} \frac{f_{\tau}}{g} N_{3 i}, \text { for } \tilde{\tau}_{2} .
\end{array}\right.
\end{aligned}
$$


Remember the $c(1)$ and $c(2)$ were given previously in (I.154).

Sneutrino decays into neutralinos are given by:

$$
\Gamma\left(\tilde{v}_{\tau_{1 / 2}} \rightarrow v_{\tau} \tilde{Z}_{i}\right)=\frac{g^{2}}{16 \pi m_{v_{\tau_{1 / 2}}}} \tilde{\lambda}^{\frac{1}{2}}\left(m_{v_{\tau_{1 / 2}}}, 0, m_{\tilde{Z}_{i}}\right)\left(m_{v_{\tau_{1 / 2}}}^{2}-m_{\tilde{Z}_{i}}^{2}\right)\left(\frac{c(2)}{\sqrt{2} \cos \theta_{W}}\right)^{2} .
$$

For chargino decays into neutralinos and charged Higgs bosons the partial width is given by:

$$
\begin{aligned}
\Gamma\left(\tilde{W}_{1} \rightarrow H^{ \pm} \tilde{Z}_{j}\right)= & \frac{g^{2}}{32 \pi\left|m_{\tilde{W}_{1}}\right|} \tilde{\lambda}^{\frac{1}{2}}\left(m_{\tilde{W}_{1}}, m_{\tilde{Z}_{j}}, m_{H^{ \pm}}\right)\left[\left(c 1_{\tilde{W}_{1} H^{ \pm} \tilde{Z}_{j}}^{2}+c 2_{\tilde{W}_{1} H^{ \pm} \tilde{Z}_{j}}^{2}\right)\left(m_{\tilde{Z}_{j}}^{2}+m_{\tilde{W}_{1}}^{2}-m_{H^{ \pm}}^{2}\right)\right. \\
& +4 c 1_{\tilde{W}_{1} H^{ \pm}}^{2} \tilde{Z}_{j} \\
c & \left.2_{\tilde{W}_{1} H^{ \pm} \tilde{Z}_{j}}^{2} m_{\tilde{Z}_{j}} m_{\tilde{W}_{1}}\right],
\end{aligned}
$$

where:

$$
\begin{aligned}
& c 1_{\tilde{W}_{1} H^{ \pm} \tilde{Z}_{j}}=\frac{1}{g}\left[\lambda \sin \beta N_{5 j} \cos \theta_{R}+\frac{\cos \beta}{\sqrt{2}}\left(g^{\prime} N_{1 j}+g N_{2 j}\right) \cos \theta_{R}+g \cos \beta N_{4 j} \sin \theta_{R}\right], \\
& c 2_{\tilde{W}_{1} H^{ \pm} \tilde{Z}_{j}}=\frac{1}{g}\left[\lambda \cos \beta N_{5 j} \cos \theta_{L}-\frac{\sin \beta}{\sqrt{2}}\left(g^{\prime} N_{1 j}+g N_{2 j}\right) \cos \theta_{L}+g \sin \beta N_{3 j} \sin \theta_{L}\right] .
\end{aligned}
$$

For $\tilde{W}_{2}$ the formulae are the same, just make the replacements $m_{\tilde{W}_{1}} \rightarrow m_{\tilde{W}_{2}}, \cos \theta_{L / R} \rightarrow \sin \theta_{L / R}$ and $\sin \theta_{L / R} \rightarrow$ $-\cos \theta_{L / R}$.

$$
\begin{aligned}
\Gamma\left(\tilde{W}_{1} \rightarrow W \tilde{Z}_{j}\right)=\frac{g^{2}}{32 \pi\left|m_{\tilde{W}_{1}}\right|} \tilde{\lambda}^{\frac{1}{2}}\left(m_{\tilde{W}_{1}}, m_{\tilde{Z}_{j}}, m_{W}\right)[ & -12 m_{\tilde{W}_{1}} m_{\tilde{Z}_{j}} c L_{\tilde{W}_{1} W \tilde{Z}_{j}} c R_{\tilde{W}_{1} W \tilde{Z}_{j}} \\
& +\left(c L_{\tilde{W}_{1} W \tilde{Z}_{j}}^{2}+c R_{\tilde{W}_{1} W \tilde{Z}_{j}}^{2}\right)\left\{\left(m_{\tilde{W}_{1}}^{2}+m_{\tilde{Z}_{j}}^{2}-m_{W}^{2}\right)\right. \\
& \left.\left.+\left(m_{\tilde{W}_{1}}^{2}+m_{W}^{2}-m_{\tilde{Z}_{j}}^{2}\right)\left(m_{\tilde{W}_{1}}^{2}-m_{\tilde{Z}_{j}}^{2}-m_{W}^{2}\right) \frac{1}{m_{W}^{2}}\right\}\right],
\end{aligned}
$$

where:

$$
\begin{aligned}
& c L_{\tilde{W}_{1} W \tilde{Z}_{j}}=-\frac{1}{\sqrt{2}} N_{4 j} \cos \theta_{R}+N_{2 j} \sin \theta_{R}, \\
& c R_{\tilde{W}_{1} W \tilde{Z}_{j}}=\frac{1}{\sqrt{2}} N_{3 j} \cos \theta_{L}+N_{2 j} \sin \theta_{L} .
\end{aligned}
$$

Again for $\tilde{W}_{2}$ the formulae are the same, just make the replacements $m_{\tilde{W}_{1}} \rightarrow m_{\tilde{W}_{2}}, \cos \theta_{L / R} \rightarrow \sin \theta_{L / R}$ and $\sin \theta_{L / R} \rightarrow$ $-\cos \theta_{L / R}$.

\section{Appendix J. QCD Corrections to Decays}

Note, for the decays of neutral Higgs bosons to quarks or gluons, the possibility of including QCD corrections is included in the program, by default the QCD corrections are on. The formulae are those provided in HDECAY-3.4 in SUSYHIT [23, 24] and NMSSMTools -4.2.1 in NMHDECAY [13, 28]. With QCD corrections incorporated our formulae become as follows:

$$
\Gamma(h \rightarrow q q)_{Q C D \text { corr }}=\Gamma(h->q q)_{\text {tree }}\left(1+\frac{4 \alpha_{s}\left(m_{h}\right)}{3 \pi}\left[\frac{A(\tilde{\beta})}{\tilde{\beta}}+\frac{3+34 \tilde{\beta}^{2}-13 \tilde{\beta}^{4}}{16 \tilde{\beta}^{3}} \log \frac{1+\tilde{\beta}}{1-\tilde{\beta}}+\frac{3}{8 \tilde{\beta}^{2}}\left(7 \tilde{\beta}^{2}-1\right)\right]\right) .
$$

This formula applies for all the CP even neutral Higgs bosons, whether in the MSSM or NMSSM, the difference between the MSSM and NMSSM comes in the tree-level formula. Note $\alpha_{s}$ is evaluated at the mass of the decaying Higgs boson. Also note that $\tilde{\beta}$ and $A(\tilde{\beta})$ are given by:

$$
\tilde{\beta}=\sqrt{1-4 \frac{m_{q}^{2}}{m_{h}^{2}}}
$$




$$
\begin{aligned}
A(\tilde{\beta})= & \left(1+\tilde{\beta}^{2}\right)\left[4 L i_{2}\left(\frac{1-\tilde{\beta}}{1+\tilde{\beta}}\right)+2 L i_{2}\left(\frac{\tilde{\beta}-1}{\tilde{\beta}+1}\right)-3 \log \left(\frac{1+\tilde{\beta}}{1-\tilde{\beta}}\right) \log \frac{2}{1+\tilde{\beta}}-2 \log \left(\frac{1+\tilde{\beta}}{1-\tilde{\beta}}\right) \log \tilde{\beta}\right] \\
& -3 \tilde{\beta} \log \frac{4}{1-\tilde{\beta}^{2}}-4 \tilde{\beta} \log \tilde{\beta} .
\end{aligned}
$$

This is exactly as given in Eqs. (16) and (25) of [39]. $L i_{2}$ is the di-logarithm function (Spence's function).

For the CP odd Higgs bosons we have:

$$
\Gamma(A \rightarrow q q)_{Q C D \text { corr }}=\Gamma(A->q q)_{\text {tree }}\left(1+\frac{4 \alpha_{s}\left(m_{A}\right)}{3 \pi}\left[\frac{A(\tilde{\beta})}{\tilde{\beta}}+\frac{19+2 \tilde{\beta}^{2}+3 \tilde{\beta}^{4}}{16 \tilde{\beta}} \log \frac{1+\tilde{\beta}}{1-\tilde{\beta}}+\frac{3}{8}\left(7-\tilde{\beta}^{2}\right)\right]\right) .
$$

$\tilde{\beta}$ and $A(\tilde{\beta})$ are as given above but with the change $m_{h} \rightarrow m_{A}$ as appropriate. This formula is as given in Eqs. (25) and (26) of [39]. It should be noted that when QCD corrections are applied one should use the pole quark masses (as we do within SOFTSUSY here), rather than the running masses, as otherwise the formulae double count $O\left(\alpha_{s}\right)$ effects [49].

The QCD corrections for $h \rightarrow g g$ are more complicated as they involve both standard QCD corrections due to gluons being radiated, gluons in the loop, tops, bottoms and other quarks, and additional SUSY-QCD corrections due to gluinos, stops, sbottoms and other squarks. This complicates matters as whilst the usual "fermionic" QCD (FQCD) corrections apply to all particles in the loop, the SUSY-QCD (SQCD) corrections only apply to the scalar squark contributions, therefore rather than multiply the whole width by a correction factor (as was the case for $h \rightarrow q q$ ) we must now correct the SM and SUSY loop contributions separately. The usual MSSM equation for $h \rightarrow g g$ with no corrections is:

$$
\Gamma(\phi \rightarrow g g)_{1-l o o p}=\frac{\alpha_{s}^{2}\left(m_{\phi}\right)}{128 \pi^{3}} \frac{G_{F}}{\sqrt{2}} m_{\phi}^{3} \frac{9}{8}\left|\Sigma I_{\text {loop }}^{\phi}\right|^{2} .
$$

Here the $\alpha_{s}$ is run to the mass of the decaying Higgs boson. The $I_{\text {loop }}$ can be split into $I_{\text {quark }}$ and $I_{\text {squark }}$ loop contributions. So $I_{\text {looptot }}^{\phi}=I_{\text {qtot }}^{\phi}+I_{\text {sqtot }}^{\phi}$, where $I_{\text {qtot }}^{\phi}=I_{t}^{\phi}+I_{b}^{\phi}+I_{c}^{\phi}$ and $I_{\text {sqtot }}^{\phi}=I_{\tilde{t}_{1}}^{\phi}+I_{\tilde{t}_{2}}^{\phi}+I_{\tilde{b}_{1}}^{\phi}+I_{\tilde{b}_{2}}^{\phi}+I_{\tilde{c}_{L}}^{\phi}+I_{\tilde{c}_{R}}^{\phi}+I_{\tilde{s}_{L}}^{\phi}+I_{\tilde{s}_{R}}^{\phi}+I_{\tilde{u}_{L}}^{\phi}+I_{\tilde{u}_{R}}^{\phi}+I_{\tilde{d}_{L}}^{\phi}+I_{\tilde{d}_{R}}^{\phi}$.

To account for the usual QCD corrections, i.e. "FQCD" corrections, as these affect all the loop contributions, the whole partial width is multiplied by $\delta_{F Q C D}$ :

$$
\delta_{F Q C D}^{C P \text { evenHiggs }}=1+\frac{\alpha_{s}\left(m_{\phi}\right)}{\pi}\left(\frac{95}{4}-\frac{7}{6} N_{f}\right)
$$

or

$$
\delta_{F Q C D}^{\text {CPoddHiggs }}=1+\frac{\alpha_{s}\left(m_{\phi}\right)}{\pi}\left(\frac{97}{4}-\frac{7}{6} N_{f}\right) .
$$

$N_{f}$ is the number of active fermion flavours. The SUSY QCD corrections, i.e. "SQCD" corrections, apply only to the squark loop contributions. Therefore to incorporate these in the final partial width you must multiply both the squark loop squared contributions and the interference terms of the squark loops with the quark loops by the correction factor. Therefore the $\left|I_{\text {loop }}\right|^{2}$ (which comes from the matrix element squared) above with both FQCD and SQCD corrections included becomes:

$$
\left|I_{\text {looptot }}^{\phi}\right|^{2}=\delta_{F Q C D}^{\phi}\left|I_{\text {looptot }}^{\phi}\right|^{2}+\operatorname{Re}\left[\left(I_{\text {looptot }}^{\phi}\right)^{*} I_{\text {sqtot }}^{\phi}\right] \delta_{S Q C D}
$$

To be clear the $\left(I_{\text {looptot }}^{\phi}\right)^{*}$ here means the complex conjugate of $I_{\text {looptot }}^{\phi}$, given this is an interference term. The $\delta_{S Q C D}$ correction factor is the same for CP even and CP odd neutral Higgs bosons and is given by (J.9) below, note that $\alpha_{s}$ is run to the mass of the decaying Higgs boson:

$$
\delta_{S Q C D}=\frac{17 \alpha_{s}\left(m_{\phi}\right)}{6 \pi} .
$$

Consequently in the MSSM the overall formula for the QCD and SUSY-QCD corrected $h \rightarrow g g$ decay (at 2-loop) is:

$$
\Gamma(\phi \rightarrow g g)_{1-\text { loop }+Q C D c o r r}=\frac{\alpha_{s}^{2}\left(m_{\phi}\right)}{128 \pi^{3}} \frac{G_{F}}{\sqrt{2}} m_{\phi}^{3} \frac{9}{8}\left[\delta_{F Q C D}^{\phi}\left|I_{\text {looptot }}^{\phi}\right|^{2}+\operatorname{Re}\left[\left(I_{\text {looptot }}^{\phi}\right)^{*} I_{\text {sqtot }}^{\phi}\right] \delta_{S Q C D}\right] .
$$


$\phi$ is a CP even neutral Higgs here as CP odd Higgs bosons do not have squark loop contributions because of CP invariance of the decays. For the CP odd Higgs $A$ in the MSSM we therefore only have the quark loops and FQCD corrections:

$$
\Gamma(A \rightarrow g g)_{1-\text { loop }+Q C D c o r r}=\frac{\alpha_{s}^{2}\left(m_{A}\right)}{128 \pi^{3}} \frac{G_{F}}{\sqrt{2}} m_{A}^{3} \frac{9}{8}\left[\delta_{F Q C D}^{A}\left|I_{q t o t}^{A}\right|^{2}\right] .
$$

The FQCD corrections for CP even and CP odd neutral Higgs bosons in the MSSM are as given in Ref. [40] in Eqs. (7) and (15) but with the log terms dropped as the scale of alphas is run to the masses of the decaying Higgs. As the corrections are purely coloured and the NMSSM only alters the Higgs and neutralino sectors, the form of the QCD corrections is exactly the same in the NMSSM. The alterations to the Higgs sector in the NMSSM however result in the couplings of the neutral Higgs bosons to other particles, and therefore the leading order (i.e. 1-loop) formula for the loop contributions to $h \rightarrow g g$, being altered. The formula in the uncorrected NMSSM (i.e. at 1-loop) is (as detailed previously) as follows, with the $\alpha_{s}$ evaluated at the scale of the decaying Higgs boson:

$$
\Gamma\left(h_{i} \rightarrow g g\right)_{1-\text { loop }}=\frac{G_{F} m_{h_{i}}^{3} \alpha_{s}^{2}\left(m_{h_{i}}\right)}{64 \sqrt{2} \pi^{3}}\left|M_{g g}\right|^{2}
$$

where

$$
\begin{aligned}
\left|M_{g g}^{\phi}\right|^{2}= & {\left[J_{t}^{r}+J_{b}^{r}+J_{c}^{r}+J_{\tilde{u}_{L}}^{r}+J_{\tilde{u}_{R}}^{r}+J_{\tilde{d}_{L}}^{r}+J_{\tilde{d}_{R}}^{r}+J_{\tilde{c}_{L}}^{r}+J_{\tilde{c}_{R}}^{r}+J_{\tilde{s}_{L}}^{r}+J_{\tilde{s}_{R}}^{r}+J_{\tilde{t}_{1}}^{r}+J_{\tilde{t}_{2}}^{r}+J_{\tilde{b}_{1}}^{r}+J_{\tilde{b}_{2}}^{r}\right]^{2} } \\
& +\left[J_{t}^{i}+J_{b}^{i}+J_{c}^{i}+J_{\tilde{u}_{L}}^{i}+J_{\tilde{u}_{R}}^{i}+J_{\tilde{d}_{L}}^{i}+J_{\tilde{d}_{R}}^{i}+J_{\tilde{c}_{L}}^{i}+J_{\tilde{c}_{R}}^{i}+J_{\tilde{s}_{L}}^{i}+J_{\tilde{s}_{R}}^{i}+J_{\tilde{t}_{1}}^{i}+J_{\tilde{t}_{2}}^{i}+J_{\tilde{b}_{1}}^{i}+J_{\tilde{b}_{2}}^{i}\right]^{2},
\end{aligned}
$$

where the $J_{X}$ contributions are different to those in the MSSM as the couplings are different. Here the ${ }^{r}$ and ${ }^{i}$ were used as shorthand for real and imaginary parts. The $\left|M_{g g}\right|^{2}$ is therefore just the mod square of the sum of the complex loop contributions.

In order to incorporate the FQCD and SQCD corrections we again group the loop contributions into quark and squark so that $J_{q t o t}^{\phi}=J_{t}^{\phi}+J_{b}^{\phi}+J_{c}^{\phi}, J_{s q t o t}^{\phi}=J_{\tilde{u}_{L}}^{\phi}+J_{\tilde{u}_{R}}^{\phi}+J_{\tilde{d}_{L}}^{\phi}+J_{\tilde{d}_{R}}^{\phi}+J_{\tilde{c}_{L}}^{\phi}+J_{\tilde{c}_{R}}^{\phi}+J_{\tilde{s}_{L}}^{\phi}+J_{\tilde{s}_{R}}^{\phi}+J_{\tilde{t}_{1}}^{\phi}+J_{\tilde{t}_{2}}^{\phi}+J_{\tilde{b}_{1}}^{\phi}+J_{\tilde{b}_{2}}^{\phi}$ and $J_{\text {looptot }}^{\phi}=J_{\text {qtot }}^{\phi}+J_{\text {sqtot }}^{\phi}$. Then $\left|M_{\text {gg }}\right|^{2}$ becomes:

$$
\left|M_{g g}^{\phi}\right|^{2}=\left[\delta_{F Q C D}^{\phi}\left|J_{\text {looptot }}^{\phi}\right|^{2}+\operatorname{Re}\left[\left(J_{\text {looptot }}^{\phi}\right)^{*} J_{\text {sqtot }}^{\phi}\right] \delta_{S Q C D}\right]
$$

because the FQCD and SQCD corrections apply to the loop contributions exactly as in the MSSM, however the loop contributions themselves have changed between the MSSM and NMSSM. So overall in the NMSSM, the QCD corrected partial width for neutral Higgs decays to gluons is as follows, again note the $\alpha_{s}$ is evaluated at the scale of the mass of the decaying Higgs boson:

$$
\Gamma\left(h_{i} \rightarrow g g\right)_{1-\text { loop }+Q C D \text { corr }}=\frac{G_{F} m_{h_{i}}^{3} \alpha_{s}^{2}\left(m_{h_{i}}\right)}{64 \sqrt{2} \pi^{3}}\left[\delta_{F Q C D}^{\phi}\left|J_{\text {looptot }}^{\phi}\right|^{2}+\operatorname{Re}\left[\left(J_{\text {looptot }}^{\phi}\right)^{*} J_{\text {sqtot }}^{\phi}\right] \delta_{S Q C D}\right] .
$$

Again $\phi$ here is a CP even neutral Higgs boson as CP odd Higgs bosons do not have squark loop contributions because of CP invariance of the decays, as in the MSSM. Therefore CP odd Higgs bosons have only quark loop contributions and so only receive FQCD corrections, without corrections the formula was:

$$
\Gamma\left(A_{i} \rightarrow g g\right)_{1-\text { loop }}=\frac{g^{2} \alpha_{s}^{2}\left(m_{A_{i}}\right) m_{A_{i}}^{3}}{128 \pi^{3} m_{W}^{2}}\left|M_{A_{i} g g}\right|^{2},
$$

remembering that the $\alpha_{s}\left(m_{A_{i}}\right)$ means $\alpha_{s}$ evaluated at the mass of the decaying CP odd Higgs boson $A_{i}$. Here $\left|M_{A_{i} g g}\right|^{2}$ is:

$$
\left|M_{A_{i} g g}\right|^{2}=\left(\mathcal{R}_{t}^{r}+\mathcal{R}_{b}^{r}+\mathcal{R}_{c}^{r}\right)^{2}+\left(\mathcal{R}_{t}^{i}+\mathcal{R}_{b}^{i}+\mathcal{R}_{c}^{i}\right)^{2} .
$$

The corrections are incorporated by multiplying by $\delta_{F Q C D}^{A}$, so with the QCD corrections the CP odd Higgs decays in the NMSSM are given by:

$$
\Gamma\left(A_{i} \rightarrow g g\right)_{1-\text { loop }+Q C D \text { corr }}=\frac{g^{2} \alpha_{s}^{2}\left(m_{A_{i}}\right) m_{A_{i}}^{3}}{128 \pi^{3} m_{W}^{2}}\left|M_{A_{i} g g}\right|^{2} \delta_{F Q C D}^{A} .
$$

Throughout, the formulae used are those given in HDECAY-3.4 [24] (and hence SUSYHIT [23]) and NMSSMTools -4.2.1 [2, 27. 28]. 


\section{Bibliography}

[1] B. C. Allanach, SUSY Predictions and SUSY Tools at the LHC, Eur. Phys. J. C59 (2009) 427-443. arXiv:0805.2088 doi:10.1140/ epjc/s10052-008-0695-2

[2] B. C. Allanach, SOFTSUSY: a program for calculating supersymmetric spectra, Comput. Phys. Commun. 143 (2002) 305-331. arXiv: hep-ph/0104145 doi:10.1016/S0010-4655(01)00460-X

[3] B. C. Allanach, P. Athron, L. C. Tunstall, A. Voigt, A. G. Williams, Next-to-Minimal SOFTSUSY, Comput. Phys. Commun. 185 (2014) 2322-2339. arXiv: 1311.7659 doi:10.1016/j.cpc.2014.04.015

[4] B. C. Allanach, M. A. Bernhardt, Including R-parity violation in the numerical computation of the spectrum of the minimal supersymmetric standard model: SOFTSUSY, Comput. Phys. Commun. 181 (2010) 232-245. arXiv:0903.1805 doi:10.1016/j.cpc. 2009.09.015

[5] B. C. Allanach, C. H. Kom, M. Hanussek, Computation of Neutrino Masses in R-parity Violating Supersymmetry: SOFTSUSY3.2, Comput. Phys. Commun. 183 (2012) 785-793. arXiv:1109.3735 doi:10.1016/j.cpc.2011.11.024

[6] B. C. Allanach, A. Bednyakov, R. Ruiz de Austri, Higher order corrections and unification in the minimal supersymmetric standard model: SOFTSUSY3.5, Comput. Phys. Commun. 189 (2015) 192-206. arXiv:1407.6130 doi:10.1016/j.cpc.2014.12.006

[7] B. C. Allanach, S. P. Martin, D. G. Robertson, R. R. de Austri, The Inclusion of Two-Loop SUSYQCD Corrections to Gluino and Squark Pole Masses in the Minimal and Next-to-Minimal Supersymmetric Standard Model: SOFTSUSY3.7arXiv:1601.06657

[8] P. Athron, J.-h. Park, D. Stckinger, A. Voigt, FlexibleSUSYA spectrum generator generator for supersymmetric models, Comput. Phys. Commun. 190 (2015) 139-172. arXiv:1406.2319 doi:10.1016/j.cpc.2014.12.020

[9] F. E. Paige, S. D. Protopopescu, H. Baer, X. Tata, ISAJET 7.69: A Monte Carlo event generator for pp, anti-p p, and e+e- reactionsarXiv: hep-ph/0312045

[10] A. Djouadi, J.-L. Kneur, G. Moultaka, SuSpect: A Fortran code for the supersymmetric and Higgs particle spectrum in the MSSM, Comput. Phys. Commun. 176 (2007) 426-455. arXiv: hep-ph/0211331 doi:10.1016/j.cpc.2006.11.009

[11] W. Porod, SPheno, a program for calculating supersymmetric spectra, SUSY particle decays and SUSY particle production at e+ e- colliders, Comput. Phys. Commun. 153 (2003) 275-315. arXiv: hep-ph/0301101 doi:10.1016/S0010-4655(03) 00222-4

[12] S. Heinemeyer, W. Hollik, G. Weiglein, FeynHiggs: A Program for the calculation of the masses of the neutral CP even Higgs bosons in the MSSM, Comput. Phys. Commun. 124 (2000) 76-89. arXiv: hep-ph/9812320 doi:10.1016/S0010-4655(99) 00364-1

[13] U. Ellwanger, J. F. Gunion, C. Hugonie, NMHDECAY: A Fortran code for the Higgs masses, couplings and decay widths in the NMSSM, JHEP 02 (2005) 066. arXiv : hep-ph/0406215 |doi:10.1088/1126-6708/2005/02/066

[14] U. Ellwanger, C. Hugonie, NMSPEC: A Fortran code for the sparticle and Higgs masses in the NMSSM with GUT scale boundary conditions, Comput. Phys. Commun. 177 (2007) 399-407. arXiv: hep-ph/0612134 doi:10.1016/j.cpc.2007.05.001

[15] F. Staub, SARAH 4 : A tool for (not only SUSY) model builders, Comput. Phys. Commun. 185 (2014) 1773-1790. arXiv:1309.7223. doi: 10.1016/j.cpc.2014.02.018

[16] J. Baglio, R. Grber, M. Mhlleitner, D. T. Nhung, H. Rzehak, M. Spira, J. Streicher, K. Walz, NMSSMCALC: A Program Package for the Calculation of Loop-Corrected Higgs Boson Masses and Decay Widths in the (Complex) NMSSM, Comput. Phys. Commun. 185 (12) (2014) 3372-3391. arXiv:1312.4788 doi:10.1016/j.cpc.2014.08.005

[17] B. C. Allanach, S. Kraml, W. Porod, Theoretical uncertainties in sparticle mass predictions from computational tools, JHEP 03 (2003) 016. arXiv: hep-ph/0302102 doi:10.1088/1126-6708/2003/03/016

[18] B. C. Allanach, A. Djouadi, J. L. Kneur, W. Porod, P. Slavich, Precise determination of the neutral Higgs boson masses in the MSSM, JHEP 09 (2004) 044. arXiv: hep-ph/0406166 doi:10.1088/1126-6708/2004/09/044

[19] F. Staub, P. Athron, U. Ellwanger, R. Grber, M. Mhlleitner, P. Slavich, A. Voigt, Higgs mass predictions of public NMSSM spectrum generators, Comput. Phys. Commun. 202 (2016) 113-130. arXiv: 1507.05093 doi:10.1016/j.cpc.2016.01.005

[20] D. de Florian, et al., Handbook of LHC Higgs Cross Sections: 4. Deciphering the Nature of the Higgs SectorarXiv: 1610.07922

[21] T. Sjstrand, S. Ask, J. R. Christiansen, R. Corke, N. Desai, P. Ilten, S. Mrenna, S. Prestel, C. O. Rasmussen, P. Z. Skands, An Introduction to PYTHIA 8.2, Comput. Phys. Commun. 191 (2015) 159-177. arXiv:1410.3012 doi:10.1016/j.cpc.2015.01.024

[22] M. Bahr, et al., Herwig++ Physics and Manual, Eur. Phys. J. C58 (2008) 639-707. $\operatorname{arXiv:0803.0883}$ doi:10.1140/epjc/ s10052-008-0798-9

[23] A. Djouadi, M. M. Muhlleitner, M. Spira, Decays of supersymmetric particles: The Program SUSY-HIT (SUspect-SdecaY-HdecayInTerface), Acta Phys. Polon. B38 (2007) 635-644. arXiv: hep-ph/0609292

[24] A. Djouadi, J. Kalinowski, M. Spira, HDECAY: A Program for Higgs boson decays in the standard model and its supersymmetric extension, Comput. Phys. Commun. 108 (1998) 56-74. arXiv: hep-ph/9704448 doi:10.1016/S0010-4655(97) 00123-9

[25] A. Djouadi, M. Muhlleitner, Y. Mambrini, SDECAY: a Fortran code for the decays of the supersymmetric particles in the MSSM, Comput. Phys. Commun. 168 (2005) 46-70. arXiv: hep-ph/0311167 doi:10.1016/j.cpc.2005.01.012

[26] M. D. Goodsell, S. Liebler, F. Staub, Generic calculation of two-body partial decay widths at the full one-loop leve arXiv:1703.09237

[27] D. Das, U. Ellwanger, A. Teixeira, NMSDECAY: A Fortran Code for Supersymmetric Particle Decays in the Next-to-Minimal Supersymmetric Standard Model, Comput. Phys. Commun. 183 (2012) 774-779. arXiv:hep-ph/1106.5633 doi:10.1016/j.cpc.2011.11.021

[28] U. Ellwanger, C. Hugonie, NMHDECAY 2.0: An updated program for Sparticle masses, Higgs masses, couplings and decay widths in the NMSSM, Comput. Phys. Commun. 175 (2006) 290-303. arXiv: hep-ph/0508022 doi:10.1016/j.cpc. 2006.04 .004

[29] I. Jack, D. R. Timothy Jones, S. P. Martin, M. T. Vaughn, Y. Yamada, Decoupling of the -scalar mass in softly broken supersymmetry, Phys. Rev. D. 50 (1994) 5481-5483. arXiv: hep-ph/9407291

[30] W. H. Press, S. A. Teukolsky, W. T. Vettering, B. P. Flannery, Numerical Recipes in Fortran 77: The Art of Scientific Computing, Vol. 1, Cambridge University Press, 1992.

[31] G. Passarino, M. Veltman, One-loop corrections for e+e annihilation into + in the Weinberg model, Nuc. Phys. B. 160 (1979) 151-207. doi:10.1016/0550-3213(79) 90234-7

[32] J. F. Gunion, H. E. Haber, G. Kane, S. Dawson, The Higgs Hunter's Guide, Vol. 1, Frontiers in Physics, 2000. 
[33] A. Djouadi, Y. Mambrini, M. Muhlleitner, Chargino and Neutralino Decays Revisited, Eur. Phys. J. C 20 (2001) 563-584. arXiv : hep-ph/ 0104115 doi:10.1007/s100520100679

[34] H. Baer, C. Chen, M. Drees, M. Paige, X. Tata, Supersymmetry Reach of Tevatron Upgrades: The Large tan $\beta$ Case, Phys. Rev. D 58. arXiv:hep-ph/9802441 doi:10.1103/PhysRevD.58.075008

[35] W. Porod, SPheno 3.1: extensions including flavour, CP-phases and models beyond the MSSM, Comput. Phys. Commun. 183 (2012) 24582469. arXiv:hep-ph/1104.1573 doi:10.1016/j.cpc.2012.05.021

[36] P. Z. Skands, et al., SUSY Les Houches accord: Interfacing SUSY spectrum calculators, decay packages, and event generators, JHEP 07 (2004) 036. arXiv:hep-ph/0311123 doi:10.1088/1126-6708/2004/07/036

[37] B. C. Allanach, et al., SUSY Les Houches Accord 2, Comput. Phys. Commun. 180 (2009) 8-25. arXiv:0801.0045 doi:10.1016/j . cpc. 2008.08.004

[38] J. Alwall, R. Frederix, S. Frixione, V. Hirschi, F. Maltoni, O. Mattelaer, H.-S. Shao, T. Stelzer, P. Torrielli, Z. M., The automated computation of tree-level and next-to-leading order differential cross sections, and their matching to parton shower simulations, JHEP 07 (2014) 079. arXiv:1405.0301 doi:10.1007/JHEP07(2014) 079

[39] M. Spira, Higgs Boson Production and Decay at Hadron CollidersarXiv: hep-ph/1612.07651

[40] A. Djouadi, M. Spira, P. Zerwas, QCD Corrections to Hadronic Higgs Decays, Z.Phys. C 70 (1996) 427-434. arXiv: hep-ph/9511344

[41] H. Baer, X. Tata, Weak Scale Supersymmetry: From Superfields to Scattering Events, Vol. 1, Cambridge University Press, 2006.

[42] A. Djouadi, P. Gambino, QCD corrections to Higgs boson self-energies and fermionic decay widths, Phys. Rev. D 51 (1995) $218-228$.

[43] M. Spira, Higgs Boson Production and Decay at the Tevatror arXiv: hep-ph/9810289

[44] A. Djouadi, The Anatomy of ElectroWeak Symmetry Breaking II: The Higgs bosons in the Minimal Supersymmetric Model, Phys.Rept. 459 (2008) 1-241. arXiv: hep-ph/0503173 doi:10.1016/j.physrep.2007.10.005

[45] A. Denner, S. Heinemeyer, I. Puljak, D. Rebuzzi, M. Spira, Standard Model Higgs-Boson Branching Ratios with Uncertainties, Eur. Phys. J. C 71 (2011) 1753. arXiv:hep-ph/1107.5909 doi:10.1140/epjc/s10052-011-1753-8

[46] A. Buckley, PySLHA: a Pythonic interface to SUSY Les Houches Accord datalarXiv: hep-ph/1305.4194

[47] C. Patrignani, et al., Review of Particle Physics, Chin. Phys. C40 (10) (2016) 100001. doi:10.1088/1674-1137/40/10/100001

[48] A. Djouadi, Y. Mambrini, M. Muhlleitner, Supersymmetry Reach of Tevatron Upgrades: The Large tan $\beta$ Case, Eur. Phys. J. C 20 (2001) 563-584. arXiv:hep-ph/0104115 doi:10.1007/s100520100679

[49] M. Drees, K. Hikasa, Note on QCD Corrections to Hadronic Higgs Decays, Phys. Lett. B 240 (1990) 455-464. doi:10.1016/ 0370-2693(90) 91130-4 